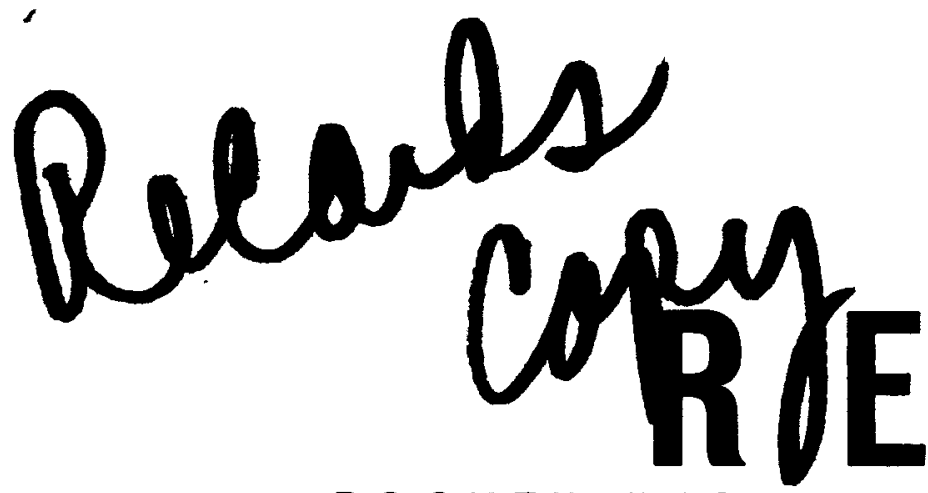

SNPO-C

Ricanda

ROCKET ENGINE OPERATIONS - NUCLEAR

RN-S-0146

A Report To

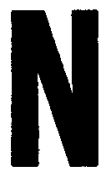

NUCLEAR

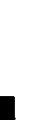




\section{DISCLAIMER}

This report was prepared as an account of work sponsored by an agency of the United States Government. Neither the United States Government nor any agency Thereof, nor any of their employees, makes any warranty, express or implied, or assumes any legal liability or responsibility for the accuracy, completeness, or usefulness of any information, apparatus, product, or process disclosed, or represents that its use would not infringe privately owned rights. Reference herein to any specific commercial product, process, or service by trade name, trademark, manufacturer, or otherwise does not necessarily constitute or imply its endorsement, recommendation, or favoring by the United States Government or any agency thereof. The views and opinions of authors expressed herein do not necessarily state or reflect those of the United States Government or any agency thereof. 


\section{DISCLAIMER}

Portions of this document may be illegible in electronic image products. Images are produced from the best available original document. 


\section{$\underline{\text { ABSTRACT }}$}

This report describes in detail the data analysis techniques developed during Contract Year 1964. An evaluation of these techniques was made by their application to the NRX-Al test data. These techniques proved useful for analyzing that data, although a need for improvements and continued development was indicated.

New data analysis techniques currently under development are also described. These techniques are primarily concerned with comparing model predictions to actual test data, and interpreting this comparison when both the data and the model are subject to error. Computer Job No. 364, which is described in a companion report, is the basis for this comparison. The purpose of the comparison is to utilize the results of actual tests to upgrade Aerojet's analytical representation of systems behavior.

Hardware useful for acquisition, processing, and analysis of engine test data is also discussed.

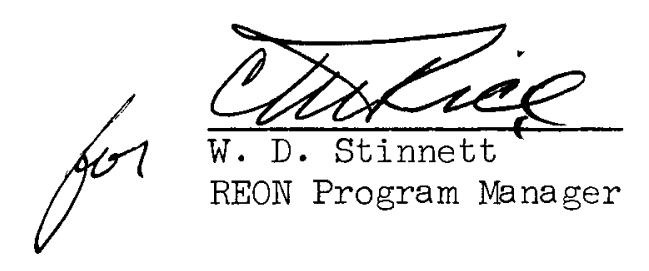




\section{PAGE BLANK}

\section{iii}


$\mathrm{RN}-\mathrm{S}-0146$

\section{FOREWORD}

This report is submitted in partial fulfillment of SNP-1 Contract Task Item 1.8, which states in part: "Complete and issue report on data analysis method for nuclear rocket engine test data" and which calls for the contractual milestone "Issue Report on XE Data Analysis Method." 
I. INTRODUCTION

A. Behavior Analyses of Systems

B. Validation and Improvement of Models

II. NATURE OF AVAILABLE TEST DA.A

A. NRX Data Flow

1. Processing of Narrow-Band (Digital) Data

2. Processing of Wide-Band (Analog) Data

B. CFDTS Data Flow

1. Digital Data System

2. On-line Recording for Display

3. Analog (FM) Data System

C. Engine Test Data Flow

III. ANALYSIS OF DIGITAL DATA

A. Description of Available Data

1. N2 Data Format (Raw Data)

16

2. N3 Data Format (Reduced Data)

3. Nature and Estimation of Experimental Errors

B. Quantitative Methods of Describing Information Content of Data

20

1. Statistical Examination of Raw Data (N2 Tapes)

2. Statistical Analysis and Processing of Reduced Data (N3 Tape)

3. Statistical Analysis of Asymmetry

C. Comparison of Test Results with Analytical Models

1. Data Analysis Using Computer Job No. 364 32

2. Statistical Tests of Validity of Model 
$\mathrm{RN}-\mathrm{S}-0146$

TABLE OF CONTENTS (cont.)

$\underline{\text { Page }}$

3. Effects of Input and Parameter Errors on

Systems Model Predictions

44

IV. ANALYSIS OF ANALOG DATA

47

A. Qualitative Analysis of Wide-Band Data

47

1. Transients

47

2. Oscillatory Modes

48

3. Cause-Effect Relations

48

4. Instrumentation Errors Unique with Wide-Band Data

48

B. Quantitative Methods of Describing Wide-Band Data Properties

1. Statistical Properties

49

2. Correlation Techniques

50

3. Power Spectral Density

51

4. Transfer Function Analysis

51

C. Comparison of Test with Computer Model

53

D. Possible Computer Methods of Obtaining Data Description

54

1. Digital Computer

54

2. Analog Computer

55

3. Hybrid Computer

E. Detail Description of Analog Computer Method of Analysis __ 56

1. Mathematical Relations to be Computed _ 56

2. Computer Mechanization _ـ 58

3. Investigation to Identify Problems and Limitations __ 64

V. CONCLUSIONS

64

A. Techniques for Digital Data

64

B. Techniques for Analog Data

66

References

68 
Table

EP-IV Liquid into Gas (1.25 Remp) Core Inlet Plenum Temperatures

EP-IV Liquid into Gas ( 1.25 Raup) Core Inlet Plenum Temperatures Adjusted for Effect of Time

Analysis of Variance for Unadjusted Data.

EP-IV Liquid into Gas ( $1.25 \mathrm{Ramp}$ ) Core Inlet Plenum Temperatures Adjusted for Effects of Time and Radius Analysis of Adjusted Data

Typical Narrow-Band (CALCOMP) Plot

NRX-Al Pressure Characteristics

NRX-Al Data Flow

Data Processing (RDH)

Calibration Curve for Narrow-Band Data System

Envelope Plot Showing a 50-cps Step in Calibration

High-Speed Recording of Part of a 50-cps Step in Calibration

CFDTS Data Flow

Output Format for N2 Logical Records

Transfer Function Analyzer Block Diagram 10

Programmable Reference Oscillator

Programmable Active Tuned Filter

APPENDIX A - ANALOG-TO-DIGITAL DATA ANALYSIS PROGRAM (ADDA)

APPENDIX B - STATISTICAL EDIT AND SMOOTH PROGRAM

APPENDIX C - ETS-1 DATA SYSTEM

APPENDIX D - AN EXAMPLE OF ERROR PROPAGATION IN A HEATTRANSFER MODEL 
RN-S-0146

SUMMARY 
RN-S -0146

\section{STMMARY}

An essential step in the development of effective techniques for analyzing test data is the use of available techniques with similar data. An evaluation of these techniques was made by their application to the NRX-Al test data.

When the analytical model and test results are both subject to error, information regarding the characteristics of both sources of errors is essential to permit separation of their respective effects in any comparison of calculated values with test results for the purpose of validation and improvement of analytical models. During Contract year 1964, the REON Data Analysis activity under Subsubtask 1.8.7, was aimed primarily at developing mathematical-computer techniques for deducing the characteristics of these errors. These programs include the following:

A. Analog-to-Digital Data Analysis Program (ADDA). This program, described in detail in Appendix $A$, was used to analyze over 50 million narrow-band data points generated in the NRX-Al test series. The program determined means and variances of the data at 1 -sec intervals; if the variance was sufficiently large, the linear trend and frequency content of the data was further calculated. Information on data quality such as the frequency of missing time values, outliers, and off-scale or off-spec signals, was also obtained. It was found that the NRXAl data quality was very high with regard to defects that could be detected by the tools available to the test operations organization.

B. Statistical Edit and Smooth. This program, described in detail in Appendix B, was used to detect and correct outlying observations, to smooth and time-synchronize the values analyzed. This program was quite useful for transforming the data into a format useful for asymmetry studies of NRX-Al. The output results will be used as input to the data analysis version of Computer Job No. 364 .

C. Computer Éob No. 364. This digital program for simulation of nuclear powered rocket engines is described in detail in a companion report (RN-S-0047). This program proved very useful for analyzing the NRX-Al data since it could represent detailed feedline and nozzle models useful for analysis of postulated 


\section{SUMMARY (cont.)}

mechanisms for asymmetry. A version of this program being developed especially for data analysis involves a technique for comparing experimental data with values computed by the program. The new part of the program will permit a statistical evaluation of some types of model and instrumentation errors and their effects on the comparison of models with data.

D. Analysis of Wide-Band Data. This involves the use of spectral analyzers which are useful in the evaluation of the information on magnetic tapes when conditions are relatively steady and when the frequency content represents perturbations and deviations from a steady state. Digital cross-correlation and spectral analysis techniques have merit but are rather expensive for extensive analysis of data having high frequency content. The use of hybrid and of pure analog computers for correlation analysis of wide-band data helps to offset the cost disadvantage.

The data acquisition and data processing hardware specified for engine test data are described in Appendix $C$. The data flow through that system is compared with that used in the other systems tests in the NERVA program, and the similarities are noted. 
$\mathrm{RN}-\mathrm{S}-\mathrm{O} 146$

\section{INTRODUCTION}

The Nuclear Engine for Rocket Vehicle Application (NERVA) is a system being developed by Aerojet-General Corporation (AGC) under contract to the Space Nuclear Propulsion Office (Reference 1). Tests of major subsystems are being conducted at the Nuclear Rocket Development Station, Jackass Flats, Nevada, and at Aerojet's Sacramento, California facility. A large number of measurements are necessary to describe adequately the phenomena occurring in the systems being tested, and their interactions (References 2 and 3 ). The evaluation and analysis of the resulting mass of data is a rather formidable task unless effective computer techniques are used. Thus, the initial objectives of the engine data analysis effort were: (a) the development of effective computer techniques for evaluating and interpreting the data produced by systems tests, (b) the use of these techniques for the analysis of data similar to future engine test data to evaluate effectiveness and to identify and define problem areas with regard to the use of these techniques, and (c) the providing of quality test data input for the upgrading of engine models.

The REON data analysis effort stems from a requirement to evaluate and interpret the results of the various system tests related to the NERVA program. There is a further requirement to make the results of these evaluations and interpretations available to the designers, engineers, and scientists who are contributing to the NERVA program, as well as the technical management of the entire nuclear rocket (Rover) program. In responding to this demand, the need for a digital program for simulation on nuclear-powered rocket engines became apparent. It was observed that the Computer Program 364 (Reference 4), originally intended as a performance analysis code, could be modified to become an exceptionally powerful tool for analyzing data. The program was designed so that models could be specified with much detail in one portion of the system to conform with intensity of instrumentation being considered and/or the complexity of phenomena under study. 
$\mathrm{RN}-\mathrm{S}-0146$

The initial REON data analysis effort was aimed at devising an economical method of translating the test data from the form in which it would be available from data reduction facilities (Reference 5) to a form more convenient for analysis. The processing required wolld include the detection of data points which were inconsistent with the trend of the data (i.e., switch points and outliers) and which might seriously perturb any attempt at an automatic data analysis procedure.

Hiarly in this effort, when the Kiwi $B 4 K$ cold flow test results became available, it was noted that a high noise level was present in that data (Reference 6). Recause of this high noise level, it was necessary to provide for a smoothing as well as an editing function. Since Computer job No. 364 was intended for simulating frequencies lower than $1 \mathrm{cps}$, a very low cutoff frequency was desirable for the data being analyzed under that program. The program developed for this application is called "Statistical Edit and Smooth," and is described in detail in Appendix B. A cutoff frequency of $I$ cps was chosen since the cost of digital computation using Job No. 364 could easily become prohibitive for simulating higher frequencies.

While Westinghouse Astronuclear Laboratory (WANL) was concentrating its data analysis effort under this contract on the analog (wideband) data (Reference 7), Aerojet's development effort was initially limited to the narrow-band data. However, since the narrow-band data was sampled 40 times per sec, it does contain information regarding frequencies higher than 1 cps. Therefore, an economical technique for analyzing this intermediate frequency range in the narrow-band data was a primary goal of another digital computer program developed. A secondary objective of this program was the ability to obtain information regarding data quality from the data generated during the data system calibrations. This program is called ADDA, and is described in detail in Appendix A.

Test data is generated for a number of purposes. It is first used by the test operations organization to determine if the test objectives have been met and whether the specified tests have been satisfactorily performed. It is later used by component design groups to determine (a) conditions that were experienced by their component, (b) the adequacy of the component design, and (c) the validity of the design methods used. 
Systems test results are used to compare pretest predictions with post-test calculations, and to detect interactions of components and phenomena that should be more correctly described or included in the systems models.

\section{A. BEHAVIOR ANALYSES OF SYSTEMS}

The determination of the phenomena interactions occurring is a primary function of a systems test. To describe completely, by measurements alone, everything that occurs would require an infinite amount of instrumentation. Since the maximum possible amount of instrumentation is finite, some technique is essential for making inferences regarding occurrences between transducers. Typically this technique uses some analytical formulation of the events occurring between points of measurement (transducer locations). This is simpler than the four-dimensional representation (three space dimensions plus time) required to describe completely the phenomena involved. It is much more complete than the usual plots of measurements at a single point in the system versus time (Figure 1). A somewhat more meaningful picture is obtained from a multiple plot such as shown in Figure 2; this figure comes from the NRX-Al 36-day report (Reference 8).

An example of system behavior analysis is presented in the analysis of apparent asymmetries which was reported in Reference 3. The presentation of the data in a form convenient for making this analysis was a difficult problem, and several techniques of presentation were used. Each method had relative advantages and disadvantages. Statistical techniques useful for an asymmetry analysis are discussed in Section III,B,3.

\section{B. VALIDATION AND IMPROVEMENT OF MODELS}

A systems model is viewed as either an equation or set of equations (using physical and mathematical theory in conjunction with empirical data) which attempts to abstract from reality the essential features of some physical phenomena. A model usually represents a continuous process in time and can be assumed to have the form

$$
Y_{t}=\xi_{t}+\epsilon_{t}
$$

where $Y_{t}$ is the model value at time $t, \xi_{t}$ is the true value of the physical phenomena at time $t$, and $\epsilon_{t}$ is a measure of the model's inability to represent 
faithfully the actual physical situation. The $\epsilon_{t}$ is often referred to as either "model error" or "equation error," and describes the accuracy of the model.

The evaluation of systems models requires the determination of the accuracy of the predicted values. In practice, the evaluation of a model is accomplished by a comparison of predicted versus observed quantities. The outcome of a series of experiments, designed to shed light upon a particular physical phenomenon, usually has the general form

$$
x_{t}=\xi_{t}+e_{t}
$$

in which $x_{t}$ is the observed value at time $t, \xi_{t}$ is the true value of the physical phenomena at time $t$, and $e_{t}$ is the unavoidable measurement error associated with $x_{t}$. The measurement errors $e_{t}$ are usually of a complex statistical nature and arise from several sources. In particular, the primary measuring devices (transducers, thermocouples, strain gages, etc.) are members of a population of such devices and, therefore, do not possess precisely predetermined outputs. Additionally, the recorded measurements are the output of a complex data system which is not completely free of error, and thus system errors are often confounded with the errors of the primary measuring devices.

Because of system complexity and extreme operating conditions, the goal of an ideal model which would predict the true value, $\xi_{t}$ with insignificant error must be approached by going through a sequence of corrections and improvements based on observed system operation. The true value $\xi_{t}$ cannot be directly determined from either measurements or model, and any comparison of the model with the observations will involve both of the quantities $\epsilon_{t}$ and $e_{t}$. The most meaningful comparison is in terms of the deviation of the predicted value $Y_{t}$ from the observed value $x_{t}$. In evaluating either the measuring system or the system model it is therefore necessary to work with the differences

$$
X_{t}-Y_{t}=e_{t}-\epsilon_{t}
$$

which contain all of the information on the nature of experimental or model errors. The crucial problem is somehow to obtain separate estimates of the $\epsilon_{t}$ and $e_{t}$, since the difference between model and the true value is sought. 
$\mathrm{RNN}-\mathrm{S}-0146$

For many years the problem of separating observed errors into their various components has confronted people working in the field of missile tracking. Various solutions to this problem have been proposed (Reference 9). However, for the physical situation under consideration the problem becomes more complicated. There are more physical entities involved, the model is less accurate, and there are portions of it which are quite uncertain (e.g., two-phase flow). In addition, a significant portion of the instrumentation is subject to human as well as equipment errors, malfunctions, and deterioration due to severe environmental conditions. With all of these complications it is apparent that the validation and improvement of system models will require involved and sophisticated techniques.

\section{NATURE OF AVAILABLE TEST DATA}

The data produced by a test comes in a variety of forms and media. It includes the following items:

Visual observations made before, during, and after the test

All measurements made of the test article geometry and physical characteristics before, and after, the test

On-line information displays made during pretest checkout and during the conduct of the test

Recordings in computer-readable formats made during checkout, while the test is in progress and after the test

Recordings in a form intended for human use (strip charts, etc.)

Observations on types of instrumentation and their location

in the test article and facility

Instrumentation failure reports.

When the data analysis is performed entirely by human beings without the aid of electronic computers, then only the data recorded in computer-readable format is not directly accessible. This situation can be remedied by preparing listings or graphs of such information. In the situation under discussion in this report, the number of measurements and test durations are such that a single test would consume the activities of the entire analytical staff for many years. Therefore, extensive use is made of electronic computers in this analysis to bring the task down to more reasonable proportions.

When computers do perform the extensive data processing involved in analyzing test results, only the information recorded on computer-readable media is 
directly useful. Hence a substantial amount of information which is initially obtained in a human-readable form must be converted into computer-readable form. In the case of the systems tests with which we are concerned, the amount of information involved from all sources is so great that there are many opportunities for both human and computer error. If the lines of communications are kept short and with a minimum of interfaces to minimize increase in entropy, the error rate can be relat1vely low. It $1 \mathrm{~s}$ of interest to observe that an obsolete IBM 704 computer and four comparatively slow mechanical plotters were able to reduce the test results to engineering units and plot them in much less time, and with fewer human errors in the data reduction phase, than was true with the much faster IBM 7090 computer and an expensive electronic plotter. (With the IBM 7090, much longer and more complicated communications paths had to be followed.)

The instrumentation that is used to generate the computer-readable data was discussed at an instrumentation review held last year (Reference 7). At this meeting, the planned processing of this data was also discussed. The specifications for the instruments used are covered in the Instrumentation Data Book (Reference 10). Of interest to the systems analysis effort are such instruments as pressure transducers, differential pressure transducers, temperature transducers, fluid flow rate transducers, accelerometers, strain gages, displacement transducers, and nuclear and gamma radiation transducers.

The signals from the transducers are processed through the data acquisition system for recording. These records typically included "on-line" recordings intended for human use, digital recordings, and analog recordings. Since the details of the equipment differ among NRX, CFDTS, and ETS-I acquisition systems, digital data will be defined here as data which is obtained through a sampling system such as a commutator or multiplexer (i.e., sampled data). The term "digital" is used since the sampled data is normally converted into digital form for processing by a digital computer; for this purpose it must be sampled data.

Techniques for the acquisition of this type of data are reviewed on the next few pages, and analytical techniques are discussed in Section III. Techniques for the acquisition of analog data are reviewed later on in Section II. Analysis techniques are discussed in Section IV. 
Although the use of the adjective "digital" rather than "sampled" in referring to data may seem somewhat arbitrary, it should be noted that it is rather unusual to sample analog data because of the loss of information which is caused by the sampling. This loss is unnecessary with analog techniques, and hence is usually avoided whenever possible. Sampling, and thus information loss, is unavoidable with digital techniques since the digital computer requires a finite amount of time to process each value. With proper data system design (i.e., the use of a suitable filter before the sampling), the loss of information can be limited to the frequencies above the cut-off for the filter. Thus digital data acquisition systems are used primarily with test data for which the primary interest is in the very low frequencies. When higher frequencies are of interest, analog types of recording and analysis are usually far more efficient.

A. NRX DATA FLOW

The overall flow of the NRX data is summarized in Figure 3. The onsite data processing was discussed in detail in References 11 and 12 and will be reviewed in this section. The results of the off-site data analyses performed by Aerojet are presented in Reference 3 and the techniques used are discussed in this report.

Basic to the data flow is the generation of the data which begins with the transducers. These are devices (e.g., thermocouples, strain gages, swept pots, resistance thermometers) which produce an electrical output in response to external stimuli. Each transducer has a signal conditioner which operates on the signal from the transducer in order to make it compatible with the rest of the data system. Calibration voltages are introduced at the signal conditioner. The data is recorded in both narrow band (where the frequency response is from 0 to $5 \mathrm{cps}$ ) and wide band (where the response is from 0 to $1000 \mathrm{cps}$ ).

\section{1. $\quad$ Processing of Narrow-Band (Digital) Data}

The narrow-band system is divided into a low-level and highlevel system as defined by the full-scale voltage input to the multiplexers (electrical or mechanical devices for sampling from a number of simultaneous voltage inputs, and outputting the samples in series). The input signals to the highlevel system are 0 to $5 \mathrm{v}$ full scale. They emanate from Offner amplifiers or 
Inherently high-level transducers. The input signals to the low-level system have a full-scale input level of $0-10 \mathrm{mv}$ to $0-100 \mathrm{mv}$; they emanate primarily from thermocouples. The Offner amplifiers have a second output of $0-10 \mathrm{v}$ full scale which are used to drive meters and Sanborn (multiple pen strip chart) recorders. By action of the multiplexer ( 14 were used), the input data channels are sampled most channels at 40 times per second, some at 10 times per second - and pulseamplitude-modulated signals (PAM) are produced. These are further frequency modulated (FM) by a voltage controlled oscillator (VCO), and the resulting PAM/FM signal is fed through 2 miles of cable to the Control Point Building into Ampex magnetic tape recorders (see Reference 11).

The resultant tape is called $\mathrm{N}-1$ in Section 21 of the NRX-Al Test Specification (Reference 2). This portion of the data flow is shown in greater detail in Figure 4. The $\mathrm{N}-1$ tape is converted to an $\mathrm{N}-2$ tape by processing it on the Astrodata A-D converter which converts the PAM/FM analog signals on the $N-1$ tape to raw digital data on the $N-2$ tape. The resulting $N-2$ tapes are fed into the IBM 704 digital computer for processing channels using the RDH (Rover Data Handling) code (Reference 5). At the same time, a card deck containing calibration times, plot times, transducer calibrations, scaling information, etc., is fed into the IBM 704 for converting the data to engineering units. The resulting output is a time history tape $(N-3)$. This tape, together with a "plot parameter tape," Is used to develop a plot tape to enable the CDC 160-A computer to operate the four CALCOMP incremental digital plotters. This plotting operation is the limiting step. For a typical experimental plan of NRX-Al, it took 4 hours per multiplexer out of a total of 8 hours per multiplexer.

In addition to producing $\mathrm{N}-1$ tapes, a Sanborn record is made during the actual running of the test. The Sanborn is a multiple-pen strip chart recorder. The data thus recorded can be used for a "quick look" (preliminary" analysis of data) by the Data Review Board during data holds (at which time the complex is in a static state).

The present $\mathrm{RDH}$ code, which sorts the data from multiplexer order to channel order in the process of reducing the data to engineering units, consumes $1-1 / 2$ hours per multiplexer for five data points per channel per second, making a total of 21 hours of IBM 704 time to process all 14 multiplexers. Therefore, a new code, which is designed to process the data in multiplexer order, is 
$\mathrm{RN}-\mathrm{S}-0146$

being written by NTO (NERVA Test Operations). Called NDRS (NERVA Data Reduction System), its estimated saving of time over the present code is in the ratio of 10 to 1 ( 2.1 hours versus 21.0 hours).

Copies of the N-3 tapes were sent to WANL (Westinghouse Astronuclear Laboratory, Large, Pennsylvania) for their backup reduction and plotting function and to AGC (Aerojet-General Corporation) for analysis. Copies of the CALCOMP plots are duplicated and distributed to WANL, Aerojet, and NTO for examination and analysis. Figure 1 shows the CALCOMP plot for the nozzle manifold inlet pressure during the second liquid-hydrogen ramp (flow profile, which is a programmed excitation to the system) for EP-II. Figure 5 shows the same channel. during a pretest calibration. In addition to direct duplication, the CALCOMP plots are microfilmed. The microfilm is reproduced and a copy is shipped to Aerojet and to WANL. As part of the distribution effort at Aerojet, the microfilms were mounted on aperture cards (so called because a hole in the IBM card is provided in the space where the film is to be mounted) and copies of requested plots are furnished.

Copies of the Sanborn recordings were distributed with the ThreeDay report which is issued three days after the completion of an experimental plan and which contains a summary of the plan and some analysis. When an entire plan is completed, all the duplicated $N-1$ tapes are converted to digital form and the resulting $\mathbb{N}-2$ digital tapes are shipped to Aerojet for analysis.

\section{Processing of Wide-Band (Analog) Data}

At NTO, the wide-band signals after being conditioned are amplified by the Dynamics Model 2640 amplifier. The signals are then frequency-modulated at the VCO, but preceding this, each channel of a group of five is filtered so that the upper frequency cut-off point corresponds to the band pass of one of the five IRIG (inter-range instrumentation group) channels ( 14 through 18). The resulting five FM signals are mixed linearly at the mixing amplifier and are then fed through the 2 miles of long lines to the Control Point Building and onto one of the Ampex magnetic tapes (see Reference 11).

The wide-band data tapes are designated in the Test Specification as W-1. They are duplicated at NTO, and a copy is shipped by NTO to WANL and Aerojet for further processing. The next step in the processing procedure is 
the preparation of envelope plots which are a recording of the data at a low paper speed. Aerojet used 5/8-in. per sec paper and generated a mile of paper for each experimental plan. Figure 6 shows the wide-band envelope plot during part of the pre-test calibration for EP-II, recorded at a paper speed of $5 / 8$ in. per sec. Figure 7 shows a small part of the data in Figure 6 recorded at a paper speed of 100 in. per sec.

The envelope plots are examined, and specific intervals on the plots are selected for more processing. The $W-1$ tapes can be converted to $W-2$ tapes using an analog-to-digital converter and associated discriminators and filters. The information on the $W-2$ tapes can be converted to engineering units on the IBM 704 using the RDH program. The $\mathrm{W}-2$ tapes can be used for digital spectral analysis.

In addition to the $W-1$ tapes, CEC records are produced to be used for "quick look" analysis. The CEC is a photo recording galvanometer used to record wide-band data because of its high-frequency response. It is manufactured by the Consolidated Electrodynamics Corporation.

B. CFDTS DATA FLOW

The preliminary test plan for the Cold Flow Development Test System (CFDTS) for the NERVA Engine is reported in Reference 13. As stated in that test plan, the primary objectives of the CFDTS test series are "to verify the control functions and startup sequence of operations previously determined by transient analysis of a mathematical model" and "to evaluate flow characteristics of a nonnuclear engine system to determine chilldown time, temperatures throughout the system, flow rates, and pressure drop data."

The planned data flow for the CFDTS tests is shown in Figure 8 . It parallels the NRX data flow by starting at the transducers. After signal conditioning, it is split into the categories described below.

1. Digital Data System

The digital data system currently used for NERVA testing in Sacramento uses a Beckman 210 system which provides commutation and analog to digital ( $A D$ ) conversion. At the current sampling rate of 3750 samples per sec, this system samples 2 channels at 288 samples per sec each, 20 at 36 samples per 
RN-S-0146

sec, 70 at 18 samples per sec, and 96 at 9 samples per sec. The system also provides for recording of 4 channels which enter the system in digital form at a sampling rate of 36 samples per sec each. These rates are based upon a recording density of 200 bytes per inch. The recording system can also be operated at a total sampling rate of 10,400 samples per sec, which corresponds to a recording density of 556 bytes per inch. Another digital data system similar to the present one is being installed and is scheduled for completion prior to the CFDTS testing, doubling the above specified capacity.

After the test, the digital tapes are played back into the IBM 7040 in the Sacramento Test Area. At the same time, ID information necessary to apply calibrations is read in from cards. The output from this processing includes summary tabulations, data listing, and plots which are reviewed for qualification (validation) before any of the data is released. Further analysis of the reduced data tapes is performed using an IBM 7094. These further analyses would make use of the techniques discussed in Section III. In addition, the results are compared with pretest predictions for validation and improvement of analytical models. When significant anomalies are observed, such as the asymmetry for the nozzles tested in the INRX-Al series, more detailed analytical models and analyses would be required. In that case, detailed digital nozzle models were used to evaluate proposed asymmetry mechanisms and the work was reported in Reference 3.

\section{On-line Recording for Display}

The on-line displays provide information essential for test operations and for quick look. This information is necessary for safety considerations and for those controls which are performed by the test operators. They quickly provide the engineers involved with preliminary information regarding the conduct of the test. This information is superseded by the validated reduced data as soon as it is available.

\section{Analog (FM) Data System}

The data system for recording of CFDTS analog data now contains one 32-track FM tape recorder similar to the type described in Appendix $\mathrm{C}$ for engine testing. An additional tape recorder will be installed before CFDTS testing starts. Each track of these FM tape recorders can faithfully record frequencies up to $10 \mathrm{kc}$. After the test, the FM tapes should be played back for recording 


$$
\mathrm{RN}-\mathrm{S}-0146
$$

of the signals at a low paper speed to provide "time plots." These plots are compared with pre-test analog predictions and the observed deviations are the basis for model improvements. Some of the plots are also examined by the component design groups to validate the performance of their designs.

Further analog analyses is the expression used in Figure 8 to cover any analyses performed directly with the signals generated when the tapes are played back. Typical analyses of this kind are discussed in section IV. Since the CFDTS tests are entirely transient, this work should consist primarily of analyses using subsystem models. These subsystem models are necessary to provide more detail regarding the behavior of a portion of the system tested than can be provided in a system analog model due to equipment capacity limitations. This parallels the use of a detailed digital model of a portion of the NRX-Al system since the digital system model taxed the capacity of available digital computers. The subsystem models need to be supplied with boundary conditions. For the analog models, these boundary conditions would be supplied by tape playback equipment now on order.

\section{ENGINE TEST DATA FLOW}

The flow of the engine test data should be similar to that shown in Figure 8 for the CFDTS data. Due to equipment differences between the test facilities, a CDC 3200 would be used with the engine test data in place of the IBM 7040 used with the CFDTS data. There would be no transmission line since the digital data recording and $\mathrm{AD}$ conversion are performed in the digital data system described in detail in Appendix $\mathrm{C}$, and reviewed below.

The ETS-1 Digital Data System (DDS) was designed by the Aerojet REONI and C group. Procurement specifications were prepared by EG and G (Reference 14). The system is presently being fabricated by SEL Corporation, Fort Lauderdale, Florida.

The initial system design specified an input capability of 1440 analog data channels, 156 binary events, and 9 counter channels. To prevent a complete loss of data in the event of a catastrophic instrumentation failure, the system was divided into three independent but completely identical subsystems of 480 input channels each. The use of redundant channel recording is not planned. However, the loss of a subsystem during a test run would not represent a complete loss 
$\mathrm{RN}-\mathrm{S}-0146$

of all test data as might occur if only one exceedingly large system were used. Presently only one subsystem has been purchased with the second subsystem funding: expected in the near future. Although two subsystems will provide approximately the same recording capability as the system installed in Test Cell A or $\mathrm{C}$, much greater accuracy and ease of data processing will be achieved.

Each DDS subsystem has the characteristics described below.

Input - 480 channels sampled at a total rate of 10,000 data samples per sec distributed as follows: 400 channels at 10 samples per sec $(\mathrm{s} / \mathrm{s})$, 40 channels at $50 \mathrm{~s} / \mathrm{s}$, and 40 channels at $100 \mathrm{~s} / \mathrm{s}$.

Input Level - the DDS will accept either high-level signals (0 to \pm 5 VFS) or low-level signals ( 0 to $\pm 10,20,30,40,50$ and $100 \mathrm{mv}$ FS). Highlevel signals enter through the DDS high-level commutators at the desired sampling rate (i.e., 10, 50, $100 \mathrm{~s} / \mathrm{s}$ ). Low-level signals are sampled at low-level and at $10 \mathrm{~s} / \mathrm{s}$ only.

Input Sampling - the sampling plan is arranged as follows:

160 differential input low-level channels at $10 \mathrm{~s} / \mathrm{s}$ 240 differential input high-level channels at $10 \mathrm{~s} / \mathrm{s}$ 40 differential input high-level channels at $50 \mathrm{~s} / \mathrm{s}$ 40 differential input high-level channels at $100 \mathrm{~s} / \mathrm{s}$

By forfeiting 40 channels at $50 \mathrm{~s} / \mathrm{s}$ and 40 channels at $100 \mathrm{~s} / \mathrm{s}$, a high-speed super commutation plan may be utilized to provide 3 channels at 2000 $\mathrm{s} / \mathrm{s}, 12$ channels at $500 \mathrm{~s} / \mathrm{s}, 30$ channels at $200 \mathrm{~s} / \mathrm{s}$, or any other reasonable arrangement of channels and sampling rate producing a product of $6000 \mathrm{~s} / \mathrm{s}$.

In addition to the analog sampling, each DDS subsystem also records range time, test identification, operating mode, subsystem identifier, 12 binary events at $100 \mathrm{~s} / \mathrm{s}$, and 40 binary events at $10 \mathrm{~s} / \mathrm{s}$.

Information from pulse or frequency output transducers is recorded by three channels in each subsystem. Data from turbine-flow meters or RPM counters are input to 10,000-count BCD channel positions. This count resolution provides sufficient storage area so that the count value cannot cycle through the range more than once per sample period. The sample period is 0.1 sec. Thus the actual rotation of the measuring device is stored, and provides the best measurement accuracy. Since this technique does not permit flow determination by reference 
to only one record, these transducers are also sampled, at the same rate, by Vidar frequency-to-dc converters. These measurements are stored in the same manner as any other high-level transducer. The Vidar channels are used for most calculations unless maximum accuracy is desired.

Data Recording - data is recorded in a bipolar, 4-character, BCD Format ( \pm 9999$)$. Record lengths are $0.1 \mathrm{sec}$ and contain exactly 1000 data words. Recording format is gapless with record markers identified by double unique characters at the end of each record. Data is recorded on 7-track CDC604s. Two handlers are assigned to a subsystem with a third handler as a reliability backup. End of tape sensing is provided to permit continuous recording. Track assignments are as follows: 8-4-2-1-A-B-C. The "A" track is used for the record marker bits, the " $B$ " track for word sign value, and the "C" track for even lateral parity bit. Recording rate is approximately $556 \mathrm{bpi}$ at 75 ips. Character rate is exactly 40,000 characters per sec.

Data Output - since the ETS-I DDS records in a gapless BCD data mode it is necessary that the ETS-I Data Processor (DP) provide data reformating, correction, and conversion prior to transmittal. Primary raw ETS-I DDS data tapes are not shipped off-site, and only the output tapes from the ETS-I DP are transmitted. This prevents format and validity problems.

System Control - the DDS may be controlled from the Test Cell Building, Forward Control Room, or the Data Processor Room of ETS-1. The master control console and magnetic tape switch unit, along with magnetic tape handlers, are located in the Data Processor Room. System master control has been provided with a manual switching capability which permits the use of any digital tape handler in any system recording position of either the DDS or DP.

System Accuracy - the static accuracy of the DDS is divided into three classes as indicated below.

Class I Channels: $\pm 0.15 \%$ full scale for standard millivolt level channels individually preamplified and sampled by any high-level multiplexer.

Class 2 Channels: $+0.1 \%$ full scale for standard high-level signals sampled by any high-level multiplexer. 


$$
\mathrm{RN}-\mathrm{S}-0146
$$

Class 3 Channels: $\pm 0.5 \%$ full scale for standard millivolt level channels directly sampled by the low level, 10 sample per sec multiplexer. These accuracy figures represent the root-sum-square combination of zero and gain instability, hysteresis, and noise or scatter typical of what was called random errors in Section II. Other system errors such as zero transfer, gain accuracy, linearity and common mode conversion may be accounted for during calibration and subsequent analysis by the data processor. Therefore, their contribution to the accuracy of the final data may be virtually eliminated.

III. ANALYSIS OF DIGITAL DATA

To have proven data analysis techniques available in time for engine testing, it is essential that the techniques being developed be thoroughly evaluated prior to that testing. Hence, it is desirable to evaluate thoroughly the programs being developed by using them with the available data. This was done using the data generated during the NRX-Al test series, which is similar to the kinds of data to be generated by engine testing. The detailed data format and reduction procedures for NRX-Al data are different from that expected for ETS-1. The amount of data involved was very large; therefore, it was much quicker and less expensive to write programs specifically for the NRX -Al data. It is likely that major changes will be required in these programs as the problem areas become defined, and economical solutions to these problems are developed. Under these conditions, it is much quicker and cheaper to use the block concept, with new programs being written for each major change incorporating the proven features of previous programs and abandoning inefficient features.

This section of the report describes the digital programs that were used in analyzing the NRX-Al test data for Reference 3 and the digital techniques which are currently under development for use in connection with NRX and CFDTS experimentation during the next contract year.

\section{A. DESCRIPTION OF AVAILABLE DATA}

The overall flow of the NRX-Al reactor test data is described in References 3 and 7 , and is summarized in Figure 3. For the digital data, this flow - up to its receipt at Aerojet for analysis - was as follows. The data was generated by the transducers imbedded in the hardware and transmitted through the PAM/FM data 


$$
\mathrm{RN}-\mathrm{S}-0146
$$

system to magnetic tape recorders where narrow-band analog recordings of the data were made. These tapes (NI tapes) were then converted from analog to digital form at NRDS using their CDC-160A digital computer and peripheral equipment. Copies of these tapes (N2 tapes) were made and sent to Aerojet for analysis. The N2 tapes at NRDS were further processed using the IBM 704 computer to convert each recorded channel into engineering units and thereby to put the data into a more meaningful form. These tapes (N3 tapes) were also sent to Aerojet for analysis.

\section{N2 Data Format (Raw Data)}

The data on the $\mathbb{N} 2$ tapes are in multiplexer order, in digitized form (called counts), and are packed three 12-bit CDC-160A words in each IBM 704 36-bit word. The $\mathrm{N} 2$ tapes are recorded at high density ( 556 bytes per in.), where each byte contains 6 bits of information and a parity bit recorded in parallel on the seven-track magnetic tape.

Each multiplexer represented one file on tape. For each multiplexer, the first physical record is header information recorded in binary coded decimal form. This header identifies the test, the multiplexer number, and the pin locations for the recorded channels. The header is followed by physical records, each of which contains ten logical records. Each logical record contains a series of packed 36-bit words, the length of which depends upon the number of recorded channels. The first 36-bit word contains three major time words, each of which contains 12 bits including flag bits and two binary coded decimal digits. The second 36-bit word in each logical record contains one 12-bit vernier time word in millisec and two 12-bit data recordings. These words are in binary form and include two flag bits. Each subsequent word contains three 12-bit data words, in binary form with flag bits provided, until all channels are listed. If the number of recorded channels is such that the last channel does not end so as to fill completely a 36-bit word, zeros are added to fill that word. The major time word for the next logical record immediately follows the last data word. The output format for a single logical record is shown by Figure 9 . An end of file record is placed between multiplexers. The sampling rate for all high-level multiplexers

and some low-level multiplexers, was 40 points per sec; other low-level multiplexers were sampled at 10 points per sec. 
$\mathrm{RN}-\mathrm{S}-0146$

\section{N3 Data Format (Reduced Data)}

The data on the $\mathbb{N 3}$ tapes are stored as a single physical record for each channel processed. The tapes are at low density (200 bytes per in.), since this is the only tape density acceptable to the IBM 704 used.

The N3 tapes begin with information identifying the particular channel and continue with time-word, data-word until all reduced data points have been exhibited. The identifying information gives the channel association number (its location with respect to record on tape), the number of recorded data points, 12 words (each containing six alphanumeric characters of information, with the REC card information coming from the RDH-D code $\mathrm{e}^{*}$, and six words of alphanumeric information (title card information from the RDH-D code). The REC card information gives the transducer number, the multiplexer number and pin position at which the data was recorded, and the theoretical range of the transducer recordings. The title card information gives the name and date of the test. Time is recorded in seconds. The sampling rate for the $N 3$ tapes was five points per sec for NRX-Al.

\section{Nature and Estimation of Experimental Errors}

This section considers the general nature of the experimental errors, presenting methods both for reducing the effect of these errors and the estimation of a component of the experimental error. In general, the observed data are regarded as a sample from a continuous time series (that is, the measurements constitute a discrete time series). It is initially assumed that the observations have the form

$$
x_{t}=\xi_{t}+e_{t}
$$

where $\xi_{t}$ is the actual value of the phenomenon being observed at time $t$, and $e_{t}$ is the expected experimental error associated with the observation $\mathrm{X}_{t}$. These expected errors are classified into two categories: random errors and systematic errors. The distinction is based primarily on the frequency components of the errors; for this discussion, the errors which have high-frequency components are called random errors and those with low-frequency characteristics are classified as systematic errors. The random errors are related to the precision of the measurement process, while the systematic errors yield information about the bias of the observational

* See Reference 5 . 
process. It is assumed that the high-frequency errors will exhibit truly random characteristics and fluctuate around some average value. Additionally, since the systematic errors can vary from test to test, it is assumed that they are representative observations on some random variable.

There are several methods available for the modeling of the overall error term $e_{t}$. In many cases of practical interest, it is sufficient to regard $e_{t}$ as square additive. That is, to assume

$$
e_{t}^{2}=e_{t}^{2}(r)+e_{t}^{2}(s)
$$

where $e_{t}(r)$ is the random component and $e_{t}(s)$ is the systematic component. Some reflection indicates that Equation (5) may be interpreted as the squared length of the hypotenuse of a right triangle. As is well known from elementary trigonometry, one generalization of the Pythagorean theorem is the law of cosines for oblique triangles. According to this formulation, the overall error of a set of measurements may be viewed as

$$
e_{t}^{2}=e_{t}^{2}(r)+e_{t}^{2}(s)+p\left[e_{t}(r), e_{t}(s)\right]
$$

in which $e_{t}^{2}(r)$ and $e_{t}^{2}(s)$ are the same as above, and $\rho\left[e_{t}(r), e_{t}(s)\right]$ is the correlation coefficient between the random and systematic errors. The term $\rho\left[e_{t}(r)\right.$, $\left.e_{t}(s)\right]$ may be viewed as a measure of the interaction between the random and systematic components. If there is no interaction between $e_{t}(r)$ and $e_{t}(s)$, then $\rho\left[e_{t}(r)\right.$, $\left.e_{t}(s)\right]=0$, and Equation (6) reduces to Equation (5); that is, the error triangle is a right triangle. If the error triangle is a right triangle, then the errors $e_{t}(r)$ and $e_{t}(s)$ are often called independent errors. Thus, if the errors $e_{t}(r)$ and $e_{t}(s)$ are independent, the covariance $\rho\left[e_{t}(r), e_{t}(s)\right]=0$. However, it is known from elementary probability theory that if $\rho\left[e_{t}(r), e_{t}(s)\right]=0$, it does not necessarily follow that $e_{t}(r)$ and $e_{t}(s)$ are independent. It is assumed in this treatment and as a first approximation that the error components $e_{t}(r)$ and $e_{t}(s)$ are independent so that Equation (5) is used as the basic error model.

Each of the components $e_{t}(r)$ and $e_{t}(s)$ is generally composed of several constituent elements. Usually the systematic component $e_{t}(s)$ contains many complex subcomponents, and it is the estimation and evaluation of these constituent 
effects that presents the principal problem in systems model evaluation. In practice, the evaluation of $e_{t}$ requires first a reduction in the effects of the random component $e_{t}(r)$ and then a very stringent analysis of the residual $e_{t}(s)$. The process of reducing the effects of $e_{t}(r)$ consists of passing the data through a low-pass filter, allowing only a minimum of random errors and a maximum of systematic errors to remain in the data. This procedure is aptly called "smoothing."

Smoothing is a mathematical procedure for reducing the effects of the high-frequency or random-error components in a series of data points. It is assumed that the underlying time series function can be expressed locally by an elementary function - usually a low-order polynomial. This function is usually called a smoothing function or a numerical filter. The process of smoothing fits the smoothing function to the data by some mathematical method (usually least squares) so that a smoothed value can be obtained by evaluating the smoothing function at the time point of interest. If this time point is near the midpoint of the span over which the smoothing function was fitted, then the estimated variance of the smoothed value is given by $s_{t}{ }^{2}(r) / N$, where $s_{t}{ }^{2}(r)$ is the residual variance of the fit and $N$ is the number of points to which the smoothing function was fitted. Further, if the smoothing function is a very good approximation to the true time series function over the smoothing interval, then $s_{t}{ }^{2}(r)$ is an excellent estimate of $\mathrm{e}_{\mathrm{t}}{ }^{2}(r)$. Thus, the net effect of smoothing is a reduction of the squared random error component by a factor of $1 / \mathbb{N}$. This result is subject to the condition that the systematic component, $e_{t}(s)$, remain constant throughout the smoothing interval; $e_{t}(s)$ may, however, vary from interval to interval.

It is necessary to remark that the smoothing procedure does not completely eliminate the high-frequency effects; it only minimizes it. Thus, from a practical viewpoint, it is highly desirable to have estimates of the remaining error components. There are, under suitable assumptions, two methods for estimating the random component: the variate difference method, and the least squares method. The estimation of the systematic error is considerably more difficult; in fact, it is necessary to have several time records available in order to estimate effectively the systematic error. Consequently, only the estimation of the random component will be discussed and it will be assumed that the serial correlation of the random errors is zero. 
$\mathrm{RN}-\mathrm{S}-0146$

In the variate difference method, the series of observations is partitioned into spans of $\mathbb{N}$ points. For each span, $\mathrm{k}$ successive differences are taken. The random component of variance for each difference is then estimated by

$$
s_{r}^{2}=\frac{\sum_{j=i}^{n-i}\left(\Delta_{j}^{i}\right)^{2}}{(n-1)\left(\begin{array}{c}
2 i \\
i
\end{array}\right)} \quad(i=1, \ldots, k)
$$

where

$$
\begin{aligned}
\Delta^{i} & =\text { the ith aifference } \\
n & =\text { the number of data points in the span } \\
\left(\begin{array}{c}
2 i \\
i
\end{array}\right) & =\frac{(2 i) !}{(i) !(i) !}=\frac{(2 i) !}{(i !) 2}
\end{aligned}
$$

It may be shown (Reference 15) that the "best" estimate of the random component of variance for each span is given by the lowest ordered difference for which the variance estimates of higher ordered differences do not vary (within sampling limits). In most practical situations, these variance estimates tend to stabilize around the third to fifth difference, depending upon the phenomena and the length of the span.

In the least squares method, a polynomial is fitted to the reduced data by standard least squares curve fitting techniques. Let $x_{t_{i}}(i=1, \ldots, n)$ be the observations at time, $t_{i}$, and suppose a polynomial of degree $p$ is fitted to these data. Let $\mathrm{X}_{t_{i}}^{*}$ denote the corresponding points on the fitted curve. Then

$$
s_{x}^{2}=\left[(n-p-1)^{-1} \sum_{i=1}^{n}\left(x_{t_{i}}-x_{t_{i}^{*}}^{*}\right)^{2}\right]
$$

is an estimate of the variance of $x_{t_{i}}$ around the polynomial. If the fitted polynomial is a good approximation to the true time function, the $s_{x}^{2}$ becomes a very good estimate of the random component of variance.

B. QUANTITATIVE METHODS OF DESCRIBING INFORMATION CONTENT OF DATA

1. Statistical Examination of Raw Data (N2 Tapes)

To judge the adequacy of the collected data, quantitative methods must be established for measuring data quality. Data quality can be viewed in terms of the following considerations: 
The frequency of occurrence of outlying observations and other types of errors detected during each stage of data processing

The accuracy of the data (i.e., the closeness of agreement between the recorded value determined by the measuring system and the true value of the phenomena occurring)

The precision of the data (i.e., the repeatability of measurements, where each observation is an assessment of the same value of the phenomenon)

The periodicities encountered in the data. Certain frequencies may be induced by the data system and should not be interpreted as cyclical patterns in the phenomena.

Clearly, the raw calibration data in the form of counts can be very useful in determining the quality level of the data. In generating the calibration data, known voltages are applied to the measuring system. These voltages are held constant over specified periods of time, and measurements of these voltages are made by the system. The precision of the data could then be determined from these measurements by estimating the variance of data over intervals of constant applied voltage. The accuracy of the data could be determined by testing the means of the observations over these periods with the known voltage inputs. Outlying observations could be counted as could the occurrences of error flag bits.

For tests composed of a series of linear ramps and steady-state holds, such as the NRX-Al series, a similar analysis could be provided for the run data. During the period of ramps, linear equations could be fitted to the data. Precision could be measured by the standard error of estimate, and accuracy could be measured by comparing the equation of the fitted line with pre-test objectives. For cases showing large standard errors of estimate, the frequency content of the data should be determined. During periods of steady state, the behavior of the data should be similar to that experienced during calibration.

To provide measures useful in determining the quality level of the N2 data, the program ADDA was developed. This program gives, by channel number, a compilation of successive calculated means and calculated variances, and, at the occurrence of a major break in time, some summary statistics for that 
time interval. These include the "start-stop" times for the interval, the number of data points per channel, the number of missing time points, the number of occurrences of various flag bits, the number of outliers, the number of steps, and the number of times the periodicities were searched for in the data. Furthermore it provides, by pin number, a frequency distribution of calculated variances and a frequency distribution of estimated periodicities. A complete account of this program is given in Appendix A. A brief description of this program is given below.

Data is read from the $\mathbb{N 2}$ tapes in blocks of four physical records (or less if data has been exhausted). These data are then unpacked. Flags are stripped from time values; times are floated and consolidated. Flag bits in data words are masked and tallied, and the unpacked data is stored in matrix fashion for further processing. The time values are then edited for missing values. Large gaps in time signify a change in major test conditions. Assuming no major changes in time, the matrix of channel data is then processed channel by channel until all channels have been processed. At that time, four additional physical records are read from the $\mathrm{N} 2$ tape and the processing cycle is restarted. The channel-by-channel processing is carried out as follows:

a. A comparison is made between the first and last data values within a channel. If these values do not differ by more than a preset constant, $K_{l}$, it is inferred that during this time interval the data represents a constant value; the arithmetic mean, $\bar{x}$, and the variance, $s^{2}$, are then calculated for the $N$ data points.

b. The estimated variance, $s^{2}$, is then compared to a second preset constant, $\mathrm{K}_{2}$. If $\mathrm{s}^{2}$ exceeds $\mathrm{K}_{2}$, it is inferred that ( 1 ) either the data contains one or more outliers, or (2) the data contains an excessive amount of periodicity. A test for outliers is made. If any are found, $\bar{X}$ and $s^{2}$ are adjusted; if no outliers are found and/or $s^{2}$ still exceeds $K_{2}$, the mean, $\bar{x}$, is subtracted from all data points, and a frequency analysis is performed on the data to find any possible periodicities in the data.

c. If the first and last data values differ by more than $K_{l}$, it is inferred that: (1) one of the end points may be an outlying observation; (2) the data encompass a step, as for cases in the calibration data; or (3) the 


$$
\text { RN-S-0146 }
$$

data encompass a ramp, as for cases in the run data. A test for end-point outliers is made; any outliers found are then adjusted. If this reduces the difference between the first and last data point to less than $K_{l}, \bar{x}$ and $s^{2}$ are calculated and the program proceeds as in $a$ and $b$ above. If no end-point outliers are found, or if the first and last observations still differ by more than $K_{l}$, a test is made to determine if a step has occurred within the data. If a step is found, the data are broken into two subsets and each subset is analyzed as discussed in a above. If no step is found, the program fits a linear equation to the data, since it is assumed that the run data are comprised of only linear ramps and constant holds. Again, a test of $\mathrm{s}^{2}=\mathrm{K}_{2}$ is made. If the inequality is satisfied, the data are detrended and the frequency analysis is applied to the data.

The program was applied to the N2 data generated by the NRX-Al test series. Approximately 50 million data points were interrogated arising from selected multiplexers from test plans EP-I, EP-II, EP-III, and EP-IV. A complete tabulation of the results of this application is contained in Reference 16. (Sample outputs are shown in Appendix A.) A condensed and summarized treatment of these data for EP-I and EP-II is contained in Reference 3. Useful conclusions drawn from the application of program ADDA to these data were also presented.

When evaluating frequency distributions like those generated by program $A D D A$, it may be desirable to make objective tests to determine if an observed distribution is different from some expected distribution, or if two observed distributions can be considered to represent the same underlying population. Such tests may be made using the Kolmogorov-Smirnov statistics. A discussion of this statistic is given below.

Let $\xi_{1}, \xi_{2}, \ldots, \xi_{n}$ be mutually independent random variables with a common distribution function $F(x)$, where

$$
P\left\{\boldsymbol{g}_{j}=x\right\}=F(x) \quad(j=1,2, \ldots, n) .
$$

In statistical language, $\xi_{1}, \xi_{2}, \ldots, \xi_{n}$ form a sample of size $n$ drawn from the population having the distribution function $F(x)$. Let $\xi_{1}^{*}$, $\xi_{2}^{*}, \ldots, \xi_{n}^{*}$ be the same set of variables arranged in increasing order of magnitude. The empirical distribution of the sample $\xi_{1}, \xi_{2}, \ldots, \xi_{n}$ is the step function $E_{n}(x)$ defined by 


$$
E_{n}(x)=\left\{\begin{array}{ll}
0 & \text { for } x<\xi_{1}^{*} \\
k / n & \text { for } \xi_{k}^{*} \leq x \leq \xi_{k+1}^{*} \\
1 & \text { for } x \geq \xi_{n}^{*}
\end{array} \quad(k=1,2, \ldots, n-1)\right.
$$

In other words, $\mathrm{n} \cdot \mathrm{E}_{\mathrm{n}}(\mathrm{x})$ is the number of variables $\boldsymbol{\xi}_{\mathrm{k}}$ which do not exceed $x$. According to the strong law of large numbers, for each fixed $x$, as $n \rightarrow \infty$

$$
P\left\{E_{n}(x) \rightarrow F(x)\right\}=1
$$

The maximum of the deviation

$$
D_{n}=\sup _{-\infty<x<\infty}\left|E_{n}(x)-F(x)\right|
$$

is a random variable. If

$$
P\left\{E_{n}(x) \rightarrow F(x)\right\}=1
$$

then almost surely

$$
P\left\{\lim _{n \rightarrow \infty} D_{n}=0\right\}=1,
$$

and $D_{n}$ is independent of the specified form of $F(x)$ provided only that $F(x)$ is continuous. In fact, in this case, the random variables

$$
\eta_{\mathrm{k}}=\mathrm{F}\left(\boldsymbol{\xi}_{\mathrm{k}}\right)
$$

are mutually independent and each is uniformly distributed in the interval $(0,1)$. If $n \cdot E_{n}^{*}(x)$ is the number of

$$
F\left(\xi_{j}\right) \leq x
$$

then for $j \leq n$

$$
\operatorname{Sup}_{0 \leq \mu \leq 1}\left|E_{n}^{*}(\mu)-\mu\right|=\operatorname{Sup}_{-\infty<x<\infty} \mid E_{n}(x)-F(x)
$$

Thus there is no restriction in replacing the $\boldsymbol{\xi}_{j}$ by the $F\left(\boldsymbol{\xi}_{j}\right)$, if necessary, in finding the distribution of $D_{n}$ to assume that $F(x)=x$ for $0 \leq x \leq 1$ and 


$$
\begin{gathered}
\mathrm{RN}-\mathrm{S}-0146 \\
\mathrm{D}_{\mathrm{n}}=\operatorname{Sup}_{0 \leq \mathrm{x} \leq 1}\left|\mathrm{E}_{\mathrm{n}}(\mathrm{x})-\mathrm{x}\right|
\end{gathered}
$$

The exact distribution of $D_{n}$ is unknown; however, Kolmogorov succeeded in showing that $n^{1 / 2} D_{n}$ has the following limiting distribution:

If $F(x)$ is continuous and

$$
D_{n}=\sup \left|E_{n}(x)-F(x)\right|
$$

then for every fixed $z \geq 0$, as $n \rightarrow \infty$

$$
P\left\{D_{n}=2 n^{-1 / 2}\right\} \rightarrow L(Z)
$$

where $L(Z)$ is the distribution function given by equivalent relations

$$
L(z)=\left\{\begin{array}{l}
1-2 \sum_{t=1}^{\infty}(-1)^{t-1} \exp \left(-t^{2} z^{2}\right) \\
=(2 \pi)^{1 / 2} z^{-1} \sum_{t=1}^{\infty} \exp \left(-\frac{(2 t-1)^{2}}{8 z^{2}}\right) \text { for } z=0 \\
0 \text { for } z \leq 0
\end{array}\right.
$$

The equivalence of these two forms is a well-known relationship often called the transformation formula for theta functions.

Of equal interest and utility is Smirnov's result concerning the maximum difference between the empirical distributions of two samples with the same cumulative distribution.

Let $\left(\boldsymbol{\xi}_{1}, \boldsymbol{\xi}_{2}, \ldots, \boldsymbol{\xi}_{n}\right)$ and $\left(\eta_{1}, \eta_{2}, \ldots \eta_{n}\right)$ be two samples of mutually independent random variables having a common continuous distribution function $F(x)$. Let $S_{m}(x)$ and $T_{n}(x)$ be the corresponding empirical distribution functions and define a new random variable, $D_{m n}$, by

$$
D_{m n}=\sup \left|S_{m}(x)-T_{n}(x)\right|
$$


Put $N=\frac{m n}{m+n}$ and suppose that $m \rightarrow \infty$, and $n \rightarrow \infty$ in such a manner that

$$
\frac{m}{n} \rightarrow a
$$

where a is some constant. Then for every fixed $\mathrm{z} \geq 0$

$$
\begin{aligned}
P\left\{D_{m n} \leq\right. & \left.Z N^{-1 / 2}\right\}=L(Z) \\
& =\left\{\begin{array}{l}
1-2 \sum_{t=1}^{\infty}(-1)^{t-1} \exp \left(-t^{2} z^{2}\right)(z>0) \\
0 \quad z \leq 0
\end{array}\right.
\end{aligned}
$$

It would be well at this point to consider practical applications of this approach. Suppose that a population is thought to have some specified distribution function, say $F_{0}(x)$. That is, for any specified value of $x$, the value of $F_{0}(x)$ is the proportion of individuals in the population having measurements less than or equal to $x$. The cumulative step function of a random sample of $n$ observations is expected to be fairly close to this specified distribution function. If it is not close enough, then this is evidence that the hypothetical distribution function is not the correct one. One procedure is to draw the hypothetical distribution on a graph and to draw curves a distance $D_{n}(\alpha)$ above and below the hypothetical curve. If $E_{n}(x)$ passes outside of this band at any point we shall reject, at the $\alpha$ level of significance, the hypothesis that the true distribution is $F_{0}(x)$. An alternative, and perhaps simpler, scheme is to record in a table the observed and hypothetical distributions and calculate the maximum deviation between them. If this exceeds $D_{n}(\alpha)$, the hypothetical distribution is rejected.

A short table of $L(Z)$ is given in Reference 17. A table of percentage points of the distribution of $D_{n}$ is given in Reference 18 . Derivations of the limiting distribution $L(Z)$ which is stated here are given in Reference 19.

2. Statistical Analysis and Processing of Reduced Data (N-3 Tape)

Whereas the raw $\mathrm{N} 2$ data were most useful in providing measures of data quality for NRX-A.1, analytical investigations aimed at describing engine performance are best made using the $\mathrm{N} 3$ data which is in engineering units. 


$$
\mathrm{RN}-\mathrm{S}-0146
$$

Although the N3 data format, as described in Section III, A, 2, is very suitable for plotting purposes, it is inconvenient for further data analysis. Reasons for this are as follows:

a. Almost all data analysis efforts involve investigating the behavior of various recordings at a single point in time. The formating of the N3 tapes is not readily conducive to this type of investigation.

b. Missing time values occur and are completely ignored. Hence, recordings are not available for all channels for all times.

c. No attempt has been made to identify and correct outlying or erroneous observations. Outlying observations are defined as values that seriously depart from the trend of the time series.

d. Corresponding time values for different channels do not exactly coincide. The data reduction code which applied calibrations to the data adjusted the frame time to account for the time it took the multiplexer to move from pin to pin. Since measurements on differing pins occur at different times, it is impossible to perform a standard merge on time to reach the desired multiplexer order (Item a above).

e. No smoothing of the data has been provided. Smoothing has the effect of reducing the random component of variance in the time series, thus allowing the overall trend of the data to be viewed more easily. Smoothing is desirable if the data are to be used as direct input to data analysis programs.

To overcome these deficiencies, the Statistical Edit and Smooth program was developed. A detailed write-up of this program is included as Appendix B. The program, as written, provides only situations b through e. However, on the resulting binary tape each time and corresponding smoothed channel value has associated with it an integer time which enables the merging of time and channel recordings so as to produce the desired multiplexer type formating discussed in paragraph a.

The program edits the observations by applying a moving polynomial curve fit to the data, estimating the expected value and standard deviation of the next point in time, and comparing the observed value at this point to these 
estimates on a statistical basis. Observations that are missing are replaced in the original series by $-1.0 \times 10^{16}$. These values will be judged outliers in the editing procedure, thus assuring a uniform series of outputted values. When the program notices a series of ten successive outliers, it infers that the form of the underlying time function has changed. A new polynomial is then fitted to the original data beginning with the time corresponding to the occurrence of the first "outlier" in that series (sometimes called the switch point).

Smoothing and time synchronization are accomplished by evaluating the polynomial near the midpoint of the fitted span at a point corresponding to the desired output time. The desired output sampling rate is an input to the program, and must be equal to or less than the sampling rate of the original series. In addition, the program provides an estimate of the random component of variance in the time series. This value is very useful for many data analysis programs, since it is often desirable to normalize the input variables. It also prints out information regarding switch points.

In a number of ways, this program is similar to a series of subroutines being developed at Redstone Arsenal (Reference 20). Whereas Redstone is developing a series of general-purpose subroutines aimed at performing the same general type of data analysis activity independent of data format, this program was developed as a special-purpose program for the format of the NRX-Al data. Should the format of data from future NRX or XE tests change, it would be necessary to modify the existing program to be compatible with that particular format. The mathematical principles underlying the program are valid for any type of data format.

The program was applied to all NRX-AI N3 data tapes for the EP-II and EP-IV series. The resulting tapes for each test series were merged to obtain for each a single tape giving time and all smoothed channels values for that time as a single logical record. Smoothed data values were output at a sampling rate of 1 per sec. The primary purpose for this processing was to smooth and align the data for identical times, so as to make possible an investigation of the problems of asymmetry. The results of this investigation were reported in Reference 3 . The original objective for this program was to prepare the data for validation and improvements of the engine system simulator model computer job (Reference 4 ). Work on this latter activity will be discussed further in Section III,C. 


\section{Statistical Analysis of Asymmetry}

The results of the asymmetry investigations for NRX-Al reported in Reference 3 were based primarily on engineering judgements. Detailed statistical analyses of the asymmetry data are planned for future tests. To illustrate the type of analyses planned, a sample analysis of one test time for one location is presented here. The test location chosen for this illustration is the core inlet plenum temperature for EP-IV liquid into gas ( $1.25 \mathrm{lb} / \mathrm{sec}^{2} \mathrm{ramp}$ ). The data analyzed are shown in Table 1. This particular set of data was chosen for illustration chiefly because the data displayed a constant variance over the duration of the test. Similar analyses are possible for the test location combinations not displaying a homogeneous variance. However, such analyses are somewhat more complicated and will not be discussed in this report.

As a first step in the analysis, a two-way analysis of variance was performed on the data to separate the variation due to time from the variation due to column effects (theta, radius, and instrument-to-instrument variability). This analysis of variance indicated both the time effect and the column effect to be highly significant. To view more clearly the column effect, which contains the effect of angular asymmetry (theta), the time effect was removed from the data. A time effect is certainly expected due to the transient nature of the test, and the removal of this effect will simplify the analysis by reducing it to a one-way analysis of variance. The time effect was removed by subtracting the time (row) means from each of the data points at that time. These data are displayed in Table 2. Column-to-column differences are readily apparent.

As stated above, the column effect is composed of three components: the effect due to angular asymmetry, the effect due to radius, and the effect due to instrument-to-instrument variability. A statistical test for the significance of asymmetry must be made by comparing the mean square due to theta with the mean square due to instruments. Thus, the column effect must be broken into its various components.

An examination of the theta values reveals that two recordings were made at each (or near each) of three theta values. Channels 720 and 721 were each at $1^{\circ}$ theta, channels 725 and 726 were each at $121^{\circ}$ theta, and channels 730 and 728 were at $241^{\circ}$ and $242^{\circ}$ theta, respectively. This allows the column variation 
to be separated into two components - that due to theta and that due to instruments. This is done by obtaining the between column sum of squares for all 12 columns and subtracting from this the sum of the between column sum of squares for columns $720-$ 721, 725-726, and 730-728. These components, however, contain the confounded effect of radius. The analysis-of-variance table for the data in Table 2 , ignoring the effect of radius, is given in Table 3.

It is impossible to estimate the true effect of radius due to a lack of sufficient degrees of freedom. If it can be assumed that the effect is linear, it is possible to estimate this linear effect, and to adjust the data accordingly so as to remove the influence of radius from the estimated effects of theta and instruments. This is done by calculating

$$
b=\frac{\sum_{i=1}^{12} r_{i} \bar{c}_{i}}{\sum_{i=1}^{12} r_{i}^{2}}
$$

where $r_{i}=R_{i}-\bar{R}$, with $R_{i}$ being the radius value for the ith column, and $\bar{R}$ the overall radius mean; $c_{i}$ is the mean value of the deviations from the time means for the ith column. Performing the adjustments

$$
\begin{aligned}
& \hat{y}_{i j}=y_{i j}-b r_{i} \text {, for } i=1, \ldots, 12 \\
& j=1, \ldots, 24
\end{aligned}
$$

where $\hat{y}_{i j}$ represents the adjusted values, $y_{i j}$ represents the unadjusted values, and $\mathrm{b}$ and $\mathrm{r}_{i}$ are as previously defined. For the data in Table 2 , $\mathrm{b}$ was estimated to be -1.9585. The adjusted table of values is shown as Table 4. The analysis of variance performed on the adjusted data is shown in Table 5. The adjustment for the effect of radius resulted in the loss of one degree of freedom between columns. Since there are eight unique levels of theta, with instruments degrees of freedom being obtained by subtraction in partitioning the column degrees of freedom, this loss of one degree of freedom was assigned to instruments. 


\section{RN-S-0146}

A comparison of the two analysis-of-variance tables indicates that the correction for radius reduced the total sum of squares by better than a factor of two. Further, it reduced the mean square due to instruments by a factor of four. This indicates that the effect of radius was obviously significant.

On the basis of this analysis of a single test location combination, several important facts can be established:

a. Instrument repeatability is extremely good. The standard deviation of instrument repeatability is estimated to be $2.5^{\circ}$.

b. Instrument-to-instrument variation may be slightly smaller than expected. However, in this instance it was large enough to obscure any existing asymmetry variation, although none was expected for this particular test location combination. For the adjusted data the between-instrument variances for the three equal theta values were estimated as $2.83,16.24$, and $57.96^{\circ}$ squared, for standard deviations of $1.7,4.0$, and 7.6 respectively. On the basis of viewing other data at equal theta values, it is quite possible that the value $57.96(7.6$ standard deviation) may be considered as an outlier. Further analysis would be required to establish a "good" estimate of the instrument-to-instrument standard deviation.

\section{COMPARISON OF TEST RESULTS WITH ANALYTICAL MODELS}

Systems analysis models will be evaluated by a comparison of predicted versus observed quantities. The validity of the model will be dependent on the assumed form of the equations and method of calculation, but will also be affected by the values of model parameters and by the assumed boundary conditions. Evaluation would preferably trace model lack of fit to sources despite systematic and random errors in instrumentation.

Considerable knowledge of the precision, accuracy, and time behavior of the instrumentation system will be required to separate instrumentation effects from those due to model imperfections. Proper calibration and treatment of calibration data will be an important factor. As will be seen, it appears possible to utilize a comparison of calculated and measuren temperature and pressure of the entering fluid as a partial measure of model vaidity. Errors in the model parameters do not seem easily separable from other model defects due to model complexity. 
However, a careful analysis of the pattern of deviation of model predictions from observations, combined with physical theory, may supply information concerning the nature of any deficiencies in the model.

An important adjunct to such an analysis is the determination of the effect on model predictions of errors in model input and in model parameters. The determination of this effect is essentially a sensitivity analysis which can be carried out using propagation of error techniques. Such a procedure is discussed in Section III,C,3. An example of propagation of error to a heat transfer model is given in Appendix D.

1. Data Analys is Using Computer Job No. 364

a. Methods of Compensating for Errors in Assumed or Measured Inlet Conditions

The engine system simulator, Computer Job No. 364, is based on a deterministic model which must satisfy all boundary conditions. The model used for the NRX-Al Data Analysis Report (Reference 3) was for the nozzle subsystem. For this model there are no outlet constraints; thus fluid characteristics in the nozzle are completely determined by nozzle temperature and the conditions and flow rate of the entering fluid. In this situation, there are two immediately obvious courses to follow when making downstream predictions. These courses of action are described below.

(1) The first option is to use the planned test profile to obtain inlet flow, pressure, and temperature. This is the course of action when pretest predictions are used. However, any deviations from planned inlet conditions during the actual test will cause complex deviations between predicted and observed downstream fluid flow, pressure, and temperature. It would be difficult to determine the cause of the observed deviations, and they would likely be considered due to deficiencies in the model.

(2) The second option is to use observed inlet conditions as input for the computer program when making downstream predictions. This was the course of action partially followed in Reference 3. The flow rate measurement is made some distance away from the nozzle, and differs significantly. The use of observed inlet conditions has a severe limitation when there are instrumentation 
RN-S-0146

problems. In certain ranges of operation, large systematic measurement errors can occur. When these biased observations are used to make downstream predictions, they cause extremely unrealistic predictions to be made. In addition, random errors in inlet measurements are propagated through the model to the values predicted downstream. As a result, the statistical analysis of prediction versus observation deviations is more complex and much less sensitive.

The disadvantages of the two methods cited above led to the development of a less obvious, alternative method which has certain advantages for model evaluation. This alternative method consists of selecting initial conditions which best fit the model predictions to the downstream observations in the sense of least squares. Such a procedure arbitrarily eliminates random and systematic errors in inlet conditions from downstream comparisons. This is essential because the downstream conditions are not directly dependent on measured inlet conditions, since flow rate is not measured at the model boundary. Time vectors of computed inlet pressure, temperature, and flow rate would be an output which could be compared with observed and/or planned inlet conditions. Any model inconsistencies related to initial conditions only would be exposed as relatively simple trends or biases in inlet deviations rather than as complex interactions downstream. With errors in starting conditions eliminated, deviations downstream can be directly related to the type of measurement, location in the system, and time effects. Such an analysis of deviations will be helpful in explaining and eliminating any inconsistencies in the model. This approach has been referred to as direct use of Computer Job No. 364 and is discussed below.

The method of obtaining pseudo starting conditions is described in some detail here by utilizing the approximating equations currently being evaluated.

$$
\begin{aligned}
& \text { Consider the heat-transfer equation } \\
& W C_{W} \frac{d T_{W}}{d t}=\dot{W} C_{p}\left(T_{i}-T_{O}\right)+Q_{f} Q_{m}
\end{aligned}
$$

where

$$
\begin{aligned}
\mathrm{W} & =\text { mass of wall, } \mathrm{lb} \\
\mathrm{C}_{\mathrm{W}} & =\text { specific heat of wall, Btu/lb- }{ }^{\circ} \mathrm{R} \\
\mathrm{T}_{\mathrm{W}} & =\text { wall temperature }
\end{aligned}
$$




$$
\begin{aligned}
t & =\text { time, sec } \\
Q_{f} & =\text { spatial distribution factor } \\
Q_{m} & =\text { power level } \\
\dot{\mathrm{w}} & =\text { fluid flow rate, } \mathrm{lb} / \mathrm{sec} \\
\mathrm{C}_{\mathrm{p}} & =\text { specific heat of fluid, } \mathrm{Btu} / \mathrm{Ib}-\mathrm{O}_{\mathrm{R}}
\end{aligned}
$$

and where the subscripts have the following meanings:

$$
\begin{aligned}
& 0=\text { out (fluid departing) } \\
& i=\text { in (fluid entering) } \\
& \mathrm{w}=\text { wall }
\end{aligned}
$$

Several simplifying assumptions made in developing this equation are described briefly below.

The assumed heat-transfer model is based upon fluid flow through passages in a solid material. Energy may be received by the solid material from external sources, and heat is transferred from the solid to the fluid.

The energy received is expressed as the product $Q_{f} Q_{m}$ where $Q_{m}$ refers to the energy level of the device or component that radiates the energy and where $Q_{f}$ is the proportion received by the component under consideration.

The energy transferred to the fluid is assumed to be used only to increase the temperature of the fluid. It is also assumed that the fluid flow rate is the same at the entrance and exit of the component, and that there is an insignificant change in the enthalpy content of the fluid stored in the flow passages in the component. To simplify the derivation further, the gain in enthalpy of the fluid is expressed in terms of an average specific heat for the fluid. This total gain in enthalpy of the fluid can therefore be written $C_{p} \dot{w}\left(T_{0}-T_{i}\right)$. It is further assumed that the enthalpy change of the fluid is the only significant change in energy of the fluid.

When the temperature of the solid material changes, its enthalpy changes. The rate of change of enthalpy is equal to the mass of the solid, $W$, times its average specific heat, $C_{W}$, times the rate of change of the average temperature of the solid material, $\mathrm{dT}_{\mathrm{W}} / \mathrm{dt}$. For simplicity, we assume that the rate of change of the average temperature of the solid material is equal to the rate of change of the average of the temperature measurements. Based upon these 
assumptions we can write Equation (26), noting that the first term on the righthand side of this equation is equal to the total energy transferred from the fluid to the wall.

If flow rate at a flow node, $\alpha$, is considered to be proportional to starting flow rate, say, $\dot{\mathrm{w}}(\alpha)=\mathrm{g}(\alpha) \dot{\mathrm{w}}_{\text {start }}$, then Equation ( 26 ) can be rearranged for a flow node, $\alpha$, as

$$
T_{0}-T_{i}=\frac{Q_{f}(\alpha) Q_{m}-W(\alpha) C_{W}(\alpha) \frac{d T_{w}(\alpha)}{d t}}{g(\alpha) \dot{W}_{\text {start }} C_{p}(\alpha)}
$$

All parameters are assumed to be location-dependent. The function $g(\alpha)$ would be derived from system geometry considerations.

Computer Program No. 364 can be used to sum Equation (27) over all node numbers on the flow path to location, $j$, to give

$$
\stackrel{\hat{T}}{j}_{j}-T_{S}=\frac{1}{\dot{w}_{S}} h_{j}
$$

or

$$
\hat{\mathrm{T}}_{j}=\mathrm{T}_{\mathrm{s}}+\frac{1}{\dot{\mathrm{w}}_{\mathrm{S}}} \mathrm{h}_{j}
$$

where

$$
\begin{aligned}
& \hat{\mathrm{T}}_{\mathrm{j}}=\text { approximate calculated temperature at location } \\
& \overrightarrow{\mathrm{T}}_{\mathrm{S}}=\text { starting temperature } \\
& \dot{\mathrm{W}}_{\mathrm{S}}=\text { starting flow rate } \\
& \mathrm{h}_{\mathrm{j}}=\sum_{\alpha=1}^{j} \frac{+Q_{f}(\alpha) Q_{\mathrm{m}}-\mathrm{W}(\alpha) \mathrm{C}_{\mathrm{W}}(\alpha)\left(\frac{d \mathrm{~T}_{\mathrm{W}}(\alpha)}{\mathrm{dt}}\right)}{\mathrm{g}(\alpha) \mathrm{C}_{\mathrm{p}}(\alpha)}
\end{aligned}
$$

Equation (29) can now be used to minimize the sum of the squared deviations between calculated and observed temperatures. Let

$$
R=\sum_{j=1}^{m}\left(T_{j}-\hat{T}_{j}\right)^{2}
$$


where

$$
\begin{aligned}
\mathrm{T}_{j}= & \text { observed temperature at location } \\
& (j=1,2, \ldots, \mathrm{m}) \\
\mathrm{m}= & \text { number of locations where observations are made. }
\end{aligned}
$$

Then using Equation (29)

$$
R=\sum_{j=1}^{m}\left[T_{j}-\left(T_{s}+\frac{I}{\dot{w}_{s}} h_{j}\right)\right]^{2}
$$

Taking partial derivatives with respect to $\mathrm{T}_{\mathrm{S}}$ and $\mathrm{W}_{\mathrm{S}}$ gives

$$
\begin{array}{r}
\frac{\partial R}{\partial T_{s}}=2 \sum_{j=1}^{m}\left(T_{j}-T_{s}-\frac{1}{\dot{w}_{s}} h_{j}\right)(-1) \\
\frac{\partial R}{\partial \dot{w}_{S}}=2 \sum_{j=1}^{m}\left(T_{j}-T_{s}-\frac{1}{\dot{w}_{s}} h_{j}\right)\left(\frac{1}{\dot{w}_{s}^{2}} h_{j}\right)
\end{array}
$$

Equating these derivatives to zero gives

$$
\begin{gathered}
\sum_{j=1}^{m} T_{j}-m_{s}-\frac{1}{\dot{w}_{s}} \sum_{j=1}^{m} h_{j}=0 \\
\sum_{j=1}^{m} T_{j} h_{j}-T_{s} \sum_{j=1}^{m} h_{j}-\frac{1}{\dot{w}_{s}} \sum_{j=1}^{m} h_{j}^{2}=0
\end{gathered}
$$

Equations (34) and (35) can be directly solved for the values of $T_{S}$ and $\dot{\mathrm{w}}_{\mathrm{S}}$ which will minimize $R$. This will not ordinarily be a final solution since the values of $\mathrm{h}_{j}$ used in the calculation are affected by the starting conditions of the fluid. It will therefore be necessary to use some trial starting conditions with Computer Program No. 364 to generate an initial set of $h_{j}$ values. New values of $T_{S}$ and $\dot{w}_{S}$ would then be obtained from Equations (34) and (35). These can then be used to estimate a new starting pressure as described below and the process repeated until the value of $h_{j}$ is sufficiently stable. 
The deviations between predicted and observed downstream pressures would be minimized in similar manner by using Equation (B-9) in Reference 23, which is itself a more accurate version of Equation (113) in Reference 4. Changing the notation slightly this equation is

$$
\mathrm{P}_{\mathrm{s}}^{2}-\hat{\mathrm{P}}_{\mathrm{j}}^{2}=\sum_{\alpha=1}^{\mathrm{j}} \dot{\mathrm{w}}_{\alpha}^{2}\left(2 \mathrm{k}_{2} \mathrm{~T}_{\alpha-1}+2 \mathrm{k}_{3} \mathrm{~T}_{\alpha}\right)
$$

where

$$
\begin{aligned}
\hat{P}_{S} & =\text { starting pressure } \\
\hat{P}_{j} & =\text { approximate calculated pressure at location } \\
k_{2} \text { and } k_{3} & =\text { constants defined in Reference } 23 \\
\alpha & =\text { index indicating node number }
\end{aligned}
$$

Assume that an approximate starting temperature and flow rate have been determined by using Equation (29) and the method described above. By use of Computer Program No. 364, the sum on the right-hand side of Equation (36) can then be determined from the starting inlet conditions for any location in the system. Denote this sum by $k_{j}$ for location $j$. Then

$$
\hat{P}_{j}^{2}=P_{s}^{2}-k_{j}
$$

or

$$
\hat{P}_{j}=\left(P_{s}^{2}-k_{j}\right)^{1 / 2}
$$

To simplify the notation, let

$$
\left|\mathrm{P}_{\mathrm{s}}^{2}-\mathrm{k}_{j}\right|^{1 / 2}=\mathrm{f}_{j}
$$

Starting with an approximate value of $P_{S}, f_{j}$ can be evaluated. If an adjustment to the preliminary estimate of $P_{S}$ is given by $\delta P_{S}=b$, then an improved value of $f_{j}$ is given by

$$
f_{j}+b f_{j}^{\prime}
$$

where

$$
f_{j}^{\prime}=\frac{d f_{j}}{d P_{s}}
$$


The equation

$$
\hat{\mathrm{P}}_{j}=f_{j}+b f_{j}^{\prime}
$$

can then be considered as a linear regression of $\hat{P}_{j}$ on $f_{j}^{\prime}$ with known intercept $f_{j}$ in which the coefficient $b$ gives the adjustment to $P_{S} \cdot$ Once the adjusted value, $P_{s}+b$, has been determined, new values of $f_{j}$ and $f_{j}^{\prime}$ can be found and the regression must be calculated again.

The least-squares estimate of $b$, the adjustment for $\mathrm{P}_{S}$, is obtained as follows: Let

$$
Q=\sum_{j=1}^{n}\left(P_{j}-\hat{P}_{j}\right)^{2}=\sum_{j=1}^{n}\left(P_{j}-f_{j}-b f_{j}^{\prime}\right)^{2}
$$

where

$$
\begin{aligned}
& P_{j}=\text { observed pressure at location } \\
& \mathrm{n}=\text { number of downstream locations where pressure observations } \\
& \text { are made } \\
& f_{j}=\left(P_{s}^{2}-k_{j}\right)^{I / 2} \\
& f_{j}^{\prime}=\frac{P_{S}}{\left(P_{s}^{2}-k_{j}\right)^{1 / 2}}
\end{aligned}
$$

$Q$ will be minimized with respect to $b$ when the partial derivative is zero.

$$
\begin{aligned}
& \frac{\partial Q}{\partial b}=\sum_{j=1}^{n} 2\left(P_{j}-f_{j}-b f_{j}^{\prime}\right)\left(-f_{j}^{\prime}\right) \\
& \sum_{j=1}^{n}\left[P_{j} f_{j}^{\prime}-f_{j} f_{j}^{\prime}-b\left(f_{j}^{\prime}\right)^{2}\right]=0 \\
& \mathrm{~b}=\frac{\left[\sum_{j=1}^{n}\left(P_{j_{j}} f^{\prime}-f_{\left.j_{j} f_{j}^{\prime}\right)}\right]\right.}{\sum_{j=1}^{n}\left(f_{j}^{\prime}\right)^{2}}
\end{aligned}
$$


For each improvement in $P_{S}$, new values of $f_{j}$ and $f_{j}$ ' would be computed and the process repeated until convergence on $\mathrm{P}_{\mathrm{S}}$ was obtained.

This value of $P_{S}$ would be combined with the $T_{S}$ and $\dot{w}_{S}$ obtained from Equations (34) and (35) to compute new $h_{j}$ values. The complete cycle of minimizing temperature deviations and pressure deviations would be repeated until the starting conditions were stabilized. Each time point would be handled independently so that a trace over time of pseudo starting conditions would be generated. These starting conditions are used by Computer Program No. 364 to make downstream predictions.

b. Least-Squares Estimation (Regression Analysis) When Both Variables are Subject to Random Fluctuation

The method of estimation used above requires the use of iterative regression schemes which result in least-squares estimates of inlet conditions. As each iteration is carried out, temperature and pressure observations within the nozzle are used to estimate inlet conditions which are in turn used to calculate certain parameters. The latter are considered as independent variables in each iteration, but become affected by a random component as iterations are continued. This gives rise to a problem which is not considered to be serious here but which has received widespread attention and should be recognized as a possible source of bias in the estimates. This problem is the fitting of a function when both variables are subject to random error.

Regression analysis may be defined as the estimation or prediction of the value of one variable (the dependent variable) from values of other given variables (the independent variables). Alternatively, regression analysis may be viewed as a relation between the expected value of the dependent variable and the observed values of the independent variables. It is usually assumed, in regression analysis, that the independent variables are errorless, so that their expected values and observed values coincide. If, on the other hand, the expected value of the dependent variable is a function of the expected values of the independent variables, then a regression analysis becomes considerably more complex.

A functional expression between the expected value of the dependent variable and the expected values of the independent variables implies a relationship among the parameters of the distributions of different variables. It 
is known from Reference 21 , that if both the dependent and independent variables are normally distributed, a functional relationship cannot be determined from the data. Reference 22 indicates that at least one of the distributions must necessarily be non-normal in order to estimate the relationship. To put this fact in a heuristic light, the first- and second-order sample moments exhaust all the information carried within samples from normal populations. It is necessary, therefore, that information beyond that contained in the first-and second-order moments of the distribution be available if a functional relation is to be determined. This information may be present in the sample if the distribution is not normal.

It is not considered likely that the parameters used as independent variables will have normally distributed errors, since they are nonlinear functions of variables (inlet conditions) which are expected to be more or less normally distributed.

\section{Statistical Tests of Validity of Model}

A test of the validity of the model must allow for random errors in the observed temperatures and pressure, and possibly in the predictions of these quantities. As an example of the latter, some model parameters might be based on measurements, so that random measurement errors would be included in model predictions.

For the present it will be assumed that model parameters are predetermined and do not contribute random error to predictions, although they may be a source of systematic error as previously mentioned. The predicting model will then be completely deterministic. If the model were a perfect predictor and exact inlet conditions were known, deviations between downstream predictions and measurements would be due solely to measurement (instrumentation) errors. A deterministic model is unlikely to be a perfect predictor, of course, since there will be apparently random variations in temperature of pressure at any one location in the system due to complex instabilities and turbulence in the fluid. Therefore, it will be assumed initially that the model is an unbiased predictor, with random deviations averaging to zero. Deviations over time would then be the result of a combination of actual variation in fluid characteristics and apparent variation due to measurement errors. If the precision and time behavior of a measuring instrument were known exactly, then this component could be taken out of the total to determine the statistical nature of the actual variation at that location. 


\section{RN-S-0146}

In addition to variations over time, there will be a variation between the average deviations at different locations. Under the assumption of an unbiased predicting model, variation between locations in the system would be largely due to instrument inaccuracies. If this variation could be observed over a sufficient number of locations or identical runs, it would be a measure of a random instrument calibration error. It would obviously be necessary to know the magnitude of this calibration error in order to determine whether differences between locations might be due to actual failure of the model to predict system behavior.

Under ideal conditions of kriown starting conditions and known instrumentation errors, a statistical test of model validity would thus require a hypothesis that the model is an unbiased predictor. This hypothesis would be accepted or rejected by comparing a measure of the deviations between locations in the system with a known measure of instrument calibration error. If the observed deviations were too large in magnitude, then the hypothesis would be rejected. The pattern of deviation through the system would then have to be analyzed. Deviations over time at any location would be compared with the known time behavior and precision of the instrument. Any unexplained random behavior would be attributed to essentially unpredictable flow behavior. Any unexplained non-random behavior world be attributed to an inadequacy in the predicting model.

Unfortunately, for the present analysis, starting conditions are not known and complete information is not available on irstrument accuracy and precision. Under these circumstances, the statistical analysis will be more involved and subject to more assumptions.

A suggested first step would be a preliminary analysis using a non-random input to Computer Program No. 364 which corresponded to the planned test profile. For any instrument location in the system and at a discrete point in time, a smoothed observation and its standard deviation will be obtained from the Statistical Edit and Smooth Program. Coordination of this information with the output of Computer Program No. 364 will produce a difference corresponding to the deviation of the calculated value from the observed value. Under the hypothesis of a perfect predicting model and unbiased instrumentation, these deviations will be due to random errors in instrumentation and random variation in fluid 
characteristics. The composite of these random components will be estimated by the standard deviations of the smoothed observations. If we assume independent and normally distributed errors, each deviation can be divided by the standard deviation of the observed quantity to give variates which are independently and normally distributed with zero mean ard unit variance. Under the hypothesis stated, the sum of squares of $\mathrm{Ni}$ of these standardized deviations will have a chi-square distribution with $N$ degrees of freedom. A significantly large chisquare value over all observations would therefore indicate significant lack of fit of the model, biased instrumentation, or both. In this case, a partition of the sum of squares into components due to overall bias, type of observation, location in the system, and simple time trerds within locations should result in a non-significant residual chi-square value. If so, this would verify the completeness of the analysis.

The next step would be to use the smoothed observations to determine pseudo starting conditions which would then be used to make model predictions. The differences could again be normalized by dividing by the standard deviation of the smoothed observation. In fact, because of the probable nonhomogeniety of the variances of the observations, it may be preferable to minimize the normalized differences in the least-squares fit. This could be accomplished by minor changes in Equations (30) and (41).

The quantities minimized would be

$$
E^{\prime}=\sum_{j=1}^{m}\left[\frac{T_{j}-\left(T_{S}+\frac{1}{\dot{W}_{S}} h_{j}\right)}{\sigma\left(T_{j}\right)}\right]^{2}
$$

and

$$
Q^{\prime}=\sum_{j=1}^{n}\left[\frac{P_{j}-f_{j}-b f_{j}{ }^{\prime}}{\sigma\left(P_{j}\right)}\right]^{2}
$$

where

$$
\begin{aligned}
\sigma\left(T_{j}\right)= & \text { standard deviation of the smoothed temperature } \\
& \text { observation } \\
\sigma\left(P_{j}\right)= & \text { standard deviation of the smoothed pressure } \\
& \text { observation }
\end{aligned}
$$


RN-S-0146

When a least-squares fit is used, the normalized differences would not have unit variance. This is because of random prediction errors incorporated into each difference by the least-square adjustment. If the previous analysis with fixed input achieved a non-significant residual, then the same analysis on this data would establish the magnitude of the residual to be expected with the least-squares predictions.

The basic analysis would require that an equation be fitted to the time trend within each instrument. This would be a polynomial of high enough degree to reduce the residual to the proper order of magnitude. The result would be a description of the time behavior of the system at that point.

The average behavior of the system over locations will be more difficult to determine independently of instrumentation errors. One course would ke to assume that any low degree trends through the system from inlet to outlet would be due to actual differences between system operation and model predictions. Any trends of high degree would be considered due to instrument inaccuracies. This is more likely to be true with the disturbing influence of starting condition errors removed. In fact, with the least-squares predictions, the deviations remaining may be primarily due to instrumentation errors.

Another procedure for estimating between instrument error would be to determine from an examination of the location of the instruments those which are so close together that their differences reflect instrument error rather than differences in fluid characteristics. An example of this procedure is illustrated in Section III,B,3.

The remaining part of the analysis would be to compare the pseudo starting conditions with some other estimate of actual starting conditions. This would require some statistical tests of significance and a determination of the actual cause, in terms of the model, of any definite deviations. The latter determination is not a statistical problem.

In all of the analysis described, the assumption of the statistical independence of observations between time points, within and between locations, will be critical. Strong correlations would invalidate all of the statistical analysis, including the proposed least-squares estimates of starting conditions. 
However, instrumentation will generally be far enough apart to assure independence of errors between locations. In addition, any analysis over locations will be made on averages. The use of averages will tend to eliminate the effect of serial correlations within locations; however these correlations will have to be considered as a disturbing influence when fitting time trends within locations.

A statistical analysis of the deviations between calculated and measured quantities has been started. This aralysis has the goal of separating errors in the model from errors in instrumentation. Quantitative information on the sources of both types of errors will allow corrections in the model to be made, and will be of value in evaluating irstrumentation. Some of the difficlities involved ir such a statistical analysis have been discussed. This analysis appears feasible, although many of the details remain to be worked out during the next contract period.

\section{Effects of Input and Farameter Errors on Systems Model} Predictions

There are two important sources of error present in a systems model: (a) model error which arises from ar incorrect selection of parameter values, ard (b) experimental error which arises from the use of measured data as input to the model. In addition there is the possibility that these two errors will interact with one another in such a fashion as to make a separation of their effects extremely complex. It is the purpose of this section to consider the effects of these errors on model prediction errors. Attention will first be directed to a general function of two variables, $f(x, y)$, and the effect of using erroneous functional values.

Let the function $f(x, y)$ be defined on a region containing the point $\left(x_{0}, y_{0}\right)$ and assume that $f(x, y)$ admits a Taylor series expansion in a neighbornood of the point $\left(x_{0}, y_{0}\right)$. The function $f(x, y)$ may conveniently be viewed as a mold for some physical phenomena. If $f$ is evaluated at the point ( $\left.x_{0}, y_{0}\right)$ instead of at the point $(x, y)$, then an error has been introduced irto the model value. In order to evaluate the effect of this error, it is necessary to determine whether or not $f\left(x_{0}, y_{0}\right)$ lies outside of physically plausible limits. In practice, this determination is usually accomplished by considering the standard deviation of the 
function $f(x, y)$ near the point $\left(x_{0}, y_{0}\right)$. Usually, however, the standard deviation of a function is algebraically cumbersome to manipulate, so that in practice the calculations are generally performed by using the variance of the function. The variance is, of course, simply the square of standard deviation. Accordingly, since $f$ admits a Taylor series expansion, it is possible to show (see Reference 2l) that

$$
f(x, y)=f\left(x_{0}, y_{0}\right)+P_{n}+R_{n}
$$

in which

$$
\begin{aligned}
P_{n}= & \frac{\left(x-x_{0}\right)}{1 !} \frac{\partial f}{\partial x}+\frac{y-y_{0}}{1 !} \frac{\partial f}{\partial y}+\frac{\left(x-x_{0}\right)^{2}}{2 !} \frac{\partial^{2} f}{\partial x^{2}}+\left[\frac{\left(y-y_{0}\right)}{2 !}\right]\left[\frac{\partial^{2} f}{\partial f}\right]+ \\
& {\left[\frac{\left(x-x_{0}\right)}{1 !} \mid\left[\frac{\left(y-y_{0}\right)}{1 !}\right]\left[\frac{\partial^{2} f}{\partial x \partial y}\right]+\ldots+\left[\frac{\left(x-x_{0}\right)^{n}}{n !}\right]\left[\frac{\partial^{n} f}{\partial x}\right]+\ldots\right.} \\
& +\left[\frac{\left(x-x_{0}\right)^{n-1}}{(n-1) !}\right]\left[\frac{\left(y-y_{0}\right)}{1 !}\right]\left[\frac{\partial^{n} f}{\partial x^{n-1} \partial y}\right]+\ldots+\left[\frac{\left(y-y_{0}\right)^{n}}{n !} \mid\left[\frac{\partial^{n} f}{\partial y}\right],\right.
\end{aligned}
$$

and

$$
R_{n}=\left[\frac{\left(x-x_{0}\right)^{n+1}}{(n+1) !}\right]\left[\frac{\partial^{n+1} f}{\partial x^{n+1}}\right]+\cdots+\left[\frac{\left(y-y_{0}\right)^{n+1}}{(n+1) !}\right]\left[\frac{\partial^{n+1} f}{\partial y^{n+1}}\right]
$$

The partial derivatives in Equation (48) are to be evaluated at the point ( $\mathrm{x}_{0}, \mathrm{y}_{0}$ ) and the partials in Equation (49) are evaluated at a point ( $x^{*}, y^{*}$ ) which is located on the line segment connecting $\left(x_{0}, y_{0}\right)$ and $(x, y)$.

Equations (47), (48), and (49) give an exact expression for the functional value of $f(x, y)$. In practice, it is usually sufficient, for a first approximation, to consider only the linear term in Equation (48); that is, to assume that

$$
f(x, y)=f\left(x_{0}, y_{0}\right)+\left(x-x_{0}\right) \frac{\partial f}{\partial x}+\left(y-y_{0}\right) \frac{\partial f}{\partial y}
$$

The variance of $\mathrm{f}(\mathrm{x}, \mathrm{y}), \mathrm{V}[\mathrm{f}(\mathrm{x}, \mathrm{y})]$, is defined by the relation

$$
V[f(x, y)]=E\left[f^{2}(x, y)\right]-[E(x, y)]^{2}
$$


where $E$ is the linear mathematical expectation operator. The expectation of $f(x, y), E[f(x, y)]$ is

$$
\begin{aligned}
E[f(x, y)] & =E\left[f\left(x_{0}, y_{0}\right)\right]+\frac{\partial f}{\partial x} E\left(x-x_{0}\right)+\frac{\partial f}{\partial y} E\left(y-y_{0}\right) \\
& =f\left(x_{0}, y_{0}\right)
\end{aligned}
$$

since, for a large number of trends, it is expected that $E x=x_{0}$ and $E y_{0} y_{0}$; that is, there would be no error. Consequently, to a first approximation,

$$
\begin{aligned}
& V[f(x, y)]=E\left[f^{2}(x, y)\right]-f^{2}\left(x_{0}, y_{0}\right) \\
& =E\left\{\left[f^{2}\left(x_{0}, y_{0}\right)\right]+\left(x-x_{0}\right)^{2}\left(\frac{\partial f}{\partial x}\right)^{2}+\left(y-y_{0}\right)^{2}\left(\frac{\partial f}{\partial y}\right)^{2}\right. \\
& +2 f\left(x_{0}, y_{0}\right)\left(x-x_{0}\right)\left(\frac{\partial f}{\partial x}\right)+2 f\left(x_{0}, y_{0}\right)\left(y-y_{0}\right)\left(\frac{\partial f}{\partial y}\right) \\
& \left.+2\left(x-x_{0}\right)\left(y-y_{0}\right)\left(\frac{\partial f}{\partial x}\right)\left(\frac{\partial f}{\partial y}\right)\right\}-f^{2}\left(x_{0}, y_{0}\right) \\
& V[f(x, y)]=\left|\frac{\partial f}{\partial x}\right|^{2} E\left[\left(x-x_{0}\right)^{2}\right]+\left|\frac{\partial f}{\partial y}\right|^{2} E\left[\left(y-y_{0}\right)^{2}\right]+2\left|\frac{\partial f}{\partial x}\right|\left(\frac{\partial f}{\partial y} \mid E\left[\left(x-x_{0}\right)\left(y-y_{0}\right)\right]\right. \\
& \text { since } E\left[2 f\left(x_{0}, y_{0}\right)\left(x-x_{0}\right) \frac{\partial f}{\partial x}\right]=E\left[2 f\left(x_{0}, y_{0}\right)\left(y-y_{0}\right) \frac{\partial f}{\partial y}\right]=0 \text {. }
\end{aligned}
$$$$
\text { The expressions } E\left(x-x_{0}\right)^{2} \text { and } E\left(y-y_{0}\right)^{2} \text { are simply the variance of }
$$
the quantities $\mathrm{x}$ and $\mathrm{y}$ respectively, so that Equation (54) becomes

$$
V \mid f(x, y)]=\left(\frac{\partial f}{\partial x}\right)^{2} V(x)+\left(\left.\frac{\partial f}{\partial y}\right|^{2} V(y)+\left(\frac{\partial f}{\partial x}\right)\left|\frac{\partial f}{\partial y}\right| E\left[\left(x-x_{0}\right)\left(y-y_{0}\right) \mid\right.\right.
$$

The expression $\mathrm{E}\left(\mathrm{x}-\mathrm{x}_{\mathrm{O}}\right)\left(\mathrm{y}-\mathrm{y}_{\mathrm{O}}\right)$ is knowr as the covariance of the quantities $\mathrm{x}$ and $\mathrm{y}$ and measures the tendency of $x$ and $y$ to vary together. If $x$ and $y$ are independent, then $E\left(x-x_{0}\right)\left(y-y_{0}\right)$ is zero; however, the converse is not true. In this treatment, it is assumed that $E\left(x-x_{0}\right)\left(y-y_{0}\right)$ is zero. A useful measure of relative variation is provided by the coefficient of variation of a function, $c(f)$. Here $c(f)$ is defined by the equation

$$
C(f)=\sqrt{\frac{V(f)}{[E(f)]^{2}}}
$$

Extensive use of $\mathrm{c}(\mathrm{f})$ is made in Appendix $D$ where a specific example of error propagation in a heat-transfer model is presented. 
IV. ANALYSIS OF ANALOG DATA

The analog data is obtained from a measurement system (discussed in Section II) which is capable of recording, with high fidelity, the values of physical quantities which may change rapidly. Such changes may be intentional, as in the start-up transient or they may be due to the effect of small perturbations that are introduced into the system for response determination purposes. Changes also may be unintentional as would occur in self-excited system oscillations. The wide-band data system is capable of recording these rapidly varying quantities regardless of the cause. The analysis of the wide-band data therefore must be capable of using the data to show that the system performs as predicted by the mathematical models, to demonstrate any anomalies from the predicted performance and also to describe any phenomena that are not explicitly predicted by the mathematical models.

The necessary analyses are described herein, first on a qualitative basis which should be performed before any quantitative analyses are made. Next the several widely used quantitative methods are described and assessed for use. Then the various computer methods are considered. All of this suggests the use of the general purpose analog computer for wide-band data analysis, the details of which are described below.

\section{A. QUALITATIVE ANALYSIS OF WIDE-BAND DATA}

A large amount of useful information concerning the wide-band data can be described by qualitative measures. Usually simple visual inspection of the records displayed on time plots, together with engineering judgement, is all that is required. For this qualitative analysis of the data the following topics are considered separately: transients, oscillations, cause-effect relations, and instrumentation errors unique with wide-band data.

\section{Transients}

The transient behavior of the physical quantities of the system is very important but difficult to describe. Transients are assumed here to occur during start-up, at level changes, malfunctions, and shut-down.

The qualitative description of the transient data normally would answer such questions as 
a. How fast does the system respond?

b. Does the system overshoot? (how much)

c. Does the system oscillate? (what amplitude and frequency)

a. Do all values stay within design limits?

This information is useful to determine if design changes or additional controls are required, and also what safety features would be desirable.

\section{Oscillatory Modes}

Oscillations may exist in the system for two basic reasons: self-sustained oscillations due to some feedback mechanism, or response to a noise source at a resonant frequency. Quasi-self-sustained oscillations, such as lightly damped responses to level changes, might also be included.

The approximate amplitude and frequency of the oscillations are useful in deciding if the oscillations are harmful in themselves, and also if the oscillations may couple into other systems perhaps not on a particular test. It is also desirable to determine what quantities are obviously oscillating at the same frequency to check mathematical models in the gross sense.

\section{Cause-Effect Relations}

The mathematical model of the system assumes certain cause-effect relations. Therefore a useful result of the analyses of wide-band data is to check these relations. Also useful is to determine cause-effect relations that actually exist in test but which were neglected or missed in setting up the mathematical model. By simple visual inspection of data and good engineering judgements these cause-effect relations can be seen in steady operation, in transient conditions, and when intentional perturbations are applied.

\section{Instrumentation Errors Unique with Wide-Band Data}

The usefulness of the wide-band data is sometimes limited by the instrumentation system that may be completely adequate for low-frequency or narrowband data, but may not be adequate for high frequencies.

Examples of this include the use of small tubes or lines connecting a pressure transducer to a liquid system line. If some gas is trapped in the transducer line, then a spring mass resonant system is between the transducer 
$\mathrm{RN}-\mathrm{S}-0146$

and the pressure supposedly being measured. Such a system can amplify or attenuate pressures oscillations or changes. The presence of this type of error can usually be detected by noting particular frequencies at particular transducers that are not present in other transducers, since it is unlikely that two different lines, transducers, and tap positions would produce the same resonant frequency.

B. QUANTITATIVE METHODS OF DESCRIBING WIDE-BAND DATA PROPERTIES

The wide-band data quantitative description must cover two areas: individual properties, and relations to other variables. In describing the individual properties, calculations are made which characterize the data by means of certain "descriptors" such as statistical properties and power-density spectrum. In describing the relations to other variables, one can use correlation techniques or use transfer functions. A semi-qualitative analysis of relations between variables can be made by comparing power-density spectra.

These descriptors have been well developed and are in wide use. One use for them is given in Reference 26. Descriptors have much usefulness but also some limitations which will be discussed below. For the purpose of analysis of wide-band data, the following points will be considered, described, and assessed: (1) statistical properties, (2) correlation techniques, (3) power spectral density, and (4) transfer function analysis.

\section{Statistical Properties}

One of the simplest ways to describe data is by statistical properties such as averages, variance, and moment.s. For many applications this type of description is amply adequate. For example, the analysis of the narrowband NERVA data includes the calculation of the average and variance (see section on the ADDA program). If the variance is above a given minimum, then the frequency content of the data is determined.

The primary object of the recording of the wide-band NERVA data is to obtain validation of the system mathematical model and an understanding of the system behavior. The above statistical properties of the data alone are insufficient for this purpose but are useful in the evaluation of data quality and the identification of problem areas, particularly those related to high-frequency oscillations. 


\section{Correlation Techniques}

Correlation techniques, specifically the calculation of the crosscorrelation function $R_{x y}(r)$ will show the degree of similarity or correlation that exists between two signals $x(t)$ and $y(t)$ as a function of the lag time $(\tau)$ between them.

$$
R_{x y}(\tau)=\lim _{I \rightarrow \infty} \frac{1}{T} \int_{0}^{T} x(t) y(t-\tau) d t
$$

where

$$
\begin{aligned}
& x(t) \text { and } y(t) \text { are functions of time } \\
& \tau_{\text {is }} \text { the lag time, } 0 \leq \gamma \leq \gamma_{m} \\
& \tau_{m}=\text { maximum lag time, } \leq 1 / 10 \mathrm{~T} \\
& T=\text { record length, sec }
\end{aligned}
$$

The cross-correlation function $\mathrm{R}_{\mathrm{xy}}(\tau)$ may be normalized and then it is known as the coherence function $\Gamma_{x y}(\gamma)$ which lies in the region $(-1$ to +1$)$. To calculate the coherence function, in addition to the cross-correlation function $\mathrm{R}_{\mathrm{xy}}(\boldsymbol{\omega})$, the auto-correlation functions $\mathrm{R}_{\mathrm{xx}}(0)$ and $\mathrm{R}_{\mathrm{yy}}(0)$ are needed.

$$
\Gamma_{x y}(\tau) \leq\left[R_{x x}(0) \times R_{y y}(0)\right]^{1 / 2}
$$

where

$$
\begin{aligned}
R_{x x}(0) & =\text { auto-correlation of } x(t) \text { at } \tau=0 \\
& =\lim _{T \rightarrow \infty} \frac{1}{T} \int_{0}^{T} x^{2}(t) d t \\
R_{y y}(0) & =\text { auto-correlation of } y(t) \text { at } r=0 \\
& =\underset{T \rightarrow \infty}{\lim } \frac{1}{T} \int_{0}^{T} y^{2}(t) d t
\end{aligned}
$$

The correlation function or coherence function is very difficult to use directly in verifying mathematical models and in describing system behavior. 


\section{Power Spectral Density}

If $x(t)$ is a periodic function of period $T$ which has a finite energy per period $\left.\left.\left|\int_{0}^{\mathrm{T}}\right| \mathrm{x}(t)\right|^{2} \mathrm{dt}<\infty\right)$, then by the use of Parseval's theorem (Reference 27) the time average of its energy, or its power, is equal to a sum of terms, each of which is the time average of one particular component frequency.

The power spectral density does not yield phase information of the various frequency components since two functions that have the same magnitude of Fourier coefficients, but different phases, will have the same power spectral density function.

The power spectral density function may be calculated if the auto-correlation function is first obtained, as these two functions are related to each other by a Fourier transform pair.

$$
\begin{aligned}
& P_{x}(\omega)=\int_{-\infty}^{\infty} R_{x}(t) e^{-j \omega t} d t \\
& R_{x}(\tau)=\frac{1}{2 \pi} \int_{-\infty}^{\infty} P(\omega) e^{j \omega t} d \omega
\end{aligned}
$$

where

$$
\begin{aligned}
P_{x}(\omega) & =\text { power spectral density function of } x(t) \\
R_{x}(\boldsymbol{\gamma}) & =\text { auto-correlation function of } x(t) \\
\omega & =\text { radian frequency }=2 \pi f \text { (where } f \text { is in cycles } / \mathrm{sec} \text { ) }
\end{aligned}
$$

The power density spectra can be used directly to assess system behavior and indirectly to determine relations between parameters. Therefore this method is recommended as a basis for wide-band data analysis techniques when strong signal frequencies are identified.

\section{Transfer Function Analysis}

A transfer function is a function that relates an input to the output expressed as a function of frequency. Two functions are required to describe this relationship, one giving amplitude versus frequency and the other giving phase versus frequency. The transfer function is determined by dividing the crosspower spectral density by the power-density spectrum of the variable considered as the input. 
The cross-power spectral density $P_{x y}(\omega)$, where $x(t)$ is the input and $y(t)$ is the output, is a complex quantity whose real part is called the copower spectral density function $\left[C_{x y}(\omega)\right]$ and its imaginary part is called the quadpower spectral density function $\left[Q_{x y}(\omega)\right]$. This relationship is shown in Equation $(61)$,

$$
P_{x y}(\omega)=C_{x y}(\omega)-j Q_{x y}(\omega)
$$

The amplitude function $\mathrm{A}(\boldsymbol{W})$ is given below.

$$
A(\omega)=\frac{\left[C_{x y}^{2}(\omega)+Q_{x y}^{2}(\omega)\right]^{1 / 2}}{P_{x x}(\omega)}
$$

The phase function $\phi(\omega)$ is given by

$$
\phi(\omega)=\tan ^{-1}\left[\frac{-Q_{x y}(\omega)}{C_{x y}(\omega)}\right]
$$

The co-power and quad-power spectral density functions may be obtained by two methods: (a) by the time average of the signal amplitudes that are within the frequency range $(f+\Delta f)$, or $(b)$ by obtaining the Fourier transform of the cross-correlation functions.

\section{a. Time Average of Signal Amplitudes}

To employ the time average of the signal amplitudes that are within the frequency range $(f+\Delta f)$, the following equations are used:

$$
\begin{aligned}
& C_{x y}(f)=\lim _{T \rightarrow \infty} \lim _{\Delta f \rightarrow 0} \frac{I}{T(\Delta f)} \int_{0}^{T} x_{\Delta f}(f, t) y_{\Delta f}(f, t) d t \\
& Q_{x y}(f)=\lim _{T \rightarrow \infty} \lim _{\Delta f \rightarrow 0} \frac{l}{T(\Delta f)} \int_{0}^{T} \operatorname{mun}_{\Delta f}(t, t) y_{\Delta f}(f, t) d t
\end{aligned}
$$

where

$x_{\Delta f}(f, t)$ and $y \Delta f(f, t)$ are the signal amplitudes within the fre$\mathrm{x}_{\triangle f_{\mathrm{y}}(f, t)}^{(f, t)} \mathrm{x}_{\triangle f}(f, t)$ with a phase shift of $90^{\circ}$ with respect to 


$$
\begin{aligned}
\mathrm{T} & =\text { length of record } \\
\Delta \mathrm{f} & =\text { frequency bandwiath } .
\end{aligned}
$$

b. Fourier Transform of Cross-Correlation Functions

In obtaining the Fourier transform of the cross-correlation functions, the following procedure is followed. Let $R_{x y}(\boldsymbol{\gamma})$ be the cross-correlation function of signal $x(t)$ with respect to signal $y(t)$, at a lag time $\tau$. Let $\mathrm{R}_{\mathrm{yx}}(\tau)$ be the cross-correlation function of signal $\mathrm{y}(t)$ with respect to signal $x(t)$, at a lag time $\gamma^{\prime}$. Let

Then

$$
\begin{aligned}
& A(\boldsymbol{\tau})=1 / 2\left[\mathrm{R}_{\mathrm{xy}}(\boldsymbol{\tau})+\mathrm{R}_{\mathrm{yx}}(\boldsymbol{\tau})\right] \\
& \mathrm{B}(\boldsymbol{\tau})=1 / 2\left[\mathrm{R}_{\mathrm{xy}}(\boldsymbol{\tau})+\mathrm{R}_{\mathrm{yx}}(\boldsymbol{\tau})\right]
\end{aligned}
$$

$$
\begin{aligned}
& C_{x y}(f)=\int_{0}^{\infty} A(\mathcal{\gamma}) \cos (2 \pi f \mathcal{\gamma}) d t \\
& Q_{x y}(f)=\int_{0}^{\infty} B(\mathcal{\gamma}) \sin (2 \pi f \mathcal{\gamma}) d t
\end{aligned}
$$

The transfer function method of describing relations between two variables is very highly developed and widely used. The results are in a form suitable for direct use for control system purposes. It is limited by nonstationarity and low signal-to-noise ratios. The use of transfer function description of variable relations is recommended as a useful technique for wide-band data analysis.

\section{COMPARISON OF TEST WITH COMPUIER MODEL}

The classical methods of describing quantitative signal analyses are limited in use to steady-state, linear, time-stationary operation. During nonlinear transients, a different method of verifying the mathematical model is suggested.

The mathematical model of the system is simulated on the analog computer, including effects of nonlinear relations and dynamic terms. It is possible therefore to apply test data into the computer and determine some output from the computer model. By comparison of the computed output with measurement of the same 
RN-S-0146

quantity, one can obtain an error between the measurement and the simulation. The error could be measured in terms of the maximum difference, or the integral of the difference, or its square. Other criteria also could be used.

As an example, assume the pump head rise is computed in the simulation as a function of weight flow, speed, and suction pressure. Now by using measured values of weight flow speed and suction pressure as driving functions for the simulation, a computed pump head rise may be obtained. This computed head rise would be compared with the actual measured head rise and an error determined. Engineering judgemert would have to be used to determine the significance of the error. If the error were determined to be excessive and all measurements were determined to be accurate, then the model simulated on the system would have to be changed.

This type of comparison could be made for steady operations as well as transient conditions.

This method of analysis has advantages of not depending on linearity or stationary systems. The error could be defined in any number of ways which could be determined best after the error data had been generated.

D. POSSIBLE COMPUTER METHODS OF OBTAINING DATA DESCRIPTION

1. Digital Computer

The calculation of the data descriptors (Section III,B) by the use of a digital computer will be done by calculating the correlation functions and then obtaining their Fourier transforms (References 29 and 30).

As the Fourier transforms are infinite processes, their digital solution is based upon discrete finite approximations and so the results obtained are only estimates of their true value. The accuracy of the estimates obtained may be improved by smoothing, using "Hanning" weights, for example (Reference 28).

There are two disadvantages to the digital approach:

a. Test data that is recorded as an FM analog magnetic tape must be converted into digital form and re-recorded onto a digital tape for input to the digital computer.

b. The computing time will be proportional to the number of data points processed. For a job of the magnitude of the NERVA wide-band data 


$$
\text { RN-S-0146 }
$$

processing, it becomes prohibitive. A typical digital program to calculate correlation functions, power spectral density functions, and transfer functions is described by J.S. Bendat in Reference 30. The use of standard Fourier routines takes approximately 4.835 min for 1000 points, with 200 lag times $(\boldsymbol{\gamma})$. By using the modified Fourier analysis technique (Appendix A) in the analysis of the narrow-band data, it may be possible to reduce the time to approximately 2 min for 1000 data points. Another digital computer program is described in Reference 31.

\section{Analog Computer}

Use of the analog computer in analyzing wide-band data requires that the data be made available in the form of a voltage signal that is a measure of a variable. A magnetic tape playback unit is required.

Assuming such voltages are available, then the mathematical processes of averaging, filtering, comparing, etc. are performed using conventional highly developed, widely used techniques which are further described in Section IV,E,2.

The analog computer has advantages of using the tape directly. There is no sampling rate problem. It is flexible, and it provides the results as the problems are being run. Furthermore, control analysis groups have had extensive experience with this type of computer - a factor that could prove helpful should control system changes be required.

\section{Hybrid Computer}

The hybrid computer, which is a combination of analog and digital computers, can be used for data analysis. It is expected that the digital part would be used for memory, program control, and routine arithmetic operations, while analog part would be used for operations such as integration and averaging. In following such an arrangement, it may be possible to perform the analysis with less computing time using a computer that charges a lower rate, thereby effecting a cost savings. An example estimate was made for determining cross correlation using the Hydac 2400 hybrid computer and the IBM 7094. This operation showed that the actual computer time used would be 2.5 times less on the hybrid than the digital - a difference that is quite significant. One disadvantage of the hybrid is the limited experience in its use. 
For the present there does not seem to be any advantage in using the hybrid for transfer function aralyses over the analog. However, as more experience is gained in the use of the hybrid and more is determined as to what specific methods are best for the wide-band analysis, the use of the hybrid should be reviewed. It would use the input data in the same form as the analog so that no additional special playback equipment would be required.

E. DETAIL DESCRIPIION OF ANALOG COMPUTER METHOD OF ANALYSIS

\section{Nathematical Relations to be Computed}

The quantities to be computed are power-density spectra and transfer functions. These quartities are described in section IV, $B$.

Some of the errors involved in this process are described below. a. Error

The "estimated" values of the cross-correlation function $\hat{\mathrm{R}}_{\mathrm{xy}}(\tau)$; the power-spectral densities $\hat{\mathrm{S}}_{\mathrm{x}}(f), \hat{\mathrm{S}}_{\mathrm{y}}(\mathrm{f})$; and the cross-power-spectraldensity are in error because of two factors: ( 1 ) the finite length of the record, $T$ seconds; and (2) the finite width of the noise bandwidth $B$ (cps) of the spectrum analyzers.

The error $\varepsilon$ is given by:

$$
\varepsilon=(\mathrm{BT})^{-1 / 2} \quad \text { for } \mathrm{BT} \geq 10, \mathrm{~T} \geq \gamma
$$

If $\in$ is small, about 0.30 , we can say with a $68 \%$ confidence that the true values of $E_{x y}(f), S_{x y}(f), C_{x y}(f), Q_{x y}(f)$ are within the range

$$
\begin{aligned}
& (1 \pm \varepsilon) \hat{\mathrm{R}}_{\mathrm{xy}}(\gamma) \\
& (1 \pm \epsilon) \hat{S}_{\mathrm{xy}}(f) \\
& (1 \pm \epsilon) \hat{\mathrm{C}}_{\mathrm{xy}}(f) \\
& (1 \pm \epsilon) \hat{Q}_{\mathrm{xy}}(f)
\end{aligned}
$$

b. Resolution

For the correlation functions, the functions are evaluated at different lag times $(\boldsymbol{\gamma})$, thus obtaining a plot of function vs lag time. The 
resolution is determined by the change in $\mathcal{\gamma}$ between points; $(\Delta \tau)$ should obey the following inequality:

$$
\Delta \gamma<(4 \mathrm{~B})^{-1}
$$

where $B$ is the noise bandwidth of the signal in cps.

$$
\text { For the power-spectral density functions } \hat{\mathrm{S}}_{x y}(f), \hat{\mathrm{C}}_{x y}(f) \text {, }
$$
$\hat{Q}_{x y}(f), \hat{S}_{x}(f), \hat{S}_{y}(f)$, the resolution is a function of the filter bandwiath $B$ and the frequency difference, taken at the half power points, of the peak signal one wishes to resolve. The filter bandwidth $B$ (cps) is determined by the inequality

$$
B<\frac{(\Delta f)}{4}
$$

where $\Delta f$ is the frequency width of the signal peak measured at the half-power points.

$$
\begin{aligned}
& \text { c. Scan Rate } \\
& \text { For the correlation functions } \mathrm{R}_{x y}\left(\tau^{\prime}\right), \mathrm{R}_{\mathrm{xx}}(\tau) \text { and } \mathrm{R}_{\mathrm{yy}}\left(\boldsymbol{\gamma}^{\prime}\right) \text {, }
\end{aligned}
$$
the scan rate on the lag range $\gamma^{\prime}$ is determined by the following inequality:

$$
\text { Scan Rate }(\tau)<\frac{1}{16 \mathrm{BK}}
$$

where

$$
\begin{aligned}
& B \text { is the noise bandwidth of the signal in cps } \\
& K \text { is the time constant of the RC low-pass averaging filter. }
\end{aligned}
$$

The scanning rate for the frequency range $f$ in the computation of the powerdensity functions $\hat{S}_{x y}(f), \hat{C}_{x y}(f), \hat{Q}_{x y}(f), \hat{S}_{x}(f), \hat{S}_{y}(f)$, is given by the following inequality:

$$
\text { Scan Rate }(f)<\frac{B}{4 K}
$$

d. Analysis Time

For a given range in $\tau^{\prime}$ or $f$ with the scan rates determined above, we can obtain minimum analysis time. For the cross-correlation and normalized cross-correlation functions, the minimum analysis time is found as follows:

$$
\text { Minimum Analysis Time }=16 \mathrm{BK} \gamma_{m}^{\prime}
$$


where $\gamma_{m}$ is the range in $\gamma$, and $B K$ is as previously defined. For the power-dens ity functions, using the scan rate given above, the minimum analysis time is given by

$$
\text { Minimum Analysis Time }=\frac{4 \mathrm{FK}}{\mathrm{B}}=\frac{\mathrm{F}}{\mathrm{Scan} \text { Rate }}
$$

where

$$
\begin{aligned}
& F \text { is the frequency range (cps) } \\
& B \text { and } K \text { are as previously defired. } \\
& \text { 2. Computer Mechanization }
\end{aligned}
$$

This describes the operation of a proposed transfer function analyzer. The device will operate on two time functions, $x(t)$ and $y(t)$, to compute the input power spectral density $\left(\mathrm{P}_{\mathrm{xx}}\right)$ and the output power spectral density $\left(\mathrm{P}_{\mathrm{yy}}\right)$. Also, in order to compute the trarsfer furction of the system in question, the cross co-power spectral density $\left(\mathrm{C}_{\mathrm{xy}}\right)$ and the cross quadrature power spectral density $\left(Q_{x y}\right)$ will be generated. The flow diagram (Figure 10) illustrates the basic computational procedure. The four density functions are described by the following mathematical relationships:

$$
\begin{gathered}
P_{y y}=\int\left[\operatorname{ReF}\{y(t)\}^{2}+\operatorname{ImF}\{y(t)\}^{2}\right] d t \\
P_{x x}=\int\left[\operatorname{ReF}\{x(t)\}^{2}+\operatorname{ImF}\{x(t)\}^{2}\right] d t \\
C_{x y}=\int[\operatorname{ReF}\{x(t)\} \cdot \operatorname{ReF}\{y(t)\}+\operatorname{ImF}\{x(t)\} \\
\cdot \operatorname{ImF}\{y(t)\}] d t \\
Q_{x y}=\int[\operatorname{ReF}\{x(t)\} \cdot \operatorname{ImF}\{y(t)\}-\operatorname{ReF}\{y(t)\} \\
\cdot\{\operatorname{ImF}\{x(t)] d t
\end{gathered}
$$

With these four functions we can now compute the transfer functions of the system under test. The amplitude and phase of the system is determined by the following computation:

$$
\begin{gathered}
\text { Amplitude }=\left[C_{x y}^{2}+Q_{x y}^{2}\right]^{1 / 2} \\
\text { Phase }=\text { arc tan }\left[\frac{-Q_{x y}}{C_{x y}}\right]
\end{gathered}
$$


The amplitude ratio, output/input, is determined as shown in Equation (81).

$$
\text { Amplitude Ratio }=\frac{\left[C_{x y}^{2}+Q_{x y}^{2}\right]^{1 / 2}}{P_{x x}}
$$

The above mathematical computations are readily handled by existing equipment available on analog computers.

The computing elements of the analyzer which are to be synthesized on the analog computer of greatest importance are the reference oscillator and the band-pass filter (see Figure 10).

The oscillator must provide a constant-amplitude sinusoid and its quadrature component over a wide range. The generation of these signals is accomplished by the solution of the differential equation

$$
\ddot{z}+\omega_{\beta}^{2} z=0
$$

with

$$
\begin{aligned}
& z(0)=+1 \\
& \dot{z}(0)=0
\end{aligned}
$$

Since a wide dynamic range is being sought and it is desirable to avoid the possibility of a non-constant amplitude and a non-quadature condition, we can impose an additional equation to stabilize the system. This is given by the trigonometrical relation

$$
1-\left(\sin ^{2} \omega_{\beta} t+\cos ^{2} \omega_{\beta} t\right)=0
$$

Figure $I l$ is the computer mechanization to implement this condition. The equations being solved in general are as follows:

$$
\dot{Z}=-Y-Z\left(Y^{2}+Z^{2}-1\right)
$$

with

$$
\begin{aligned}
& Z(0)=+1 \\
& \quad \dot{Y}=Z-Y\left(Y^{2}+Z^{2}-1\right)
\end{aligned}
$$

with

$$
Y(0)=0
$$

The solutions being $Z=\cos t, Y=\sin t$. 
Frequency changes are made by a programmable multiplier in the closed loop gain. The frequency will be a linear function of the time function, $\beta$, whose value lies between 0.1 and 1.0. A tabulation of frequency ranges proposed as a function of loop gains is shown below. Reference also should be made to Figure 11 .

$\begin{array}{lrr}B & \text { Integrator Gains (each) } & \text { Frequency Out (rad/sec) } \\ 1 & 10,000 & 10,000 \\ 0.5 & 10,000 & 5,000 \\ 0.1 & 10,000 & 1,000 \\ 1 & 1,000 & 1,000 \\ 0.1 & 1,000 & 100 \\ 1 & 100 & 100 \\ 0.1 & 100 & 10 \\ 1 & 10 & 10 \\ 0.1 & 10 & 1\end{array}$

Satisfactory results have been achieved for the reference oscillator on the EAI23I-R computer. Further tests are in progress to assure reliability at the higher order frequencies (100 rad/sec and greater).

The band-pass filter proposed is shown in Figure 12. Its transfer function is given by

$$
-\frac{E_{0}}{E_{1}}=\frac{\frac{\beta}{r c}}{S^{2}+\frac{\beta}{r c} S+\frac{\beta^{2}}{R^{2} C^{2}}}
$$

where $\beta$ is the programmable function which determines the center frequency of the band-pass filter and the frequency of the reference oscillator. Thus the reference oscillator and the center of the band-pass filter are in track. Shown below is a tabulation of filter characteristic under prescribed conditions.

For explanatory purposes we choose a circuit of constant $Q=10$

$$
Q=\frac{1}{2 \xi} \text { for } \xi<0.1
$$

from our transfer function (see Figure 12)

$$
\frac{\beta}{r c}=2 \xi \omega_{n}
$$


where

$$
\omega_{\mathrm{n}}=\frac{\beta}{\mathrm{RC}}
$$

Conditions $r=1 \quad R=0.1$

\begin{tabular}{llrl}
$\beta$ & \multicolumn{1}{c}{$\mathrm{C}$} & \multicolumn{1}{c}{$\omega_{\mathrm{n}}$} & Q \\
1 & 0.001 & 10,000 & 10 \\
0.1 & 0.001 & 1,000 & 10 \\
1 & 0.01 & 1,000 & 10 \\
0.1 & 0.01 & 100 & 10 \\
1 & 0.1 & 100 & 10 \\
0.1 & 0.1 & 10 & 10 \\
1 & 1.0 & 10 & 10 \\
0.1 & 1.0 & 1 & 10
\end{tabular}

Preliminary tests of the filter indicate the practicality of the proposed circuit. Further tests will be required to finalize the circuit.

In order to perform a spectral density analysis with a single filter device, it is necessary to scan through the desired frequency range. The frequency range of this analyzer is intended to be between 0.01 and $1000 \mathrm{cps}$. The scan speed is important since, if too slow, it will result in lengthy computer operation which may be expensive. A fast scan speed, however, can lead to the following difficulties:

a. Uncertainty of results will increase because all available information at a given frequency will not be viewed by the analyzer filter over the record length.

b. The narrow band-pass filter will not fully respond to sharp peaks in the signal power spectrum.

c. With $\mathrm{RC}$ averaging, the averaging filter will not fully respond to sharp peaks in the signal power spectrum.

The above difficulties impose the following limitations on the scan rate:

For true averaging

$$
\text { scan rate minimum of } \frac{B}{T_{A}} \text { or } \frac{B^{2}}{8}
$$


For RC averaging

$$
\text { scan rate minimum of } \frac{B}{4 K} \text { or } \frac{B^{2}}{8}
$$

where

$$
\begin{aligned}
\mathrm{T}_{A} & =\text { averaging time (sec) } \\
K & =\text { RC time constant ( } \mathrm{sec}) \\
B & =\text { bandwidth of filter (cps) }
\end{aligned}
$$

The scan rate determined by either set of equations is always the smaller of the two calculated.

The actual analyzer will be implemented on the EAI-23l-R computer as shown in Figures 11 and 12 .

The reference oscillator shown in Figure 11 generates a constant amplitude sin wt ard cos $\omega t$, by the solution of the differential equation

$$
\ddot{x}+\omega^{2} x=0
$$

Since the high frequencies needed would cause the output of a simple oscillator to converge, feedback and compensation are used to form an error signal given by the trigonometric relation $1-\left(\sin ^{2} \omega t+\cos ^{2} \omega t\right)=0$. The feedback forces the sine and cosıne to remain in quadrature and at constant amplitude over the frequency range of interest. The compensation is necessary to nullify the inherent frequency characteristics of the computer components. Frequency changes in this circuit are made by the multipliers in the closed loop circuit since

$$
\omega_{t}=T \times \omega \quad(\text { see Figure 12) }
$$

An alternate method for generating the sinusoids would be the utilization of a resolver which is fed by a triangular wave of appropriate frequency and amplitude. This method would have to be checked out, however, to determine if the frequency range of the EAI resolvers is sufficient.

The second component of this system is the band-pass filter shown in Figure 12. The center frequency of the filter in this application is synchrorized to the reference oscillator; the transfer function for the filter, Equation (82:, can be derived as shown below. 
It is evident that

$$
\mathrm{Q}=\frac{\mathrm{T}^{2} \mathrm{E}}{\mathrm{RCS}}
$$

Also, summing grid currents, we have

$$
\frac{E_{1} T^{2}}{r}+\frac{Q}{R}+\mathrm{CE}_{0} S+\frac{T^{2} E_{0}}{r}=0
$$

or

$$
\frac{E_{1} T^{2}}{r}+\frac{T^{2} E_{0}}{R^{2} C S}+C E_{0} S+\frac{T^{2} E_{0}}{r}=0
$$

and

$$
\frac{E_{1} T^{2}}{r}+E_{0}\left[\frac{T^{2}}{R^{2} C S}+C S+\frac{T^{2}}{r}\right]=0
$$

Thus

$$
\frac{E_{I} T^{2}}{r}=-E_{0}\left[\frac{T^{2}+R^{2} C^{2} S^{2}}{R^{2} C S}+\frac{T^{2}}{R}\right]
$$

which gives

$$
E_{1} T^{2}=-E_{0}\left[\frac{r T^{2}+R^{2} r C^{2} S^{2}+T^{2} R^{2} C S}{R^{2} C S}\right]
$$

Then

$$
\frac{E_{0}}{E_{1}}=-\frac{T^{2} R^{2} C S}{R^{2} r C^{2} S^{2}+T^{2} R^{2} C S+r T^{2}}
$$

Finally

for

$$
\frac{E_{0}}{E_{1}}=-\frac{\frac{T^{2}}{r C} S}{S^{2}+\frac{T^{2}}{r C} S+\frac{T^{2}}{R^{2} C^{2}}}
$$

$$
0<T^{2}<1
$$


Since $\omega^{2}=\frac{T}{R^{2} C^{2}}$, the center frequency $\left(\omega_{C}\right)$ as well as $\omega_{T}$ for the reference oscillator will change as a function of $T$. The circuit for the reference oscillator has beer checked on the EAI-23I-R analog computer with satisfactory results over a frequency range from 1 to $1000 \mathrm{cps}$. Further checks on the circuit to assure adequate results for the reference oscillator and the band-pass filter should be made.

\section{Investigation to Identify Problems and Limitations}

The use of the computer to determine power spectra and transfer functions of wide-band data has some problems and limitations that are krown, and also some that probably are not known. Further investigation is required to identify and describe such problems and limitations by using input signals of known properties and relations. Use should be made of noise sources, transfer function nonlinearities, nonstationary inputs and relations, etc., that can be generated in the anclog complter laboratory.

For example, two or more quantities can be related on the computer in a manner similar to relations thought to exist in the system. Then an input signal of known power density spectra (including noise) can be used as an input to one quantity. Then the power density spectra of the two quantities and the corresponding transfer function car be determined and compared to known values. Effects of nonlinear relations, signal-to-noise ratio, and stationarity can be determined. All of this effort is required to evaluate properly the analog computer technique of transfer function and spectral analysis.

V. CONCLUSIONS

An essential step in the development of effective techniques for analyzing engine test data is the use of available techniques as soon as possible with similar data. By the time that testing is started, the techniques which will be used to analyze the engine test data will already have been evaluated. Hence, only the features which are proven to be efficient in use will be retained and there will be time to find effective solutions.

\section{A. TECHNIQUES FOR DIGITAL DATA}

The digital data analysis tools developed during Contract Year 1964 are described in detail in this report. The digital programs ADDA, Statistical 


$$
\mathrm{RN}-\mathrm{S}-0146
$$

Edit and Smooth, and Computer Job 364, were used extensively with data from NRX-AI to evaluate their effectiveness. Their utility was demonstrated in the results reported in the NRX-AI Data Analysis report (Reference 3).

The program ADDA described in Appendix A was used to analyze 50 million narrow-bard data points generated during the NRX-Al test series. The voluminous output of this analysis is being presented in a report entitled Statistical Details Narrow-Band (N2) INRX-Al Test Data (Reference 16). Since this report is 16 in. thick, its distribution will be rather limited. A copy will be available at AGC, WANL, NTO and SNPO-C. The data quality characteristics measured by this program were much better than expected on the basis of pretest information (Reference 7). Eighty percent of the data had a variance less than 5, which corresponds to a standard deviation of 2 counts or less. For full scale equal to 1024, these variations are insignificant from an engineering point of view. Thus substantial savings in time could be effected in the engine test data analysis by using a tight tolerance on the first difference to screen the data and then by running further analyses only on the data which justifies more sophisticated analysis. The potential savings are particularly significant with a small computer such as the CDC 3200 which is to be used for test data processing at ETS-I. That computer can determine and analyze first differences very rapidly with a relatively small program which can easily fit within its limited memory size. The more sophisticated techniques that were used for the NRX-Al data were programmed for a much larger computer. The optimum location for that part of the data analysis is dependent upon the capabilities and availability of the central data processor currently under consideration for NRDS (Reference 32).

The program, Statistical Edit and Smooth, described in detail in Appendix B, was used in the analysis of temperature asymmetries in the NRX-Al data. It was developed to transform the data into a form in which it could be analyzed using Computer Job 364. The use of data processed by Edit-Smooth for asymmetry studies is discussed in detail in Section III,B,3 of this report. In this area, development of a special-purpose analysis of variance program which would expedite this analysis is planned for Contract Year 1964. Edit-Smooth has also been provided with a capability for detecting "switch" points (i.e., the point at which there is a change in the characteristics of the functions recorded). The present technique can detect major changes such as the start and stop of fluid flow. Further development is required to increase the sensitivity of the technique to detect more subtle changes. Work in this area is planned for Contract Year 1965. 


\section{$\mathrm{RN}-\mathrm{S}-0146$}

The theory behind comparison of models with test data when both are subject to error was discussed in Section III,C. The major difficulty in that type of analysis is separating the effects of measurement errors from those of model errors. The nature and magnitude of measurement errors must be fairly well known if the interpretation of the contribution of model errors is to be meaningful. The digital programs (ADDA and Edit-Smooth) can provide much information regarding data system errors from an analysis of calibration data and overall measurement errors as determinable from treatment of data from individual data channels. Modifications to Computer Job 364 for data analysis are being made along the lines discussed in this report. These techniques for simultaneous treatment of systematic measurement and model errors will be evaluated during Contract Year 1965 using available data such as NRX-A2 test results.

A corollary benefit from a separation of measurement and model errors is the information gained regarding systematic measurement errors. These errors include the consequences of instrumentation malfunctions. Instrumentation difficulties cannot be corrected until after they have been detected. Thus the data from the NRX-Al tests were quite free of difficulties that could be detected in the checkout and calibration procedures used. In other cases, some difficulties were not detected until after the data had been analyzed. (This was accomplished only after the series had been completed and the system disassembled.) Hence it is desirable that the data be subjected to the most rigorous instrumentation malfunction-anderror detection procedure that is feasible within the limitations of computer hardware available at the test facility and through appropriate communications with off-site data analysis. The optimal allocation of programming and computer operations in the analysis of test data is dependent upon the central data processing at NRDS study now under way (Reference 32 ).

\section{B. TECHNIQUES FOR ANALOG DATA}

Communications theory is the basis for the currently usable techniques for analysis of analog data. These techniques are reviewed in Section IV of this report, and the analog computer technique recommended for use is described. All of these techniques for analyzing signals are based upon the assumption of stationary signals (i.e., the statistical characteristics and frequency content are constart). Hence these techniques will be useful primarily during steady-state 


$$
\mathrm{RN}-\mathrm{S}-0146
$$

operations. Introduction of known perturbation signals into the system being tested increases the information content of the steady-state test results (regarding the dynamic behavior of systems tested). This in turn produces much data worthy of analysis by these techniques (Reference 33 )。

Thus, these analysis techniques can be used for steady-state test results and for equivalent results of calculations using analog models to provide a basis for comparison of their dynamic behavior. However, it should be noted that the validity (and hence the utility) of these techniques is questionable. This is because they are based upon stationary signals for analysis of "transients" such as chilldowns, which are the primary portions of the NRX-AI and CFDIS test series or startup, power level changes, and cooldown of INRX reactor and the NERVA engine.

The validation and improvement of analog models with regard to system behavior during transients is a more difficult task. It is the analog equivalent of the digital model evaluation problem discussed in Section III of this report. However, the amount of information required to describe the system dynamics during transients is too large for the digital techniques under development to be practical. When the analog model represents a complete and autonomous system, the problem then is similar to many of the problems faced and solved by controls analysis groups.

To reduce the analog model to a size and complexity that can be handled by the largest analog computer available to the NERVA project, a number of simplifications and assumptions must be made. Hence to avoid exceeding this limitation, any refinement in the model for analysis of test data will require use of a component model. This would parallel the NRX-Al data analysis where a more detailed model of the propellant feed line and nozzle was used for a study of the apparent asymmetries. It would be necessary to use the wide-band data to provide the aralog model with its boundary conditions. Tape playback equipment has been ordered to play back the wide-band data tapes and provide the voltages equivalent to boundary conditions when needed. This equipment also makes it feasible to use the analog computer analysis method described above. 
$R N-S-0146$

REFERENCES

1. William C. House, "The Development of Nuclear Rocket Propulsion in the United States," Prepared for Presentation to the British Interplanetary Society, Advanced Propulsion Symposium, London, England, Oct. 9, 1963.

2. NRX-Al Test Specifications, Westinghouse Astronuclear Laboratory, WANL-TNR121, November 1963.

3. Data Analysis Report of NRX-Al Tests (U), Aerojet-General Report RN-S-0115, JuIy 1964 (Conf/RD)。

4. A Digital Program for Simulation of Nuclear Powered Rocket Engines (Computer Job No. 364), Aerojet-General Report RN-S-0047, September 1964.

5. F. Narin, "Computer Data Processing System, Project Rover," Ios Alamos Scientific Laboratory, Report IAM 5-2845, 1963.

6. Kiwi B4B Cold Flow Test Results, Los Alamos Scientific Laboratory, September 1963.

7. Summary of NERVA Instrumentation Feview Meeting at SNPO-C - August 13 Thru 15, 1963 (U), Joint, Report REON, Aerojet-General Corporation, and Astronuclear Laboratory, Westinghouse Ellectric Corporation, Report No. 2703, September 1.963 (Conf/RD).

8. NRX-Al Thirty-Six Day Test Feport, MRA-Al-R-5, NERVA Test Operations, NRDS, Nevada, 22 May 1964.

9. N. Eash, Evaluation of MISTRAM Accuracies Through Regression Analysis, Aerospace Corporation Technical Report, TDR-930 (A2122)TN-2, 11 October 1961.

10. Instrumentation Data Book to AEC-NASA Space Nuclear Propulsion Office, NERVA Program, Contract SNF-1, Aerojet-General Report 2320, January 1964.

11. Data Acquisition and Instrumentation Systems, NRX-Al Test Series, NRX-64D-0059.

12. Memorandum on NTO Data to N. E. Erickson, "NRDS Automatic Data Processing Equipment," NTO-N-O375.

13. Preliminary Test Plan - Cold Flow Development Test System (CFDIS) for NERVA Engine, Aerojet-General Report 2556, May 1963 (Conf/RD).

14. Description of Engine Test Stand ETS-1 Instrumentation and Control Systems Capabilities, 741:Mo759.

15. M. G. Kendall, The Advanced Theory of Statistics, Vol. II, Hafner Publishing Co., New York, 1951, pp. 387-394. 


\author{
RN $-\mathrm{S}-0146$
}

\title{
REFERENCES (cont.)
}

16. Statistical Details - Narrow Band (N2) INRX-Al Test Data, Aerojet-General Report RN-S-O116 (in preparation).

17 N. V. Smirnov, "Table for Estimating the Goodness of Fit of Empirical Distributions," Ann. Math. Stat., 19 (1948), pp. 279-281.

18. L. H. Miller, "Table of Percentage Polnts of Kolmogorov Statistics," J.Amer. Stat. Assn., 51 (1956), pr. 111-12l.

19. W. Feller, "On the Kolmogorov-Smirnov Limlt Theorems for Empirical Distributions," Annals of Math. Stat., 19 (1948).

20. R. A. MacGowan, "FORTRAN Subroutines for Time Series Data Reduction," Communications of the ACM, 7, No. 3, March 1964, pp. 153-157.

21. T. Haavelmo, "The Statistical Implication of a System of Simultaneous Equations," Econometrica, II (1943), pp. 1-12.

22. D. V. Lindley, "Estimation of a Functional Relation," Biometrika, 40 (1953), pp. $47-49$.

23. Engine System Simulator, Convective Heat Transfer and Fluid Flow Considerations, TM 732:63-010.

24. T. Apostol, Calculus, Vol. II, Blaisdell, 1963.

25. E. S. Fearson and H. O. Hartley, Biometrika Tables for Statisticians, I, Cambridge University Fress, 1958.

26. NRX-Al Twelve Week Data Processing Feport, Westinghouse Astronuclear Laboratory, WANL-TME-898, July 1964.

27. P. M. Woodward, Frobability and Information Theory with Applications to Radar, Pergamon Press, 1955, Chapter II.

28. R. B. Blackman and J.W. Tukey, The Measurement of Power Spectra, Part I, Dover Publications, 1958.

29. J. S. Bendat et al., "The Applications of Statistics to Flight Vehicle Vibratior Problem, "ASTIA 271913, December 1961.

30. J. S. Bendat et al., "Analytical Study of Vibration Data Reduction Methods," Technical Products Company, Los Angeles, Report prepared for Marshall Space Flight Center, Huntsville, Alabama, 1963.

31. W. J. Dixon (Editor), "BMDO2T, Autocovariance and Power Spectral Analysis," Biomedical Computer Programs, Health Sciences Computing Facility, Department of Freventive Medicine and Public Health, School of Medicine, University of California, Los Angeles, 1964, pp. 459-481. 


\section{$\mathrm{RN}-\mathrm{S}-0146$}

\section{REFERENCES (cont.)}

32. "Staff Study on NRDS ADP Requirements," Memorandum from H. B. Finger to R. W. Schroeder/B. P. Helgeson, 20 August 1964.

33. Balcomb and Douglas, "A Crosscorrelation Method for Measuring the Impulse Response of Reactor Systems," University of California, Los Alamos Scientific Laboratory and Massachusett.s Institute of Technology, Cambridge, Massachusetts (Reprinted from Nuclear Science and Engineering, 11, No. 2, October 1961). 
TABLE I

EP-IV LIQUID IVTO GAS ( 1.25 RAMP)

CORE TNLET PLENUM TEEMPERATURES

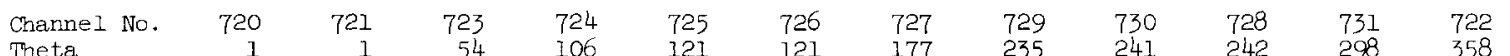

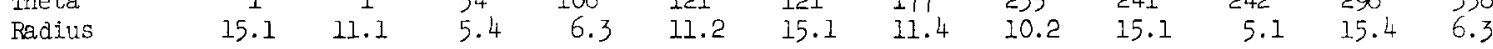

\begin{tabular}{|c|c|c|c|c|c|c|c|c|c|c|c|c|c|c|}
\hline \multirow{2}{*}{$\frac{\text { Time }}{3 \text { I } 681}$} & \multicolumn{12}{|c|}{ Temperature, ${ }^{\circ} R$} & \multirow{2}{*}{$\frac{\text { Mean }}{448.83}$} & \multirow{2}{*}{$\begin{array}{l}\text { St. Dev. } \\
10.23\end{array}$} \\
\hline & 447 & 454 & 458 & 451 & 451 & 448 & 457 & 452 & 423 & 457 & 435 & 453 & & \\
\hline 31682 & 445 & 453 & 455 & 450 & 450 & 447 & 456 & 451 & 423 & 456 & 432 & 454 & 447.67 & 10.22 \\
\hline 31683 & 443 & 451 & 454 & 450 & 448 & 447 & 456 & 449 & 422 & 455 & 430 & 453 & 446.50 & 10.40 \\
\hline 31684 & 442 & 451 & 454 & 450 & 447 & 446 & 456 & 447 & 419 & 454 & 430 & 452 & 445.67 & 10.92 \\
\hline 31685 & 441 & 451 & 454 & 449 & 446 & 444 & 454 & 446 & 416 & 453 & 431 & 451 & 444.67 & 11.18 \\
\hline 31.686 & 440 & 450 & 453 & 447 & 445 & 443 & 452 & 446 & 415 & 452 & 429 & 451 & 443.58 & 11.25 \\
\hline 31687 & 440 & 447 & 450 & 447 & 445 & 442 & 453 & 445 & 413 & 451 & 427 & $45 I$ & 442.58 & 11.62 \\
\hline 31688 & 439 & 446 & 448 & 444 & 443 & 440 & 451 & 444 & 413 & 450 & 424 & 448 & 440.83 & 11.33 \\
\hline 31689 & 436 & 444 & 446 & 441 & 440 & 437 & 447 & 441 & 413 & 447 & 419 & 444 & 437.92 & 10.96 \\
\hline 31690 & 427 & 437 & 440 & 434 & 432 & 430 & 439 & 434 & 410 & 441 & 412 & 437 & 431.08 & 10.25 \\
\hline 31691 & 416 & 427 & 430 & 424 & 422 & 419 & 431 & 424 & 402 & 432 & 402 & 428 & 421.42 & 10.26 \\
\hline $3 \geq 692$ & 405 & 417 & 420 & 414 & 411 & 408 & 420 & 414 & 393 & 422 & 393 & 418 & 421.25 & 9.90 \\
\hline 31693 & 391 & 405 & 408 & 401 & 399 & 397 & 408 & 403 & 382 & $4 I I$ & 382 & 408 & 399.58 & 9.95 \\
\hline 32694 & 379 & 391 & 394 & 386 & 385 & 384 & 394 & 390 & 368 & 398 & 371 & 395 & 386.25 & 9.52 \\
\hline 31695 & 372 & 384 & 386 & 378 & 378 & 376 & 386 & 383 & 360 & 390 & 359 & 388 & 378.33 & 10.26 \\
\hline 31696 & 366 & 378 & 381 & 374 & 373 & 370 & 381 & 378 & 356 & 386 & 350 & 383 & 373.00 & 10.97 \\
\hline 31697 & 358 & 370 & 373 & 367 & 365 & 362 & 372 & 369 & 349 & 378 & 341 & 375 & 364.92 & 10.98 \\
\hline 31698 & 348 & 359 & 363 & 355 & 353 & 353 & 361 & 358 & 339 & 367 & 332 & 364 & 354.33 & 10.39 \\
\hline 31699 & 337 & 349 & 353 & 345 & 343 & 344 & 351 & 348 & 329 & 357 & 321 & 354 & 344.25 & 10.64 \\
\hline 31700 & 330 & 340 & 343 & 335 & 334 & 334 & 341 & 339 & 320 & 346 & 310 & 344 & 334.67 & 10.55 \\
\hline 31701 & 321 & 330 & 333 & 326 & 324 & 322 & 330 & 329 & 311 & 336 & 300 & 333 & 324.58 & 10.29 \\
\hline 31702 & 310 & 320 & 325 & 316 & 313 & 311 & 319 & 318 & 301 & 325 & 290 & 323 & 314.25 & 10.34 \\
\hline 31703 & 297 & 309 & 317 & 306 & 301 & 300 & 308 & 307 & 291 & 313 & 278 & 313 & 303.33 & 10.87 \\
\hline 31704 & 282 & 295 & 303 & 292 & 289 & 286 & 295 & 296 & 278 & 299 & 265 & 300 & 290.00 & 10.83 \\
\hline
\end{tabular}


TABLE 2

EP-IV LIQUID INTO GAS ( 1.25 RAMP) CORE INLET PLENUM TEMPERATURES ADJUSTED FOR EFFECT OF TIME

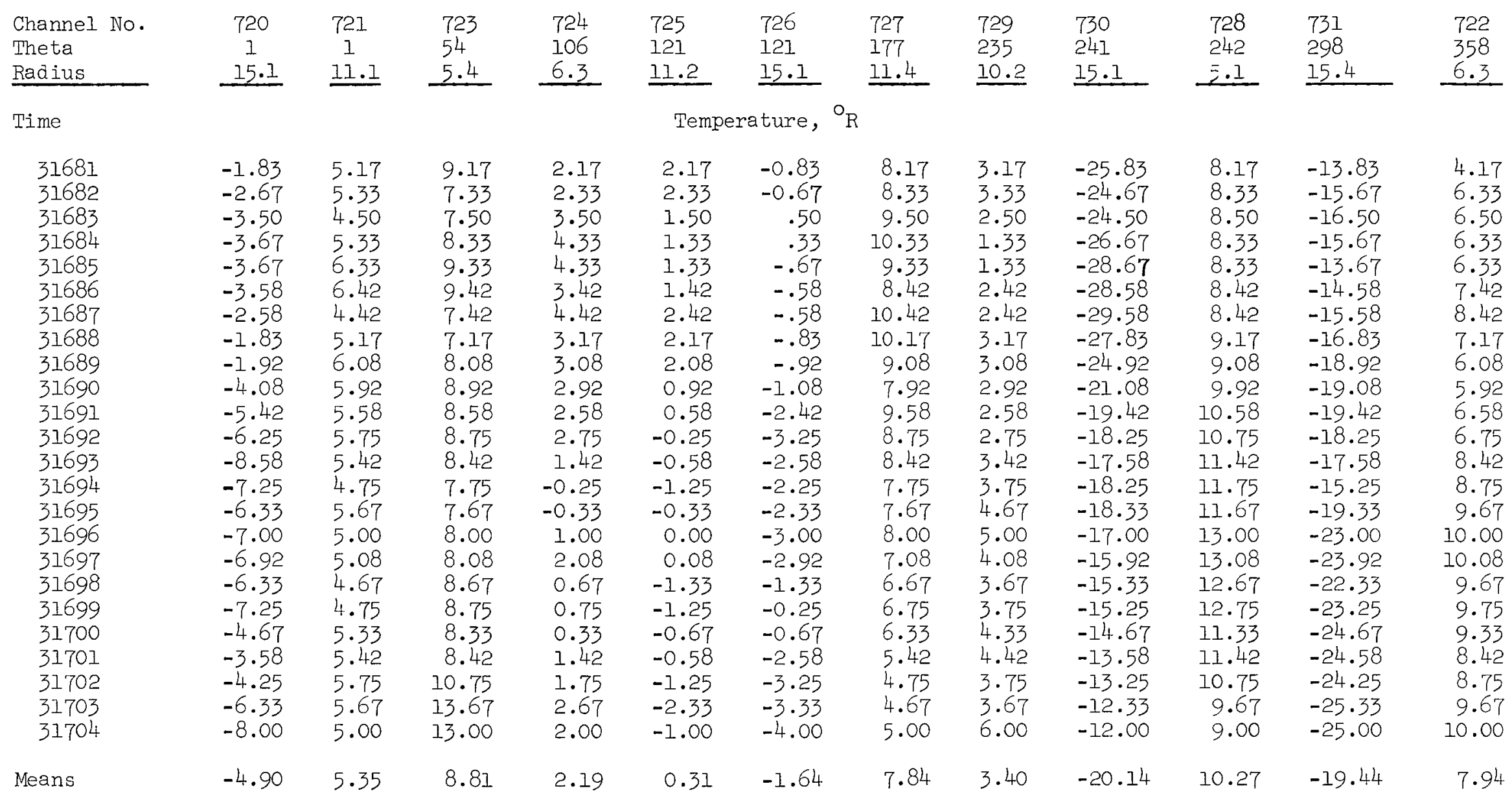




\section{RN-S-0146}

\section{TABLE 3}

\section{ANALYSIS OF VARIANCE}

FOR UNADJUSTED DATA

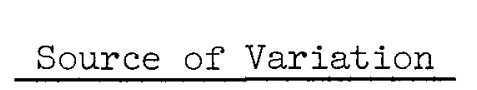

Between Columns

Due to Theta*

Due to Instruments ${ }^{*}$

Within Columns

Total 287

Degrees of

Freedom

11

8

3

276

29575.33
1656.79

Sum of Squares

27918.54

15509.67

12408.85

Mean Squares

2538.05

1938.71

4136.28

6.00
F-Ratio

0.47

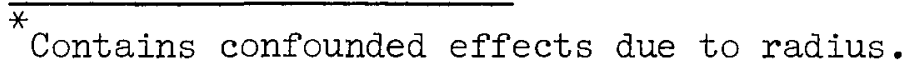


TABLE 4

EP-IV LIQUID INTO GAS (1.25 RAMP) CORE INLEI PLENUM TEMPERATURES ADJUSTED FOR EFFECTS OF TIME AND RADIUS

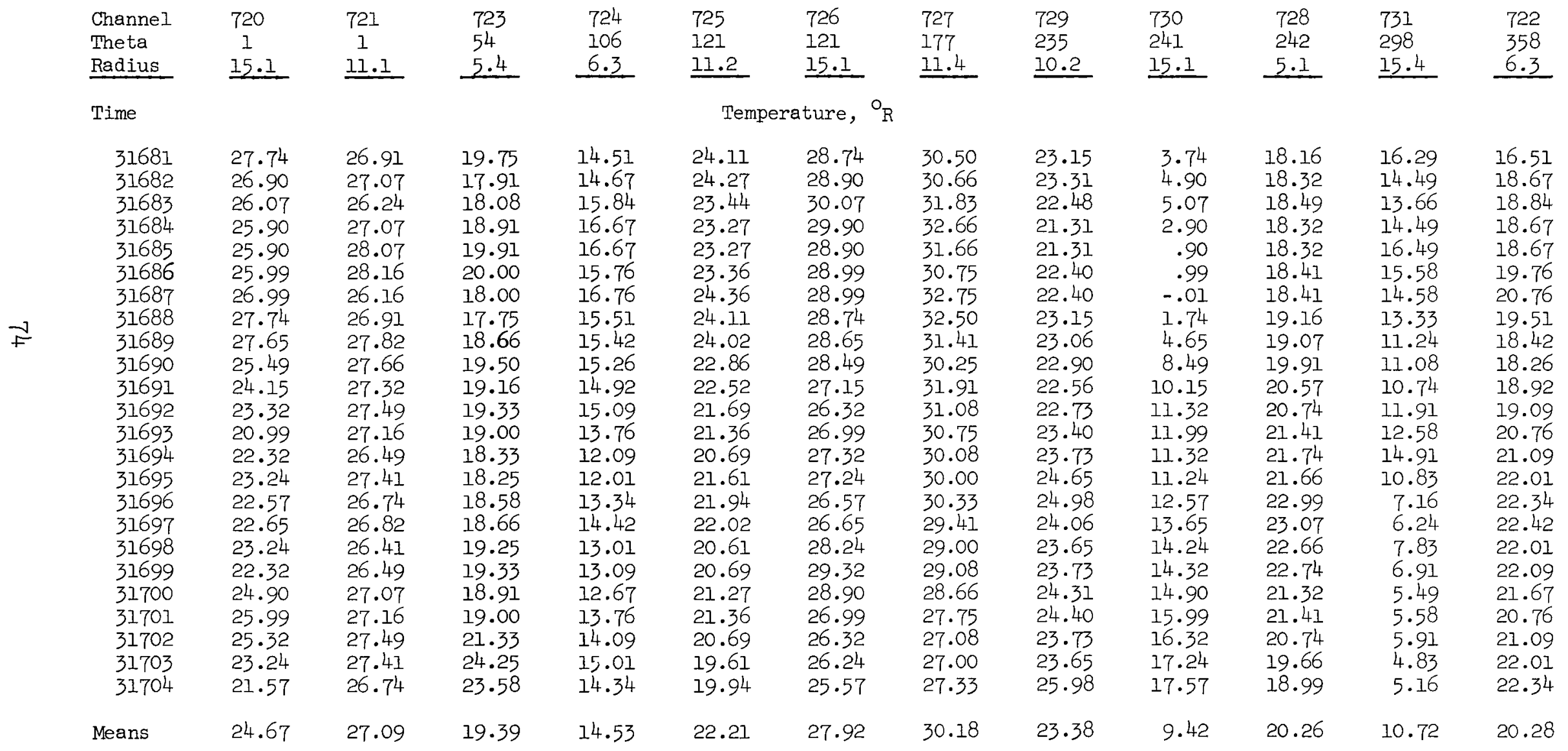


$\mathrm{RN}-\mathrm{S}-0146$

\section{TABLE 5}

ANALYSIS OF VARIANCE FOR ADJUSTED DATA

\begin{tabular}{|c|c|c|c|c|}
\hline Source of Variation & $\begin{array}{l}\text { Degrees of } \\
\text { Freedom }\end{array}$ & Sum of Squares & Mean Squares & F-Ratio \\
\hline Between Columns & 10 & 11398.50 & 1139.85 & \\
\hline Due to Theta & 8 & $9527 \cdot 31$ & 1190.91 & 1.27 \\
\hline Due to Instruments & 2 & 1871.19 & 935.60 & \\
\hline Within Columns & 276 & 1656.79 & 6.00 & \\
\hline Total & 286 & 13055.29 & & \\
\hline
\end{tabular}




\section{PAGE BLANK}





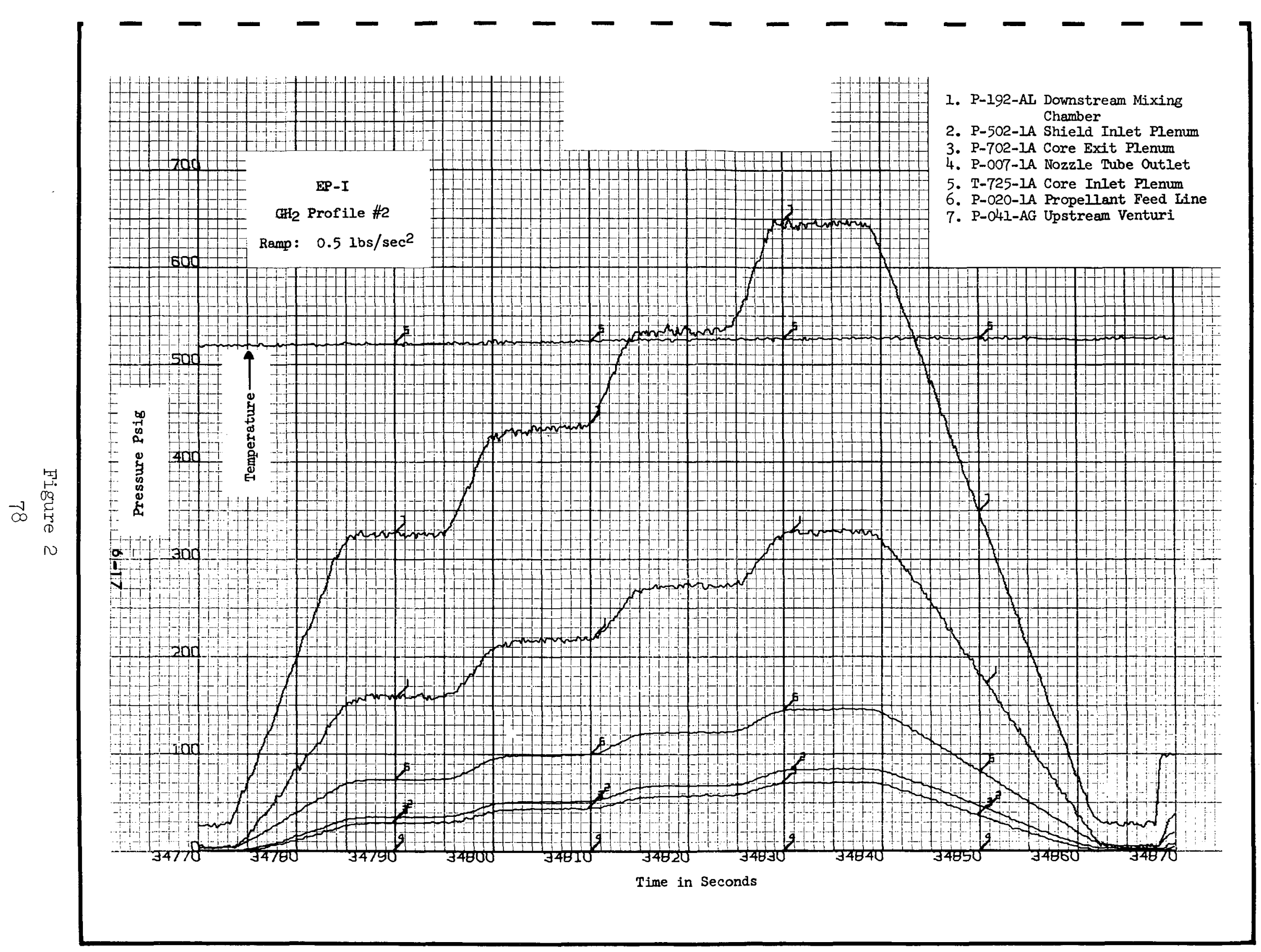




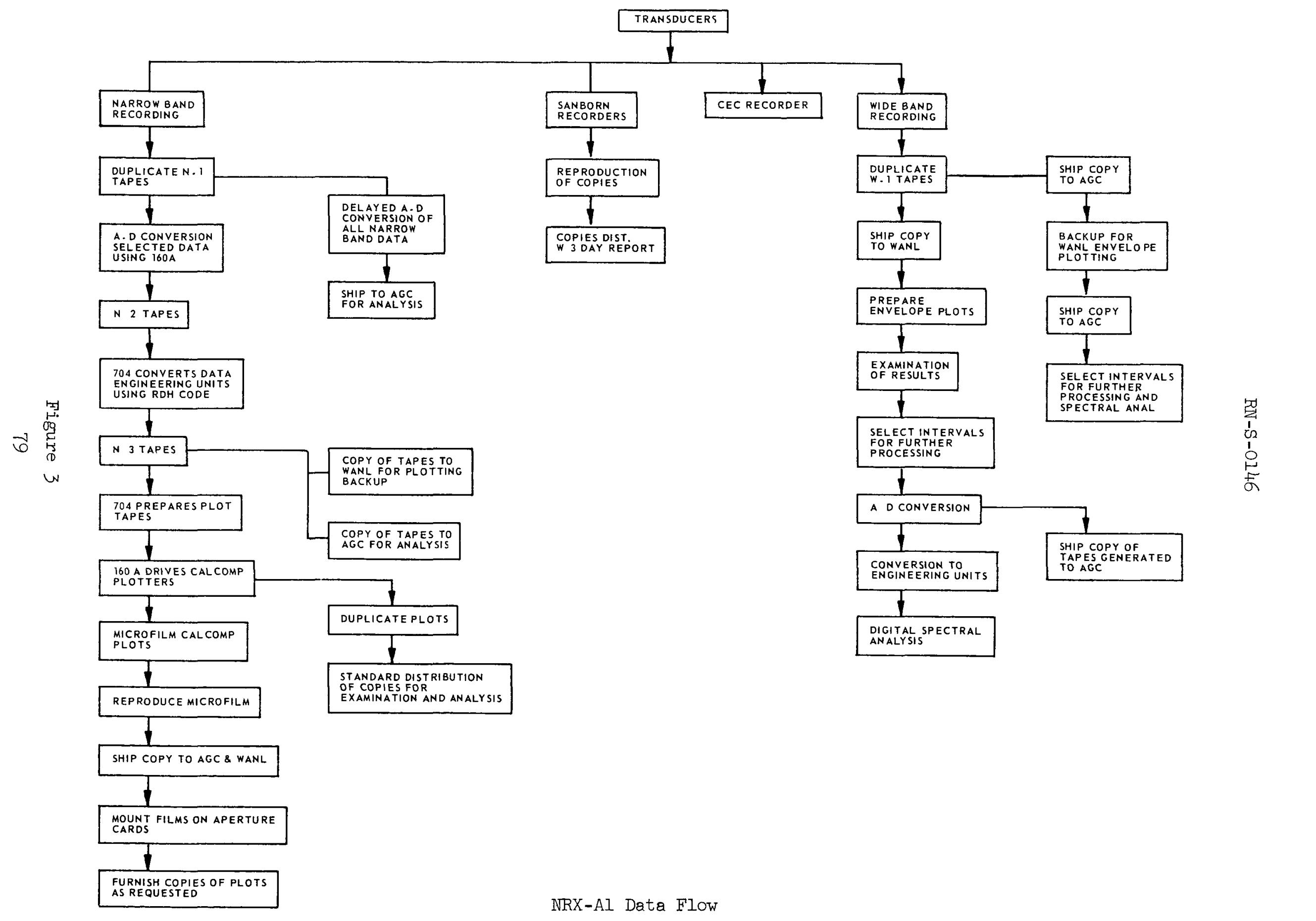




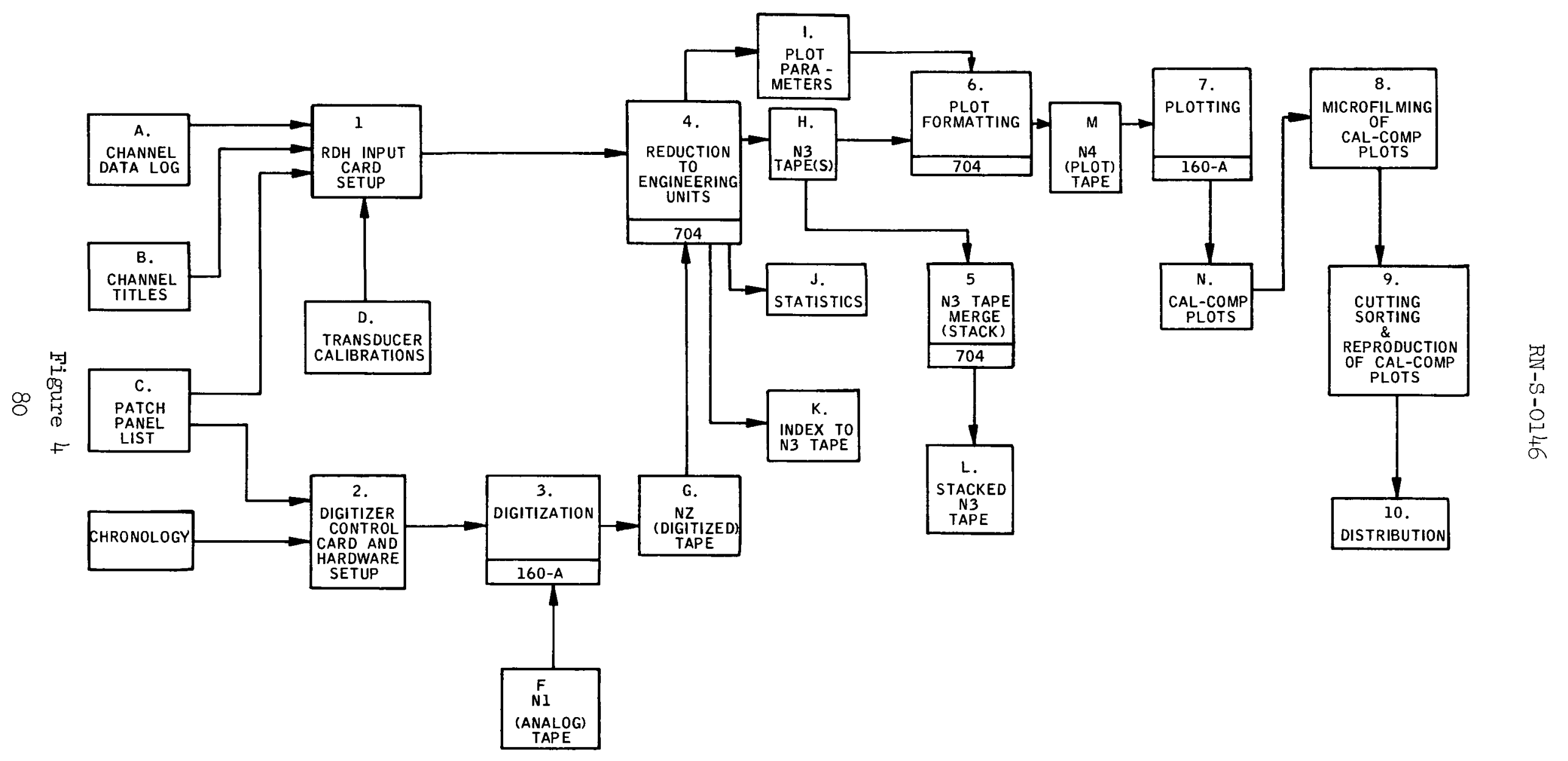

Data Processing (RDH) 


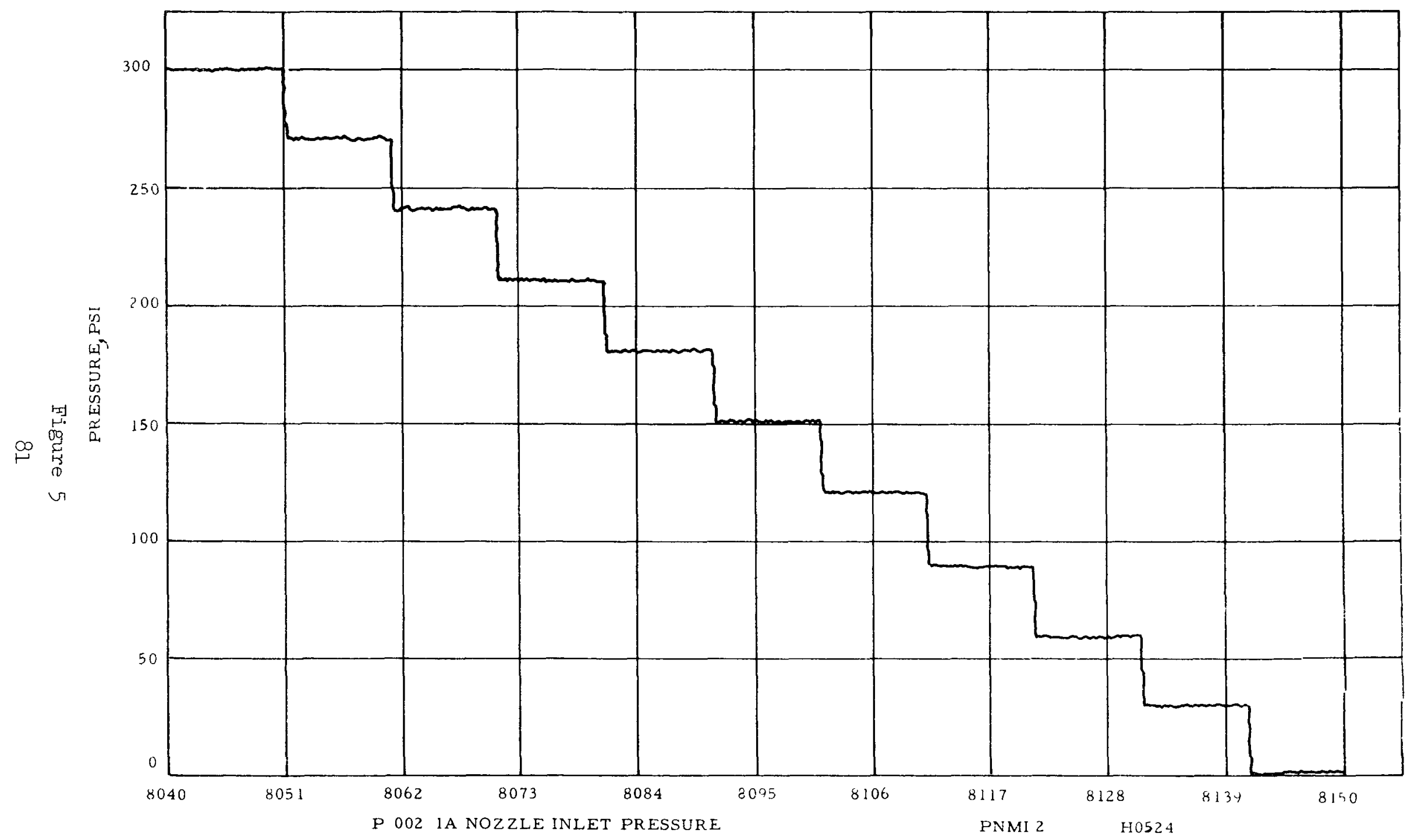

Calibration Curve for Narrow-Band Data System 
$\mathrm{RN}-\mathrm{S}-\mathrm{O} 146$

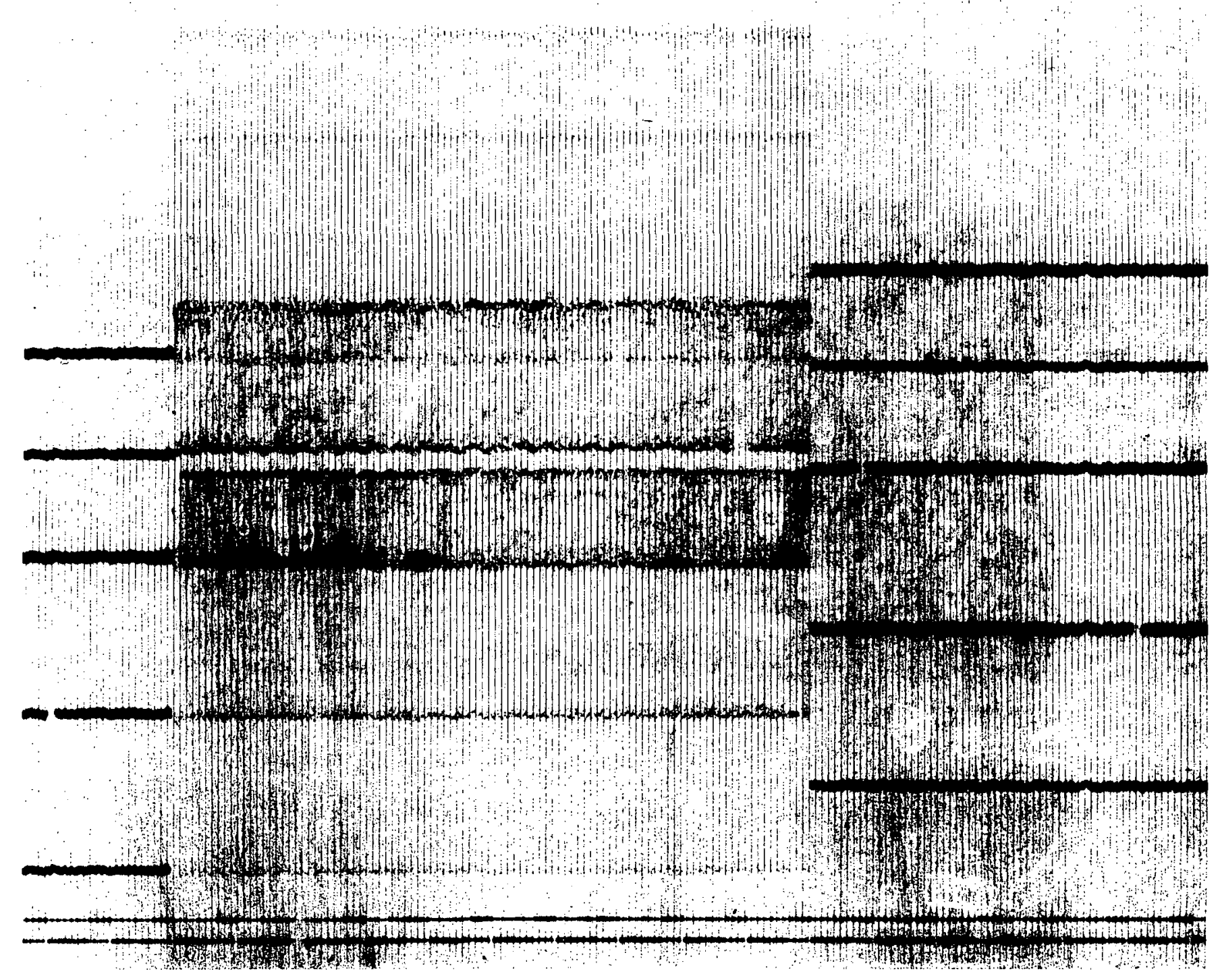

Figure 6

Envelope Plot Showing a 50-cps Step in Calibration 


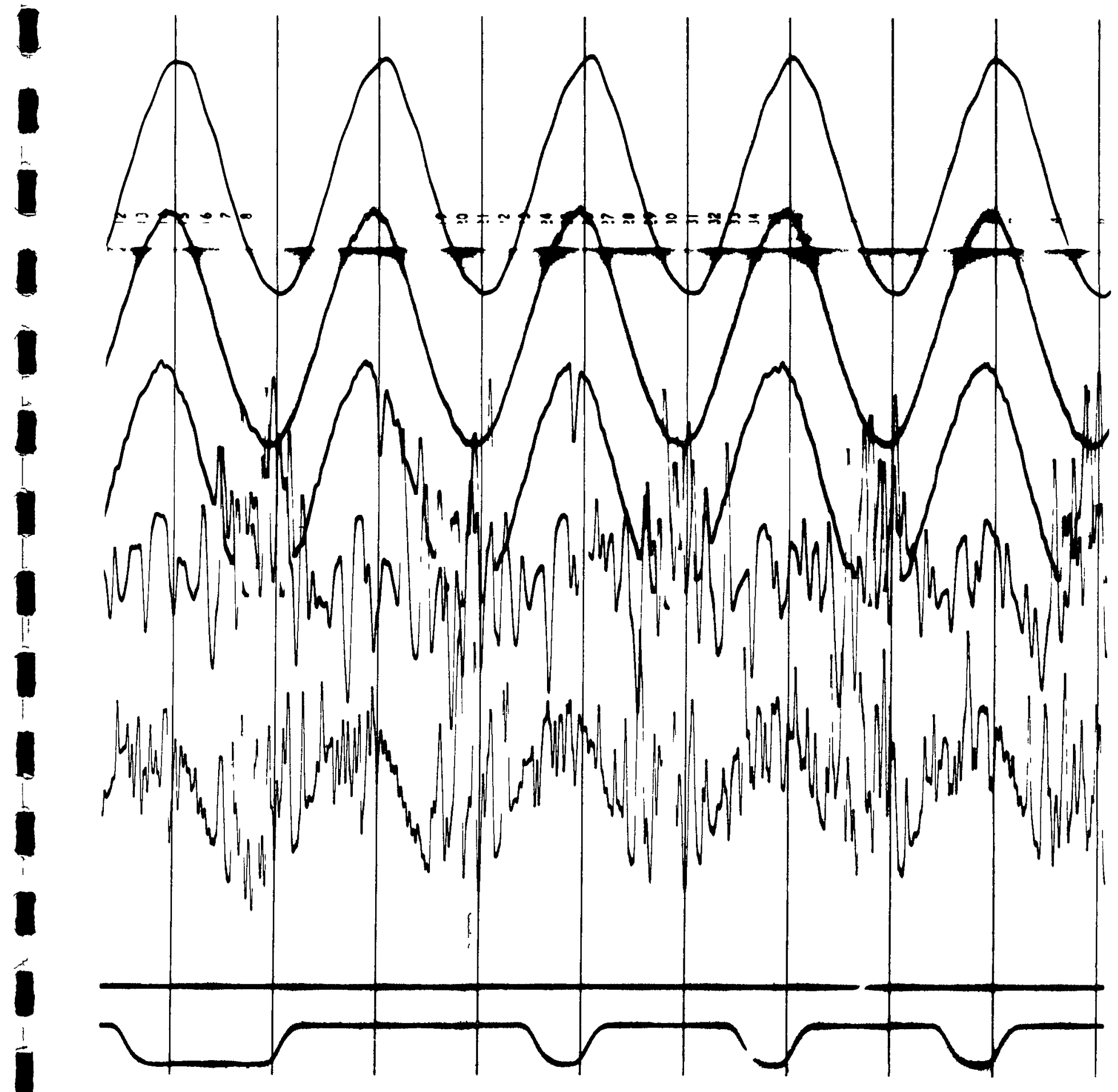

Figure 7

High-Speed Recording of Part of a 50-cps Step in Calibration 


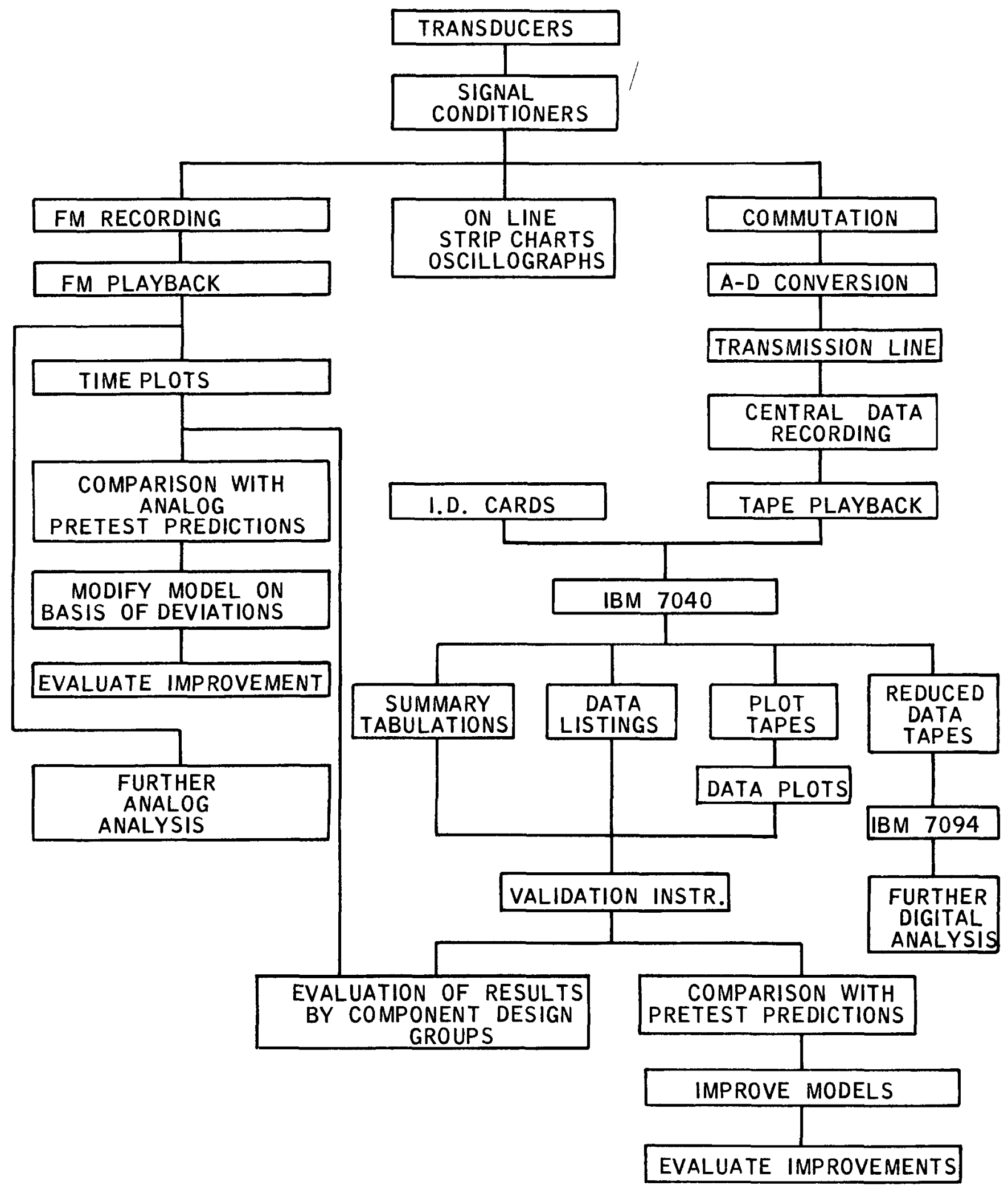

Figure 8

CFDTS Data Flow 


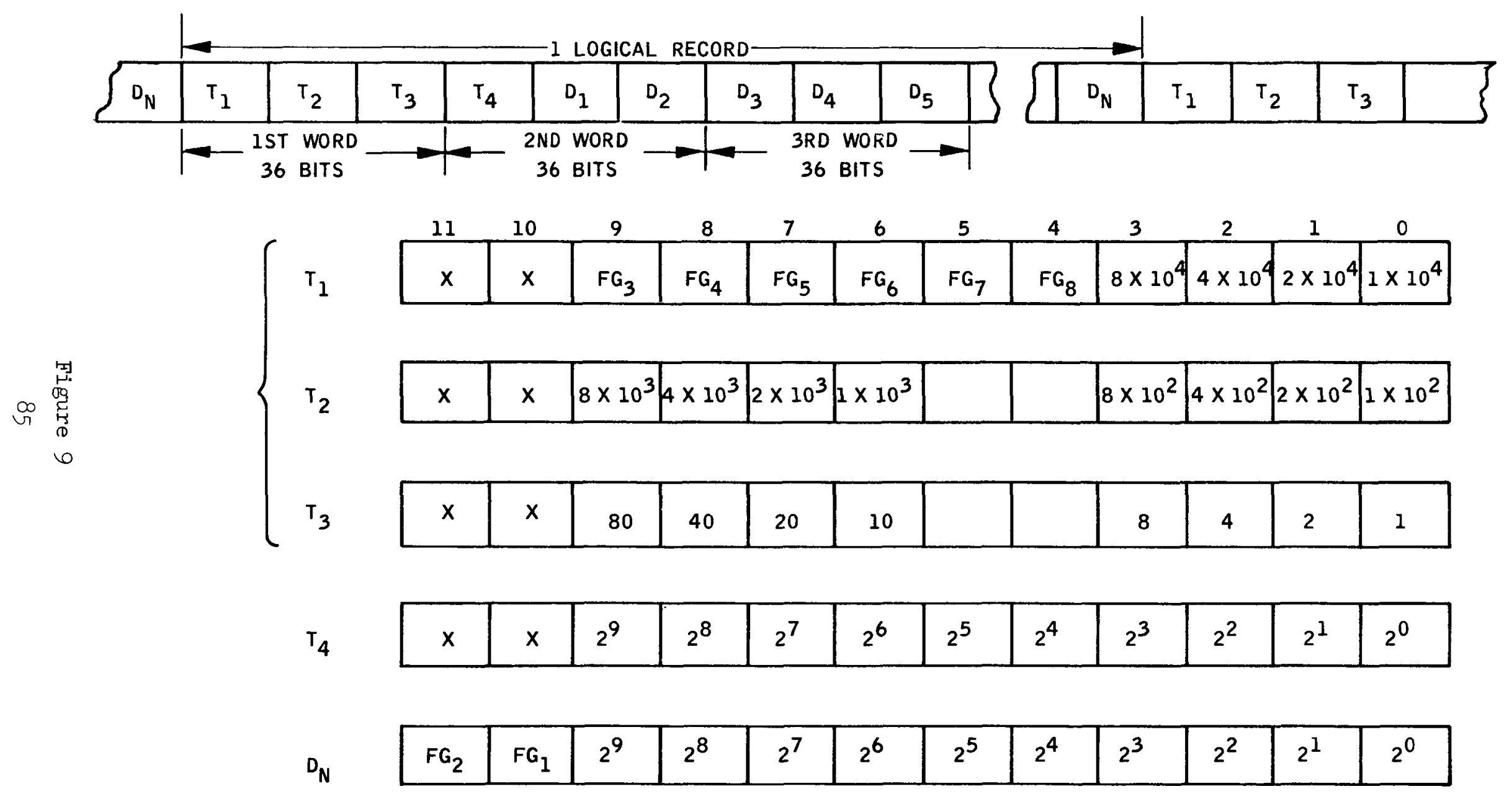

Output Format for N2 Logical Records 


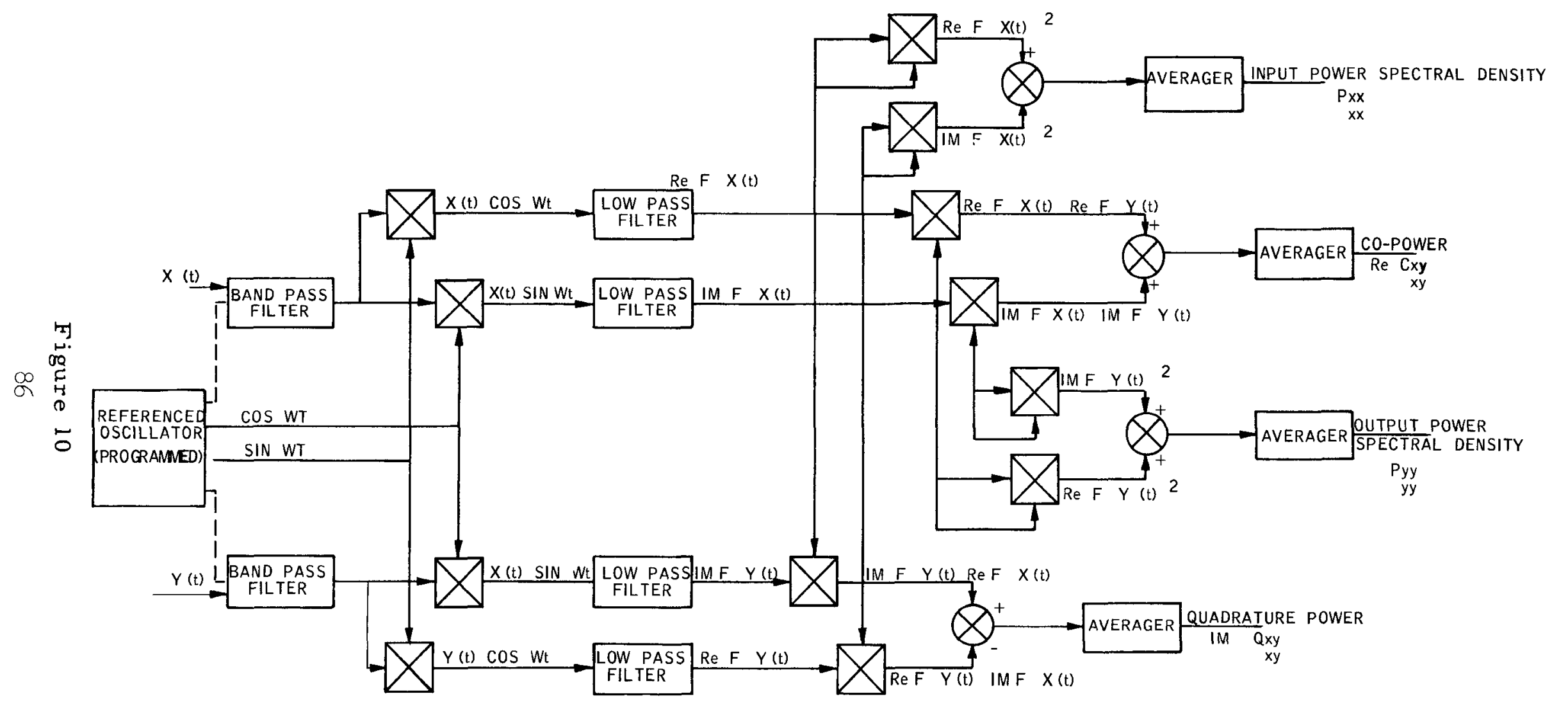

Transfer Function Analyzer Block Diagram 


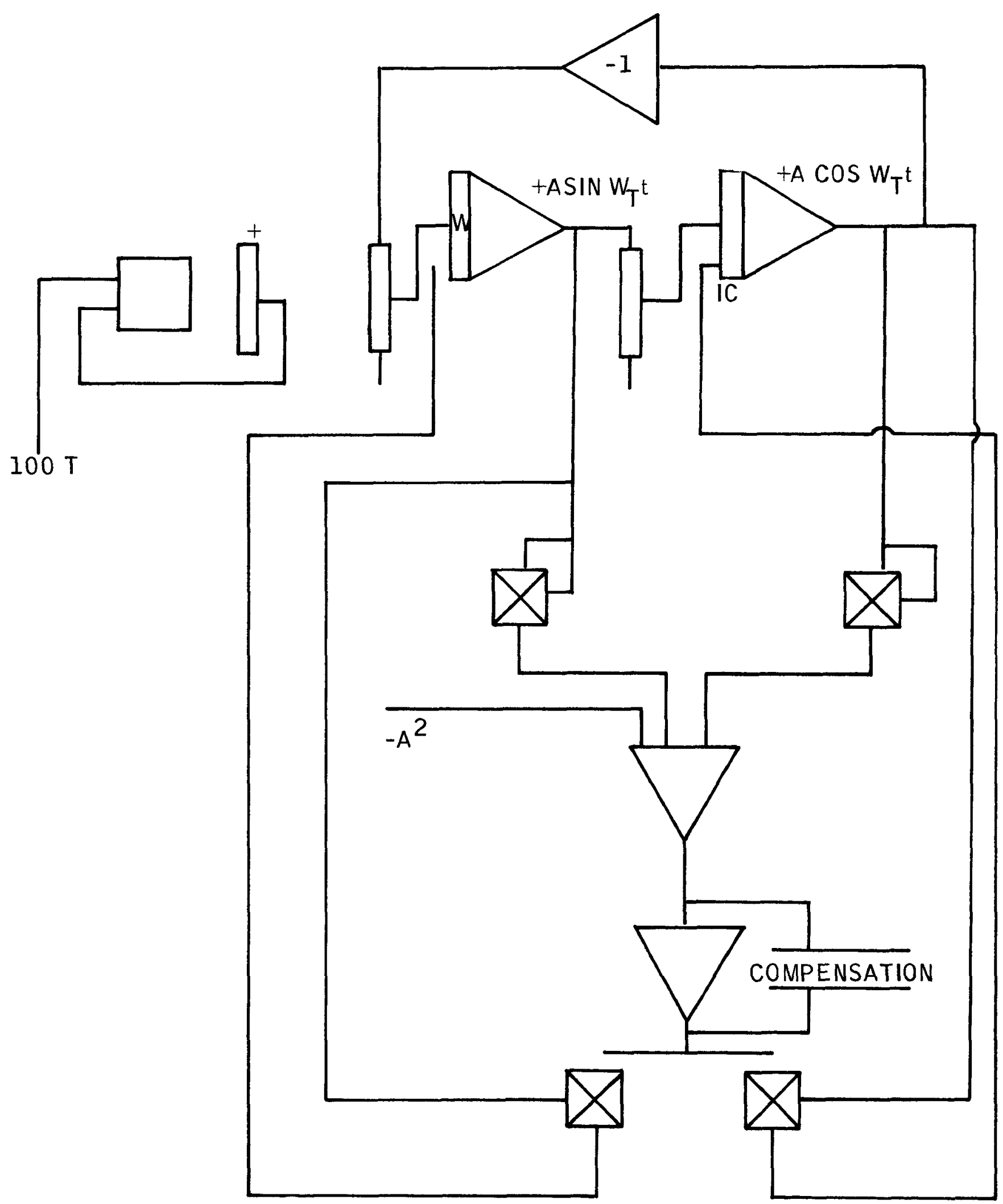

Figure 11

Programmable Reference Oscillator 


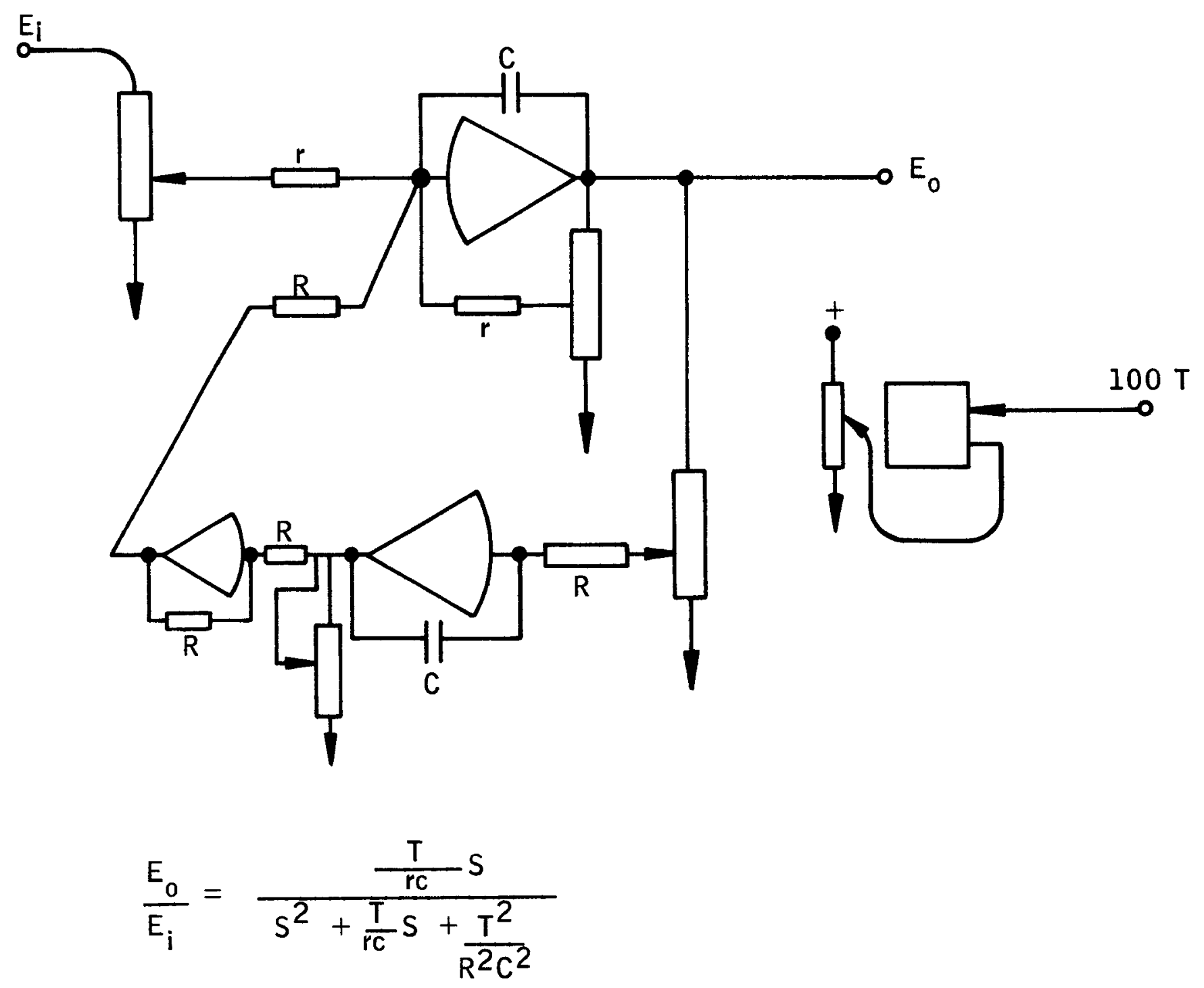

Figure 12

Programmable Active Tuned Filter 
RN-S-0146

APPENDIX A

ANALOG-TO-DIGITAL DATA ANALYSIS PROGRAM (ADDA) 
Abstract

ii

I. STATEMENT OF PROBLEM

A-1

II. DESCRIPTION OF PROGRAM

$A-2$

III. DESCRIPTION OF SUBROUTINES

A -3

IV. INPUT

$A-10$

V. OUTPUT

A-11

VI. PROGRAM LIMITATIONS

$A-11$

VII. OPERATIONAL PROCEDURE

VIII. FREQUENCY ANALYSIS OF THE NERVA NARROW-BAND DATA

$A-20$

IX. DICTIONARY OF FORTRAN TERMS

$A-41$

X. LISTING OF PROGRAM SOURCE DECKS, PROGRAM ADDA

$A-45$

XI. DETAIL FLOW DIAGRAM OF PROGRAM AND SUBROUTINES

A-78 
$\mathrm{RN}-\mathrm{S}-0146$

\section{$\underline{\text { ABSTRACT }}$}

This program processes the narrow-band NERVA test data, in the form in which it comes directly from analog-to-digital conversion (N2 tapes), to provide measures of data quality. In this regard the program gives, by channel number, a compilation of successively calculated means and variances. The basic sample size for these estimates is 40 points. However, for cases of steps within these intervals, the sample size may be less. The start time for each sample interval is also outputted. At the occurrence of a major break in time, the program outputs some summary statistics for that time interval. These include the start-stop times for the interval, the number of data points per pin, the number of missing time points, the number of occurrences of various flag bits, the number of outliers, the number of steps, and the number of times periodicities are searched for in the data. In addition, it provides by pin number a frequency distribution of calculated variances, and a frequency distribution of estimated periodicities. 
$\mathrm{RN}-\mathrm{S}-0146$

I. STATEMENT OF PROBLEM

During the conduct of the NRX-Al reactor tests, many measurements were made on the various physical phenomena occurring. A portion (narrow-band data) of these measurements were reduced to digital form, calibrated, and released to engineering personnel to aid in determining the overall performance of the reactor. While the fully reduced data is probably most useful as an aid in making these determinations, the data in its form (N2 tape) directly after analog-to-digital conversion is very useful in determining the basic quality of the data. Quality of the data can be viewed in terms of the following considerations:

The frequency of occurrence of outlying observations (erroneous signals), and other types of error flags tacked on to the data during analog-to-digital conversion.

The accuracy of the data, or the closeness of agreement between the recorded value determined by the measuring system and the true value of phenomena occurring.

The precision of the data, or the repeatability of measurements where each observation is an assessment of the same value of the phenomena.

Clearly, the raw calibration data in the form of counts can be very useful in determining the quality level of the data. In generating the calibration data, known voltages are applied to the measuring system. These voltages are held constant over specified periods of time, and measurements of these voltages are made by the system. The precision of the data could then be determined from these measurements by estimating the variance of data over intervals of constant applied voltage. The accuracy of the data could be determined by testing the means of the observations over these periods with the known voltage inputs. Outlying observations could be counted as could the occurrences of other error flag bits. Since the run data for the IRX-Al series was composed of a series of linear ramps and steady-state holds, similar aralyses could be provided for the run data. During the 
RN-S-0146

periods of ramps, linear equations could be fitted to the data. Precision could then be measured by the standard error of estimate, and accuracy could be measured by comparing the equation of the line with pre-test objectives.

Periodicities in the data are further measures of data quality since certain frequencies may be induced by the data system and should not be interpreted as cyclical patterns in the phenomena.

To provide measures useful in determining the quality level of the N2 data, the program analog-to-digital data analysis (ADDA) was developed.

\section{DESCRIPTION OF PROGRAM}

Data is read from the $\mathbb{N} 2$ tapes in blocks of four physical records (or less if data has been exhausted). This data is then unpacked; flags are stripped from time values; times are floated and consolidated; flag bits in data words are masked and tallied; and the unpacked data is stored in matrix fashion for further processing. The time values are then edited for missing values. Large gaps in time signify a change in major test conditions. Assuming no major changes in time, the matrix of channel data is then processed channel-by-channel until all channels have been processed. At that time, four additional physical records are read from the N2 tape and the processing cycle is restarted. The channel-by-channel processing is carried out as follows:

A. A comparison is made between the first and last data values within a channel. If these values do not differ by more than a preset constant, $K_{1}$, it is inferred that during this time interval the data represents a constant value; the arithmetic mean, $\overline{\mathrm{X}}$, and the variance, $\mathrm{s}^{2}$, are then calculated for the $\mathrm{N}$ data points.

B. The estimated variance, $s^{2}$, is then compared to a second preset constant, $\mathrm{K}_{2}$. If $\mathrm{s}^{2}$ exceeds $\mathrm{K}_{2}$ it is inferred that ( 1 ) either the data contains one or more outliers, or (2) the data contains an excessive amount of periodicity. A test for outliers is made. If any are found, $\bar{x}$ and $s^{2}$ are adjusted; if none are found and/or $s^{2}$ still exceeds $K_{2}$, the mean, $\bar{x}$, is subtracted from all data points, and a frequency analysis is performed on the data to find any possible periodicities in the data. 
C. If the first and last data values differ by more than $K_{1}$, it is inferred that: ( 1 ) one of the end points may be an outlying observation; (2) the data encompass a step, as for cases in the calibration data; or (3) the data encompasses a ramp, as for cases in the run data. A test for end-point outliers is made; any outliers found are then adjusted. If this reduces the difference between the first and last data point to less than $K_{l}, \bar{x}$ and $s^{2}$ are calculated and the program proceeds as in $A$ and $B$ above. If no end-point outliers are found, or if the first and last observations still differ by more than $K_{1}$, a test is made to determine if a step has occurred within the data. If a step is found, the data are broken into two subsets and each subset is analyzed as discussed in $A$ above. If no step is found, the program fits a linear equation to the data, since it is assumed that the run data are comprised of only linear ramps and constant holds. Again, a test of $\mathrm{s}^{2}>\mathrm{K}_{2}$ is made, and if the inequality is satisfied, the data are detrended, and the frequency analysis applied to the data.

A generalized flow diagram of the program is given in Figure A-1. A description of the various subroutines is given in the following section, and the dictionary of FORTRAN terms is given in Section IX.

\section{DESCRIPTION OF SUBROUTINES}

This section is concerned with describing the function and/or mechanics of the various subroutines contained in ADDA.

A. SUBROUTINE INITIAL

This subroutine initializes various flags and counters used throughout the program.

B. SUBROUTINE BRAIN

The subroutine BRAIN supervises the reading of the N2 input tapes and the loading of the matrix JAZZ for processing. It is called by the main program and, in turn, calls several subroutines to accomplish its function. The interaction of BRAIN and its associated subroutines is illustrated in tho sketch at the top of page $A-5$. 
1 READ INPUT

2 CALL INITAL

3 CALL BRAIN

$$
\begin{gathered}
I=1 \\
N=40 \\
L I T E 4=0
\end{gathered}
$$$$
4 \quad I=1 k
$$

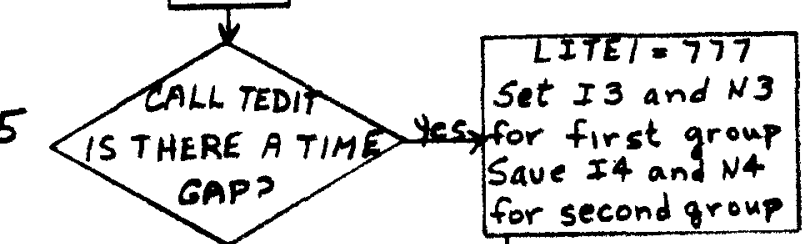

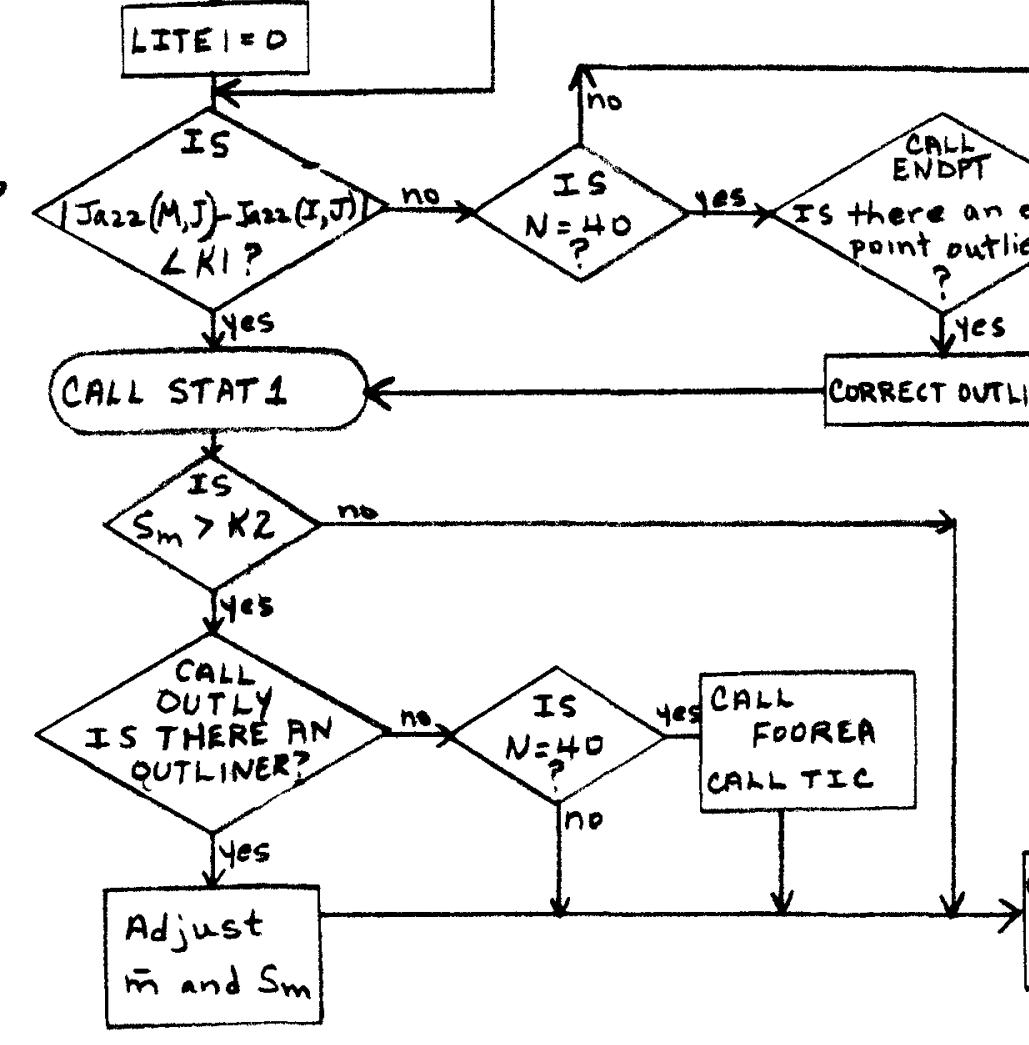

Figure A-I

General Flow Diagram of Program ADDA

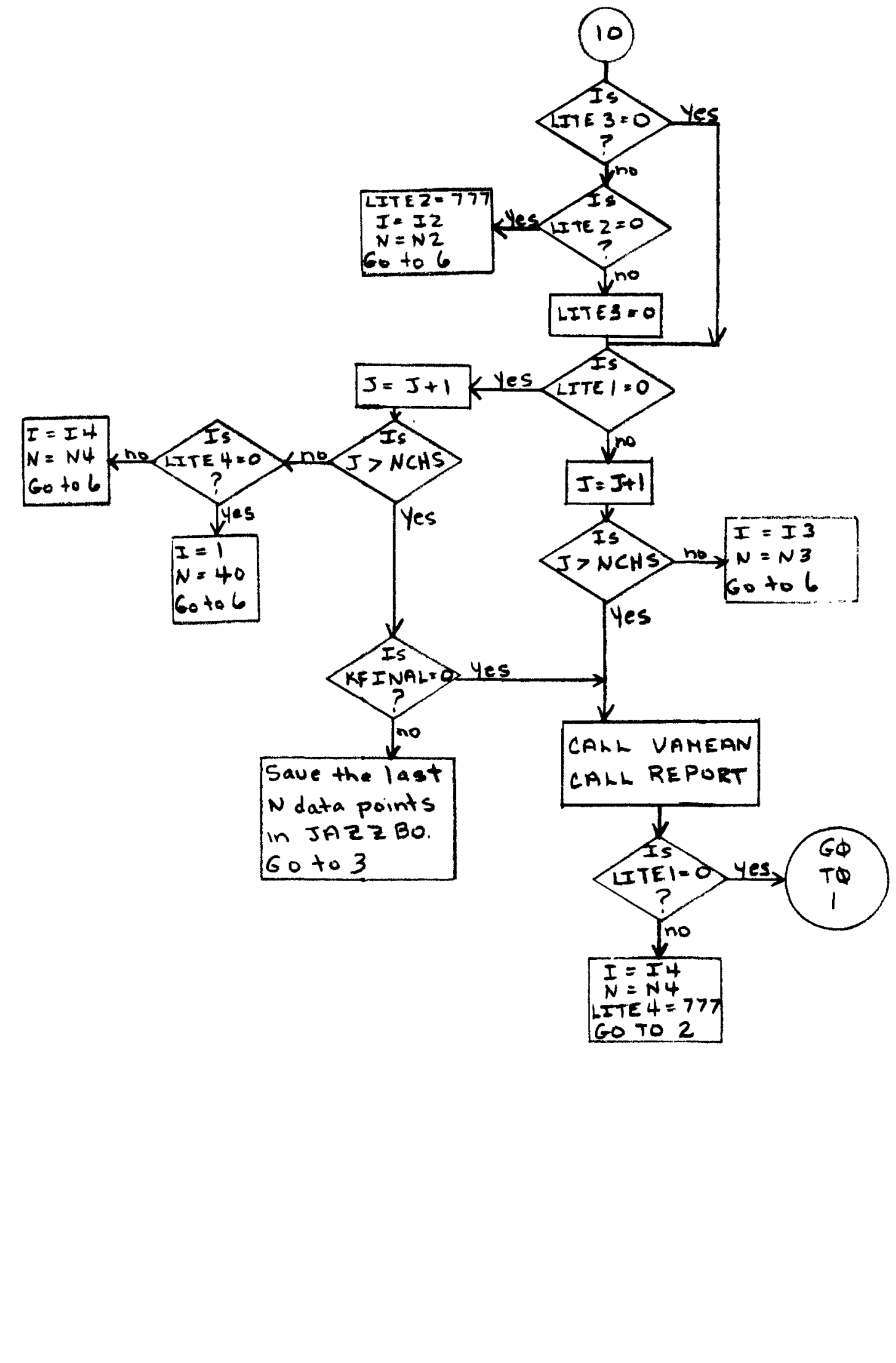




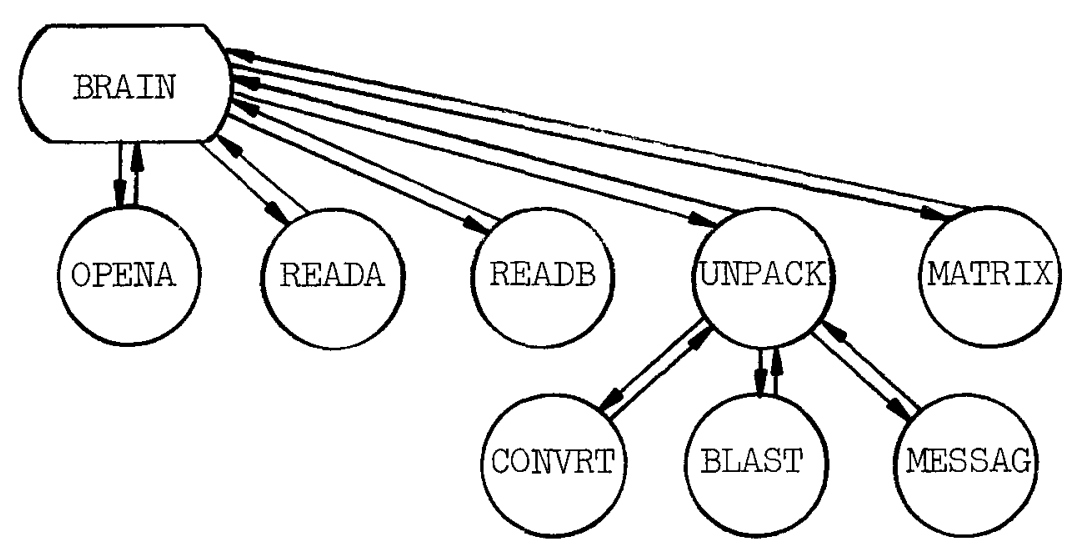

\section{SUBROUTINE OPENA}

The first subroutine called by subroutine BRAIN is OPENA. This subroutine initializes the tape unit on which the original data tape (N2 tape) is placed. The subroutine is called only once for each tape.

\section{SUBROUTINE READA}

The second subroutine called by subroutine BRAIN is READA. This subroutine reads the header record which is a short physical record describing the multiplexer data following it. The subroutine is called only once for each file.

\section{E. SUBROUTINE READB}

READB is the third subroutine called. The function of READB is to read one physical record of multiplexer data.

F. SUBROUTINE UNPACK

The fourth subroutine called is UNPACK. The purpose of UNPACK is to remove various flags imbedded in the time data, and to convert the time data from its coded form to floating point form. The multiplexer data is also unpacked, stripped of flags, and stored in vector fashion. To accomplish its function, subroutine UNPACK has three associated minor subroutines: CONVRT, BLAST, and MESSAG. Subroutine CONVRT is used to change time from BCD form to binary integer form. Subroutine BLAST, in turn, converts the binary integer form to floating point form, while combining the major time in seconds with the vernier time in millisec. Subroutine MESSAG is called to tally the various types of flag bits found imbedded in the $\mathrm{N} 2$ data. 


\section{G. SUBROUTINE MATRIX}

MATRIX is the fifth and last subroutine called by subroutine BRAIN. This subroutine positions the time data in a vector, TIME, and the multiplexer data in a matrix, JAZZ.

\section{H. SUBROUTINE TEDIT}

This subroutine tests the successive times in each interval. These times should differ by TAU, an irput variable. If this difference is only slightly larger than TAU it represents only one missing time point, and these missing points are counted by the variable, NICK.

If the difference is significantly larger than TAU, it represents a major time break. In this case the accumulated output is printed out by subroutines VAMEAN and REPORT, the flags and counters are reinitialized, and the data processing is continued.

\section{SUBROUTINE STATI}

This subroutine computes the arithmetic mean, BARM, and the variance, STERRY, of $N$ data points in column $J$ of matrix JAZZ, starting at row position $I$. The mean is calculated by

$$
\operatorname{BARM}=\frac{\sum_{k=I}^{I+N-I} \operatorname{JAZZ}(k, J)}{\mathbb{N}}
$$

and the variance by

$$
\text { STERRY }=\frac{\sum_{k=I}^{I+N-I}[\operatorname{JAZZ}(k, J)]^{2}-\operatorname{BARM} \sum_{k=I}^{I+N-I} \operatorname{JAZZ~}(k, J)}{N-I}
$$

\section{J. SUBROUTINE OUTLY}

A given time interval may include up to 40 data points for each channel or pin. This subroutine tests the inner points (excluding the end points) of each of these sets of points to locate outliers. A data point is considered to be an outlier if it differs from the two points surrounding it by a given input value 
$\mathrm{RN}-\mathrm{S}-0146$

$\mathrm{K} 4$, and if the two surrounding points differ by less than a smaller input constant K6. The value of such an outlier is returned to the main program by the variable, JOUT, so that the variance and mean of the set of data may be corrected; the value of the data point in the JAZZ matrix is then replaced by the arithmetic average of its two surrounding points. The total of such outliers is counted by the variable, NUM 1 .

\section{K. SUBROUTINE ENDPT}

This subroutine is called after a full set of data points (40) has been tested and the difference between the first and last point has been determined to be larger than a constant input $\mathrm{Kl}$. The purpose of the routine is to ascertain whether either of the end points is an outlier. Three variations from the usual smooth set of data have been found:

1. If the first point differs from the second point by more than $\mathrm{K} 4$, it is replaced by the arithmetic average of the last point in the previous set of data and the second point in the present set. This outlier is counted by $\mathrm{NUM} 1$.

2. If the last point differs from the previous point by more than $\mathrm{K} 4$, this point is discarded and the set is thereafter considered to have 39 points. This outlier is also counted by NUM 1 .

3. If the difference between the last two points is less than $K 4$ but more than $\mathrm{K} 6$, and if the $38 \mathrm{th}$ and 39 th points differ by less than $K 6$, then the last point is considered to be a one-point step and is discarded, and the set is considered to contain 39 points. The last point is not counted as an outlier.

If none of these possibilities will explain the original difference between the first and last points, the set of data points is input to SUBROUTINE STEP.

\section{SUBROUTINE STEP}

This subroutine tests for a step or ramp in a set of data. The set must contain at least three points. A one-point step at the end of the set cannot be identified by this routine. The difference between each two consecutive points is tested successively throughout the set of data. Whenever a difference 


$$
\mathrm{RN}-\mathrm{S}-0146
$$

larger than $\mathrm{K} 6$ is found that is not nullified or corrected by the following difference, that point is considered to be the beginning of a step or ramp in the data. The following differences are then tested until the point is found where the data values level off (i.e., the number of points in the ramp is determined). When a ramp contains more than one point, the midpoint of the ramp is chosen as a breaking point. The points before the midpoint are set equal to the value at the beginning of the ramp, and the points after the midpoint are set equal to the value at the end of the ramp. The variable, JFIAG, counts the number of points in the ramp.

The routine then returns to the main program with an indicator that causes the original set of points to be divided into two subsets which will be analyzed separately and with counters to tell the size of each subset. The second subset will be interrogated again by SUBROUTINE STEP if the main program determines that this is necessary. The number of steps or ramps is counted by the variable, NUM3.

\section{SUBROUTINE FITI}

This routine fits a linear trend to 40 points of data and returns with A (the mean of the observations), B (the slope of the trend), and STERRY (the variance of estimate). Furthermore, a test of STERRY VS K2 is made, where K2 is the constant used to judge excessive variance for possible periodicities. If STERRY is greater than K2, a flag (FLAG 17) is set to 777 to direct the program to subroutine FOOREA upon return to the main program; otherwise, FLAG 17 is set to zero.

Fitting of the line to the data is done using orthogonal polynomials and standard least-squares techniques.

\section{N. $\quad$ SUBROUTINE FIT2}

This subroutine fits a linear trend to $\mathbb{N}$ points of data, where $3<\mathbb{N}<40$. The subroutine returns with A (the mean of the observations), B (the slope of the trend), and STERRY (the variance of estimate).

Fitting of the line to the data is done using orthogonal polynomials and standard least-squares techniques. The program computes the required orthogonal weights for the linear term each time entered. These weights depend on the length of the data span, $\mathbb{N}$. 


\section{O. SUBROUTINE FOOREA}

This routine finds the frequencies present in 39 detrended data points. The total number of frequencies allowed is 20 .

The frequencies in cycles per second, their sine and cosine components, and the sum of the squares of the sine and cosine terms are returned. For the mathematical details of the subrouting, see section VIII.

\section{P. SUBROUTINE VAMEAN}

This subroutine stores the calculated values of the variances and the arithmetic means in two separate two-way arrays dimensioned by channel numbers (horizontally) and time values (vertically). After 52 time points have been processed, these two arrays are printed out and refilled. The printout occurs while the data is being processed. This output is formatted for $1 I-I / 2$ by $14 \mathrm{in}$. paper.

\section{Q. SUBROUTINE TALLY}

This subroutine performs a tabulation of the frequency distribution of the calculated variances. A running count is made for each channel of the number of times the variance falls in the intervals 0 to 1,1 to 2 , etc., up to $9+$. Totals are kept for each channel and each interval. These counts are printed out at each major time break by SUBROUIINE REPORT in tabular form with each interval in a column followed by its total, and each channel in a row followed by its total.

\section{R. SUBROUTINE TIC}

This subroutine performs a tabulation of the frequency distribution of the estimated periodicities found in the data by SUBROUTINE FOOREA. A running tally is made for each channel of the number of times the periodicities (or frequencies) in the data fell in the intervals 0 to 1,1 to 2,2 to 3 , etc., up to 19 to 20. Running totals are also stored for each channel and each interval. These counts are printed out at each major time break by SUBROUTINE REPORT in the same general format as that used for SUBROUTINE TALLY.

\section{S. SUBROUTINE REPORT}

This subroutine prints out the accumulated output at each major time break. This output consists of the following items: 
1. An input descriptive title

2. The name of each multiplexer (input).

3. The number of data points processed for each channel.

4. The start time of the group.

5. The last time of the group.

6. The number of missing time points.

7. The number of 01 flags on the data.

8. The number of 10 flags on the data.

9. The number of 11 flags on the data.

10. The number of outliers found in the group.

11. The number of times the program called SUBROUTINE FOOREA.

12. The number of steps found in the group.

13. The table of the frequency distribution of the calculated variances (see SUBROUTINE TALLY).

14. The table of the frequency distribution of estimated periodicities in the data (see SUBROUTINE TIC).

The output format is arranged to be printed on $11-1 / 2$ by $14 \mathrm{in}$. paper. IV. INPUT

A. Header - a descriptive title.

B. K4 - If the difference between two successive data points is larger than $\mathrm{K} 4$, the second point is either an outlier or the beginning of a step in the data.

C. K6 - This value is smaller than $\mathrm{K} 4$, and is used to differentiate between outliers and steps. If the point following the two points discussed above differs from the first point by less than $K 6$, the second point is an outlier; otherwise the second point is the beginning of step or ramp in the data.

D. Kl - If the first and last values within a channel do not differ by more than $\mathrm{Kl}$, the data is following a constant trend during this time interval, and the mean and the variance are calculated for the $\mathbb{N}$ data points by Subroutine STATl. If the difference is larger than $\mathrm{Kl}$, the $\mathbb{N}$ data points are investigated for a possible step or outlier. 


\section{$\mathrm{RN}-\mathrm{S}-0146$}

E. K2 - After the variance for $\mathbb{N}$ data points is determined in STATl, this value is compared with $\mathrm{K} 2$. If it is larger than $\mathrm{K} 2$, the $\mathrm{N}$ data points are tested to determine whether an outlier is present and should be corrected.

F. MULTPX - the name of the multiplexer being processed.

G. NCHS - the number of channels present in the multiplexer.

H. IBITE - the number of logical records per physical record of data.

I. ISIZE - the number of words in each physical record.

J. TAU - the time between successive samples.

Sample input is shown directly below.

A. First card - HEADER - Format $12 A 6$

Example: EPI NRX-Al TCA

B. Second card - K4, K6, Kl, K2 - Format 3I6, F6.0

Example: $100 \quad 20 \quad 30 \quad 5$

c. Next $n$ cards - MULTPX, NCHS, IBITE, ISIZE, TAU - Format A6, 3I6, F6.0

Example: HLOI $49 \quad 10 \quad 180 \quad .025$

$\begin{array}{lllll}\text { HLO2 } & 49 & 10 & 180 & .025\end{array}$

STOP

Note: The final card should have STOP input as MULTPX in order to rewind the input data tape and halt the program.

V. OUTPUT

Descriptions of the various outputs by Program ADDA were discussed in Section III under Subroutines VAMEAN, TALIY, TIC, and REPORT. Examples of these outputs are shown on the next few pages.

VI. PROGRAM LIMITATIONS

The labels on the N2 data input tapes received from the test site are often incomplete and occasionally are not correct. Thus, to ensure successful execution of this program, it is necessary to dump the first two physical records of each file on the N2 data input tape. 
ANALOG-DIGITAL DATA ANALYSIS

EPI NRX-AI TCA CALIBRATION CATA

MULTIPLEXER NUMBER HLOI

NUMBER OF DATA POINTS FOR EACH PIN

4392

START TIME $=84040.016$

STOP TIME $=84149.990$

NUMBER OF MISSING TIME POINTS

NUMBER OF OI FLAGS ON DATA 1

NUMBER OF 10 FLAGS ON DATA 0

NUMBER OF 11 FLAGS ON DATA 0

NUMBER OF OUTLIERS 36

NUMBER OF TIMES PROGRAM WENT TO FOOREA

NUMBER OF STEPS 315 


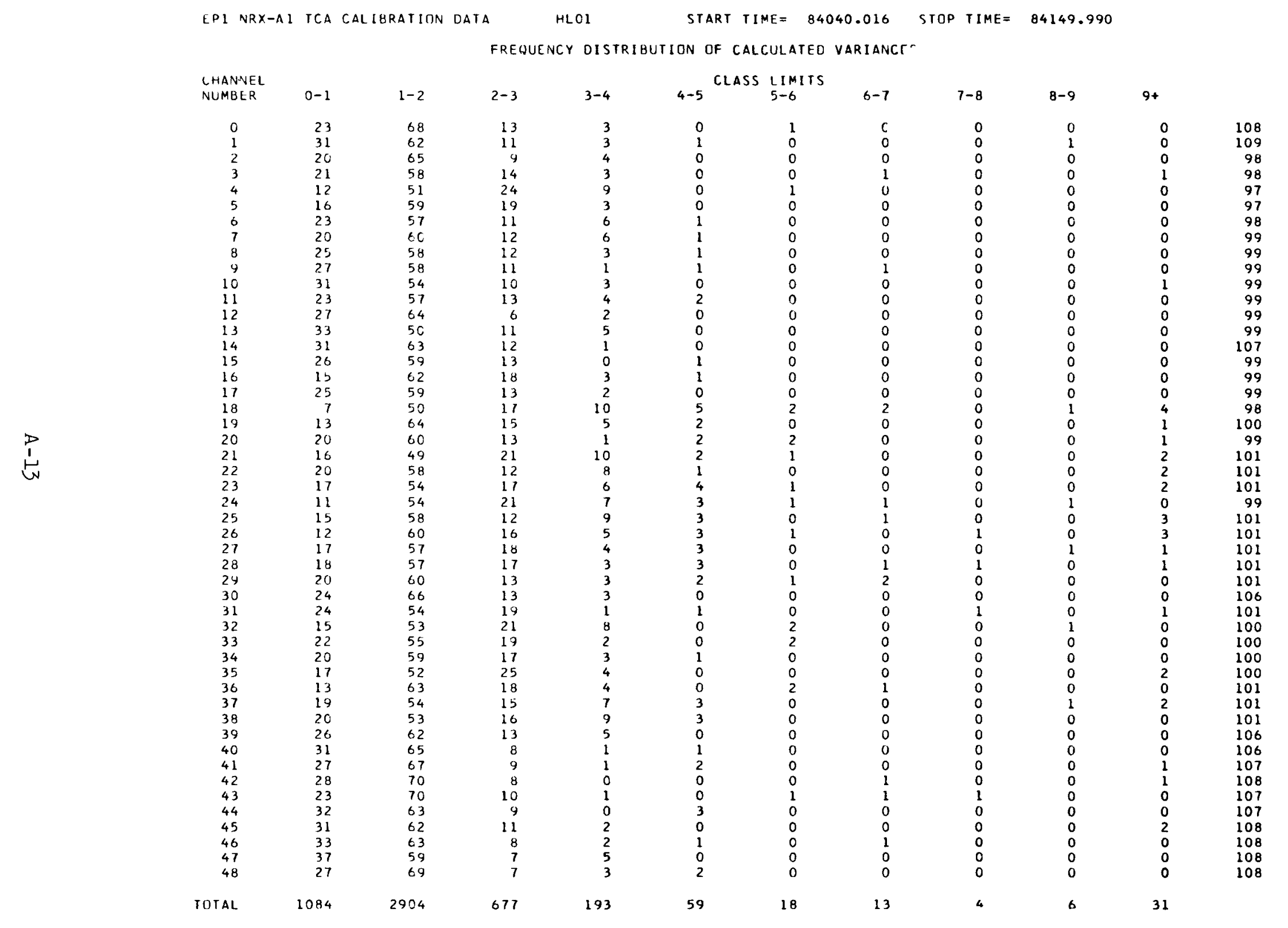


EPI NRX-AI TCA CALIBRATION DATA HLOI

START TIME $=84040.016$

STOP TIME $=84149.990$

FREQUENCY OISTRIBUTION OF ESTIMATED PERIODICITIES IN OATA

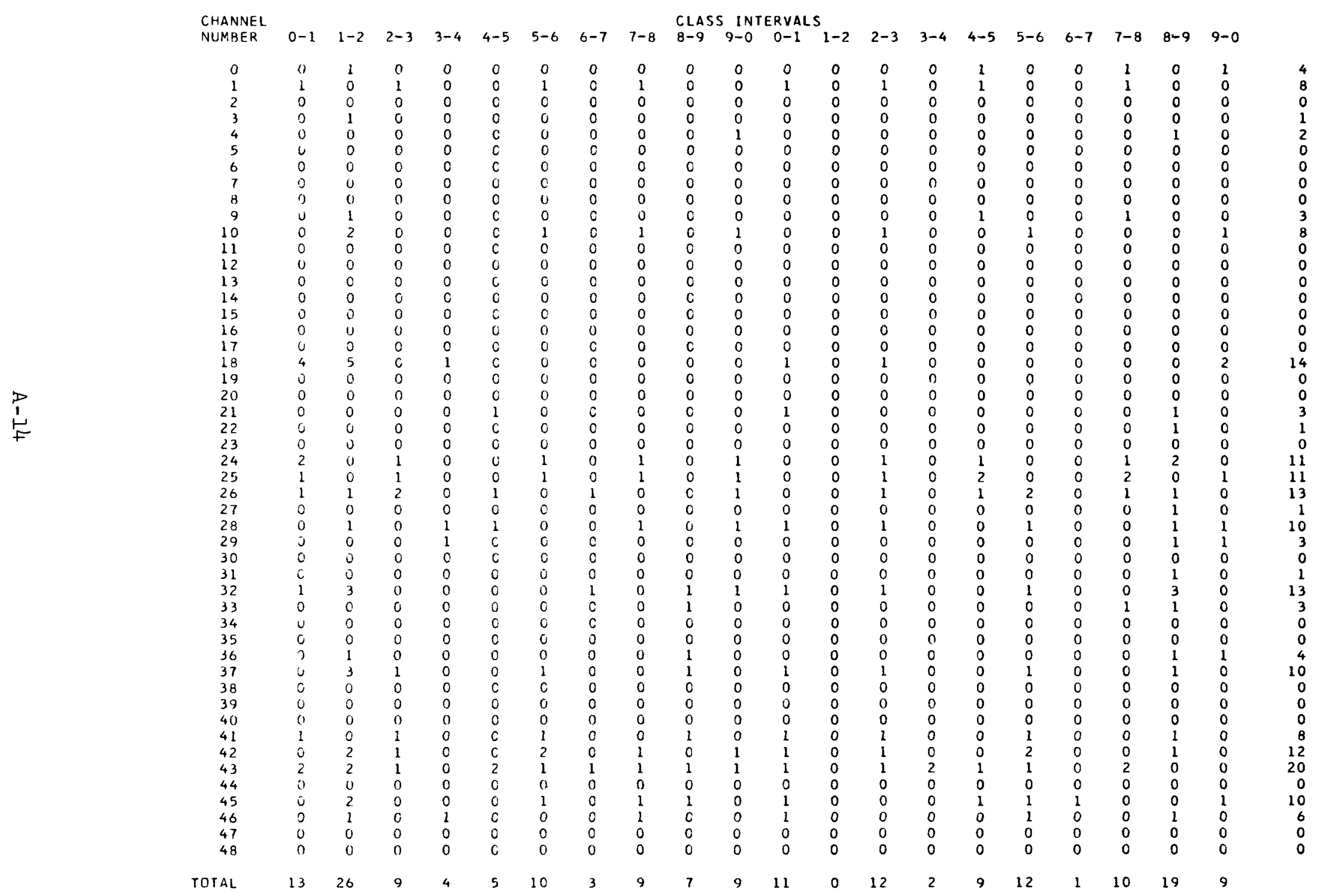


EPI NRX-AI ICA CALIBRATION CATA

I I ME

84040.016 84041.016 84042.016 84043.016 84044.016 84045.016 84046.016 84048.016 4052.016 84053.016 84054.016 84055.016 84056.016 84057.016 84058.016 84059.016 34060.016 84061.040 84062.040 84063.065 84064.065 84065.065 84066.065 84067.065 84068.065 84069.065 84071.09 84072.091 84073.091 84074.115 84075.115 84076.115 84077.115 84078.115 84079.115 84080.115 84081.115 84082.115 84083.115 84084.115 84085.115 84086.115

84088.115

4408.115

84000.115
COMPILATION OF SUCCESSIVE CALCULATED VARIANCES

\begin{tabular}{|c|c|c|c|c|c|c|c|c|c|c|c|c|c|c|}
\hline \multicolumn{15}{|c|}{ CHANNEL NUMBERS } \\
\hline 10 & Il & 12 & 13 & 14 & 15 & 16 & 17 & 18 & 19 & 20 & 21 & 22 & 23 & \\
\hline 1 & 0 & 0 & 1 & 0 & 1 & 1 & 0 & 4 & 1 & 0 & 0 & 0 & 0 & \\
\hline 0 & 1 & 1 & 1 & 1 & 1 & 1 & 2 & 1 & 1 & 0 & 1 & 1 & 1 & \\
\hline 0 & 0 & 0 & 0 & 1 & 1 & $i$ & 2 & 4 & $i$ & 1 & 0 & 1 & 1 & \\
\hline 0 & 1 & 1 & 1 & 0 & 0 & 0 & 0 & 8 & 0 & 0 & 0 & 0 & 1 & \\
\hline 0 & 1 & 0 & 0 & 0 & 1 & 1 & 0 & 5 & 1 & 0 & 1 & 0 & 1 & \\
\hline 0 & 1 & 0 & 1 & 1 & 1 & 3 & 1 & 14 & 1 & 1 & $i$ & 0 & 1 & \\
\hline I & 2 & 0 & 0 & 0 & $i$ & 1 & 3 & 2 & 1 & i & l & 1 & 0 & \\
\hline 0 & 0 & 0 & 1 & 0 & 1 & 0 & 1 & 1 & $i$ & 0 & $i$ & 0 & 0 & \\
\hline 1 & 1 & 1 & 1 & 1 & 1 & 0 & 1 & 1 & 1 & 0 & 0 & 1 & 0 & \\
\hline$i$ & 0 & 0 & 0 & 0 & 1 & 2 & 0 & 1 & 0 & 1 & 1 & 0 & 0 & \\
\hline 0 & 0 & 0 & 0 & 1 & 1 & 1 & 0 & $i$ & 0 & 0 & 0 & $i$ & 1 & \\
\hline 1 & 2 & 2 & 1 & 2 & 2 & 1 & 2 & 5 & 2 & 43 & 1 & 14 & 10 & \\
\hline 1 & 2 & 1 & 1 & 1 & 4 & 1 & 1 & 3 & 1 & 1 & 1 & 0 & 1 & \\
\hline 1 & 1 & i & $i$ & 0 & 1 & i & 0 & 2 & 1 & 1 & 2 & 2 & 2 & \\
\hline 1 & 3 & 1 & 3 & 0 & $i$ & $i$ & 0 & 2 & $i$ & i & 1 & $i$ & $i$ & \\
\hline 1 & 1 & 2 & 2 & 3 & 1 & 1 & 1 & 4 & $i$ & $i$ & $i$ & 1 & 1 & \\
\hline 1 & 0 & 1 & 1 & 0 & 1 & 1 & 0 & 6 & 1 & 1 & 1 & 1 & 2 & \\
\hline 1 & 1 & i & 2 & 1 & $i$ & $i$ & 1 & 2 & $i$ & $i$ & $i$ & $i$ & $i$ & \\
\hline 1 & 1 & 0 & 1 & 0 & 1 & 1 & 1 & 1 & 1 & 2 & 3 & 2 & 3 & \\
\hline 2 & 4 & 2 & 1 & 1 & 2 & 3 & 2 & 1 & 1 & 1 & l & 1 & 1 & 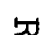 \\
\hline 1 & i & 3 & $i$ & 0 & 1 & 1 & 1 & 1 & 1 & 1 & $i$ & 1 & 2 & 己 \\
\hline 4 & 1 & 2 & 1 & 1 & 2 & 2 & 1 & 2 & 1 & 8 & 14 & 2 & 2 & 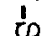 \\
\hline 3 & 2 & 1 & 3 & 1 & 1 & 1 & 2 & 2 & 1 & 1 & 1 & 1 & 1 & $0 \Omega$ \\
\hline 1 & 4 & 1 & 2 & 0 & 0 & 1 & 1 & 3 & $i$ & 2 & 2 & 2 & 3 & 0 \\
\hline 1 & 2 & $i$ & 1 & 0 & 1 & 1 & $i$ & 3 & 2 & 2 & 3 & 3 & 4 & \\
\hline 2 & 1 & 1 & 1 & 1 & 0 & l & 1 & 4 & 2 & 1 & 3 & 3 & 4 & $\mp$ \\
\hline 1 & 1 & $i$ & 1 & 0 & 1 & $i$ & $i$ & 1 & $i$ & 2 & 3 & 3 & 3 & \\
\hline
\end{tabular}


EPI NRX-AI TCA CALIBRATION DATA

TIME

84040.016
84041.016
84042.016
84043.016
84044.016
84045.016
84046.016
84047.016
84048.016

84049.016

84050.016

84051.016

84052.016

84053.016

84054.016

84055.016

84057.016

84058.016

84059.016

.016

84061.040

84062.040

84063.065
84064.065

84064.065
84065.065

84066.065

84067.065

84068.065

84070.091

84071.091

84072.091

84073.091

84074.115

84075.115

84076.115

84077.11

84078.115

84079.115

84080.115

84081.115

84082.115

84083.115

84084.115

84085.115

84086.115

84088.115

84089.115

84090.115

84091.115
COMPILATION OF SUCCESSIVE CALCULATED VARIANCES

$$
\text { CHANNEL NUMBERS }
$$

\begin{tabular}{|c|c|c|c|c|c|c|c|c|c|c|c|c|c|}
\hline 34 & 35 & 36 & 37 & 38 & 39 & 40 & 41 & 42 & 43 & 44 & 45 & 40 & \\
\hline 2 & 1 & 1 & 1 & 2 & 0 & 1 & 1 & $\begin{array}{r}r_{0} \\
\end{array}$ & 0 & 0 & 1 & $\begin{array}{r}40 \\
1\end{array}$ & 4 \\
\hline 1 & 2 & 2 & 1 & 1 & 1 & 1 & 0 & 1 & 1 & 0 & 0 & 0 & \\
\hline 1 & 2 & 1 & 0 & 0 & l & I & 0 & 1 & $i$ & 0 & 2 & 1 & \\
\hline 0 & 0 & $\mathfrak{1}$ & 1 & 0 & 1 & 1 & 1 & 1 & 0 & 1 & 1 & 1 & \\
\hline 1 & 1 & $i$ & 1 & 1 & $i$ & 0 & $i$ & 0 & 1 & 0 & l & 1 & \\
\hline 3 & 1 & $i$ & 1 & 0 & $i$ & 1 & 0 & 1 & $i$ & i & $i$ & 0 & \\
\hline 1 & 0 & 1 & 0 & 0 & 1 & 2 & 1 & 1 & 2 & 2 & 2 & 2 & \\
\hline 1 & 1 & 0 & 2 & 1 & l & 1 & 0 & 0 & 1 & 1 & 1 & 1 & \\
\hline 1 & 1 & I & 1 & 0 & 0 & 0 & 0 & 0 & $i$ & 1 & 1 & 1 & \\
\hline 2 & 1 & 0 & 1 & i & 1 & 1 & 1 & 1 & 2 & 2 & 2 & 2 & \\
\hline 1 & 1 & 1 & 1 & 1 & 0 & 0 & 1 & 1 & 1 & l & 1 & 2 & \\
\hline 2 & 8 & 2 & 6 & 2 & 1 & 0 & 0 & $i$ & i & $i$ & 1 & 1 & \\
\hline 1 & 1 & 1 & 1 & 1 & 1 & 1 & 1 & 1 & 1 & 1 & 1 & 2 & \\
\hline 2 & 2 & 1 & 1 & 1 & 0 & 0 & 1 & 1 & 1 & 1 & 1 & 1 & \\
\hline 2 & 1 & 1 & 1 & 1 & 0 & 0 & 1 & 0 & 1 & 0 & 0 & 0 & \\
\hline 0 & 0 & 1 & 0 & 1 & 1 & 0 & 2 & 1 & 1 & 2 & 2 & 2 & \\
\hline 1 & 1 & 1 & 1 & 2 & 0 & 0 & 0 & 0 & 0 & 1 & 1 & 1 & \\
\hline 0 & 1 & 0 & $i$ & 1 & 0 & 1 & 1 & 2 & 1 & 2 & i & 1 & \\
\hline 2 & 2 & 2 & i & $i$ & 0 & 0 & 0 & 0 & 0 & 0 & 0 & 2 & \\
\hline 1 & 1 & 2 & 1 & 0 & 1 & 1 & 1 & 1 & 0 & 1 & 1 & 0 & \\
\hline 1 & 1 & 1 & 1 & 1 & 1 & 1 & 1 & 1 & 1 & 1 & 2 & 1 & \\
\hline 3 & 4 & 4 & 9 & 3 & 1 & 1 & 2 & 1 & 2 & $i$ & 1 & $i$ & \\
\hline 1 & 1 & 2 & 1 & 3 & 1 & 2 & 3 & 2 & 2 & 0 & 0 & 1 & \\
\hline 1 & 1 & 1 & 1 & 1 & 1 & 1 & 1 & 2 & 1 & 1 & 1 & 1 & \\
\hline 1 & 1 & $i$ & $i$ & $i$ & $i$ & 1 & 2 & 1 & 1 & 4 & i & 1 & \\
\hline i & 3 & 1 & 2 & 1 & 1 & 0 & 0 & 0 & 0 & 4 & i & 1 & \\
\hline 2 & 3 & 1 & 1 & 2 & 0 & 0 & 0 & 0 & 1 & 2 & $i$ & 0 & \\
\hline
\end{tabular}


EPI NRX-A1 TCA CALIBRATION DATA

TIME

0

$12 \quad 3 \quad 4 \quad 4 \quad 5$

56

COMPILATION OF SUCCESSIVE ARITHMETIC AVERAGES

$\begin{array}{lllllllllllllll}84041.016 & 983 & 36 & 973 & 384 & 978 & 985 & 958 & 984 & 979 & 978 & 978 & 978 & 982 & 979 \\ & 983 & 37 & 973 & 984 & 978 & 986 & 958 & 985 & 979 & 978 & 978 & 979 & 981 & 980\end{array}$

$\begin{array}{lllllllllllllll}84042.016 & 983 & 38 & 973 & 984 & 977 & 985 & 958 & 984 & 979 & 978 & 978 & 979 & 982 & 979\end{array}$

$\begin{array}{lllllllllllllll}84043.016 & 983 & 37 & 973 & 984 & 976 & 985 & 958 & 985 & 979 & 978 & 978 & 978 & 982 & 979 \\ 84044.016 & 984 & 37 & 973 & 984 & 976 & 985 & 958 & 985 & 979 & 978 & 978 & 979 & 982 & 980\end{array}$

$\begin{array}{lllllllllllllll}84045.016 & 984 & 38 & 973 & 984 & 977 & 985 & 958 & 984 & 979 & 978 & 978 & 978 & 982 & 980\end{array}$

$\begin{array}{lllllllllllllll}84046.016 & 984 & 38 & 973 & 984 & 978 & 985 & 958 & 984 & 979 & 978 & 978 & 978 & 981 & 979\end{array}$

$\begin{array}{lllllllllllllll}84047.016 & 984 & 38 & 973 & 984 & 976 & 985 & 958 & 984 & 979 & 978 & 978 & 978 & 982 & 979 \\ 84048.016 & 984 & 37 & 972 & 984 & 975 & 985 & 958 & 984 & 979 & 978 & 979 & 978 & 982 & 980\end{array}$

$\begin{array}{lllllllllllllll}84049.016 & 985 & 38 & 972 & 984 & 977 & 985 & 958 & 984 & 979 & 978 & 978 & 978 & 982 & 979\end{array}$

$\begin{array}{lllllllllllllll}84050.016 & 984 & 38 & 973 & 984 & 978 & 984 & 958 & 984 & 979 & 978 & 979 & 978 & 982 & 979\end{array}$

$\begin{array}{lllllllllllllll}84051.016 & 986 & 39 & 881 & 892 & 883 & 891 & 867 & 891 & 886 & 886 & 885 & 885 & 889 & 887 \\ 84052.016 & 986 & 39 & 880 & 891 & 884 & 891 & 866 & 891 & 886 & 885 & 885 & 885 & 888 & 887\end{array}$

$\begin{array}{lllllllllllllll}84052.016 & 986 & 39 & 880 & 891 & 884 & 891 & 866 & 891 & 886 & 885 & 885 & 885 & 888 & 887 \\ 84053.016 & 986 & 39 & 880 & 891 & 885 & 892 & 867 & 891 & 886 & 885 & 885 & 885 & 888 & 886\end{array}$

$\begin{array}{lllllllllllllll}84053.016 & 986 & 39 & 880 & 891 & 885 & 892 & 867 & 891 & 886 & 885 & 885 & 885 & 888 & 886 \\ 84054 & 986 & 39 & 880 & 891 & 883 & 891 & 867 & 891 & 886 & 885 & 885 & 885 & 888 & 886\end{array}$

$\begin{array}{lllllllllllllll}84054.016 & 986 & 39 & 880 & 891 & 883 & 891 & 867 & 891 & 886 & 885 & 885 & 885 & 888 & 886 \\ 84055.016 & 985 & 39 & 880 & 890 & 883 & 891 & 866 & 891 & 886 & 886 & 885 & \mathbf{8 8 5} & \mathbf{8 8 9} & \mathbf{8 8 7}\end{array}$

$\begin{array}{llllllllllllllll}84056.016 & 986 & 39 & 880 & 890 & 884 & 891 & 866 & 891 & 886 & 885 & 885 & 885 & 889 & 888\end{array}$

$\begin{array}{lllllllllllllll}84057.016 & 985 & 39 & 880 & 890 & 884 & 891 & 867 & 891 & 886 & 885 & 885 & 885 & 889 & 888 \\ 84058.016 & 985 & 39 & 880 & 890 & 882 & 891 & 866 & 891 & 886 & 885 & 885 & 885 & 890 & 888\end{array}$

$\begin{array}{lllllllllllllll}84058.016 & 985 & 39 & 880 & 890 & 882 & 891 & 866 & 891 & 886 & 885 & 885 & 885 & 890 & 888 \\ 84059.016 & 986 & 39 & 880 & 890 & 884 & 891 & 867 & 891 & 886 & 885 & 885 & 885 & 890 & 889\end{array}$

$\begin{array}{lllllllllllllll}84059.016 & 986 & 39 & 880 & 890 & 884 & 891 & 867 & 891 & 886 & 885 & 885 & 885 & 890 & 889 \\ 84060.016 & 985 & 39 & 880 & 890 & 884 & 891 & 866 & 891 & 886 & 885 & 885 & 886 & 891 & 888\end{array}$

84061.040986

84063.065987

84064.065987

84065.065987

84066.065987

$84068.065 \quad 987$

84069.065987

84070.091987

84071.091988

84072.091989

84073.091988

$84075.115 \quad 488$

$84076.115 \quad 988$

$84077.115 \quad 988$

$84078.115 \quad 989$

$84079.115 \quad 988$

$84080.115 \quad 988$

84081.115990

84082.115990

84083.115990

84084.115990

84085.115990

84086.115990

84087.115990

84089.115 991

84090.115992

84091.115994 $\begin{array}{lllllllllllll}40 & 788 & 798 & 791 & 798 & 776 & 797 & 794 & 792 & 792 & 793 & 797 & 795 \\ 40 & 787 & 797 & 791 & 798 & 776 & 798 & 793 & 792 & 792 & 794 & 797 & 795\end{array}$

\begin{tabular}{lllllllllllll}
40 & 788 & 796 & 790 & 797 & 775 & 798 & 793 & 792 & 793 & 794 & 797 & 795 \\
\hline
\end{tabular}

$\begin{array}{lllllllllllll}40 & 788 & 797 & 790 & 797 & 775 & 798 & 792 & 792 & 793 & 794 & 797 & 795\end{array}$

$\begin{array}{lllllllllllll}40 & 788 & 797 & 792 & 797 & 776 & 798 & 793 & 792 & 794 & 794 & 797 & 795\end{array}$

$\begin{array}{lllllllllllll}41 & 788 & 797 & 789 & 797 & 776 & 798 & 793 & 793 & 794 & 794 & 797 & 795\end{array}$

$\begin{array}{lllllllllllll}40 & 788 & 797 & 791 & 797 & 776 & 798 & 793 & 792 & 794 & 794 & 797 & 795 \\ 40 & 788 & 797 & 791 & 798 & 776 & 798 & 793 & 793 & 793 & 794 & 797 & 795\end{array}$

$\begin{array}{lllllllllllll}40 & 788 & 797 & 791 & 798 & 776 & 797 & 793 & 792 & 794 & 794 & 797 & 795\end{array}$

$\begin{array}{lllllllllllll}42 & 695 & 705 & 699 & 704 & 685 & 704 & 700 & 701 & 702 & 701 & 704 & 702\end{array}$

$\begin{array}{lllllllllllll}43 & 695 & 704 & 699 & 705 & 686 & 705 & 701 & 702 & 702 & 702 & 704 & 702\end{array}$

$\begin{array}{lllllllllllll}43 & 696 & 704 & 699 & 705 & 686 & 705 & 700 & 701 & 701 & 701 & 704 & 702\end{array}$

$\begin{array}{lllllllllllll}42 & 696 & 704 & 698 & 705 & 685 & 705 & 700 & 701 & 701 & 702 & 704 & 702 \\ 42 & 696 & 704 & 698 & 704 & 686 & 705 & 701 & 701 & 702 & 701 & 704 & 702\end{array}$

$\begin{array}{llllllllllllll}42 & 695 & 704 & 698 & 705 & 685 & 705 & 702 & 701 & 702 & 701 & 704 & 702\end{array}$

$\begin{array}{lllllllllllll}42 & 696 & 704 & 698 & 705 & 685 & 706 & 702 & 701 & 701 & 701 & 704 & 702\end{array}$

$\begin{array}{llllllllllllll}42 & 695 & 704 & 698 & 704 & 687 & 706 & 702 & 701 & 701 & 701 & 704 & 702\end{array}$

$\begin{array}{lllllllllllll}42 & 695 & 703 & 698 & 705 & 687 & 706 & 701 & 701 & 701 & 701 & 704 & 702\end{array}$

$\begin{array}{lllllllllllll}44 & 604 & 612 & 606 & 612 & 596 & 613 & 609 & 608 & 609 & 608 & 611 & 609\end{array}$

$\begin{array}{lllllllllllll}44 & 603 & 611 & 608 & 613 & 596 & 613 & 609 & 608 & 609 & 609 & 611 & 610\end{array}$

$\begin{array}{lllllllllllll}44 & 603 & 612 & 608 & 613 & 596 & 613 & 609 & 608 & 608 & 608 & 611 & 610\end{array}$

$\begin{array}{lllllllllllll}44 & 603 & 613 & 609 & 614 & 596 & 613 & 609 & 608 & 608 & 608 & 611 & 610\end{array}$

$\begin{array}{llllllllllllll}44 & 604 & 613 & 608 & 613 & 596 & 613 & 609 & 608 & 609 & 608 & 611 & 610\end{array}$

$\begin{array}{lllllllllllll}45 & 604 & 613 & 607 & 613 & 596 & 613 & 608 & 608 & 608 & 608 & 611 & 610\end{array}$

$\begin{array}{lllllllllllll}45 & 605 & 613 & 607 & 613 & 596 & 613 & 608 & 608 & 609 & 608 & 611 & 610 \\ 45 & 605 & 613 & 607 & 613 & 596 & 613 & 608 & 608 & 608 & 608 & 611 & 609\end{array}$

$\begin{array}{llllllllllllllll}45 & 605 & 613 & 600 & 613 & 596 & 613 & 608 & 608 & 608 & 608 & 611 & 609\end{array}$

$\begin{array}{lllllllllllll}47 & 514 & 521 & 515 & 520 & 506 & 520 & 517 & 516 & 517 & 516 & 519 & 517\end{array}$ $\begin{array}{lllllllll}15 & 16 & 17 & 18 & 19 & 20 & 21 & 22 & 23\end{array}$

$\begin{array}{llllllllll}5 & 976 & 975 & 981 & 982 & 975 & 978 & 986 & 977 & 982\end{array}$ $\begin{array}{llllllllll}5 & 977 & 975 & 982 & 982 & 976 & 978 & 986 & 978 & 982\end{array}$ $\begin{array}{llllllllll}5 & 977 & 975 & 982 & 983 & 975 & 978 & 986 & 977 & 981\end{array}$ $\begin{array}{llllllllll}4 & 977 & 975 & 983 & 983 & 976 & 978 & 986 & 978 & 981 \\ 5 & 977 & 975 & 983 & 982 & 976 & 978 & 986 & 978 & 982\end{array}$ $\begin{array}{llllllllll}4 & 976 & 975 & 983 & 982 & 976 & 978 & 986 & 977 & 981\end{array}$ $\begin{array}{llllllllll}14 & 976 & 976 & 984 & 983 & 976 & 978 & 986 & 977 & 981\end{array}$ $\begin{array}{llllllllll}4 & 977 & 976 & 984 & 982 & 975 & 978 & 986 & 977 & 981\end{array}$ $\begin{array}{llllllllll}15 & 977 & 977 & 983 & 983 & 975 & 978 & 986 & 977 & 981\end{array}$ $\begin{array}{llllllllll}15 & 977 & 977 & 983 & 983 & 976 & 978 & 985 & 977 & 981\end{array}$ $\begin{array}{llllllllll}15 & 978 & 977 & 983 & 983 & 976 & 978 & 986 & 978 & 981\end{array}$ $\begin{array}{llllllllll}18 & 885 & 884 & 890 & 889 & 883 & 887 & 892 & 886 & 889\end{array}$ $\begin{array}{lllllllllll}18 & 886 & 884 & 890 & 890 & 883 & 885 & 892 & 885 & 888\end{array}$ $\begin{array}{llllllllll}19 & 886 & 885 & 890 & 889 & 883 & 885 & 892 & 885 & 888\end{array}$ $\begin{array}{llllllllll}19 & 886 & 884 & 890 & 888 & 883 & 885 & 892 & 885 & 888\end{array}$ $\begin{array}{llllllllll}19 & 886 & 884 & 889 & 890 & 883 & 885 & 892 & \mathbf{8 8 4} & \mathbf{8 8 8} \\ 19 & 886 & 884 & 890 & 888 & 883 & 885 & 892 & 885 & \mathbf{8 8 9}\end{array}$ $\begin{array}{llllllllll}19 & 886 & 884 & 890 & 889 & 883 & 885 & 892 & 885 & 888\end{array}$ $\begin{array}{llllllllll}20 & 886 & 884 & 890 & 889 & 883 & 885 & 892 & 885 & 888\end{array}$ $\begin{array}{llllllllll}20 & 886 & 884 & 890 & 889 & 883 & 885 & 892 & 885 & 888\end{array}$ $\begin{array}{llllllllll}19 & 886 & 884 & 890 & 890 & 883 & 885 & 892 & 885 & 888 \\ 21 & 793 & 791 & 796 & 796 & 791 & 793 & 799 & 792 & 795\end{array}$ $\begin{array}{llllllllll}22 & 793 & 791 & 796 & 796 & 790 & 792 & 798 & 792 & 794\end{array}$ $\begin{array}{llllllllll}21 & 793 & 791 & 796 & 797 & 790 & 792 & 799 & 792 & 795\end{array}$ $\begin{array}{llllllllll}22 & 793 & 791 & 796 & 796 & 790 & 792 & 799 & 792 & 795\end{array}$ $\begin{array}{llllllllll}22 & 793 & 791 & 796 & 797 & 790 & 793 & 799 & 792 & 795\end{array}$ $\begin{array}{llllllllll}22 & 793 & 792 & 796 & 795 & 790 & 793 & 798 & 792 & 795\end{array}$ $\begin{array}{llllllllll}22 & 793 & 792 & 796 & 798 & 790 & 792 & 799 & 792 & 795 \\ 22 & 793 & 792 & 796 & 795 & 790 & 793 & 799 & 792 & 795\end{array}$ $\begin{array}{llllllllll}22 & 793 & 791 & 796 & 794 & 790 & 792 & 798 & 792 & 795\end{array}$ $\begin{array}{llllllllll}25 & 701 & 700 & 704 & 704 & 698 & 701 & 706 & 700 & 702\end{array}$ $\begin{array}{llllllllll}25 & 701 & 700 & 703 & 702 & 699 & 700 & 706 & 700 & 703\end{array}$ $\begin{array}{llllllllll}25 & 700 & 699 & 703 & 703 & 697 & 700 & 705 & 699 & 702\end{array}$ $\begin{array}{llllllllll}25 & 701 & 699 & 703 & 703 & 698 & 700 & 706 & 700 & 702\end{array}$ $\begin{array}{llllllllll}25 & 700 & 699 & 703 & 706 & 698 & 700 & 705 & 699 & 702\end{array}$ $\begin{array}{llllllllll}25 & 700 & 700 & 703 & 704 & 698 & 700 & 706 & 699 & 702\end{array}$ $\begin{array}{llllllllll}25 & 700 & 699 & 703 & 704 & 698 & 700 & 706 & 699 & 702 \\ & 700 & 699 & 703 & 704 & 698 & 700 & 705 & 699 & 703\end{array}$ $\begin{array}{llllllllll}25 & 700 & 699 & 703 & 704 & 698 & 700 & 706 & 700 & 704\end{array}$

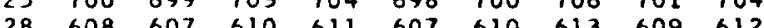
$\begin{array}{llllllllll}28 & 608 & 607 & 610 & 611 & 607 & 610 & 613 & 609 & 612\end{array}$

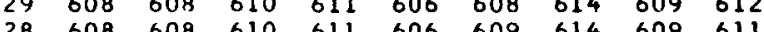

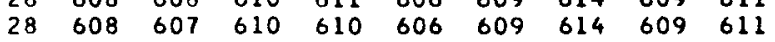
$\begin{array}{lllllllllll}28 & 608 & 608 & 610 & 611 & 607 & 609 & 614 & 609 & 611\end{array}$ $\begin{array}{llllllllll}28 & 607 & 607 & 611 & 613 & 607 & 609 & 614 & 609 & 610\end{array}$ $\begin{array}{llllllllll}28 & 608 & 608 & 611 & 613 & 608 & 609 & 614 & 608 & 611\end{array}$ $\begin{array}{llllllllll}28 & 608 & 609 & 611 & 612 & 607 & 608 & 614 & 608 & 611\end{array}$ $\begin{array}{llllllllll}32 & 518 & 518 & 519 & 520 & 516 & 518 & 522 & 517 & 519\end{array}$ 
EPI NRX-A1 TCA CALIBRATION DATA

IIME

$\begin{array}{rrrrrrr}84040.016 & 24 & 25 & 26 & 27 & 28 & 29\end{array}$

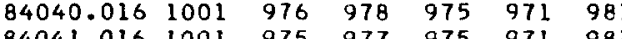
$\begin{array}{lllllll}84042.016 & 1001 & 976 & 977 & 975 & 970 & 981\end{array}$ $\begin{array}{lllllll}84043.016 & 1001 & 975 & 977 & 975 & 971 & 982\end{array}$ $\begin{array}{lllllll}84044.016 & 1001 & 975 & 977 & 975 & 971 & 982\end{array}$ $\begin{array}{lllllll}84045.016 & 1001 & 976 & 977 & 975 & 971 & 982\end{array}$ $\begin{array}{lllllll}84046.016 & 1001 & 976 & 977 & 975 & 971 & 982\end{array}$ $\begin{array}{lllllll}84047.016 & 1002 & 975 & 977 & 975 & 971 & 982\end{array}$ $\begin{array}{llllllll}84048.016 & 1001 & 975 & 977 & 975 & 970 & 981\end{array}$ $\begin{array}{lllllll}84049.016 & 1002 & 975 & 977 & 975 & 970 & 981\end{array}$ $\begin{array}{lllllll}84050.016 & 1002 & 975 & 977 & 975 & 971 & 982\end{array}$ $\begin{array}{lllllll}84051.016 & 906 & 883 & 885 & 882 & 879 & 888\end{array}$ $\begin{array}{lllllll}84052.016 & 906 & 883 & 884 & 882 & 878 & 888\end{array}$ $\begin{array}{lllllll}84053.016 & 907 & 883 & 885 & 883 & 879 & 888\end{array}$ $\begin{array}{lllllll}84054.016 & 906 & 883 & 885 & 882 & 878 & 888\end{array}$ $\begin{array}{llllllll}84055.016 & 907 & 883 & 885 & 882 & 878 & 888\end{array}$ $\begin{array}{llllllll}84056.016 & 907 & 833 & 885 & 883 & 879 & 889\end{array}$ $\begin{array}{llllllll}84057.016 & 907 & 883 & 884 & 882 & 879 & 889 \\ 8495 & 889 & 879 & 890\end{array}$ $\begin{array}{lllllll}84058.016 & 907 & 884 & 885 & 883 & 879 & 890 \\ 84059.016 & 907 & 883 & 884 & 882 & 879 & 890\end{array}$ $\begin{array}{llllllll}84059.016 & 907 & 883 & 884 & 882 & 879 & 890 \\ 84060.016 & 906 & 883 & 885 & 883 & 880 & 890\end{array}$ $\begin{array}{llllllll}84060.016 & 906 & 883 & 885 & 883 & 880 & 890 \\ 84061.040 & 811 & 791 & 792 & 791 & 788 & 797\end{array}$ $\begin{array}{llllllll}84062.040 & 811 & 790 & 792 & 791 & 787 & 796\end{array}$ $\begin{array}{lllllll}84063.065 & 811 & 791 & 792 & 791 & 788 & 797\end{array}$ $\begin{array}{lllllll}84064.065 & 811 & 791 & 793 & 791 & 788 & 797\end{array}$ $\begin{array}{lllllll}84065.065 & 812 & 791 & 792 & 791 & 783 & 797\end{array}$ 84066.065 812 7910793791078879 $\begin{array}{lllllll}84068.065 & 811 & 791 & 792 & 791 & 788 & 796\end{array}$ $\begin{array}{lllllll}84069.065 & 811 & 791 & 792 & 792 & 788 & 796\end{array}$ $\begin{array}{llllllll}84070.091 & 811 & 791 & 792 & 791 & 788 & 796\end{array}$ $\begin{array}{lllllll}84071.091 & 716 & 699 & 701 & 699 & 696 & 704\end{array}$ $\begin{array}{lllllll}84072.091 & 716 & 699 & 699 & 699 & 696 & 704\end{array}$ $\begin{array}{lllllll}84073.091 & 715 & 699 & 702 & 699 & 697 & 703\end{array}$ $\begin{array}{lllllll}84074.115 & 716 & 649 & 701 & 699 & 696 & 704\end{array}$ $\begin{array}{llllllll}84075.115 & 716 & 700 & 701 & 699 & 696 & 703\end{array}$ $\begin{array}{llllllll}84076.115 & 717 & 700 & 700 & 699 & 696 & 703\end{array}$ $\begin{array}{llllllll}84077.115 & 717 & 700 & 701 & 699 & 696 & 703\end{array}$ $\begin{array}{lllllll}84078.115 & 717 & 700 & 701 & 699 & 696 & 703\end{array}$ $\begin{array}{lllllll}84079.115 & 717 & 700 & 701 & 698 & 696 & 703\end{array}$ $\begin{array}{lllllll}84080.115 & 717 & 700 & 700 & 698 & 696 & 703 \\ 84081.115 & 623 & 609 & 609 & 607 & 605 & 611\end{array}$ $84082.115 \quad 623 \quad 610 \quad 608 \quad 606 \quad 604 \quad 610$ $84083.115 \quad 623 \quad 608 \quad 608 \quad 606 \quad 604 \quad 611$ $\begin{array}{lllllll}84084.115 & 623 & 608 & 608 & 606 & 604 & 611\end{array}$

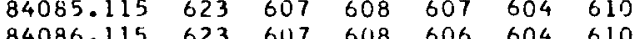
$\begin{array}{lllllll}84086.115 & 623 & 607 & 608 & 606 & 604 & 610\end{array}$ $\begin{array}{lllllll}84087.115 & 623 & 607 & 608 & 606 & 604 & 611 \\ 84088.115 & 623 & 607 & 608 & 606 & 604 & 611\end{array}$ $\begin{array}{lllllll}84088.115 & 623 & 607 & 608 & 606 & 604 & 611 \\ 84089.115 & 623 & 607 & 608 & 606 & 604 & 610\end{array}$ $\begin{array}{lllllll}84089.115 & 623 & 607 & 608 & 606 & 604 & 611 \\ 84090.115 & 623 & 607 & 608 & 606 & 604 & 610\end{array}$ $\begin{array}{lllllll}84091.115 & 529 & 516 & 517 & 515 & 514 & 518\end{array}$
COMPILATION OF SUCCESSIVE ARITHMETIC AVERAGES

\begin{tabular}{|c|c|c|c|c|c|c|c|c|c|c|c|}
\hline \multicolumn{12}{|c|}{ CHANNEL NUMBERS } \\
\hline 30 & 31 & 32 & 33 & 34 & 35 & 36 & 37 & 38 & 39 & 40 & 41 \\
\hline 35 & 984 & 988 & 981 & 975 & 986 & 983 & 1004 & 974 & 34 & 42 & 46 \\
\hline 35 & 984 & 988 & 981 & 975 & 985 & 984 & 1004 & 974 & 35 & 42 & 46 \\
\hline & 984 & 987 & 981 & 975 & 985 & 984 & 1003 & 974 & 35 & 42 & 46 \\
\hline 35 & 984 & 988 & 981 & 975 & 985 & 984 & 1003 & 974 & 35 & 42 & 47 \\
\hline 35 & 984 & 988 & 981 & 975 & 985 & 984 & 1003 & 974 & 35 & 42 & 47 \\
\hline 35 & 983 & 987 & 981 & 975 & 985 & 983 & 1003 & 974 & 34 & 42 & 46 \\
\hline 35 & 483 & 988 & 982 & 976 & 985 & 983 & 1003 & 973 & 34 & 42 & 46 \\
\hline 35 & 984 & 988 & 983 & 976 & 985 & 984 & 1003 & 974 & 34 & 42 & 46 \\
\hline 35 & 983 & 988 & 983 & 975 & 985 & 984 & 1003 & 974 & 34 & 42 & 46 \\
\hline 35 & 984 & 989 & 983 & 975 & 985 & 984 & 1003 & 974 & 34 & 43 & 46 \\
\hline 35 & 984 & 989 & 983 & 976 & 984 & 984 & 1003 & 974 & 34 & 42 & 46 \\
\hline 37 & 891 & 895 & 890 & 883 & 892 & 891 & 908 & 882 & 37 & 44 & 48 \\
\hline 38 & 892 & 895 & 889 & 883 & 891 & 891 & 908 & 882 & 38 & 45 & 49 \\
\hline 39 & 892 & 895 & 889 & 883 & 892 & 890 & 908 & 882 & 37 & 4 & 48 \\
\hline 39 & 893 & 895 & 889 & 883 & 892 & 890 & 907 & 882 & 37 & 45 & 48 \\
\hline 39 & 892 & 895 & 889 & 883 & 892 & 890 & 907 & 882 & 38 & & 49 \\
\hline 39 & 892 & 896 & 889 & 883 & 892 & 890 & 908 & 882 & 37 & 4 & 48 \\
\hline 40 & 893 & 895 & 889 & 883 & 891 & 890 & 908 & 882 & 37 & 45 & 49 \\
\hline 40 & 893 & 895 & 890 & 883 & 891 & 891 & 908 & 881 & 38 & & 48 \\
\hline 39 & 893 & 895 & 889 & 883 & 891 & 890 & 907 & 882 & 37 & 4 & 48 \\
\hline 40 & 892 & 895 & 889 & 883 & 891 & 890 & 907 & 882 & 38 & 45 & 48 \\
\hline 42 & 799 & 800 & 795 & 791 & 798 & 797 & 812 & 789 & 40 & 4 & 51 \\
\hline 43 & 799 & 800 & 795 & 790 & 798 & 797 & 812 & 789 & 41 & 47 & 51 \\
\hline 42 & 798 & 801 & 796 & 790 & 798 & 796 & 812 & 789 & 40 & 4 & 51 \\
\hline 42 & 799 & 800 & 796 & 790 & 798 & 796 & 812 & 789 & & & 51 \\
\hline 42 & 798 & 800 & 796 & 790 & 798 & 797 & 812 & 789 & 40 & 4 & 50 \\
\hline 42 & 799 & 800 & 795 & 790 & 798 & 797 & 812 & 789 & 40 & 4 & 50 \\
\hline 42 & 798 & 800 & 795 & 790 & 798 & 796 & 812 & 789 & 40 & & 51 \\
\hline 42 & 799 & 800 & 796 & 790 & 798 & 796 & 813 & 789 & 40 & 4 & 51 \\
\hline 43 & 799 & 801 & 796 & 790 & 797 & 796 & 812 & 789 & 40 & 47 & 51 \\
\hline 43 & 799 & 801 & 796 & 790 & 798 & 796 & 812 & 78 & 41 & 4 & 51 \\
\hline 45 & 706 & 707 & 703 & 698 & 705 & 703 & 717 & 697 & 43 & 50 & 53 \\
\hline 45 & 706 & 707 & 703 & 698 & 705 & 704 & 717 & 697 & 42 & 5 & 53 \\
\hline 5 & 706 & 706 & 702 & & 705 & 703 & 717 & 69 & 4 & & 54 \\
\hline 45 & 706 & 706 & 703 & 698 & 705 & 704 & 717 & 697 & 44 & 5 & 54 \\
\hline 45 & 706 & 707 & 703 & 697 & 705 & 703 & 717 & 69 & 4 & & 54 \\
\hline+3 & 706 & 706 & 703 & & 705 & 703 & 717 & 69 & 4 & & 55 \\
\hline 45 & 706 & 706 & 703 & 698 & 705 & 702 & 716 & 69 & 4 & 5 & 55 \\
\hline 45 & 706 & 706 & 703 & 698 & 705 & 703 & 717 & 69 & 4 & 5 & 5 \\
\hline 45 & 705 & 106 & $7 C_{3}$ & 698 & 705 & 703 & 717 & 697 & 42 & 5 & 55 \\
\hline 45 & 705 & 706 & 703 & 698 & 705 & 703 & 716 & 69 & 4 & 5 & 55 \\
\hline 49 & 613 & 613 & 611 & 606 & 612 & 610 & 622 & 60 & 4 & & 57 \\
\hline 49 & 613 & 613 & 611 & & 611 & & & & 4 & & 57 \\
\hline 49 & 613 & 613 & 611 & 606 & 612 & 610 & 624 & 606 & 49 & 54 & 57 \\
\hline 49 & 613 & 613 & 611 & 605 & 611 & 611 & 625 & 607 & 49 & 54 & 57 \\
\hline 49 & 613 & 613 & & & & & 624 & & 4 & & \\
\hline 49 & 613 & 613 & 610 & 606 & 613 & 611 & 625 & 607 & 49 & 54 & 57 \\
\hline 49 & 613 & 613 & 611 & 607 & 613 & 612 & 624 & 607 & 49 & & \\
\hline 49 & 613 & 613 & 611 & 60 & 613 & 61 & 624 & & 48 & 54 & 57 \\
\hline 48 & 613 & 613 & 612 & 607 & 613 & 612 & 624 & 606 & 48 & 54 & 57 \\
\hline 48 & 613 & 614 & 612 & 607 & 613 & 612 & 624 & 60 & 48 & & 57 \\
\hline 3 & 521 & 522 & 520 & 516 & 522 & 520 & 530 & 515 & 52 & 57 & 60 \\
\hline
\end{tabular}


$\mathrm{RN}-\mathrm{S}-0146$

The first record will be a header that will tell in BCD code the title of the test and the name of the multiplexer. (Each multiplexer is one file.) This information will provide the input data HEADER and MULTPX. The second record is actual data, and can be read to determine the input data NCHS, IBITE, ISIZE and. TAU in the following manner:

ISIZE: The total number of words in each physical record.

NCHS, IBITE, TAU: The first sixteen octal characters (1-1/3 words) of the record represents a time value. This time is followed by a certain quantity of data values, and then another time will appear which will differ from the first time in its last three characters. The difference between these two times is TAU. However, the subtraction must be performed octally and the difference changed to a decimal number. The total number of such times in each physical record is the input value IBITE. Every four characters of the data between the times represents one channel or pin on the multiplexer. The total number of channels between two successive times is the input value NCHS. However, the last one or two channel data values sometimes are all zeros, and, in this case, NCHS is accordingly reduced by one or two.

NCHS, IBITE, ISIZE and TAU should remain constant throughout the entire set of data from one multiplexer. However, it has happened at least once that the number of channels and size of a physical record were changed part way through a multiplexer. This program cannot handle such a situation. An error routine was included in the main program to guard against such a possibility. If such a change occurs, the processing of the multiplexer in question is terminated, an on-line message is printed to this effect, the data in the rest of the file is passed over, and the processing resumes with the next multiplexer (i.e., file)。

\section{OPERATIONAL PROCEDURE}

The program, as listed in Section $X$, was written to be run on an IBM 7094 . Programming was done in FORTRAN IV - MAP. The program requires one special tape drive. The input data (N2 data tape) is to be placed on drive B7 (logical tape 13). A pause statement is included to permit time for mounting this tape. 
VIII. FREQUENCY ANALYSIS OF THE INERVA NARROW-BAND DATA

The analysis of the NERVA narrow-band data for its frequency content, because of the large number of data channels and data points per channel, requires the processing of a very large number of data points. The usual digital computer approaches for either the harmonic analysis (Fourier series coefficients) or Fourier integral calculations which take a relatively short time for a small amount of data, became prohibitive when applied to the amount of NERVA test data available.

The data is recorded after being sampled at 10 or 40 samples per second. The maximum frequency that may be determined is 20 cycles per second, at the higher sampling rate with higher frequencies folded into this frequency band. A basic unit of 40 data points for processing was chosen, corresponding to 1 sec and 4 sec with the used sampling rates used (40 and 10 samples/sec).

The first approach to the frequency analysis was by the use of a standard harmonic analysis (Fourier series); the method used was a modification of the standard harmonic analysis routines modified to reduce the total number of multiplications and diminish the total computer time. The output consisted of 20 sine and 20 cosine coefficients. Since the actual frequencies present may not be in a harmonic relation to each other, additional computation is required to convert the 40 Fourier coefficients (20 cosine and 20 sine coefficients) to the actual frequencies present in the data.

A second approach to the frequency analysis, described by Lanczos ${ }^{*}$ and based upon the use of the Fourier integral, permits the computations of the actual frequencies present in the data. This method was the one chosen and used to perform the analysis of the NERVA narrow-band data.

The data was assumed to belong to a stationary time series, and no test was performed to verify this assumption. Although the first approach (Fourier harmonic analysis) was not used, a description of the method and its modifications that permit a significant reduction in its running time, as compared with standard procedures, is included in this report.

* C. Lanczos, Applied Analysis, Prentice Hall, Inc., 1961, Chapter IV, Section 22. 


\section{A. MODIFIED HARMONIC ANALYSIS}

For the application of the harmonic analysis to the narrow-band NERVA data, it was assumed that the usual "Dirichlet Conditions" existed in the NERVA data and thus we could expand the experimental results in a convergent Fourier series.

\section{Fourier Coefficients}

Taking $\mathbb{N}$ data points - $\mathbb{N}$ being an even number - the discrete representation of the Fourier series is

$$
f(t)=a_{0}+\sum_{p=1}^{N / 2}\left[a_{p} \cos \left(\frac{2 \pi}{N} n p\right)+b_{p} \sin \left(\frac{2 \pi}{N} n p\right)\right]
$$

where

$$
\begin{aligned}
\mathrm{p} & =\text { order of the harmonic } \\
\mathrm{n} & =\text { digital time increment } \\
\mathrm{a}_{\mathrm{o}}, \mathrm{a}_{\mathrm{p}}, \mathrm{b}_{\mathrm{p}} & =\text { Fourier coefficients }
\end{aligned}
$$

The Fourier coefficients are given by

$$
\begin{gathered}
a_{0}=\frac{I}{N} \sum_{i=1}^{N} y_{i} \\
a_{p}=\frac{2}{N} \sum_{i=1}^{N} y_{i} \cos \left(\frac{2 \pi n_{i}}{N}\right), n=1,2, \ldots N / 2 \\
b_{p}=\frac{2}{N} \sum_{i=1}^{N} y_{i} \sin \left(\frac{2 \pi n_{i}}{\mathbb{N}}\right), n=1,2, \ldots N / 2
\end{gathered}
$$

The calculation of the $a_{p}$ and $b_{p}$ coefficients by the commonly used digital routines is accomplished by a calculating loop in which a running sum is kept of the product of a generated sine or cosine and the data ordinate $y_{i}$. We see that most of the elapsed calculation time is used in generating the trigonometric functions and in performing the required multiplications. 
For a fixed number of points, $N$, the required sines and cosines can be inserted into the program as fixed data, thus reducing the computing time. If the total number of multiplications can be reduced appreciably, then the computing time becomes reasonable for a job of the magnitude of the NERVA test analysis. A description of the method employed is given in the next section.

\section{Modification for Faster Computation}

The number of multiplications can be reduced by first adding up all the ordinates that have the same sine or cosine factor and performing only one multiplication per group.

Unfortunately there are no general formulae that permit the calculation of the ordinates that are used as factors for the multiplication of each sine and cosine. These factors are obtained by writing down explicitly Equations 2,3 , and 4 and collecting the common terms for each sine and cosine.

This was done for the cases of 40 and 10 data samples/sec. For a large number of points it would be more expedient to write a compiler-type program to obtain the desired factors.

A careful examination of the data ordinates that were used in each sine or cosine factor for each harmonic showed that certain combinations of sums and differences of the data ordinates appeared again and again and thus it was necessary to calculate them only once.

a. Data for 40 Samples/sec

The total number of sines and cosines used is 9. Let

$\mathrm{Cl}=\cos 9^{\circ}=\mathrm{S} 9=\sin 81^{\circ}=0.98768834$

$\mathrm{C} 2=\cos 18^{\circ}=\mathrm{S} 8=\sin 72^{\circ}=0.95105652$

$\mathrm{C} 3=\cos 27^{\circ}=\mathrm{S} 7=\sin 63^{\circ}=0.98100652$

$\mathrm{C} 4=\cos 36^{\circ}=\mathrm{S} 6=\sin 54^{\circ}=0.80901699$

$\mathrm{C} 5=\cos 45^{\circ}=\mathrm{S} 5=\sin 45^{\circ}=0.70710678$

$\mathrm{C} 6=\cos 54^{\circ}=\mathrm{S} 4=\sin 36^{\circ}=0.58778525$

$\mathrm{C} 7=\cos 63^{\circ}=\mathrm{S} 3=\sin 27^{\circ}=0.45399050$

$\mathrm{C} 8=\cos 72^{\circ}=\mathrm{S} 2=\sin 18^{\circ}=0.30901699$

$\mathrm{C} 9=\cos 81^{\circ}=\mathrm{SI}=\sin 9^{\circ}=0.15643447$ 
For example $a_{0}, a_{1}$ and $b_{4}$, are explicitly written as follows:

$$
\begin{aligned}
& a_{0}=\frac{1}{40} \sum_{i=1}^{40} y_{i} \\
& a_{1}= \frac{1}{20}\left[\left(y_{1}-y_{21}\right)+\left(y_{2}-y_{20}-y_{22}+y_{40}\right) c 1+\left(y_{3}-y_{19}-y_{23}+y_{39}\right) c 2\right. \\
&+\left(y_{4}-y_{18}-y_{24}+y_{38}\right) c 3+\left(y_{5}-y_{17}-y_{25}+y_{37}\right) c 4+\left(y_{6}-y_{16}\right. \\
&\left.-y_{26}+y_{36}\right) c 5+\left(y_{7}-y_{15}-y_{27}+y_{35}\right) c 6+\left(y_{8}-y_{14}-y_{28}+y_{34}\right) c 7 \\
&\left.+\left(y_{9}-y_{13}-y_{29}+y_{33}\right) c 8+\left(y_{10}-y_{12}-y_{30}+y_{32}\right) c 9\right] \\
& b_{4}+\frac{1}{20}\left[\left(y_{2}+y_{5}-y_{7}-y_{10}+y_{12}+y_{15}-y_{17}-y_{20}+y_{22}+y_{25}-y_{27}-y_{30}\right.\right. \\
&\left.+y_{32}+y_{35}-y_{37}-y_{40}\right) s_{4}+\left(y_{3}+y_{4}-y_{8}-y_{9}+y_{13}+y_{14}-y_{18}-y_{19}\right. \\
&\left.\left.+y_{23}+y_{24}-y_{28}-y_{29}+y_{33}+y_{34}-y_{38}-y_{39}\right) s_{8}\right]
\end{aligned}
$$

Obtaining the following sums and differences:

$$
\begin{aligned}
& \begin{array}{llllllllll}
y_{1} & y_{2} & y_{3} & y_{4} & \cdots & -\cdots & y_{17} & y_{18} & y_{19} & y_{20}
\end{array} \\
& \mathrm{y}_{21} \mathrm{y}_{40} \mathrm{y}_{39} \mathrm{y}_{38}-\cdots-\cdots-\cdots \mathrm{y}_{25} \quad \mathrm{y}_{24} \quad \mathrm{y}_{23} \quad \mathrm{y}_{22} \\
& \text { Sums } \\
& { }_{1} \mathrm{~S}_{1} I_{2} \mathrm{~S}_{2} \mathrm{~S}_{3} I_{1}^{\mathrm{S}_{4}}-\cdots-\cdots-\cdots{ }_{1} \mathrm{~S}_{17} I_{18} \mathrm{~S}_{1} \mathrm{~S}_{19} I_{20} \\
& \operatorname{Diff} \quad \triangle_{1} \triangle_{1} \Delta_{2} \triangle_{3} \Delta_{4}-\cdots-\cdots \cdots{ }_{17} \triangle_{18} \triangle_{19} \triangle_{20}
\end{aligned}
$$

where

$$
\begin{aligned}
& \text { sums }{ }_{1} S_{2}=y_{2}-y_{40} \\
& \text { Diff }{ }_{1} \triangle_{17}=y_{17}-y_{25}
\end{aligned}
$$




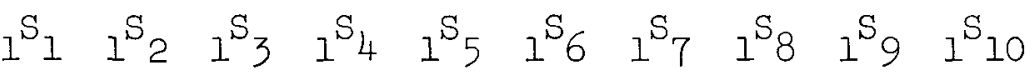

$\begin{array}{lllllllllll}1^{\mathrm{S}} 11 & 1^{\mathrm{S}} 20 & 1^{\mathrm{S}} 19 & 1^{\mathrm{S}} 18 & 1^{\mathrm{S}} 17 & 1^{\mathrm{S}} 16 & 1^{\mathrm{S}} 15 & 1^{\mathrm{S}} 14 & 1^{\mathrm{S}} 13 & 1^{\mathrm{S}} 12\end{array}$

Sums

$\begin{array}{llllllllllll}2^{S_{1}} 1 & 2^{S_{2}} & 2^{S_{3}} & 2^{S_{4}} & 2^{S_{5}} & 2^{S_{6}} & 2^{S_{7}} & 2^{S_{3}} & 2^{S} 9 & 2^{S_{10}}\end{array}$

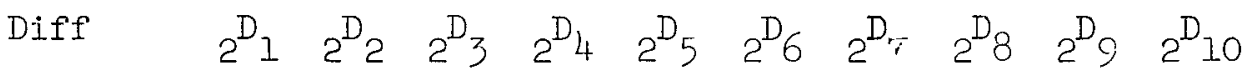

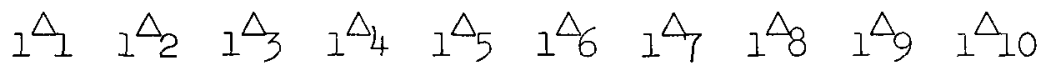

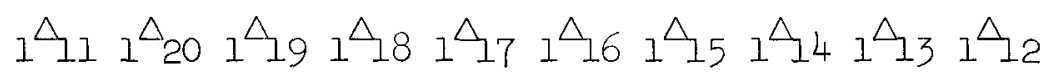

Sums

$\begin{array}{lllllllllll}2^{\sigma_{1}} & 2^{\sigma_{2}} & 2^{\sigma_{3}} & 2^{\sigma_{4}} & 2^{\sigma_{5}} & 2^{\sigma_{6}} & 2^{\sigma_{7}} & 2^{\sigma_{8}} & 2^{\sigma_{9}} & 2^{\sigma_{10}}\end{array}$

Diff

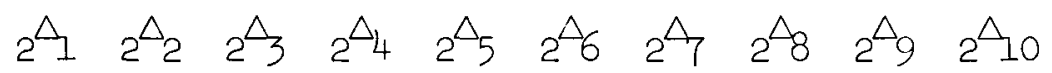

$\begin{array}{lllll}2^{S_{1}} 1 & 2^{\mathrm{S}_{2}} 2 & 2^{\mathrm{S}_{3}} & 2^{\mathrm{S}_{4}} & 2^{\mathrm{S}_{5}}\end{array}$

$2^{\mathrm{S}} 62^{\mathrm{S}} 10 \quad 2^{\mathrm{S}} 92^{\mathrm{S}} 82^{\mathrm{S}_{7}}$

Sums

$3^{\mathrm{S}_{1}} \quad 3^{\mathrm{S}_{2}} \quad 3^{\mathrm{S}_{3}} \quad 3^{\mathrm{S}_{4}} \quad 3^{\mathrm{S}_{5}}$

Diff

$3^{D_{1}} 3^{D_{2}} \quad 3^{D_{3}} 3^{D_{4}} 3^{D_{5}}$

$2^{\triangle} \Omega_{2} \Delta_{2} \triangle_{3} 2^{\Delta_{4}} 2^{\Delta_{5}}$

$2^{\Delta} \Delta_{6} \Delta_{10} \Delta_{9} \Delta_{2} \Delta_{8} 2^{\Delta}$

Sums

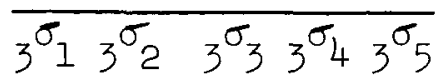

Diff $\quad 3 \Delta^{\Delta} 3^{\Delta_{2}} \quad 3_{3}^{\Delta_{3}} 3^{\Delta_{4}} 3^{\Delta_{5}}$

Sums

$3^{D_{2}} 3^{D_{3}} \quad 3^{\Delta_{2}} 3_{3}^{\triangle_{3}} \quad 3^{S_{2}} 23^{S_{3}}$

$\frac{3_{5}^{D_{5} D_{4}}}{\cdots \cdots} \frac{3^{\Delta_{5}} 3^{\Delta_{4}}}{4^{\sigma_{1} \sigma_{2}}} \frac{3^{S_{5}} 3^{S_{4}}}{5^{S_{1}} 5^{S_{2}}}$

$4_{1}^{D_{1}} 4_{2}^{D_{2}} \quad 4 A_{4} \Delta_{2} \quad 5^{D_{1}} 5_{2}^{D_{2}}$ 


\section{RN-S-0146}

Let

$$
\begin{aligned}
& v_{1}=\sum_{i=1}^{5} 3^{S_{i}} \\
& V_{2}={ }_{1} \triangle_{1}-2_{5}+{ }^{D_{9}} \\
& V_{3}=2_{2}-2_{4} D_{2} D_{6}+2^{D_{8}}+2^{D_{10}} \\
& v_{4}={ }_{1} \Delta_{11}+{ }_{2} \sigma_{3}-2_{7} \sigma_{7} \\
& v_{5}=2_{2} \sigma_{2} \sigma_{4}-2_{6} \sigma_{2} \sigma_{8}+{ }_{2} \sigma_{10} \\
& v_{6}=2_{1} D_{2} D_{3}+2^{D_{5}}-2^{D_{7}}+2^{D_{9}} \\
& v_{7}=2_{2} \Delta_{2} \Delta_{4}+2 \Delta_{6}-2 \Delta_{8}+{ }_{2} \Delta_{10} \\
& V_{8}=2_{1} S_{1}-2_{2}+2_{3} S_{2} S_{4}+2^{S_{5}}-2^{S_{6}}+2_{7} S_{2} S_{8}+2^{S_{9}}-2^{S_{10}}
\end{aligned}
$$

Then

$$
\begin{aligned}
& a_{0}=(1 / 40)\left(V_{1}\right) \\
& \mathrm{b}_{\mathrm{O}}=0 \\
& a_{1}=(1 / 20)\left[\left({ }_{1} \Delta_{1}+2^{D_{3}} \times C_{2}+{ }_{2} D_{5} \times C_{4}+{ }_{2} D_{7} \times C 6+2_{9} \times C 8\right)\right. \\
& \left.+\left({ }_{2} D_{2} \times C 1+2_{4} \times C 3+2^{D} \times C 5+2^{D_{8} \times C 7}\right)\right] \\
& a_{1}=(1 / 20)\left(w_{1}+w_{2}\right) \\
& a_{19}=(1 / 20)\left(w_{1}-w_{2}\right) \\
& a_{2}=(1 / 20)\left[\left(2_{1}+3 D_{3} \times C 4+3 D_{5} \times C 8\right)\right. \\
& \left.+\left({ }_{3} D_{2} \times C 2+{ }_{3} D_{4} \times C 6\right)\right] \\
& a_{2}=(1 / 20)\left(w_{3}+w_{4}\right) \\
& a_{18}=(1 / 20)\left(w_{3}-w_{4}\right) \\
& a_{3}=(1 / 20)\left[\left({ }_{1} \triangle_{1}-2^{D_{7}} \times C 2-{ }_{2} D_{9} \times C 4+2^{D_{3}} \times C 6-2_{5}\right.\right. \\
& x \mathrm{C} 8)-\left(2^{D_{8}} \times \mathrm{Cl}-2_{2} \mathrm{D}_{2} \times \mathrm{C} 3+2^{D_{6}} \times \mathrm{C} 5+{ }_{2} \mathrm{D}_{10} \times \mathrm{C} 7\right. \\
& \left.-2^{D} \times(9)\right]
\end{aligned}
$$


RN-S-0146

$$
\begin{aligned}
& a_{3}=(1 / 20)\left(w_{5}-w_{6}\right) \\
& a_{17}=(1 / 20)\left(w_{5}+w_{6}\right) \\
& a_{4}=(1 / 20)\left({ }_{3} D_{1}+{ }_{5} D_{1} \times C_{4}+{ }_{5} D_{2} \times c 8\right) \\
& a_{5}=(1 / 20)\left(v_{2}+v_{3} \times C 5\right) \\
& a_{5}=(1 / 20)\left(v_{2}+w_{7}\right) \\
& a_{15}=(1 / 20)\left(v_{2}-w_{7}\right) \\
& a_{6}=(1 / 20)\left[\left({ }_{2} D_{1}-3_{3} D_{5} \times C 4+{ }_{3} D_{3} \times C 8\right)-\left({ }_{3} D_{4}\right.\right. \\
& \left.x \mathrm{C} 2-3_{2} \times(\mathrm{C})\right] \\
& a_{6}=(1 / 20)\left(w_{8}-w_{9}\right) \\
& a_{14}=(1 / 20)\left(w_{8}+w_{9}\right) \\
& a_{7}=(1 / 20)\left[\left({ }_{1} \triangle_{1}+{ }_{2} D_{7} \times C 2-{ }_{2} D_{9} \times C 4-{ }_{2} D_{3} \times C 6-{ }_{2} D_{5} \times C 8\right)\right. \\
& \left.-\left({ }_{2} D_{4} \times C 1+2^{D} 10 \times C 3-2^{D} D_{6} \times C 5-2_{2} \times C 7-2_{8} \times C 9\right)\right] \\
& a_{7}=(1 / 20)\left(w_{10}-w_{11}\right) \\
& a_{13}=(1 / 20)\left(w_{10}+w_{11}\right) \\
& a_{8}=(1 / 20)\left[\left({ }_{3} S_{1}+\left({ }_{5} S_{1} \times C 4-5_{2} \times c 8\right)\right]\right. \\
& a_{8}=(1 / 20)\left(S_{1}+w_{12}\right) \\
& a_{16}=(1 / 20)\left(S_{1}-w_{12}\right) \\
& a_{9}=(1 / 20)\left[{ }_{1} \triangle 1-{ }_{2} D_{3} \times C 2+{ }_{2} D_{5} \times C 4-2_{7} \times C 6\right. \\
& \left.+2^{D_{9}} \times \mathrm{C} 8\right)+\left({ }_{2} D_{10} \times \mathrm{Cl}-2^{D_{8}} \times \mathrm{C} 3+2^{D_{6}} \times \mathrm{C}_{5}\right. \\
& \left.\left.-2^{D_{4}} \times \mathrm{C7}+2^{D_{2}} \times \mathrm{C9}\right)\right] \\
& a_{9}=(1 / 20)\left(w_{13}+w_{14}\right)
\end{aligned}
$$


RN-S-OI 46

$$
\begin{aligned}
& a_{11}=(1 / 20)\left(w_{13}-w_{14}\right) \\
& \mathrm{a}_{10}=(1 / 20)\left(\mathrm{v}_{6}\right) \\
& a_{12}=(1 / 20)\left({ }_{3} D_{1}+{ }_{5} D_{2} \times C 4-5_{1} \times C 8\right) \\
& a_{20}=(1 / 20)\left(v_{8}\right) \\
& b_{1}=(1 / 20)\left(\Delta_{11}+2 \Delta_{2} \times S 1+{ }_{2} \Delta_{3} \times S 2+{ }_{2} \Delta_{4} \times S 3+\Delta_{5} \times S 4\right. \\
& \left.+2 \Delta_{6} \times \mathrm{S} 5+2 \Delta_{7} \times \mathrm{S} 6+{ }_{2} \Delta_{8} \times \mathrm{S} 7+{ }_{2} \Delta_{9} \times \mathrm{S} 8+2 \mathrm{~A}_{10} \times \mathrm{S} 9\right) \\
& b_{2}=(1 / 20)\left[\left({ }_{2} \Delta_{6}+{ }_{3} \sigma_{2} \times \mathrm{S} 2+{ }_{3} \sigma_{4} \times \mathrm{S} 6\right)-\left({ }_{3} \sigma_{3} \times \mathrm{S} 4+{ }_{3} \sigma_{5} \times \mathrm{S} 8\right)\right] \\
& b_{2}=(1 / 20)\left(w_{15}-w_{16}\right) \\
& b_{18}=(1 / 20)\left(w_{15}+w_{16}\right) \\
& \mathrm{b}_{3}=(1 / 20)\left[\left(-{ }_{2} \sigma_{8} \times \mathrm{Sl}+{ }_{2} \sigma_{2} \times \mathrm{S} 3+{ }_{2} \sigma_{6} \times \mathrm{S} 5-{ }_{2} \sigma_{10} \times \mathrm{S} 7+{ }_{2} \sigma_{4} \times \mathrm{S} 9\right)\right. \\
& \left.-\left({ }_{1} \triangle_{11}-2^{\sigma_{9}} \times \mathrm{S} 4-2_{2} \sigma_{3} \times \mathrm{S} 6-2_{5} \sigma_{5} \times \mathrm{S} 8-{ }_{2} \sigma_{7} \times \mathrm{S} 2\right)\right] \\
& b_{3}=(1 / 20)\left(w_{17}-w_{18}\right) \\
& \mathrm{b}_{17}=(1 / 20)\left(\mathrm{w}_{17}+\mathrm{w}_{18}\right) \\
& \mathrm{b}_{4}=(1 / 20)\left({ }_{4} \sigma_{1} \times \mathrm{S} 4+{ }_{4} \sigma_{2} \times \mathrm{S} 8\right) \\
& b_{5}=(1 / 20)\left[\left(v_{5} \times 55\right)+v_{4}\right] \\
& \mathrm{b}_{5}=(1 / 20)\left(\mathrm{w}_{19}+\mathrm{v}_{4}\right) \\
& \mathrm{b}_{15}=(1 / 20)\left(\mathrm{w}_{19}-\mathrm{v}_{4}\right) \\
& b_{6}=(1 / 20)\left[\left(-{ }_{2} \Delta_{6}+{ }_{3} \sigma_{4} \times S 2+3_{2} \sigma_{2} \times S 6\right)-\left({ }_{3} \sigma_{5} \times S 4\right.\right. \\
& \left.\left.-{ }_{3} \sigma_{3} \times \mathrm{s} 8\right)\right] \\
& b_{6}=(I / 20)\left(w_{20}-w_{2 I}\right) \\
& b_{14}=(1 / 20)\left(w_{20}+w_{21}\right) \\
& \mathrm{b}_{7}=(1 / 20)\left[\left(-{ }_{2} \sigma_{4} \times \mathrm{S} 1-2_{2} \sigma_{10} \times \mathrm{S} 3-{ }_{2} \sigma_{8} \times \mathrm{S} 5+{ }_{2} \sigma_{2} \times \mathrm{S} 7+\right.\right. \\
& \left.\left.2^{\sigma_{8}} \times \mathrm{S} 9\right)-\left({ }_{1} \mathrm{~A}_{1}-2^{\sigma_{7}} \times \mathrm{S} 2-2^{\sigma_{9}} \times \mathrm{S} 4-2_{3} \times \mathrm{S} 6+{ }_{2} \sigma_{5} \times \mathrm{S} 8\right)\right] \\
& b_{7}=(1 / 20)\left(w_{22}-w_{23}\right) \\
& b_{13}=(1 / 20)\left(w_{22}+w_{23}\right)
\end{aligned}
$$




\section{RN-S-OI 46}

$$
\begin{aligned}
& b_{3}=(1 / 20)\left({ }_{4} \triangle_{2} \times 54+{ }_{4} \triangle_{1} \times 58\right) \\
& \mathrm{b}_{9}=(1 / 20)\left[\left({ }_{2} \sigma_{10} \times \mathrm{S} 1-2^{\sigma_{8}} \times \mathrm{S} 3+2^{\sigma_{6}} \times \mathrm{S} 5-2_{4} \times \mathrm{S} 7+{ }_{2} \sigma_{2}\right.\right. \\
& \left.\mathrm{xS9})+\left({ }_{1} \Delta_{11}-2^{\sigma_{3}} \times \mathrm{S} 2+{ }_{2} \sigma_{5} \times \mathrm{S} 4-2^{\sigma_{7}} \times \mathrm{S} 6+{ }_{2} \sigma_{9} \times \mathrm{S} 8\right)\right] \\
& \mathrm{b}_{9}=(1 / 20)\left(\mathrm{w}_{24}+\mathrm{W}_{25}\right) \\
& \mathrm{b}_{11}=(1 / 20)\left(\mathrm{w}_{24}-\mathrm{w}_{25}\right) \\
& \mathrm{b}_{10}=(1 / 20)\left(\mathrm{V}_{7}\right) \\
& b_{12}=(1 / 20)\left(-{ }_{4} \sigma_{2} \times S 4+{ }_{4} \sigma_{1} \times S 8\right) \\
& \mathrm{b}_{16}=(1 / 20)\left({ }_{4} \triangle_{1} \times 54+{ }_{4} \triangle_{2} \times 58\right) \\
& b_{19}=(1 / 20)\left(-{ }_{1} \Delta_{11}+{ }_{2} \sigma_{2} \times S 1-{ }_{2} \sigma_{3} \times S 2+{ }_{2} \sigma_{4} \times S 3-\sigma_{5} \times S 4\right. \\
& \left.+2^{\sigma_{6}} \times \mathrm{S} 5-2_{7} \sigma_{7} \times \mathrm{S} 6+2^{\sigma_{8}} \times \mathrm{ST}-2^{\sigma_{9}} \times \mathrm{S} 8+2^{\sigma_{10}} \times \mathrm{S} 9\right) \\
& b_{20}=0 \text {. }
\end{aligned}
$$

b. Data for 10 Samples/sec

The total number of sines and cosines is 2 . Let

$$
\begin{array}{ll}
\mathrm{C} 1=\cos 36^{\circ}=0.80901699 & \mathrm{~S} I=\sin 36^{\circ}=0.58778525 \\
\mathrm{C} 2=\cos 72^{\circ}=0.30901699 & \mathrm{~S} 2=\sin 72^{\circ}=0.95105652
\end{array}
$$

Obtaining the following sums and differences:

$$
\begin{aligned}
& \begin{array}{lllll}
\mathrm{y}_{1} & \mathrm{y}_{2} & \mathrm{y}_{3} & \mathrm{y}_{4} & \mathrm{y}_{5}
\end{array} \\
& \begin{array}{lllll}
\mathrm{y}_{6} & \mathrm{y}_{10} & \mathrm{y}_{9} & \mathrm{y}_{8} & \mathrm{y}_{7}
\end{array}
\end{aligned}
$$

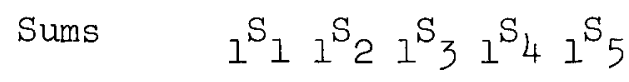

$$
\begin{aligned}
& \text { Diff }{ }_{1} \triangle_{1} \triangle_{2}{ }_{1} \triangle_{3}{ }_{1} \triangle_{4}{ }_{1} \triangle_{5} \\
& I_{2}^{S_{2}} \quad 1_{3}^{S_{3}} \quad 1_{2} \quad I_{3} \\
& \frac{1^{S_{5}} \quad 1^{S_{4}}}{2^{S_{1}} \quad 2^{S_{2}}} \quad \frac{1^{\triangle_{5}} \quad 1^{\triangle_{4}}}{2^{\sigma_{1}} \quad 2^{\sigma_{2}}} \\
& \text { Diff } \quad 2^{D_{1}} \quad 2^{D_{2}} \quad 2^{\triangle} \quad 2^{\triangle_{2}}
\end{aligned}
$$


Let

$$
\begin{aligned}
& V_{1}={ }_{1} S_{1}+2 S_{1}+2 S_{2} \\
& V_{2}=1 \triangle_{1}-2 \triangle_{1}+2 \Delta_{2}
\end{aligned}
$$

Then

$$
\begin{aligned}
& a_{0}=(1 / 10)\left(V_{1}\right) \\
& \mathrm{b}_{0}=0 \text {. } \\
& a_{1}=(1 / 5)\left({ }_{1} \triangle_{1}+{ }_{2} D_{1} \times C l+{ }_{2} D_{2} \times C 2\right) \\
& b_{1}=(1 / 5)\left({ }_{2} \sigma_{1} \times \mathrm{SI}+2_{2} \times \mathrm{S} 2\right) \\
& a_{2}=(1 / 5)\left({ }_{1} S_{1}+2^{S_{1}} \times C_{2}-2_{2} S_{2} \times C l\right) \\
& \mathrm{b}_{2}=(1 / 5)\left({ }_{2} \triangle 1 \times S 2+{ }_{2} \triangle_{2} \times S I\right) \\
& a_{3}=(1 / 5)\left({ }_{1} A_{1}-2^{D_{1}} \times C_{2}-2_{2} D_{2} \times l\right) \\
& b_{3}=(1 / 5)\left({ }_{2} \sigma_{1} \times S 2-2_{2} \sigma_{2} \times S 1\right) \\
& \mathrm{b}_{4}=(1 / 5)\left({ }_{1} \mathrm{~S}_{1}-2_{1} \mathrm{~S}_{1} \mathrm{Cl}+{ }_{2} \mathrm{~S}_{2} \times \mathrm{C}_{2}\right) \\
& b_{4}=(1 / 5)\left(\triangle_{1} \times S 1-\triangle_{2} \times \mathrm{S} 2\right) \\
& a_{5}=(1 / 5)\left(V_{2}\right) \\
& b_{5}=0 \text {. }
\end{aligned}
$$

\section{B. FOURIER TRANSFORM FREQUENCY ANALYSIS}

In a system as complex as NERVA with test results that show oscillations, the possibility exists that if several frequencies are present simultaneously, they will not be harmonically related to each other.

A method based upon the Fourier Transform, discussed by Lanczos* was selected for use in the frequency analysis of the NERVA data.

The analysis is performed on $(2 \mathrm{~N}+1)$ points, an odd number, separated by $(\Delta t)$ sec. The group of data points is assumed to form part of a stationary time series, so that the origin can be translated to the midpoint of the $(2 \mathrm{~N}+1)$ points and the data is known at the following digital times:

\footnotetext{
*. Lanczos, Applied Analysis, Prentice Hall, Inc., 1961, Chapter IV, Section 22.
} 


$$
\begin{gathered}
\mathrm{RN}-\mathrm{S}-0146 \\
t_{k}=0, \pm 1, \pm 2, \ldots, \pm N
\end{gathered}
$$

The oscillatory data is represented by

$$
f(t)=\sum_{i=1}^{M}\left(A_{i} \cos \theta_{i} t+B_{i} \sin \theta_{i} t\right)
$$

where

$M$ is the number of periodic components

$\theta_{i}$ is the angular frequency, related to the true angular frequency $\boldsymbol{\omega}_{i}$ by $\boldsymbol{\omega}_{i}=\theta_{i} /(\Delta t)$

$(\Delta t)=$ time interval between data points.

The Fourier transform $F(\omega)$ of $f(t)$ contains all the harmonic components of $f(t)^{*}$

$$
F(\boldsymbol{\omega})=\int_{-\infty}^{\infty} f(t) e^{-j \omega t} d t
$$

as $e^{-j \omega i}=\cos \omega t-j \sin \omega t$, the function $F(\omega)$ is complex

$$
F(\boldsymbol{\omega})=R(\boldsymbol{\omega})+j X(\boldsymbol{\omega})=A(\boldsymbol{\omega}) e^{j \phi(\boldsymbol{\omega})}
$$

where

$A(\boldsymbol{\omega})$ is called the Fourier or frequency spectrum of $f(t)$, containing all the harmonic components of $f(t)$

$A^{2}(\boldsymbol{\omega})$ is called the energy spectrum

$\phi(\boldsymbol{\omega})$ is called the phase-angle

If $f(t)$ is real in Equation (75), the real and imaginary parts of $F(\boldsymbol{\omega})$ are

$$
\begin{aligned}
& R(\omega)=\int_{-\infty}^{\infty} f(t) \cos \omega t d t \\
& X(\omega)=\int_{-\infty}^{\infty} f(t) \sin \omega t d t
\end{aligned}
$$

*A. Papoulis, The Fourier Integral and Its Applications, McGraw-Hill Book Co., 1962, Chapters I and II. 
And we see that $R(\boldsymbol{\omega})$ is an even function while $X(\boldsymbol{\omega})$ is an odd function. An arbitrary function of $t, f(t)$ may be decomposed into an odd $f_{O}(t)$ and even $f_{E}(t)$ functions of $t$ as

$$
\begin{aligned}
& 2 f_{0}(t)=f(t)+f(-t)=2 \sum_{i=1}^{M} A_{i} \cos \theta_{i} t \\
& 2 f_{E}(t)=f(t)+f(-t)=2 \sum_{i=1}^{M} B_{i} \sin \theta_{i} t
\end{aligned}
$$

Thus we can define two new sets of numbers $\left(u_{k}\right.$ and $\left.v_{k}\right)$ as follows:

$$
\begin{aligned}
& u_{k}=f_{k}+f_{-k} \\
& v_{k}=f_{k}-f_{-k}
\end{aligned} \quad, k=0,1,2, \ldots, N
$$

where $f_{k}$ signifies the data point at digital time $t=k$.

By restricting the angular frequencies $\theta_{i}$ in Equation (74) to be less than $\pi$, these can be written as

$$
\theta_{i}=\frac{\pi}{N} p_{i}, \quad p_{i}=0,1,2, \ldots, N
$$

The amplitudes of the sine and cosine components are obtained by

$$
\begin{aligned}
& a_{k}=\sum_{i=0}^{N} u_{i} \cos \left(\frac{\pi}{N} i k\right), \quad k=0,1,2, \ldots, \mathbb{N} \\
& b_{k}=\sum_{i=1}^{N-1} v_{i} \sin \left(\frac{\pi}{N} i k\right), \quad k=1,2, \ldots, N-1
\end{aligned}
$$

where the symbol $\sum^{*}$ denotes that in the sum the elements $u_{0}$ and $u_{N}$ are entered with a weight of $1 / 2$. 


\section{$\mathrm{RN}-\mathrm{S}-\mathrm{O} \mathrm{I} 46$}

As the number of data points $N$ is increased, the accuracy of the method increases. Where $\mathbb{N}$ is a small number, Equations (81) and (82) are modified by the use of a focusing or weighting function $\mathrm{S}_{k}$, and used on Equations (84) and (85).

$$
\begin{aligned}
& \bar{u}_{k}=u_{k} \times S_{k} \\
& \bar{v}_{k}=v_{k} \times S_{k} \\
& S_{k}=\frac{\sin (k \pi / N)}{(k \pi / N)}
\end{aligned}
$$

The amplitudes of the cosine and sine components are given by Equations (84) and (85). Examination of $a_{k}$ and $b_{k}$ in the range $k=0$ to $k=\mathbb{N}$ will show a function that has peaks, one for each frequency present.

If a peak appears on an integral value of $\mathrm{k}$, and the adjacent values of $k$ show zero or very small amplitude, then the frequency in cycles/second $\left(F_{k}\right)$, and the actual cosine and sine amplitudes $\left(A_{k}, B_{k}\right)$ are given by

$$
\begin{aligned}
& F_{k}=\frac{k}{2 N(\Delta t)} \\
& A_{k}=\frac{a_{k}}{N} \\
& B_{k}=\frac{b_{k}}{N}
\end{aligned}
$$

If a peak appears between two values of $\mathrm{k}$, then a parabola is passed through the maximum point and the two adjacent points, one on each side of the maximum. Let $k=m$ at the maximum, $k=m-1$ to the left of the maximum, and $k=m+1$ to the right of the maximum. Then

$$
\begin{aligned}
& \mathrm{k}_{i}=\mathrm{m}+\epsilon \\
& \epsilon_{\mathrm{a}}=(1 / 2)\left(a_{m+1}-a_{m-1}\right) /\left(2 a_{m}-a_{m+1}-a_{m-1}\right) \\
& \epsilon_{b}=(1 / 2)\left(b_{m+1}-b_{m-1}\right) /\left(2 b_{m}-b_{m+1}-b_{m-1}\right) \\
& a_{\mu}=a_{m}+\left(\epsilon_{a} / 4\right)\left(a_{m+1}-a_{m-1}\right) \\
& b_{\mu}=b_{m}+\left(\epsilon_{b} / 4\right)\left(b_{m+1}-b_{m-1}\right)
\end{aligned}
$$




$$
\text { RN-S-0146 }
$$

$$
\begin{aligned}
& F_{i}=\frac{k i}{2 N(\Delta t)} \\
& A_{i}=1.6963 a_{\mu} / \mathrm{N} \\
& B_{i}=1.6963 \mathrm{~b}_{\mu} / \mathrm{N}
\end{aligned}
$$

where

$$
\begin{aligned}
k_{i}= & \text { location of the maximum } \\
\epsilon= & \text { amount by which maximum is displaced from an inte- } \\
& \text { gral value of } k \\
a_{\mu}, b_{\mu}= & \text { maximum values (by parabolic calculation) of } a_{k}, b_{k} \\
F_{i}= & \text { frequency in cycles/second } \\
A_{i}= & \text { cosine amplitude } \\
B_{i}= & \text { sine amplitude }
\end{aligned}
$$

In general, because of noise and experimental errors in the test data, $\boldsymbol{\epsilon}_{a}$ is not equal to $\boldsymbol{\epsilon}_{b}$ as the peaks of $a_{k}$ and $b_{k}$ do not appear at the same $k_{i}$. When this happens we calculate two values of frequency and average them. Frequencies closer than 0.8 cycles/sec cannot be resolved. A discussion of frequency resolution and accuracy that can be obtained using $N=39$ points is given under a separate heading.

1. Modification of Equations for Cosine-Sine Amplitudes for Faster Computation

By using the scheme discussed in Section VIII,A,2 with Equations (84) and (85) (with $N=39$ points), we can calculate $a_{k}$ and $b_{k}$ very rapidly. The total number of sine and cosines is 9.

$$
\begin{array}{lll}
\mathrm{C} 1=0.9863 & 6129, & \mathrm{~S} 1=0.16459459 \\
\mathrm{C} 2=0.94581724, & \mathrm{~S} 2=0.32469947 \\
\mathrm{C} 3=0.87947375, & \mathrm{~S} 3=0.47594739 \\
\mathrm{C} 4=0.78914050, & \mathrm{~S} 4=0.61421271 \\
\mathrm{C} 5=0.67728157, & \mathrm{~S} 5=0.73572391 \\
\mathrm{C} 6=0.54694815, & \mathrm{~S} 6=0.83716648 \\
\mathrm{C} 7=0.40169542, & \mathrm{~S} 7=0.91577332 \\
\mathrm{C} 8=0.24548548, & \mathrm{~S} 8=0.96940026 \\
\mathrm{C} 9=0.082579343, & \mathrm{~S} 9=0.99658449
\end{array}
$$


Forming the following sums and differences

$$
\begin{aligned}
& \begin{array}{llllllllll}
\bar{u}_{1} & \bar{u}_{2} & \bar{u}_{3} & \bar{u}_{4} & \bar{u}_{5} & \bar{u}_{6} & \bar{u}_{7} & \bar{u}_{8} & \bar{u}_{9} & \bar{u}_{10}
\end{array} \\
& {\underline{\bar{u}_{20}}}_{\bar{u}_{19} \bar{u}_{18} \bar{u}_{17} \bar{u}_{16} \bar{u}_{15} \bar{u}_{14} \bar{u}_{13} \bar{u}_{12} \bar{u}_{11}} \\
& \text { Sums } \quad u^{S_{1}} u^{S} 2 u^{S_{3}} u^{S_{4}} u^{S_{5}} u^{S_{6}} u^{S} 7 u^{S_{8}} u^{S_{9}} u^{S_{10}}
\end{aligned}
$$

Diff $\quad u_{1}^{D_{1}} u^{D_{2}} u^{D_{3}} u^{D_{4}} u^{D_{5}} u^{D_{6}} u^{D_{7}} u^{D_{8}} u^{D_{9}} u^{D_{10}}$

$$
\begin{aligned}
& \begin{array}{lllllllll}
\bar{v}_{2} & \bar{v}_{3} & \bar{v}_{4} & \bar{v}_{5} & \bar{v}_{6} & \bar{v}_{7} & \bar{v}_{8} & \bar{v}_{9} & \bar{v}_{10}
\end{array} \\
& \bar{v}_{19} \bar{v}_{18} \bar{v}_{17} \bar{v}_{16} \bar{v}_{15} \bar{v}_{14} \bar{v}_{13} \bar{v}_{12} \bar{v}_{11} \\
& \mathrm{v}_{1} \mathrm{v}_{2} \mathrm{v}_{3} \mathrm{v}_{4} \mathrm{v}_{5} \mathrm{v}_{6} \mathrm{v}_{7} \mathrm{v}_{8} \mathrm{v}_{9}
\end{aligned}
$$

Sums

Diff $\quad v_{1}^{D_{1}} v_{2}^{D_{2}} v_{3}^{D_{3}} v_{4}^{D_{4}} v_{5}^{D_{5}} v_{6} v_{7}^{D_{7}} v_{8}^{D_{8}} v_{9}^{D_{9}}$

Let

$$
v_{1}=\sum_{i=1}^{10} u_{i}^{s_{i},} v_{2}=\sum_{i=1}^{10} u_{i}^{D_{i}}
$$

Then:

$$
\begin{aligned}
& a_{0}=V_{1} \\
& a_{1}=u_{1}+u_{2} \times C l+u_{3} \times C 2+u_{4} \times C_{3}+u_{5} \times C_{4} \\
& +u_{6} \times C^{2}+u_{7} \times C 6+\mathrm{D}_{8} \times \mathrm{C} 7+\mathrm{u}_{9} \times \mathrm{C} 8+\mathrm{u}_{10} \times \mathrm{c} 9 \\
& a_{2}=u^{S_{1}}-u_{10}^{S_{10}} \times C_{1}+u_{2} S_{2} c_{2}-u^{S} \times C_{3}+u_{3} \\
& \mathrm{xC}_{4}-\mathrm{uS}_{8} \times \mathrm{C} 5+\mathrm{uS}_{4} \times \mathrm{C6}-\mathrm{u}_{7} \times \mathrm{C} 7+\mathrm{u}_{5} \times \mathrm{C} 8 \\
& -u^{s} 6 \times c 9 \\
& a_{3}=u_{1}-u_{7} \times C l-u_{8} \times C 2+u_{2} \times C 3-u_{6} \times C_{4} \\
& -u_{9} \times C 5+u_{3} \times C 6-u_{5} \times C 7-u_{10}^{D} \times C 8+u^{D} \times C 9
\end{aligned}
$$




\section{$\mathrm{RN}-\mathrm{S}-0146$}

$$
\begin{aligned}
& a_{4}=u_{1}^{S_{1}}-u_{6}^{S_{6}} \times C_{1}+u^{D_{10}} \times C_{2}-u_{5}^{D_{5}} \times C_{3}+u_{2} \times C_{4} \\
& -u_{7} \times C_{5}+u_{9} \times C 6-u_{4} \times C 7+u_{3} \times C 8 \\
& -\mathrm{u}_{8} \times \mathrm{C} 9
\end{aligned}
$$

$$
\begin{aligned}
a_{5}= & u_{1}^{D_{1}}-u_{5} \times C 1+\mathrm{D}_{9} \times \mathrm{C}_{2}+\mathrm{D}_{8} \times \mathrm{C}_{3}-\mathrm{u}_{4} \times \mathrm{C}_{4} \\
& +\mathrm{u}_{2} \times \mathrm{C}_{5}-\mathrm{D}_{6} \times \mathrm{C} 6+\mathrm{D}_{10} \times \mathrm{C} 7+\mathrm{D}_{7} \times \mathrm{C} 8 \\
& -\mathrm{u}_{3} \times \mathrm{C} 9
\end{aligned}
$$

$$
\begin{aligned}
& a_{6}=u^{S_{1}}-u_{4} \times C 1+u_{7} \times C_{2}-u^{S}{ }_{10} \times C_{3}+u_{8} \times C_{4} \\
& -u_{5} \times C_{5}+u_{2} S_{2} \times 6-u_{3} \times C 7+u_{6} \times C 8 \\
& -\mathrm{u}_{9} \times \mathrm{c} 9
\end{aligned}
$$

$$
\begin{aligned}
& a_{7}=u_{1}-u_{9}^{D_{9}} \times C l-u_{4} \times C 2+u_{6} \times C 3+u_{7} \times C 4 \\
& -u_{3}^{D_{3}} \times C_{5}-u_{10}^{D_{10}} \times C_{6}+u_{2} \times C_{7}-u_{8} \times c 8 \\
& -u_{5} \times C 9
\end{aligned}
$$

$$
\begin{aligned}
a_{8}= & u_{1} S_{1} \mathrm{~S}_{8} \times \mathrm{Cl}+\mathrm{uS}_{6} \times \mathrm{C}_{2}-\mathrm{uS}_{3} \times \mathrm{C} 3+\mathrm{uS}_{10} \times \mathrm{C}_{4} \\
& -\mathrm{uS}_{4} \times \mathrm{C} 5+\mathrm{uS}_{5} \times \mathrm{C6}-\mathrm{u}_{9} \times \mathrm{C} 7+\mathrm{u}_{2} \times \mathrm{C} 8 \\
& -u^{\mathrm{S}_{7}} \times \mathrm{C} 9
\end{aligned}
$$

$$
\begin{aligned}
& a_{9}=u_{1}-u_{3} \times C 1+u_{5} \times C_{2}-u_{7} \times C 3+u_{9} \times C_{4} \\
& +u^{D_{10}} \times C_{5}-u_{8} \times c 6+u_{6} \times c 7-u_{4} \times c 8 \\
& +\mathrm{u}_{2} \times \mathrm{C} 9
\end{aligned}
$$

$$
\begin{aligned}
& a_{10}=u^{S_{1}}-u_{3}^{S_{3}} \times C_{1}+u_{5}^{S_{5}} \times C_{2}-u_{7}^{S_{7}} \times C_{3}+u_{9}^{S_{9}} \times C_{4} \\
& -u^{S_{10}} \times \mathrm{C}_{5}+u^{S_{8}} \times \mathrm{c} 6-u_{6} \times \mathrm{C}_{6}+u_{4} \mathrm{~S}_{4} \times \mathrm{C} 8 \\
& -u_{2} \times c 9
\end{aligned}
$$


$\mathrm{RN}-\mathrm{S}-0146$

$$
\begin{aligned}
& a_{11}=u_{1}+u_{8} \times C 1-u_{6} \times C 2-u_{3} \times C 3-u_{10} \times C_{4} \\
& +u_{4} \times C 5+u_{5} \times C 6-u_{9} \times C 7-u_{2} \times C 8 \\
& -\mathrm{u}_{7} \times \mathrm{C} 9
\end{aligned}
$$

$$
\begin{aligned}
& a_{12}=u^{S} 1-u_{9}^{S} \times C l+u_{4} \times C 2-u_{6} \times C 3+u_{7} \times C 4 \\
& -u_{3} \times C 5+\mathrm{u}_{10} \times \mathrm{C} 6-\mathrm{u}_{2} \times \mathrm{C} 7+\mathrm{u}_{8} \times \mathrm{C} 8 \\
& -u_{5} \times C 9
\end{aligned}
$$

$$
\begin{aligned}
& a_{13}={ }_{u} D_{1}+{ }_{u} D_{4} \times C l+{ }_{u} D_{7} \times C_{2}+{ }_{u} D_{10} \times C_{3}-D_{8} \times C_{4} \\
& -u_{5} \times C_{5}-u_{2} \times C 6-u_{3} \times C 7-u_{6} \times c 8 \\
& -{ }^{\mathrm{D}} \mathrm{9} \times \mathrm{C} 9
\end{aligned}
$$

$$
\begin{aligned}
& a_{14}=u_{1}^{S_{1}}-u_{5} S_{5} C_{1}+u_{9} S_{9} C_{2}-u_{8} S_{8} C_{3}+u_{4} \times C_{4} \\
& -u_{2} \times C 5+u_{6} \times C 6-u_{10} \times C 7+u_{7} \times C 8 \\
& -\mathrm{u}_{3} \times \mathrm{C} 9
\end{aligned}
$$

$$
\begin{aligned}
& a_{15}=u_{1}^{D_{1}}+u_{6}^{D_{6}} \times C 1-u_{10}^{D_{10}} \times C_{2}-u_{5} \times C_{3}-u_{2} \times C_{4} \\
& -u_{7}^{D_{7}} \times \mathrm{C}_{5}+\mathrm{u}^{D_{9}} \times \mathrm{C} 6+\mathrm{u}_{4} \times \mathrm{C}_{7}+\mathrm{u}_{3} \times \mathrm{C} 8 \\
& +\mathrm{u}_{8} \times \mathrm{C} 9
\end{aligned}
$$

$$
\begin{aligned}
& a_{16}=u_{1}-u_{7} \times C_{1}+u_{8} \times C_{2}-u_{2} \times C_{3}+u_{6} \times C_{4} \\
& -u_{9}^{S_{9}} \times \mathrm{C}_{5}+\mathrm{uS}_{3}+\mathrm{C} 6-\mathrm{u}_{5} \times \mathrm{C} 7+\mathrm{u}_{10} \times \mathrm{C} 8 \\
& -\mathrm{u}_{4} \times \mathrm{C} 9
\end{aligned}
$$

$$
\begin{aligned}
& a_{17}=u_{1}^{D_{1}}+u_{10}^{D_{10}} \times C l_{-} \mathrm{u}_{2} \times \mathrm{C}_{2}-\mathrm{u}_{9} \times \mathrm{C}_{3}+\mathrm{u}_{3} \times \mathrm{C}_{4} \\
& +u_{8} \times C 5-u_{4} \times C 6-u_{7} \times C 7+u_{5} \times C 8 \\
& +\mathrm{u}_{6} \times \mathrm{C} 9
\end{aligned}
$$




$$
\begin{aligned}
& a_{18}=u_{1} S_{1} s_{2} \times C l+u_{3} \times C 2-u_{4} \times C 3+u_{5} \times C_{4} \\
& -u^{S} 6 \times C_{5}+u_{7} \times c 6-u_{8} \times C_{7}+u_{9}^{S} \times c 8 \\
& -u^{S} 10 \times c 9 \\
& a_{19}=v_{2} \\
& b_{0}=0 \text {. }
\end{aligned}
$$

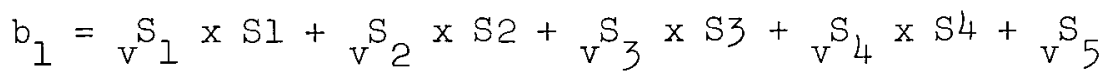

$$
\begin{aligned}
& \mathrm{xS} 5+\mathrm{v}_{6} \times \mathrm{s} 6+\mathrm{vS}_{7} \times \mathrm{s} 7+\mathrm{vS}_{8} \times \mathrm{s} 8+\mathrm{v}_{9} \times \mathrm{s} 9 \\
& \mathrm{~b}_{2}=\mathrm{v}_{9} \mathrm{SS1}+\mathrm{v}_{1} \times \mathrm{S} 2+\mathrm{v}_{8} \times \mathrm{S} 3+\mathrm{v}_{2} \times \mathrm{S} 4+\mathrm{v}_{7} \\
& \mathrm{xS} 5+\mathrm{v}_{3} \times \mathrm{S} 6+\mathrm{v}_{6} \times \mathrm{S} 7+\mathrm{v}_{4} \times \mathrm{S} 8+\mathrm{v}_{5} \times \mathrm{S} 9 \\
& \mathrm{~b}_{3}=\mathrm{v}_{6} \mathrm{~S} \mathrm{~S} 1-\mathrm{v}_{7} \times \mathrm{S} 2+\mathrm{v}_{1} \times \mathrm{S} 3+\mathrm{vS}_{5} \times \mathrm{S} 4-\mathrm{v}_{8} \\
& \mathrm{xS} 5+\mathrm{vS}_{2} \mathrm{x} \mathrm{S6}+\mathrm{v}_{4} \mathrm{~S} \mathrm{S7}-\mathrm{v}_{9} \mathrm{~S} \mathrm{~S} 8+\mathrm{v}^{\mathrm{S}} \mathrm{x} \mathrm{S} 9 \\
& b_{4}=-v^{D} \times S 1-v_{9} \times S 2+v_{v} D_{4} \times S 3+v^{D} \times S 4-v^{D} 6 \\
& \mathrm{x} S 5-\mathrm{v}_{8} \times \mathrm{s} 6+\mathrm{v}_{3} \times \mathrm{S} 7+\mathrm{v}_{2} \times \mathrm{s} 8-\mathrm{v}_{7} \times \mathrm{s} 9 \\
& \mathrm{~b}_{5}=-\mathrm{v}_{4} \times \mathrm{S} 1+\mathrm{v}_{8} \times \mathrm{S} 2-\mathrm{v}_{7} \times \mathrm{S} 3+\mathrm{v}_{3} \times \mathrm{S}_{4}+\mathrm{v}_{1} \\
& \mathrm{x} S 5-\mathrm{vS}_{5} \times \mathrm{S} 6+\mathrm{v}_{9} \times \mathrm{S} 7-\mathrm{v}_{6} \times \mathrm{S} 8+{ }_{\mathrm{v}} \mathrm{S}_{2} \mathrm{x} \mathrm{S} 9 \\
& b_{6}=v_{3} \times S 1-D_{6} \times S 2+{ }_{v} D_{9} \times S 3+{ }_{v} D_{7} \times S 4-D_{4} \\
& \mathrm{x} 55+\mathrm{v}_{1} \times \mathrm{S} 6+\mathrm{v}_{2} \times \mathrm{S} 7-\mathrm{v}_{5} \times \mathrm{S} 8+\mathrm{v}_{8} \times \mathrm{S} 9 \\
& b_{7}=v^{S} 8 \mathrm{Sl}-\mathrm{v}_{3} \times \mathrm{S} 2-\mathrm{v}_{5} \times \mathrm{S} 3+\mathrm{vS}_{6} \times \mathrm{S} 4+\mathrm{v}_{2} \\
& \mathrm{x} S 5-\mathrm{vS}_{9} \times \mathrm{S} 6+\mathrm{vS}_{1} \times \mathrm{S} 7+\mathrm{vS}_{7} \times \mathrm{S} 8-\mathrm{vS}_{4} \times \mathrm{S} 9
\end{aligned}
$$




$$
\begin{aligned}
& \mathrm{b}_{8}={ }_{\mathrm{v}} \mathrm{D}_{7} \times \mathrm{SI}+{ }_{\mathrm{v}} \mathrm{D}_{5} \times \mathrm{S} 2+{ }_{\mathrm{v}} \mathrm{D}_{8} \times \mathrm{S} 3-\mathrm{v}_{9} \mathrm{D}_{9} \times \mathrm{S}_{4}-\mathrm{v}_{3} \\
& \mathrm{x} S 5-\mathrm{v}_{4} \times \mathrm{S} 6-\mathrm{v}_{8} \times \mathrm{S} 7+\mathrm{v}_{1} \times \mathrm{S} 8+\mathrm{v}_{6} \times \mathrm{S} 9 \\
& b_{9}=v_{2} S_{2} \times S 1-v_{4} \times S 2+v_{v} s_{6} \times S_{3}-v^{S} \times S_{4}+v^{S} 9
\end{aligned}
$$

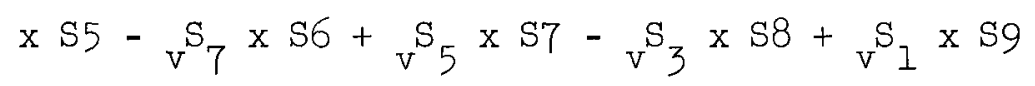

$$
\begin{aligned}
b_{10}= & -v_{2}^{D} \times S 1+v_{4} \times S 2-v_{6} \times S 3+v_{8} \times S 4+v_{9} \\
& \times S 5-v_{7} \times S 6+v_{5} \times S 7-v_{3} \times S 8+v_{1} \times S 9
\end{aligned}
$$$$
b_{11}=v_{7}^{S_{7}} \times \mathrm{S} 1+v_{5} \times S_{2}-v_{2} \times S_{3}-v_{9}^{S_{9}} \times S_{4}-v_{v} S_{3}
$$$$
\mathrm{x} 55+\mathrm{v}_{4} \times \mathrm{S} 6+\mathrm{vs}_{8} \times \mathrm{s} 7+\mathrm{v}_{1} \times \mathrm{s} 8-\mathrm{vs}_{6} \times \mathrm{s} 9
$$

$$
\begin{aligned}
b_{12}= & -v_{8} \times S 1-D_{3} \times S 2-D_{5} \times S 3-D_{6} \times S 4-D_{2} \\
& \times S 5-v D_{9} \times S 6+{ }_{v} \times S 7+D_{7} \times S 8+{ }_{v} \times S 9
\end{aligned}
$$

$$
\begin{aligned}
& b_{13}=v_{v_{3}} \times \mathrm{S} 1+v_{v} \mathrm{~S}_{6} \times \mathrm{S} 2+{ }_{\mathrm{v}} \mathrm{S}_{9} \times \mathrm{S} 3+\mathrm{v}_{7} \times \mathrm{S} 4+\mathrm{v}_{4} \\
& \mathrm{xS} 5+\mathrm{v}_{1} \mathrm{~S} \mathrm{~S} 6-\mathrm{v}_{2}^{\mathrm{S}} \times \mathrm{S} 7-\mathrm{v}_{5} \mathrm{~S} \text { S8 }-\mathrm{v}_{8} \times \mathrm{S} 9
\end{aligned}
$$

$$
\begin{aligned}
b_{14}= & v D_{4} \times S 1-v_{8} \times S 2-D_{7} \times S 3+v_{3} \times S 4+v D_{1} \\
& \times S 5-v_{5} \times S 6+v_{9} \times S 7+{ }_{v} D_{6} \times S 8-v_{2} \times S 9
\end{aligned}
$$$$
b_{15}=-v_{5} \times S 1-v_{9} \times S 2-v_{4} \times S 3+v_{1} S_{1} \times S_{4}+v^{S} 6
$$$$
\mathrm{xS} 5+\underset{v^{\mathrm{S}} 8}{\mathrm{~S}} \mathrm{x} \mathrm{S} 6+\mathrm{v}_{3} \times \mathrm{S} 7-\underset{v_{2}}{\mathrm{~S}} \times \mathrm{S} 8-\mathrm{v}_{7} \times \mathrm{S} 9
$$

$$
\begin{aligned}
b_{16}= & -v_{6} \times S 1-D_{7} \times S 2+D_{1} \times S 3+v D_{5} \times S 4+v D_{8} \\
& \times S 5-D_{2} \times S 6-D_{4} \times S 7-D_{9} \times S 8+v_{3} \times S 9
\end{aligned}
$$

$$
\begin{aligned}
b_{17}= & v_{9} S_{9} S_{1}+v_{1} \times S_{2}-v_{8} \times S_{3}-v_{2} \times S_{4}+v_{7} S_{7} \\
& \times S 5+v_{3} \times S 6-v_{6} \times S 7-v_{4} \times S 8+v_{5} \times S 9
\end{aligned}
$$




$$
\begin{aligned}
b_{18}= & v_{1} \times S 1-v_{2} \times S 2+{ }_{v} \times S 3-D_{4} \times S 4+v_{5} \\
& \times S 5-v^{D_{6}} \times S 6+{ }_{v} \times S 7-D_{8} \times S 8+D_{9} \times s 9 \\
b_{19}= & 0 .
\end{aligned}
$$

\section{Frequency and Amplitude Discrimination}

To determine the frequency and amplitude discrimination and accuracy of the method used on the NERVA narrow-band data analysis, a test problem was run on the computer. The following function was computed at 39 points, at intervals of $0.025 \mathrm{sec}$ (corresponding to the 40 samples/sec rate). These 39 points were used as the data from which the frequency and amplitude was computed.

$$
y(t)=A_{1} \sin \left(2 \pi f_{1} t+\phi_{1}\right)+A_{2} \sin \left(2 \pi f_{2} t+\phi_{2}\right)
$$

where

$$
\begin{aligned}
& A_{I}=1.0 \\
& 0.25 \leq A_{2} \leq 1.0, \text { in } 0.25 \text { steps } \\
& f_{1}=3.0 \text { cycles } / \mathrm{sec} \\
& 3.1 \leq f_{2} \leq 5.0 \text { cycles } / \mathrm{sec}, \text { in } 0.1 \text { cycles } / \mathrm{sec} \text { steps } \\
& \phi_{1}=30^{\circ}, 60^{\circ}, 90^{\circ} \\
& \phi_{2}=-30^{\circ},-60^{\circ},-90^{\circ}
\end{aligned}
$$

Frequencies were separable in 578 cases. The lowest consistent separation frequency was 1.0 cycles/sec, although a small number of cases showed a frequency separation of $0.9 \mathrm{cycle} / \mathrm{sec}$. The average error in the frequency determination was found to be 0.119 cycles/sec. The average error in the amplitude determination was 0.041 units.

The error diminishes as the frequency separation increases, being largest at the threshold separation of 0.9 to $1.0 \mathrm{cycle} / \mathrm{sec}$, and showing its greatest values at this threshold separation point. When two frequencies are close together and one has an amplitude of the order of four times the other, the larger error is associated with the frequency with the smallest amplitude.

The frequencies and amplitudes calculated are estimates of the true frequencies and amplitudes. The accuracy of these estimates increases as 


\section{$\mathrm{RN}-\mathrm{S}-0146$}

the number of points used increases. When a large number of points is used ( $>39$ used in the present program), the use of a focusing function is no longer required.

As the number of samples/second is fixed in the data acquisition system, an increase in accuracy may be obtained by the use of a more efficient focusing function and a more sophisticated way of obtaining the peaks of the cosine and sine amplitudes. Most of the search for focusing functions has been done empirically on a trial and experiment basis. An evaluation was made of the functions given by Blackman and Tuckey, ${ }^{*}$ the one recommended by Lanczos, ${ }^{* *}$ and also several other variations. The function given by Lanczos was found to give the most consistent results and was therefore used in the digital program.

* R. B. Blackman and J.W. Tuckey, The Measurement of Power Spectra, Dover Publications, Inc., New York.

${ }^{*}$ C. Lanczos, Applied Analysis, Prentice Hall, Inc., 1961, Chapter IV, Section 22. 
A

$A C(20)$

$A C(20)$

ACHOMP

ADUD

AT IME

BARN

$B C(20)$

$\mathrm{CC}(20)$

CONST

CSS

$F(20)$

FLAG

FLAG 10

FLAG 17

FLAG 44

FLAG 99

HEADER $\$ 12$ !

I

( A (200)

I B $(540)$

I B I TE

ICHOMP

IDUD

IFLUB

IGAG

IGOTA

I JKL

ILABEL (100)

I REDWD

I $S$ ICK
THE ESTIMATE OF THE INTERCEPT OF THE LINEAR CURVE FIT TO THE DATA AREA IN CORE INTO WHICH THE FLOATING POINT TIMES ARE STOREO BY SUBROUTINE UNPACK * NOT USED IN MAIN PROGRAM

THE SINE COMPONENTS OF FREQUENCY PRESENT WITHIN THE $39 \%$ I

THF NUMBER OF CHANNELS PER MULTIPLEXER IN FLOATING POINT FORMAT

FLOATING POINT TIME IN SECONOS IIDUD\&IFLUB)

TIME CF OCCURANCE DF FLAG ISTCK

THE ESTIMATE OF THE SLOPE OF THE I.INEAR CURVE FTT TC THE DATA

THE MEAN OF THE SAMPLE OF DATA POINTS BEING PROCESSED

THE COSINE COMPONENTS OF FREOUENCY PRESENT WITHIN THE 39 YII

$A C(1) * 2+B C(1 * 2$

EGUIVALENT TO K?

THE CCRRECTED SUM OF SQUARES OF THE SAMPLE DATA POENTS BETNG PROCESSED

THE VECTOR CF FREQUENCIES (CYCLES/SEC I FOR THE 39 YUT)

A FLAG TO DENOTE AN INTERNAL OUTLIER

A FLAG TO DENOTE TYPES OF END POINT OUTLIERS OR STEPS

A FLAG TO DENOTE EXCESSIVE VARIANCE AFTER A LINEAR TREND HAS BEEN

FIT TO A RAMP

A FLAG TO DENOTE A BREAK IN TIME

A FLAG TO DENOTE FIRST DATA FOR A MULTIPLEXER

A DESCRIPTIVE TITLE OF THE TEST

THE INDEX VALUE OF THE FIRST DATA POINT IN THE SET BEING ANALIZED

AREA IN CORE INTO WHICH ONE PHYSICAL RECORD OF DATA IS READ BY SUBROUTINE READP

AREA IN CORE INTO WHICH UNPACKED DATA IS STORED BY SUBROUTINE UNPACK

THE NUMBER OF LOGICAL RECORDS PER PHYSICAL RECCRD

THE NUMBER OF CHANNELS PER MULTIPLEXER

FIXED POINT TIME IN SECCNDS

FIXED PCINT TIME IN MILLISECONDS

DATA VALUE STRIPED OF FLAGS

THE NUMBER OF LOGICAL RECORDS PER PHYSICAL RECORD WHEA THE PHYSICAL RECORE IS SMALLER THAN NORMAL

A POINTER TO DENOTE POSIIION IN LOADING OF MATRIX JAZZ

AREA IN CORE INTO WHICH THE MULTIPLEXER HEADER RECCRD IS READ EY SUBROUTINE READA

NUMBER OF WORDS OF DATA IN PHYSICAL RECORD READ BY SUBRDUTINE READB

THE FLAG RIT CONFIGURATION STRIPED FROM THE DATA WORD 


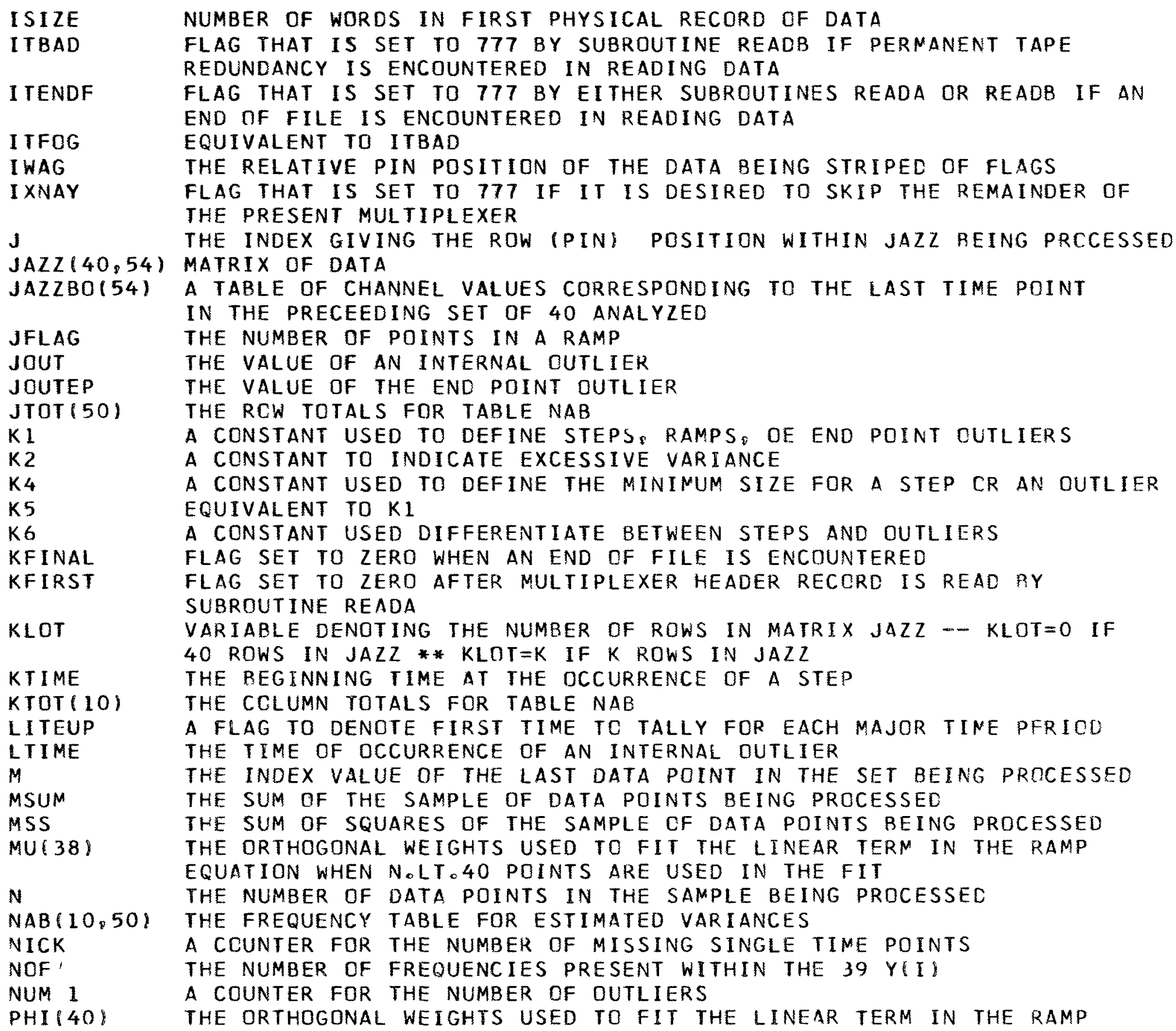


STERRY

TAU

TIME 4 40

Y 1391
EQUATIDN WHEN 40 POINTS ARE USED IN THE FIT

THE VARIANCE OF THE SAMPLE OF DATA POINTS BEING PROCESSED

TIME BETWEEN SUCCESSIVE SAMPLES

VECTOR OF FLOATING POINT TIMES CORRESPONDING TO THE RCWS OF JAZZ THE VECTOR OF DETRENDED DATA FOR WHICH IT IS DESIRED TO DETERMINE THE NUMBER AND NATURE OF THE FREQUENCIES WITHIN 


\section{RN-S-0146}

X. IISTING OF PROGRAM SOURCE DECKS, PROGRAM ADDA 
$\$$ IBFTC ADDA M94,XR7,LIST

C A TO D DATA ANALYSIS PROGRAM

C PROGRAM TO ANALIZE REON N-2 TAPES

DIMENSICN JAZZ(40,54), TIME (40), Y (39), F(20),AA(20),BB(20),CSC(20)

DIMENSICN JAZZBO(54)

COMMON/NAME/LITE2,ITFOG,MARK, LATERN,KZOW,LITEUP,NICK,FLAG99, NUMI, 1NUM2,HEADER (12), MUL TPX,NAB(10,50), JTOT(50), KTOT(10), NCHS \& NCR 20,49

2) \& KTOTAL $(20)$ \& MTOTAL (49) \& NUM3 \& TFIRST, TFINAL\&KNT \& LINE

COMMON MOUSE (3)

COMMON/SKIP/IXNAY

REAL K2,K92

INTEGER FLAG FLAG10, FLAG17, FLAG44, FLAG99

EQUIVALENCF (A\&BARM)

EQUIVALENCE (NCHS, ICHOMP)

FQUIVALENCE $(J, N C H)$

TOUIVALENCE (K5,K1), (CONST,K92,K2)

DATA KICK/GHSTOP,

CALL TIMEI

READ $\{5,9993\}$ (HEADER $([), I=1,12\}$

9993 FRRMAT $(12 A 6)$

READ 5.9991$) K 4, K 6: K 1, K 2$

9991 FORMAT(3I6,F6.0)

1 REAC $(5,9990)$ MULTPX, NCHS, IOITE, ISIZE,TAU

9990 FORMAT $(A 6,316, F 6.0)$

CALL TIME2 (NNN)

NNN $=$ NNN $/ 60$

PRINT 919, NNN

919 FORMAT $\left(9 X_{0} 6 H * * * *\right.$ (16)

CALL TIMEI

IF(MULTPX。EQ。KICK) GO TO 2773

NLAST $=40$

IXNAY $=0$

LITE $1=0$

LITE $3=0$

$K 78=39$

$T B I G=5.0$

TDEL $=0.030$ 
CALL INITAL

FLAG99=1

IF ILITEI。NE。O\& GO TO 7

2 CALL BRAIN IIBITE,NCHS, JAZZ,KFINAL,KLOT,ITFOG,KZOW, TIME, ISIZE: IF (IKFINAL。EQ, O)。AND。(KLOT。EQ.0)

IFIITFOG。EQ 0 \& GO TO 13

IT FOG $=0$

13 IF(KLOT。EG。O) GO TO 1.4

$N N=K L O T$

GO TO 15

$14 \mathrm{NN}=40$

$15 \mathrm{I}=1$

$N=N N$

$\operatorname{LITE} 4=0$

$7 \mathrm{~J}=1$

CALL TEQIT \&TIMESTLAST,FLAG44,FLAG99, TBIG,TDEL, I3, N3, I4, N4, I, N) IF(KNT,EQ.0) TFIRST $=$ TIME I I

LITE I $=0$

$N L A S T=N$

$K N T=K N T+N$

GO TO 299

20 LITE $1=777$

$K N T=K N T+N 3$

IF( $\{N 3 . L T .3\} \circ A N D .(N L A S T \circ L T .3) \backslash G O$ TO 21

$1000 \quad N=N 3$

NLAST $=$ N3

$$
I=I 3
$$

GO TO 299

21

I XNAY $=777$

GO TO 2

$8 \quad 1=14$

$\mathrm{N}=\mathrm{N} 4$

$9 \quad \mathrm{I}=1$

Go ro 299

$$
\mathrm{N}=\mathrm{NN}
$$

$299 M=N+I-1$

300 IT $=I A B S(J A Z Z(M, J)-J A Z Z(I, J)$

IFIIT.GT.KI) GO TO 4300

400 CALL STATI A JAZZ, I $J_{\nabla} N_{0}$ MSUM,MSS,BARM,CSS, STERRY)

IF SSTERRY。LT。K2) GO TO 700

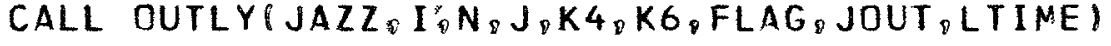


IF(FLAG。NE。1) GO TO 820

FMSUM $=$ MSUM-JOUT

$\mathrm{N}=\mathrm{N}-1$

$\mathrm{FN}=\mathrm{N}$

MSS $=$ MSS $-J O U T * 2$

$B A R M=F M S U M / F N$

CSS =FLOAT (MSS :-BARM*FMSUM

STERRY $=$ CSS $/(F N-1$.

TIMEE $=$ TIME (I)

NUM $1=$ NUM $1+1$

IFIN.LE。K78) GO TO 121

CALL TALLYILITEUP,STERRY,NAB\& KTOT\&JTOT:J)

121 CONTINUE

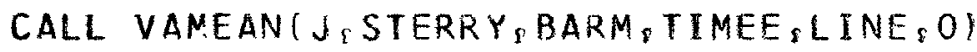

GO TO 7600

700 TEMP $=$ TIME II

IF $(N . L E, K 78)$ GO TO 122

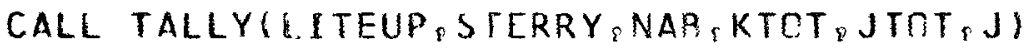

122 CONTINUE

CALL VAMEAN $\left(J_{2} S T E R R Y, B A R M_{8}\right.$ TEMP \& LINE 50$)$

GO TO 7600

820 IF $\left(N_{0} E Q .40\right)$ GO TO 840

9405 TEMP $=$ TIME (I)

IF $(N \propto L E \circ K 78)$ GO TO $\$ 23$

CALL TALLY(LITEUP, STERRY \&NAB \&KTOT\& JTOT\&J)

123 CONTINUE

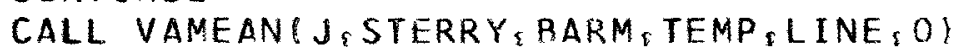

GO TO 7600

840 DO $844 \quad K=1,39$

$844 Y(K)=F L C A T(J A Z Z$ (K o ) $)$-BARM

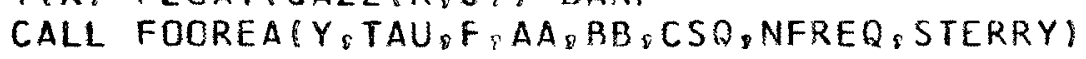

TEMP $=T$ IME \{\}

NUM2 $=$ NUM $2+1$

IFINFREQ.EQ.0: GO TO 33

DO $9406 \mathrm{~K}=1$, NFREQ

$F K=F(K)$

$A A K=A A(K)$

$B B K=B B(K)$

CSQK $=$ CSQ $(K)$

9406 CALL TIC (LATERN,FK \& NOB \& KTOTAL,MTOTAL,J)

33 CALL VAMEAN $\left.J_{\otimes} S T E R R Y_{\triangle} B A R M, T E M P \& L I N E, O\right)$ 
IF $\left(N_{0} L E_{0} K 78 \geqslant G O\right.$ TO 124

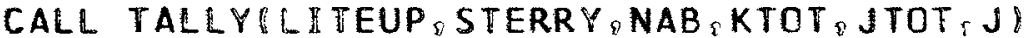

124

CONTINUE

GO 107600

4300 IF $(N \circ N E 0401$ GO TO 2131

CALL ENDPTE JAZZ, $N, J 6, K 4, F L A G 10, J O U P E, J A Z Z B O)$

GO TO $\left(3460_{1} 4320,4360,299\right), F L A G 10$

4320 JACK $=J A Z Z(1, J$

NUMI $=$ NUM $1+1$

$M=N+I-1$

$I T=I A B S\left(J A Z Z\left(M_{\{} J\right)-J A Z Z\left(I_{2} J\right)\right)$

IFIIT:GT KKI\& GO TO 3440

GO TO 400

$4360 \mathrm{JACK}=J A Z Z\left(40_{\&} \mathrm{~d}\right)$

NUM $1=$ NUM $1+1$

$N=39$

$M=N+I \cdots 1$

$I T=\mathbb{A} A B S\left(J A Z Z\left(M_{i} J\right)=J A Z Z\left(I_{R} J\right)\right)$

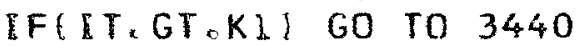

GD TO 400

2131 IF(N,GT。3) GO TO 3440

CALL STATI JAZE, I JEN,MSUM \&MSS, PARM C CSS STERRY)

TEMP $=$ TIMECI :

IFCNOLEOK78\&GO TO 125

CALL TALLYGLITEUP \& STERRY, NAB\& KTOT, JTOT\& J)

125 CONTINUE

CALL VAMEAN $\mathcal{S}$ STERRY, BARM, TEMP, LINE, 0 )

GO TO 7600

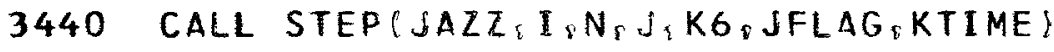

IF I JFLAG。GT 0 :GO TO 3448

IF $(N \circ E Q=40)$ GO TO 4710

2130 CALL FIT $2\left(\downarrow A Z Z_{q} d_{\varepsilon} I_{\&} N_{\&} A_{\&} B_{\&} S T E R R Y\right)$

TEMP $=$ TIME I I

IF(N.LE。K78) GO TO 126

CALL TALLY(LITEUP \& STERRY,NAB,KTOT,JTOT\& J)

126 CONTINUE

CALL VAMEAN $J_{\varepsilon} S T E R R Y_{\&} B A R M, T E M P \& L I N E$ \& $\left.O\right\}$

GO TO 7600

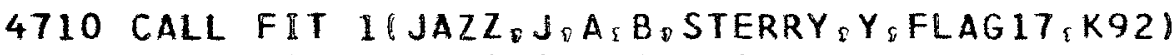

IFIFLAGIT。NE,O) GO TO 9221

IF(N.LE $0 K 78)$ GO TO 127

CALL TALLYILITEUP, STERRY NAB \&KTOT,JTOT\& J) 
127 CONTINUE

TEMP $=$ TIME $(1)$

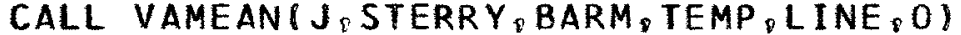

GO TO 7600

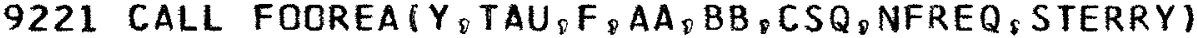

TEMP $=$ TIME $(1)$

NUM2 $=$ NUM 2+1

IFINFREQ。EQ.0 \& GO TO 34

DO $9233 \mathrm{~K}=1$, NFREQ

$F K=F(K)$

$A A K=A A(K)$

$B B K=B B(K)$

$\mathrm{CSQK}=\mathrm{CSQ}(\mathrm{K})$

9233 CALL TIC (LATERN,FK, NOB,KTOTAL\&MTOTAL。J)

34 CALL VAMEAN $\left(J_{\varepsilon}\right.$ STERRY, BARM TEMP \& LINE, 0$)$

IFINOLE。K78) GD TO 128

CALL TALLYILITEUP \& STERRY SNAR, KTOT JTOT:JI

128 CONTINUE

GO TO 7600

3448 LITE $3=777$

LITE $2=0$

NUM $3=$ NUM $3+1$

$11=\mathbf{I}$

$N 1=K T I M E-I$

$I 2=K T I M E$

$\mathrm{N} 2=\mathrm{N}-\mathrm{N} 1$

$\mathrm{N}=\mathrm{N} 1$

$I=11$

GO TO 299

7600 IFILITE 3.EQ.0 80 TO 7650 IFILITE 2.EQ.0) GO TO 7800 LITE $3=0$

7650 IFILITE $1 . E Q .01$ GO TO 7900

$8000 \mathrm{~J}=\mathrm{J}+1$

IF(J.GT.NCHS) GO TO 1919

GO TO 1000

7800 LITE $2=777$

$\mathrm{I}=\mathrm{I} 2$

$\mathrm{N}=\mathrm{N} 2$

GO TO 299

$7900 \mathrm{~J}=\mathrm{J}+1$ 
IF(S.GT.NCHS) GO TO 1767

IFILITE4。NE。O) GO TO 8

GO TO 9

1767 IF(KFINAR NE O O GO TO 1700

1919 TFINAL = TIME (M)

IF IIXNAY NEOO GO TO 2772

CALL VAMEAN $(0,0,0,0,0,0.0, L I N E \& 777)$

CALL REPORT

IFILITEI。EQ.0) 60 TO 1

$\mathrm{I}=\mathrm{I} 4$

$\mathrm{N}=\mathrm{N} 4$

LITE $4=777$

GO TO 5

1700 DO $1717 \mathrm{~K}=I_{8}$ NCHS

$1717 \mathrm{JAZZBO}(K)=J A Z Z\left(40_{\Sigma} K\right)$

TLAST $=$ TIME $(M)$

TFINAL $=$ TIME $(M)$

GO TO 2

2772 PRINT 977, MULTPX,TFINAL

977 FORMAT $\left(9 X_{₹}\right.$ ILHMULTIPLEXER \& $1 A 6_{\&} 19 H$ TERMINATED AT TIME $\left., F 11,3\right)$ GO TO 1

2773 CALL CLOSEA

RETURN

END

\$IBFTC NSOB M94:XR7\&LIST

SUBROUTINE INITAL

C THIS SUBROUTINE INITIAIZES VARIOUS FLAGS AND COUNTERS.

COMMON/NAME/LITE2\&ITFOG \&MARK, LATERN,KZOW,LITEUP,NICK, FLAG99,NUM1. INUM 2 \& HEADER $(12)$, MULTPX,NAB $(10,50)$, JTOT $(50), K$ TOT $(10)$,NCHS, NOR $(20,49$

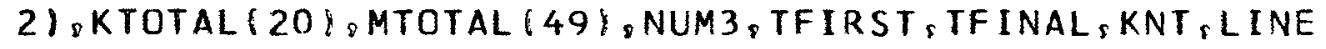

LINE $=0$

LITE2 $=777$

IT $F O G=0$

MARK $=0$

LATERN $=0$

$\mathrm{KZOW}=0$

LI TEUP $=0$

NI CK $=0$ 
NUM1 $=0$

NUM2 $=0$

NUM $3=0$

KNT $=0$

RETURN

END

\$IBFTC OTTOI LIST

SUBROUTINE BRAINIIBITE, ICHOMP, SAZZ,KFINAL\&KLOT,ITFOG,KZOW \&TIML, IS

IIZEY

C SUBROUTINE BRAIN * A SECONDARY MAIN PROGRAM WHICH CAILS IN TURN

C SEVERAL SUBROUTINES TO ACCOMPLYSH ITS FUNCTION. ITS FUNCTION PEING

C TO SUPPLY THE DATA IN A MATRIX JAZZ ANO THE ASSOCIATED FLCATING PO

C INT TIMES.

DIMENSION IA(200), IB (540), HLABEL(100), JAZZ(40,54), TIME(40), AC(20)

COMMON/SKEP/IXNAY

KFINAL $=777$

IREDWD $=0$

$K L O T=0$

ITBAD $=0$

IF I IXNAY NE $\odot O)$ GO TO 33

IFIKFIRST,EG.O) GO TO 8

CALL OPENA

CALL READAIITENDF \& ILABEL)

KFIRST $=0$

$A C H O M P=I C H O M P$

IGOTTA $=(A C H C M P+4.0) / 3.0+0.8$

$81 \mathrm{JKL}=1$

99 CALL READE (ITENDF, IA, IREDWD, ITBAD, ISIZE)

IF(ITBAO) $100,101,100$

100 ITBAD $=0$

I TFOG $=777$

GO YO 99

101 IF(ITENCF) $3,2,3$

2 IFIIREDWO.EQ.1/GO TO 66

IF(IREDWO.NE , 0$)$ GO TO 77

CALL UNPACK (IBITE, ICHOMP, IA, IB, AC, KZOW)

4 CALL MATRIXIICHOMP, IB, JAZZ,$A C$, TIME \& IJKL

IF(KLOT.NE。O) GO TO 88 


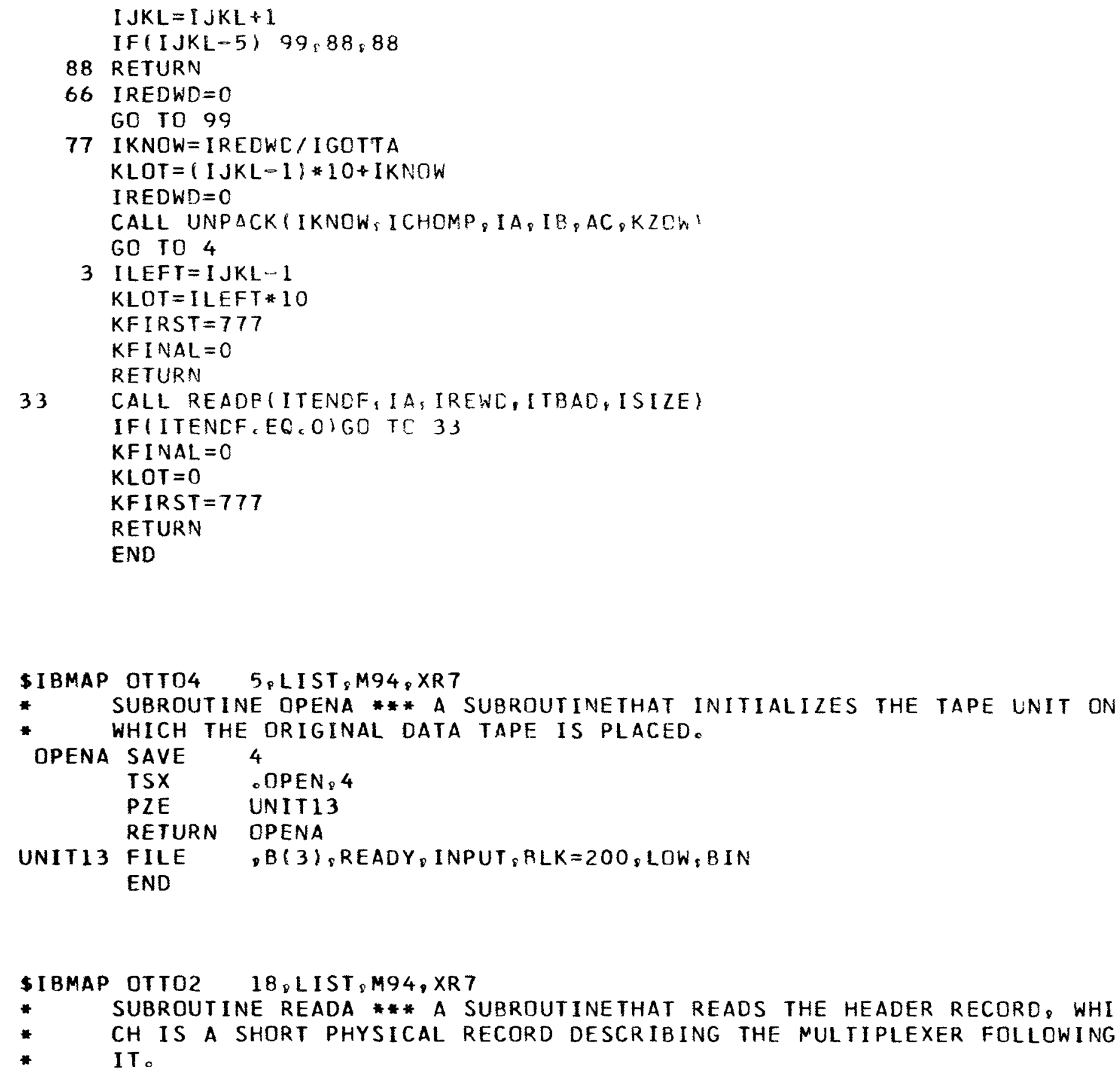




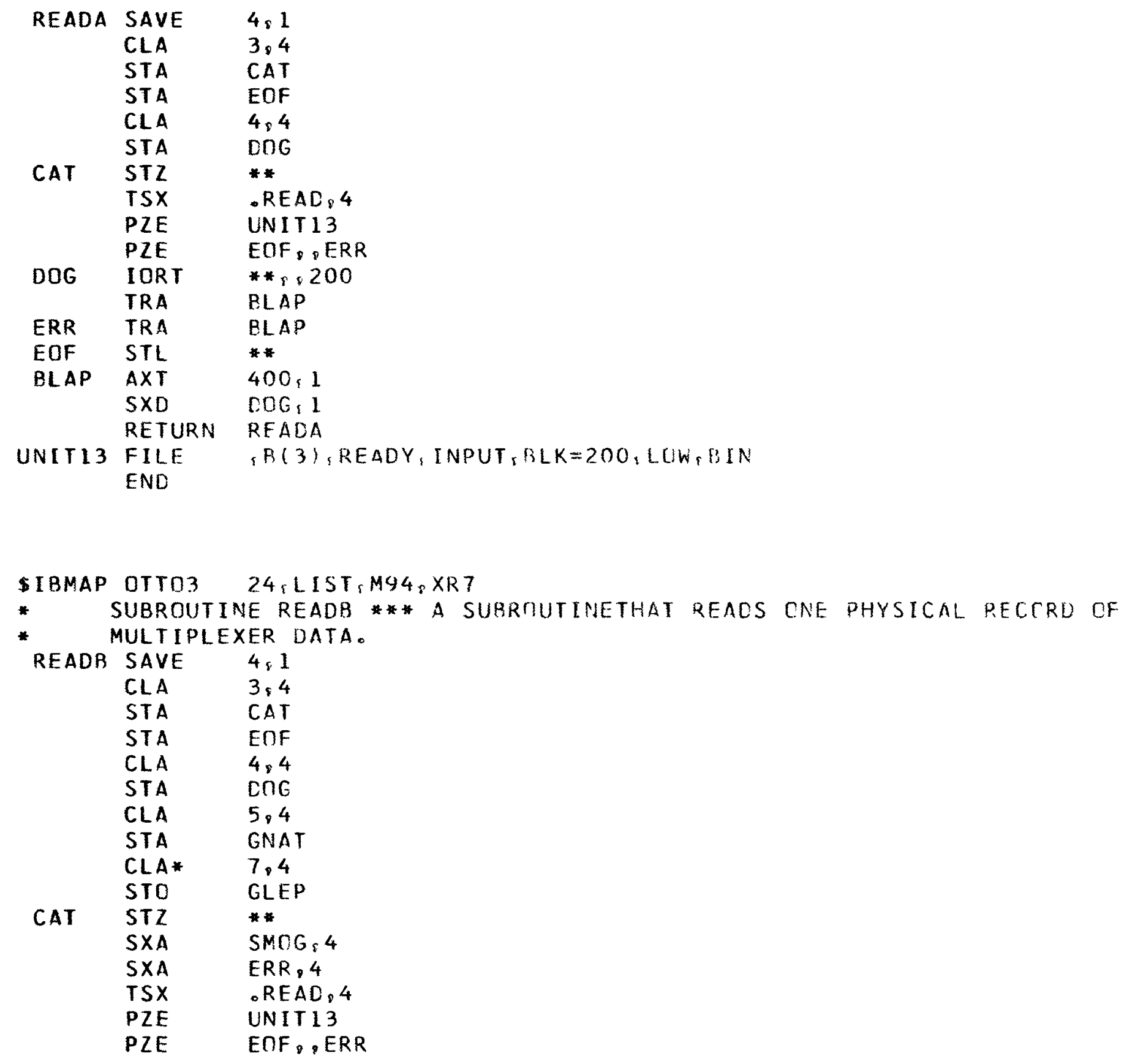




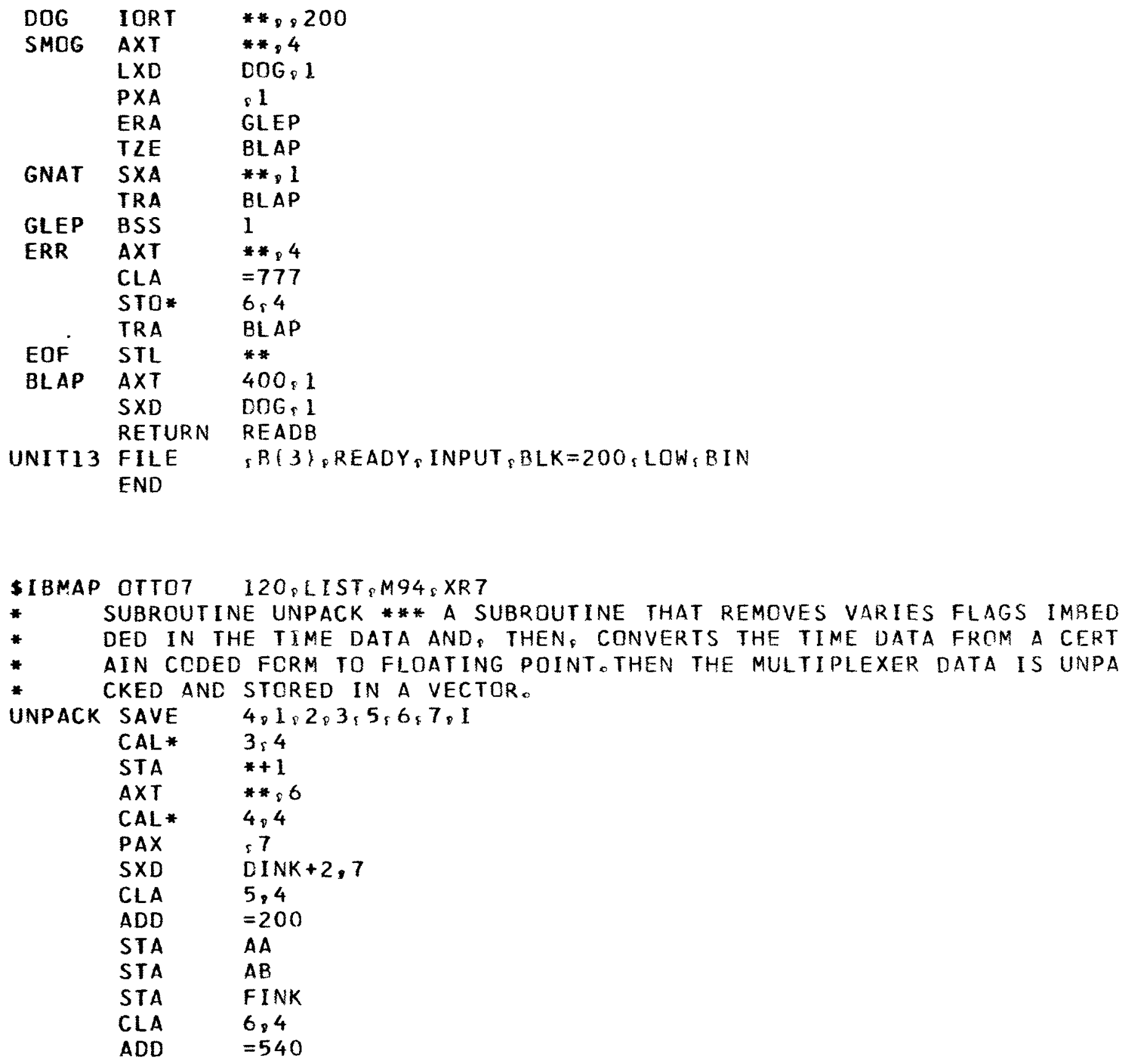




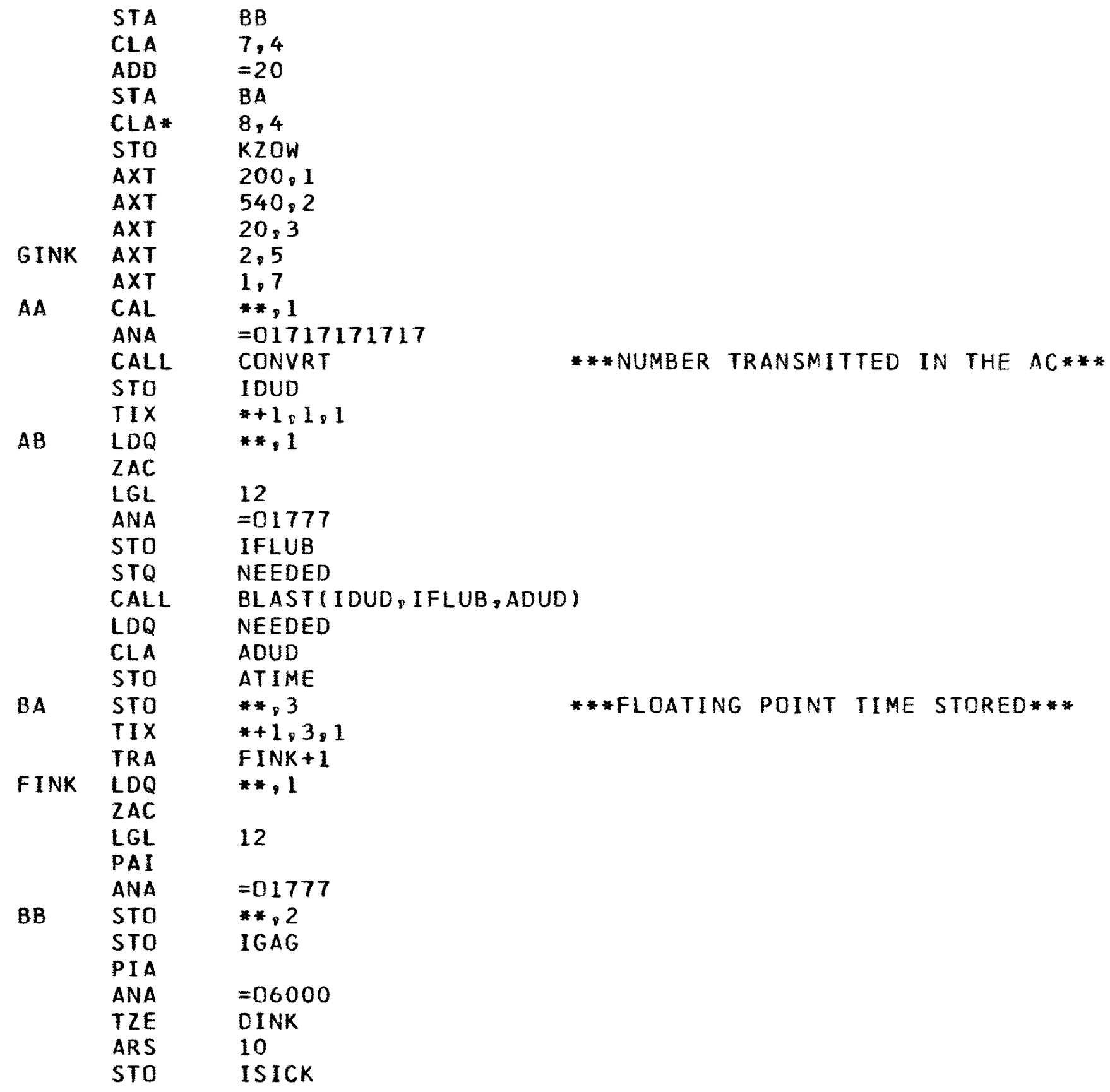




\begin{tabular}{|c|c|c|}
\hline \multirow{9}{*}{ DINK } & $S \times A$ & IWAG $\_7$ \\
\hline & CALL & NESSAGIIGAG, ISICK,IWAG,ATINE,KZON) \\
\hline & TIX & $*+1 ; 2,1$ \\
\hline & $\operatorname{TXI}$ & $*+1,7,1$ \\
\hline & $T \times H$ & SLINKr $7_{\mathrm{g}} *$ * \\
\hline & TIX & FINK $+1,5,1$ \\
\hline & $A X T$ & 3.5 \\
\hline & TIX & $*+1,1,1$ \\
\hline & TRA & FINK \\
\hline \multirow[t]{10}{*}{ SLINK } & $\operatorname{TIX}$ & $*+1,1 ; 1$ \\
\hline & TIX & GINK,6, 1 \\
\hline & $S \times A$ & $I B \Delta G: 1$ \\
\hline & $S \times A$ & IHAS, 2 \\
\hline & SXA & $\operatorname{INA3,3}$ \\
\hline & $S \times A$ & $I L A G, 4$ \\
\hline & $S \times A$ & INAG, 5 \\
\hline & $S \times A$ & $I S A G \times 6$ \\
\hline & $S \times A$ & ITAG, 7 \\
\hline & RETURN & UNPACK \\
\hline ADUD & BSS & 1 \\
\hline I DUD & BSS & 1 \\
\hline IFLUB & BSS & 1 \\
\hline I GAG & BSS & 1 \\
\hline ISICK & BSS & 1 \\
\hline IWAG & BSS & 1 \\
\hline ATIME & BSS & 1 \\
\hline I BAG & BSS & 1 \\
\hline IHAG & BSS & 1 \\
\hline I JAG & BSS & 1 \\
\hline ILAG & BSS & $\mathbf{1}$ \\
\hline I NAG & BSS & 1 \\
\hline I SAG & BSS & $\mathbf{1}$ \\
\hline I TAG & BSS & 1 \\
\hline KZOW & BSS & 1 \\
\hline \multirow{2}{*}{ NEEDED } & BSS & 1 \\
\hline & END & \\
\hline
\end{tabular}

\$IBMAP OTTOB 22,LIST,M94,XR7

* Subroutine Convrt ***a subroutine that converts bco time data to

* integer time data。 


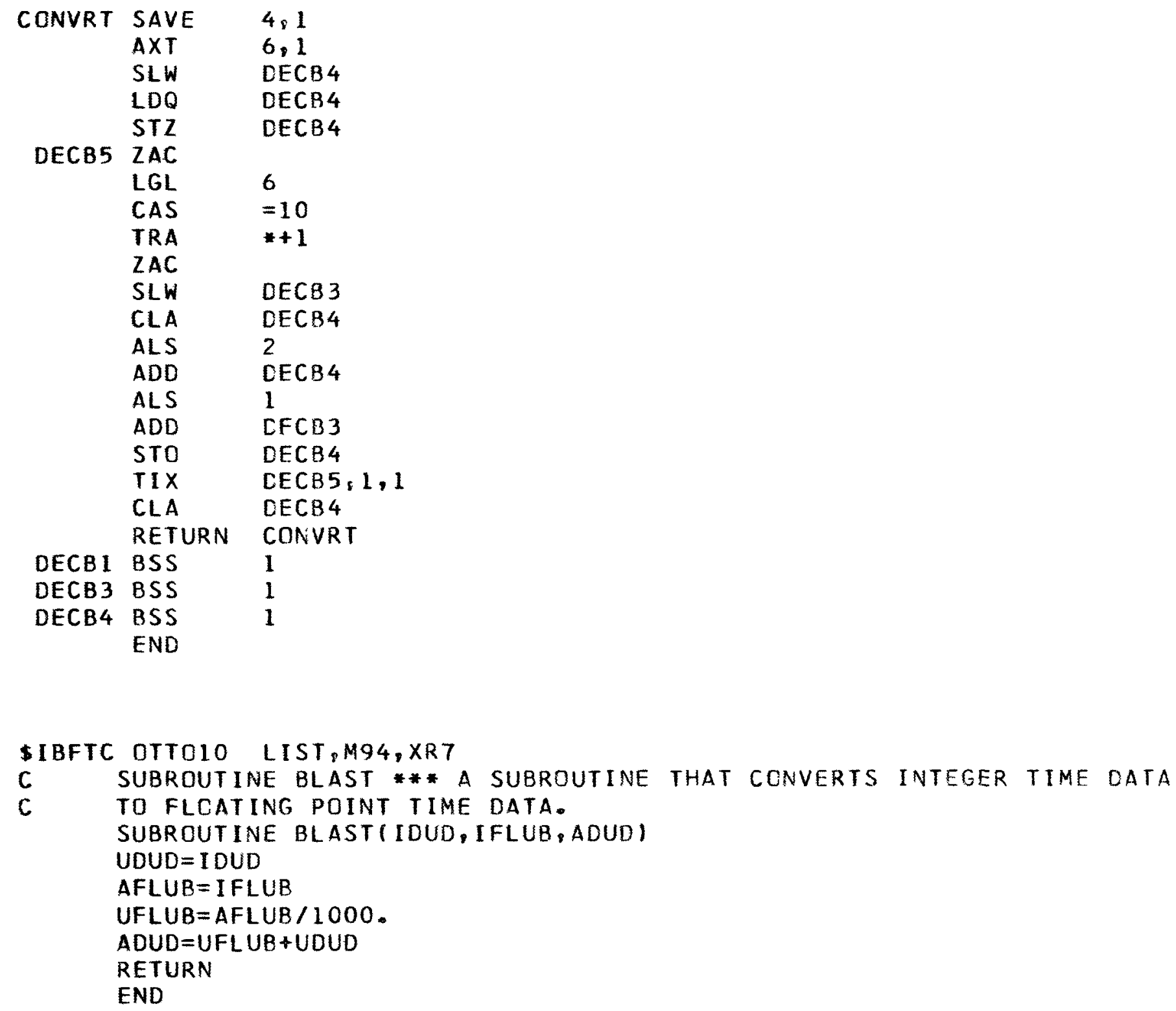


C SUBROUTINE MESSAGE *** A SUBROUTINE TO COUNT THE FREQUENCY OF

C VARIOUS FLAG BITS

SUBROUTINE MESSAGE (IGAG, ISICK I IWAG ,ATIME „KZOW)

COMMON MOUSE ( 3 :

IF(KZOWONE。O) GO TO 25

DO $2 M=1 \& 3$

2 MOUSE $(M)=0$

25 MOUSE (ISICK $)=$ MOUSE (ISICK $)+1$

RETURN

END

\$IBFTC OTTO6 LISTEM94:XRT

C SUBROUTENE MATRIX * a SUBRDUTINE THAT STORES THE TIME cate IN A

C VECTOR ANC THE MULTIPLEXER DATA IN A MATRIX.

SUBROUTINE MATRIXIICHOMP, IB,JAZZ,AC,TIME,IJKL)

DIMENSION IB(540), JAZZ $(40,54), A C(20)$, TIME $(40)$

GO TO $(6,7,8,9), \mathrm{JKL}$

6 INK $=1$

$M I N K=10$

I $M=0$

I $N=0$

GO TO 5

7 INK $=11$

MINK $=20$

IM=0

IN $=0$

GO TO 5

8 INK $=21$

$M I N K=30$

I $M=0$

$I N=0$

GO TO 5

9 INK $=31$

MINK $=40$

$1 M=0$

I $N=0$

5 CONTINUE

DO 1 IK $=$ INK, MINK

$I M=I M+I$ 
TIME (IK $)=A C(I M)$

DO 1 IL $=1$, ICHOMP

IN $=1 N+1$

I JAZZ IIK, IL) $=$ IB (IN)

RETURN

END

$\$ I B F T C$ NSO7 M94,XR7,LIST

SUBROUTINE TEDIT TTIME,TLAST,FLAG44,FLAG99,TBIG,TDEL, I3, N3, I4,N4, (I. N)

C TEDIT DETERMINES WHEN A MAJOR BREAK IN TIME HAS OCCURRED, PRINTS

C THE ACCUMULATED OUTPUT, INITIALIZES FLAGS AND COUNTERS, AND

C CONTINUES THE DATA PROCESSING.

C NICK COUNTS THE NUMBER OF SINGLE IIME POINTS THAT ARE MISSING. COMMON MOUSE 3 :

COMMON/NAME/LITE2,ITFOG ,MARK \& LATERN,KZOW,LITEUP,NICK,FLAG99,NUMI, INUM2,HEADER (12), MULTPX,NAB $(10,50)$, JTOT (50),KTOT(10), NCHS,NOB $(20,49$ 2),KTOTAL (20),MTOTAL 449 ), NUM3, TFIRST, IFINAL,KNT, LINE DIMENSION TIME (40) INTEGER FLAG44.FLAG99

C FLAG99=1 FOR FIRST SET OF POINTS.

C FLAG99=0 FOR ALL OTHER SETS

C FLAG44=2 WHEN A BREAK IN TIME OCCURS, OTHERWISE FLAG44=1. FLAG44=1

IF(FLAG99.NE.0) GO TO 77

TEST=ABS ( TIME (I)-TLAST)

IF(TEST.GT.TBIG) GO TO 36

IF(TEST.GT.TDEL) NICK $=N I C K+1$

77 CONTINUE

TO 50

CALL VAMEAN $(0,0.0,0.0,0.0, L 1 N E, 777)$ 
CALL REPORT

CALL INITAL

GO TO 35

40

FLAG $44=2$

I $3=$ I

$N 3=K-1$

I $4=\mathrm{K}$

50

$N 4=N-N 3$

END

\section{\$IBFTC RWVI LIST M94:XRT}

SUBROUTINE STAT I (JAZZ, I I J N, MSUM \&MSS BARM, CSS, STERRY)

C THIS SUBRCUTINE COMPUTES THE ARITHMETIC MEAN, BARM: AND THE

C VARIANCES STERRY, OF N DATA POINTS IN COLUMN J GF MATRIX JAZZ,

C STARTING AT ROW POSITION I

DIMENSICN JAZZ(40,54)

MSUM $=0$

$M S S=0$

NLAST $=I+N \cdot I$

DO $17 \mathrm{~K}=\mathrm{I}_{\mathrm{S}} \mathrm{NLAST}$

$M S U M=M S U M+J A Z Z(K F J)$

$17 \quad M S S=M S S+J A Z Z\left(K_{r} J\right) * 2$

$F N=N$

FMSUM $=$ MSUM

$B A R M=F M S U M / F N$

CSS $=F L O A T(M S S)-B A R M * F M S U M$

STERRY $=$ (CSS/(FN-10))

RETURN

END

$\$ I B F T C$ NSO2 LIST,M94,XR7

SUBRDUTINE OUTLY(JAZZ, I, $\left.N_{8} J_{8} K 4_{\&} K K_{,} F L A G_{8} J O U T, L T I M E\right)$

C THIS SUBROUTINE FINDS DUTLIERS IN THE DATA AND REPLACES THEM WITH AVERAGES OF THE SURROUNDING TWO DATA POINTS.

C FLAG $=1$ INDICATES AN OUTLIER

DIMENSION JAZZ (40,54) 


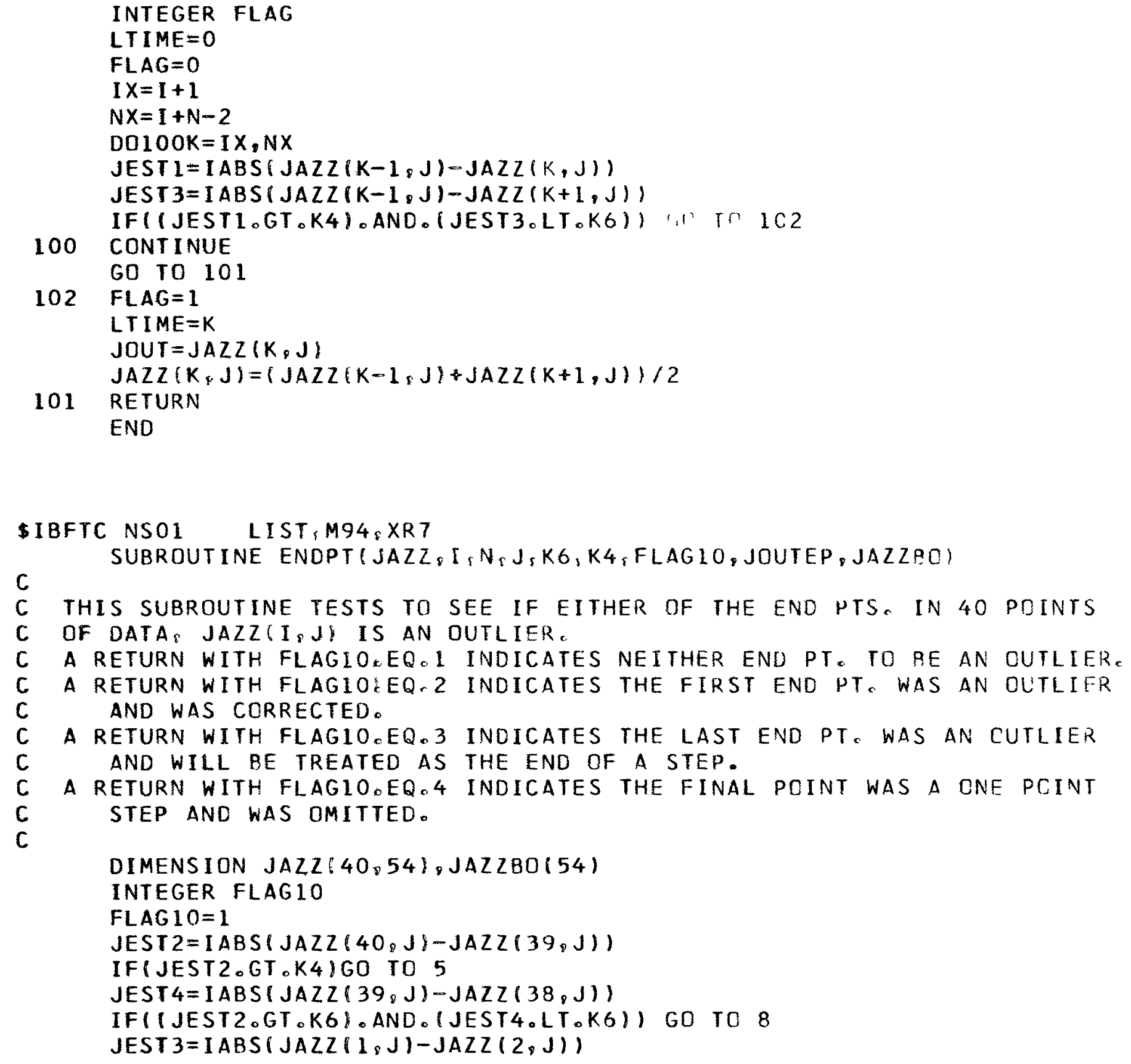


IFIJEST3。Gr。K4/GO TO 6

GO TO 10

FLAG $10=3$

JOUTEP $=J A Z Z(40, J)$

GO TO 10

6

FLAG $10=2$

JOUTEP $=J A Z Z(1, J)$

$J A Z Z(1, J)=(J A Z Z B O(J)+J A Z Z(2, J)) / 2$

GO TO 10

$8 \mathrm{I}=1$

$N=39$

FLAG $10=4$

10

RETURN

END

\$IBFTC NSOG M94,XR7.LIST

SUBRDUTINE STEP (JAZZ, I \& $\left.N_{\&} \mathcal{J}_{\uparrow} K K_{\approx} J F L A G, K T I M E\right)$

C THIS SUBRDUTINE TESTS TO FIND A STEP OR RAMP IN THE DATA.

C THE VALUE OF JFLAG TELLS THE NUMBER OF POINTS IN THE RAMP. DIMENSIDN JAZZ 40,54$)$

KTIME $=0$

$J F L A G=0$

$N X=I+N-2$

DO $100 \quad K=I, N X$

$J 1=J A Z Z\left(K K^{8} J\right)-J A Z Z(K+1, J)$

IFIIABS(JI).LT:K6)GO TO 100

IF.(K.EQ.39)GO TO 102

$\mathrm{J} 5=I A B S\left(J A Z Z\left(K_{i} J\right)-J A Z Z(K+2, J)\right)$

IF( (IABS $(J 1) \circ G T \circ K 6)$.AND。(J5.LT.K6))GO TC 100

$J 2=J A Z Z(K+1, J)-J A Z Z(K+2, J)$

$J 3=J A Z Z(K+2 \& J)-J A Z Z(K+3, J)$

$J 4=J A Z Z(K+3, J)-J A Z Z(K+4, J)$

GO TO 105

100 CONTINUE

GO TO 101

105 IF(IABS $(J 2) \cdot L T \circ K 6) G 0$ TO 102

$I F(K \circ E Q .38) G 0$ TO 103

IFIIABS $\left.(J 3) \cdot L T_{0} K 6\right) G 0$ TO 103

IF (K.EQ.37)GO TO 104 
IF(IABS $(J 4) . L T . K 6) G 0$ TO 104

JFLAG $=4$

KTIME $=K+3$

$J A Z Z(K+1, J)=J A Z Z(K, J)$

$J A Z Z(K+2, J)=J A Z Z\left(K_{0} J\right)$

$J A Z Z(K+3, J)=J A Z Z(K+4, J)$

JFLAG 101

KTIME $=K+1$

GO TO 101

103 JFLAG $=2$

KTIME $=K+1$

$J A Z Z(K+1, J)=J A Z Z(K+2, J)$

GO TO 101

$104 \mathrm{JFLAG}=3$

$K T I M E=K+2$

$J A Z Z(K+1 ; J)=J A Z Z\left\{K_{8} J\right\}$

$J A Z Z(K+2, J)=J A Z Z(K+3, J)$

101 RETURN

END

\section{\$IBFIC RWV2 LISTIM94:XRT}

SUBROUTINE FET I JAZL,J,A,B,STERRY \& Y FLAG 17 \& CONST)

C

C THIS SUBROUTINE FITS A LINEAR TREND TO 40 POINTS CF DATA

C A RETURN WITH FLAGI7,EQ*0 INDICATES S* 2,LT。CONST - A A RETURN WITH

C FLAG17。EQ.777 INDICATES $S * 2 . G T$, CONST

\section{$\mathrm{C}$}

DIMENSICN JAZZ(40,54),PHI 440$\}, Y(39)$

INTEGER FLAG 17 \& PHI

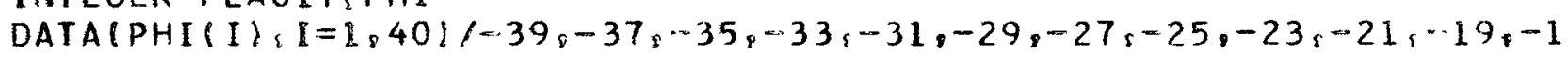

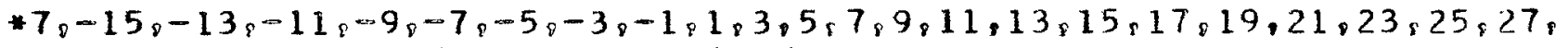

*29,31.33,35.37,39/, SSPHI/21320.0/

$M A=0$

$M B=0$

MSS $=0$

DO $19 K=1,40$

$M A=M A+J A Z Z\left(K_{\&} J\right)$

$M B=M B+J A Z Z(K, J) * P H I(K)$ 
$19 M S S=M S S+J A Z Z\left(K_{r} J\right) * * 2$

$A=F L O A T(M A) / 40.0$

$B=F L O A T(M B) / S S P H I$

CORR $=A * 2 * 40 x+B * 2 * S S P H I$

CSS $=$ FLOAT (MSS) - CORR

STERRY $=$ CSS $/ 38$ 。

IF(STERRY.LT.CONST) GO:;97

$c$

THIS PORTICN OF THE PRCGRM .COMPUTES RESIOUALS TC EETRENC DATA FCA

C

THE FOURIER ANALYSIS TC DETERMINE PERITDICITIES

LO $44 K=1,39$

$44 Y(K)=F L C A T: J A Z Z: K_{\&} J A-A-B \# F L C A T: P H I I * ;$

FLAGIT $=777$

GO TO 98

97 FLAG $17=\mathrm{C}$

98 RETURN

ENL

DIBFTC RWV3 LISTPMO4,XR7

SUBROUTINE FIT 2 (JAZZ, J, I N N, A,R, STERRY)

C THIS SUBRCLTINE FITS a LEAST SCUARES LINEAR LINE TC N POINTS OF CATA

C WHEN 3.GE.N.LT.40

DIMENS ICN JAZZ $(40,54)$, MU (48)

$M A=0$

$M B=0$

$M S S=0$

MUSS $=0$

DO $4 K=4,40,2$

4 IF(K.EQ.N) GO TO 30

$N I X=-(N-1) / 2$

D017 $L=1, N$

MU (L) $=N I X$

MUSS $=$ MUSS $+N I X * 2$

$17 N I X=N I X+1$

GO TO 600

30 NIX $=-N+1$

D018 $L=1, N$

$M U(L)=N I X$

MUSS $=$ MUSS + N I $X * * 2$ 
18 NIX $X=N I X+2$

600 INDEX $=1$

DO $19 K=1, N$

$M A=M A+J A Z Z(I N D E X, J)$

$M B=M B+J A Z Z(I N D E X, J) * M U(K)$

$M S S=M S S+J A Z Z($ INDEX, J)**2

19 INDEX $=$ INDEX +1

$F N=N$

$A=F L O A T(M A) / F N$

$B=F L O A T$ (MB) /FLOAT (MUSS)

CORR $=A * 2 * F N+B * 2 * F L O A T$ (MUSS)

CSS $=F L O A T(M S S)-C O R R$

STERRY = $(C S S /(F N-20)$

RETURN

END

\$IBFTC NSO5 M94,XR7,LIST

SUBROUTINE FOOREA ( $Y_{\&} T A U_{\&} F_{\&} A C_{\&} B C_{F} C C_{Y}$ NOF, STERRY)

$C$ THIS SUBRDUTINE FINDS THE FREQUENCIES PRESENT IN THE 39 DFTRENDED DATA PDINTS

DIMENSICN S(9),C(9), SI (20), Y (39),U(20), V(20), US(10),UD(10),VS(9): IVD $(9)$, A(20), B(20), NA(20), LA(20), NB(20), LB(20), FA(20), AA(20), FB(20) $2, B B(20), A C 620) \& B C(20)$ \&CC(20)\& $F(20) \& B A(20) \& A B(20)$

DATAIS(I),C(I) I $=1$ \& 9$) / 0.16459459,0,98636129,0.32469947,0.945817248$ $10.47594739,0.87947375,0,61421271,0.78914050,0,73572391,0,67728157$, $20 \times 83716648,0,54694815,0.91577332,0,40169542,0 \times 96940026 \& 0.24548548$ \& $30.99658449 .0 .82579343 E \cdot 01 /$

DATAESIfI /, I =2,20!/0,99546646,0.98187297,0,95949214,0.92867239,0.8 $18991513,0.84384815,0.79121346,0.732853,0.66969234,0.60272311,0.532$ $2984,0.46154118,0.38946837 \& 0.31782681,0.24764597,0,17990476,0.11551$ $3445: 0.55302744 \mathrm{E}-01.0 .0 \%$

DATA FP,FAA/0.26315789E-01,0.89278947E-01/

ZIP $=S T E R R Y / 10.0$

NOF $=0$

$00300 \mathrm{I}=2.20$

$K=19+I$

$J=21-I$

$U(I)=(Y(K)+Y(J)) * S I \& I)$ 
$U(1)=Y(20)$

$U(20)=0.5 * U(20)$

$V(1)=0.0$

$A(1)=0.0$

$A(20)=0.0$

$J=2$

$K=19$

D0400I $=2$ : 10

US $(I)=U(J)+U(K)$

$U D(I)=U(J)-U(K)$

$A(1)=U S(I)+A(1)$

$A(20)=U D(I)+A(20)$

$L=I-1$

$V S(L)=V(J)+V(K)$

$V D(L)=V(J)-V\{K\}$

$J=J+1$

$400 \quad K=K-1$

US $(1)=U(1)+U(20)$

UD $(1)=U(1)-U(20)$

$A(1)=U S(1)+A(I)$

$A(20)=U D(1)+A(20)$

$A(2)=U D(1)+C(1) * U D(2)+C(2) * U D(3)+C(3) * U D(4)+C(4) * U D(5)+C(5) * U C(6)+$

$1 C(6) * U D\{7)+C(7) * U D(8)+C(8) * U D(9)+C(9) * U D(10)$

$A(3)=U S(1)=C(I) * U S(10) * C(2) * U S(2)-C(3) * U S(9)+C(4) * U S(3)-C(5) * U S(8)$

$1 * C(6) * U S(4) \cdots C(7) * U S(7)+C(8) * U S(5)-C(9) * U S(6)$

$A(4)=U D(1)-C(1) * U D(7)-C(2) * U D(8)+C(3) * U D(2)-C(4) * U D(6)-C(5) * U D(9) *$

$1 C(6) * U D(3)-C(7) * U D(5)-C(8) * U D(10)+C(9) * U D(4)$

$A(5)=U S(1)-C(1) * U S: S)+C(2) * U S(10)-C(3) * U S(5) * C(4) * U S(2)-C(5) * U S(7)$

$1+C(6) * U S(9)-C(7) * U S(4)+C(8) * U S(3) * C(9) * U S(8)$

$A(6)=U D(1)-C(1) * U D(5)+C(2) * U D(9)+C(3) * U D(8)-C(4) * U D(4) * C(5) * U D(2)=$

$1 C(6) * U D(6) * C(7) * U D(10) * C(8) * U D(7) \cdot C(9) * U D(3)$

$A(7)=U S(1)-C(1) * U S(4) * C(2) * U S(7)-C(3) *$ US $(10) * C(4) * U S(8) \cdots C(5) * U S(5)$

$1+C(6) * U S(2)-C(7) * U S(3)+C(8) * U S(6)-C(9) * U S(9)$

$A(8)=U D(1)-C(1) * U D(9)-C(2) * U D(4)+C(3) * U D(6)+C(4) * U D(7)-C(5) * U D(3)-$

$1 C(6) * U D(10)+C(7) * U D(2)-C(8) * U D(B)-C(9) * U D(5)$

$A(9)=U S(1) \cdots C(1) * U S(8)+C(2) * U S(6) \cdots C(3) * U S(3)+C(4) * U S(10)-C(5) * U S(4)$

$I+C(6) * U S(5)-C(7) * U S(9) * C(8) * U S(2)-C(9) * U S(7)$

$A(10)=U D(1)-C(1) * U D(3)+C(2) * U D(5)-C(3) * U D(7)+C(4) * U D(9)+C(5) * U D(10$

$1)-C(6) * U D(8)+C(7) * U D(6)-C(8) * U D\{4\}+C(9) * U D\{2\}$

$A(11)=U S(1)-C(1) * U S(3)+C(2) * U S(5)-C(3) * U S(7) * C(4) * U S(9)-C(5) * U S(10$

$1)+C(6) * U S(8)-C(7) * U S(6)+C(8) * U S(4)-C(9) * U S(2)$ 
$A(12)=U D(1)+C(1) * U D(8)-C(2) * U D(6)-C(3) * U D(3) * C(4) * U D(10)+C(5) * U D(4$ $1)+C(6) * U D(5)-C(7) * U D(9)-C(8) * U D(2)-C(9) * U D(7)$

$A(13)=U S(1)-C(1) * U S(9)+C(2) * U S(4)-C(3) * U S(6)+C(4) * U S(7)-C(5) * U S(3)$

$1+C(6) * U S(10)-C(7) * U S(2)+C(8) *$ US $(8)-C(9) *$ US $(5)$

$A(14)=U D(1)+C(1) * U D(4)+C(2) * U D(7)+C(3) * U D(10)-C(4) * U D(8)-C(5) * U D(5$ $1)-C(6) * U D(2)-C(7) * U D(3)-C(8) * U D(6)-C(9) * U D(9)$

$A(15)=U S(1)-C(1) * U S(5)+C(2) * U S(9)-C(3) * U S(8)+C(4) * U S(4)-C(5) * U S(2)$ $1+C(6) * U S(6)-C(7) * U S(10)+C(8) * U S(7)-C(9) * U S(3)$

$A(16)=U D(1) * C(1) * U D(6)-C(2) * U D(10) * C(3) * U D(5) * C(4) * U D(2) \cdots C(5) * U D(7$ 1) $\{C(6) * U D(9)+C(7) * U D(4)+C(8) * U D(3)+C(9) * U D(8)$

A $(17)=U S(1)-C(1) * U S(7)+C(2) * U S(8)-C(3) * U S(2)+C(4) * U S(6)-C(5) * U S(9)$ $1+C(6) * U S(3)-C(7) * U S(5)+C(8) * U S(10)-C(9) * U S(4)$

$A(18)=U D(1) * C(1) * U D(10)-C(2) * U D(2)-C(3) * U D(9)+C(4) * U D(3)\{C(5) * 1)(8)$ $1)=C(6) * U D(4)-C(7) * U D(7)+C(8) * U D(5) * C(9) * U D(6)$

$A(19)=\operatorname{US}(1)-C(1) * U S(2)+C(2) * U S(3) \cdot C(3) * U S(4)+C(4) * U S(5)-C(5) * U S(6)$ $1+C(6) * U S(7)-C(7) * U S(8)+C(8) * U S(9) \cdot C(9) * U S(10)$

$B(1)=0.0$

$B(2)=S(1) * V S(1) * S(2) * V S(2)+5(3) * V S(3)+5(4) * V 5(4)+S(5) * V S(5)+S(6) *$ IVS $(6)+S(7) * V S(7)+S(8) * V S(8)+S(9) * V S(9)$

$B(3)=S(1) * V D(9)+S(2) * V D(1)+S(3) * V D(3)+S(4) * V D(2)+S(5) * V D(7)+S(6) *$ IVD $(3)+S(7) * V D(6)+5(8) * V D(4)+S(9) * V D(5)$

$B(4)=S(1) * V S(6) \cdot S(2) * V S(7)+S(3) * V S(1)+5(4) * V S(5) \cdot S(5) * V S(8)+5(6) *$ IVS $(2)+S(7) * V S(4) * S(8) * V S(9) * S(9) * V S(3)$

$B(5)=-S(1) * V D(5)-S(2) * V D(9)+S(3) * V D(4)+S(4) * V D(1)-S(5) * V D(6) * S(6) *$ IVD $(8)+S(7) * V D(3) * S(8) * V D(2)-S(9) * V D(7)$

$B(6)=-S(I) * V S(4) * S(2) * V S(8)-S(3) * V S(7)+S(4) * V S(3)+S(5) * V S(1)-S(6) *$ IVS $(5)+S(7) * V S(9)-S(8) * V S(6)+S(9) * V S(2)$

$B(7)=S(1) * V D(3)-S(2) * V D(6)+S(3) * V D(9)+S(4) * V D(7) \cdot S(5) * V D(4)+S(6) *$ IVD $(1)+S(7) * V D(2)-S(8) * V D(5)+S(9) * V D(B)$

$B(8)=S(1) * V S(B)-S(2) * V S(3)-S(3) * V S(5) * S(4) * V S(6) * S(5) * V S(2)-S(6) *$ IVS $(9)+S(7) * V S(1)+S(8) * V S(7)-S(9) * V S(4)$

$B(9)=S(1) * V D(7)+S(2) * V D(5)+S(3) * V D(2)-S(4) * V D(9)-S(5) * V D(3)-S(6) *$ IVD $(4)-S(7) * V D(8)+S(B) * V D(1)+S(9) * V D(6)$

$B(10)=S(1) * V S(2)-S(2) * V S(4) * S(3) * V S(6)-S(4) * V S(8) * S(5) * V S(9)-S(6) *$ IVS $(7)+S(7) * V S(5)-S(8) * V S(3)+S(9) * V S(1)$

$B(1)=-S(1) * V D(2) * S(2) * V D(4)=S(3) * V D(6)+S(4) * V D(8)+S(5) * V D(7)-S(6)$ $1 * V D(7)+S(7) * V D(5)-S(8) * V D(3)+S(9) * V D(1)$

$B(12)=S(1) * V S(7)+S(2) * V S(5)-S(3) * V S(2)-S(4) * V S(9)-S(5) * V S(3)+S(6) *$ IVS $(4)+S(7) * V S(8)+S(8) * V S(1)-S(9) * V S(6)$

$B(13)=-S(1) * V D(8)-S(2) * V D(3)-S(3) * V D(5)-S(4) * V D(6)-S(5) * V D(2)-S(6)$ $1 * \operatorname{VD}(9)+S(7) * \operatorname{VD}(1)+S(8) * \operatorname{VD}(7)+S(9) * \operatorname{VD}(4)$ 
$B(14)=S(1) * V S(3) * S(2) * V S(6)+S(3) * V S(9)+S(4) * V S(7)+S(5) * V S(4)+S(6) *$ IVS $(1)-S(7) * V S(2)=S(8) * V S(5)-S(9) * V S(8)$

$B(15)=S(1) * V D(4)-S(2) * V D(8)-S(3) * V D(7)+S(4) * V D(3)+S(5) * V D(1)-S(6) * \quad 15$

IVD $(5)+S(7) * V D(9)+S(8) * V D(6)-S(9) * V D(2)$

$B(16)=-S(1) * V S(5) \cdot S(2) * V S(9)-S(3) * V S(4)+S(4) * V S(1) * S(5) * V S(6) * S(6)$
$I * V S(8) * S(7) * V S(3)-S(8) * V S(2)-S(9) * V S(7)$

$B(17\}=-S(1) * V D(6)-S(2) * V D(7) * S(3) * V D(1)+S(4) * V D(5)+S(5) * V D(3) \cdot S(6) \quad 17$

$1 * V D(2)=S(7) * V D(4)=S(8) * V D(9) * S(9) * V D(3)$

$B(18)=S(1) * V S(9) * S(2) * V S(1)=S(3) * V S(8) * S(4) * V S(2) * S(5) * V S(7) * S(6) * \quad 18$

IVS $(3)-S(7) * V S(6)-S(8) * V S\{4)+S(9) * V S(5)$

$B(19)=S(1) * V D(1)-S(2) * V D(2)+S(3) * V D(3) \cdot S(4) * V D(4)+S(5) * V D(5)-S(6) * \quad 19$

IVD $(6)+S(7) * V D(7)-S(8) * V D(8)+S(9) * V D(9)$

$26 \quad K A=1$

$B(20\}=0.0$

$J N A=0$

$K A A=0$

$K B=1$

$J N B=0$

$K B B=0$

DD200I $=1.19$

IF $(A\{I) * A(I+I\}) 27,27,28$

$27 \quad K A=1$

$\mathrm{JNA}=0$

GOTO 31

28 IFI JNA $30,29,30$

$29 \quad K A A=K A A+1$

$J N A=1$

$30 \quad K A=K A+1$

$N A(K A A)=K A$

$L A(K A A)=I+1$

$31 \quad$ IF $(B(I) * B(I+I)) 32,32,33$

$32 \mathrm{~KB}=1$

$\mathrm{JNB}=0$

GOTO 200

33 IF IJNB I $35,34,35$

$34 \quad K B B=K B B+1$

$J N B=1$

$35 \quad K B=K B+1$

$N B(K B B)=K B$

$L B(K B B)=I+I$ 
$\angle L B=0$

D0700I $=18 K A A$

$K T=N A(I)$

$K T M=K T-1$

$D 0700 \mathrm{~J}=1, \mathrm{KTM}$

$I I=L A(I)-K T+J$

$I P=I I+1$

$I M=I I-1$

IFIIM I T0,39,70

39 TESTB=ABS $(A(I E)-A(I P))-1.0 E-6$

IF (TESTB $700,700,75$

75 TESTC $=A B S(A(11))=A B S(A(I P))$

IFITESTC $>74,700,700$

$74 \quad$ IF $(K T-2) 72,72,40$

$70 \quad$ IFIKT-2)7I,71,40

40 TEST $1=A B S(A)$ II $)-A B S(A(I M))$

TEST2 $=A B S(A(I I)) \cdot A B S(A(I P))$

IFIITEST L.GT:0,0).AND. (TEST2,GT,0,0)!GO TO 43 GO TO 700

43

$E P=0.5 *(A(I P)-A(I M)\} /(2.0 * A(I I)-A(I P)-A(I M))$

$E P 04=0.25 * E P$

$A U=A(I I)+E P 04 \#(A(I P)-A(I M))$

$B U=B([I)+E P O 4 *(B)(P)-B(I M))$

$P=F L O A T(I M)+E P$

$42 \quad L L A=L L A+1$

$F A(L L A)=P * F / T A U$

$A A(L L A)=F A A * A U$

$B A(L L A)=F A A * B U$

700 CONTINUE

$\mathrm{DO} 800 \mathrm{I}=1, \mathrm{KBB}$

$K T=N B(I)$

$K T M=K T-1$

$D 0800 \mathrm{~J}=1, \mathrm{KTM}$ 
$I I=L B(I)-K T+J$

$I P=I I+1$

$I M=I I-1$

IFI IMI 80 \& 48,80

48 TESTB=ABS (B(II)-B(IP) $\{-1.0 E-6$

IF TESTB $) 800_{8} 800_{1} 85$

85 TESTC $=A B S(B(I I))-A B S(B(I P))$

IFITESTC $\$ 84 \times 800,800$

$84 \quad$ IF (KT-2) $82,82,50$

80 IFIKT-2) $81,81,50$

81 IF (ABS(B)(IP) )-ABS(BIII) $) 51,51,82$

$82 \quad I I=I P$

$I M=I-I$

$I P=I I+I$

GO TO 51

50 TEST $1=A B S(B(I I))=A B S(B(I M))$

TEST2 $=A B S(B(I I))-A B S(B(I P))$

IF( (TEST1,GT.0.0), AND。(TEST2.GT.0.0))GO TO 51

$51 E P=0.5 *(B($ IP $)-B($ IM) $) /(2.0 * B(I I) \cdot B($ IP $) \cdot B(I M))$

$E P O 4=0.25 * E P$

$B U=B(I I) * E P O 4 *(B(I P) * B(I M)$

$A U=A(I I)+E P O 4 *(A C I P) * A(I M))$

$P=F L O A T(I M)+E P$

$52 \quad \angle L B=L L B+1$

$F B(L L B)=P * F P / T A U$

$B B(L L B)=F A A * B U$

$A B(L L B)=F A A * A U$

800 CONTINUE

\section{$\mathrm{LF}=0$}

D0900I = I, LLA

$D 0900 J=1, L L B$

59

IF(ABS (FA(I)-FB(J))-0.8) 59,59,900

\section{$L F=L F+1$}

$F(L F)=0.5 *(F A(I)+F B(J))$

$A C(L F)=0.5 *(A A \mid I)+A B(J)$

$B C(L F)=0.5 *(B A(I)+B B(J))$

$C C(L F)=A C\{L F) * 2 * B C(L F) * * 2$

IF(CC(LF) OLT OZIP) GO TO 900

$N O F=N O F+1$

$F(N O F)=F(L F)$ 
$A C(N D F)=A C(L F)$

$B C(N O F)=B C(L F)$

CC $($ NOF $)=C C\{L F)$

$F A(I)=-1.0$

$F B(J)=-1.0$

900 CONTINUE

DOBBOI $=1, L L A$

IF(FAII)/8BO,90,90

90

\section{$L F=L F+1$}

$C(L F)=A A(I) * 2+B A(I) * * 2$

IF(CCILF).LT。ZIP) G0 TO 880

$N O F=N D F+1$

$F(N O F)=F A(I)$

$A C(N O F)=A A\{I\}$

$B C(N O F)=B A \&$ I $)$

$C C(N O F)=C C\{L F\}$

880 CONTINUE

DOB $80 I=1, \angle L B$

IFIFBIII) $870,95,95$

$95 \quad L F=L F+1$

$C C(\& F)=A B(I) * 2+B B(I) * 2$

IF (CC (LF) \& T \& $Z$ IP) GO TO 870

$N O F=N D F+1$

$F(N D F)=F B(I)$

$A C(N O F)=A B(I)$

$B C(N O F)=B B(I)$

$C C\{N O F\}=C C\{L F\}$

870 CDNTINUE

1000 RETURN

END

\section{$\$ I B F T C$ NSIO M94,XR7,LIST}

SUBROUTINE VAMEANR \& STERRY \&BARM,TIMEX,LINE,NIP!

$C$ THIS SUBROUTINE COMPILES AND PRINTS TABLES OF MEANS AND VARIANCES

C FOR EACH CHANNEL AND TIME。

COMMON/NAME/LITE2,ITFOG \& MARK \& LATERN, KZOW,LITEUP \& NICK,FLAG99,NUMLs

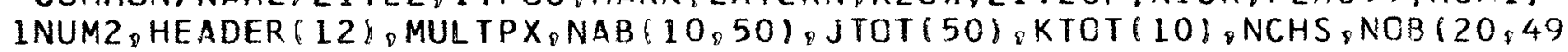

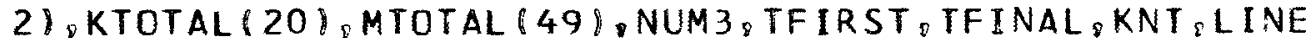

DIMENSICN KARY 52.49$)$ 
DIMENSIDN MEAN $(52 \div 49)$

DIMENSION TIMER $(52)$

$\mathrm{NXCH}=\mathrm{NCHS}$

IF (NCHS $, G E 。 49: N X C H=N C H S-1$

IF $(N I P, N E 。 O)$ GO TO 7

$I F\left(J_{0} E Q=I\right\}$ GO TO 5

GO TO 6

$L I N E=L I N E+1$

TIMER $\{L$ INE $\}=$ T IMEX

MEAN $(L I N E \& J)=B A R M$

KARY $\left\{\left[I N F_{\{} J\right\}=S T E R R Y\right.$

IF ( ILINE。EQ, 52) AND。 (J.GE。NXCH) GO TO 7

GO TO 9

7 WRITE $(6,30)(H E A D E R(K), K=I, 12)$, MULTPX

30 FORMAT $\left(1 H 1,4 X_{\varepsilon} 12 A 6 / 5 X_{5}\right.$ IA6, $37 X_{8} 45 \mathrm{HCOMPILATION}$ OF SUCCESSIVE ARITHME ITIC AVERAGES;

WRITE $(6,50)$

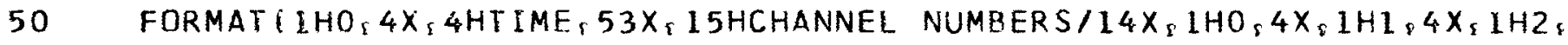
$A 4 X_{\{} 1 H 3,4$

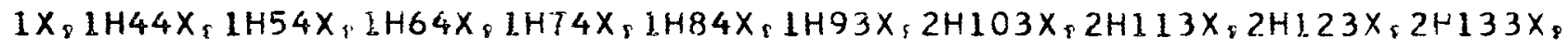
$22 H 143 X_{\&} 2 H 153 X_{\&} 2 H 163 X_{s} 2 H 173 X_{\&} 2 H 183 X_{\&} 2 H 193 X_{\&} 2 H 203 X_{\&} 2 H 213 X_{1} 2 H 223 X_{\&} 2 H 2$ $33 / / 1$

$N A K=24$

IF INCHS.LT, 24, NAK=NCHS

DO8 $K=1$ \& LINE

8

WRITE $(6,100)$ TIMER $(K),(M E A N(K, M), M=1, N A K)$

100 FORMAT (IXPF 9.3,24I5)

IFINCHS.LE.24) GO TO 88

WRITE $(6,30)$ ( HEADER $(K) \& K=1,12)$, MUL TPX

WRITE 66,51$\}$

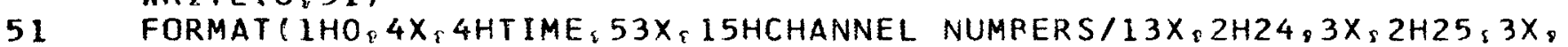
A2H26,3 $\mathrm{X}_{5}$

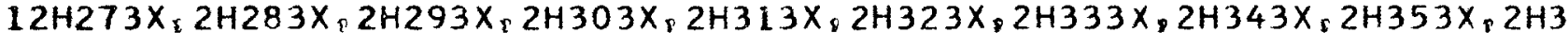
$263 X_{8} 2 H 373 X_{\diamond} 2 H 383 X_{\&} 2 H 393 X_{\diamond} 2 H 403 X_{s} 2 H 413 X_{s} 2 H 423 X_{\&} 2 H 433 X_{\&} 2 H 443 X_{*} 2 H 453 X$ $3,2 \mathrm{H} 463 \mathrm{X}, 2 \mathrm{H} 473 \mathrm{X}$ :

DO $11 K=1$, LINE

11 WRITE $(6,100)$ TIMER $(K),(M E A N(K, M), M=25, N \times C H)$

88 WRITE $(6,40)$ (HEADER $(K), K=1,12), M U L T P X$

40 FORMAT $1 \mathrm{HI}_{0} 4 \mathrm{X}_{2} 12 \mathrm{2A6} / 5 \mathrm{X}_{0} 1 \mathrm{A6}, 36 \mathrm{X}_{8} 46 \mathrm{HCOMPILATION}$ DF SUCCESSIVE CALCULA ITED VARIANCES I

WRITE $(6,50)$

DO $10 \mathrm{~K}=1, \mathrm{LINE}$ 
IF(NCHS $\left.L E_{\circ} 24\right)$ GO TO 89

WRITER 6 \& 40 ) \& HEADER \&K \& $8=1,121$, MULTPX

WRITE 6,51 )

12

DO $12 K=1, L I N E$

89 LINE $=0$

END

\section{\$IBFTC RWV8 XR7,M94,LIST}

SUBROUTINE TIC\&LATERN,FK。NOB, KTCTAL,MTOTAL\&J)

$\mathrm{C}$

THIS SUBROUTINE STORES A TARULATION OF THE FREQUENCY OISTREBUTION

OF ESTIMATED PERIODICITIES IN THE DATA.

DIMENSION NOB $(20,49)$ \& KTOTAL $(20)$, MTOTAL $(49)$

IFILATERN,NE, O) GO TO 25

DO $10 K=1,20$

DO $10 \quad M=1.49$

$10 N O B(K, M)=0$

DO $73 K=1$ \& 20

$73 K T O T A L(K)=0$

DO $75 \quad M=1,49$

75 MTOTAL $(M)=0$

LATERN $=777$

$25 \quad M=F K$

$M=M+1$

IF $\left.M M_{0} G T .20\right) \quad M=20$

NOB $\left(M_{\varepsilon} J\right)=N O B\left(M_{\varepsilon} J\right)+1$

KTOTAL $(M)=$ KTOTAL $(M)+1$

MTOTAL $(J)=$ MTOTAL $(J)+1$

RETURN

END

SIBFTC NSO4 M94:XR7,LIST

SUBROUTINE TALLYSLITEUP,STERRY,NAB,KTOT OJTOT \&J

C THIS SUBROUTINE STORES A TABULATION OF THE FREQUENCY DISTRIBUTION

C DF CALCULATED VARIANCES。 
DIMENSION KTOT ( 10 \% JTOT $(50)$

DIMENSICN NAB $(10,50)$

IFILITEUP \& NE。O\} GO TO 25

DOLOK $=1,10$

DOIOI $=1 ; 50$

10

$N A B(K, I)=0$

DO $73 K=1.10$

$73 \mathrm{KTOT}(K)=0$

DO $75 \quad I=1,50$

75 JTOT I I $=0$

LITEUP $=1$

$25 \quad K=S T E R R Y$

$K=K+1$

$I F(K \circ G T .10) K=10$

IF $(S T E R R Y, L T, 0, O) K=1$

$N A B(K, J)=N A B(K, j)+1$

KTOT $(K)=K$ TOT $(K)+1$

JTOT $(J)=J \operatorname{TOT}(S)+1$

RETURN

END

DIBFTC NSO9 M94,XR7.LIST

SUBRDUTINE REPORT

C THIS SUBROUTINE PRINTS THE ACCUMULATED OUTPUT AT FACH MAJCR TIME

C BREAK AND AT THE END OF EACH MULTIPLEXER. COMMON MOUSE 3 \})

COMMON/NAME /LITE2, ITFOG \&MARK, LATERN, KZOW L I TEUP, NICK, FL AG99, NUMI, INUM2, HEADER (12), MULTPX NAB $110,50 \%$ JTOT $(50)$, KTOT 10$)$, NCHS, NOR $(20,49$ 2) \& KTOTAL 20$\}$ \& MTOTAL $\{49\}$, NUM3, TFIRST, TFINAL, KNT \& LINE

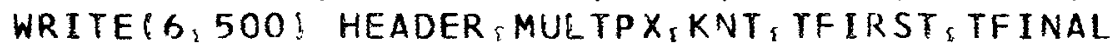

500 FORMAT \& IHI 19X,28HANALOG DIGITAL DATA ANALYSIS/20X,12A6/20X, 18HMUL ITIPLEXER NUMBER $146 / 20 X_{2} 34$ HNUMBER OF DATA POINTS FOR EACH PIN, I20/ $220 X_{0} 1$ IHSTART TIME $=F 11.3 / 20 X_{8}$ 1OHSTOP TIME $=F 12.3$ \%

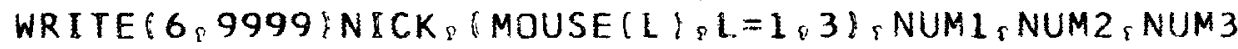

9999 FORMATI 1HO, 19X, 29HNUMBER OF MISSING TIME POINTSE $6 / 120 \mathrm{X}, 26 \mathrm{HNUMBER}$ IOF OI FLAGS ON DATA $166 / 20 X_{8}$ 26HNUMBER OF 10 FLAGS ON DATA, $16 / 120 X_{8}$ 226HNUMBER OF 11 FLAGS ON DATA $16 / / 20 \mathrm{X}_{5} 18$ HNUMBER OF OUTLIERS, $16 / 1$ $320 X_{8} 38$ HNUMBER OF TIMES PROGRAM WENT TO FOOREA, $16 / / 20 X_{1} 15$ HNUMBER OF 4 STEPS 168 
IF LITEUP, EQ,D) GO TO DOO

WRITE $(6,505)$ (HEADER $(K), K=1,6)$, MULTPX, TFERST, TF) NAI

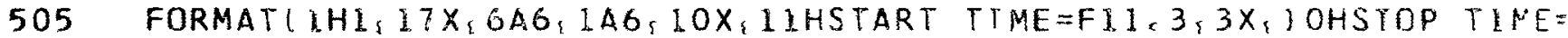

IFII $\times 3$ !

WRITE 6,502$\}$

502 FORMAT(LHO, $48 \times, 46 H F R E Q U F N C Y$ DISTRIBUTION CF CAICULATFD VARHARLES/

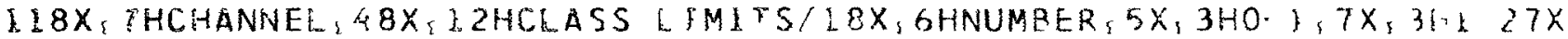

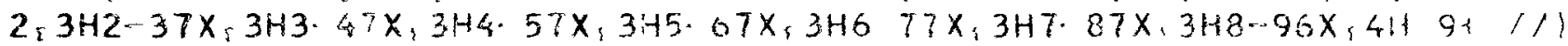
DO3OOL $=1$ \& NCHS

$L M=1 \cdot 1$

300 WRITE (G,8920IIM, (NABIK,L),K=1,IO), ITOTLL)

8920 FORMAT $(20 \%,[2 \leqslant 1.1) 10)$

WRTTE $(6,892:)(K T O T(K), K=1,10)$

892L FORMAT(JHO, )6K, 5HTOTAL, LOLIOI

100 CONTINUF

IF(LATERN,EG,O) GO IO 200

WRITE $(0,505)($ IH ADER (K),K. I, 6), MULTPX, TFIRST, TIINAI

WRTTE $(6,501)$

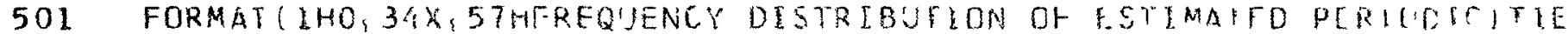

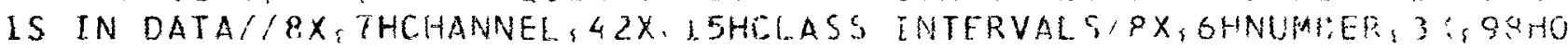
$\begin{array}{lllllllllllllllllllllll}2-1 & 1 & 2 & 2 & 3 & 3-4 & 4 & 5 & 5 & 6 & 6 & 7 & 7 & 3 & 8.9 & 9 & 0 & 0 & 1 & 1-2 & 3 & 3-\end{array}$ $\begin{array}{lllllllll}34 & 4.5 & 5.6 & 6-7 & 7.8 & 8.9 & 9-0 & 1 / 1\end{array}$

DO 6741 KRANK $=1$ NCHS

KRINK =KRANK $\cdot 1$

6741 WRITE 6,6742$)$ KRINK, (NOR (KR,KRANK) \&KR=1, 2C), MTUTAE (KRANK)

6742 FORMAT $\left(10 X_{\{} I 2,2 X_{1} 20\left(1 X_{2} I 4\right), 3 X_{1} 15 !\right.$

WRITE $(6,6743)(K T O T A L(K R), K R=1,20)$

6743 FORMAT (IHO, $6 X_{1}$ 5HTOTAL, $\left.2 X_{5} 20(2 X, 14\}\right)$

200 CONTINUE

RETURN

END

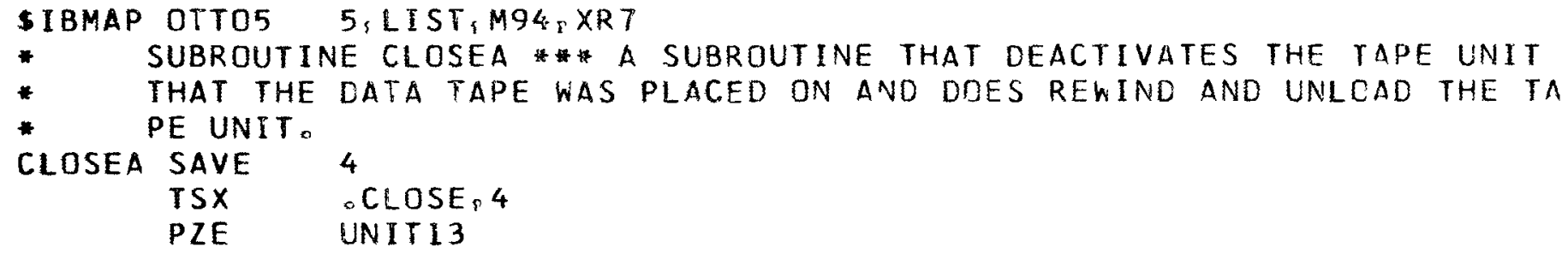


RETURN CLOSEA

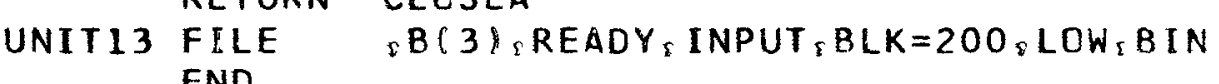


XI. DETAIL FLOW DIAGRAMS OF PROGRAM AND SUBROUTINES

\section{INDEX TO PART XI}

Subroutine

Page No.

Main Frogram

A-79

BRAIN

A-89

BLAST

A-91

MESSAGE

A-92

MATRIX

A-93

INITIAL

A -94

TEDIT

A-95

VAMEAN

A-97

REF $\varnothing \mathrm{RT}$

A-99

TIC

A. -100

$\phi U_{-L Y}^{m}$

A-101

TALLY

A-102

ENDFT'

A-103

STEP

A-105

STAT I

$A-107$

FIT 1

$A-108$

FIT 2

A-109

$F \varnothing \varnothing \mathrm{REA}$

$A-110$ 

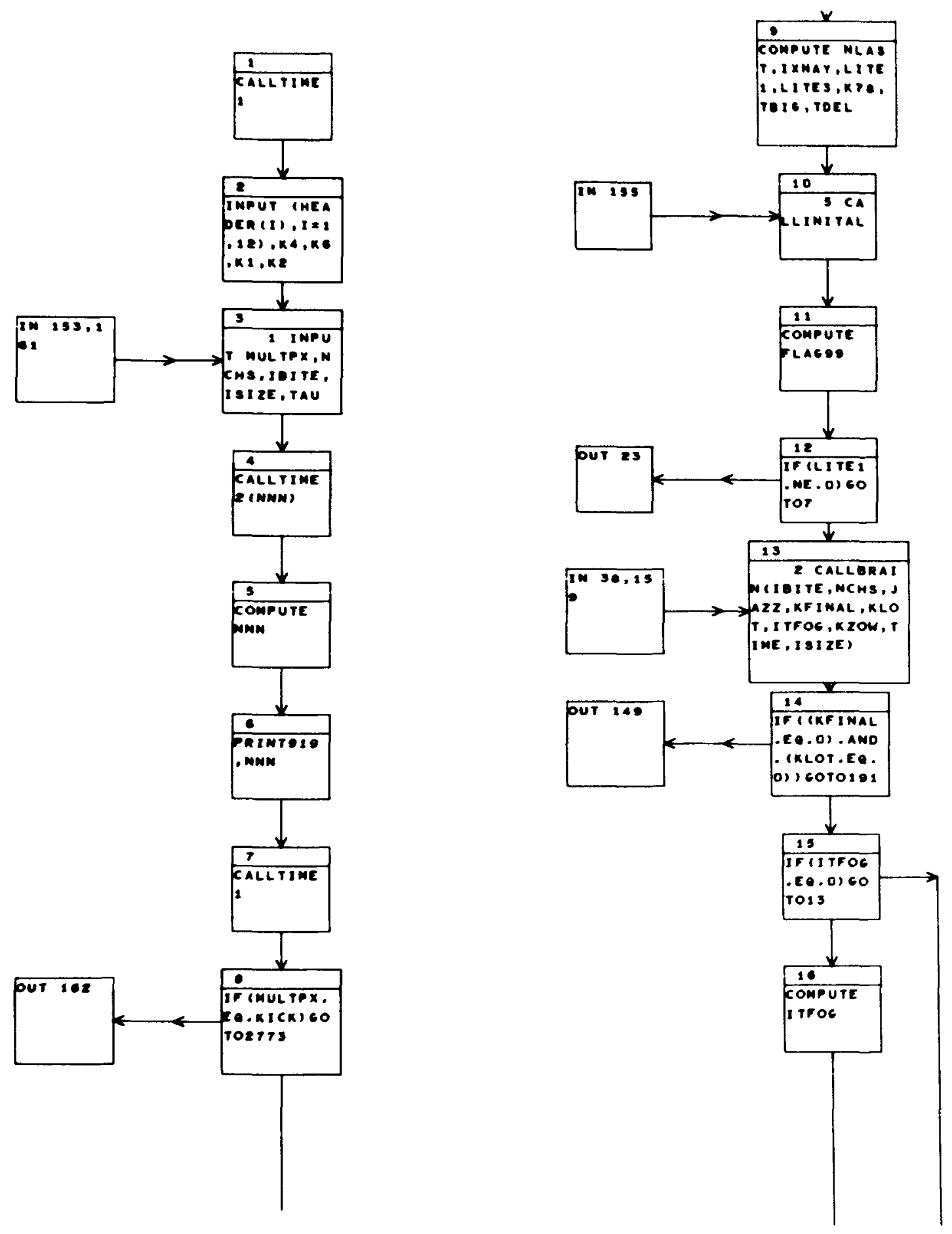

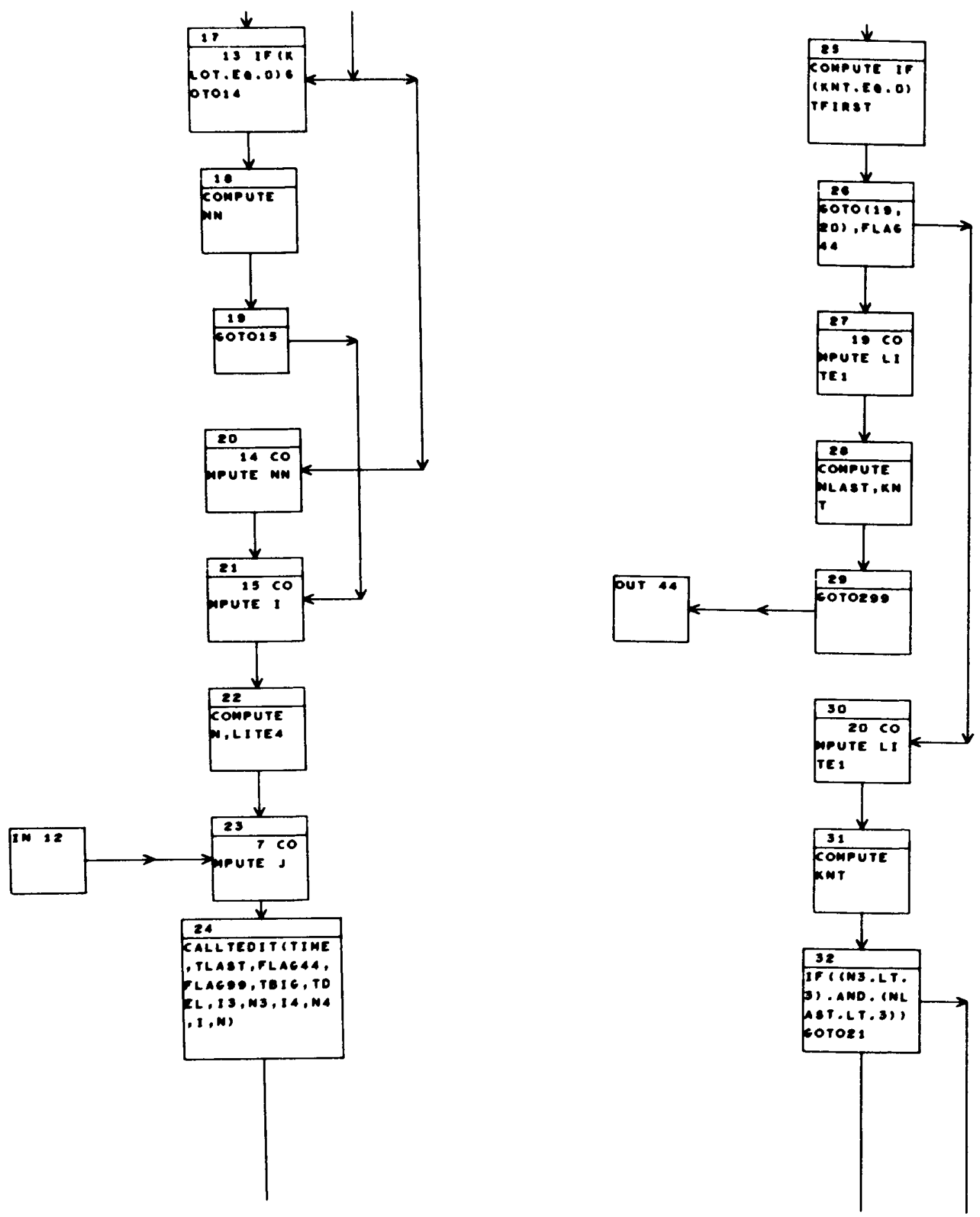
RN-S-0.146

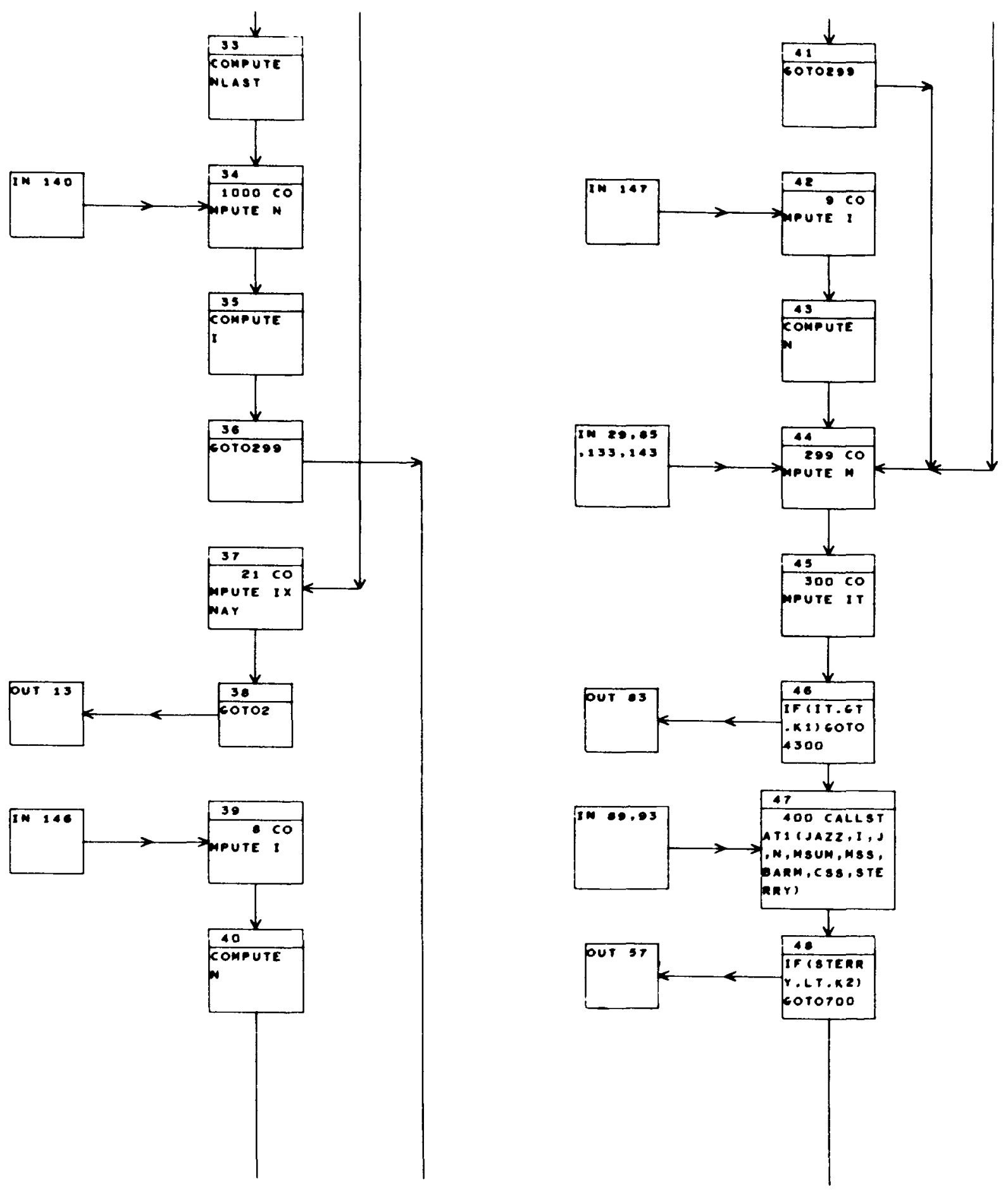



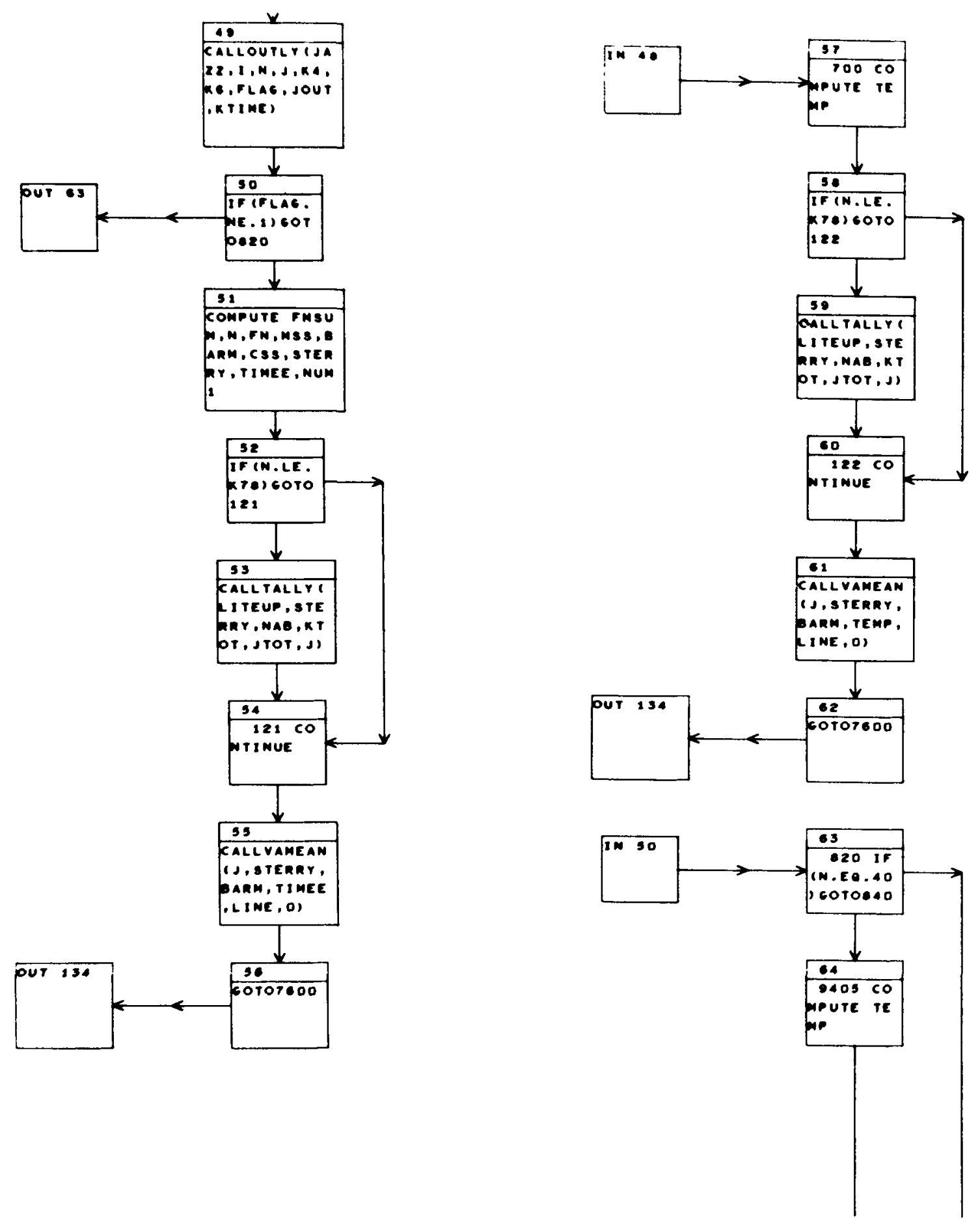

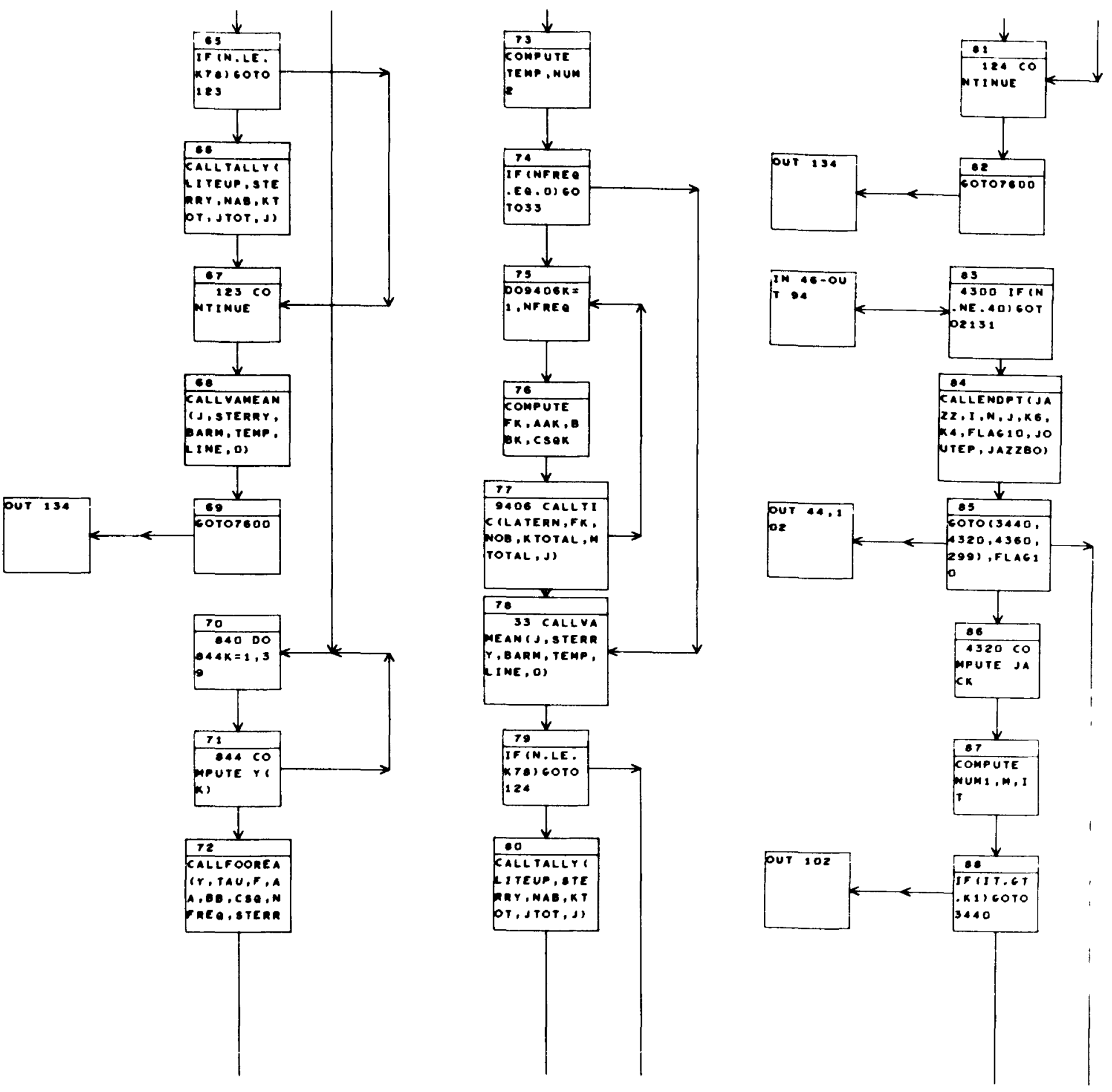

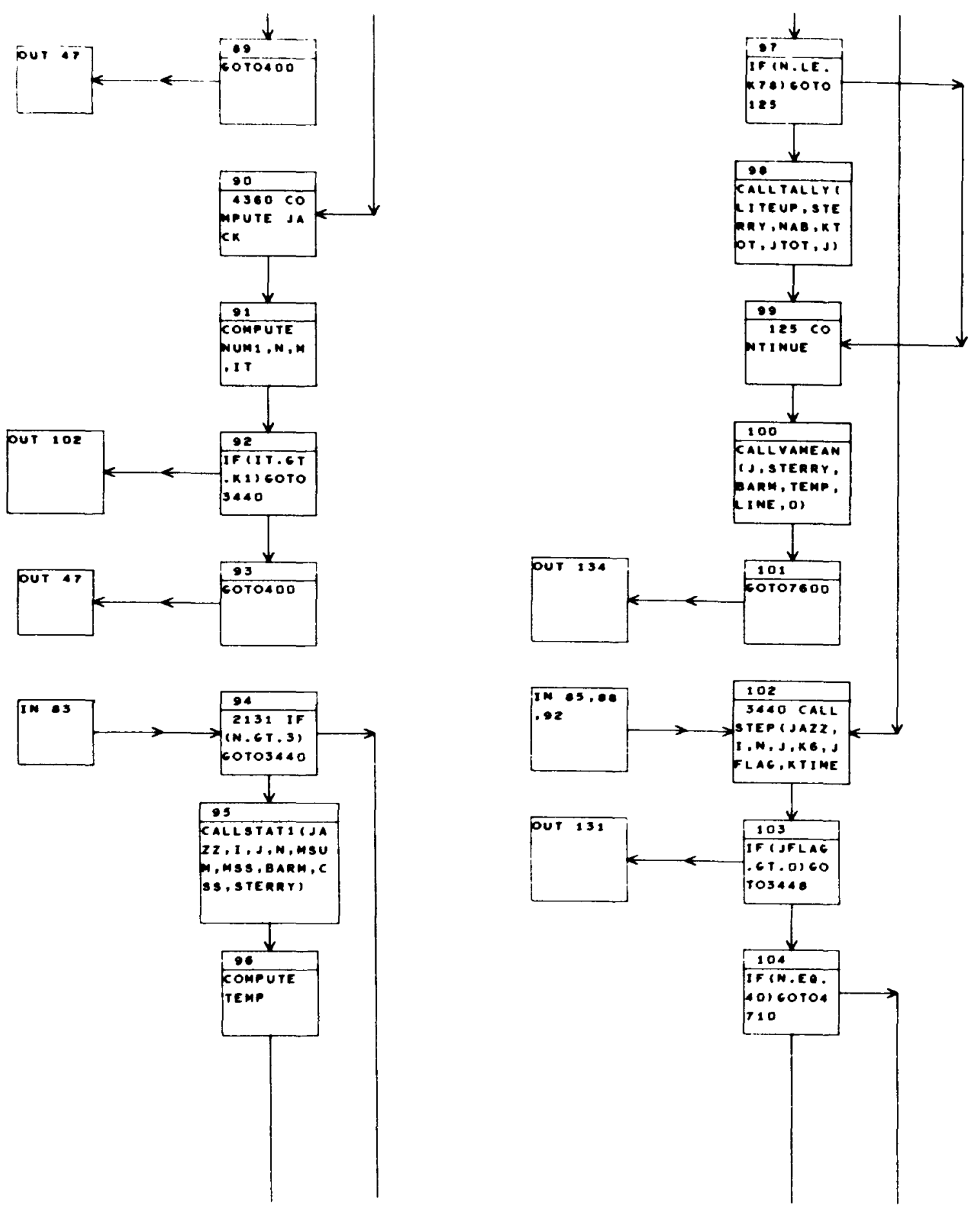
$\mathrm{RN}-\mathrm{S}-0146$
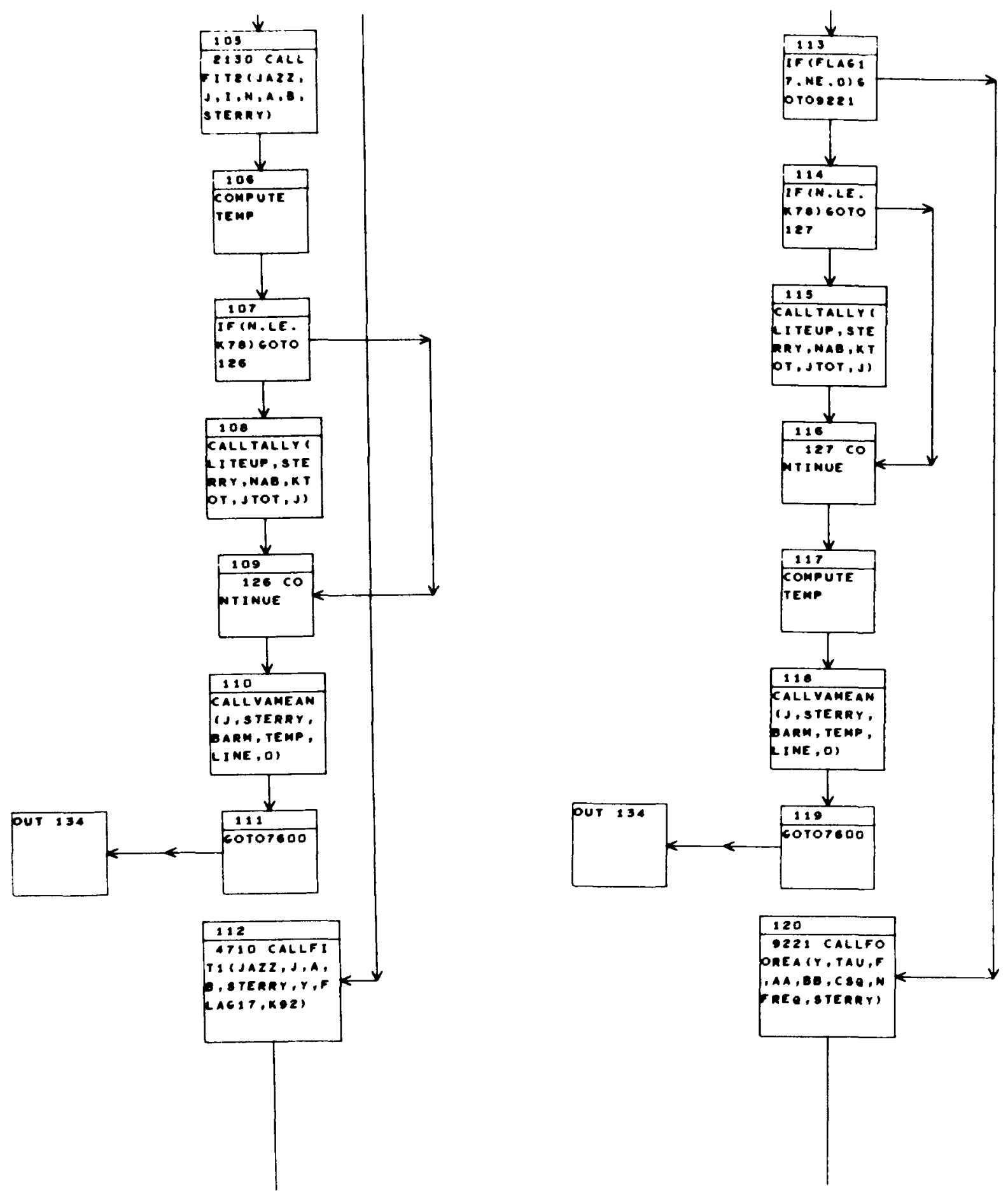

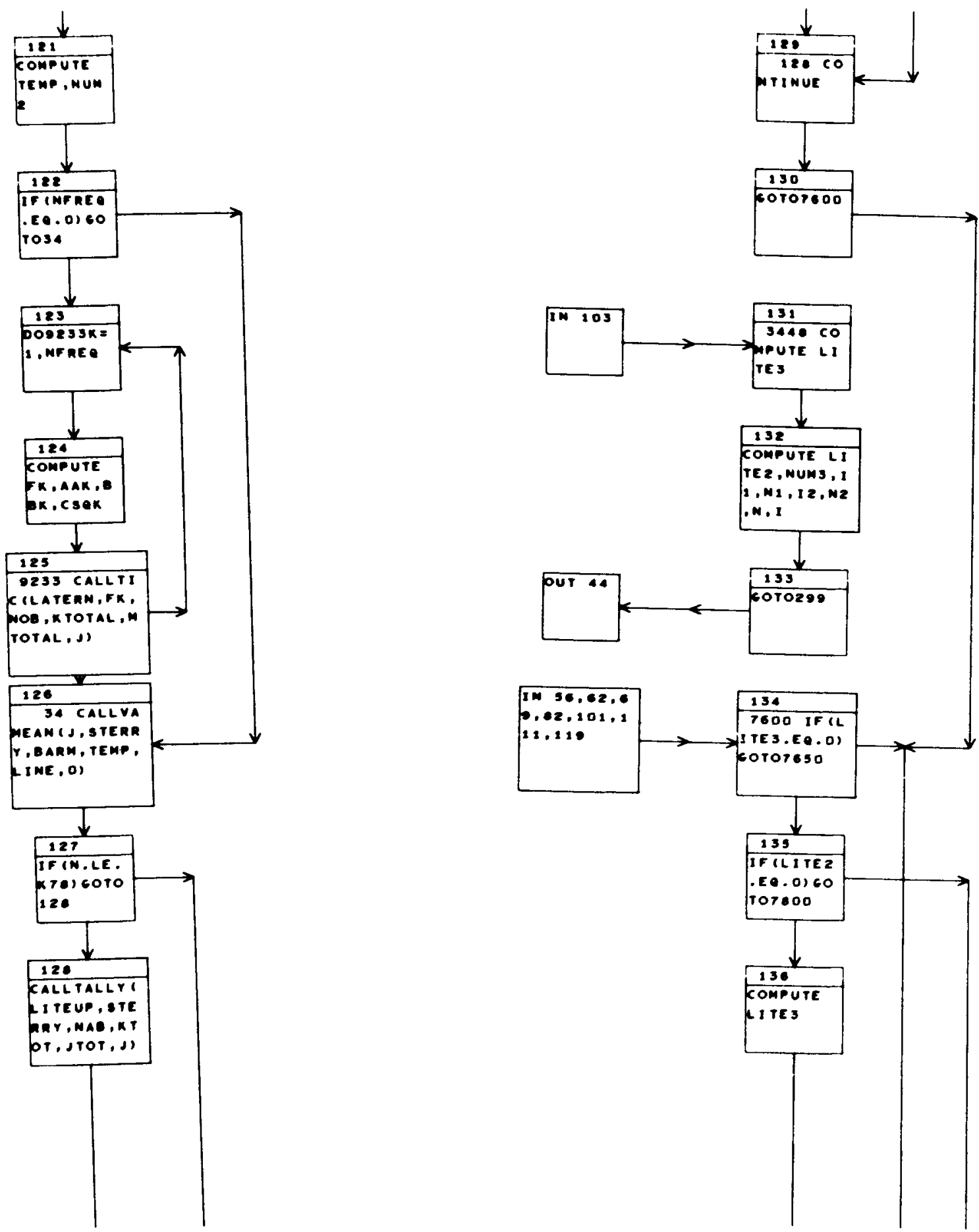
$\mathrm{RN}-\mathrm{S}-0146$
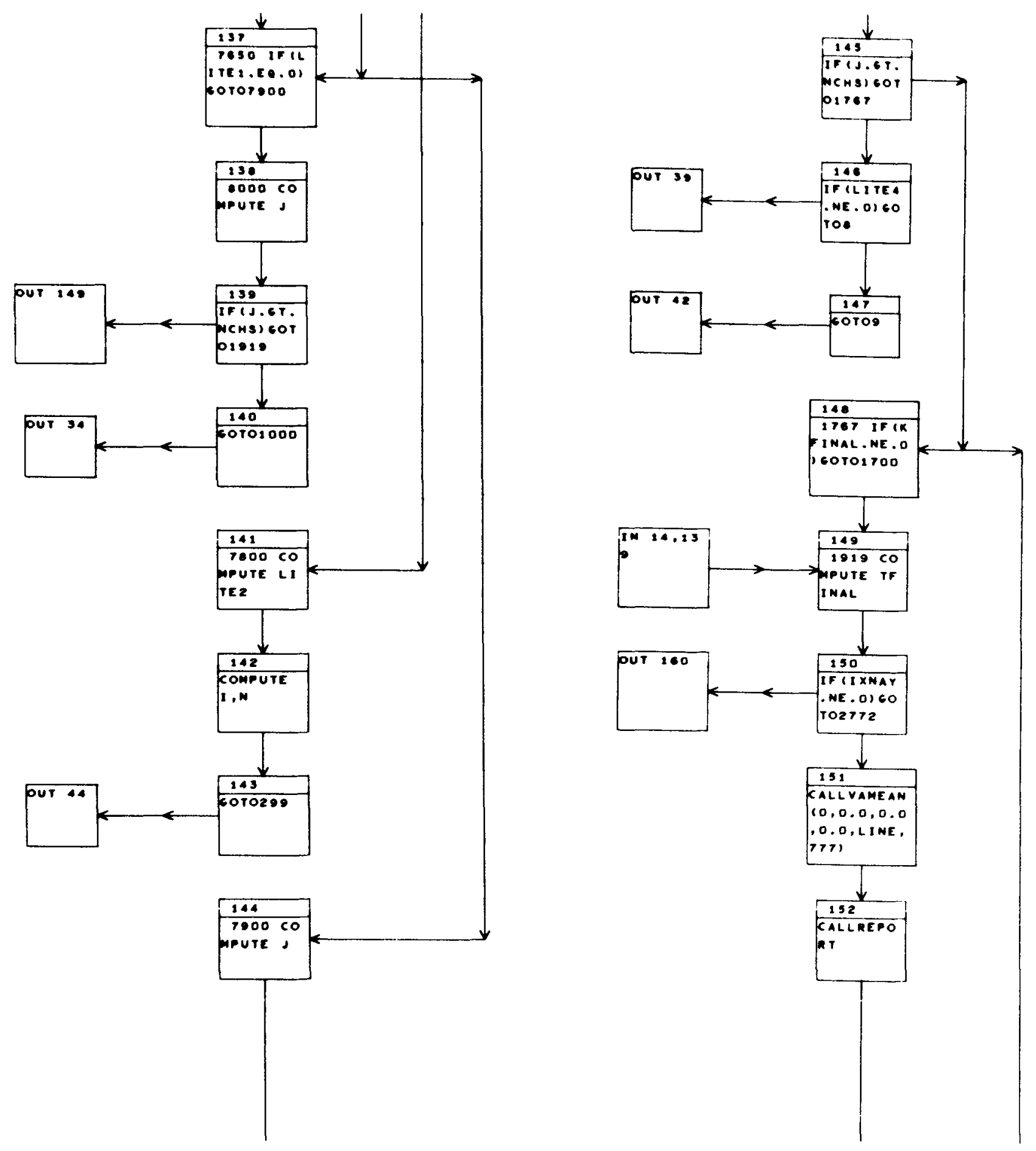
RN-S-0146
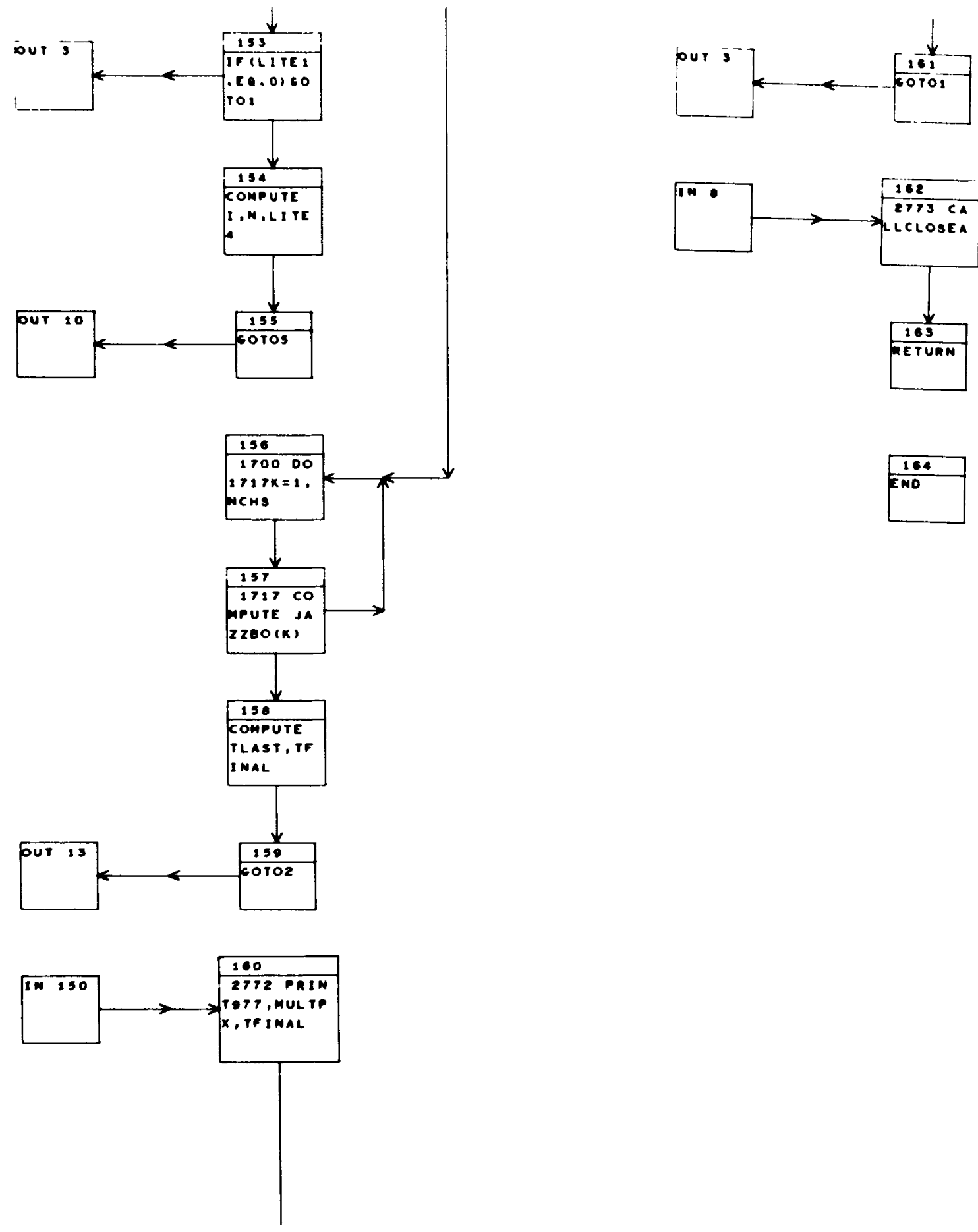

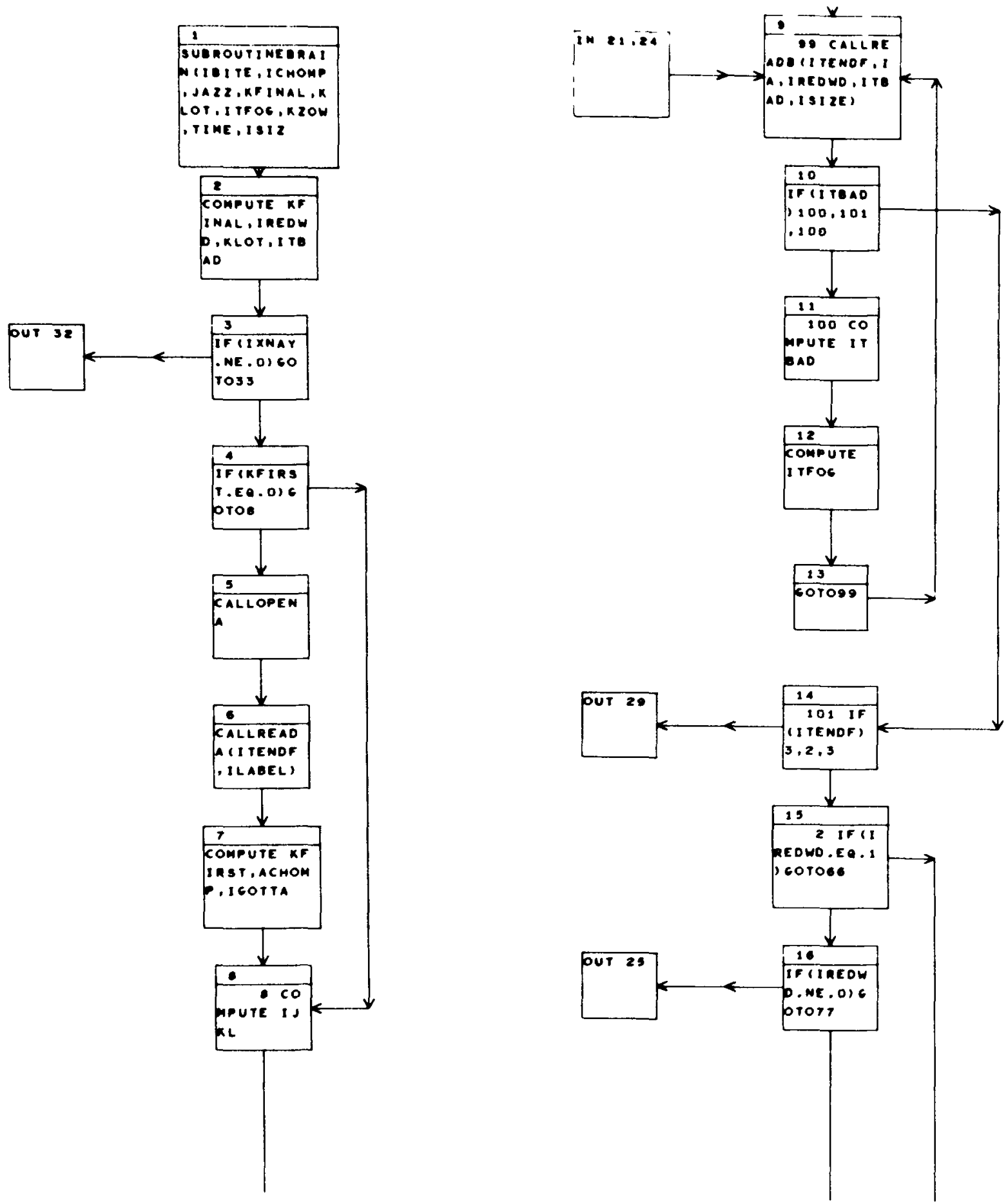

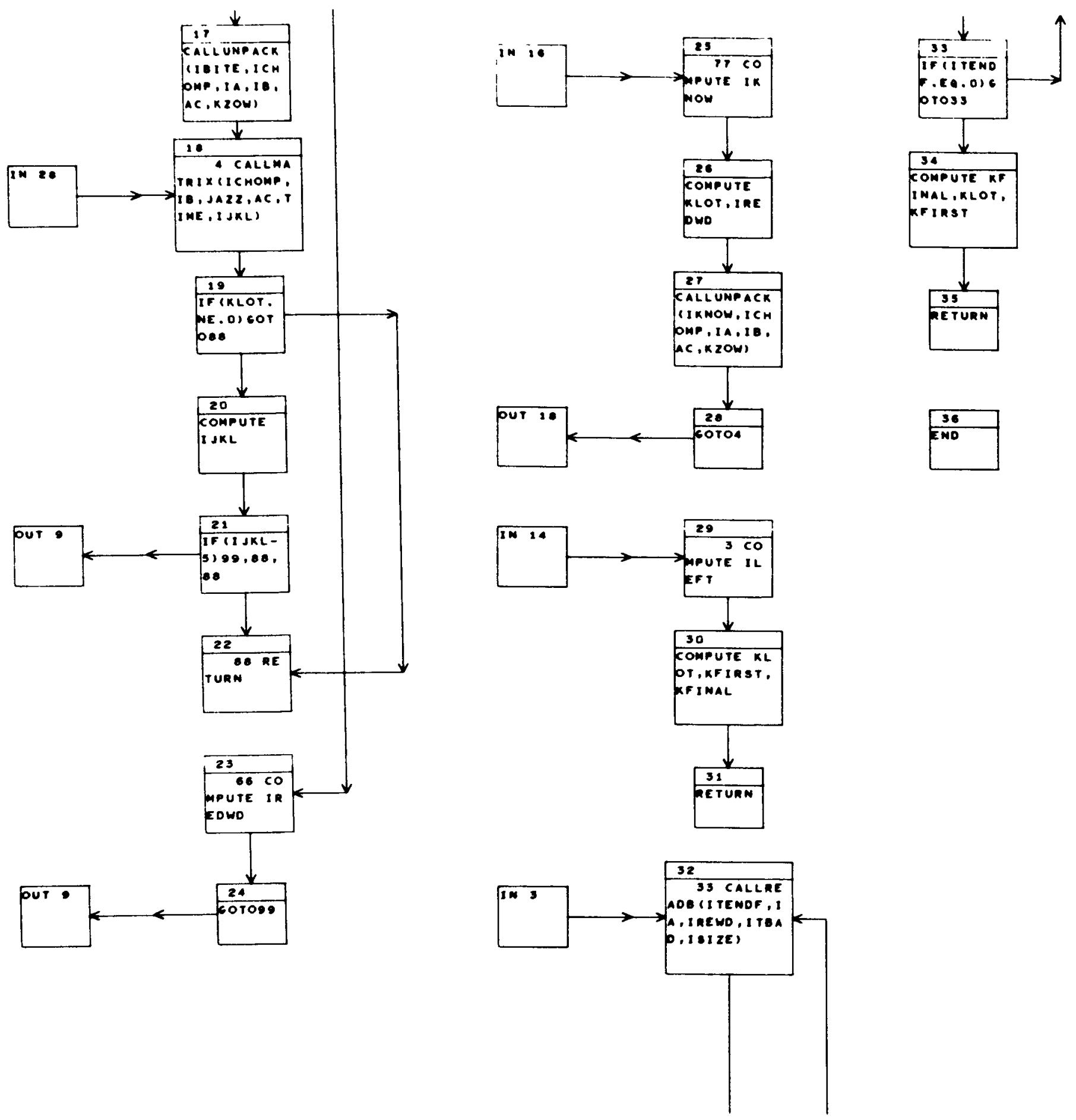
RN-S-0146

BUBROUTINE BLAST *\#* a gUROUTINE iHAT CONVERTS INTEGER TIME DATA

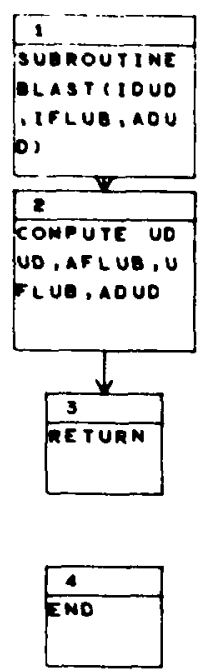


SUBROUTINE OLABS *\#* A SUBROUTINE THAT COMVERTS INTEGER TIME OATA

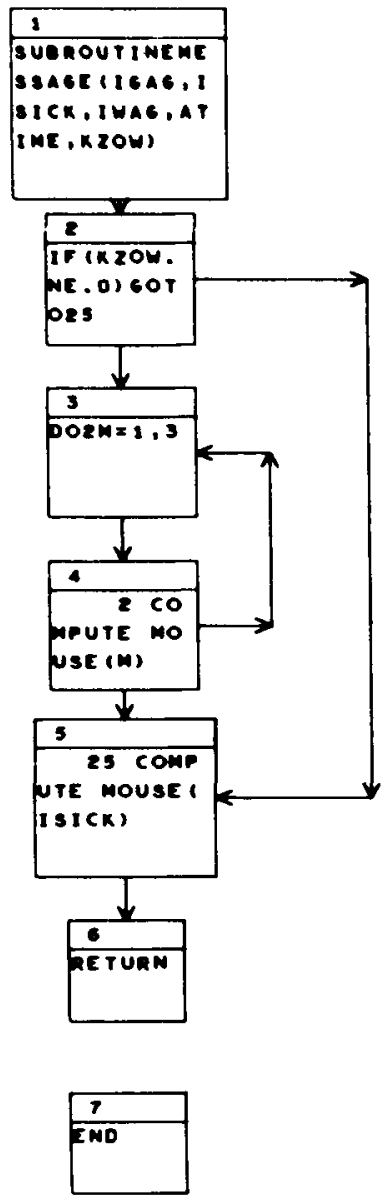



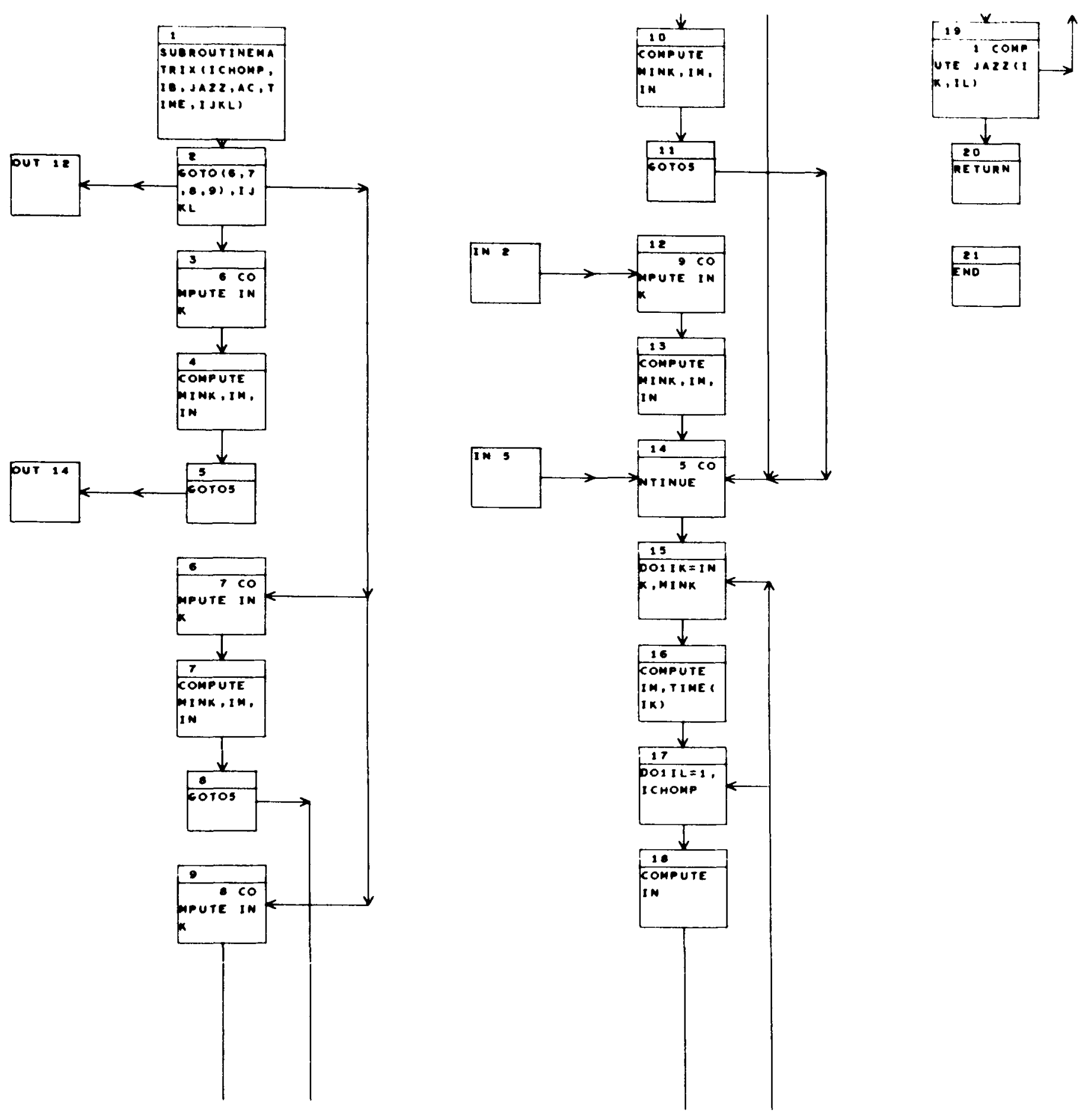
RN-S-O146

IHIS SUEROUTINE INITIALEE VARIOUB FLAGS AND COUNTERS

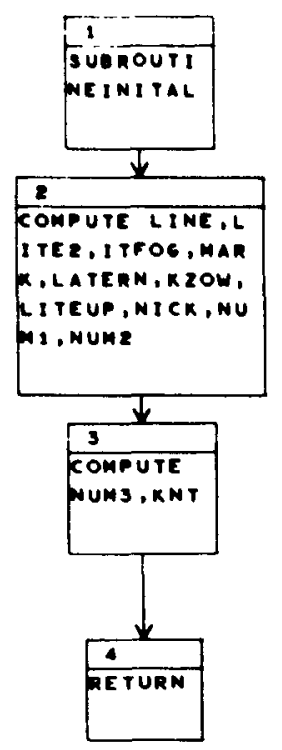

5

END 
RN-S-O146

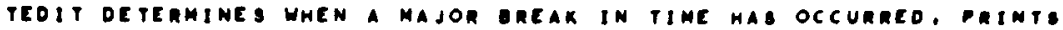
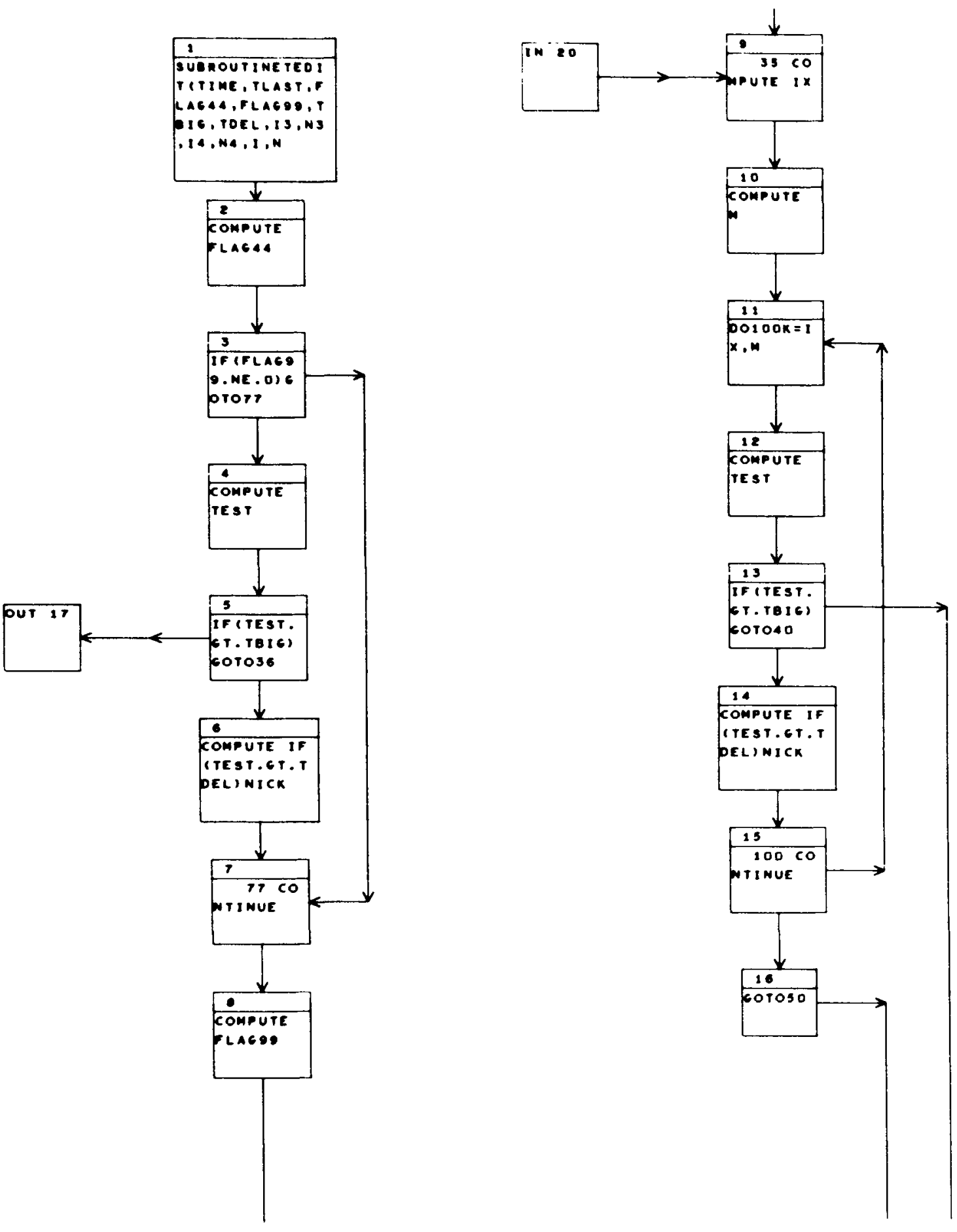

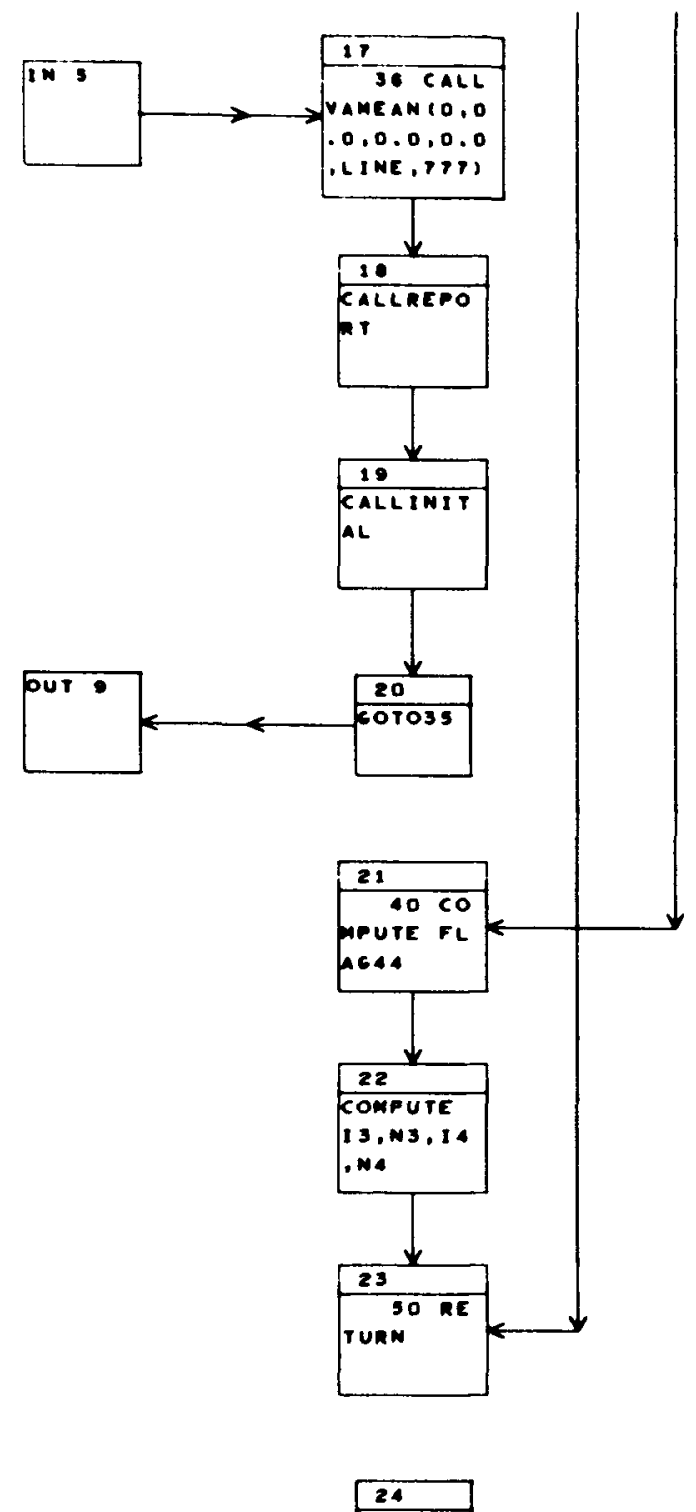

200

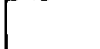


RN-S-0146

THIS SUEROUTINE COMPILE ANO PRINTS TABLES OF MEANE ANO VARIANCES
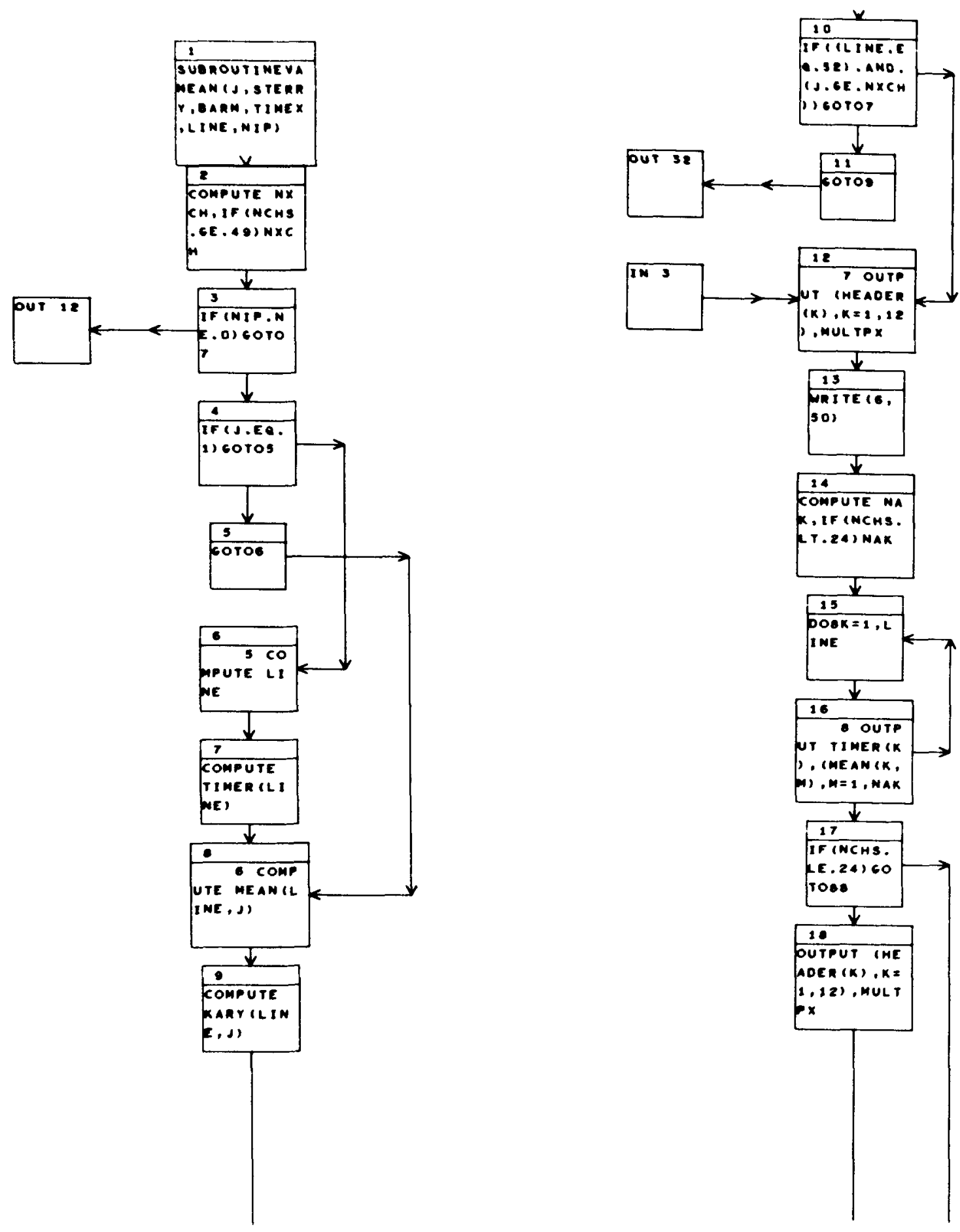

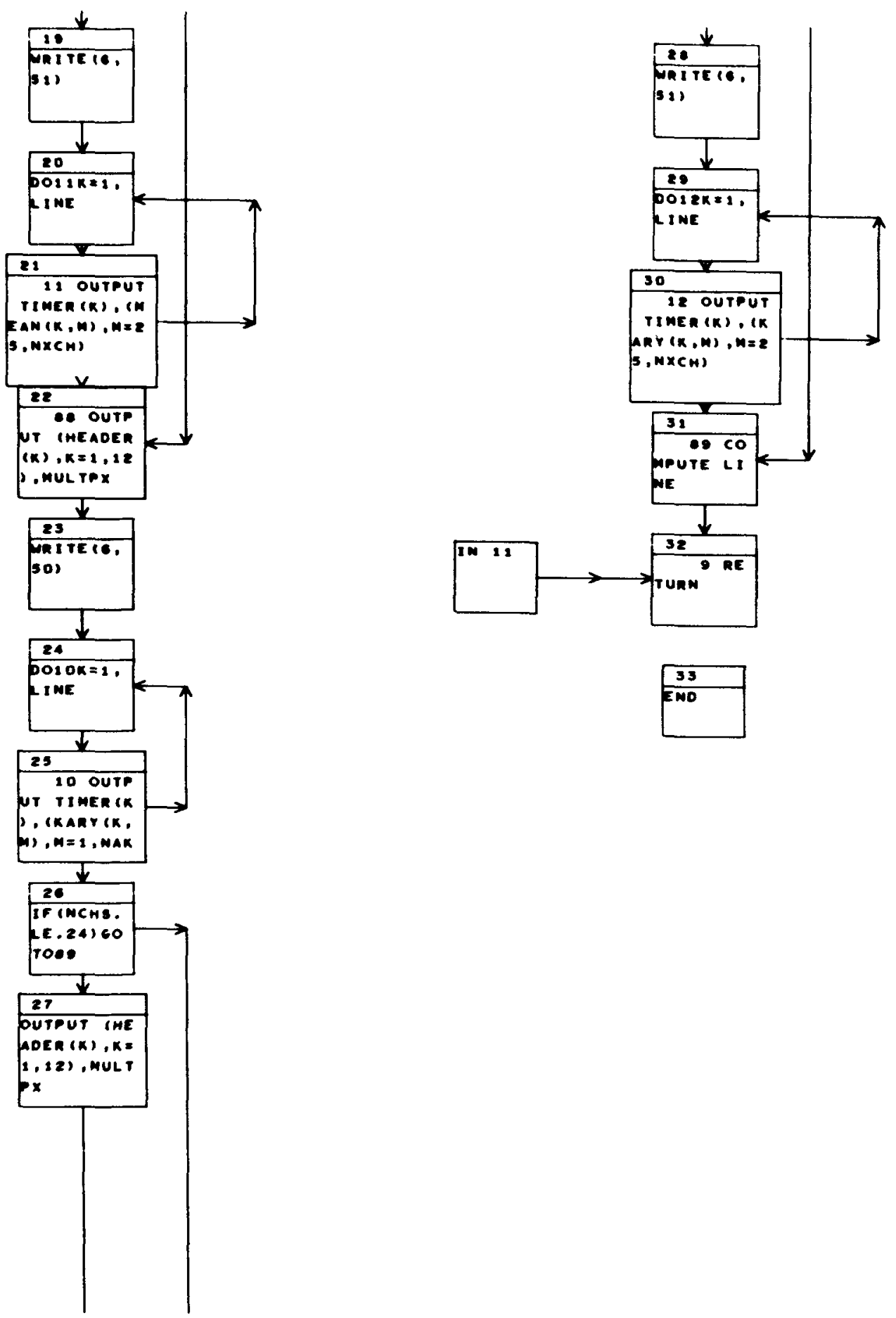

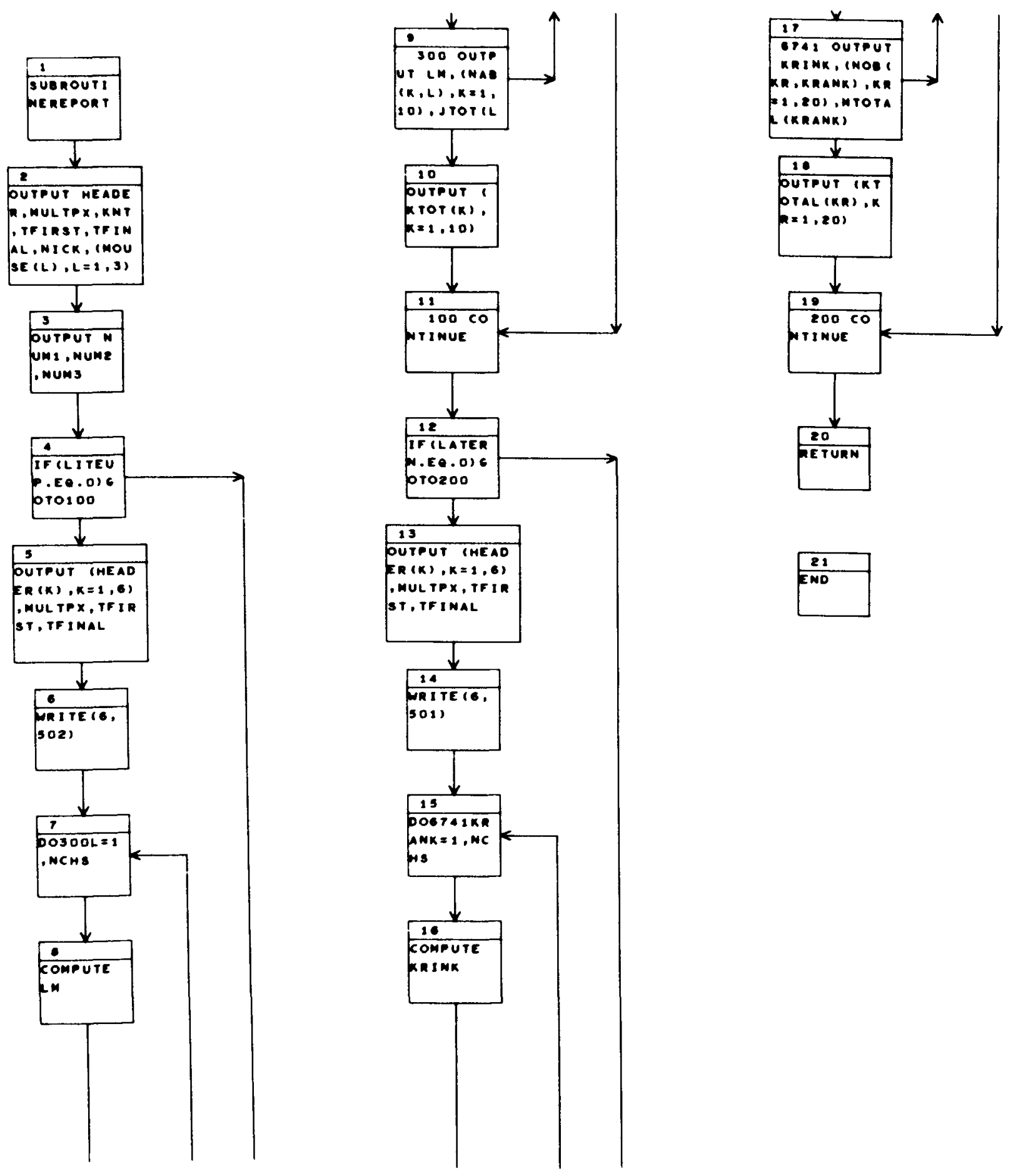
RN-S-0.146

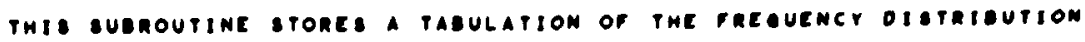
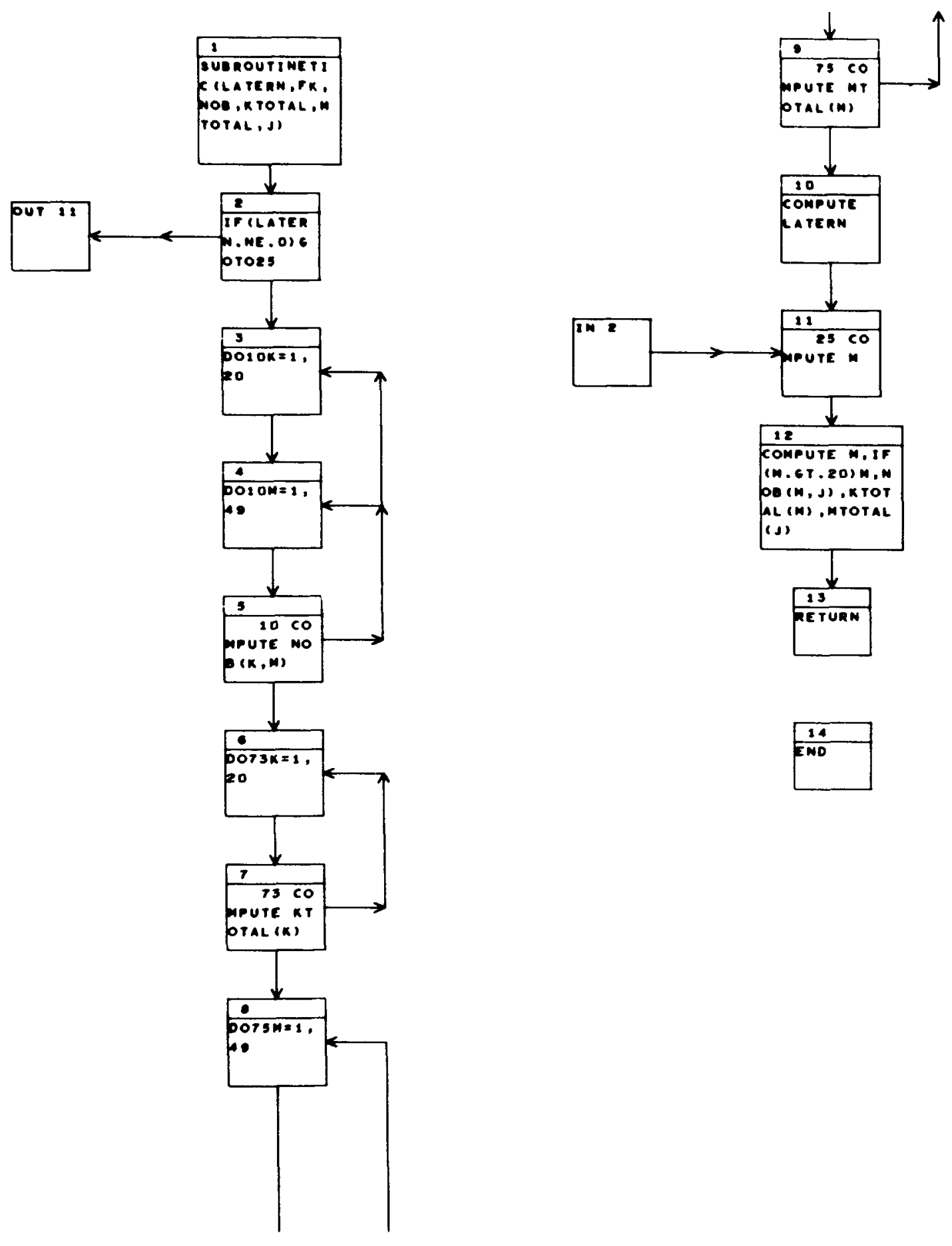
RN-S-0146

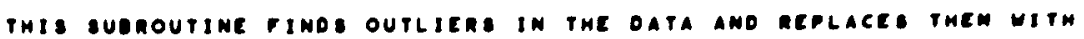
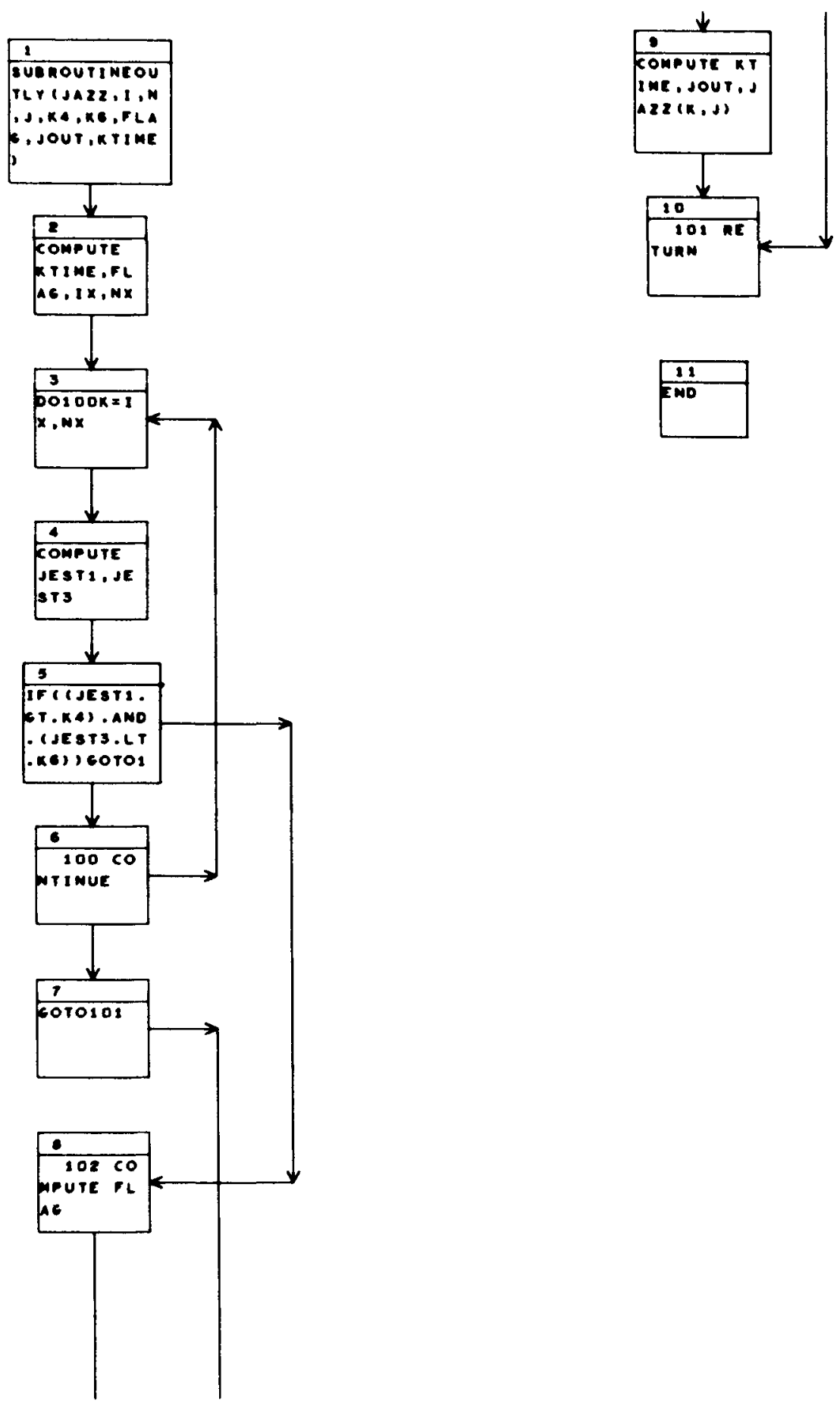

FND 


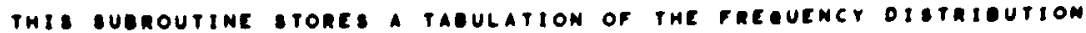
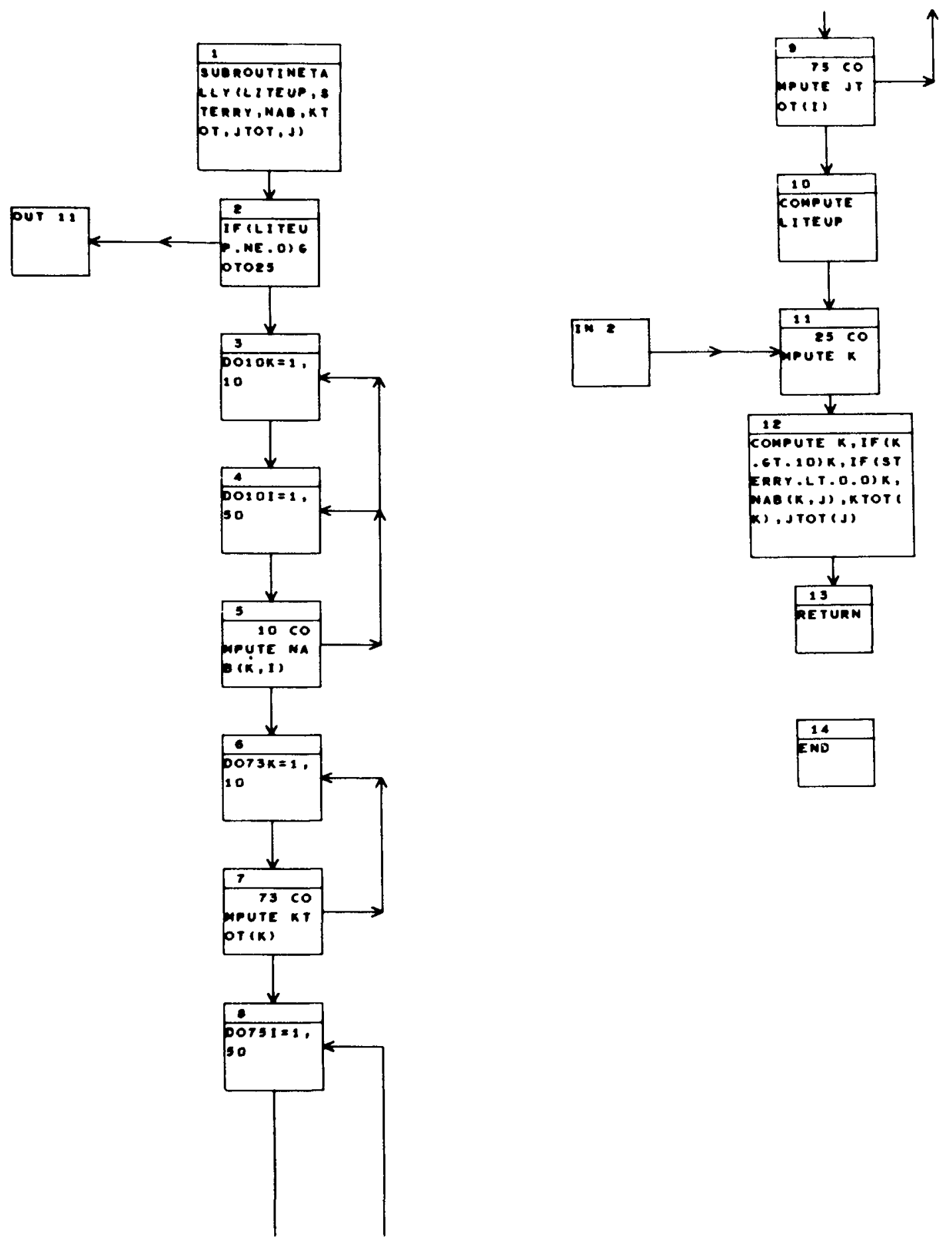
RN-S-0146
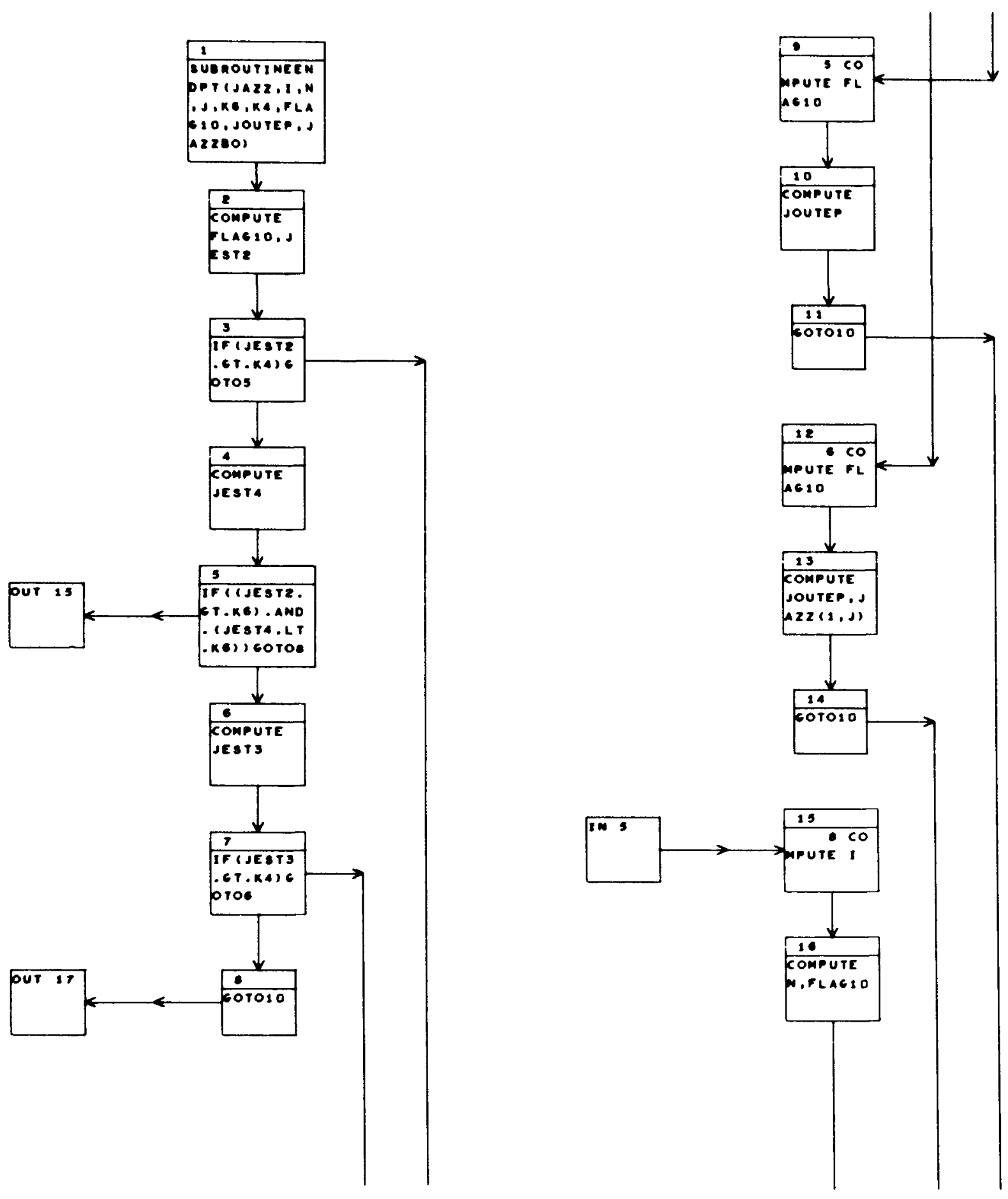
RN-S-0146
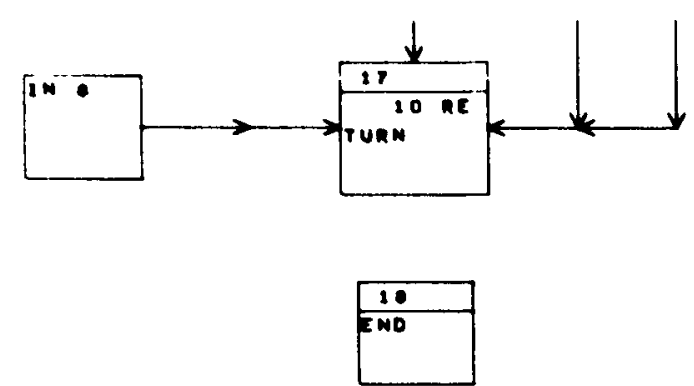

A -104 
RN-S-OI46

THIS SUEROUTINE TESTS TO FIND A SEP OR MAMP IN THE OATA.
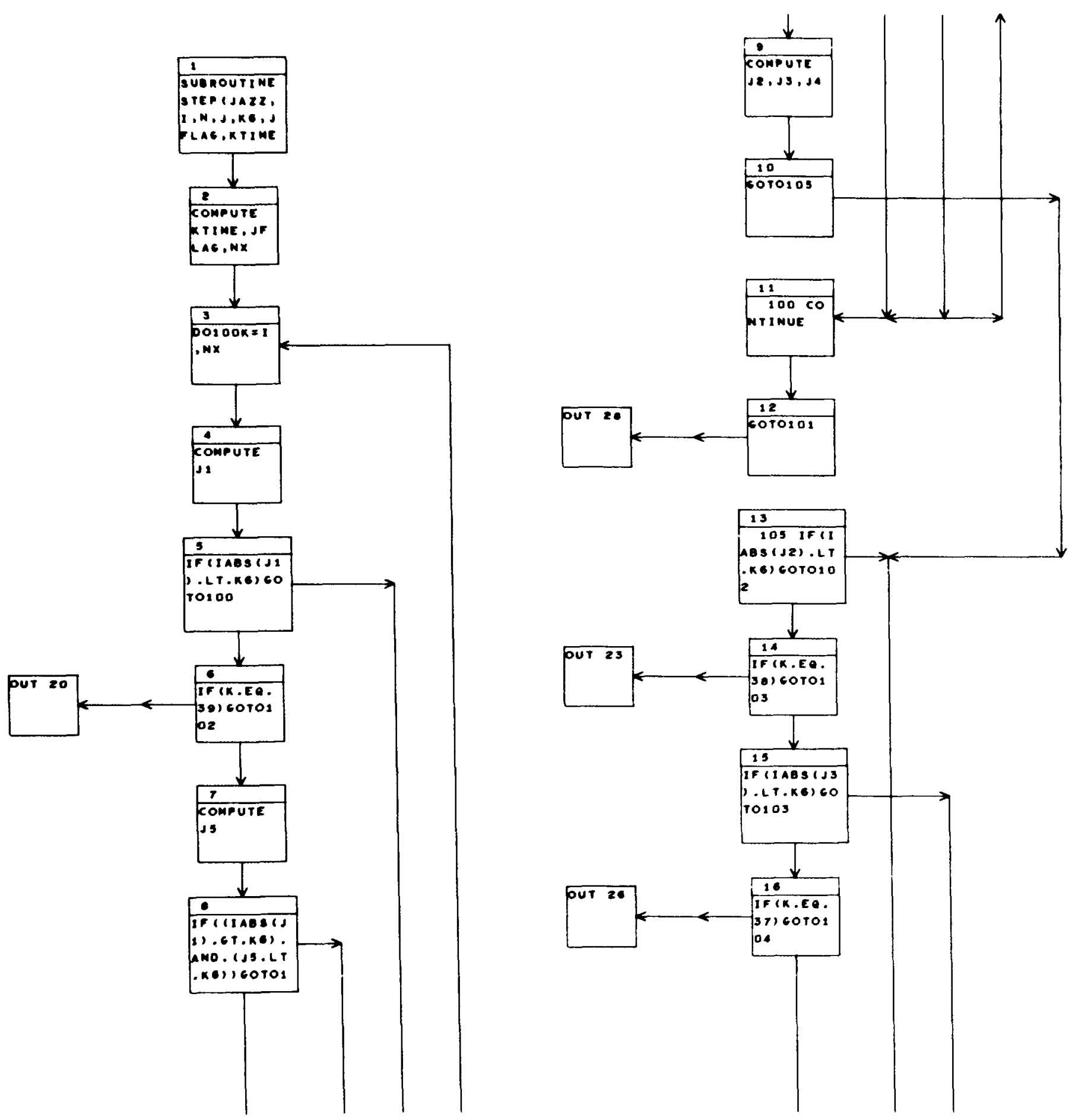

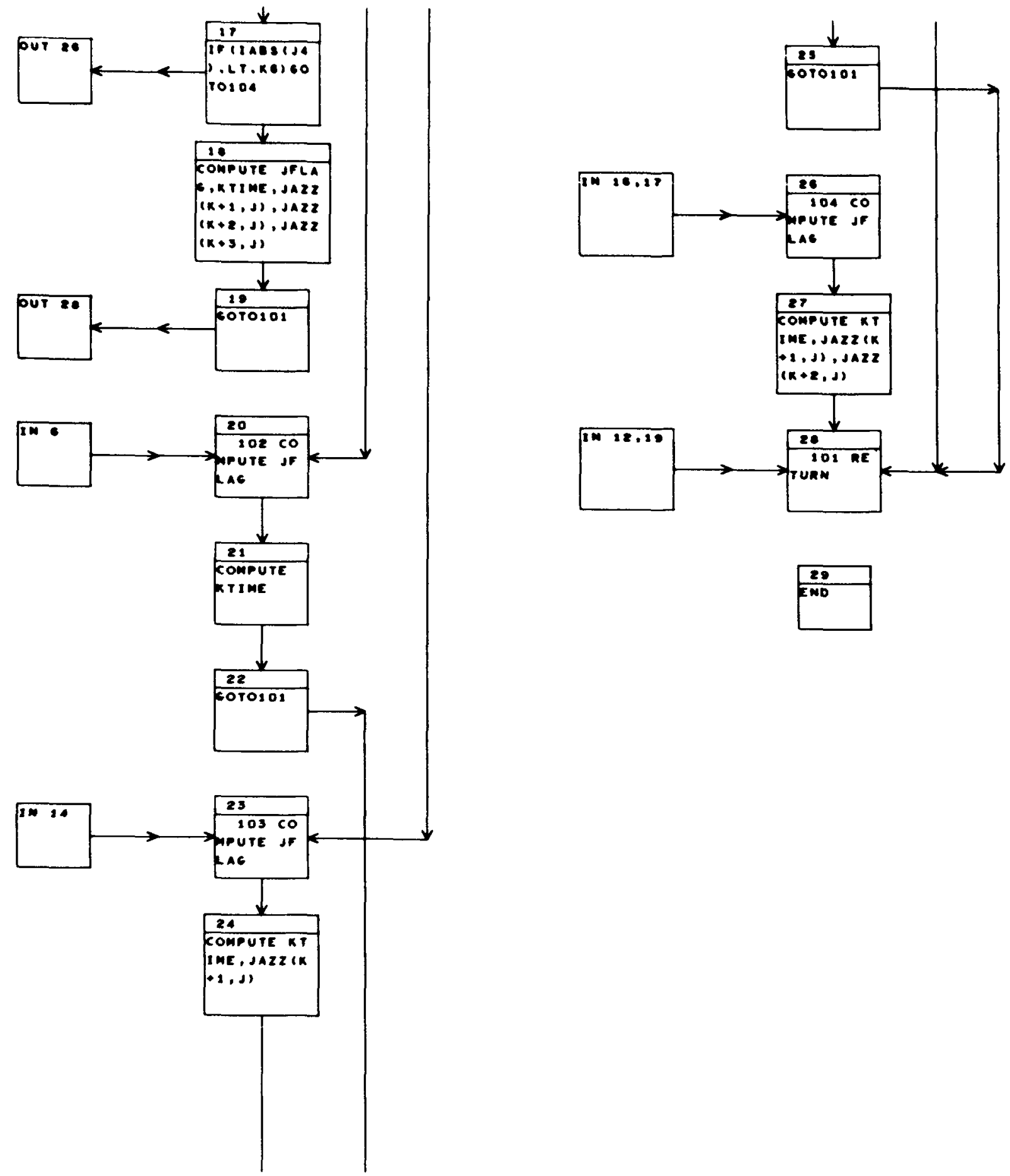
$\mathrm{RN}-\mathrm{S}-0146$

THIS SUDROUTINE FinDE THE MEAN (OARM) ANO VARIANCE (STERAT) IM
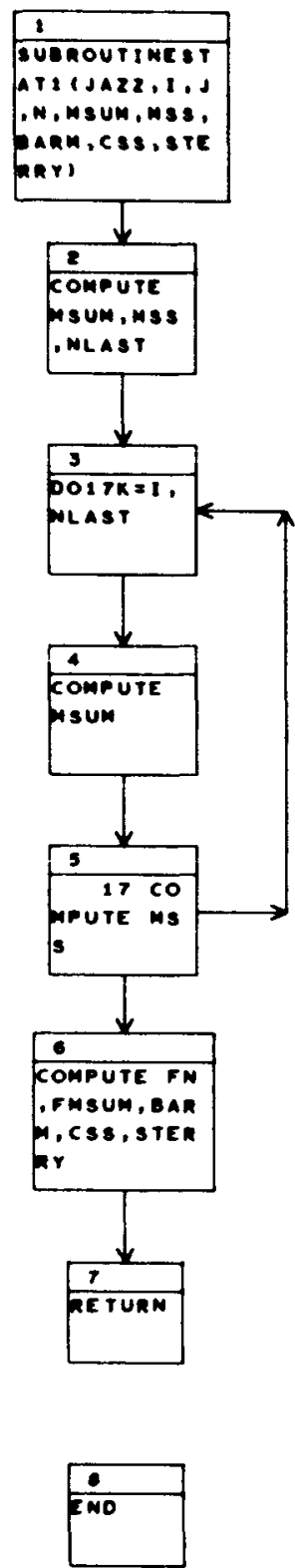
RN-S-0146
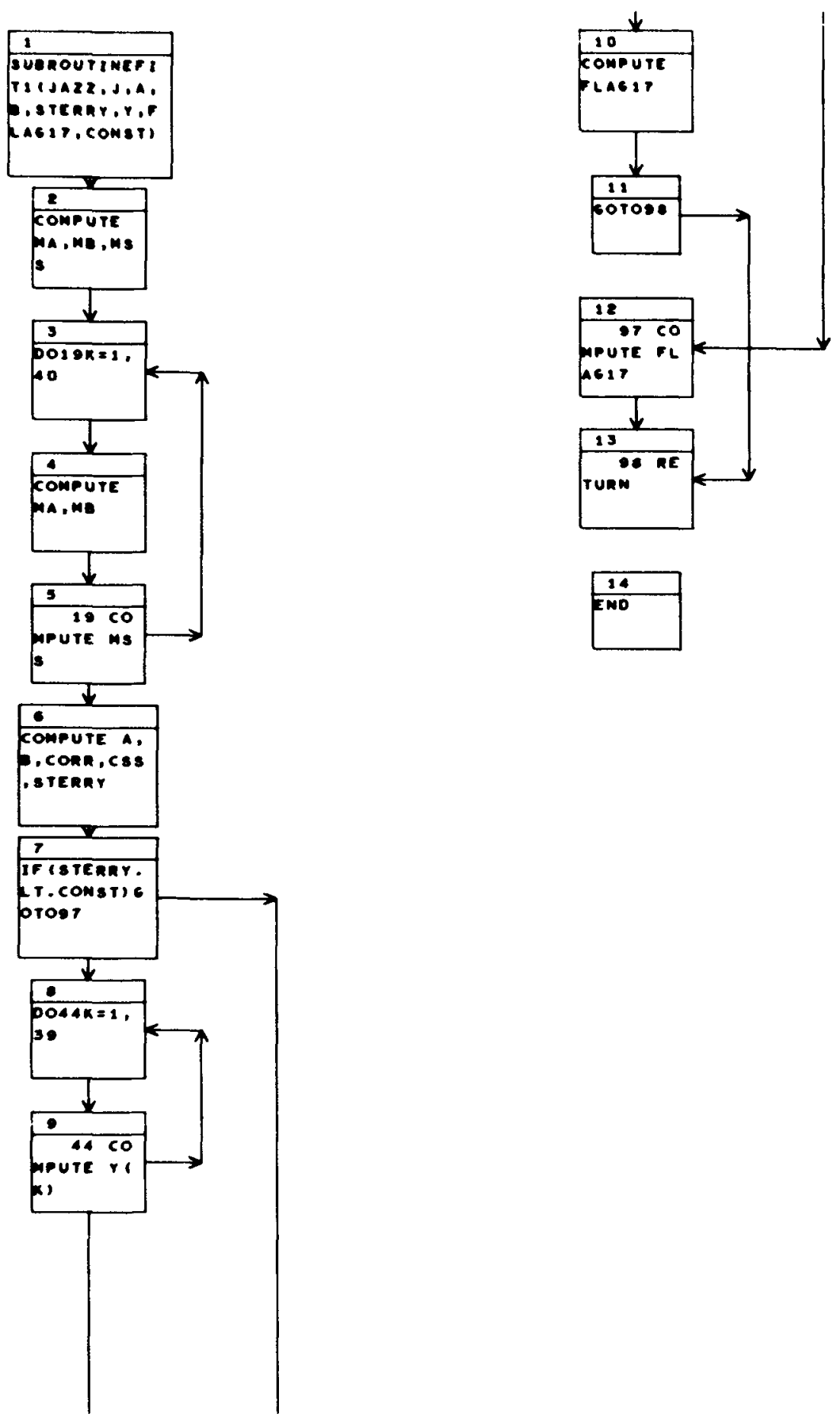

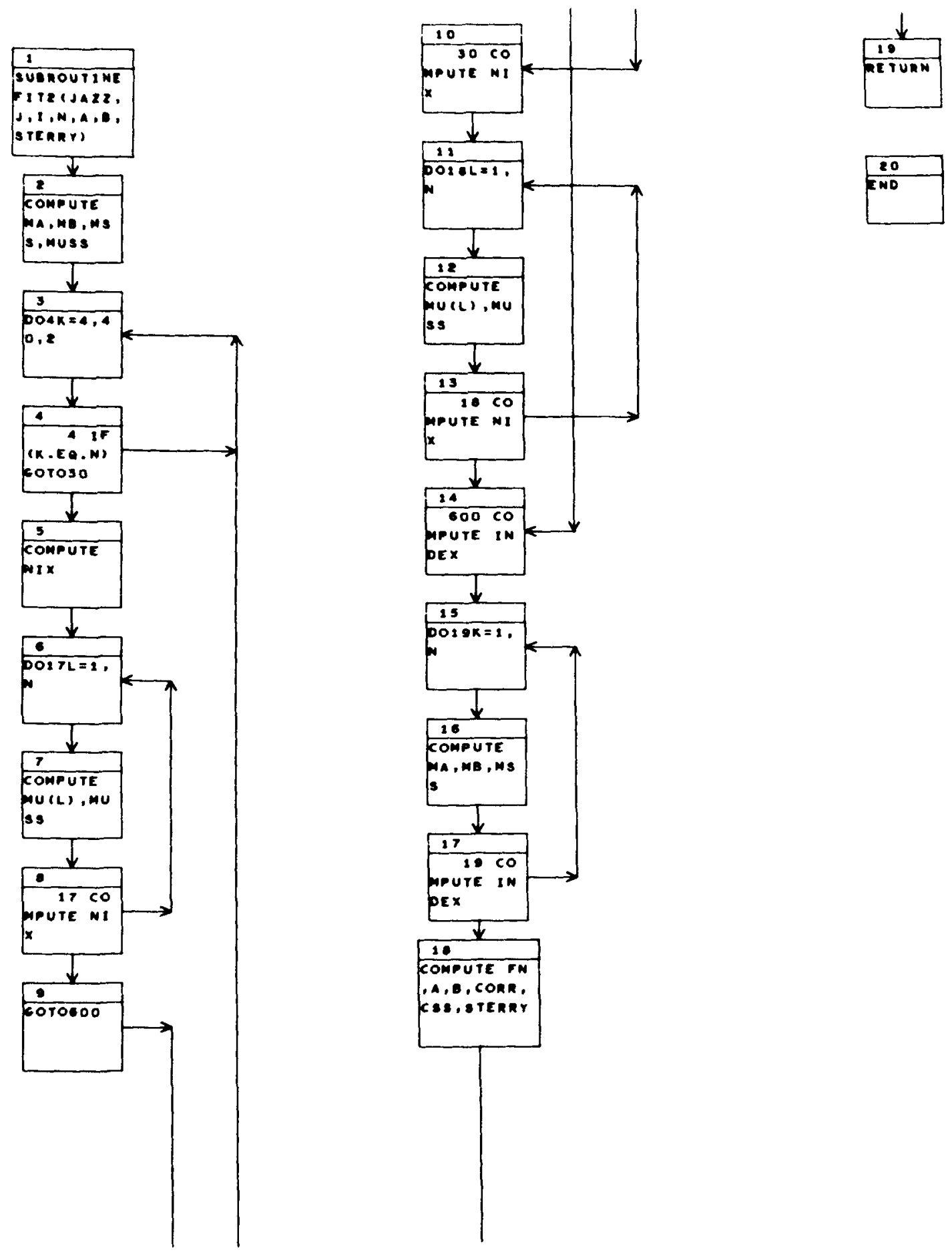

$\mathbf{x}$
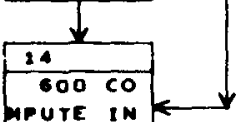

pex

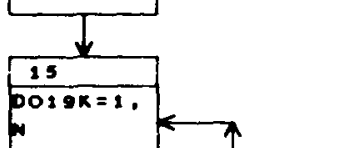

NDD

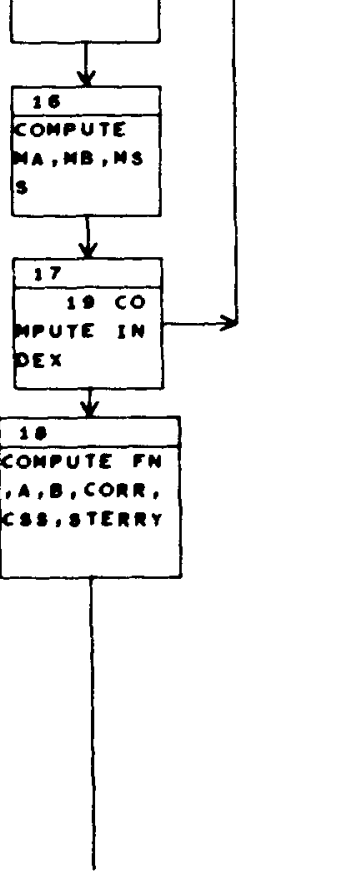



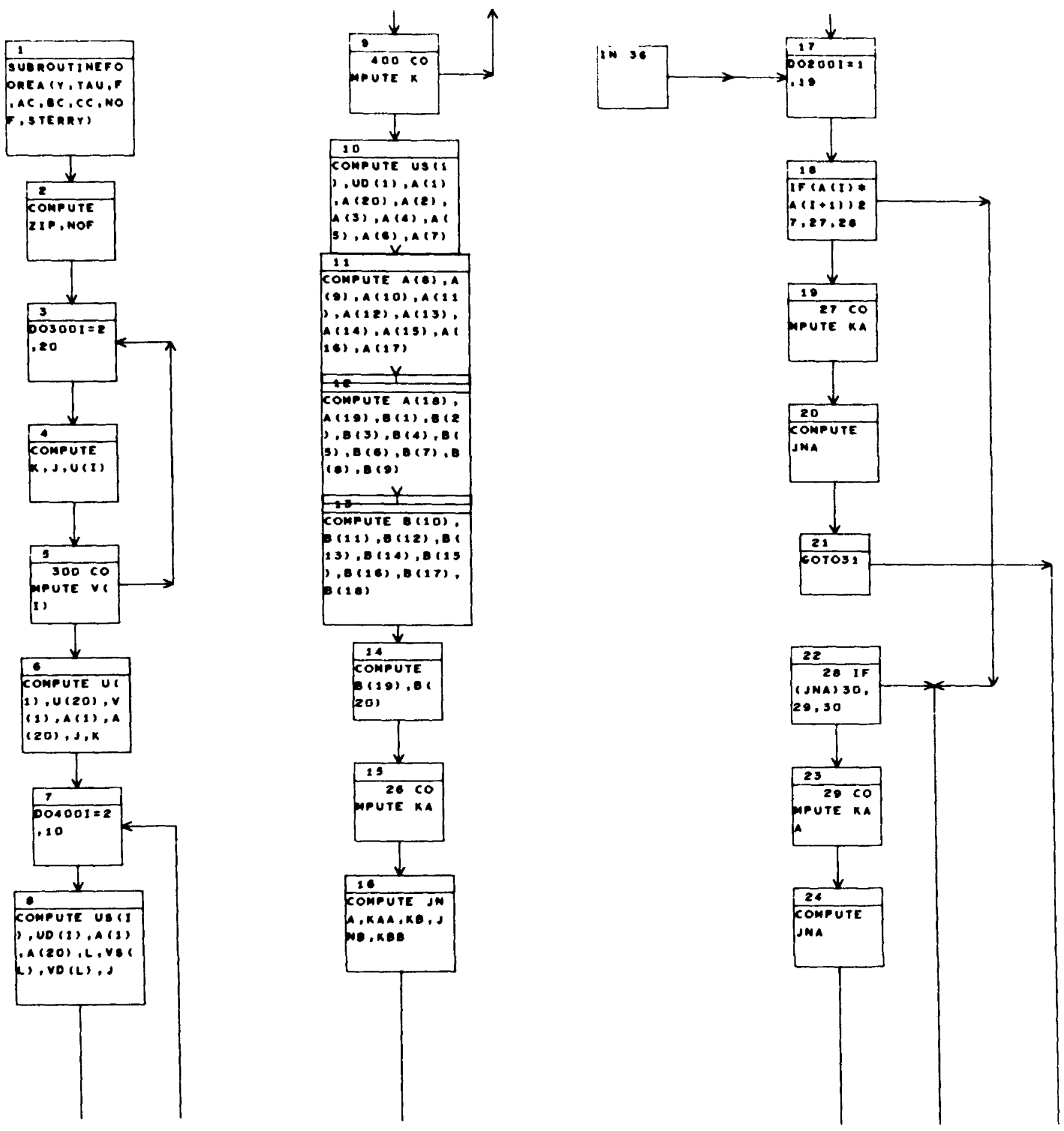

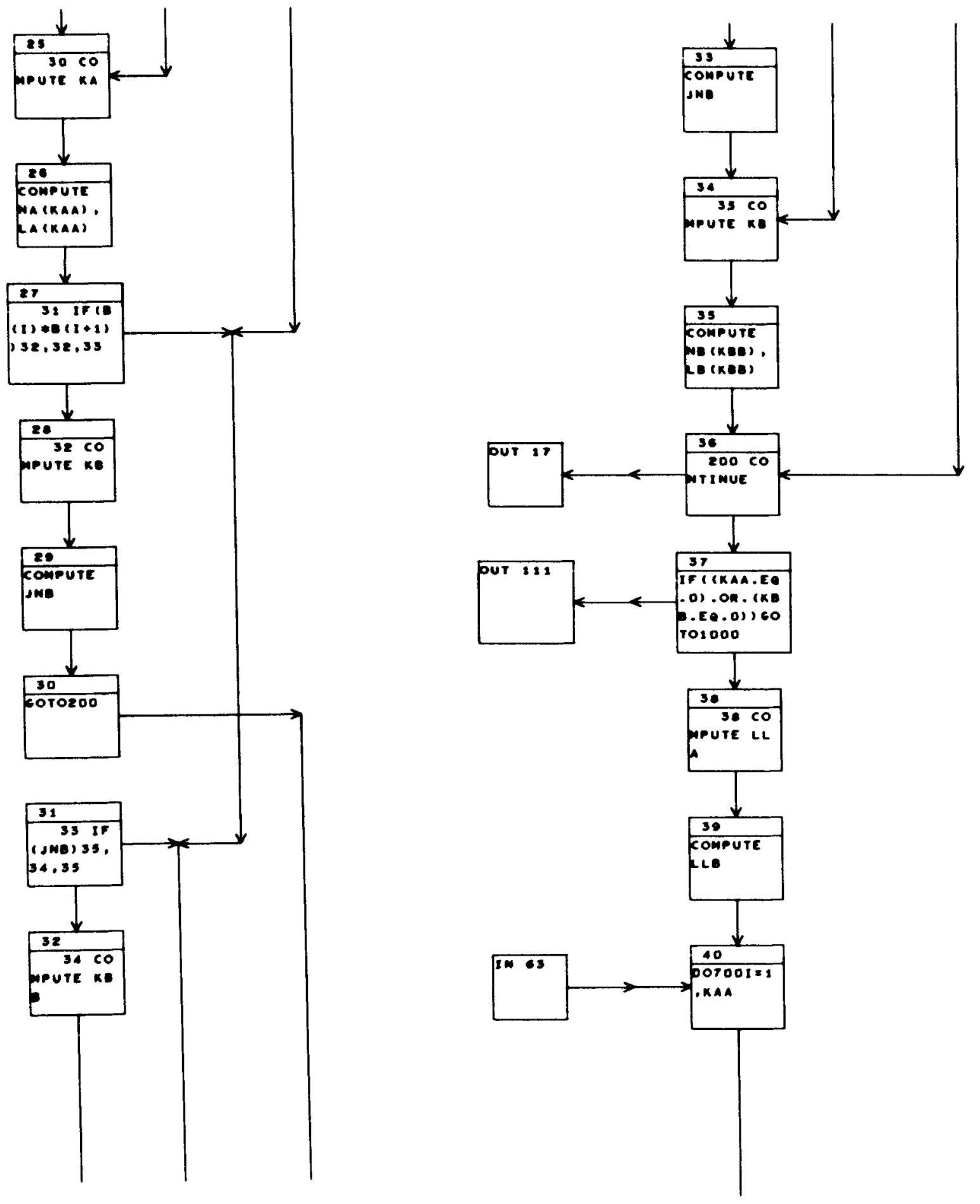
RN-S-0146
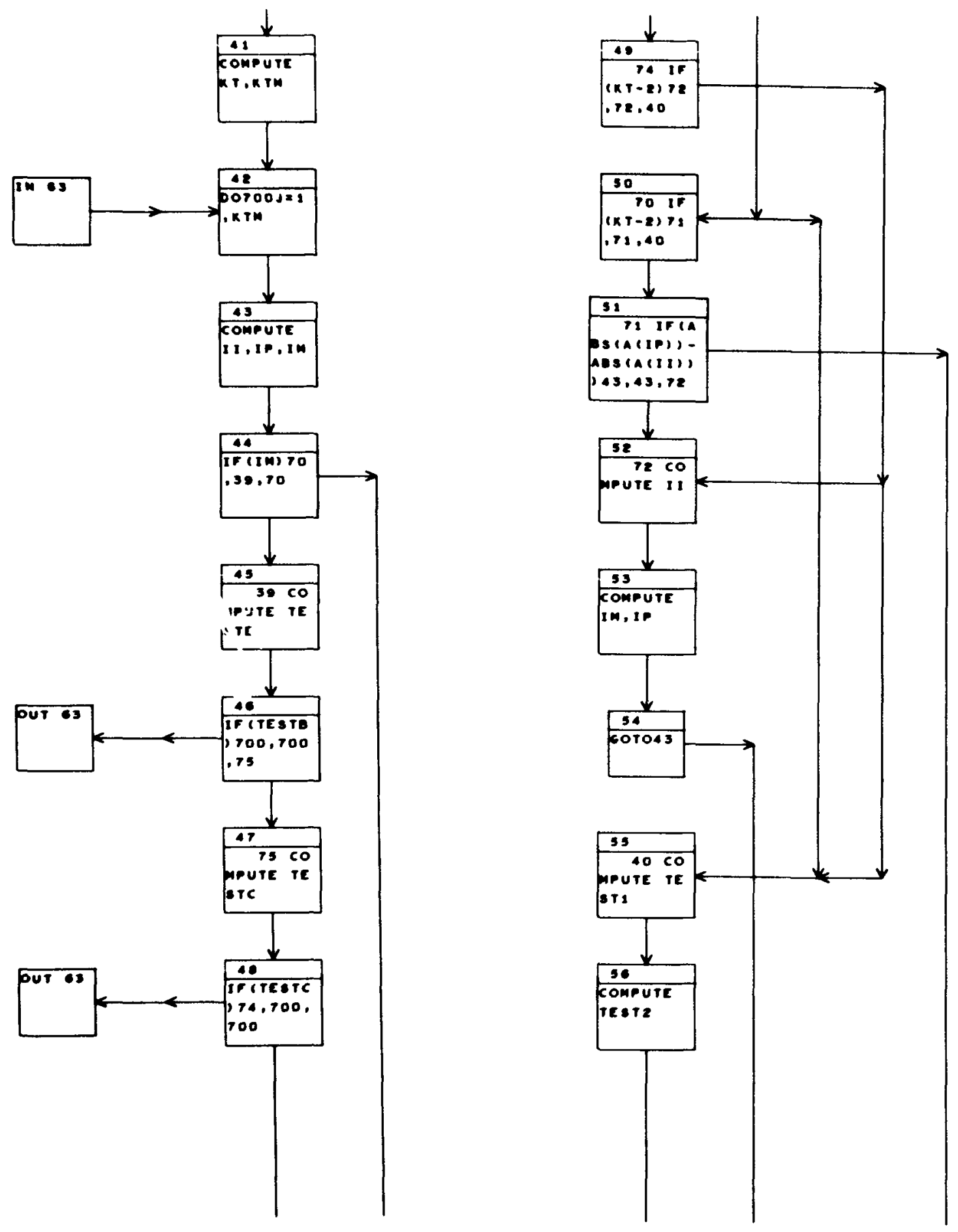
$\mathrm{RN}-\mathrm{S}-0146$
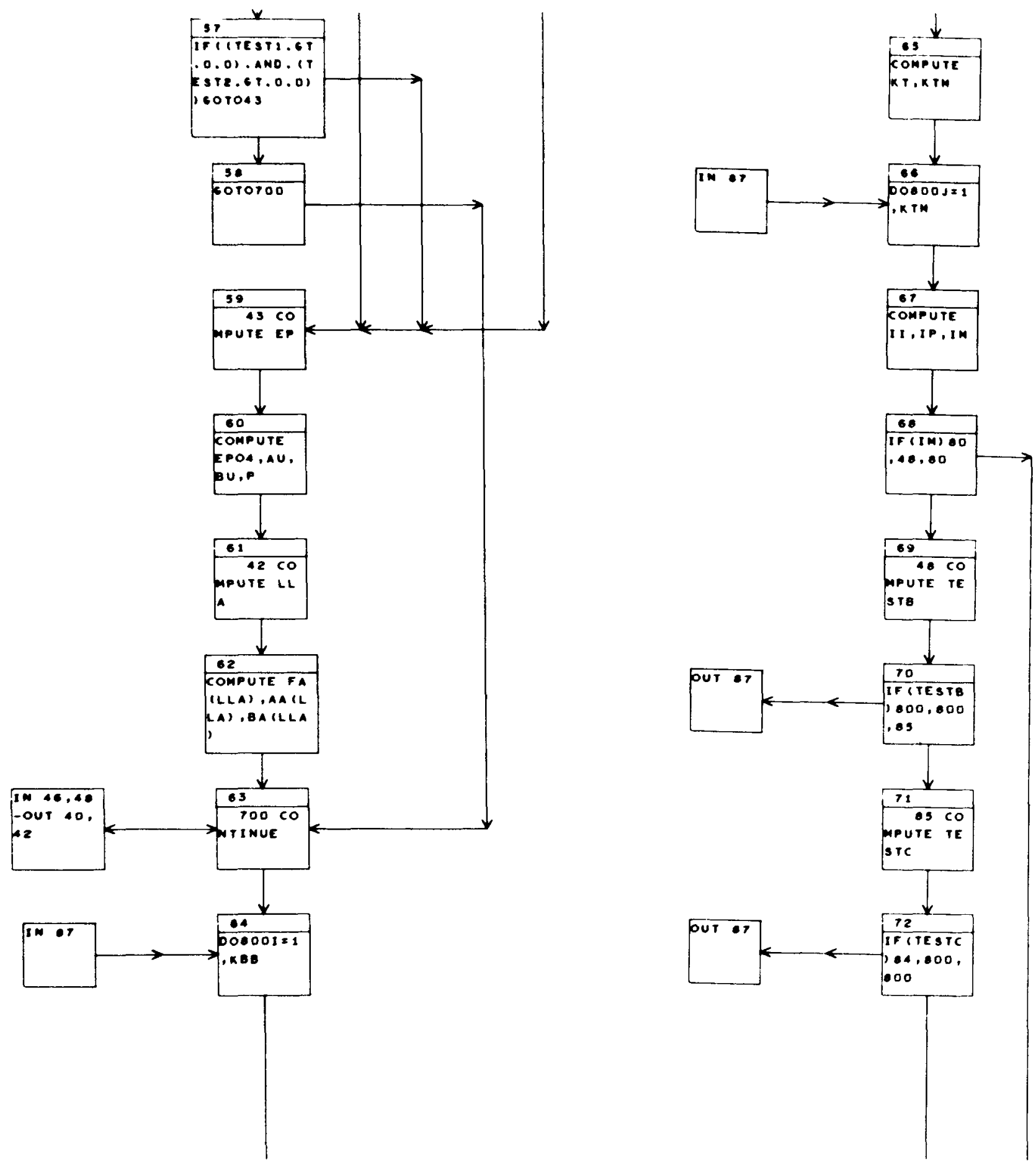
RN-S-0146
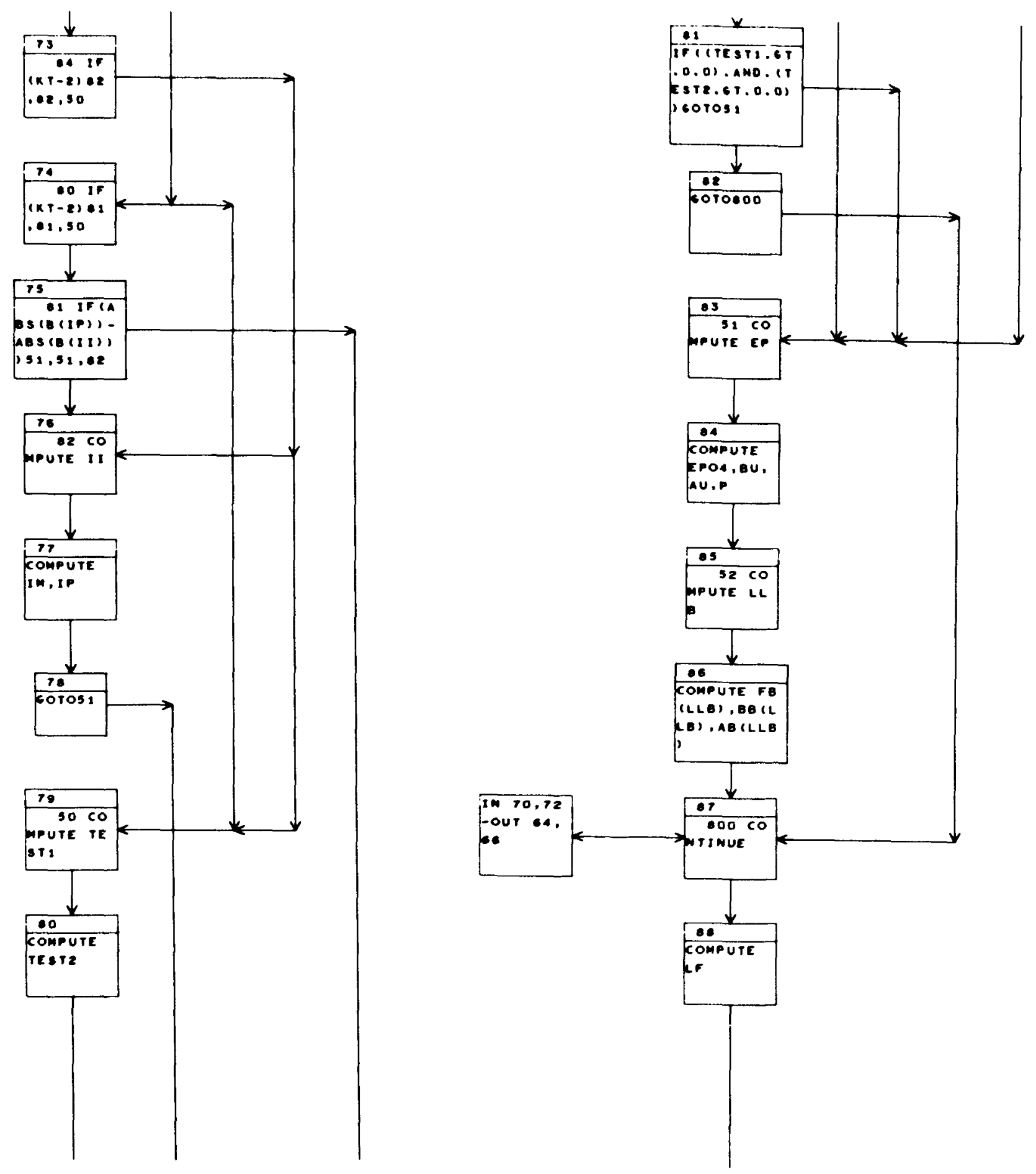
RN-S-0146
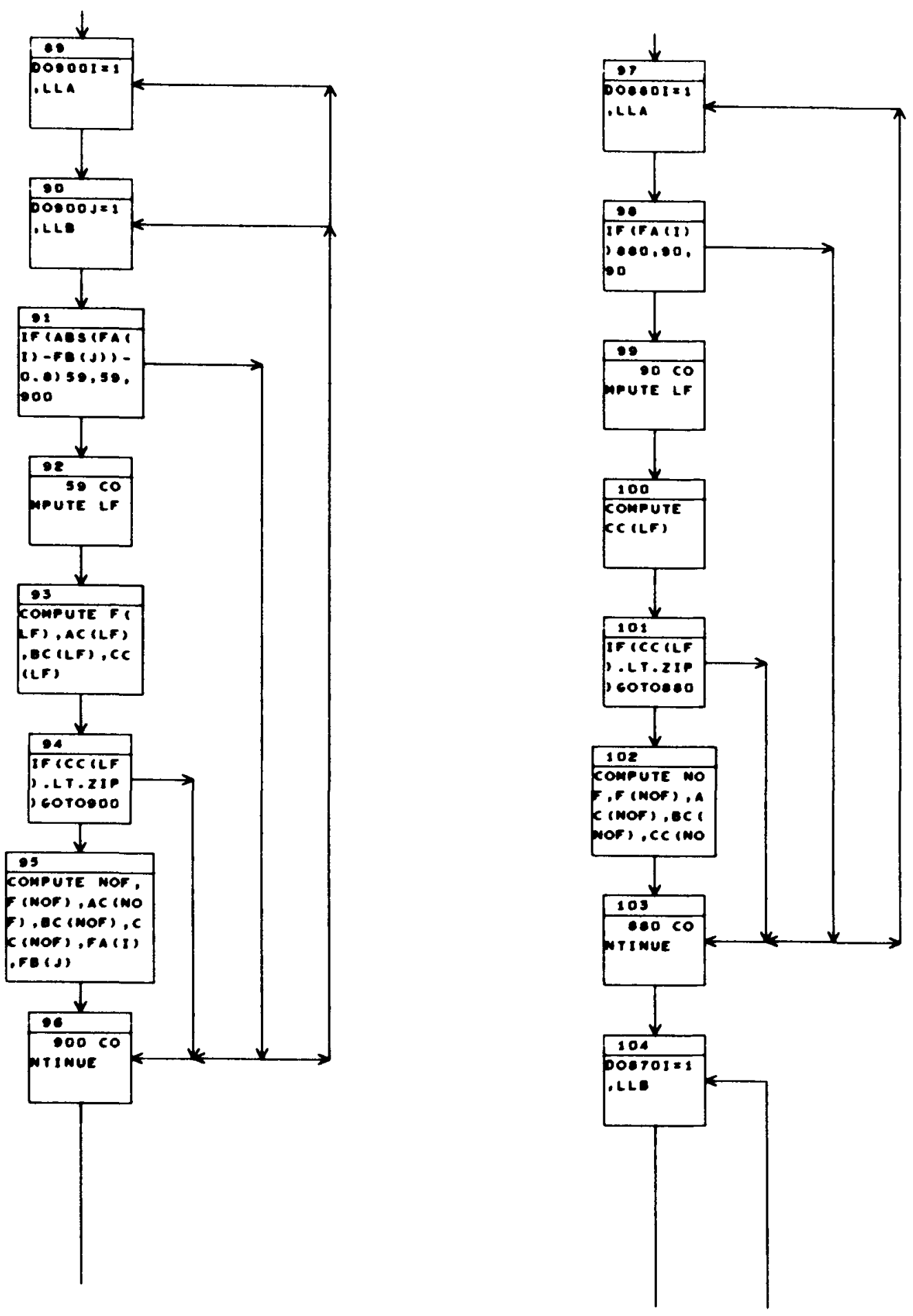

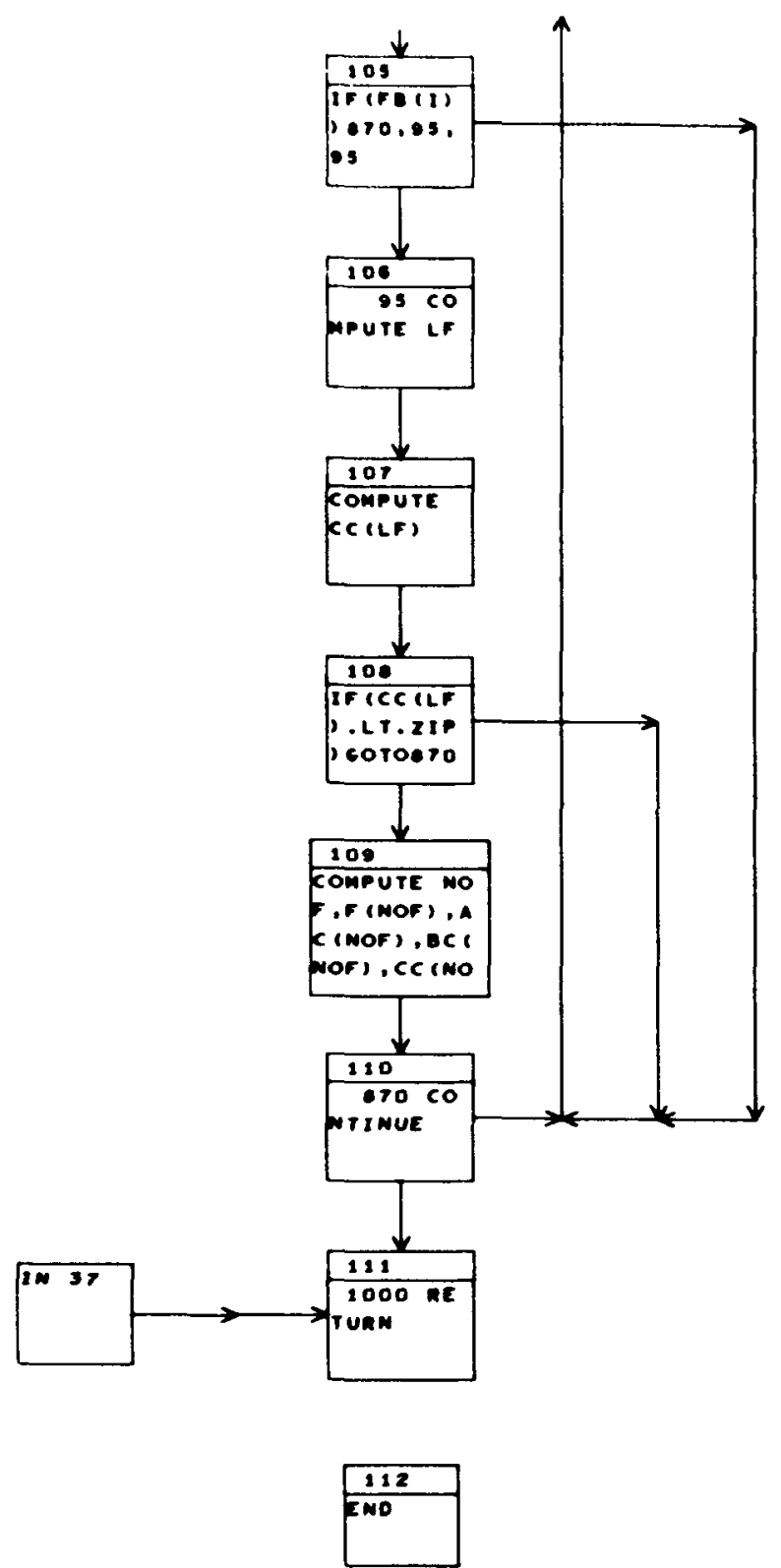
RN-S-O146

\section{APPENDIX B}

STATISTICAL EDIT AND SMOOTH PROGRAM 


\section{$\mathrm{RN}-\mathrm{S}-0146$}

CONTENTS, APPENDIX B

Page No.

Abstract

B-ii

I. STATEMENT OF PROBLEM

B-1

II. DESCRIPTION OF PR $\varnothing \mathrm{GRAM}$

B-2

III. PROGRAM MATHEMATICS

B-8

IV. INPUT REQUIREMENTS

B-II

V. PR $\emptyset$ GRAM LIMTTATIONS

B-12

VI. OPERATIONAL PROCEDURE

B-12

VII. OUTPUT B-12

VIII. DERIVATION OF FACTORS TO ADJUST SUCCESSIVE ESTIMATES OF THE COEFFICIENTSS OF THE SMOOTHING FUNCTION

B-15

IX. DICTIONARY OF FØRTRAN TERMS

B-19

X. LISTING OF PROGRAM SOURCE DECKS

B-23

XI. RECOPY PROGRAM FOR NERVA N3 DATA TAPES

B- 41

XII. DETAIL FLOW DIAGRAM OF STATISTICAL EDIT AND SMOOTH PROGRAM

B- 48 
$\mathrm{RN}-\mathrm{S}-0146$

\section{ABSTRACT}

This program edits the NERVA N3 data to: (1) identify missing time values; (2) identify and correct outlying (erroneous) observations; and (3) identify significant shifts in the trend of the data. Editing of the time for missing values is done by differencing successive values over desired time intervals. Editing of data values is done by fitting a 3rd-degree polynomial to the data, extrapolating the polynomial one point advanced in time, estimating the standard deviation of the extrapolated point, and comparing the observed and extrapolated values on a statistical basis. The judgment of a significant change in the trend of the data is made when these two values significantly differ over ten successive times. Smoothing of the data is provided by evaluating the polynomial near its midpoint at a point corresponding to a desired output time. This assures that corresponding smoothed values for all channels will be for identical times. In addition, the program provides an estimate of the random component of variance in the time series. 


\section{STATEMENT OF PROBIEM}

Prior to its receipt at Aerojet, the NERVA N3 data had been converted to digital form, corrected for calibration, and transformed into engineering units.

The data is contained on one or several reels of tape, and is broken into single records for each channel sampled. Fach record begins with alphanumeric information identifying the particular channel with information contained on the REC and TITLE cards from the RDH-D code. The record cortinues with ordered (although not necessarily consecutive) values of time and data. A further description of the formating of the $\mathrm{N} 3$ data, along with the programs which generate this data, is contained in a Los Alamos Scientific Laboratory Report.

Although the degree of data processing and the formating of the N3 data tapes are adequate for plotting purposes, they are inadequate for use as direct input to data analysis programs for the following reasons:

A. Missing time points are completely ignored.

B. No attempt has been made to edit the data to identlfy and correct outlying observations.

C. Time values for different data channela do not agree. Hence there is the possibility that some chanrels will be sampled at rates differing from those of cther channels.

D. No smoothing of the data has been provided. Smoothing has the effect of reducing the random component of variance. This is very desirable when the data is to be used as direct input in many data analysis programs.

\footnotetext{
${ }^{*}$ F. Narin, Computer Data Processing System, Project Rover, IAMS-2845, Ios Alamos Scientific Laboratory, 1962.
} 
$\mathrm{RN}-\mathrm{S}-\mathrm{Cl} 46$

E. Most data analysis programs require the data format to be all channel recordings for a single point in time as opposed to the present formating.

Deficiencies A through $D$ are overcome by the present program; deficiency $E$ is considered in a subsequent program, Sort and Merge (Job No. 509). The present program dces the following things:

Edits the time values to locate and identify any missing time values.

Edits the data observations to: (a) locate, identify and correct outlying observations; and (b) locate and identify radical shifts in the trend of the data.

Smooths the observed data to reduce the random component of variance in the "smoothed" sequence of observations and includes as a packed portion of the smoothed observation an estimate of relative error of that measurement.

Adjusts the outputted timed sequence of data to assure that all corresponding data points from different channels are for identical times.

II. DESCRIPIION OF PROGRAN:

Each test usually consists of several runs which follow back to back within each channel even though the tImes of successive runs may be several hours apart. Since it may not always be desirable to process all runs, the program must have some way of identifying the desired runs. This is done by inputting the startstop times (TMIN - TMAX) for each desired time interval. However, since the program must also know the desired times for outputting smoothed data values, the IMIN - TMAX values represent those start-stop times for each interval. consequently the TMIN - TMAX times inputted must be contained within those corresponding times on tape. Within each channel the TMIN - TMAX intervals control the major looping. After the program:(a) reads the desired TMIN - TMAX intervals; (b) initializes program constants; and (c) computes the orthogonal weights and adjusting 
coefficients which will be used to fit the smoothing function to the data, it then computes and stores on tape (as dummy channel number 998) all desired times for outputting smoothed data values. This is done by looping through the TMIN TMAX intervals, incrementing time by DELTAT (the desired time between successive outputs). The major purpose for this is to record the actual output times only once and associate with each an outputted integer time. In a later phase of processing wich aligns all corresponding channel data with time, merging of the channels requires integer time values. Also provided at this point is an option to shift this sequence of time values by one and store on tape (as a second dummy channel number 999) the values of the next time on tape. This is very useful in an analysis program using the data as input to judge the beginning and end times for different runs.

Processing of the channel data begins with reading from cards the channel association number (NCHB6), and the upper and lower limits for the data (YHIGH and YLOW). One purpose of the NCHB6 number, other than that of identifying the channel, is to determine when to terminate processing of the tape. To terminate processing, a card is supplied with NCHB6 equal to 9999. When this is encountered, a special end of file is written on the output tape, the tapes are rewound, and the program pauses to allow dismounting of the input-output tapes. The purpose of YHIGH and YIOW is to define full scale. The standard deviation of the smoothed outputted value is expressed as a percentage of full scale.

The title information and data are read from binary tape A5, channel flags and counters are initialized, and the channel sampling rate is calculated.

As mentioned previously, one of the deficiencies in the data is that isolated time values may be completely missing. Not only should such occurrences be located and identified, but it is desirable to substitute a value for those times so as to maintain a uniform time scale. A uniform time scale permits the smoothing function to be fitted to the data using orthogonal polynomials (a much more rapid computational procedure than would otherwise be possible).

These needs are provided by Subroutine YUK YÜK. This subroutine brings in data from $T(I)$ and $Y(I)$ - the storage locations for the original data. It also 


$$
\mathrm{RN}-\mathrm{S}-0146
$$

checks for missing time values and loads two temporary tables - TAK(I) and YAK(I). If a missing time point is observed, the value of that time point is placed in TAK and -1.El6 is placed correspondingly in YAK. The tables TAK and YAK are used in fitting the smoothing function. In editing the data the value -1.El6 will certainly be identified as an outlier, and hence missing time points can be identified in the outputted list of outliers by this unique value of original data.

A convenient method of editing the series for outlying values is to: (a) apply a moving polynomial curve fit to the data; (b) estimate the expected value, YNEXT, and standard duration, SYNEXT, of the next point removed from the fitted set; (c) compute the normalized ratio

$$
R E S=\frac{\mid Y(\text { observed })-Y N E X T \mid}{\text { SYNEXT }}
$$

and (d) compare RES with some preassigned constant or constants. If RES exceeds this preassigned constant, either of the two following conclusions can be drawn: (a) the observed value at the next point in time is an outlier with respect to the preceding set and should be ignored, or (b) the functional form of the data is changing (or has changed) and the fitted polynomial no longer is an adequate representation of this function. If the first alternative were true, one would expect the value of RES to be somewhat larger than that for the second alternative (with the exception of cases involving large discontinuous steps in the function). Moreover, since outliers are considered to be random happenings, one would not expect to find a lengthly series of successive occurrences. However, in the case of a change in functional form, one would expect a lengthly series of successive occurrences.

To distinguish between the two outcomes, the program uses two critical values of RES, CRITI, and CRIT2 - with CRITl being smaller than CRIT2. (CRITI and CRIT2 were set at 3 and 4.5 , respectively, for use with the NRX-Al data.) A test is first made to compare RES with CRITI. If RES exceeds CRITI, a counter, K申UNT, is advanced to denote the possibility of a change in functional form, and 
the program advances on to compare RES with CRIT2. If RES is less than CRITI, $K \emptyset$ UNT is set to zero; Y (observed) is considered to be a valid point and will be considered as the next data value in the fitting set as the polynomial slides along the time axis. The test of RES VS CRIT2 is to consider if Y(observed) is to be considered as an outlier. If RES is greater than CRIT2, Y(observed) is considered to be an outlier and will be replaced in the series by YNEXT. If RES is less than CRIT2, no substitution is made, and $Y$ (observed) will be used in the next sequence.

The decision that the fitted polynomial is no longer a valid representation of the functional form of the series is made when the value of K申UNT reaches ten. When this occurs, the program restores all substituted values starting where $K \phi U N T$ was equal to one. A new polynomial is then fitted to the next (NSPAN) set of data points beginning with this time value.

Smoothing of the data at the desired times is provided by evaluating the polynomial at the point where the time at the midpoint of the smoothing set just exceeds the time of desired output. The standard deviation of the smoothed value is estimated by the standard error of estimate divided by the length of the smoothing span. These values, along with a provision for error flags, are packed into a single word and stored in table $Y \phi_{\perp}$, to be outputted when the number of entries, IMNUN, reaches 128 .

A generalized block diagram of the program is presented in Figure B-1. 


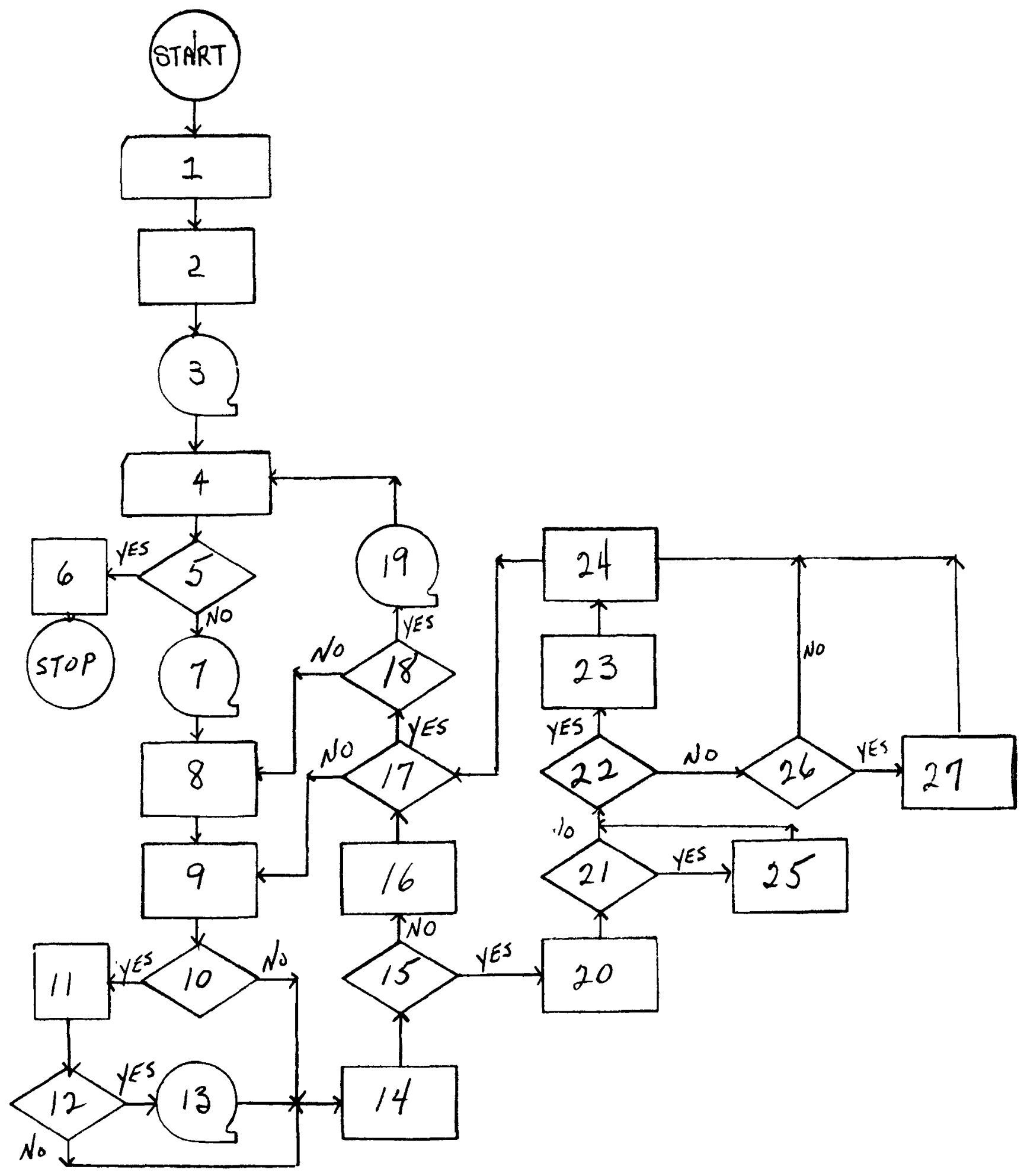

Figure B-1

Block Diagram of Statistical

Edit and Smooth Program 
1. Read control cards and initialize program constants.

2. Compute the orthogonal weights and adjusting coefficients used to fit the smoothing function to the data.

3. Compute and store on tape as dummy channel 998 all desired times for outputting smoothed data values, and, if desired, compute and store on tape as a second dummy channel 999 the times associated with data values one step in advance (the time for the next set of data values to be outputted).

4. Read the channel association number, and the upper and lower limits for the data of the channel to the processed.

5. Is the channel association number equal to 9999 ?

6. If yes, write end of file on output tape, rewind input and outputs, and call exit.

7. If no, read channel data from binary tape, initialize channel flags and counters, and compute the channel sampling rate constants.

8. Call subroutine YUK YUK to load temporary tables which are used in fitting the smoothing function. The times values are edited in this routine for missing times, and if discovered the correct time value is inserted and -1.E16 supplied for the data value. This is done to maintain a uniform time scale and ensure that the supplied data value will be counted as an outlier when the data values are edited.

9. Call subroutine FIT to fit a 3rd degree polynomial to NSPAN points of data, extrapolate to estimate the value at the next point in time, and estimate the variance of that estimate.

10. Has the time at the midpoint of the span exceeded the desired output time?

11. If yes, call SM $\phi \phi \mathrm{TH}$ to compute the smoothed value corresponding to the desired output time; obtain the appropriate estimate of the relative error in that smoothed value; determine appropriate error flags; pack this information into a single word, and store data word in temporary output table.

12. Has output table built up to 128 entries?

13. If yes, call BARF to write output table on binary tape.

14. If no, compute the absolute difference between the observed value at the next point in time and the corresponding estimated value (residual).

15. Is the residual greater than CRIT 1 times the standard deviation of the estimated value?

16. If no, set $K \phi U N T$ equal to zero, and update appropriate indices.

17. Has all the data in the temporary fitting table been interrogated?

18. Has all the data for the channel been interrogated?

19. If yes, call BARF to write any remaining entries in output table on binary tape.

20. If yes, increase K $\phi \mathrm{UNT}$ by one, and store suspicious data value in temporary table.

21. Is residual greater than CRIT 2 times the standard deviation of the estimated value?

22. If no, is K $\phi$ UNT equal to one?

23. If yes, save time value of suspicious data value.

24. Update appropriate indices.

25. Substitute estimated value of data point for the observed value, set appropriate error flags, and write out error message.

26. Is KфUNT equal to ten?

27. Write out error message, smooth to fill in for all required output times, and reset indices to begin new span. 
$\mathrm{RNV}-\mathrm{S}-0146$

\section{PROGRAM MATHEMATICS}

As was stated in the previous section, both editing and smoothing depend on fitting a moving polynomial to the observed data. For the NERVA NRX-Al data it was assumed that a $3 r d$ degree polynomial would adequately represent the functional form of the data over any small interval of time.

$$
y_{t} \approx b_{0}+b_{1} t+b_{2} t^{2}+b_{3} t^{3}
$$

In fitting this polynomial to the data, special attention is given to the fact that the data come at equal intervals of time. For such cases, it is possible to transform the time scale by the transformation

$$
u_{i}=\frac{t_{i}-t_{m}}{w}
$$

where $t_{m}$ is the midpoint of $N$ (odd) time values and $w$ is the time increment so as to reduce it to a sequence of the form

$$
-\frac{N-1}{2},-\frac{N-3}{2}, \ldots,-1,0,1, \ldots, \frac{N-1}{2} \text {. }
$$

This enables the polynomial to be expressed as

$$
y_{u}=A_{0} \phi_{0}(u)+A_{1} \phi_{1}(u)+A_{2} \phi_{2}(u)+A_{3} \phi_{3}(u)
$$

where the $A_{i}(i=0,1,2,3)$ are constants and the $\phi_{i}(u)$ are the orthogonal polynomials. *

$$
\begin{aligned}
& \phi_{0}(u)=1 \text { for } u=-\frac{N-1}{2}, \ldots, 0, \ldots \frac{N-1}{2} \\
& \phi_{1}(u)=u \\
& \phi_{2}(u)=u^{2}-\frac{1}{12}\left(N^{2}-1\right) \\
& \phi_{3}(u)=u^{3}-\frac{u}{20}\left(3 N^{2}-7\right) .
\end{aligned}
$$

\footnotetext{
* See Pearson and Hartley, Biometrika Tables for Statisticians, Vol. I, Cambridge University Press, 1958.
} 
The least squares estimates of the $A_{i}$ are given by

$$
A_{i}=\frac{\sum_{t} y_{t} \phi_{i}\left(u_{t}\right)}{\sum_{t}\left\{\phi_{i}\left(u_{t}\right)\right\}^{2}}
$$

and the standard error of estimate by

$$
s_{e}=\left\{\frac{\sum_{t} y_{t}^{2}-\sum_{i}\left\{A_{i} \sum_{t}\left[\phi_{i}\left(u_{t}\right)\right]^{2}\right\}}{N-4}\right\}^{1 / 2} \text {. }
$$

To extrapolate this polynomial one point advanced in time we merely evaluate it using the estimated coefficients and the values of $\phi_{i}(u)$ for $u=\frac{N+I}{2}$. The standard error of this estimate used in making statistical comparisons is given by

$$
s_{\hat{y}}=s_{e}\left\{1+\frac{1}{N}+\sum_{i} \frac{\left[\phi_{i}\left(\frac{N+1}{2}\right)\right]^{2}}{\sum_{t}\left[\phi_{i}\left(u_{t}\right)\right]^{2}}\right\}^{1 / 2}
$$

If $t_{m}$ is defined as the time at the midpoint of the smoothing set, and $t_{0}$ is the desired time of output, then smoothing is performed at the time when $t_{0}-t_{m}$ becomes positive. Smoothing is done by evaluating the polynomial using the estimated coefficients, and the values of $\phi_{i}(u)$ for $u=\left(t_{0}-t_{m}\right) / w$. The relative error in the new series of smoothed values is expressed as

$$
\frac{200 * s_{e}}{\text { FULSKL * N }}
$$

The smoothed data value, this estimate of relative error, and a provision for flags are then packed into a single word for outputting.

As the smoothing set is slid along the time axis, successive estimates of the $A_{i}$ need not be calculated by Equation (6). In viewing Equation (6) it can be seen that the denominators of the estimates depend only on the values of the orthogonal polynomials, and hence are constant over all sets of data values. Moreover, as is illustrated by Figure B-2, successive data spans differ by only two data points; the deletion of $\mathrm{y}_{-\mathrm{n}}$ and the addition $\mathrm{y}_{\mathrm{n}+\mathrm{l}}$. Based upon this relationship between successive sets of estimates, an algorithm was developed to allow adjusting successive estimates of the $A_{i}$ in such a manner as to considerably reduce the amount of calculations required. These adjusting coefficients and their deviation are given in Section VIII. 
RN-S-0146
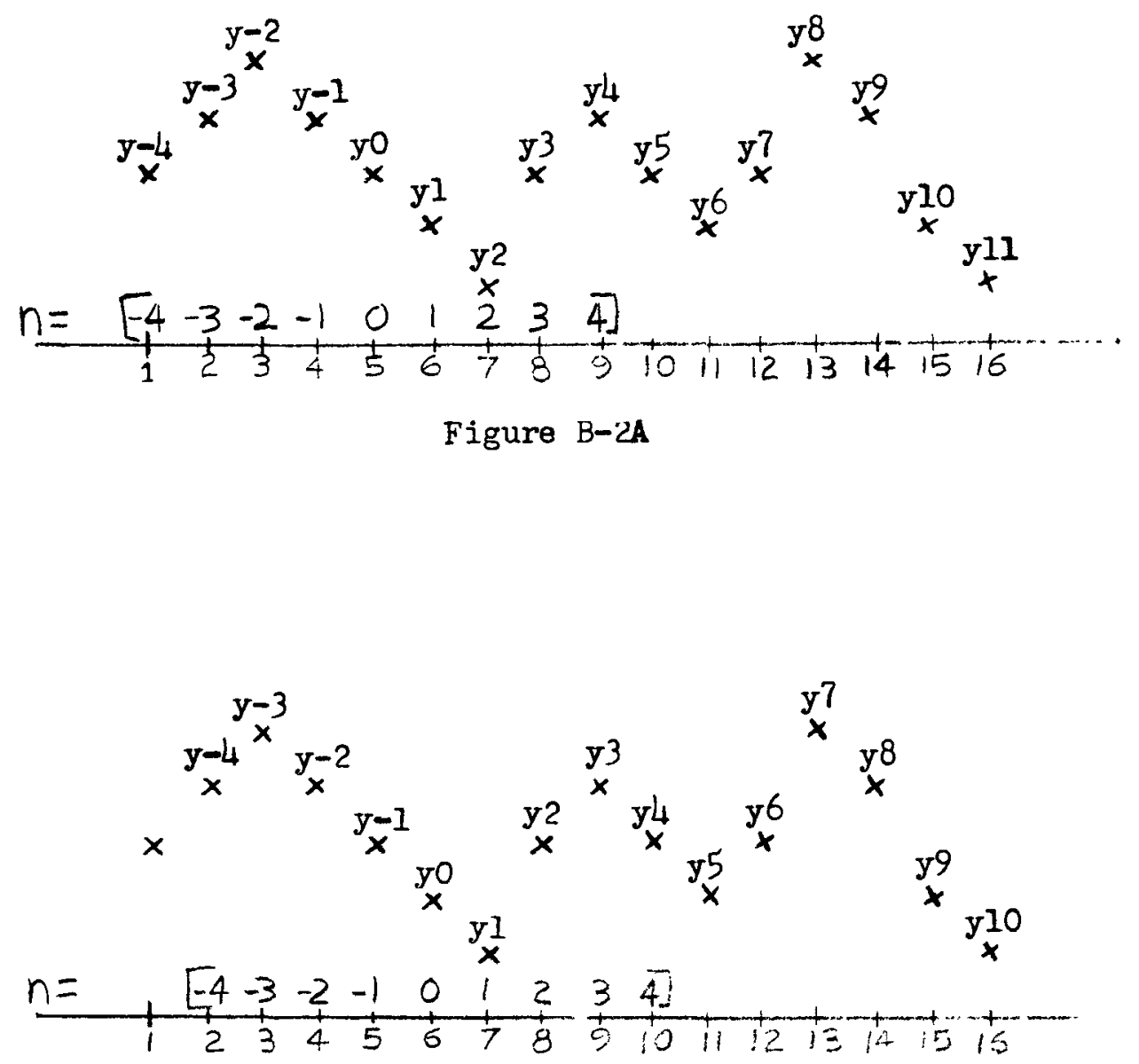

Figure $B-2 B$

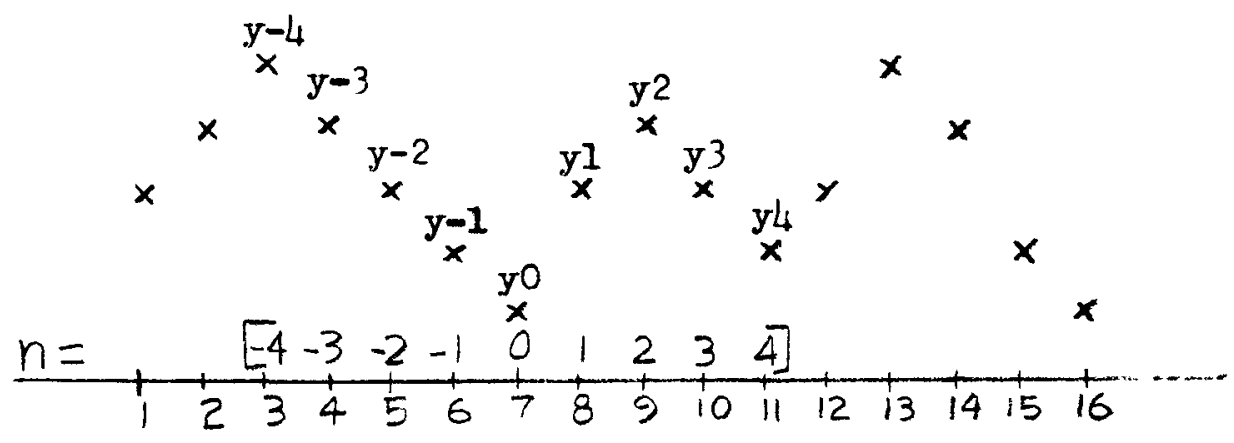

Figure B-2C

Illustration of Smoothing Process 


$$
\mathrm{RN}-\mathrm{S}-0146
$$

IV. INPUT REQUIREMENTS

The following lists state the order and format of the required input cards for processing of each tape.

A. PROBIEM CARD

\begin{tabular}{|c|c|c|}
\hline Colums & Variable Name & Description \\
\hline $1-6$ & KBAT & $\begin{array}{l}\text { The number of TMIN-TMAX intervals } \\
\text { to be processed. }\end{array}$ \\
\hline $7-12$ & $I \phi \mathrm{P}$ & $\begin{array}{l}\text { An option code to store on the out- } \\
\text { put tape, as dummy channel } 999 \text {, the } \\
\text { value of the next point in time. } \\
\text { I } \phi \mathrm{P}=0 \text {, do not store; I } \phi \mathrm{P} \neq 0 \text {, } \\
\text { do store. }\end{array}$ \\
\hline $13-18$ & DELTAT & $\begin{array}{l}\text { The desired time interval. (seconds) } \\
\text { between successive smoothed output } \\
\text { values. }\end{array}$ \\
\hline
\end{tabular}

B. TIME INTERVAL CARD (S )

Colums Variable Name

Description

$1-18 \quad \operatorname{TMTN}(I)$

19-36 TMAX(I)

The desired start time for output of the i-th time interval to be processed.

The desired stop time for output of the $i$-th time interval to be processed.

C. CHANNEL IDENTIFICATION $\operatorname{CARD(S)}$

Columns Variable Name

Description

\begin{tabular}{|c|c|c|}
\hline $1-6$ & NCHB6 & $\begin{array}{l}\text { The channel association number on } \\
\text { the } B 6 \text { tape. }\end{array}$ \\
\hline $7-12$ & YL $\phi W$ & $\begin{array}{l}\text { The lower limit on the theoretical } \\
\text { range of recordings for this } \\
\text { channel. }\end{array}$ \\
\hline $13-18$ & YHIGH & $\begin{array}{l}\text { The upper limit on the theoretical } \\
\text { range of recordings for this } \\
\text { channel. }\end{array}$ \\
\hline
\end{tabular}




$$
\mathrm{RN}-\mathrm{S}-0 \mathrm{I} 46
$$

D. FINAL CARD

$\underline{\text { Columns }}$

$$
1-4
$$

\section{PROGRAM LIMITATIONS}

The following limitations are based upon the program in its form of I september 1964:

A. The input data must be in the format of the NERVA $N 3$ tapes, but must be in a format acceptable by FORTRAN IV. The NERVA N3 NRX-AI tapes were in a format for FфRTRAN II. Section XI describes a recopy program used for the NRX-Al data to reformat those tapes.

B. The number of input-output (TMIN-TMAX) intervals cannot exceed 9 .

C. The number of significant changes in the shape of the curve cannot exceed 50 .

D. With the exception of isolated missing time values, the time code must be correct. No other provisions are made to edit and correct time values.

\section{OPERATIONAL PROCEDURE}

The program, as listed in Appendix C, was written to be run on an IBM 7094 . Programming was done in FORTRAN IV - MAP. The program requires two speciaI tape drives. The input data (N3 data tape) is to be placed on drive B6 (logical tape 12) and the outputted series of smoothed values is outputted on drive A5 (logical tape 9). Pause statements are supplied to permit time for hanging and dismounting of these tapes.

\section{OUTPUT}

For each channel processed, the program outputs:

A. ON THE REGULAR OUTPUT TAPE

1. A listing of time, original data, and corrected data for each point judged to be an outlier. RES $>$ CRIT 2

2. A listing of time and original data at those points where it was judged that the shape of the curve had changed. 


\section{$\mathrm{RN}-\mathrm{S}-0146$}

If neither of these happenings occur, this is also noted. A sample output for one channel is given in Figure B-3.

B. ON BINARY TAPE A5 IN THIS ORDER:

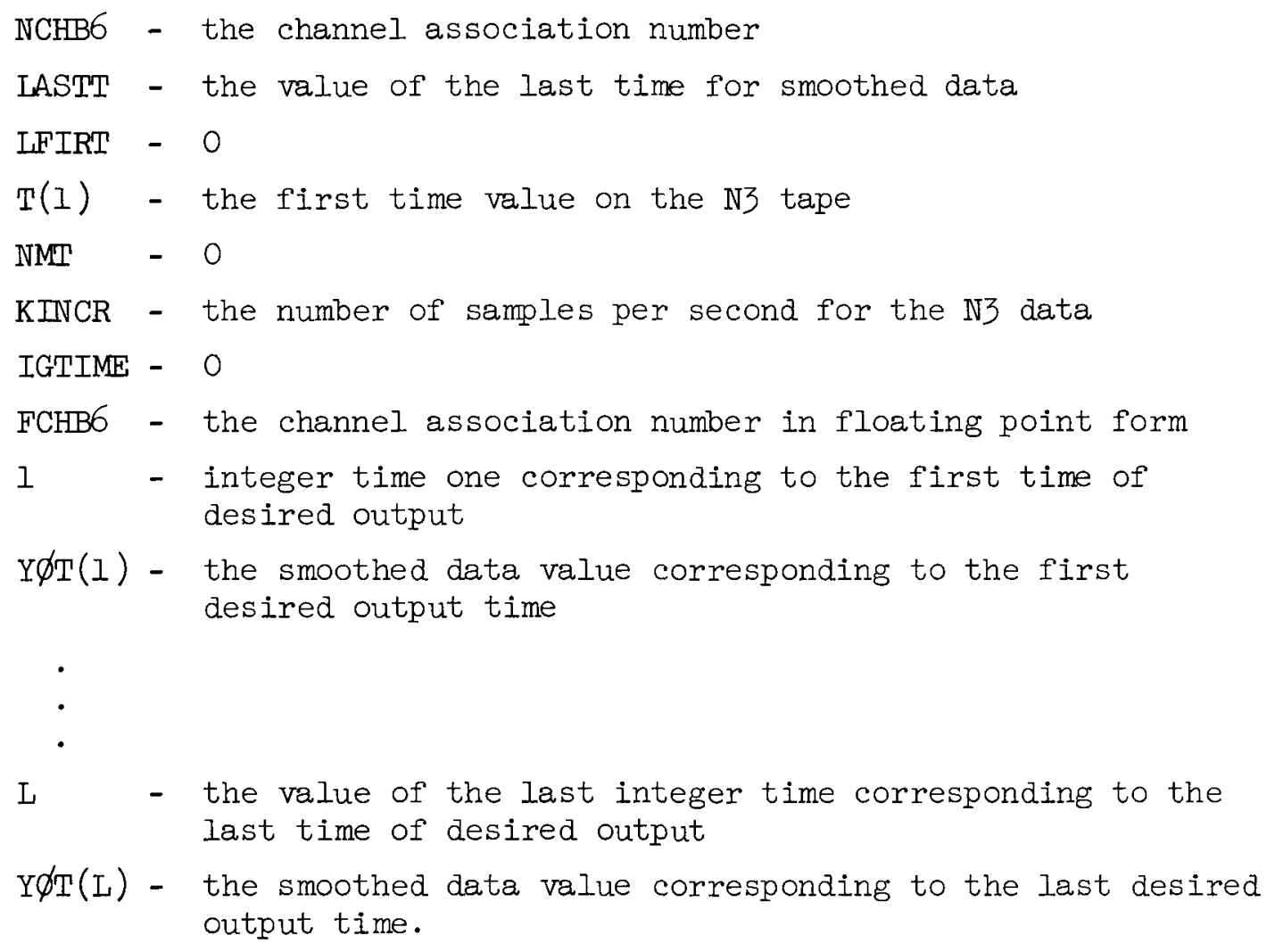

The quantities NCHB6, IASTT, IFIRT, T(I), NMT, KINCR, and IGTIME are supplied because they are (or were at time of first programming) needed by $J \emptyset \mathrm{B}$ No. 509, $S \varnothing \mathrm{RT}$ and MERGE.

After all channels have been processed, the program writes a 256 word record of BCD ENDFIL. This is also done to satisfy a need of J $\varnothing \mathrm{B}$ No. 509.

C. ON-LINE PRINTER

As each channel is processed, an on-line message will give the channel association number and time of processing in seconds. 
CHANNEL NO.

5

THE FOLLOWING POINTS WERE JUUGED OUTLIERS AND WERE CORRECTED

(ORIGINAL DATA EQUAL TO - IE 16 REPRESENT MISSING ORIGINAL VALUES)

\section{TIME DRIGINAL DATA CORRECIED DATA}

$\begin{array}{lll}15463.61 & 160.80529185 & 214.83481598 \\ 15463.81 & 166.63207436 & 219.66547585 \\ 15464.01 & 179.36704254 & 226.02061272 \\ 15464.21 & 155.62575722 & 234.15541458 \\ 15464.41 & 139.67620087 & 243.35387230 \\ 15464.61 & 142.56316949 & 253.28547096 \\ 15464.81 & 141.06509209 & 263.11946487 \\ 15465.01 & 102.32545185 & 273.52293396 \\ 15465.21 & 115.83861542 & 284.22933960\end{array}$

CHANNEL NO.

5

SIGNIFICANT CHANGES IN THE SHAPE (OR VARIANCE) OF THE CURVE WERE LOCATED AT TIME ORIGINAL DATA

15463.41137695

200.05558014

Sample Output From Statistical Edit and Smooth Program 
VIII. DERIVATION OF FACTORS TO ADUUST SUCCESSIVE ESTIMATES OF THE COEFFICIENTS OF THE SMOOTHING FUNTCTION

Consider the cubic smoothing function

$$
y(t)=b_{0}+b_{1} t+b_{2} t^{2}+b_{3} t^{3}
$$

where $y$ is data and $t$ is time. If the t's progress by constant intervals, the $t$-scale can be standardized by a simple transformation, and $y(t)$ can then be fitted as a weighted sum of orthogonal polynomials

$$
y(t)=a_{0} \phi_{0}(u)+a_{1} \phi_{1}(u)+a_{2} \phi_{2}(u)+a_{3} \phi_{3}(u) \text {. }
$$

For a span of $N=2 n+I$ points, the $\phi_{i}(u)$ considered are given by

$$
\begin{aligned}
& \phi_{0}(u)=1 \\
& \phi_{1}(u)=u \\
& \phi_{2}(u)=u^{2}-\left(\mathbb{N}^{2}-1\right) / 12 \\
& \phi_{3}(u)=u^{3}-\frac{u}{20}\left(3 N^{2}-7\right),
\end{aligned}
$$

where

$$
u=-n, \cdot ., \quad o,+n
$$

The least-squares estimates of the $a_{i}$ are given by the relations

$$
\begin{aligned}
& a_{0}=N^{-1} \sum_{i=-n}^{n} y_{i} \\
& a_{1}=\frac{\sum_{i=-n}^{n} i y_{i}}{\sum_{i=-n}^{n} i^{2}} \\
& a_{2}=\frac{\sum_{i=-n}^{n}\left(i^{2}-\frac{N^{2}-1}{12}\right)_{i}^{y_{i}}}{\sum_{i=-n}^{n}\left(i^{2}-\frac{N^{2}-1}{12}\right)^{2}}
\end{aligned}
$$




$$
a_{3}=\frac{\sum_{i=-n}^{n}\left[i^{3}-\frac{i}{20}\left(3 N^{2}-7\right)\right] y_{i}}{\sum_{i=-n}^{n}\left[i^{3}-\frac{i}{20}\left(3 N^{2}-7\right)\right]^{2}}
$$

From these relations it is easily seen that for successive estimates of these quantities only the numerators will change; the denominators depend only on the length of the smoothing span, $\mathbb{N}$, and remain constant for all successive estimates.

Denoting $a_{0}^{\prime}$ as the next successive estimate of $a_{0}$, then

$$
\begin{aligned}
a_{0}^{\prime} & =\frac{\sum_{i=-n+1}^{n+1} y_{i}}{N} \\
& =\frac{\sum_{i=-n}^{n} y_{i}+y_{n+1}-y_{-n}}{N} \\
& =a_{0}+\frac{y_{n+1}-y_{n}}{N}
\end{aligned}
$$

Denoting $a_{1}^{\prime}$ as the next successive estimate of $a_{1}$, and its constant denominator by $A$, then

$$
a_{1}^{\prime}=\frac{\sum_{i=-n+1}^{n+1}(i-1) y_{i}}{A}
$$

The numerator of $a_{I}^{\prime}$ reduces as

$$
\sum_{i=-n+1}^{n+1}(i-1) y_{i}=\sum_{i=-n}^{n} i y_{i}+(n+1) y_{n+1}+n y_{-n}-N a_{0}^{\prime}
$$


$\mathrm{RN}-\mathrm{S}-0146$

Hence,

$a_{1}^{\prime}=a_{1}+\frac{(n+1) y_{n+1}+n y_{-n}-N a_{0}^{\prime}}{A}$

Denoting $a_{2}^{\prime}$ as the next successive estimate of $a_{2}$, and its constant denominator by $B$, then

$a_{2}^{\prime}=\frac{\sum_{i=-n+1}^{n+1}\left[(i-1)^{2}-\frac{\left(N^{2}-1\right)}{12}\right] y_{i}}{B}$

The numerator of $a_{2}^{\prime}$ reduces to

$$
\begin{aligned}
& \sum_{i=-n}^{n}\left[i^{2}-\frac{\left(N^{2}-1\right)}{12}\right] y_{i}+\left[(n+1)^{2}+\frac{\left(N^{2}-1\right)}{12}\right] y_{n+1} \\
& -\left[n^{2}-\frac{\left(N^{2}-1\right)}{12}\right] y_{-n}-2 \sum_{i=-n+1}^{n+1} i y_{i}+\sum_{i=-n+1}^{n+1} y_{i},
\end{aligned}
$$

so

$$
\begin{aligned}
a_{2}^{\prime} & =a_{2}+\left\{\left[(n+1)^{2}+\frac{\left(N^{2}-1\right)}{12}\right] y_{n+1}-\left[n^{2}-\frac{\left(N^{2}-1\right)}{12}\right] y_{-n}\right. \\
& \left.-2 A a_{1}^{\prime}-N a_{0}^{\prime}\right\} / \mathrm{B}
\end{aligned}
$$

Denoting $a_{3}^{\prime}$ as the next successive estimate of $a_{3}$, and its constant denominator by $\mathrm{C}$, then

$$
a_{3}^{\prime}=\frac{\sum_{i=-n+1}^{n+1}\left[(i-1)^{3}-\frac{(i-1)}{20}\left(3 N^{2}-7\right)\right] y_{i}}{c}
$$

The numerator of $a_{3}^{\prime}$ reduces to 
$\mathrm{RN}-\mathrm{S}-\mathrm{O} \mathrm{I} 46$

$$
\begin{aligned}
& \sum_{i=-n+1}^{n+1}\left[i^{3}-\frac{i}{20}\left(3 N^{2}-7\right)\right] y_{i}-3 \sum_{i=-n+1}^{n+1}\left[(i-1)^{2}-\frac{1}{12}\left(N^{2}-1\right)\right] y_{i} \\
& -3 \sum_{i=-n+1}^{n+1}(i-1) y_{i}+\left[\frac{1}{20}\left(3 N^{2}-7\right)-\frac{1}{4}\left(N^{2}-1\right)-1\right] \sum_{i=-n+1}^{n+1} y_{i}
\end{aligned}
$$

Hence,

$$
\begin{aligned}
a_{3}^{\prime} & =a_{3}+\left\{\left[(n+1)^{3}-\frac{n+1}{20}\left(3 N^{2}-7\right) y_{n+1}\right.\right. \\
& +\left[n^{3}-\frac{n}{20}\left(3 N^{2}-7\right)\right] y_{-n}-3 B a_{2}^{\prime} \\
& \left.-3 A a_{1}^{\prime}+N\left[\frac{1}{20}\left(3 N^{2}-7\right)-\frac{1}{4}\left(N^{2}-1\right)-1\right] a_{0}^{\prime}\right\} / C .
\end{aligned}
$$

In estimating the standard error of estimate it is necessary to calculate the total sum of squares of the $y^{\prime} s$. This quantity can likewise be adjusted for successive calculations by

$$
\mathrm{SSY}^{\prime}=\mathrm{SSY}+\mathrm{y}^{2} \mathrm{n+1}-\mathrm{y}^{2}-\mathrm{n}
$$


$\mathrm{RN}-\mathrm{S}-0146$

IX. DICTIONARY OF FORTRAN TERMS 


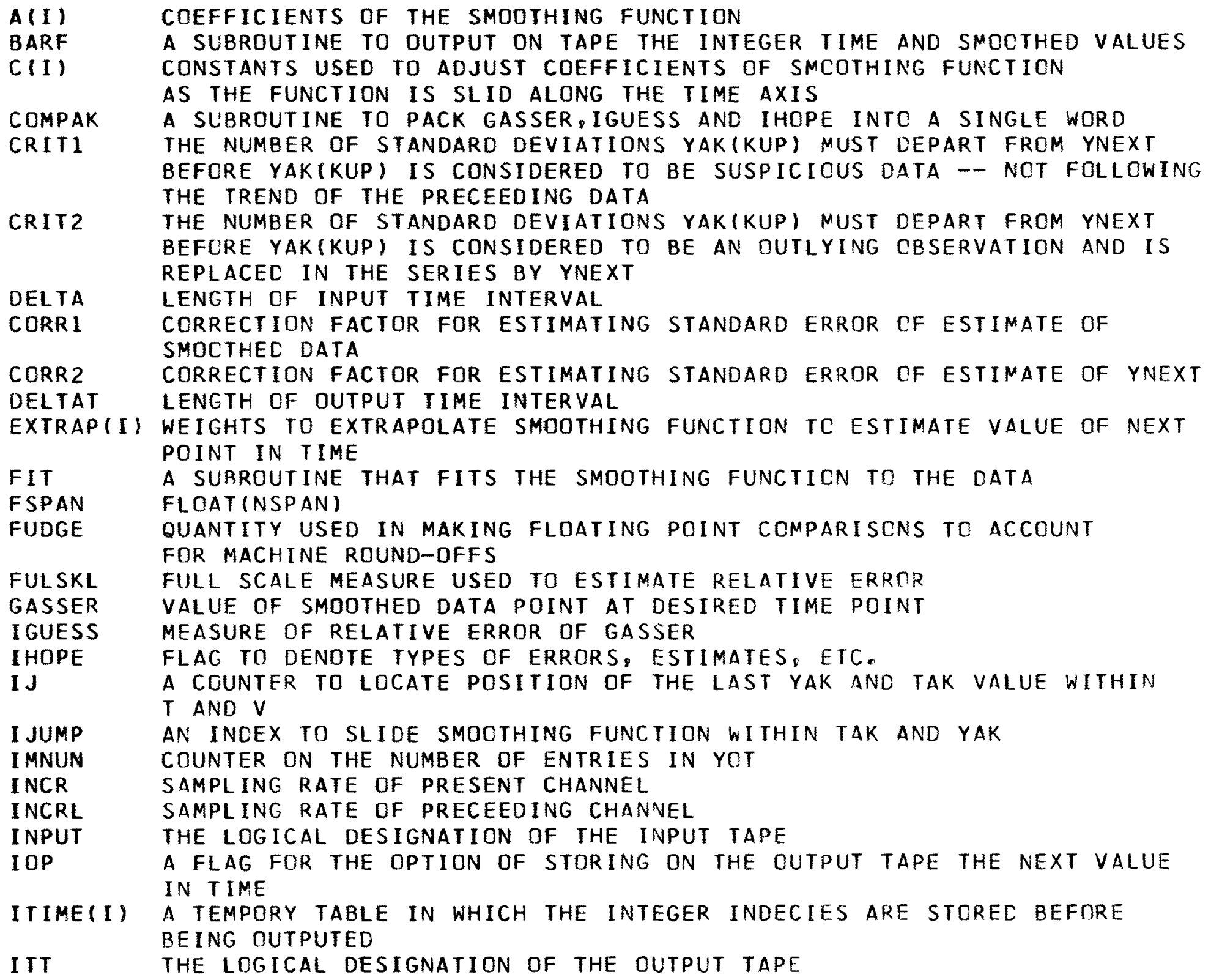




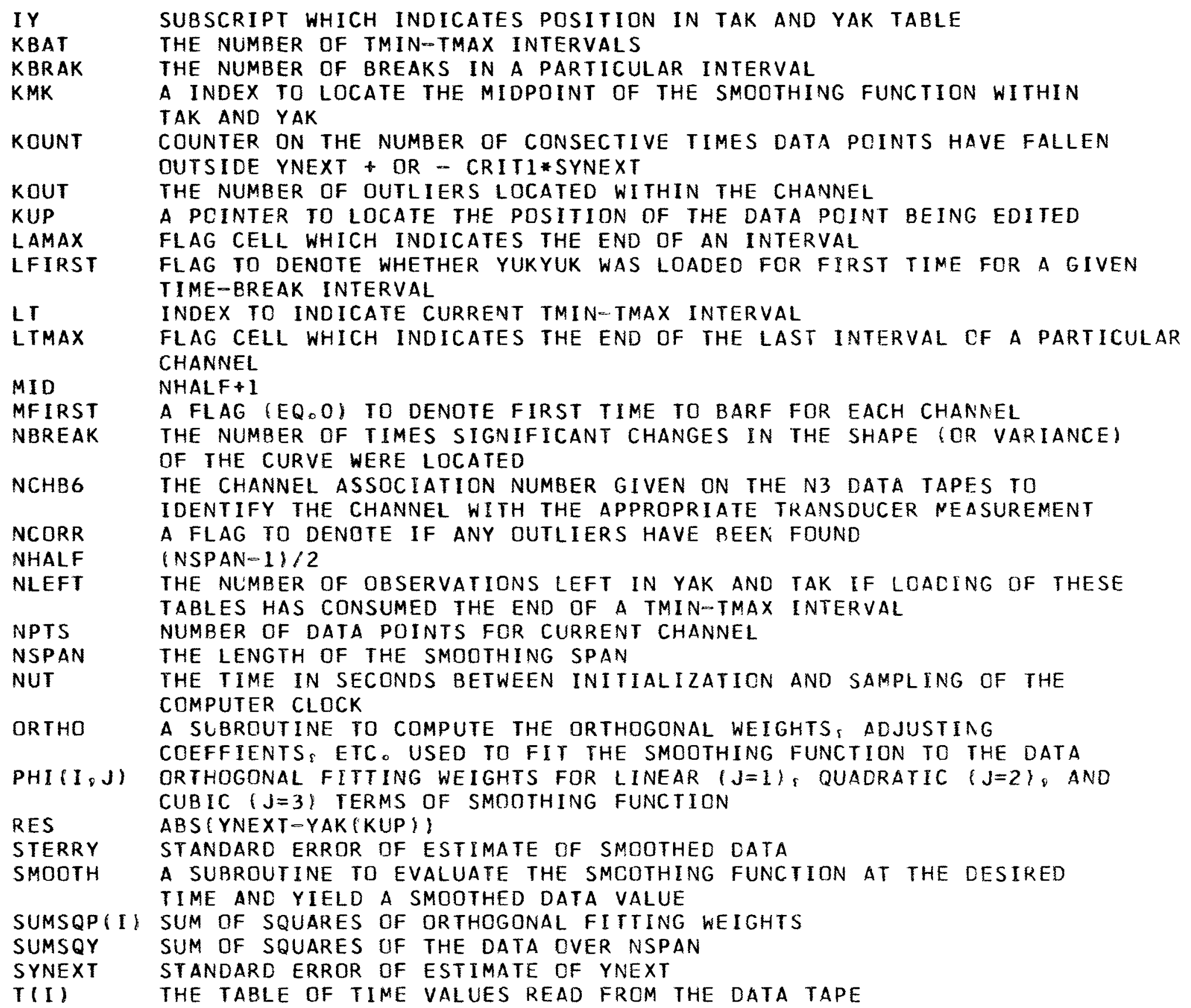


TEMPORARY TABLE INTO WHICH TIMES ARE READ

TBREAK

TEMP

THE TIME CORRESPONDING TO THE OCCURANCE OF THE I-TH BREAK IN THE CURVE

TWELVE WORDS OF BCD INFORMATION GIVING THE REC CARD INFCRMATION

FROM THE RDH-D CODE

T IME I

TIME2

A LIBRARY SUBROUTINE TO INITIALIZE THE CCMPUTER CLOCK

TITLE

TMAXII)

TMID

TMINII)

TOUPEE

A LIBRARY SUBROUTINE TO SAMPLE THE COMPUTER CLOCK

SIX WORDS OF BCD INFORMATION GIVING THE TITLE CARD INFORMATION

FROM THE RDH-D CODE

THE LAST DESIRED DUTPUT TIME FOR THE I-TH TMIN-TMAX INTERVAL

VALUE OF TIME AT MIOPOINT OF SMOOTHING SPAN

TSAVE THE TIME AT WHICH THE FIRST DF SUCCESSIVE CCCURANCES OF ABSIRES.GT. CRITI*SYNEXT) OCCURS

Y(I) THE TABLE OF DATA VALUES READ FROM THE DATA TAPE

YAK

YBREAK

YHIGH

YLOW

YNEXT

YOT II)

YSAVE (I)

TEMPORARY TABLE INTO WHICH DATA IS READ

THE VALUE OF THE ORIGINAL DATA POINT AT THE I-TH BREAK IN THE CURVF

THE UPPER RANGE OF THE CURRENT RECORDINGS

THE LOWER RANGE OF THE CURRENT RECORDINGS

ESTIMATE OF DATA POINT FOR NEXT TIME POINT

TEMPORARY STORAGE FOR OUTPUT VALUES OF PACKED DATA WORD

YUKYUK

THE I TH ENTRY IN A TEMPORARY TABLE USED TO STORE ORIGIONAL DATA VALUES WHEN ABS (RES.GT.CRITI*SYNEXT)

A SUBROUTINE TO LOAD TEMPORARY TABLES TAK AND YAK WHICH ARE USEC IN FITTING THE SMOOTHING FUNCTION 
$\mathrm{RN}-\mathrm{S}-\mathrm{O} 146$

X. LISTIIGGS OF FORTRAN SOURCE DECKS 


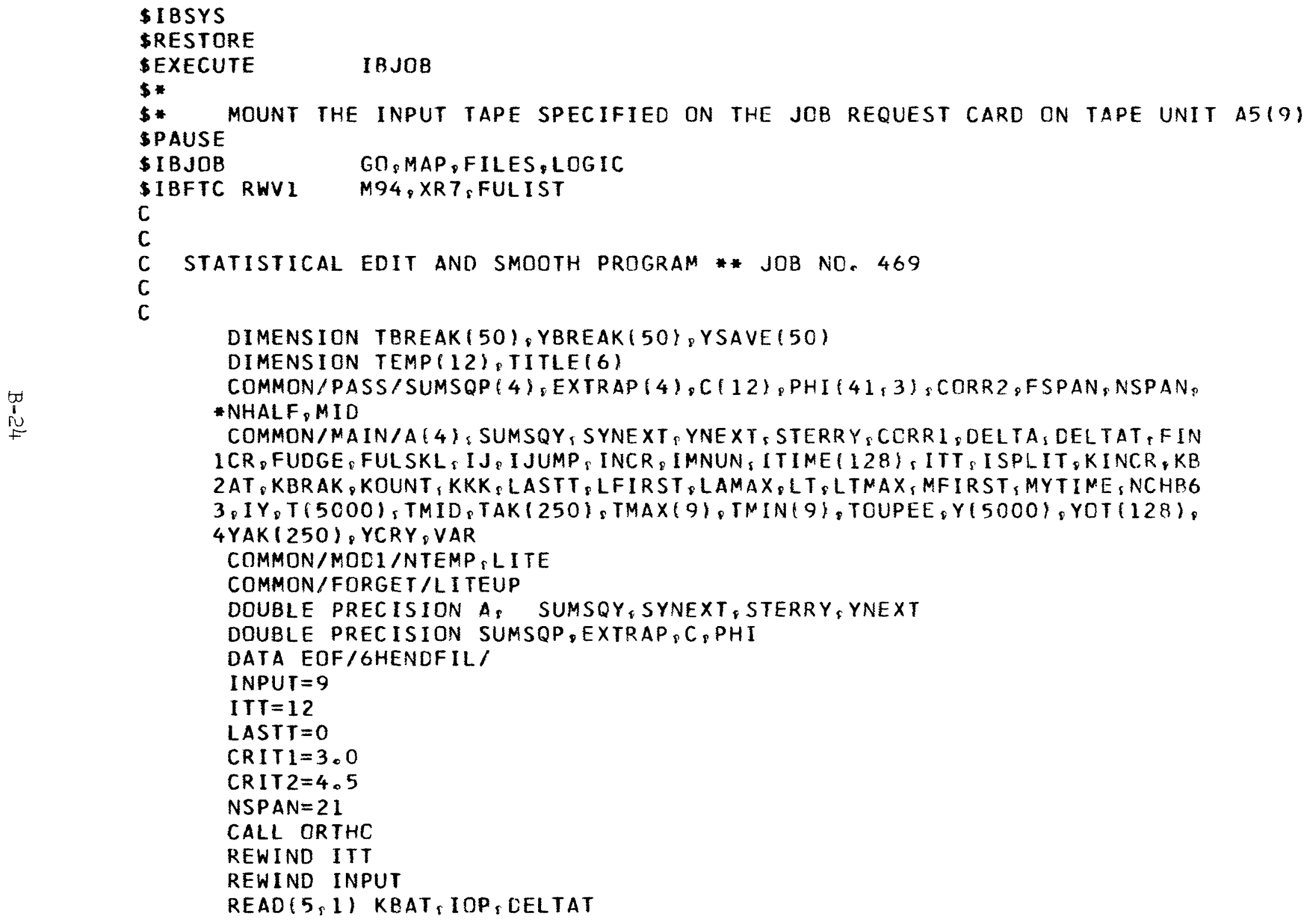

DIMENSION TBREAK (50), YBREAK (50), YSAVE (50) 
1 FORMAT $(216, F 6.0)$

READ (5,3) (TMIN(LA), TMAX (LA), LA=1,KBAT)

3 FORMAT $(2 F 18.0)$

$C$
$C$
$C$
$C$
$C$
$C$

THIS SEGIMENT DF THE PROGRAM COMPUTES AND STORES ON TAPE ALL THE DESIRED OUTPUT TIMES。 ITS CHANNEL ASSOCIATION NUMBER (NCHBG) IS 998 .

DO $3515 \quad I=1, K B A T$

NCOUNT = IFIX ( $($ TMAX (I) - TMIN (I))/DELTAT $)+1$

3515 LASTT $=$ LASTT +NCOUNT

NCHB $6=998$

$T(1)=T M I N(1)$

KINCR $=4$

MFIRST $=0$

MYTIME $=0$

I MNUN $=0$

NTIMES $=0$

I $\mathrm{TCH}=1$

3653 NCOUNT $=$ IF $[X(1$ TMAX $(I T C H)-T M I N(I T C H) / / D E L T A T)+1$

NTIMES = NT IMES + NCOUNT

NIN $=1$

I MNUN $=$ I MNUN+1

YOT (IMNUN) $=$ TMIN $(I T C H)$

IF(IMNUN-128)3661,3662,3661

3661 IF(NIN-NCOUNT) $3663,3671,3663$

3663 I MNUN $=$ IMNUN + 1

IF( I MNUN-1) 3682,3681,3682

3681 YOT $(1)=Y E K+D E L T A T$

GO TO 3683

3682 YOT (IMNUN) $=$ YOT $($ I MNUN-I + +DELTAT

$3683 N I N=N I N+1$

IFIIMNUN-128)3661,3662,3661

3662 YEK $=$ YOT $(128)$

CALL BARF

IFININ-NCOUNT $>3663,3671,3663$

3671 IF(ITCH-KBAT) $3678,3679,3678$

3678 I TCH $=$ I TCH +1

GO TO 3653

3679 CALL BARF 


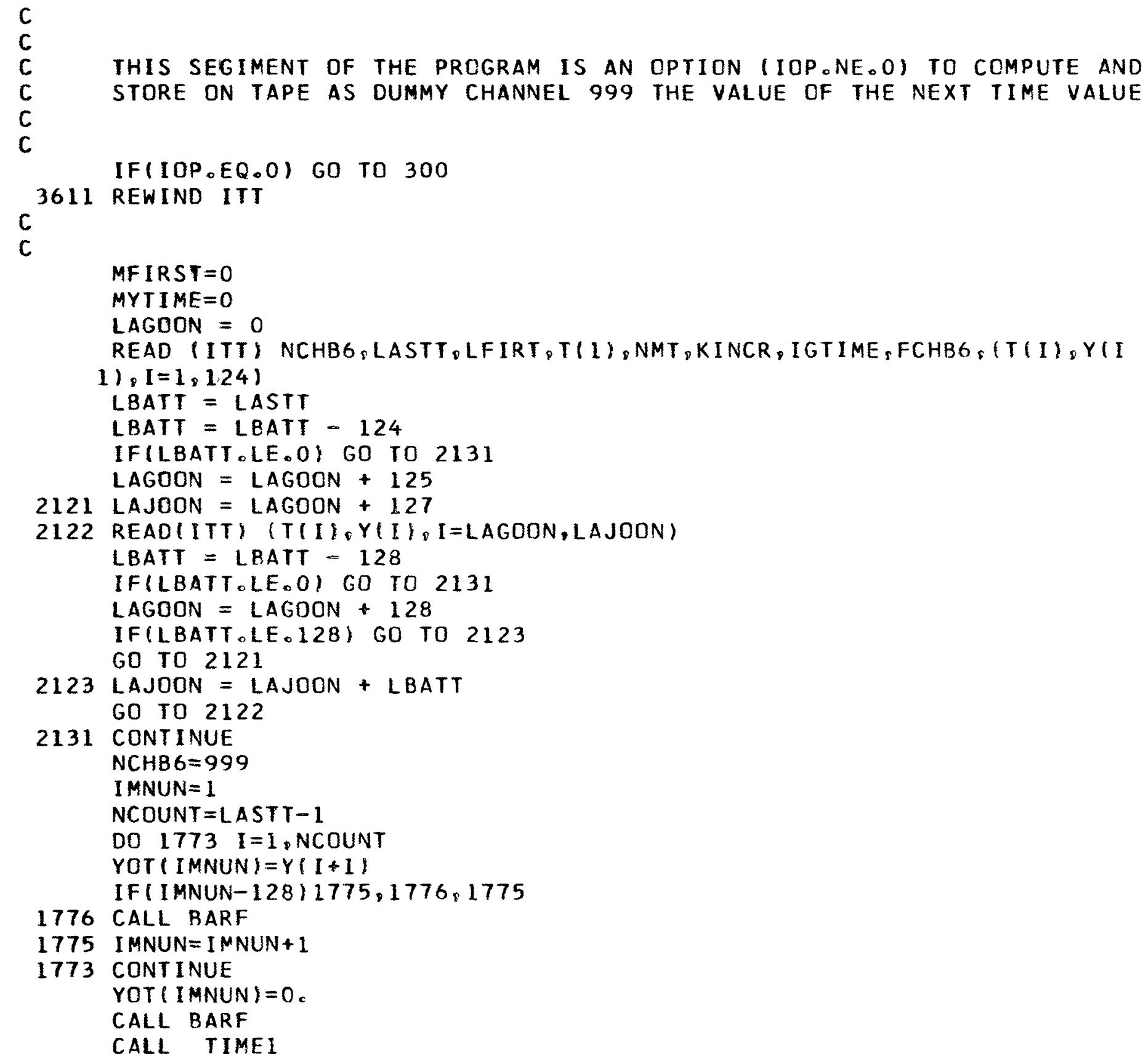




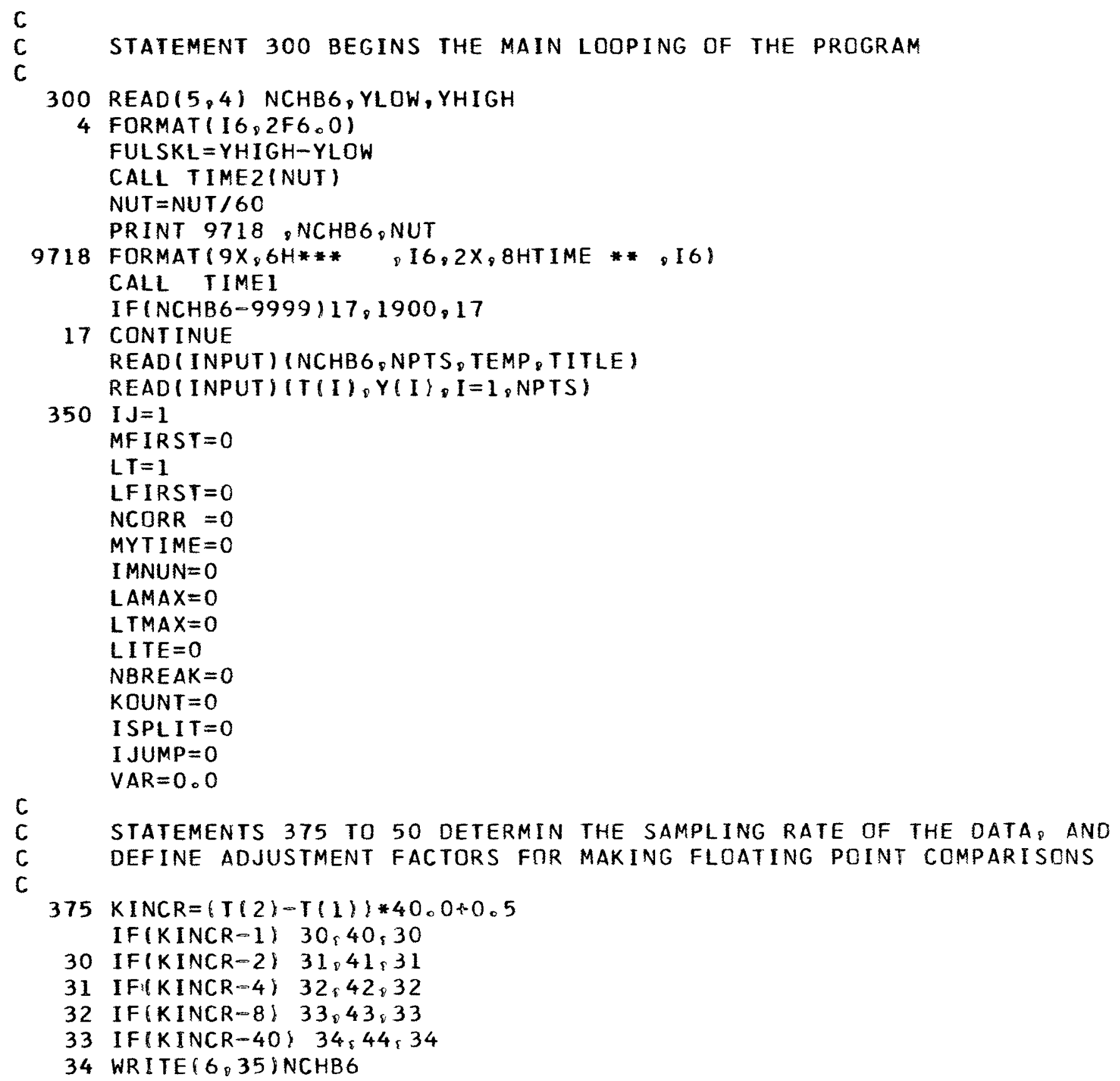

c

C STATEMENTS 375 TO 50 DETERMIN THE SAMPLING RATE OF THE DATA: AND DEFINE ADJUSTMENT FACTORS FRR MAKING FLOATING POINT COMPARISONS

$375 K I N C R=\{I(2)-T(1)) * 40.0 * 0.5$

IF (KINCR -1$) \quad 30,40,30$

30 IFIKINCR-2) $31,41,31$

31 IF $\{(K I N C R-4) \quad 32,42,32$

32 IFIKINCR-8) $33,43,33$

33 IFIKINCR-40) $34,44,34$

34 WRITE $(6,35)$ NCHB6

C 
35 FORMAT 1 IHI,81H ILLEGAL SAMPLING RATE,PRESENT CHANNEL WILL NOT BE P IPROCESSED。CHANNEL NUMBER IS, I4)

GO TO 300

40 INCR $=40$

FUDGE $=.0005$

DELTA $=.025$

GO TO 50

41 INCR $=20$

FUDGE $=.005$

DELTA $=.05$

GO TO 50

42 INCR $=10$

FUDGE $=.05$

DELTA $=.1$

GO TO 50

43 INCR $=5$

FUDGE $=.1$

DELTA $=.2$

GO TO 50

44 INCR $=1$

FUDGE $=.5$

DELTA $=1$

50 CONTINUE

FINCR = INCR

600 CALL YUKYUK

IF(LITE) $625,700,625$

625 NSPAN $=$ NTEMP

700 CALL FI

NSPAN $=$ NSPAN

$K K K=K K K$

I JUMP = I JUMP

IF (LITE) $750,800,750$

750 IF(TMID-TOUPEE) $795,775,775$

775 CALL SMOOTH

IF( I MNUN-128)776,777,776

777 CALL BARF

776 GO TO 750

795 LITE $=0$

800 IF(TMID-TOUPEE) $1200,900,900$

900 CALL SMOOTH

1000 IFIIMNUN-128)1200,1100,1200 


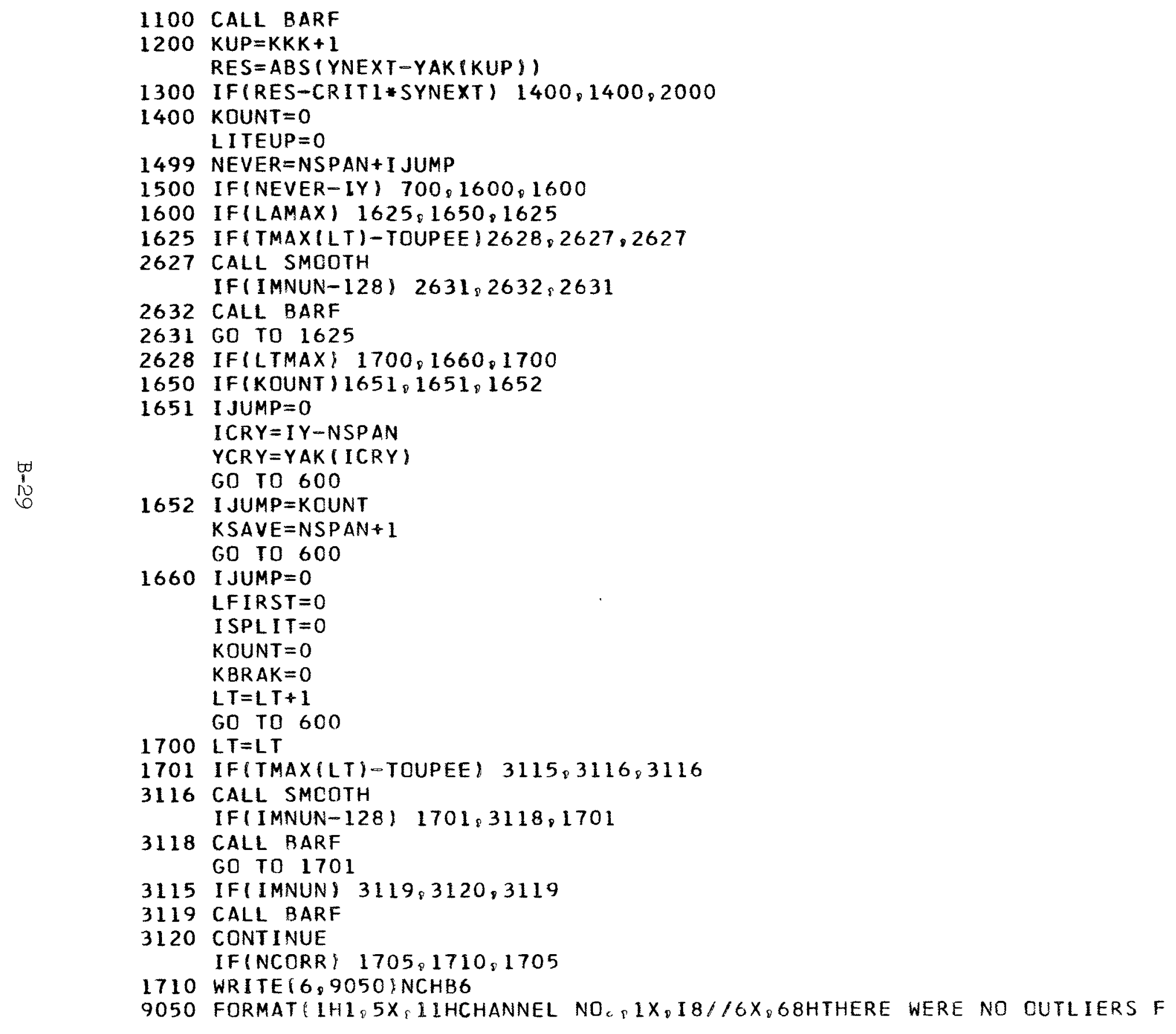


IOUND IN THE ORIGIONAL DATA FOR THIS CHANNEL。)

1705 WRITE 6,9055$)$ NCHB6

9055 FORMAT $\left(1 H 1=5 X_{0} 11\right.$ HCHANNEL NO., $1 X_{8}[8 / /)$

IF(NBREAK! $1715,1720,1715$

1715 WRITE $(6.9060)$

9060 FORMAT $(6 \times, 78 H S I G N I F I C A N T$ CHANGES IN THE SHAPE (OR VARIANCE) CF THE 1 CURVE WERE LOCATED AT //12X,4HTIME,7X,13HORIGINAL DATA//) DO $1725 \quad I=1$, NBREAK

1725 WR I TE $(6,9065)$ TBREAK(I), YBREAK(I)

9065 FORMAT $\left(5 X_{2} 2\left(5 X_{0} F 15.8\right)\right)$ GO TO 1800

1720 WRITE $(6,9070)$

9070 FORMAT $16 X$ 94HNO SIGNIFICANT CHANGES IN THE SHAPE (OR VARIANCE) OF ITHE CURVE WERE OBSERVED FOR THIS CHANNEL -

1800 GO TO 300

$\mathrm{C}$

C

1900 WRITE(ITT) (EOF $I=1.256)$

END FILE ITT

REWIND ITT

54 REWIND INPUT

PRINT 9191

9191 FORMATI 97HREMOVE TAPE FROM DRIVE BG\& LABLE IT AS INDICATED ON THE

* JOB REQUEST CARD AND RETURN IT TO LIBRARY/2X 55 HALSO REMOVE AND

* RETURN TO LIBRARY THE TAPE ON UNIT A5.:

PAUSE

RETURN

2000 KOUNT $=$ KOUNT +1

YSAVE (KOUNT $)=$ YAK $(K U P)$

2100 IFIRES-CRIT2*SYNEXT) $2200,2500,2500$

2500 CONTINUE

C

C IF DESIRED\& THE APPROPRIATE ERROR FLAGS SHOULD BE SET AT THIS POINT

IF INCORR $\$ 2501,2502,2501$

2502 WRITE 6.90011 NCHB6

9001 FORMATIIH1,5X,11HCHANNEL NO, $1 X_{0} I 8 / 16 X_{,} 60 H T H E$ FOLLOWING POINTS WER IE JUDGED CUTLIERS AND WERE CORRECTED $/ 6 \mathrm{X}$, 64HICRIGINAL DATA FGUAL T 20 - IEI6 REPRESENT MISSING ORIGINAL VALUES $/ / 1 / 12 X_{8} 4 H T I M E_{8} 8 X_{8} 13$ HORIG 3 INAL DATA $6 X_{0}$ 14HCORRECTED DATA/I 
$\$ 501$ IF (YAK(KIP).LT $-1 . E 14\}$ Bn Tr: 2503

WRITE $(6,9 C O 2)$ TAK(KUP), YAK(KUP), YVIXT

9002 FORMAT $(5 X, \Gamma 10.2,3 X, 2(4 X, F 15.8)$

GO TC, 2504

2503 WRITL $\left.(6,9 C O S) T A K(K U)^{3}\right)$, YAK(KUP), YNLXI

3003 FPRNAT(5X,F10,2,7X,[15,8,4X,F15.8)

2504 CONTIAUE

$Y A K(K U P)=Y N E X Y$

NEORR $=777$

LITEUP $=777$

$V A K=S Y N E X T$

2200 IF(KOUNT-1) $2300,2300,25,00$

300 iSAVE $=K . U P$

TSAVE $=T A K(K L . P)$

CC RC 14.39

2600 IF(KOUNT-10) i45, $2700,27 \mathrm{CC}$

2700 IFILAMAX.EC.0) 60 Tr, 2750

MLEFT $=I Y-K S A V E$

IF(NLFFT.CTrNSPAV) GO II, 2750

GC TR. 1499

2750 NBREAK=A BRT $A K+1$

YPREAK(N PREAK) = YSAV[(1)

IBREAK (NPRE AK) = TSAVE

$\checkmark A R=0.3$

KCUNT $=0$

DO $2714 \quad I=1,10$

YAK(KSAVE) = YSAVE (I)

$2714 K S A V E=K S A V R+1$

KSAVE $=K S A V E-11$

$\therefore 31$ IF(TAK(KSAVE) - TQUPEE) 272:92727.2727

2727 CALL SMCOTH

IF (INNUN-12?)2731,2732.2731

2732 CALL BARF

2731 GO TC 2721

2728 CONTINUE

LITE $=777$

NTEMP $=$ NSPAN

NSPAN $=I Y-K S A V E$

[ JUMP $=0$

I SH'LIT $=5$

KOUNT $=0$ 
60 TO 600

END

\$IBFTC RWV2 M94,XR7。LIST

SUBROUTINE FIT

C

C THIS SUBROUTINE FITS THE SMOOTHING FUNCTION TO THE DATA

CDMMON/PASS/SUMSQP (4),EXTRAP (4),C (12),PHI(41,3)ःCORR2,FSPAN.NSPAN -NHALF\&MIO

COMMON/MAIN/A (4), SUMSQY, SYNEXT, YNEXT, STERRY \&CORR 1,DELTA, DELTAT \& FIN

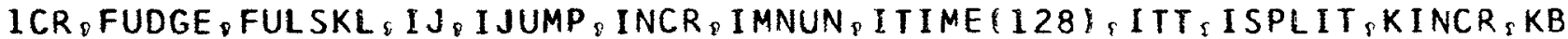

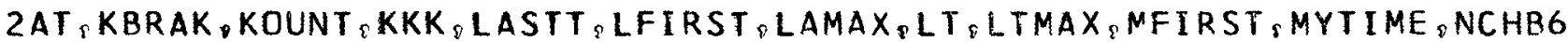

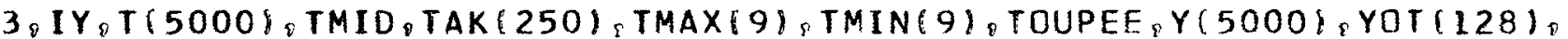
$4 Y A K(250), Y C R Y_{Q}$ VAR

COMMON/FORGET/LITEUP

DOUBLE PRECISION A, SUMSQY, SYNEXT,STERRY, YNEXT

DOUBLE PRECISION SUMSQP EXTRAP ${ }_{5}$, PHI

DOUBLE PRECISION DSQRT

DOUBLE PRECISION SUMSQ

$\operatorname{CORR} 1=0_{0} 0$

YNEXT $=0,0$

IFIISPLIT_NE。O\& GO TO 60

SUMSQY $=0.0$

DO $16 \quad J=1.4$

$16 \quad \mathrm{~A}(\mathrm{~J})=0.0$

DO 17 I $=1$ 。NSPAN

$A(1)=A(1)+Y A K(I)$

$A(2)=A(2)+Y A K(I) * P H I\{I \leq I)$

$A\{3\}=A(3)+Y A K(I) * P H I\{I=2\}$

$A(4)=A(4)+Y A K(I) * P H I(1) 3\}$

17 SUMSQY $=$ SUMSQY $* Y A K(I) * 2$

DO $18 \quad J=1,4$

$18 A(J)=A(J) / \operatorname{SUMSQP}(J)$

$T M I D=T A K$ IMID)

I SPLIT $=777$

$K K K=N S P A N$

I JUMP $=1$

GO TO 70 
60 IF(IJUMP。EQ。0) GO TO 85 $K K K=N S P A N+I J U M P$

$K M K=M I D+I J U M P$

$A(1)=A(1)+(Y A K(K K K)-Y A K(I J U M P)) / S U M S Q P(1)$

$A(2)=A(2)+C(1) * Y A K(K K K)+C(2) * Y A K(1 J U M P)-C(3) * A(1)$

$A(3)=A(3)+C(4) * Y A K(K K K)-C(5) * Y A K(I J U M P)-C(6) * A(2)-C(7) * A(1)$

$A(4)=A(4)+C(8) * Y A K(K K K)+C(9) * Y A K(I J U M P)-C(10) * A(3)-C(11) * A(2)+C(12$

1)*A(1)

SUMSOY=SUMSQY+YAK $(K K K) * * 2-Y A K(I J U M P) * * 2$

$T M I D=T A K(K M K)$

I JUMP $=$ I JUMP+1

GO TO 70

$85 A(1)=A(1)+(Y A K(N S P A N)-Y C R Y) / S U M S Q P(1)$

$A(2)=A(2)+C(1) * Y A K(N S P A N)+C(2) * Y C R Y-C(3) * A(1)$

$A(3)=A(3)+C(4) * Y A K(N S P A N)-C(5) * Y C R Y-C(6) * A(2)-C(7) * A(1)$

$A(4)=A(4)+C(8) * Y A K(N S P A N)+C(9) * Y C R Y-C(10) * A(3)-C(11) * A(2)+C(12) * A($

$11)$

SUMSQY = SUMSQY +YAK $(N S P A N) * * 2-Y C R Y * * 2$

TMID $=$ TAK $(M I D)$

$K K K=N S P A N$

I $J U M P=1$

70 DO $19 \mathrm{~J}=1,4$

CORR $1=$ CORRI+A $(J) * 2 * \operatorname{SUMSQP}(\mathrm{J})$

19 YNEXT $=Y N E X T+A(J) * E X T R A P(J)$

SUMSQ $=$ SUMSQY - CORR 1

IF(SUMSQ.LT.0.0) GO TO 99

STERRY =DSORT ( SUMSQ $/($ FSPAN-4.) )

SYNEXT = STERRY*CORR2

IF(LITEUP.EQ.0) GO TO 99

IF(SYNEXT。GE。VAR) GO TO 99

SYNEXT $=V A R$

99 RETURN

END

\$IBFTC RWV3 M94,XR7,LIST

SUBROUTINE SMOOTH

C

C THIS SUBROUTINE COMPUTES THE SMOOTHED POINT CORRESPONDING TC THE

C DESIRED OUTPUT TIME \& COMPUTES ESTIMATE OF RELATIVE ERRCR, DETERNINES 
C APPROPRIATE FLAGS, PACKS INFORMATION INTO DATA WORD, AND STCRES TIME C AND DATA WORD INTO A TEMPORARY STORAGE TABLE

C

COMMON/PASS/SUMSQP(4),EXTRAP $(4), C(12), P H I(4), 3), C O R R 2, F S P A N, N S P A N$, *NHALF,MIO

COMMON/MAIN/A (4) \& SUMSQY \& SYNEXT Y YNEXT\& STERRY, CORR I DELTA\& CELTAT \& FIN

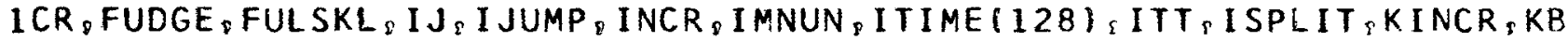
$2 A T_{2} K B R A K$,KOUNT, KKK, LASTT,LFIRST,LAMAX, LT,LTMAX, MFIRST,MYTIME,NCHBG 3.IY,T(5000),TMID,TAK (250) DTMAX(9),TMINI9) TOUPEE,Y(5000):YOT(128): $4 Y A K(250), Y C R Y_{2} V A R$

DOUBLE PRECISION A\& SUMSQY SYNEXT S STERRY, YNEXT

DOUBLE PRECISION SUMSQP,EXYRAP ${ }_{r} C_{\nabla} P H I$

I MNUN $=$ I MNUN +1

$X=($ TOUPEE $-T M I D) * F I N C R$

$Y=X * 2-0.08333333 *($ FSPAN**2-1。!

$Z=(X * 3-.05 *(3.0 * F S P A N * 2 \cdot 7) * X$.

GASSER $=A(1)+A(2) * X * A(3) * Y * A(4) * Z$

$S T E R R Y=S T E R R Y / F S P A N$

IGUESS $=200$ 。 $*$ TERR $Y / F U L S K L$

IF(IGUESS-63) $19.19,20$

20 I GUESS $=63$

I HOPE $=17$

GO TO 14

19 IHOPE $=0$

14 CALL COMPAK (IGUESS, IHOPE, GASSER ?

YOT ( IMNUN) $=$ GASSER

TOUPEE = TOUPEE +DELTAT

RETURN

END

\$IBFTC RWV4 M94:XR7:LIST

SUBROUTINE YUKYUK

C

THIS SUBRDUTINE LOADS TEMPORARY TABLES TAK AND YAK - SUPPLYENG - I. E16 FOR MISSING VALUES SO AS TO MAINTAIN A UNIFORM TIME SCALE - WHICH ARE USED IN FITTING THE SMOOTHING FUNCTION

COMMON/PASS/SUMSQP (4), EXTRAP (4), C (12), PHI $(41,3), C O R R 2, F S P A N, N S P A N$ 


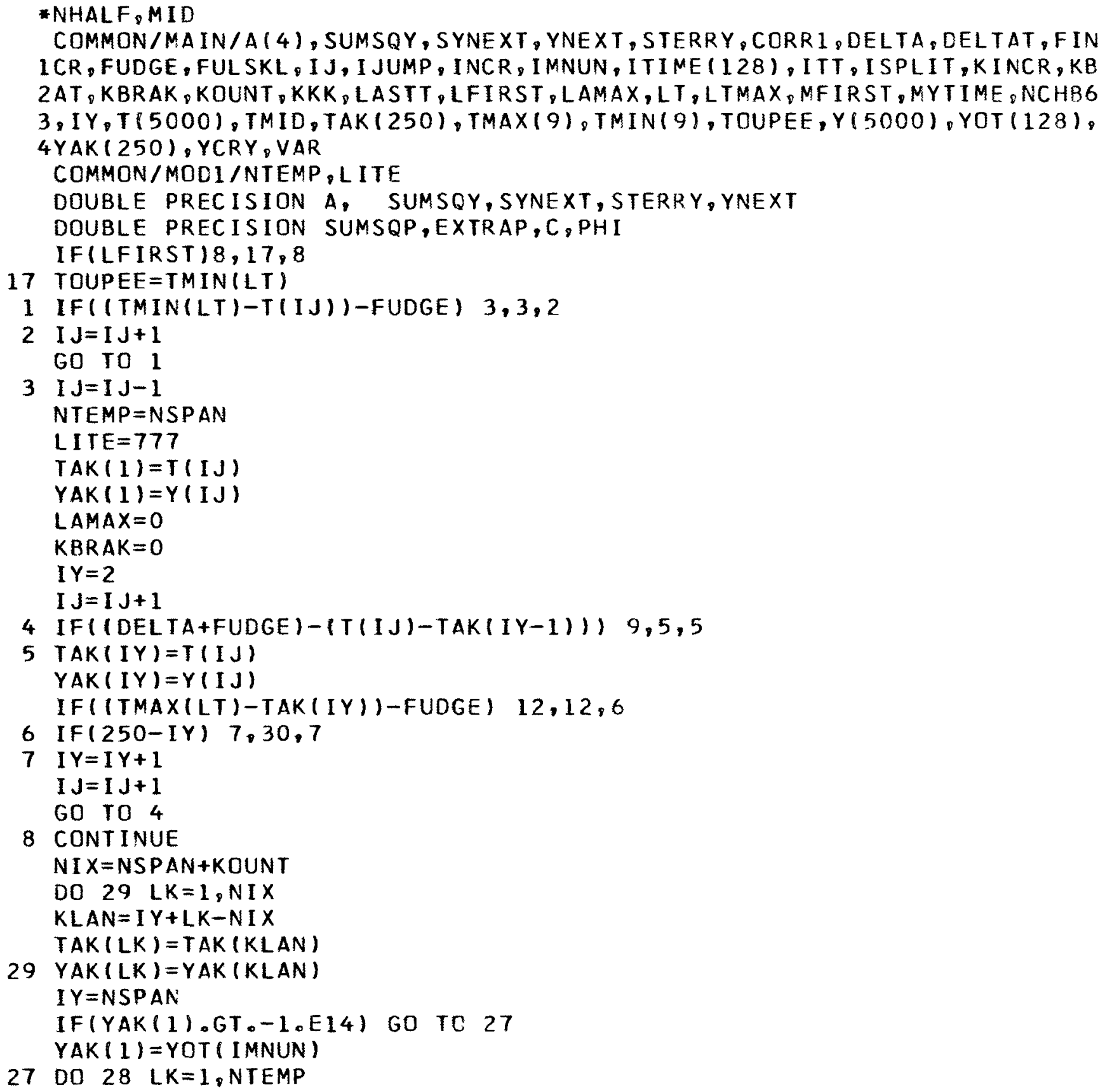




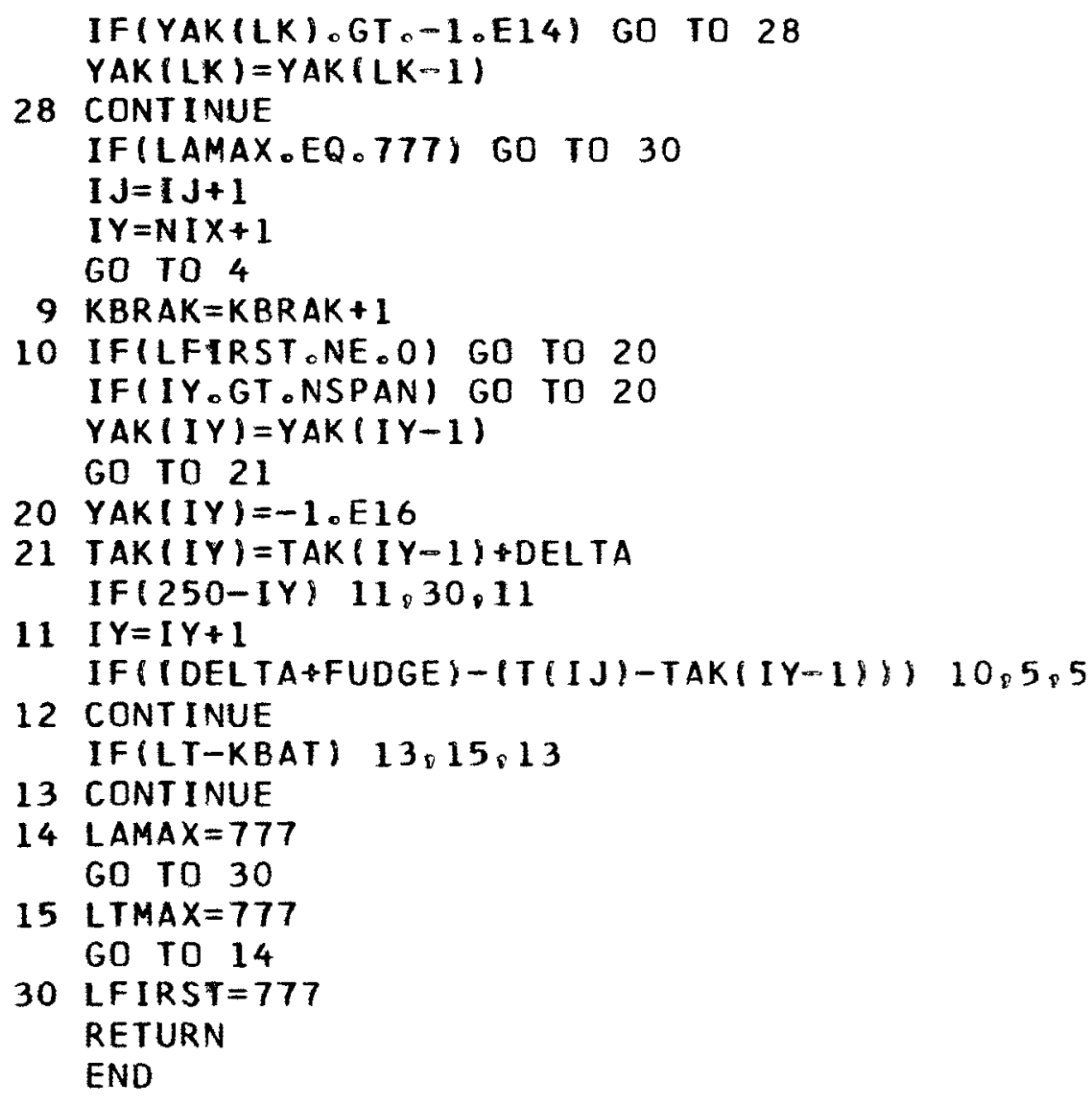




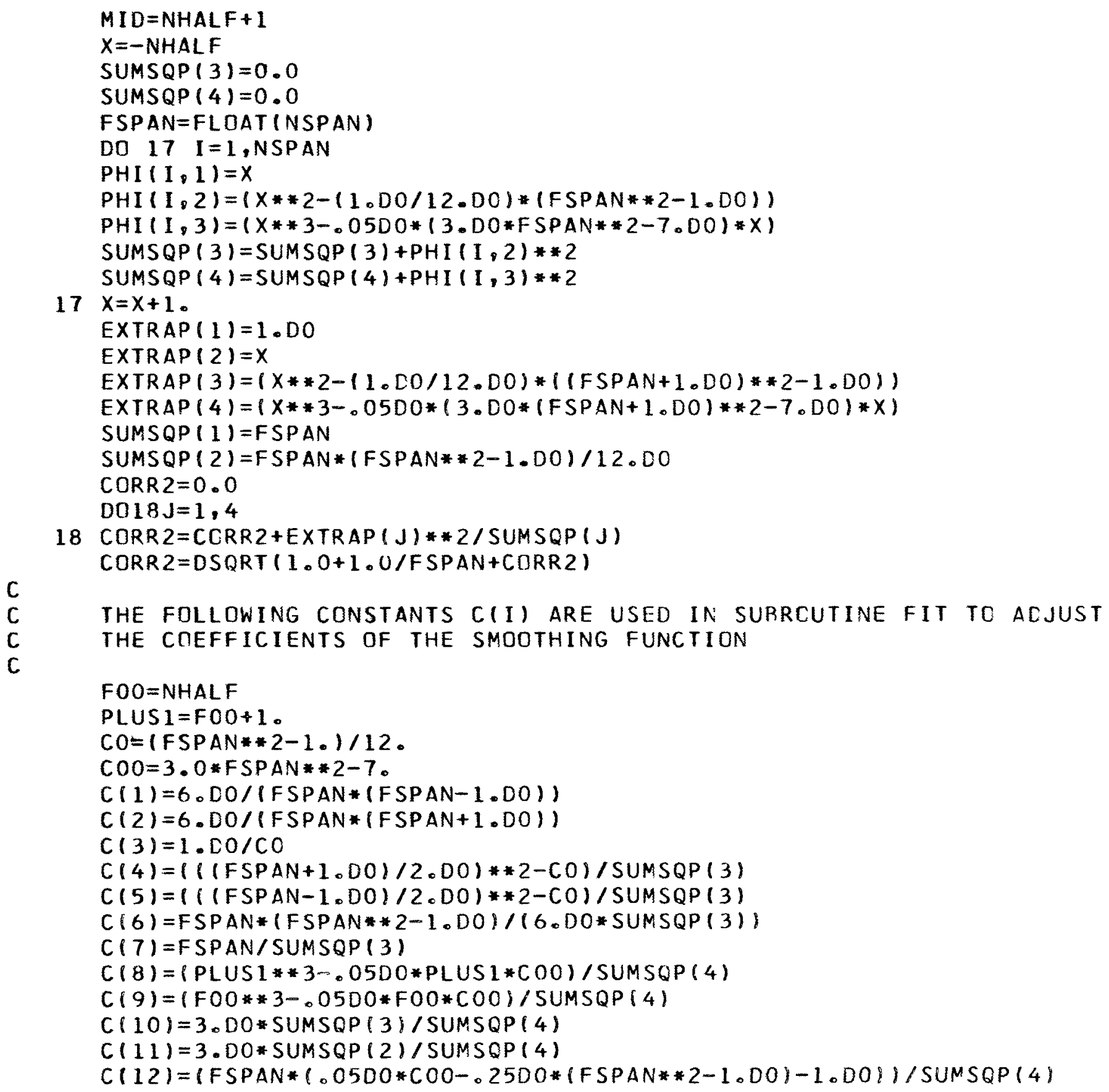


RETURN

END

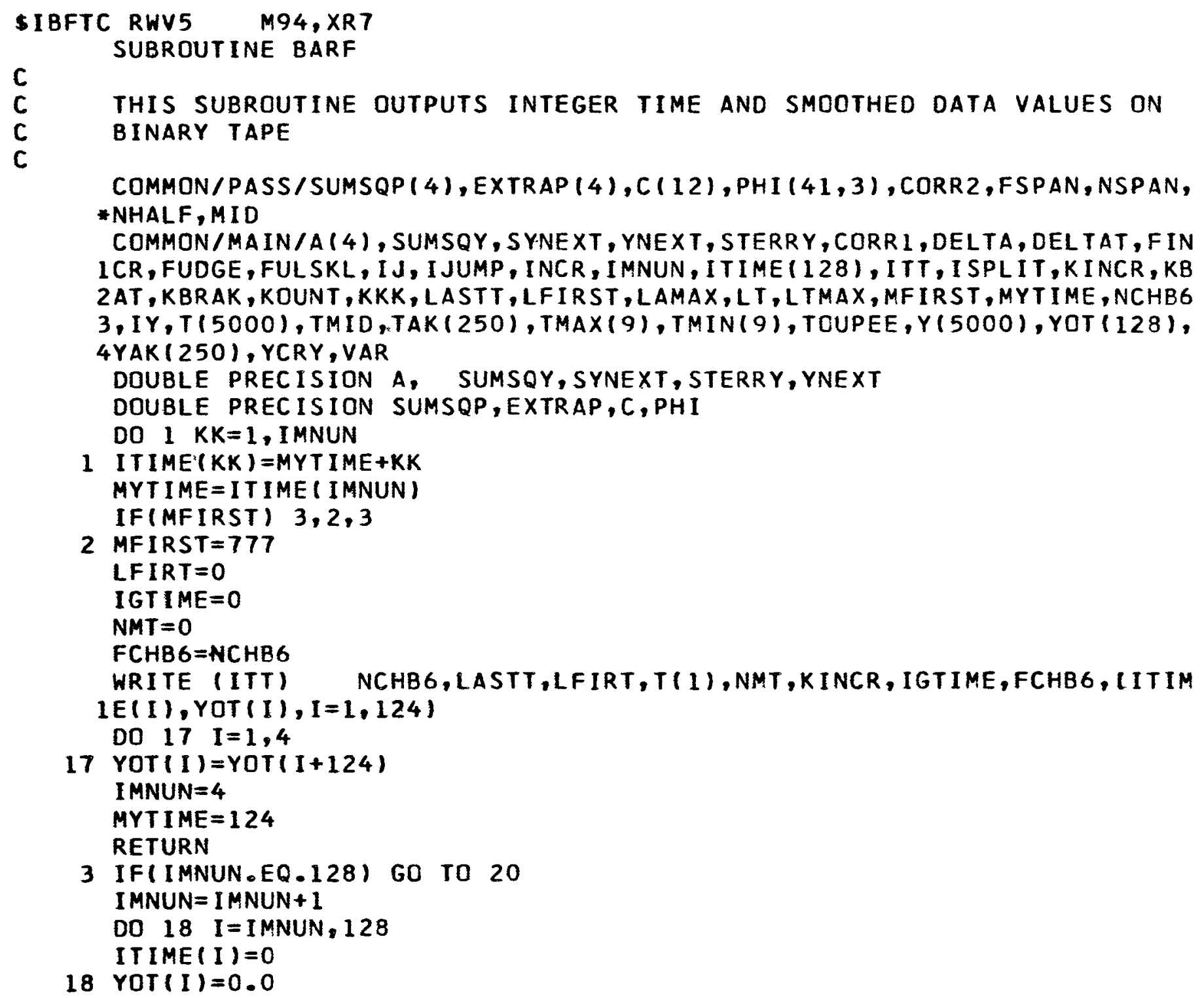


20 CONTINUE

WRITE (ITT)(ITIME(I),YOT(I), I=1,128)

I MNUN $=0$

RETURN

END

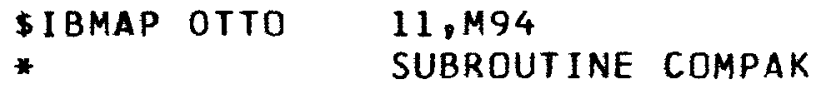

$\begin{array}{lll} & \text { ENTRY } & \text { COMPAK } \\ \text { COMPAK } & \text { CAL* } & 5,4 \\ \text { ANA } & =0777777770000 \\ \text { SLW* } & 5,4 \\ \text { CAL* } & 3,4 \\ \text { ARS } & 12 \\ \text { DRS* } & 5,4 \\ \text { CAL* } & 4,4 \\ \text { ARS } & 18 \\ \text { ORS* } & 5,4 \\ \text { TRA } & 1,4 \\ \text { END } & \end{array}$

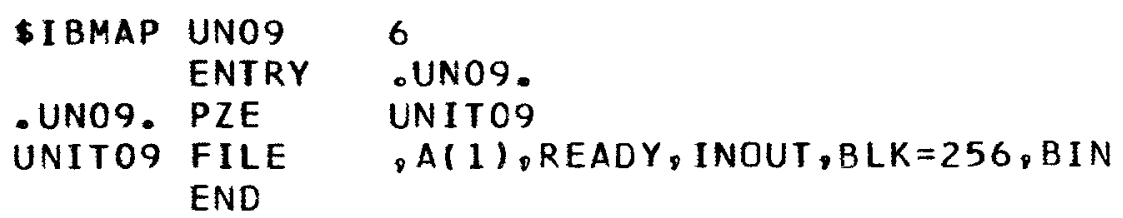



- IBMAP UN12
ENTRY OUN12。
-UN12. PZE UNITI2
UNITI2 FILE B(2),READY, INOUT,BLK=257, BIN
END 


\section{RECOPY PROGRAM FOR NERVA N3 DATA TAPES}

The purpose of this program is to recopy the NERVA N3 data tapes from a format written in FORTRAN II - FAP to a format acceptable by FORTRAN IV. (In FORTRAN IV, integer values are right-most oriented in the address field, whereas in FORTRAN II they are in the decrement field.) For each desired channel, the program lists its association number, the number of recorded data points, the transducer identification code, the multiplexer and pin numbers for which the data was recorded, and the theoretical range and units of the measurement. Since malfunctions may occur which cause the measured data to fall outside the theoretical range, the program has an option to scan the data for such occurrences and delete a given channel if more than a stated percentage of observations fall beyond these bounds. Deleted channels are identified on the output sheets.

If it is not desired to scan the data for channel deletions for excessive descripant values, then only a blank data input card is required. If scanning is desired, several data input cards are required with the stated formats.

Control Card

Columns 4-6

ICRIT - the critical percentage for which, if exceeded, causes deletion of the channel.

Channel Cards (one for each channel)

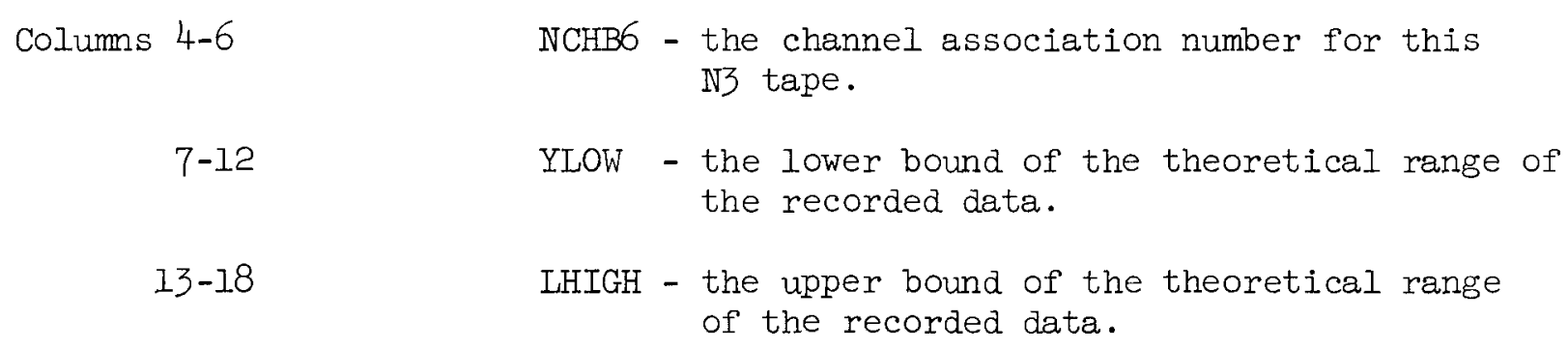

Information requested on the channel cards can be obtained from the Test Site. The original N3 (input) tape is mounted on unit $B 6$ and the recopied $N 3$ (output) tape is outputted on unit $\mathrm{A} 5$. The recopy program reads from $\mathrm{B} 6$ at a density of $556 \mathrm{BPI}$ (bits per inch).

Source listings of the recopy program and its various subroutines are included on the next several pages. 


\section{* MoUnt cesirec inPut tape cN dRIVE b6}

$*$

* pause

C PROGRAM TO RECCPY NERVA N3 TAPES FCR USE BY 469

DIMENSICN TEMP $(12)$, TITLE $(6), T(5000), Y(5 \mathrm{COC})$

COMMCN IREDUN

REWINC 12

REWINC $\mathrm{G}$

IREDUN $=\mathrm{C}$

JOKE $=C$

ICR I T $=0$

WRITE OUTPUT TAPE $6,5 \mathrm{C} 67$

5067 FCRMAT (1HI)

WRITE OLTPUT TAPE 6.60

60 FCRMAT $2 X_{2} 14 H N C H B 6$ ND。PTR, $7 X_{8} 27 H R E C$ CARC INFC. (RCH-D CCCE) REAC INPUT TAPE 5:113,ICRIT

113 FCRMAT(I6)

6 IFIICRITI16,16,16

S6 JCKE $=J O K E+1$

GC TO 98

16 READ INPUT TAPE 5,1 COC $\$$ JOKE, YLOW, YHIGH

1000 FORMAT $(16,2 F 6.0)$

SB CCNTINUE

$\mathrm{NCGO}=\mathrm{C}$

$T M I N=1 \circ E-9$

$T M A X=1 。 E+9$

8 CALL RDEG (JCKE, NPTS, TMIN, TMAX T, Y TEMP, TITLE, 1 )

IREDUN=IREDLN\$I

GO TO $\left(35 C_{3} 31 C_{5} 9\right)$, IRECUN

310 IRECUN $=\mathrm{C}$

GC TO 6

350 IRECUN $=\mathrm{C}$

$J=J C K E$

IFIICR IT $1757,753,757$ 


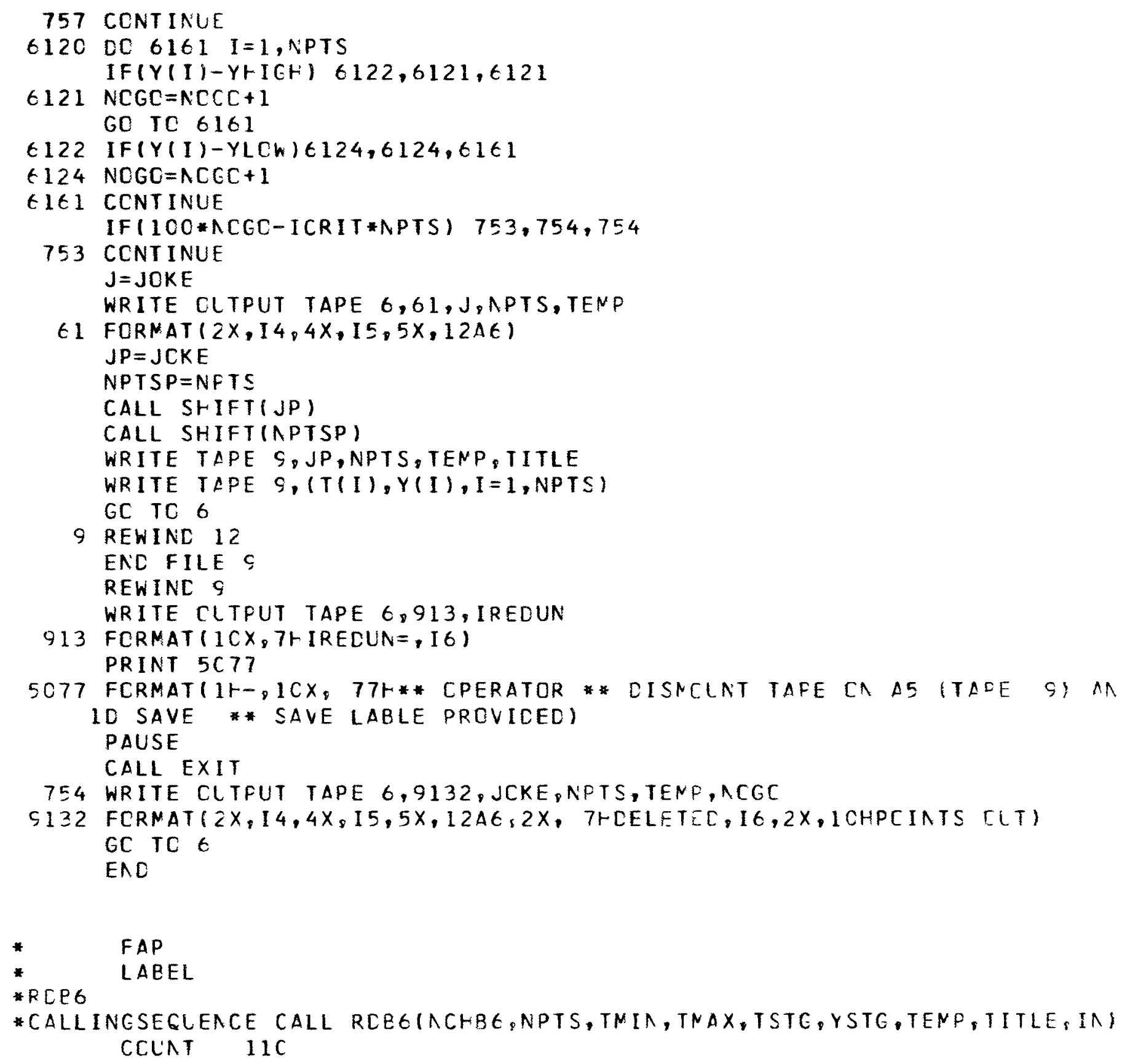




\begin{tabular}{|c|c|c|}
\hline \multirow{16}{*}{ RDE 6} & ENTRY & RDE 6 \\
\hline & $\begin{array}{l}\text { TRCB } \\
\text { TEFB }\end{array}$ & $*+1$ \\
\hline & STZ & $\begin{array}{l}\text { TH } \\
\text { TENP }\end{array}$ \\
\hline & ENB & TENP \\
\hline & SELB & $t$ \\
\hline & SXA & EXIT:1 \\
\hline & $S \times A$ & EXIT\&1,4 \\
\hline & CLA & ССT2 \\
\hline & STC & RCFKB \\
\hline & CLA* & 184 \\
\hline & STC & ACFBE \\
\hline & CLA & 2,4 \\
\hline & STA & NPTS \\
\hline & CLA* & 3.4 \\
\hline & STO & TMIN \\
\hline & $\begin{array}{l}\text { CLA* } \\
\text { STC }\end{array}$ & $\begin{array}{l}4.4 \\
\text { TMAX }\end{array}$ \\
\hline \multirow[t]{12}{*}{$z$} & CLA & 5.4 \\
\hline & STA & YES \\
\hline & CLA & $t=4$ \\
\hline & STA & STCDT \\
\hline & CLA & 7.4 \\
\hline & SUB & CCT13 \\
\hline & STA & RDTMP \\
\hline & CLA & $\begin{array}{l}8.4 \\
C\end{array}$ \\
\hline & SUE & $\begin{array}{l}\text { CCTS } \\
\text { RDT }\end{array}$ \\
\hline & CLA* & 9,4 \\
\hline & ARS & 18 \\
\hline & STA & INCSG \\
\hline \multirow[t]{10}{*}{ READ } & RTBB & $t$ \\
\hline & $\mathrm{RCHB}$ & RD 1 \\
\hline & TEFB & CNFNC \\
\hline & LCHB & RD2 \\
\hline & TEFB & CNFNC \\
\hline & $C L A$ & TEPP \\
\hline & SUB & $\mathrm{NCrBG}$ \\
\hline & TZE & GO \\
\hline & LCHB & RDN \\
\hline & TCCB & * \\
\hline
\end{tabular}




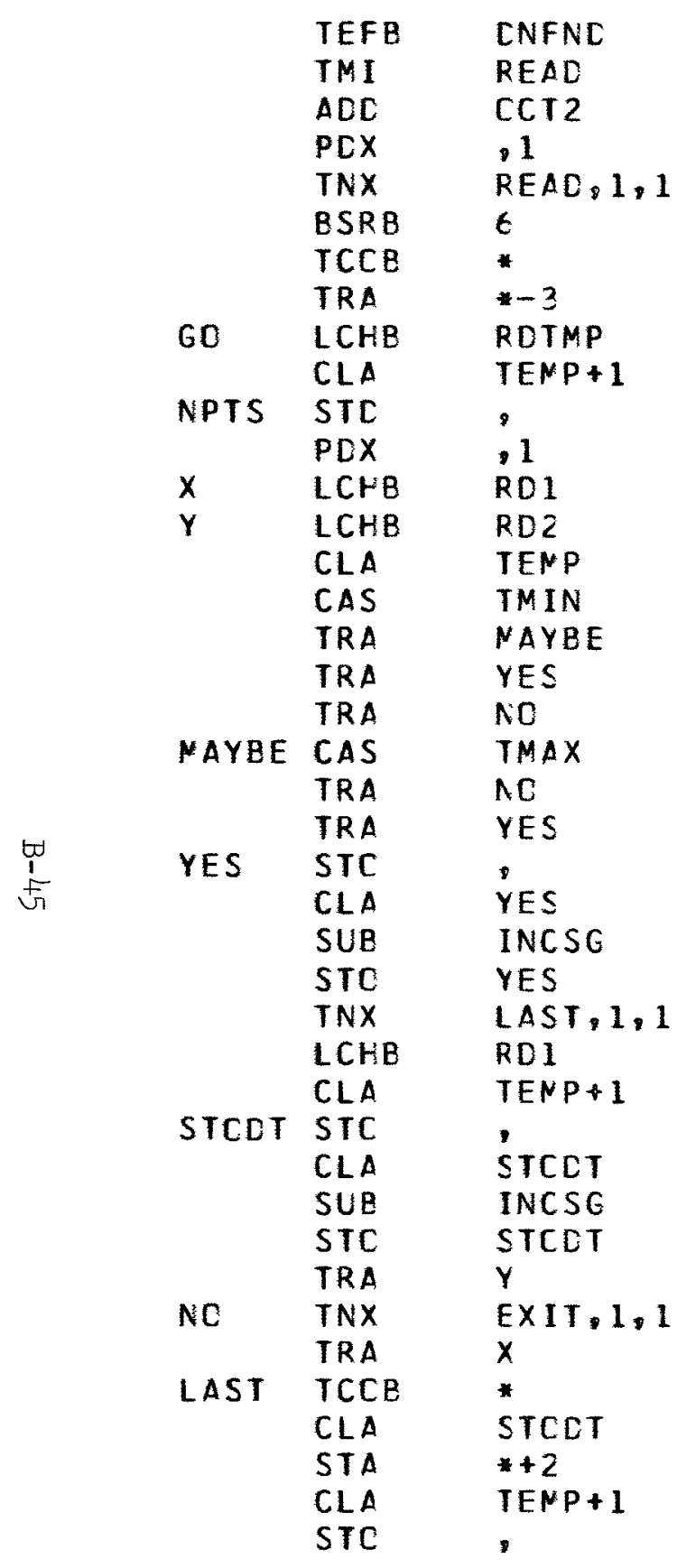




\begin{tabular}{|c|c|c|}
\hline EXIT & $A \times T$ & .1 \\
\hline & $A \times T$ & .4 \\
\hline & TCCB & * \\
\hline & TEFB & $*+1$ \\
\hline & TRCB & $*+2$ \\
\hline & TRA & 10.4 \\
\hline & NZT & RCFKB \\
\hline & TRA & CUT \\
\hline & CLA & RCFKB \\
\hline & SUE & CCT1 \\
\hline & STC & RCFKB \\
\hline & BSRB & 6 \\
\hline & TCCB & $*$ \\
\hline & TRA & $z$ \\
\hline CUT & CALL & PRINT,DCT $1 C, N C H B E$ \\
\hline & TRA & EXIT \\
\hline CNFND & IOT & \\
\hline & NOP & \\
\hline & REWB & 6 \\
\hline & CALL & PRINT,OCT $11, N C H B E$ \\
\hline & TRA & EXIT \\
\hline RDI & ICCT & TEMP:D 1 \\
\hline RD2 & IOCT & $T E N P+1,, 1$ \\
\hline RDN & IOCDN & TEMP: 1 \\
\hline RDTMP & IOCP & 8.12 \\
\hline RDTIL & IOCT & .96 \\
\hline RDBCD & IOCT & .98 \\
\hline INCSG & HTR & . \\
\hline IMIN & HTR & , \\
\hline IMAX & HTR & 8 \\
\hline NCHB 6 & HTR & 0 \\
\hline RCHKB & HTR & 3 \\
\hline TEMP & BSS & 2 \\
\hline CCT1 & CCT & $\operatorname{cococ} 1000000$ \\
\hline CCT 2 & CCT & $0000020000 C 0$ \\
\hline OCT5 & CCT & 000000000005 \\
\hline CCT10 & OCT & $C O C O 10 C 0 C 0 C 0$ \\
\hline CCT11 & OCT & $\operatorname{coc} 011000000$ \\
\hline CCT13 & OCT & $\mathrm{COCOCOCOCO} 13$ \\
\hline & END & \\
\hline
\end{tabular}




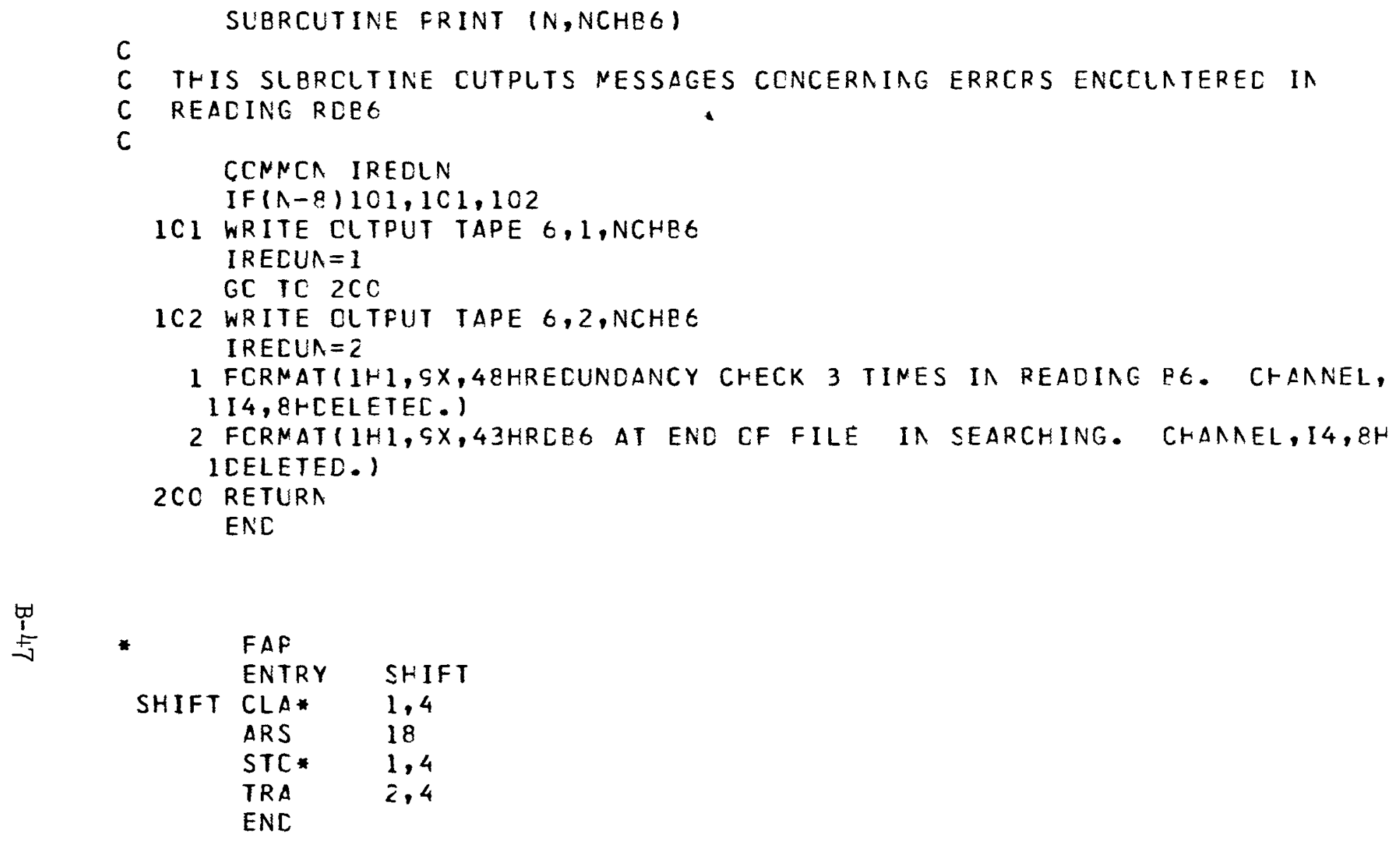


$\mathrm{RN}-\mathrm{S}-\mathrm{O} 146$

XII. DETAII FLOW DIAGRAM OF STATISTICAL EDIT AND SMOOTH PROGRAM

INDEX TO PART XII

Subroutine

Page No.

Main Program

B-49

Fit

B- 60

Smooth

B- 62

YUK YUK

B-63

$\phi \mathrm{RTH} \phi$

B-67

BARF'

B- 68 
$\mathrm{RN}-\mathrm{S}-0146$
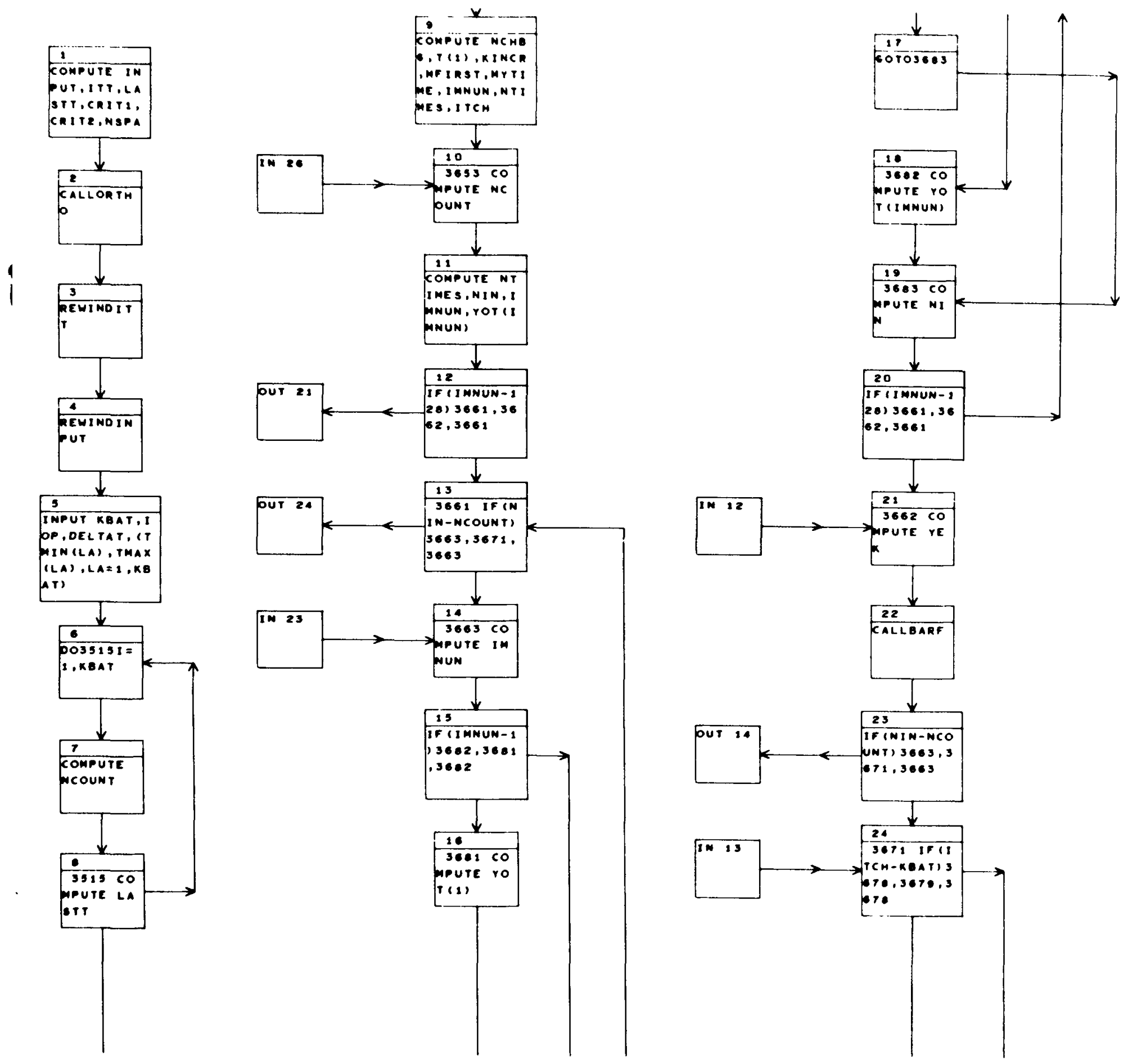
RN-S-0146
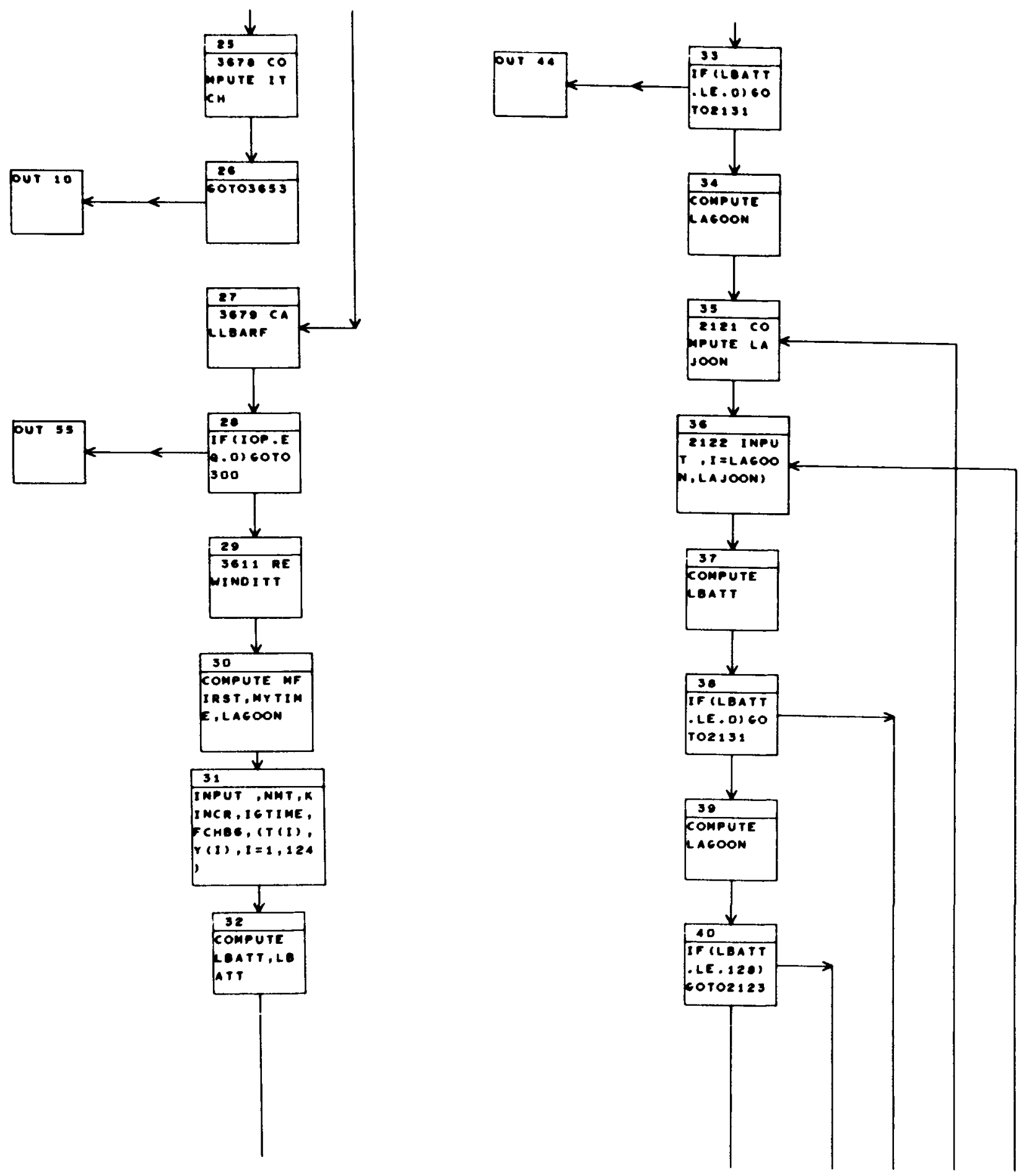
RN-S-0146
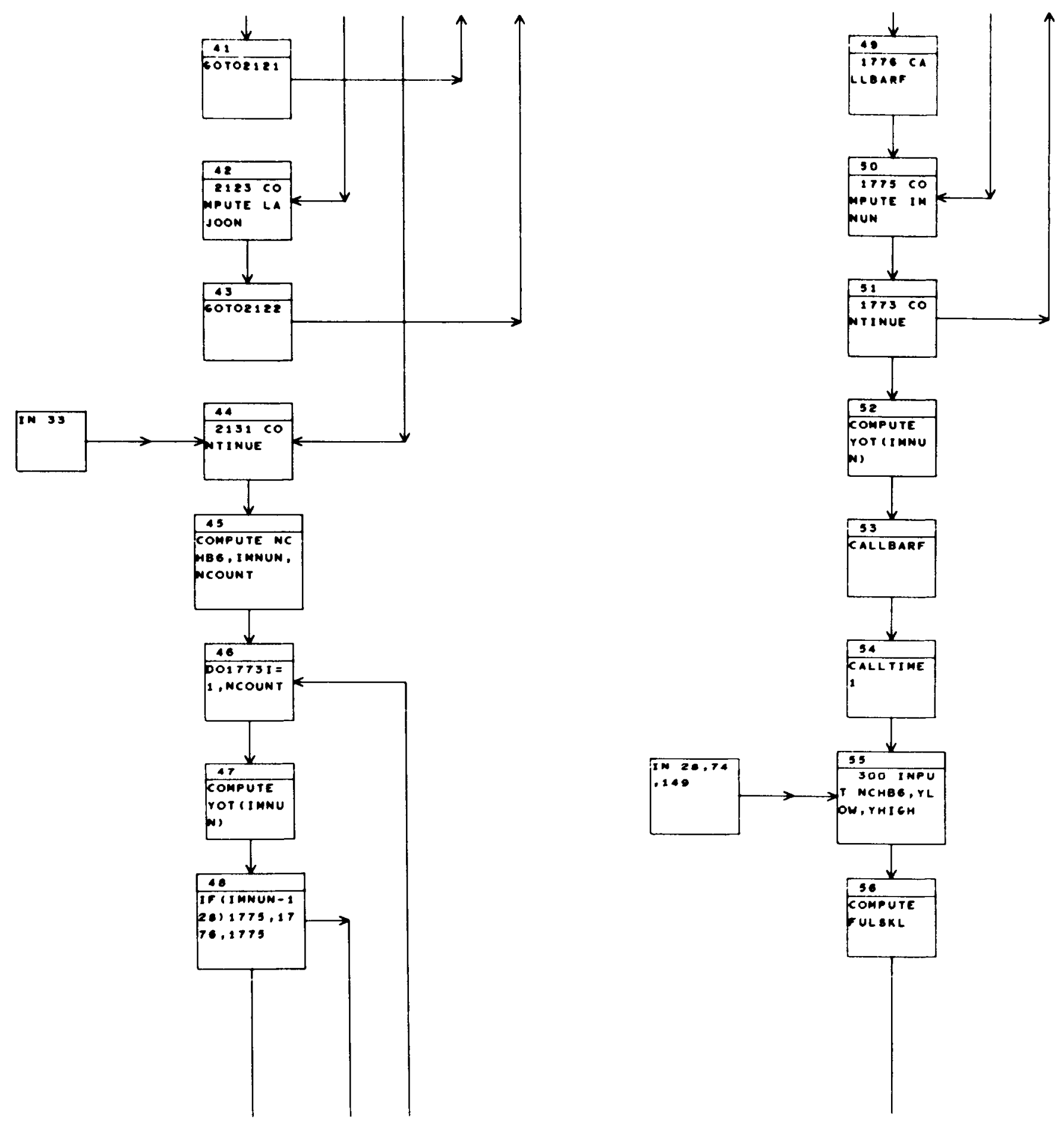

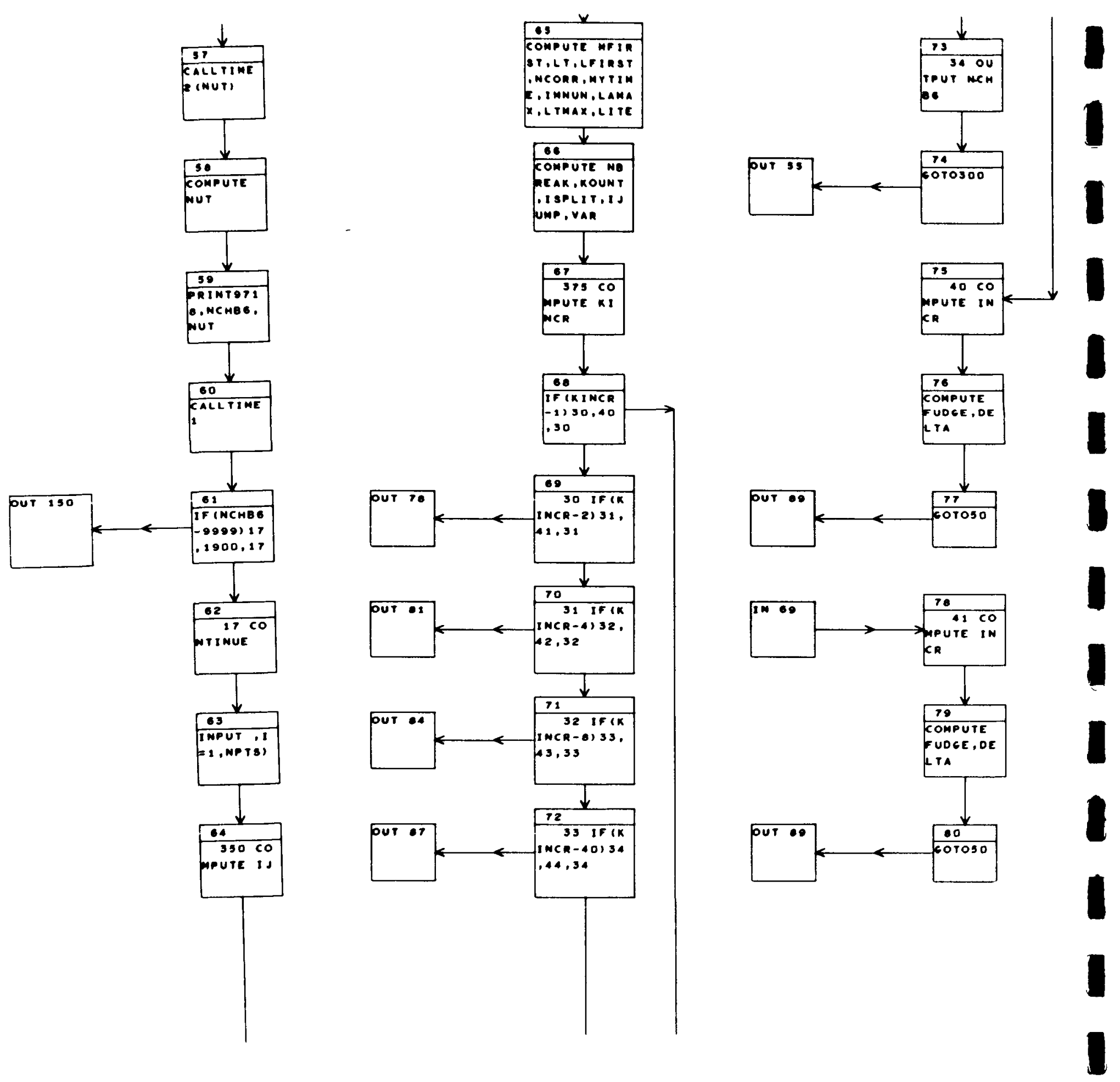
RN-S-0146
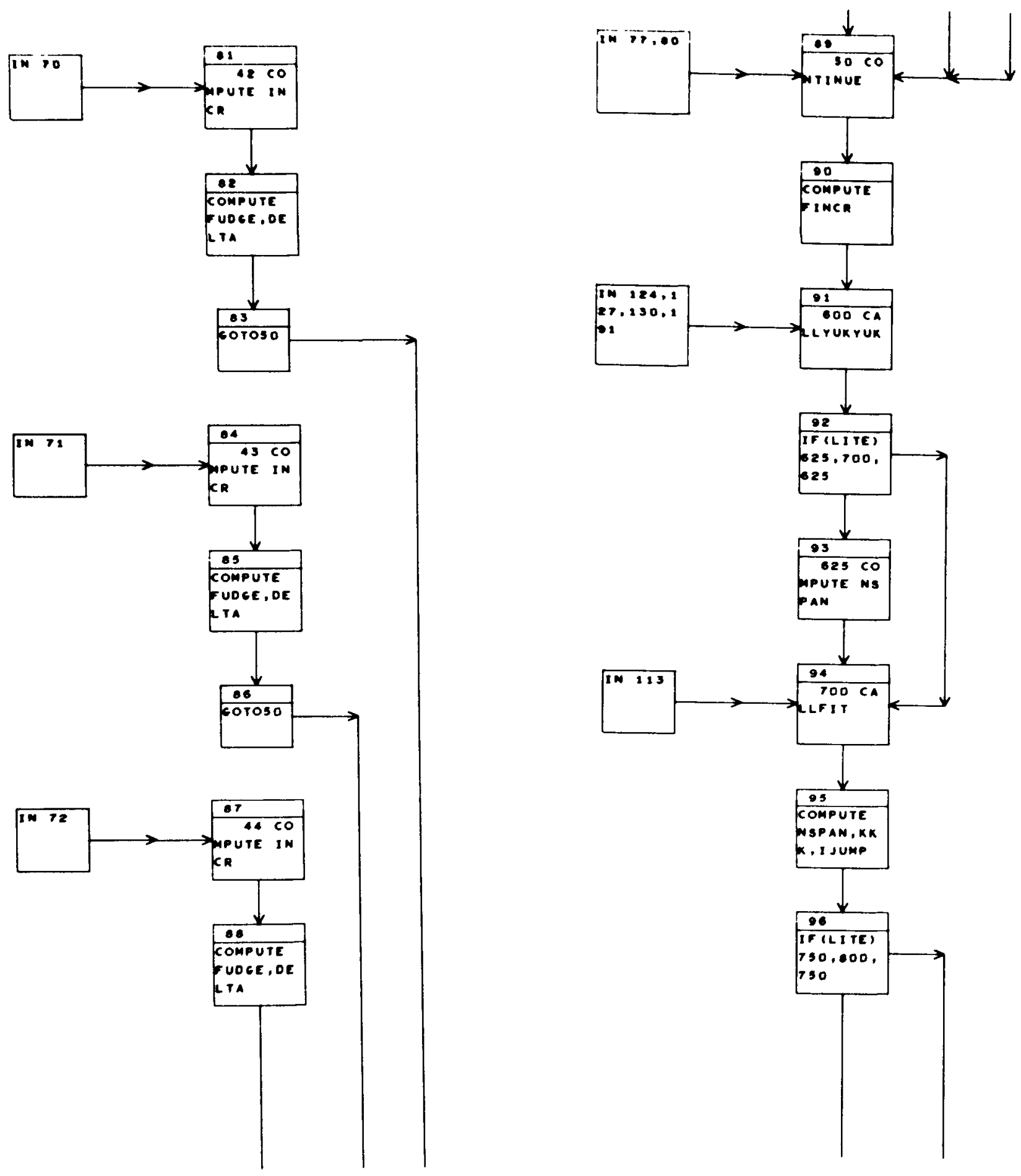
RN-S-0I46
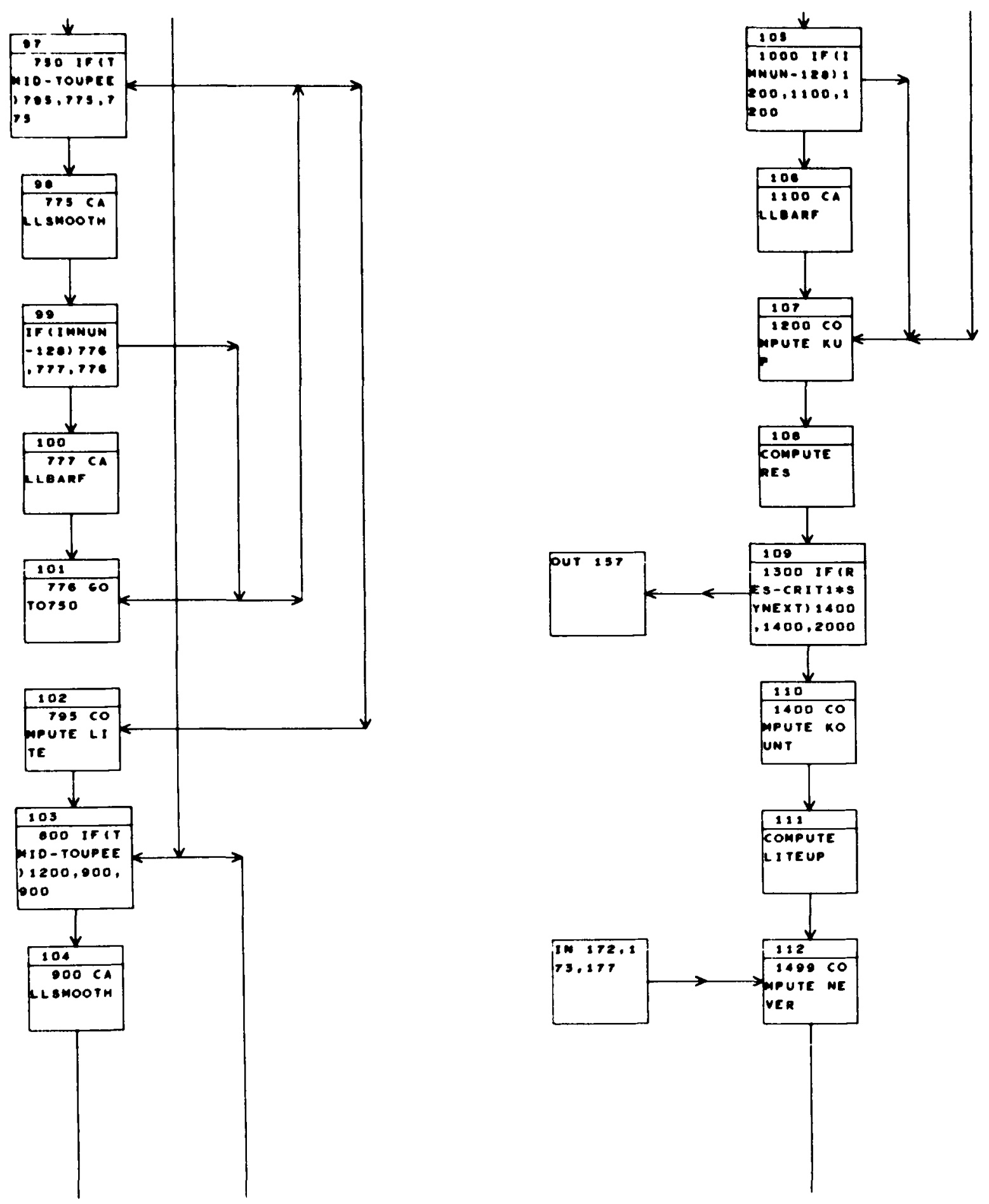

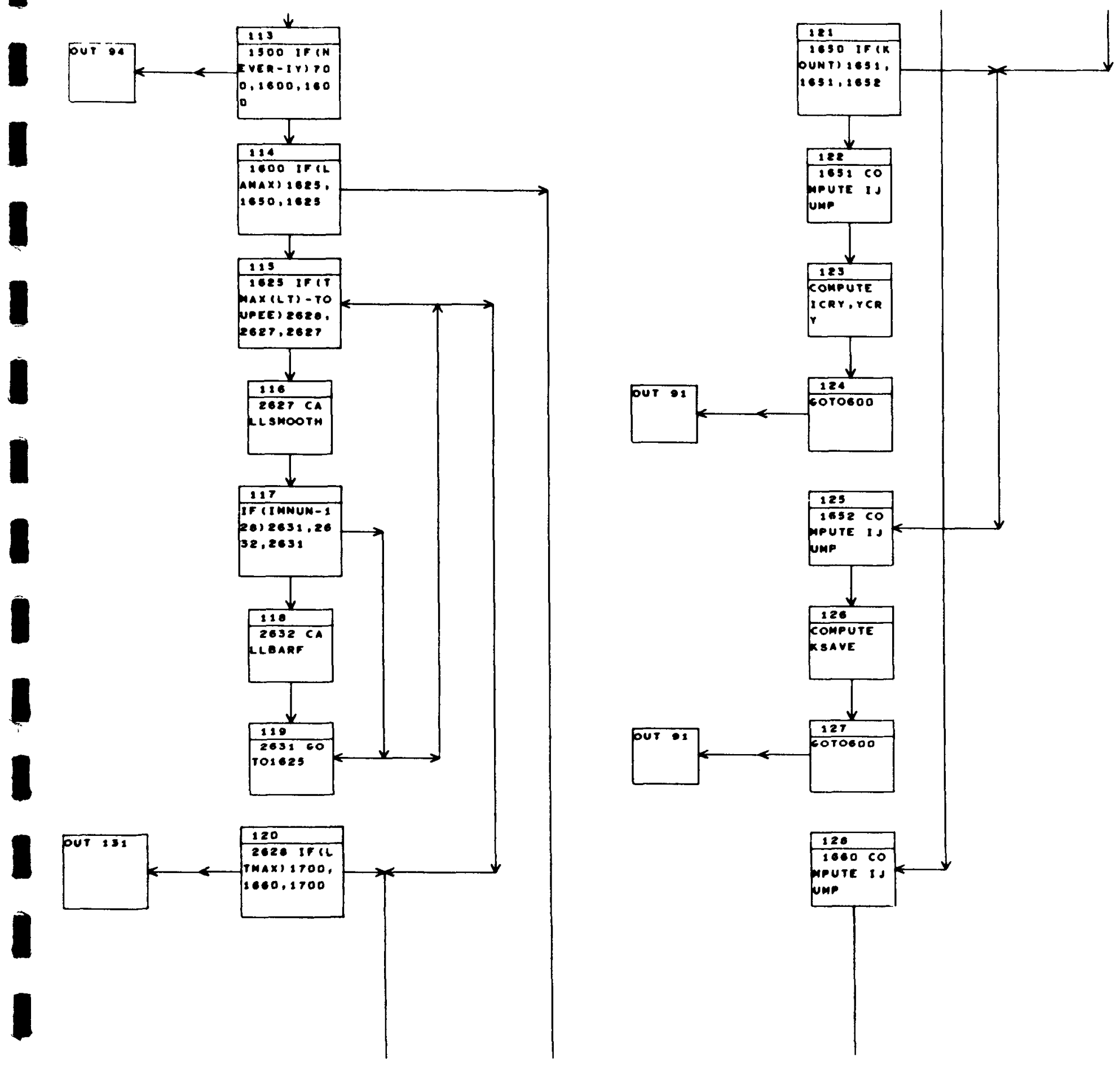

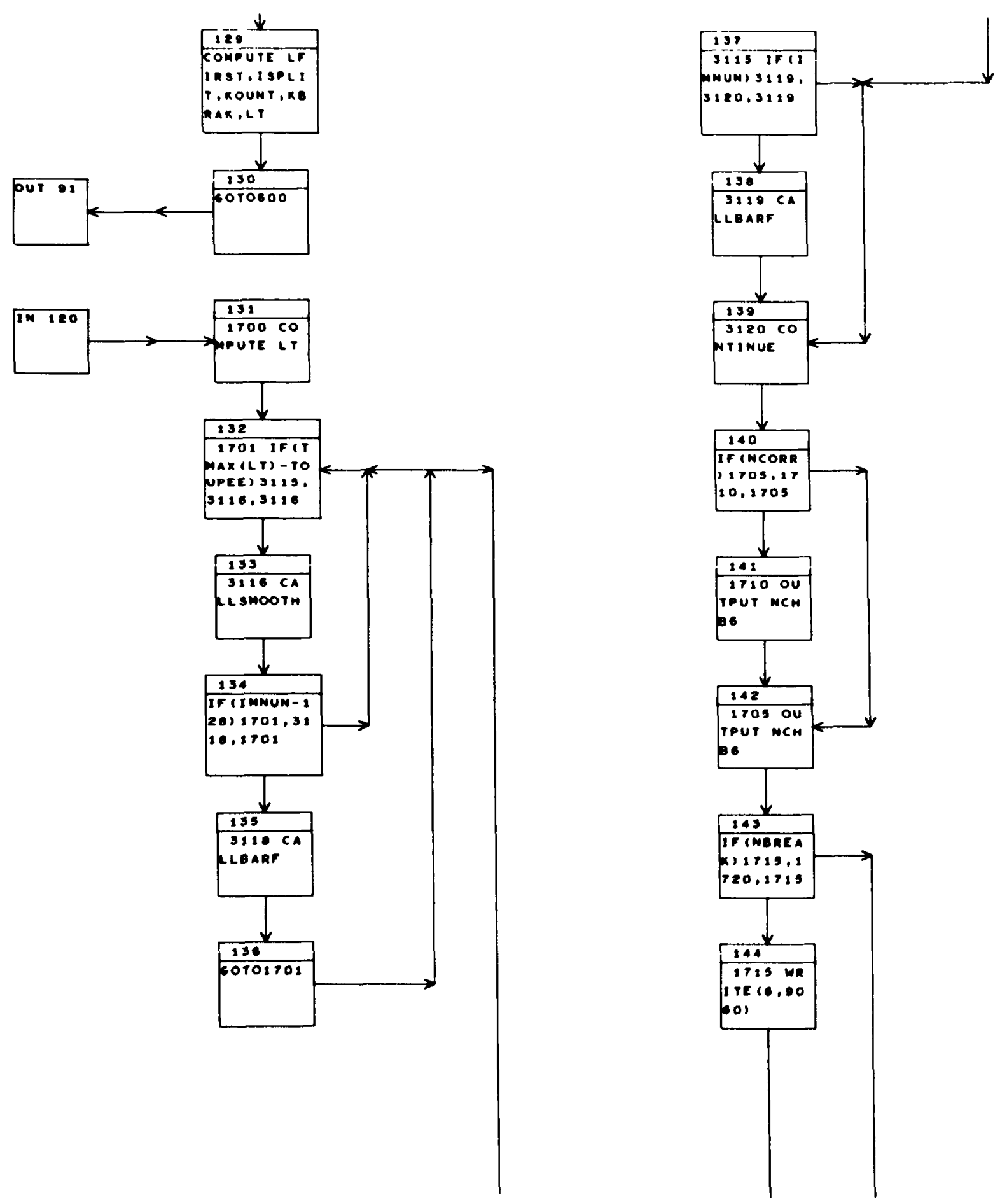
$\mathrm{RN}-\mathrm{S}-0146$
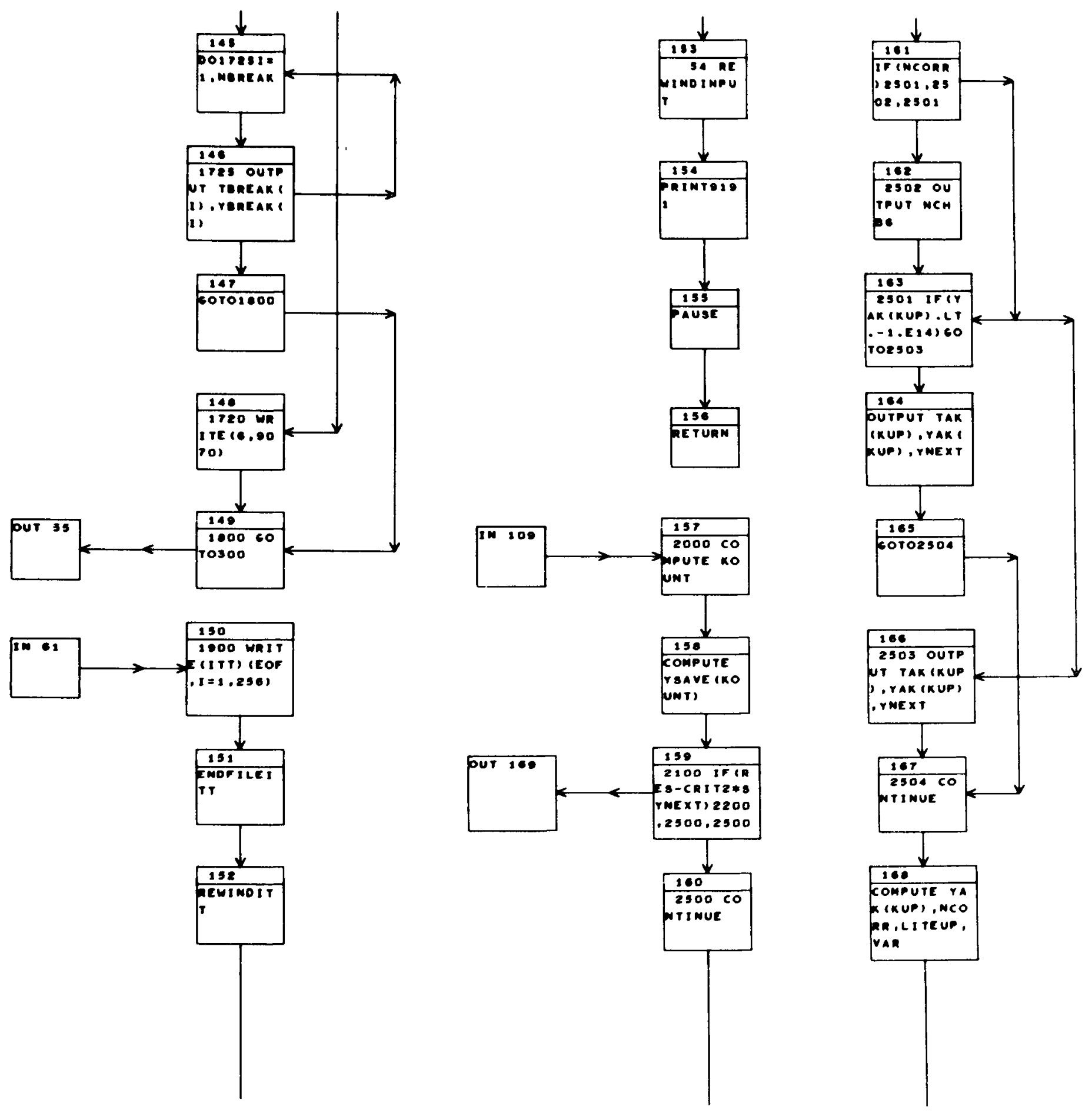

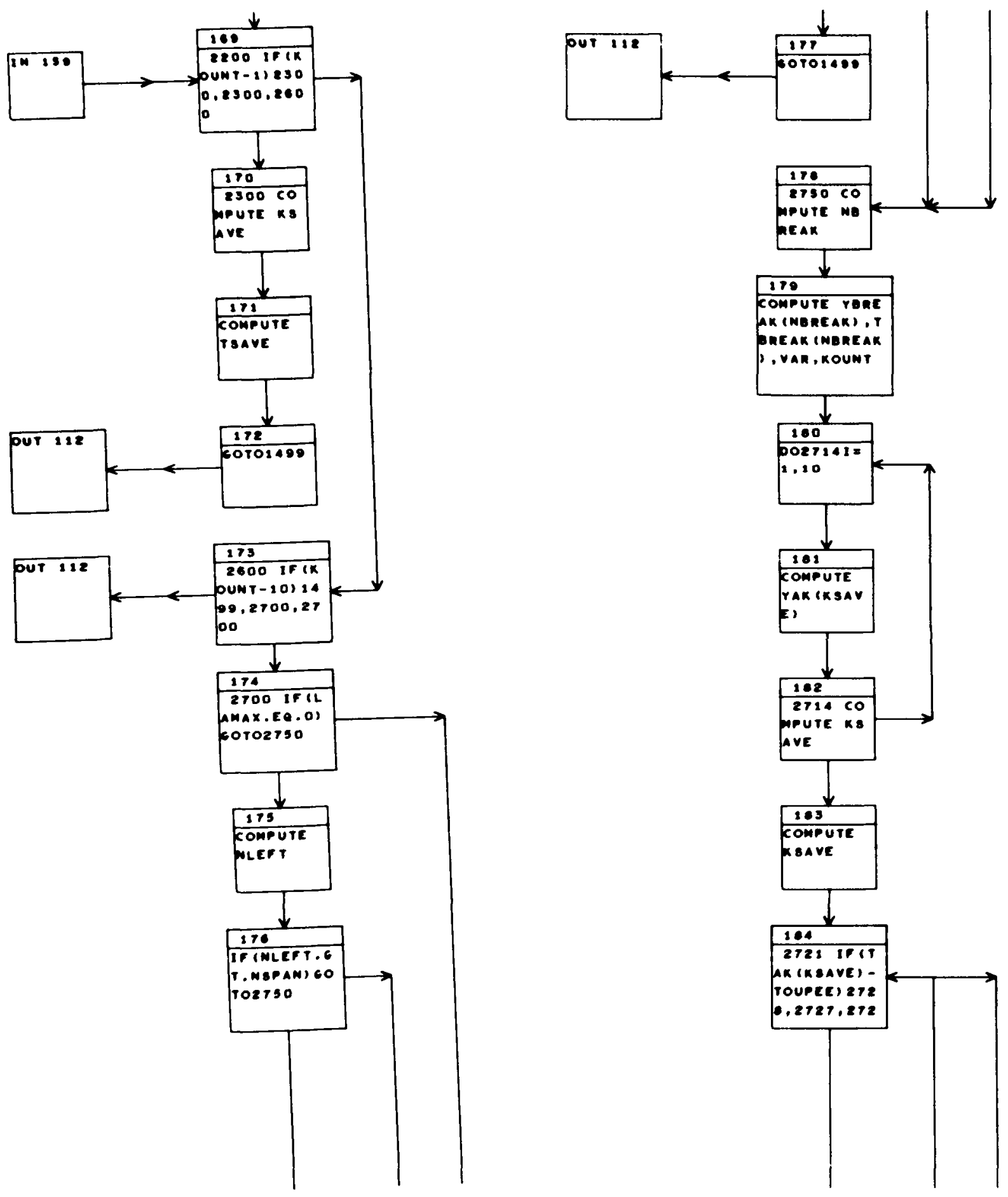


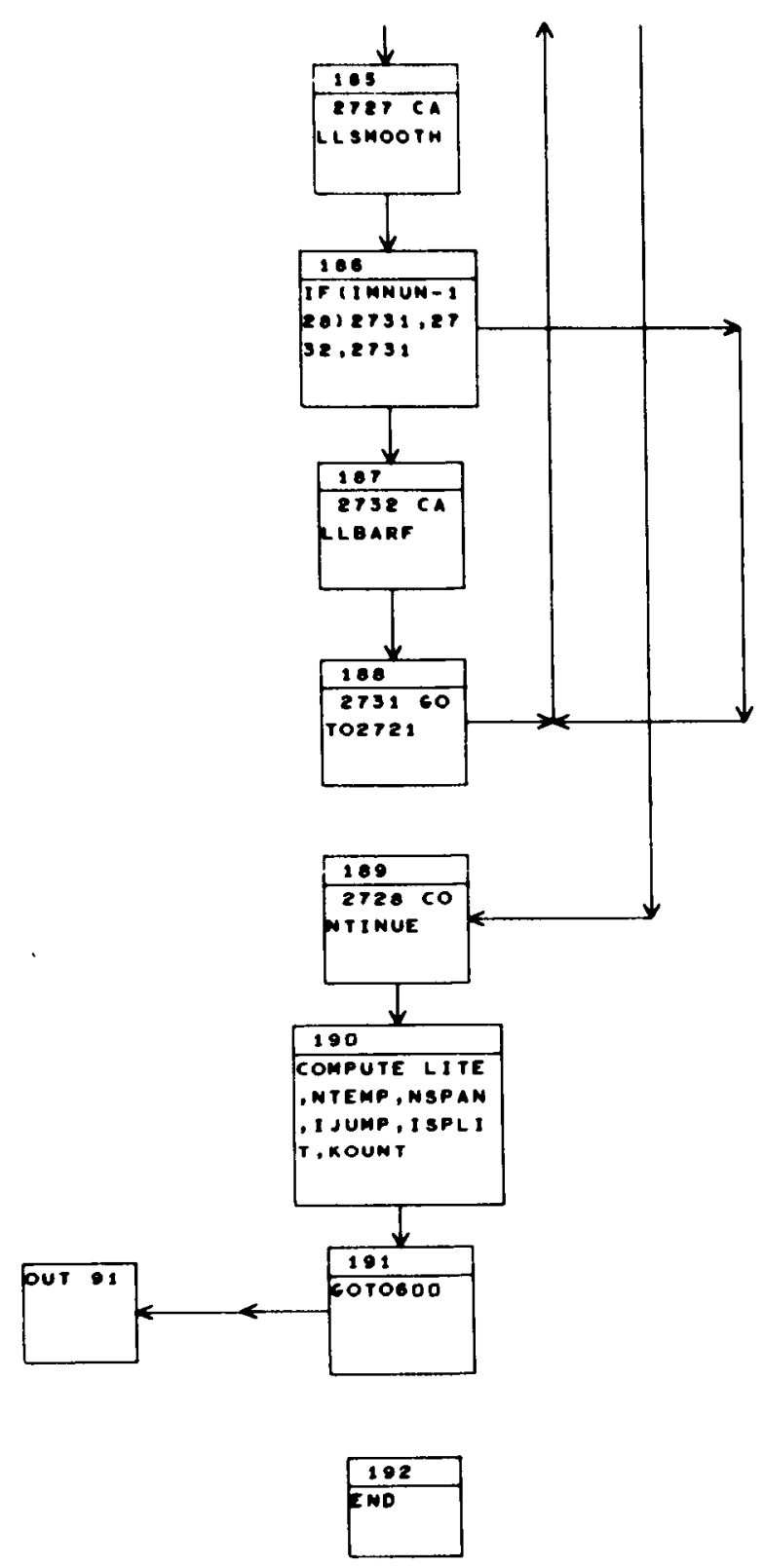


$\mathrm{RN}-\mathrm{S}-0146$
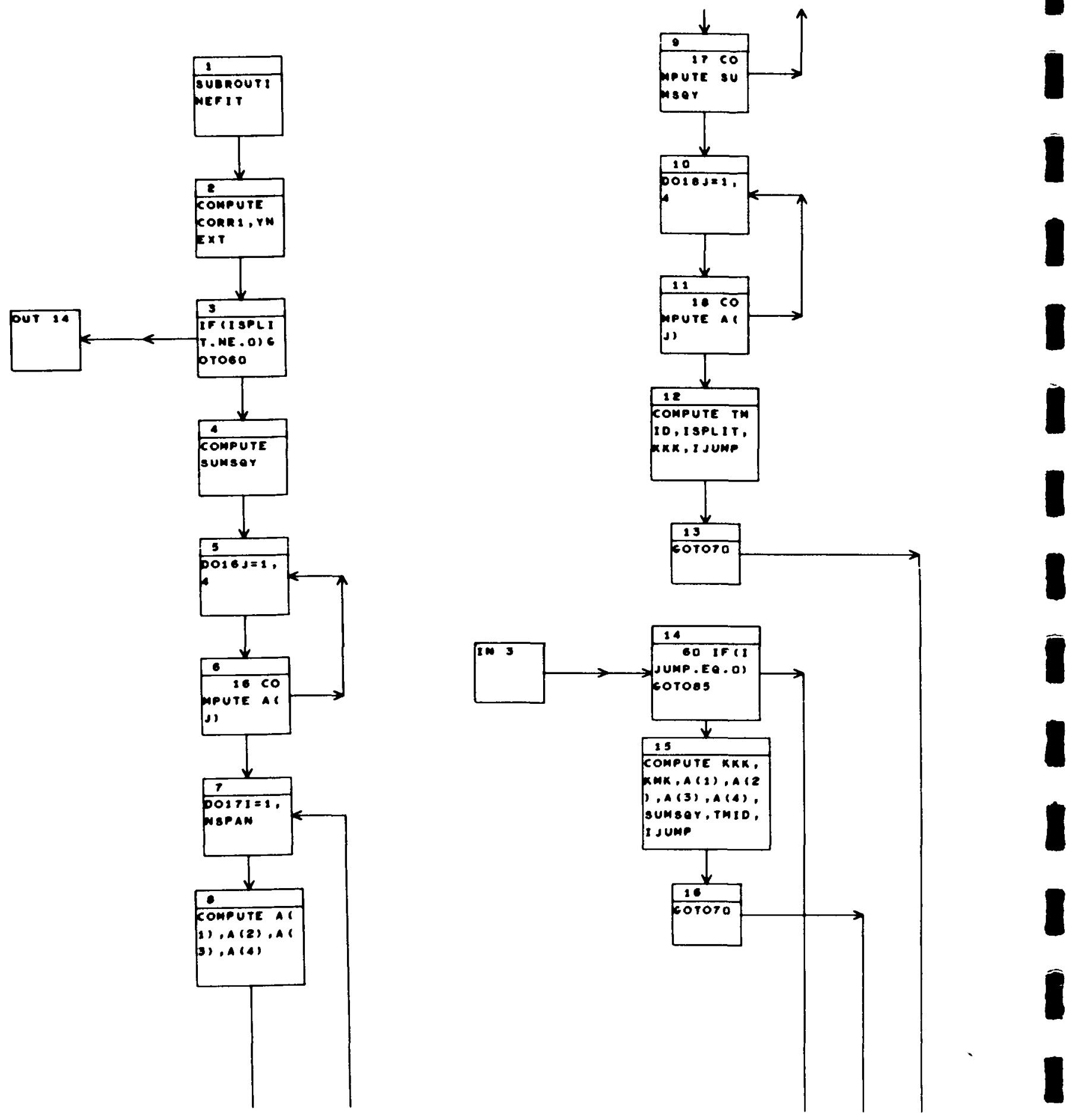

COMPUTE KKK,

Kmx , A (2) , A 12

b, A (3), A (4)

sUMser, TMID.

I J Une

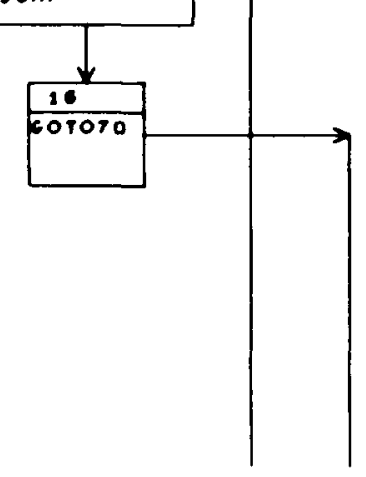



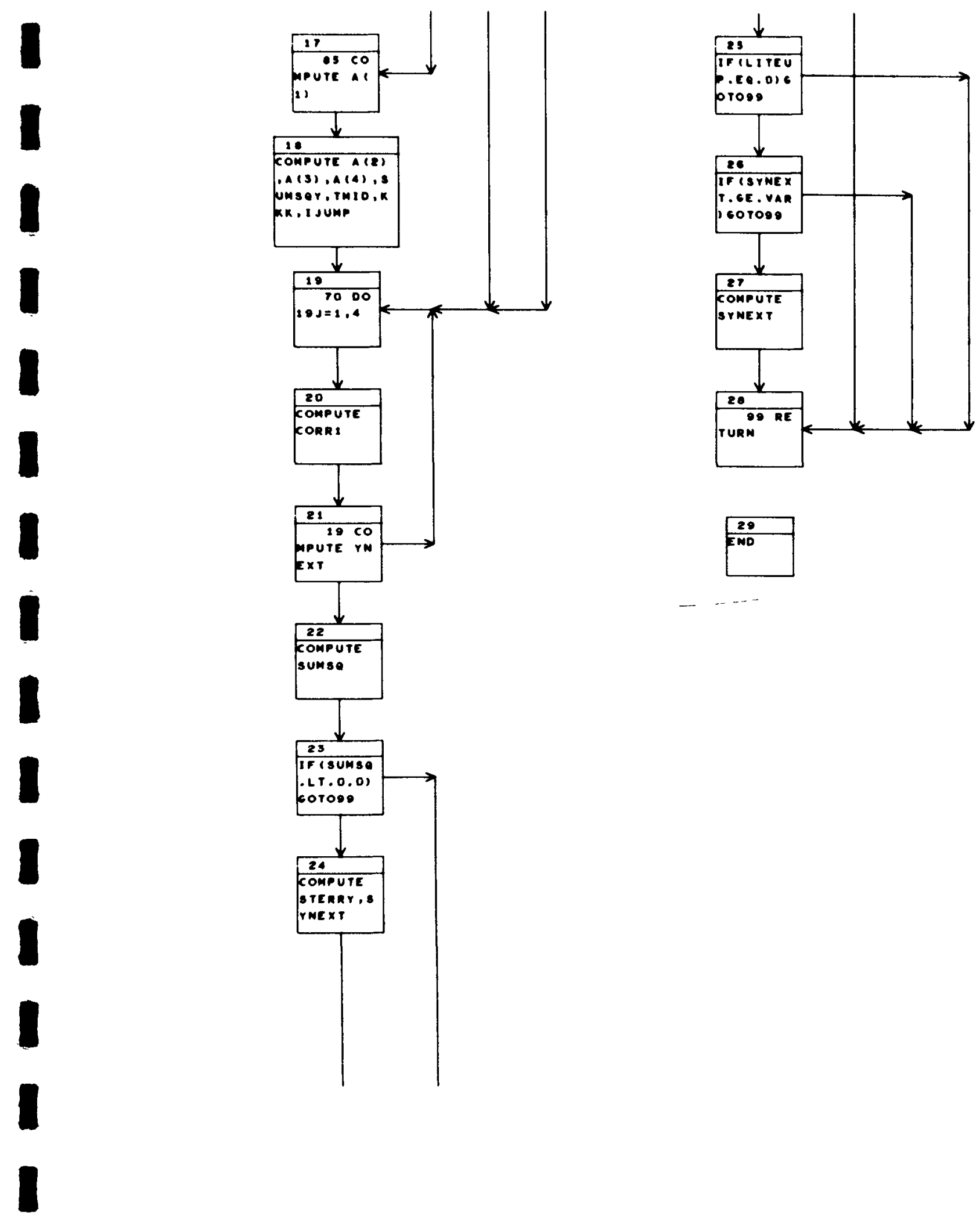
RN-S-0146
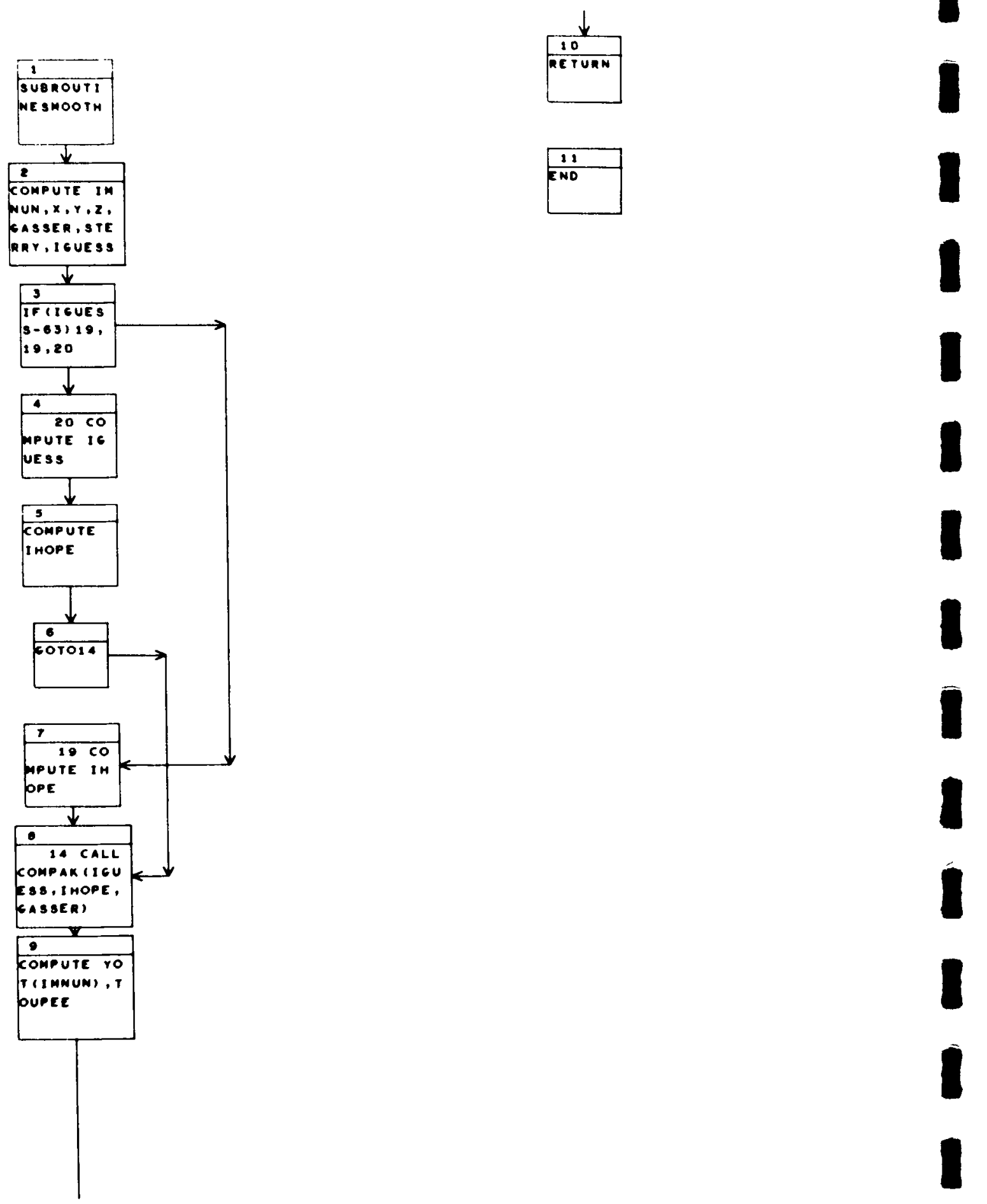

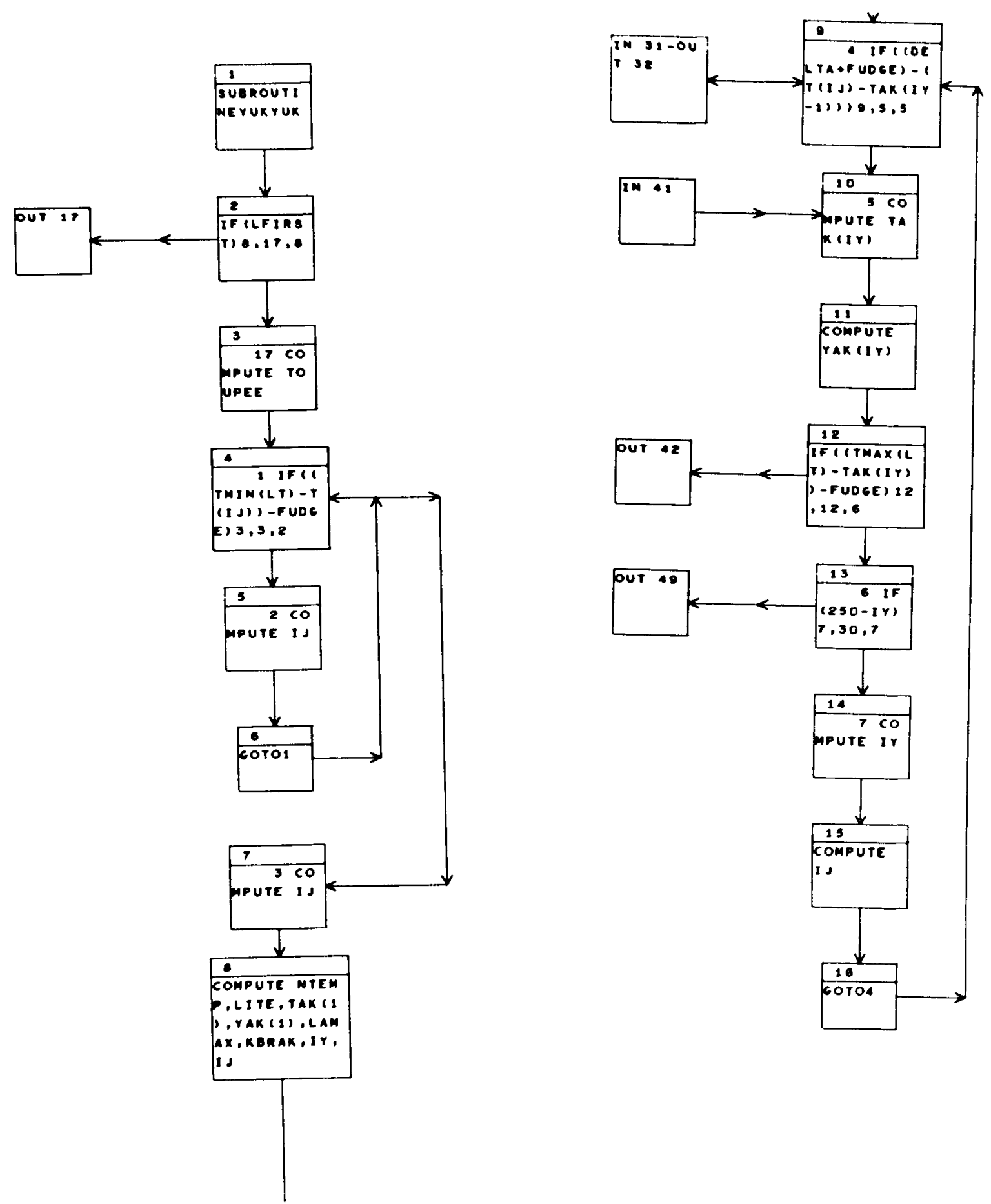
$\mathrm{RN}-\mathrm{S}-0146$
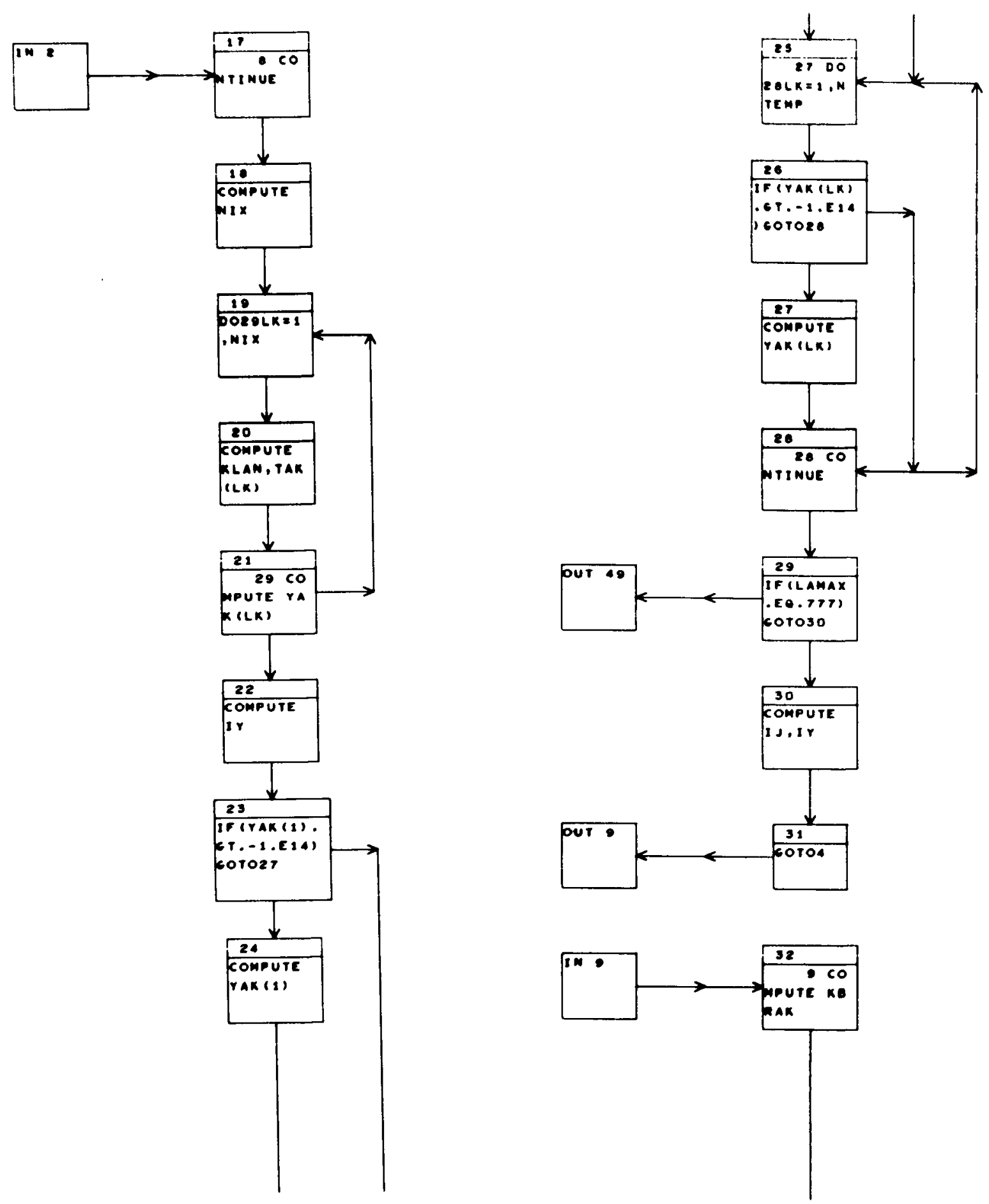

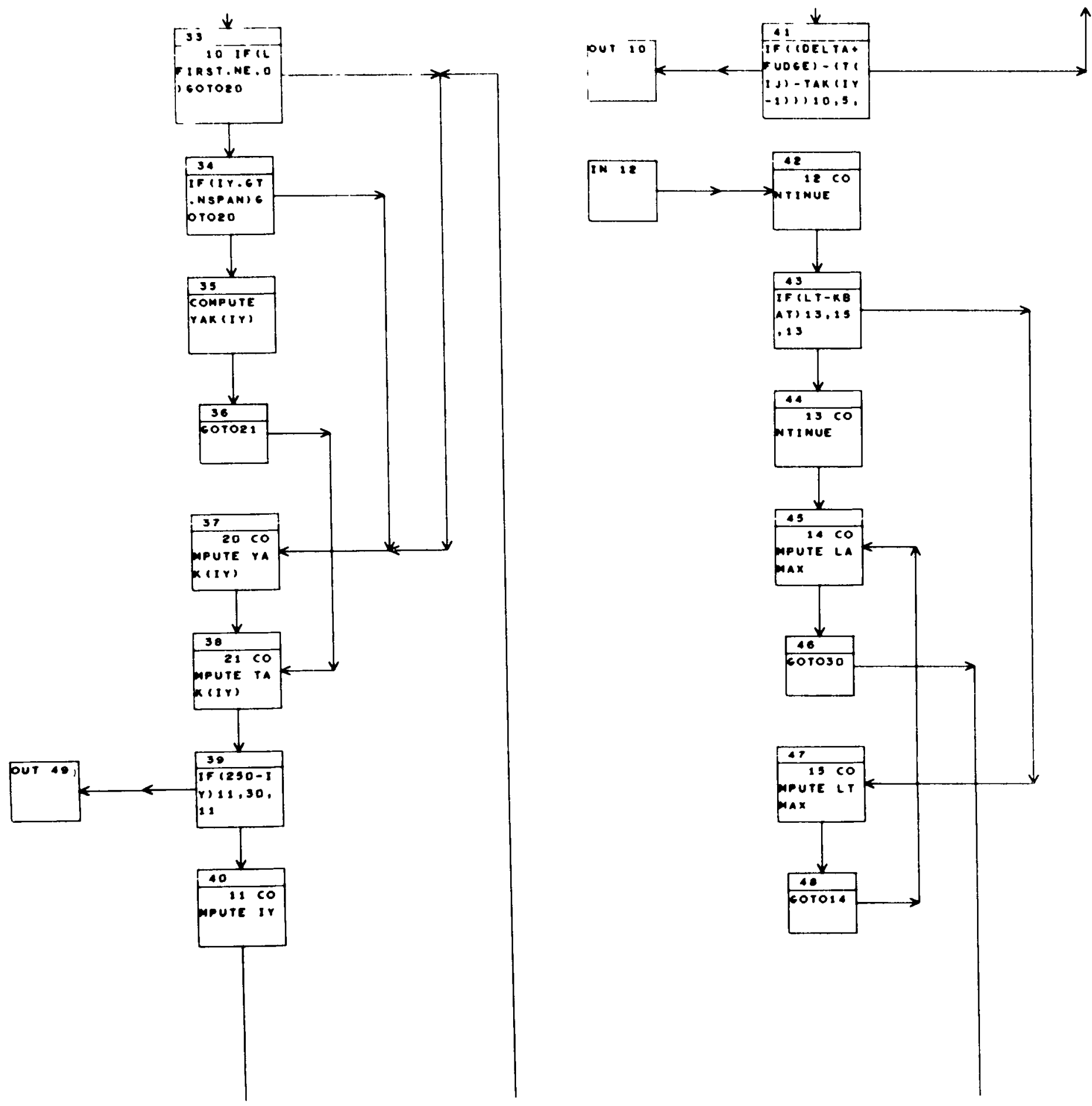

IFILT-RB

AT) 13,15

, 13

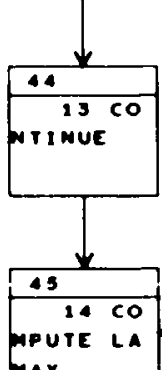

MAX

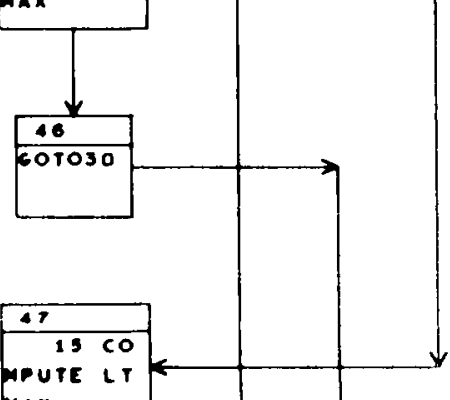

AX

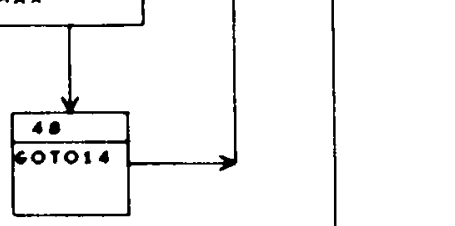


$\mathrm{RN}-\mathrm{S}-0146$
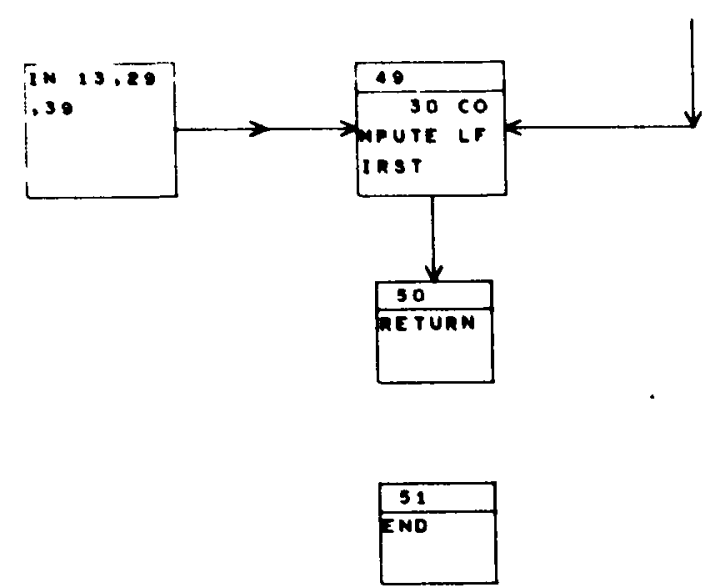

I
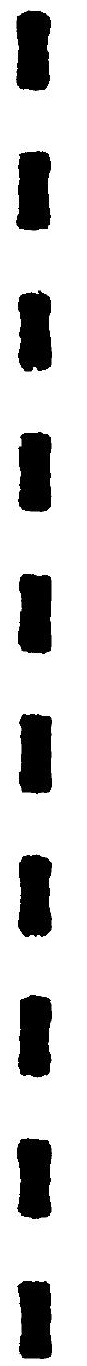

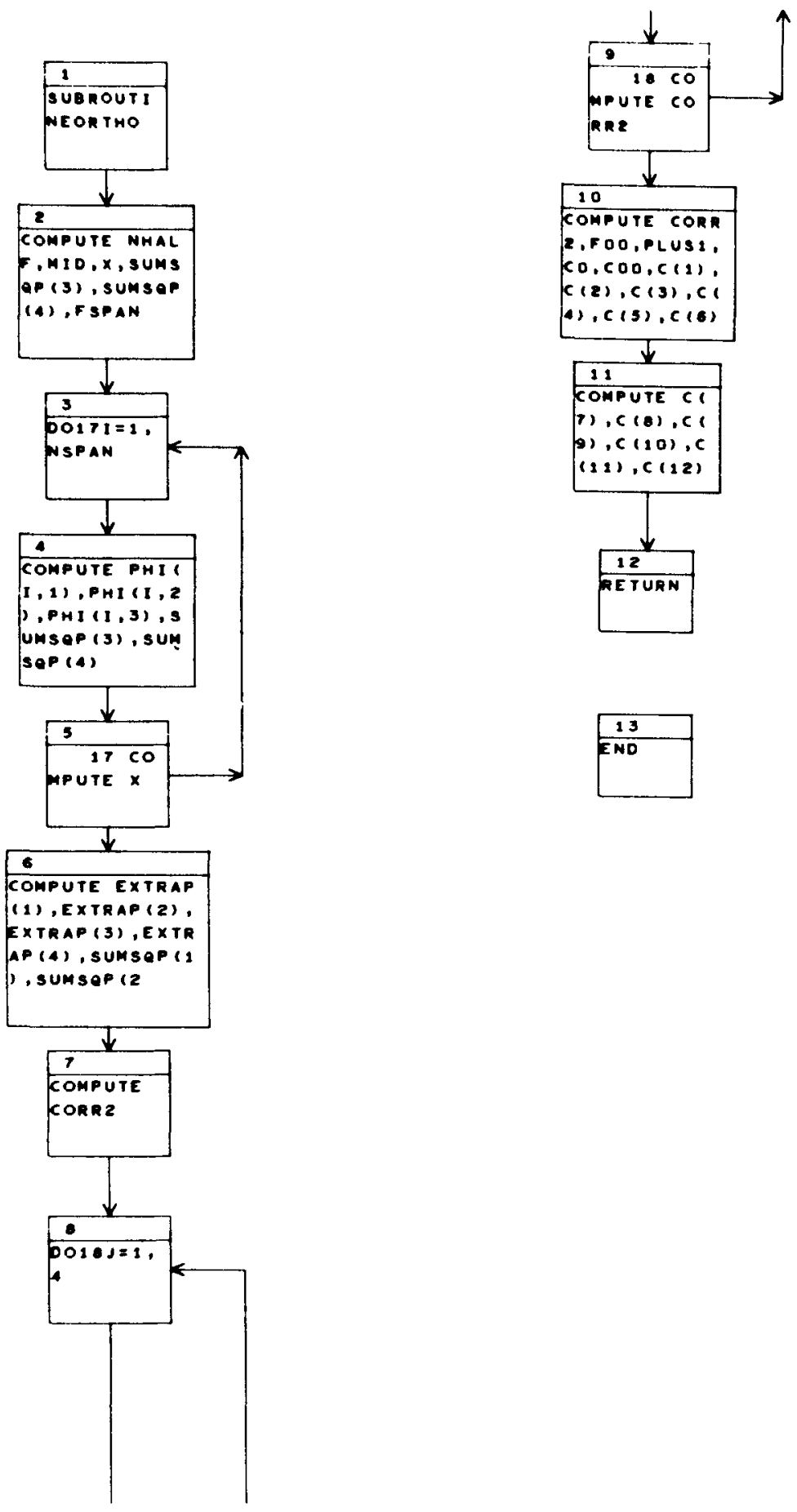

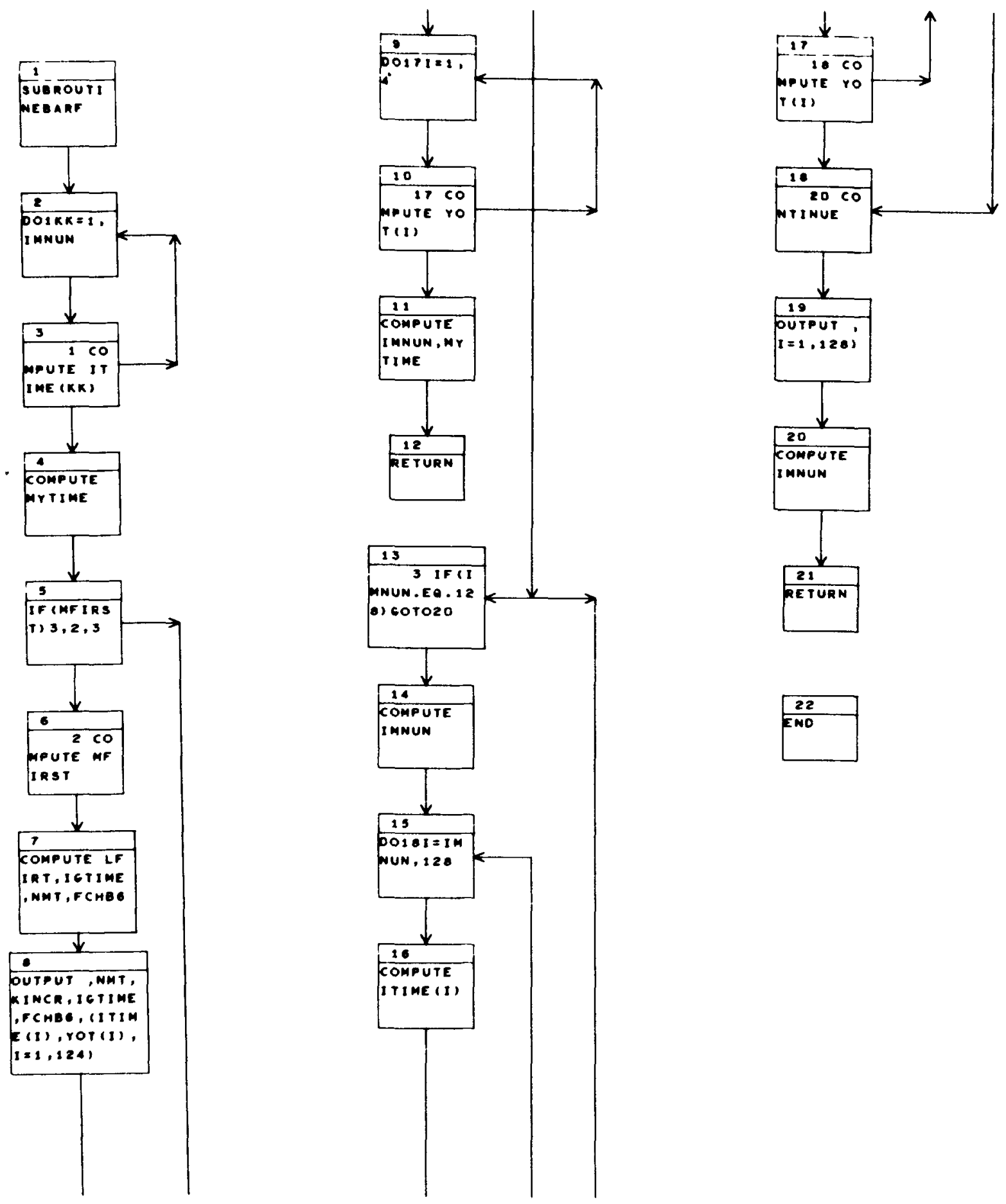
RN-S-0146

APPENDIX C

ETS-1 DATA SYSTEM 
$\mathrm{RN}-\mathrm{S}-0146$

\section{$\underline{\text { ABSTRACT }}$}

The ETS-I data system was designed by the AGC-REON Instrumentation and Controls Department, and procurement specifications were prepared by Edgerton, Germeshausen and Grier, Inc. The system is currently being installed. This appendix presents a description of this data system, including the functions of the data processor. 
APPENDIX C

ETS-1 DATA SYSTEM

I. OVERALI SYSTEM

The purpose of the Data Acquisition System (DAS) is to accept electrical signals from various transducers within the ETS-I complex, condition these signals for both analog and digital recording, and finally to convert the recorded data into usable engineering units.

There are no overall accuracy figures available at this time. However, the most recent state-of-the-art equipment is planned for use. Edgerton, Germeshausen and Grier, Inc. ( $E G$ and $G$ ) makes the following statement: "assuming idealized transducers, it is presently estimated that the systems will be capable of measurement accuracy in the range of 0.1-1\% of span for narrow-band variables (ac-10 cps) and from 1 to $5 \%$ of span for wide-band variables depending on channel type, span, and recording device." The Digital Data System is the most accurate subsystem within the $\mathrm{DA}$, and it has an overall measurement accuracy of $0.1 \%$ for high-accuracy, high-level channels, $0.15 \%$ for high-accuracy, low-level channels including amplifiers, and $0.5 \%$ for low-accuracy channels.

As may be seen from the Instrumentation Block Diagram, Figures C-I through C-4, most of the signals entering the system emanate from diagnostic type transducers associated with the engine and reactor. There are 817 of these channels plus spares available for this purpose which are broken down in accordance with the transducer type on Figure C-l. Signal sources from the exhaust system, side shields, and conventional facilities may also be identified which include an additional 113 channels plus spares.

Following the flow of data from the transducers, the signals enter the signal conditioners where desired sensitivities are established, and where channelization and calibration techniques are derived (Figure C-2). Thermocouple 


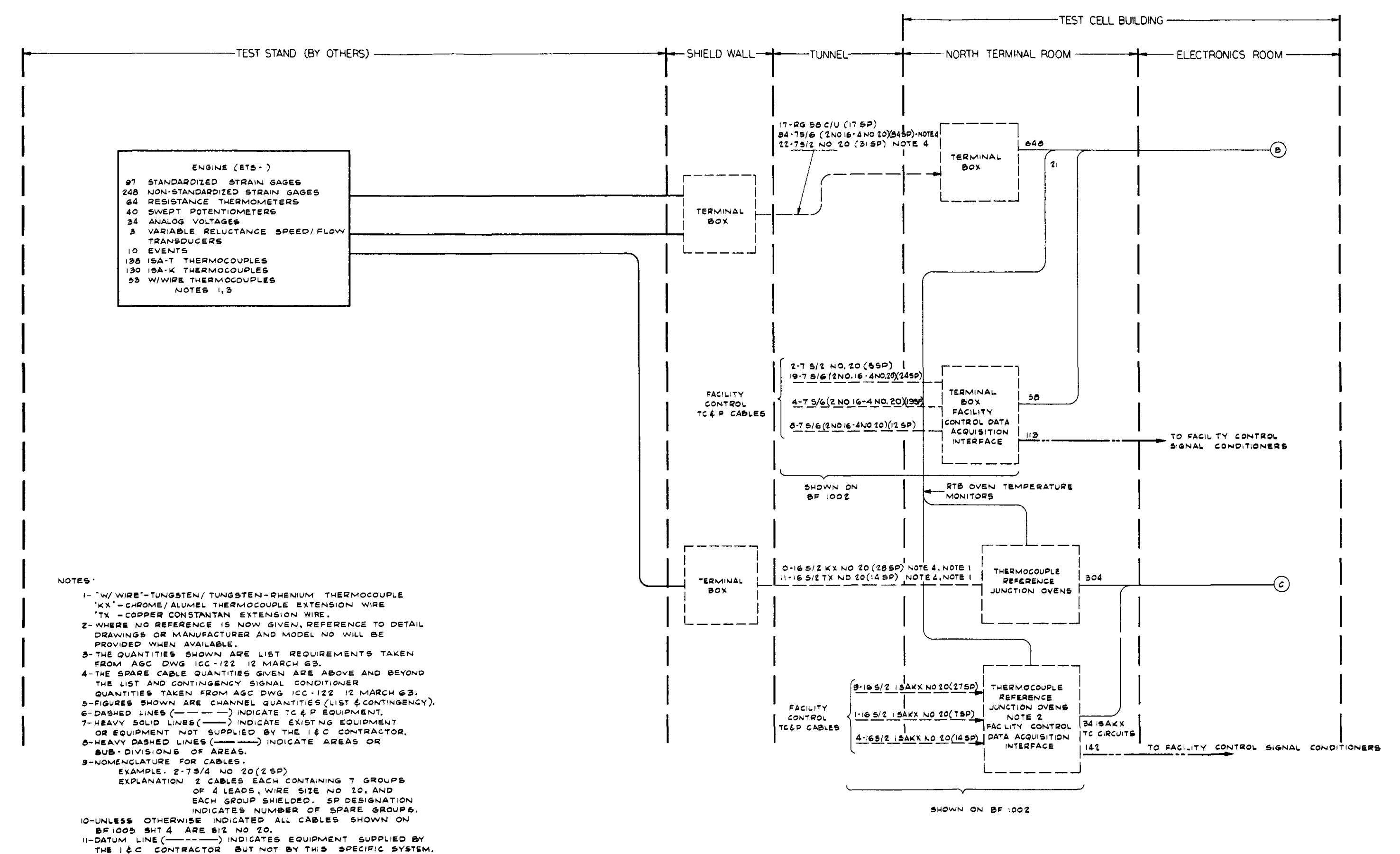

Figure C-1

First Instrumentation Block Diagram

(From EG and G Dwg No. BF-1005 sheet 1 of 4) 


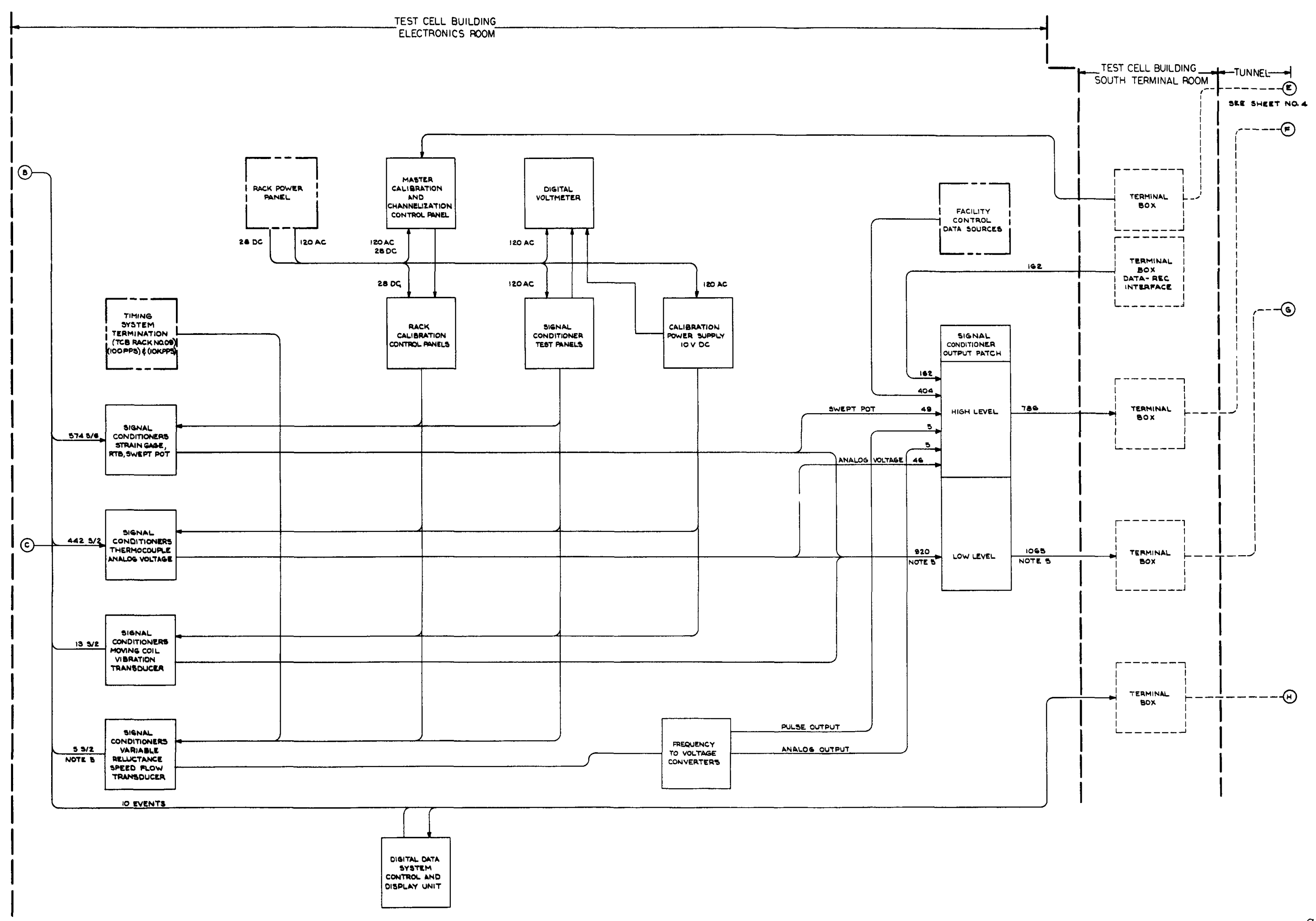

Figure C-2 


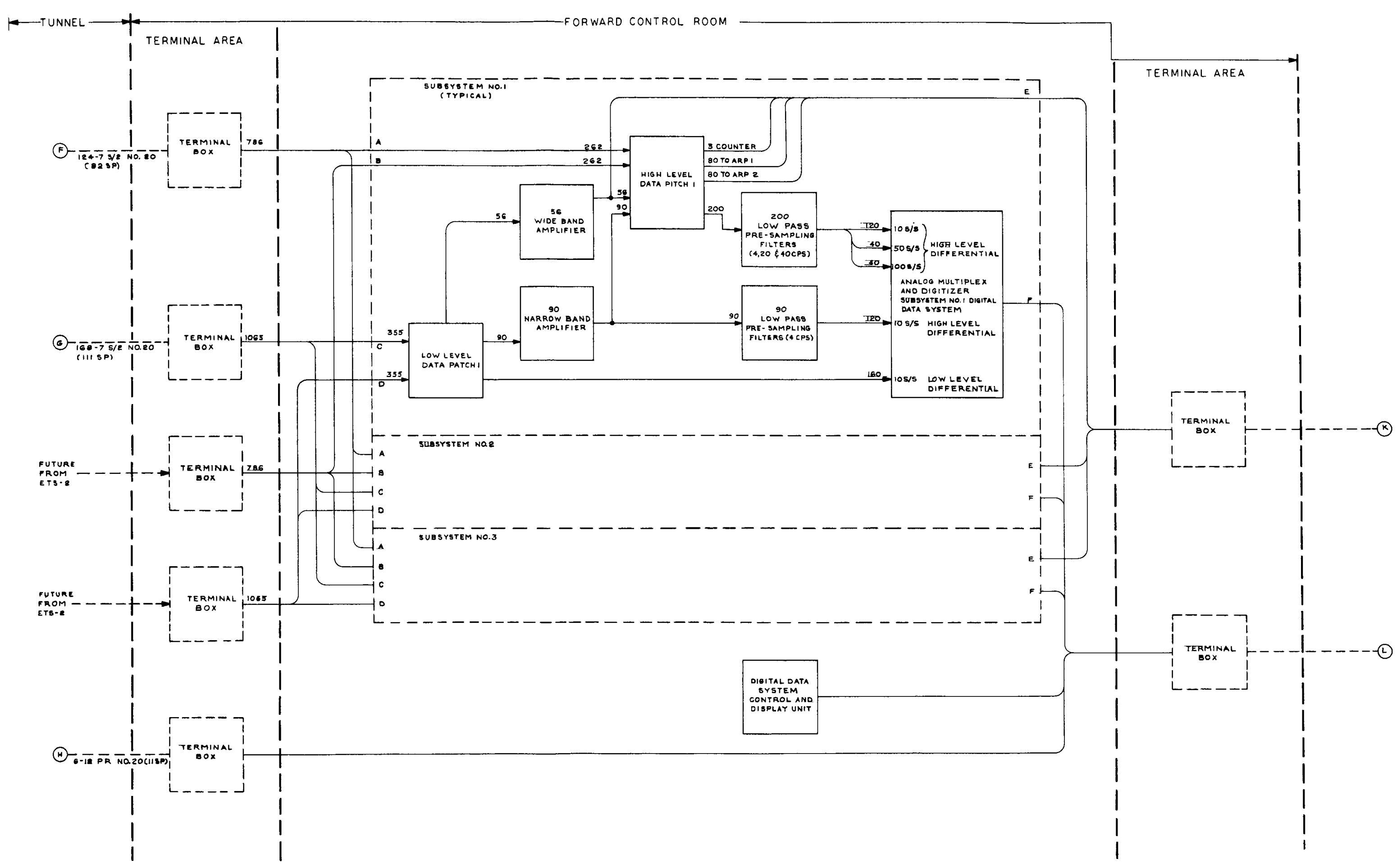

Figure C-3

Third Instrumentation Block Diagram (From EG and G Dwg No. BF-1005 Sheet 3 of 4) 


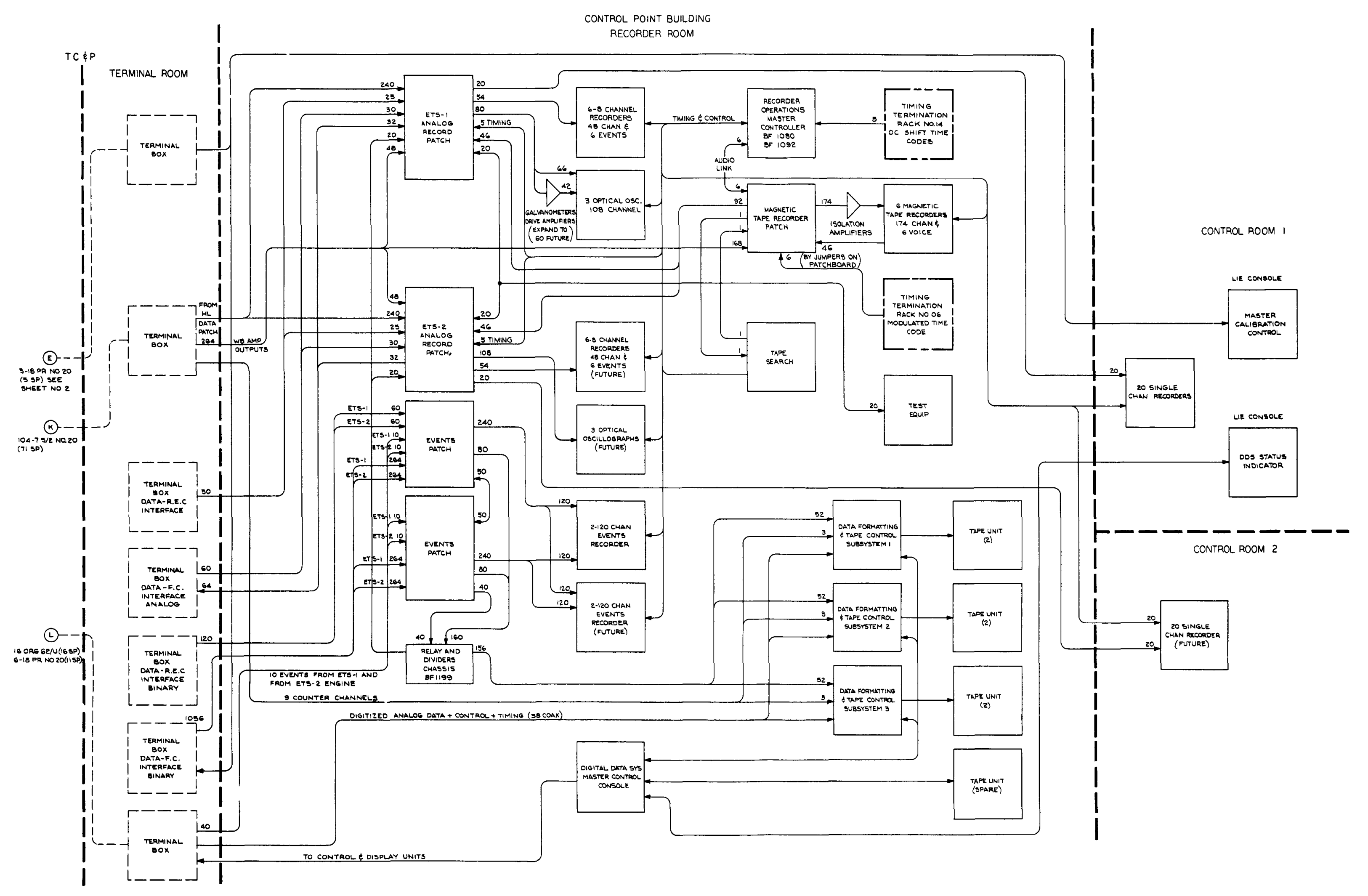

Figure $\mathrm{C}-4$ 
$\mathrm{RN}-\mathrm{S}-0146$

conditioners are preceded by reference junctions of the "heated junction" type. All the thermocouples are referenced in this manner except for $\mathrm{W} / \mathrm{W}$-Re thermocouples which are tied directly to copper transmission wires at the test stand terminal box.

Thus far, only diagnostic type channels have been discussed. However, signals emanating from control loops and other measurement sources within the Remote Engine Control System (REC) and Facility Control System (FC) also enter the DAS. The interface is shown in Figure C-2 where $162 \mathrm{REC}$ channels and $404 \mathrm{FC}$ channels are connected to the Signal Conditioner Output Patch along with the 930 diagnostic channels discussed above. It should be noted that 30 additional FC and $25 \mathrm{REC}$ channels enter the system into the Analog Record Patch as shown in Figure $\mathrm{C}-4$.

All FC and REC signals are conditioned prior to their entry, and therefore appear as 5-volt full-scale signals. Furthermore, these signals are fed through isolation networks, either active or passive, and any failure in the Data Acquisition System such as a short circuit will not impair or affect their function in the $\mathrm{FC}$ or $\mathrm{REC}$ by more than $1 \%$.

At this point in the flow of data, the overall system is subdivided into three identical subsystems as shown in Figure $\mathrm{C}-3$. The primary purpose of the subdivision is to prevent a catastrophic loss of data within the Digital Data System in the event of a major equipment failure. It should be noted that the data to be recorded from the future ETS-2 may also enter the DAS at this point.

Entrance into each identical subsystem is through either a low- or highlevel programmable patch boarả. Within each subsystem, signal amplification is accomplished and those signals entering the Digital Data System are multiplexed and digitized. Therefore, the signals emanating from each of the three subsystems are either high-level analog signals ( 5 volts full scale) to be recorded within the Analog Recording System, or digitized signals to be recorded within the Digital Data System. In the Forward Control Room area, the considerable flexibility built into the system should be noted. Through the programmable patch boards and circuit arrangement, low- or high-level signals in either the narrow- or wide-band frequency range, emanating from any selectable channel, may be directed to either the digital or analog recording facility, or to both simultaneously. 


$$
\mathrm{RN}-\mathrm{S}-\mathrm{O} 146
$$

The recording equipment is located within the Control Point Building and is shown diagrammatically in Figure $\mathrm{C}-4$. The capability can be more clearly defined in terms of three primary functions: (a) narrow-band frequency recording, (b) wide-band frequency recording, and (c) events or "on-off" data recording.

The largest and most accurate capability lies within the Digital Data System for recording narrow-band data. There are 480 channels in each subsystem, or a total of 1440 channels. Sampling rates available are 10, 50 and 100 samples/sec. Two digital tape recorders operate in series with each subsystem so that recording can be continued indefinitely. A spare tape unit is also available as a standby in the event of a recorder failure. By this method the predicted mean-timebefore-failure is increased by a factor of approximately 9. Although not shown, an on-site Data-Processor is available for operating in conjunction with the Digital System. It will produce quick-look reports and perform pre-and post-run calibrations, channelization checks, channel engineering, tape gapping and tape reproducing. As backup to the Digital Recording, there are six 8-channel strip chart recorders and three 36-channel optical oscillographs for parallel recording of certain selected channels. Critical channels may also be displayed on the 20 single-channel strip chart recorders located on the graphic display panel within the control room。

Wide-band frequency is recorded within the Analog System on FM magnetic tape recorders. There are six recorders, including one 14-channel and five 32-channel units, resulting in a total capability of 174 channels (including six timing channels). The 14-channel unit contains one direct recording track with a response of $200 \mathrm{kc}$ while the remainder are $\mathrm{fm}$ channels having a response of $20 \mathrm{kc}$. Each of the 32-channel recorders contain 31 FM tracks and one direct track having an upper frequency response of $10 \mathrm{kc}$ and $100 \mathrm{kc}$, respectively. Operating the recorders at $30 \mathrm{in} / \mathrm{sec}$, and thus obtaining a minimum response of $5 \mathrm{kc}$ on the FM channels, a continuous running time of 24 min for all channels can be obtained. If desired, the three 36-channel oscillographs can also be made available for backup or playback from the tape recorders. Galvanometers with $5 \mathrm{kc}$ response, as well as low-frequency response, will be made available. 
RN-S-OI46

The Events Recording System primarily consists of two 120-channel recorders for recording time-related "on/off" type data. However, as shown in Figure C-4, there are an additional 156 channels within the Digital Data System, thus providing a total capability of 396 event-recording channels. It should be noted that the 156 channels within the Digital system do not interfere with the 1440 data channels discussed previously. Forty channel connections, divided evenly from the Events Patch to the ETS-1 and ETS-2 Analog Record Patch, also provide added capability, or permit the user to record "on/off" events and related data signals on the same chart or oscillogram.

As mentioned previously, considerable flexibility has been built into the system by the use of effective patching. Any channel from diagnostic sources or control loops may be fed into any one of the three subsystems and then recorded graphically and/or digitally. It should also be noted that the ETS-I and the future ETS-2 analog recorders may be shared if, through an unusual circumstance, the added capability became necessary. This is made possible through switchable grounding techniques which also permit the partial or complete, independent and simultaneous, operation of the ETS-I and ETS-2 analog recorders. The three digital subsystems also have separate switchable ground busses which permit the use of one subsystem for preliminary set-up on one test stand, while the other two subsystems are devoted to the test stand nearing its firing schedule.

II. SIGNAL CONDITIONING SYSTEM

The principal function of the Signal Conditioning System is to adapt and translate transducer variables into usable electrical signals for entry into the recording systems. This is accomplished through the use of specially designed signal conditioners, transducer excitation power supplies, and signal amplifiers. Another major function of this system is to provide a rapid means for calibrating the data channels and to verify their integrity. Each of these principal functions is discussed.

The signal conditioners, as designed by EG and $G$, contain considerable flexibility so that two basic designs may be readily adapted to the various transducers through the use of plug-in cards. Figure C-5 presents a typical schematic of a 


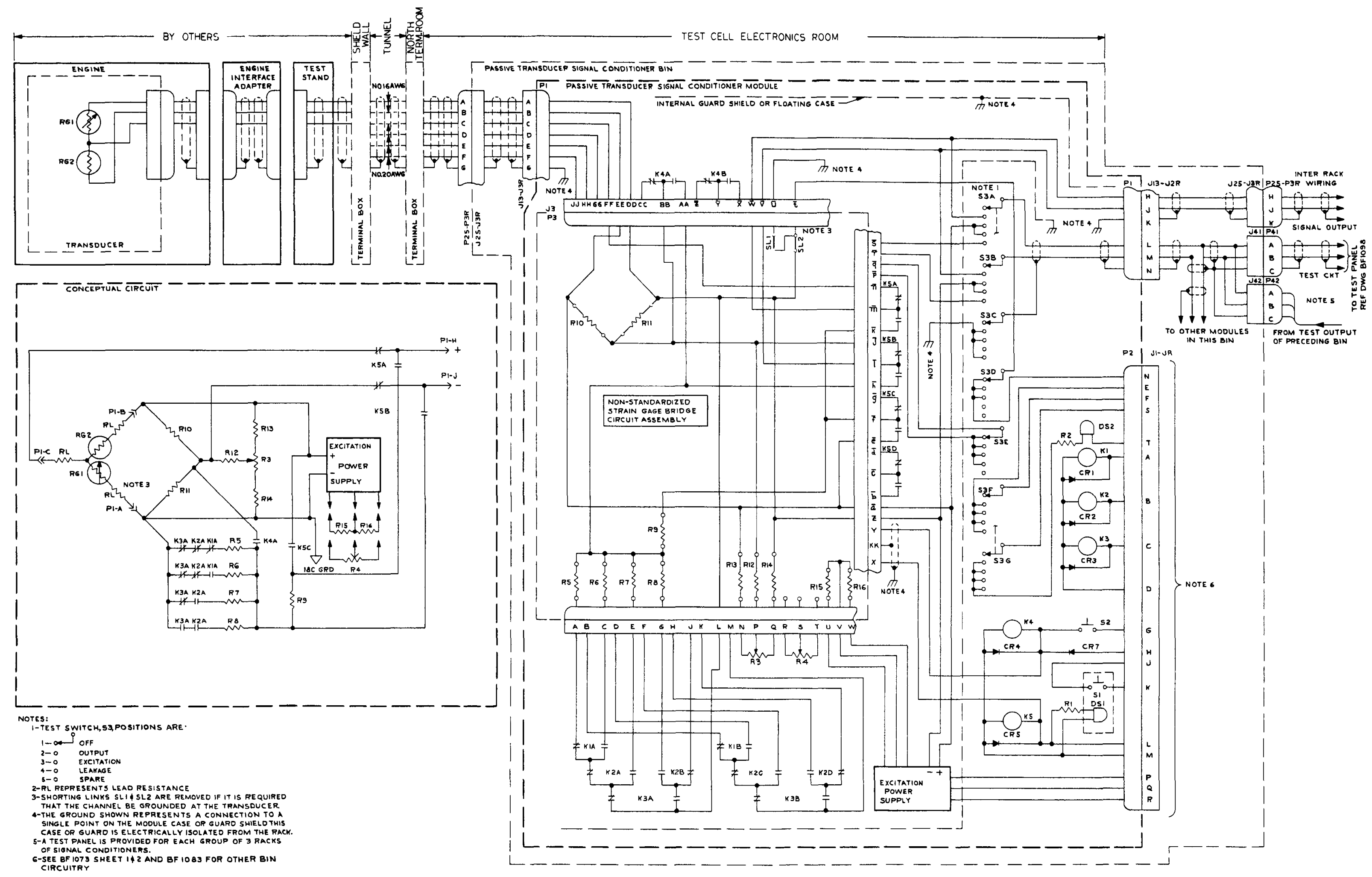


"two external arm strain gage" conditioner. This unit contains the briage completion and balance networks, shunt calibration resistors and insertion relays, relays for channelization controls, and a precision single-channel power supply for transducer excitation. By comparision, Figure C-6 depicts a thermocouple conditioner containing a voltage step divider network with insertion relays for calibration, relays for channelization controls, and a mercury cell for zero offset. Between these two examples of conditioners for passive and active transducers, respectively, there are variations to accommodate full bridge gages, swept pots, bridge-in-head RTTs, triple bridge RTTs, variable reluctance (pulse) speed and flow transducers, moving coil vibration transducers, and analog voltage signal sources. The test switch located on the front panel of each conditioner permits a rapid check of the various parameters of each channel, including leakage checks, resistance checks, zero balance, and excitation measurement, using a local meter panel or a central digital voltmeter. The design is such that an elapsed. time of not greater than I min should be sufficient to complete these adjustments and tests for each channel.

The signals emanating from the conditioners are fed to the recording systems directly or through amplifiers. Directly applied signals are either inherently high-level ( 5 volts) or millivolt signals that are accepted by a differential, low-level multiplexer into the Digital Data System. In the latter case, only lowaccuracy $(0.5 \%$ or greater) data are handled in this manner. High-accuracy (0.15\% or less) low-level data entering the Digital Data System and varying from $10 \mathrm{mv}$ to $100 \mathrm{mv}$ full scale are preamplified to a 5-volt level by isolated, differential amplifiers for wide- and narrow-band frequency ranges. The amplifiers contribute from 0.05 to $0.10 \%$ to the overall accuracy figure of $0.15 \%$, and the isolation from common mode voltage errors exceeds $120 \mathrm{db}$. $E G$ and $G$ 's design is such that a high degree of isolation is achieved throughout the system, primarily in the critical region between the transducer and amplifier or the transducer and multiplexer. At least $110 \mathrm{db}$ at $60 \mathrm{cps}$ will be achieved for system isclation in spite of the fact that numerous patch panels exist which contribute significantly to the decrease in isolation. By effectively guard-shielding the signal conditioner, channel grounding may be accomplished at either the transducer or at the signal 


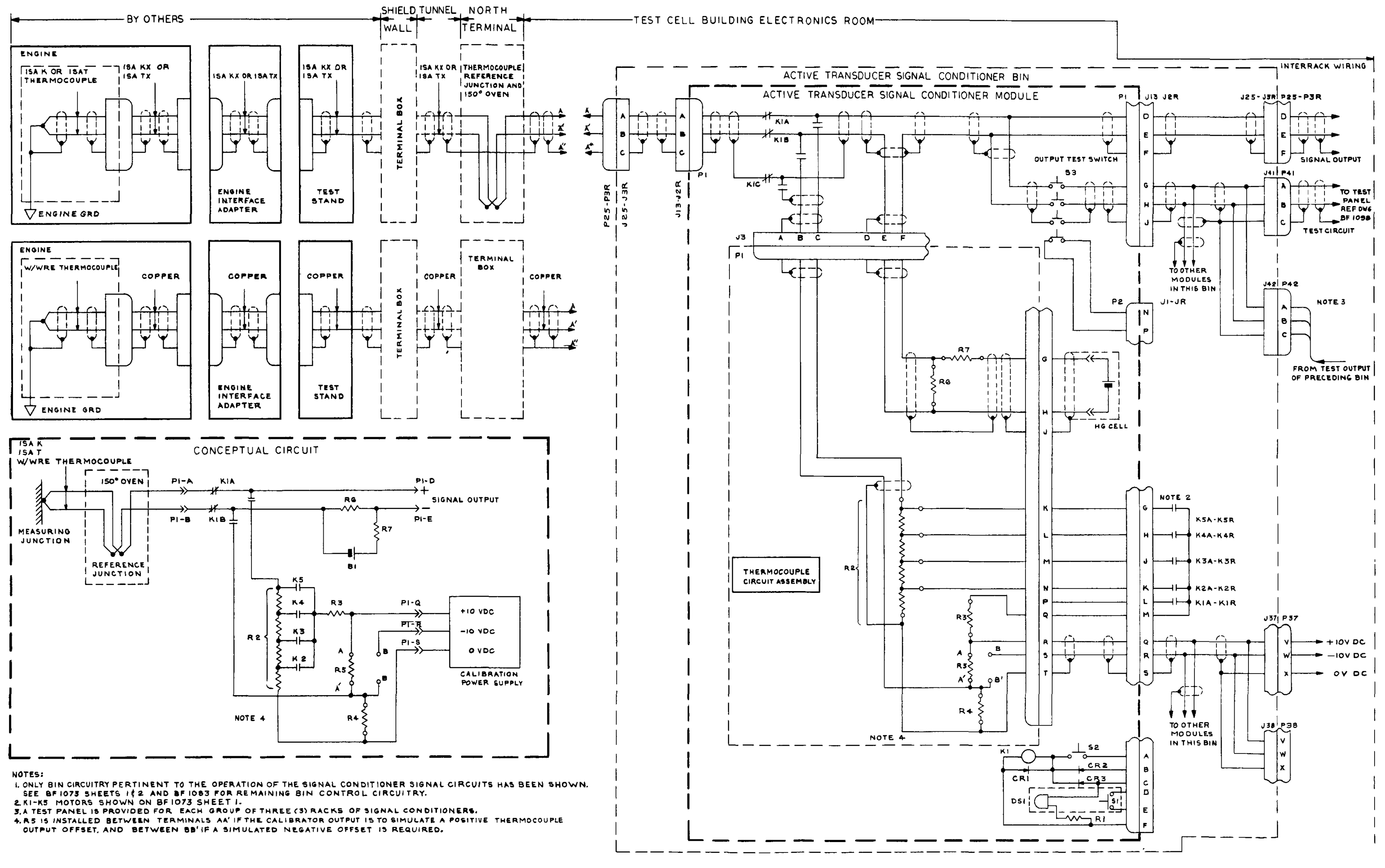

Figure C-6

Thermocouple Signal Conditioner (From EG and G Dwg No. BF-1022) 
conditioner within the terminal room - whichever proves to be desirable or necessary. Initially, the latter approach will be used for all channels except thermocouples wich are grounded at the transducer.

Channel calibration is accomplished at levels of $0,25,50,75$ and 100\% of the signal span for all channels except frequency, which is calibrated at two points only. Both automatic and manual sequencing of these levels are available from local controls in the Test Cell Building or from remote controls on the Lead Instrumentation Engineer (IIE) Console in the Control Point Building. Upon initiation of the controls, all channels within the system are individually and simultaneously subjected to a precise signal corresponding to one of the above levels. In the automatic mode the elapsed time between steps is approximately $10 \mathrm{sec}$ which permits an entire calibration to be accomplished in less than 1 min. Immediately following the initiation of each step, the Digital Data System is commanded to scan all channels and record the data on digital tape. The calibration data are then fed into the Data Processor which stores and later uses the calibration information to correct the actual data obtained during a test firing. Prior to this, however, the Data Processor may be used to compare the calibration data with pre-established limits to permit early detection of out-of-tolerance channels. Individual channel calibration and channel group calibration by rack may also be accomplished. Their controls are local only, and are used during initial channel set-up.

Because of the necessity of changing channel assignments to the recording systems through patching techniques, establishing the integrity of a channel by channelization checks is essential. For those channels to be recorded by the Analog System, individual channels may be manually checked by a pushbutton on the signal conditioner. The Digital Data System channels may also be checked manually, but normally this would be accomplished by an automatic sequencer. The automatic mode control may be operated at the Master Calibration and Channelization Control Panel, and upon initiation, a "flag" signal appears on each channel, one at a time. Since the elapsed time between channel steps is approximately $4 \mathrm{sec}$, the required time to step automatically through the entire system would be between $1-1 / 2$ and 2 hours. Tmmediately following each step, the 


\section{$\mathrm{RN}-\mathrm{S}-0146$}

Digital System is commanded to read. As with the calibration process discussed above, the resulting data are automatically compared to the channel records by the Data Frocessor, and incorrectly wired channels are immediately detected.

\section{ANAIOG RECORDING SYSTEM}

The Analog Recording System provides the means for recording and producing wide-band frequency data, displaying selected critical parameters in real time, and recording certain channels in parallel with other recording means for backup or quick-look purposes. These areas are covered respectively by FM magnetic tape recorders, single-channel strip-chart recorders, and both optical oscillograph and pen recorders. All signals entering this system will be at a level of 5 volts full scale, since each signal emanates from high-level transducers or amplifiers in the Forward Control Room. Because channel grounding to the $I$ and $C$ ground bus is accomplished at the amplifier output, the inputs to the analog recorders are floating, either inherently or through isolation amplifiers.

At the present time there are only 72 vibration measurements accounted for in the NERVA criteria, However, the wide-band recording facility far exceeds this ( 174 channels, including 6 timing channels) so that unexpected requirements, such as those developed at Test Cell A, NRDS, may be readily accommodated. Most of the data to be recorded are adequately covered by the bandwidth from dc to $5 \mathrm{kc}$. Therefore, 32-channel analog tape recorders (five total) were selected for this purpose as a means for obtaining the most channels within a minimum space, while retaining, for the most part, the performance of a 14-channel recorder. Ampex and Sangamo Electric presently produce this type of machine. A brief listing of the approximate specifications appears below.

Number of channels: $\quad 31 \mathrm{FM}$ and 1 direct

Frequency response: at $60 \mathrm{ips}$ de to $10 \mathrm{kc}$ FM and $300 \mathrm{cps}$ to $100 \mathrm{kc}$ direct; at 30 ips de to $5 \mathrm{kc}$ FM

Signal-to-noise ratio at 60 ips:

Crosstalk:

Input level:

Input impedance:

$30 \mathrm{db}$ direct and $42 \mathrm{db} \quad \mathrm{FM}$ $-40 \mathrm{db}$ at $1 \mathrm{kc}$ and $100 \mathrm{kc}$ for direct adjustable from 0.5 to 25 volts rms $100 \mathrm{k}$-ohms 
Output impedance:

Tape speeds:

Linearity:

Flutter at 60 ips:

Reel size:

Tape width:
1000 ohms single ended for FM and 100 ohms single ended direct

$60,30,15,7-1 / 2,3-3 / 4,1-7 / 8 \mathrm{in} . / \mathrm{sec}$

$1 \%$ terminal

$0.1 \%$ peak-to-peak, ac to $500 \mathrm{cps}$

$0.3 \%$ peak-to-peak, dc to $10 \mathrm{kc}$

14-in. $\mathrm{NAB}$

1 in.

Recording monitor electronics are provided for all channels. In addition, one 32-channel recorder will be provided with playback electronics for recording the data on an oscillograph, for playing back at reduced speeds into the Digital Data System, or for duplicating tape records.

Also included in the system is one 14-track tape recorder which includes 13 FM channels and one direct channel. Ihis requirement was established to cover all unusually high frequency or transient data, and such data as radiation counter outputs on the Fin channels, and pump speed on the direct channel. With the exception of signal-to-noise ratio and frequency response (which is de to $20 \mathrm{kc}$ and $300 \mathrm{cps}$ to $200 \mathrm{kc}$ at $60 \mathrm{ips}$ for the FM and direct record, respectively), the specifications for the 14 -track unit are essentially the same as those presented above. This recorder will also contain playback electronics.

The inputs to all tape recorder channels are preceded by isolation amplifiers set at a gain of one. This was a necessity since tape recorder inputs are single ended and, as mentioned above, channel grounding is accomplished at the preamplifiers (600 ft from the recorders) in the Forward Control Room.

There are 20 single-channel strip-chart recorders located on the graphic display panel in the EIS-I control center for displaying critical parameters in real time. Selection of any desired channel may be accomplished through the analog patch board as shown on Figure C-4. Either the Leeds and Northrup or Minneapolis-HoneyweIl 6-in.-wide strip charts are the type presently being considered. Each recorder is equipped with a differential preamplifier, used primarily for achieving isolation and high common mode rejection (CMR). In addition to the analog input, a time code channel is provided, allowing a serial code to be recorded on one edge of the chart. A brief listing of typical specifications (including preamplifier) appears below. 
Chart width:
Chart speed:

Perı speed:

Input :

Input impedance:

CMR:
6 in.

Two electrically switchable speeds selected from six available speeds obtainable through gear changes

Less than 1 sec for 6 in. travel

0 to +5 or 0 to $-5 \mathrm{v}$

$100 \mathrm{k}$-ohms

$80 \mathrm{db}$ from de to $60 \mathrm{cps}$ for a CMV of $10 \mathrm{v}$ and a source unbalance of 1000 ohms

Calibrated Accuracy: $\quad 0.25 \%$

For quick-look purposes or for back-up to the narrow-band digital recording or wide-band magnetic tape recording, six 8-channel strip chart recorders and three 36-channel oscillographs are provided. The oscillographs are also used to record playback data from the magnetic tape units. The type of 8-channel recorder presently under consideration is the Brush Mark 200, primarily because of its performance and high quality writing capability using pressurized ink. A brief résumé of its specifications is given below.

\footnotetext{
Capacity:

8 analog channels plus 1 event and timing channel

Channel width: $40 \mathrm{~mm}$

Accuracy:

$1 \%$

Bandwidth:

dc to $100 \mathrm{cps}$

Chart speeds:

0.25 to $100 \mathrm{~mm} / \mathrm{sec}$ in 8 steps

Input :

0 to +5 or 0 to $-5 \mathrm{v}$

CMR :

$60 \mathrm{db}$ at $\mathrm{dc}$ and $40 \mathrm{db}$ at $100 \mathrm{cps}$

The above specifications again include those of a differential preamplifier per channel used to achieve isolation and good common mode rejection.

The three 36-channel optical oscillographs will be of the type presently offered by CEC, Midwestern, or Minneapolis-Honeywell. Galvanometers will be selected to provide a series of frequency ranges up to $5000 \mathrm{cps}$. Galvanometer driver amplifiers with an input impedance of 1 megohm will be available for use; however, since galvanometers are isolated from ground, they may be driven directly from a remote source providing the low load impedance of the galvanometer is acceptable and the driving current requirements are met.
} 
$\mathrm{BN}-\mathrm{S}-0146$

I. FVENTS RECCRDING SYSTEM

The Events System is designed to accept "on-off" voltage signals which emanate from limit switches within various sensing devices and remotely operated valves, and to record these signals with respect to time. The principal items of the system include a programmable patchboard, two event recorders, and an enclosure of relays for transmitting switch closure only.

Inputs enter the system at the programmable patchboard. These include 519 channels from the Facility Control System, 55 from the Remote Engine Control System, and 10 from within the Data Acquisition System. The capability also includes provisions for accepting an equal number of input channels from the future ETS-2 complex. The level of the input signals will be either zero or $+12 \mathrm{v}$ which will be derived directly from the source or from a switch closure at the source in series with a $28-v$ supply and divider network located within the Test Cell Building.

At the present time a selected portion of the 584 (total) inputs may be programmed into two 120-channel event recorders. Expansion capability is also provided so that two additional recorders may be added for ETS-2, thus enabling 480 recording channels to be available to either test stand complex. The recorders are of the binary type. A brief listing of specifications appears below.

\footnotetext{
Capacity: 120 channels each

Speeds:

$1 \mathrm{~mm}$ per sec to $200 \mathrm{~mm}$ per sec in 8 steps

Chart width:

12 in. with printed channel identification

Chart capacity: $450 \mathrm{ft}$

Writing:

Electro-sensitive process

Input:

Differential

Input level:

0 to $+12 \mathrm{v}$

Input impedance:

Greater than $5 \mathrm{k}$-ohms

Bandwidth:

500 event changes per sec

Io provide additional recording capability and to enable selected event channels to be more conveniently correlated with other analog signals, 156 Digltal Data System channels and 40 Analog Recording System channels may be
} 
programmed into the system. The signals entering the Analog System enter directly through the Analog Record Patch (Figure C-4). However, 156 channels of relays are provided so that contact closures only may be transmitted to the Digital Data System for formatting into the digital tape recorder. For a simplified depiction of the complete Events System, including the connections to the console display lights, refer to Figure $\mathrm{C}-7$.

V. DIGITAL DATA SYSTEM

Because of its high accuracy and its capability of handing large volumes of data rapidly and semi-automatically, the Digital Data System (DDS) represents the most significant and principal recording means within the Data Acquisition System. Inputs to the system consist of both high-and low-level analog signals which are multiplexed, digitized, and fed into a formatting unit. Binary events, range time, and pre-selected identification words are also fed into the formatting unit and, together with the above digitized data, are reproduced on computercompatible magnetic tape.

The input capability of the complete system consists of 1440 analog data channels, 156 binary events, and 9 counter channels. However, as mentioned previously, in order to increase reliability and thus prevent a catastrophic loss of data in the event of a major equipment failure, the complete system is subdivided into three identical subsystems. Each one contains its own power system, controls, instrumentation ground, and timing source. Under most operating conditions all three subsystems will operate in synchronism by the central ETS-l timing system. However, any one or two may be operated at one time, or if desired, one subsystem may be assigned to the future ETS-2 Test Stand while the remaining two operate with ETS-1.

Figure C-8 describes one of the three identical subsystems. As discussed in Section I, analog signals entering the Data Patch Boards emanate from the signal conditioners and are either high level ( 0 to $\pm 5 \mathrm{VFS}$ ) or low level ( 0 to $\pm 10,20,30,40,50$ and $100 \mathrm{mv}$ FS). High-level signals enter the DDS through the high-level multiplexers according to the desired sampling rates, (i.e., 10, 50 , or 100 samples/sec). However, low-level signals are further segregated 


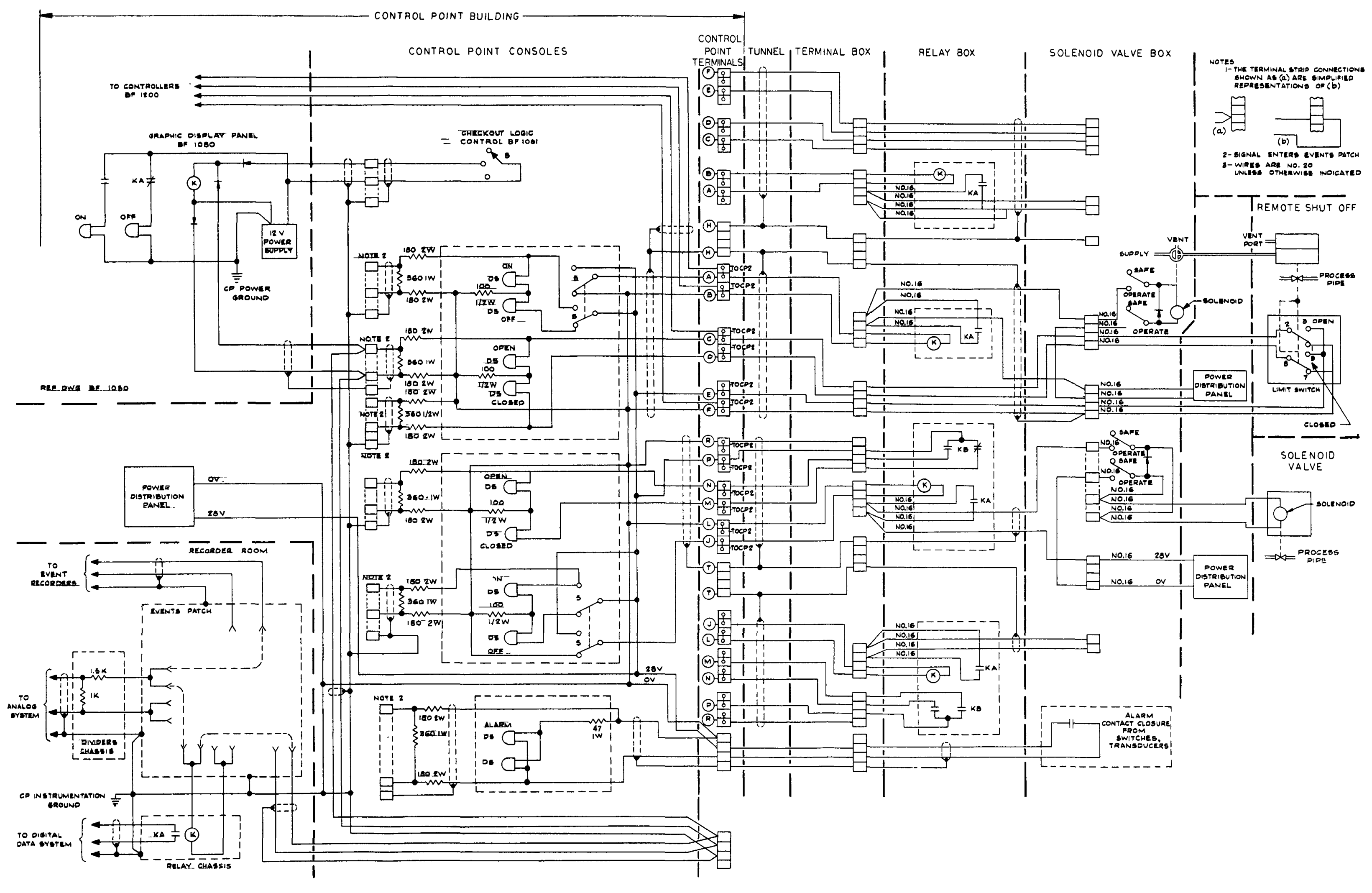

Figure $\mathrm{C}-7$

Typical Binary Control and Indication

(From EG and G Dwg No. BF-1199) 


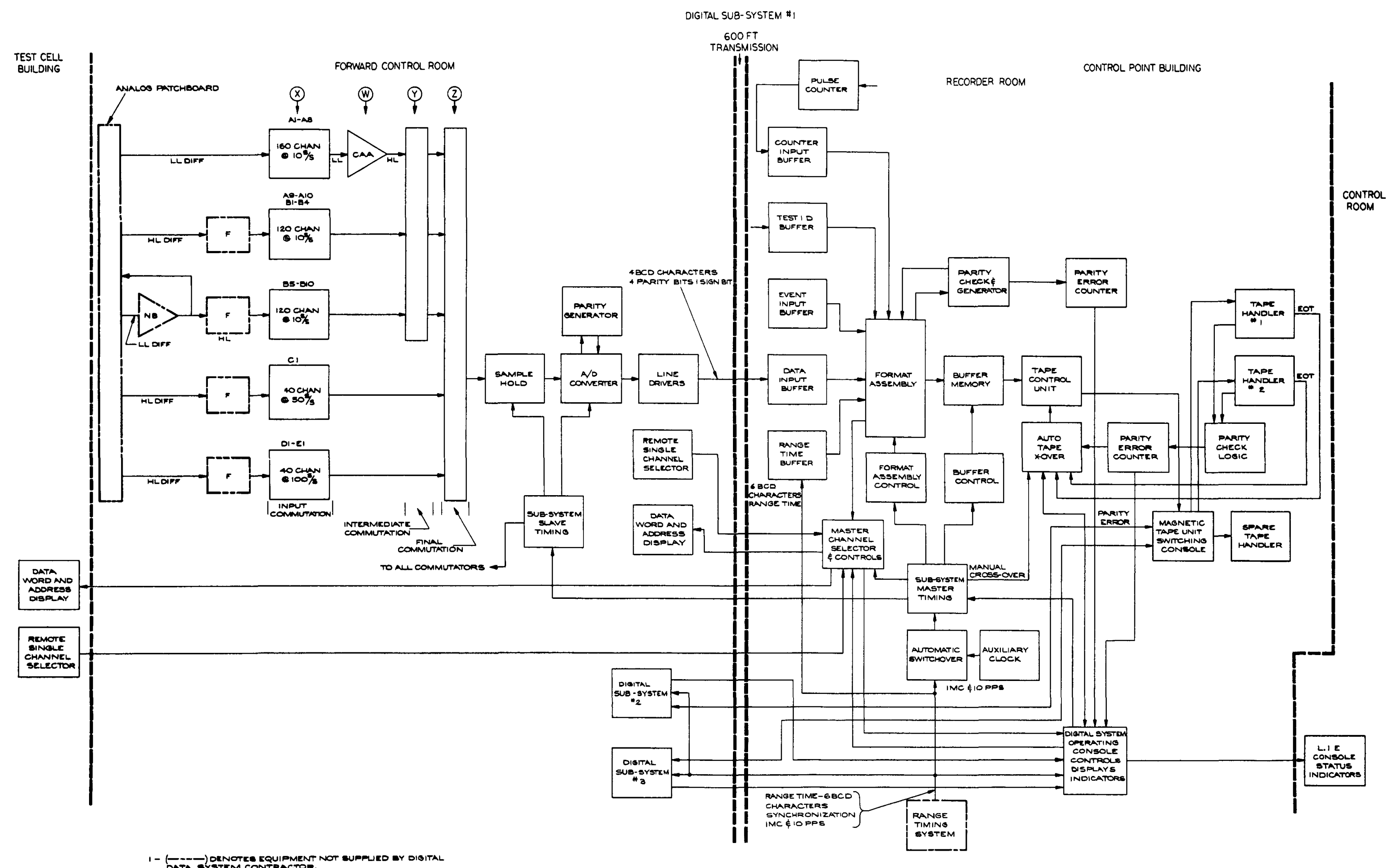

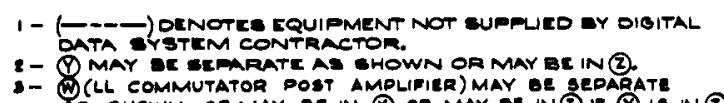


according to accuracy. Low-accuracy ( $0.5 \%$ of FS) signals are fed directly into a low-level multiplexer while high-accuracy ( $0.15 \%$ of FS) data are preamplified by narrow-band amplifiers and enter into a high-level multiplexer. As noted, the sampling rate in both cases is 10 samples/sec. If high sampling rates are desired, the signals may be routed through wide-band amplifiers as shown in Figure C-3. Through the foregoing arrangement, the criteria were satisfied for providing the following sampling rate capabilities per subsystem:

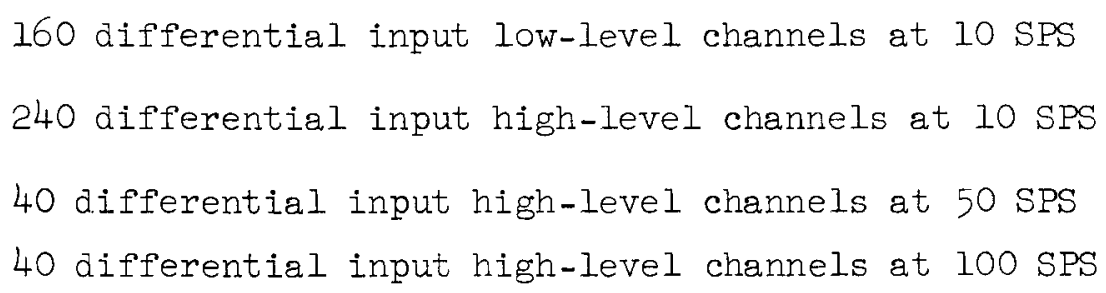

Although not shown in the drawing, a digital patchboard will be provided for achieving supercommutation. By forfeiting 40 channels at 50 SPS and 40 channels at 100 SPS, any one of the following combinations can be achieved per subsystem:

3 Channels at 2000 SPS, or

12 Channels at 500 SPS, or

30 Channels at 200 SPS

These may prove highly desirable for limited recording of higher frequency data during test runs, or for post-test playback (at reduced speeds) of analog magnetic tape recordings.

Following the digitizing and formatting, the converted data and the incoming events and other digital words are fed into one of two digital tape handlers provided with each subsystem. During normal conditions the end of the tape on the operating unit is sensed and the operation is automatically switched to the second unit. Continuous recording is possible in this manner. During abnormal conditions, more than three parity errors will cause recorder crossover. Also, one additional handler for all three subsystems is provided which may be manually switched to any one of the subsystems in the event of a simultaneous failure of both tape handlers. 
Standard 10.5-in. tape reels are used; the operating speed is $75 \mathrm{in.} / \mathrm{sec}$. With an approximate tape packing density of 556 bits/in. and a continuous recording rate of 10 frames/sec, 4000 BCD characters referenced to time are recorded per data frame. To accept the various inputs previously described, each 4000-character, 100-ms data frame contains the following:

$\begin{array}{lc}\text { Range time } & 6 \text { characters } \\ \text { Test identification number } & 4 \text { characters } \\ \text { Operating mode } & 1 \text { character } \\ \text { Subsystem number } & 1 \text { character } \\ \text { Events data } & 52 \text { characters } \\ \text { Frequency counter information } & 12 \text { characters } \\ \text { Data Samples } & 3916 \text { characters (979 words) } \\ \text { Start frame marker } & 4 \text { characters } \\ \text { End frame blank } & 4 \text { characters } \\ & -4000\end{array}$

The above data are recorded in gapless format and, upon completion, each reel of tape is temporarily stored for subsequent entry into the data processor. On-line data processing is not presently planned; however, the type of equipment and its physical location is such that on-line operation could be achieved at a future date, if desirea.

The static accuracy of the DDS is divided into three classes. According to $E G$ and G's functional specifications, the overall accuracy, millivolt or voltage input to digitized output, is as follows:

Class 1 Channels: $\pm 0.15 \%$ full scale for standard millivolt level channels individually preamplified and sampled by any high level multiplexer.

Class 2 Channels: $\pm 0.1 \%$ full scale for standard high level signals sampled by any high level multiplexer.

Class 3 Channels: $\pm 0.5 \%$ full scale for standard millivolt level channels directly sampled by the low level, 10 sample per second multiplexer. 
These accuracy figures represent the root-sum-square combination of zero and gain instability, hysteresis, and noise or scatter. Other system errors such as zero transfer, gain accuracy, linearity, and common mode conversion may be accounted for during calibration and subsequent analysis by the data processor. Therefore, their contribution to the accuracy of the final data may be virtually eliminated.

YI. ETS-1 DATA PROCESSOR

A digital data processor (DP) is installed in the Control Point Building of the EIS-1 Test Complex to handle the large volume of data acquired by the Digital Data System。

As an integral part of the Data Acquisition System, the DP is capable of performing in the following functional areas:

Providing and maintaining channel engineering records

Providing instrumentation and control system pre-test readiness checks

Eroviding DDS calibration corrections prior to and after test operations

Froviding preliminary data immediately following a test operation

Reformatting, correcting, and verifyng test data prior to final data operations

Feproducing raw, corrected, or edited digital magnetic tapes for transmittal for further processing and analysis.

The above DP required operations were expanded in the manner described below。

\section{A. CHANNEL ENGINEER ING}

Cne of the problems in operating a facility of the nature of ETS-I is the maintaining of an up-to-date wiring list and status report of all facility and data acquisition wiring. It is necessary that such information be absolutely correct and up to date. The processing of this information must be precisely 
$\mathrm{RN}-\mathrm{S}-0146$

organized and controlled by a strict discipline so that when system wiring changes are made they will be correctly routed and checked out free of human errors. Furthermore, this must be accomplished with an immediate upgrading of the wiring tabulation file. This wiring system will provide control of all EIS-l wiring including data acquisition systems, TV systems, $r f$ and wire communications systems, and facilities controls. It does not include facility power wiring, telephones, or wiring belonging to the test article. The background and techniques for design of this wiring system is a direct outgrowth of the Test Cell A and C "Chan'Tran" system.

All wiring channels are filed as records. A record contains information regarding all wires and terminations starting from a transducer plug, progressing through all connections, conditioners, patches, recording sink, use destination, and ending as a display of measurement identifier. Changes to the system are accomplished by requesting a channel of a certain type and specifying necessary information such as signal use, signal level, conditioning required, signal destination, sampling rate, and signal identifier. Frior to a new test setup, all new channels must be requested and all unused channels deleted. All unspecified channels remain as wired. New channels are added and unused channels are deleted by wiring instructions outputted by the DP line-printer. These instructions are used directly by the ETS-I wiring technicians and no engineering time, effort, or interpretations are necessary between the wiring file and actual system wiring.

\section{B。 PRE-TEST READINESS CHECKS}

System wiring integrity must be verified prior to any test operations. Unfortunately, automatic checkout capabilities do not exist for the bulk of ETS-1 wiring, and conventional checkout techniques must be utilized. However, the instrumentation system does have an automatic calibration capability which may be used for system checkout. Each DDS channel may be stepped to a discrete position while all other DDS channels remain at some other determined value. For each channel, the information is recorded on a magnetic tape by the DDS for evaluation by the DP. In this manner most wiring efforts may be determined. In some cases this technique is valid through the transducer; however, for some channels this technique is valid only to the signal conditioner. Conventional techniques must be used for further checkout when required. 


\section{CALIBRATION PROGRAM}

Prior to the test operation, the proper operation of each channel of each digital data subsystem must be verified. The operation of the channel is verified by applying a stepped calibration input signal, and recording the channel output value on digital magnetic tape. Values will be averaged over the period of each individual step and the averaged value compared against an upper and lower limit. If the value is outside of the limit band, the average value, the limits, and the channel identification will be printed on-line. Each channel is calibrated by a formula of the type

$$
y=a x+b
$$

Once these calibration constants are known, it is not necessary to retain the constants and normalize each piece of data directly. A new set of polynomial coefficients can be computed that incorporate the normalization constants. The computation involves evaluating a polynomial of the order of the $\mathrm{x}$ factor associated with each polynomial constant. This procedure will require less memory in the processor and take less computing time over each complete run.

\section{D。 PRELIMINARY DATA REPORI}

The preliminary data report will be provided in three phases as follows:

\section{Quick-Iook Eeport}

Immediately after a test operation, a report is required to identify the general achievements of the test. Selected parameters ${ }^{*}$ will, in general, be the same irrespective of engine type. This report will be presented in the form of $x-y$ plots and/or tabulations in corrected engineering units. Corrections will consist of calibration, linearity, zero-offset, and conversion to engineering units. In general, this report will be limited to 20 items plus time.

\footnotetext{
*The term "parameter" is defined as any recorded performance variable and is not exclusive of such terms as channel value, corrected measurement value, or derived parameter. A "derived parameter" is defined as a performance variable calculated by use of an equation from a number of measurement values.
} 
Fach of the 20 items will be scanned, time-identified, and printed at a rate of one per 2 sec of engine operation.

\section{General Engineering Report}

This report will consist of any parameter recorded by the digital data system or any derived parameter. In general, this report will consist of 160 items scanned, time-identified, and printed at a nominal rate of once per 2 sec of engine operation. During startup, the scan rate will be increased to one scan per 1 sec of engine run, but limited to 1 min. Capability is provided to reduce scan rate to one scan per $10 \mathrm{sec}$, or greater, of engine run during static portions of test. Test length will be considered a maximum of 30 min. Reports will be presented in the form of $x-y$ plots and/or tabulations in corrected engineering units.

\section{Detailed Engineering Report}

Based upon an evaluation of the above reports, requests may be made for detailed information upon any selected measurement record by the Digital Data System for short periods of time at maximum system resolution. A capability is provided to select any 20 channels with a maximum sample rate of 10 samples per sec for $10 \mathrm{~min}$ of engine run or any reasonable combination or multiple thereof. Reports will be presented in the form of $x-y$ plots and/or tabulations in corrected engineering units.

\section{E. REFORMAT RAW, CORRECTED, OR EDITED DIGITAL MAGNETIC TAPES}

Raw test tapes recorded by the ETS-1 DDS are recorded in a bipolar binary coded decimal (BCD) data mode. The tapes are gapless. Transmittal tape shipped off-site for further processing and analysis requires a number of modifications from the raw tape for compatibility, ease of processing, and validity. Cff-site processors are generally large-scale computers, thus being straight binary, and require gapped formats. Also any tapes leaving ETS-1 should be verified as being true data. They should be corrected for such problems as measurement scale offset and linearity, and expressed in floating point format with engineering units。 
It is important to observe that the ETS-1 DP is an extremely fast machine but is limited in memory size (8000, 24-bit words). Therefore, DP operations should use a batch processing philosophy. Extensive sorting operations are time-consuming and should be avoided.

ETS-I DF operations upon a test operation magnetic tape will be as follows.

\section{Step 1}

While the engine test is being conducted, some DP operations are possible. During this time period the calibration runs may be evaluated. Since the number of magnetic tape handlers is limited, care must be exercised not to interfere with the data acquisition operations. The ETS-I DP is not online to the DDS; therefore, simultaneous acquisition/reduction operations cannot be performed. The test run may consist of approximately $30 \mathrm{~min}$ of recorded data. This will be five rolls of magnetic tape for each DDS subsystem which represents 360,000 records of data per roll, or 18 million measurements per subsystem per test run. The output of Step 1 will be this test data tape in DDS format plus some calibration reduction constants.

$$
\text { 2. Step? }
$$

The first actual step in processing the data will be the preparation of a copy tape. It is desirable to limit the use of the raw primary data tape as much as possible. While it is slightly inefficient at this point, the best first step is to copy the run tape. Numerous operations may be conducted during this time, but data reliability requirements suggest that a simple copy is most desirable from the error or missing bit viewpoint. However, some simple data changes to permit increased efficiency of data reduction can be performed. Events data should be removed to an events summary tape file separate from other digital data. A step-up of tape efficiency may be achieved by making the copy tape in 800 bpi density rather than the initial recording density of 556 bpi. This is an important consideration since most DP operations are input-limited, and this density step-up will increase input tape efficiency by about one third. 


$$
\text { RN-S-OI } 46
$$

During this step it is also possible to produce an additional copy tape which could be shipped off-site immediately without further processing, or to produce a compendious tape by grossly editing the primary tape by whole records. A transmittal copy produced during this step is not recommended due to lack of data corrections.

\section{Step 3}

Since the tape produced in Step 2 is a BCD tape and the DP is a straight binary machine, the best next operation is to convert from BCD to straight birary. The removal of events data in Step 2 was preparatory for this operation since the conversion of the events data to straight binary is undesirable. Since the straight binary mode is one third more efficient than BCD in its tape requirement, this step will also result in increased efficiency in further input operations. Because of the high processing speed of the ETS-I $D P$, Step 3 will be input-limited; therefore it is desirable to add further processing operations to this step. The arrangement of data on the tapes to this step has not been altered. This arrangement was established by the commutation sequence of the DDS and is complex from the data processing viewpoint. Therefore a sort operation within each record is necessary. It is also desirable to reduce the record length at this time so as to more efficiently utilize the processor memory during future numerical operations.

Another function to be accomplished here will be the outputting of standardized tapes. The new records will now contain the 10 sample per second (s/s) data, the $50 \mathrm{~s} / \mathrm{s}$ data, and $100 \mathrm{~s} / \mathrm{s}$ data as individual records. Also each record will now contain time, test $\mathrm{ID}_{2}$ and events data, as the first 18 data words of each record. The $50 \mathrm{~s} / \mathrm{s}$ and $100 \mathrm{~s} / \mathrm{s}$ data records will have been sorted so that the 5 or 10 samples for each measurement occur in sequential order. Record lengths are now 1628 characters for $10 \mathrm{~s} / \mathrm{s}$ data, 872 characters for $50 \mathrm{~s} / \mathrm{s}$ data, and 1632 characters for $100 \mathrm{~s} / \mathrm{s}$ data. Each type of record must have an identifier as to the type of data contained. From this step, data may be outputted either as one sequential tape or three individual tapes containing the different sampling rates. The latter type should be preferred if some of the data acquisition tape handlexs are available to the DP during this operation. 


\section{Step 4}

The previous steps have been composed of format conversion or sort operations. The actual numerical processing can start now and is outlined in the following substeps.

\section{a. Validation}

It is necessary to know whether the information on the tape is actually data or is just noise resulting from an instrumentation failure. The first step in making this determination is to compare the pre-run and postrun calibrations. This is proof of existence for some, but not all, transducers. The next step can be the evaluation of a post-run ambient condition which will prove existence of still more of the transducers. However, these steps do not really prove that a particular measurement is, in reality, data. This problem can be handled by reviewing each data point in terms of an average of the previous values for that point. With prior knowledge of the data sampling rate, analog filters, and physical variable characteristics, it 15 possible to assign a percentage range for each measurement variation. Sequential values outside of range may be assumed to be noise or the product of an unspecified physical variable. In either case the value should be flagged as meriting further examination. The concept of a validity flag has not been allowed for previously in the ETS-l data operations. The purpose of the validity flag is to provide an identifier which travels with a given measurement throughout processing. If invalid data are utilized in succeeding calculations, then the new value is also flagged. This prevents the data user from inadvertently evaluating data which is untrue.

\section{b. Smoothing}

Data which is taken at rather high sampling rates, relative to the application interest of the physical variable, may be sufficiently dynamic to present an interpretation problem. Smoothing techniques are discussed in Appendix E, "Statistical Edit and Smooth."

\section{c. Scale Corrections}

The measurement scale provides several problems. The data input from the DDS is in the form of a percentage of full scale. This scale is bipolar and has a resolution of 10,000 parts. Data may be either positive

$$
c-28
$$




\section{RN-S-0146}

or negative, or may pass through zero going from one polarity to the other. Also, transducer outputs may be conditioned to have a zero off-set of $n$ counts. In addition, there will be a relationship of the measurement scale to the physical variable or engineering unit scale. As long as the measurement scale is linear with respect to the engineering unit scale and unreasonable nonlinearities are not entered by the DDS, the scale correction and conversion may be accomplished by the following form:

$$
Y=a x+b
$$

When non-linearity of the data system or measurement device exceeds an acceptable levels then further corrections are required.

One method of correction for non-linearities is the use of a segmented curve which intersects the physical variable at sufficient points to provide the accuracy required. To transform a given value $(x)$ into a correct scale value $(y)$ based on a linear equation, the following technique may be used:

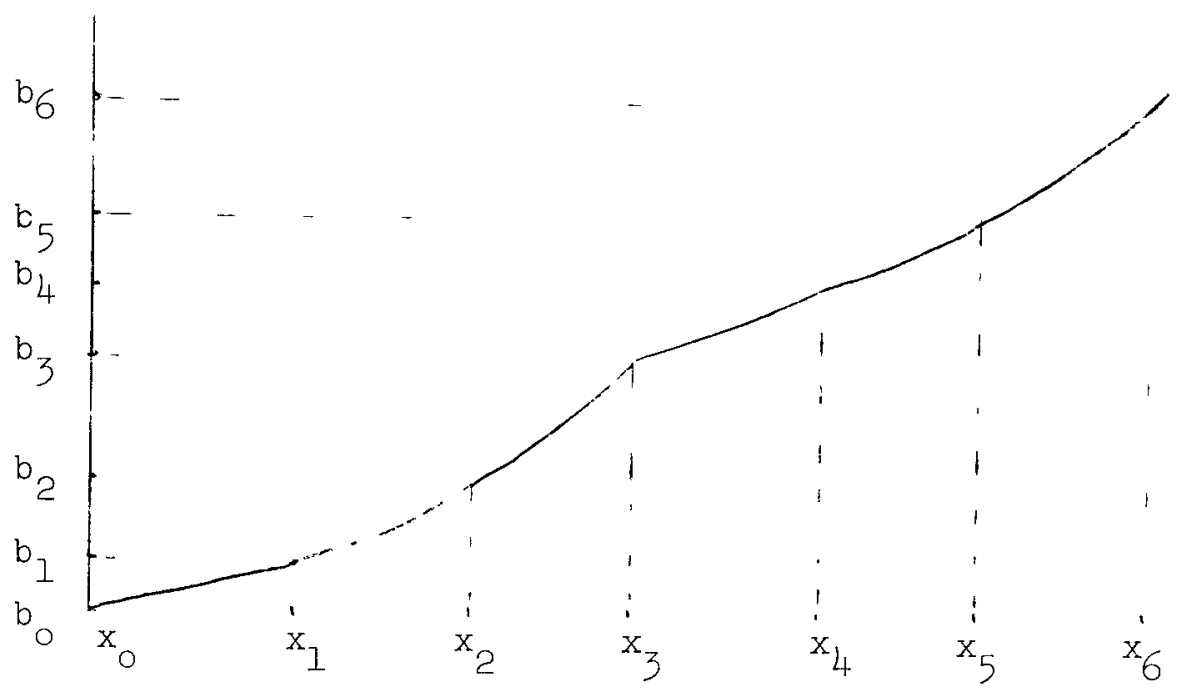

In the example shown, six segments of straight lines make up the non-linear input curve with the break points at the arbitrarily selectable limits $x_{1}$ through $x_{5}$. To completely define a straight line, two parameters are necessary. One 
of the parameters is the slope of the line and the other is any point lying on this straight line. Generally the intercept of the straight line with the $x$ axis is used. However, in the particular problem here, it is advisable to avoid the use of intercepts for describing various segments of straight lines. The proposed constants to be used are, as indicated in the figure, the breakpoints. In this arrangement, all points along the curve can be uniquely represented by the following set of equations:

$$
\begin{array}{lr}
Y=a_{0} x+b_{0} & \text { for } 0 \leq x<x_{1} \\
Y=a_{1}\left(x-x_{1}\right)+b_{1} & x_{1} \leq x<x_{2} \\
Y=a_{2}\left(x-x_{2}\right)+b_{2} & x_{2} \leq x<x_{3} \\
Y=a_{3}\left(x-x_{3}\right)+b_{3} & x_{3} \leq x<x_{4} \\
Y=a_{4}\left(x-x_{4}\right)+b_{4} & x_{4} \leq x<x_{5} \\
Y=a_{5}\left(x-x_{5}\right)+b_{5} & x_{5} \leq x
\end{array}
$$

Heres the $x_{n}$ and $b_{n}$ are the constants indicated in the figure, and a represents the slope of each straight line segment. It can be realized that all tle constants, with the exception of the $a_{n}$, are positive and the differences, delta $x$, are also all positive values. If one can further assume, that $Y$ is monotonically increasing whth increasing $x$, then all the slopes $a_{n}$ are positive. The arrangement to use the breakpoints as constants and postulate positive a offers simpler programming and better accuracy. If data scaling into engineering units is wanted together with linearization, the $b$-constants and also the resulting $y$ values can be ezther positive or negative.

Another method of correction for non-linearity and conversion involves the use of a polynomlal of the following form:

$$
\begin{aligned}
x_{\text {eng }} & =a_{1} x^{6}+a_{2} x^{5}+a_{3} x^{4}+a_{4} x^{3}+a_{5} x^{2}+a_{6} x^{x}+a_{7} \\
& =x\left[x\left(x\left\{x\left[x\left(a_{1} x+a_{2}\right)+a_{3}\right]+a_{4}\right\}+a_{5}\right)+a_{6}\right]+a_{7}
\end{aligned}
$$

The nested form shown in the second line is one of the quicker ways to evaluate this polynomial on a data processor. 


\section{RN-S-0146}

A time estimate for the above operations cannot be provided since the time required is dependent upon the number and type of corrections required. If the concept of the Aerojet standardized transducer is utilized and thus the number of correction programs is limited, then these operations could be completed in one pass. However, if many different transducer types are required, then step 4 could require many processor passes.

\section{Reproduce Raw, Corrected or Edited Tapes}

The primary data tape from the DDS will remain in storage at ETS-1. Because of its format it is nearly unusable at other data centers. This should not be considered a limitation since data users are not interested in the data tapes until sufficient reduction has been provided to correct and validate the data. Therefore, the data tapes outputted in Step 4 should be considered as the ETS-I output data tape. These tapes will have been converted, corrected, gapped, and placed in a standard engineering unit floating point format which will be acceptable by any standard data processing center.

VII. ANALOG DATA PROCESSING

In addition to processing the direct digital magnetic tapes, the ETS-I instrumentation equipment can also process the analog magnetic tapes. This is accomplished by playback of the analog tapes through a patch arrangement into the DDS to produce a digital tape. By use of a digital patch the commutator arrangement may be changed into a space-commutation arrangement. This will permit sampling one channel at $6000 \mathrm{~s} / \mathrm{s}$, three channels at $2000 \mathrm{~s} / \mathrm{s}, 30$ channels at $200 \mathrm{~s} / \mathrm{s}$, or any other reasonable sampling product of 6000 . Digital magnetic tapes produced in this manner can be processed by the same steps outlined for raw DDS data tapes.

VITI. TIMING SYSTEM

Time references in the form of serial and parallel codes and pulse rates are generated for use in recording, display, and control throughout the ETS-1 test complex and the future ETS-2. A l-mc oscillator with a stability of 1 part in $10^{8}$ per day and 5 parts in $10^{8}$ per week provides the master time base for the system from which the codes and pulse rates are derived. 
RN-S-0146

The serial time code format developed for the system is shown in Figure C-10 with the characteristics listed in the table in Figure C-9. Time is defined in seconds and tenths of seconds by the six-decade (8-4-2-1) serial code. As noted in the characteristics table, there are five sets of characteristics which establish the d-c shift codes. In addition, the 1.0-sec shift code is also provided in an amplitude-modulated carrier form using a $1 \mathrm{kc}$ carrier frequency.

The format for the parallel time code will consist of a parallel six-decade BCD (8-4-2-1) code. Six characters or 24 bits will be produced, thus enabling parallel coded time also to be read in seconds and tenths of seconds.

In addition to the serlal and parallel codes, seven pulse rates of 1,10 , $100,1000,10,000,100,000$ and 1,000,000 pulses per sec (PPS) will be produced by the time code generator (TCG).

The distribution of the time code signals and pulse rates is diagrammatically shown in Figures $\mathrm{C}-1 \mathrm{l}$ and $\mathrm{C}-12$. Further details on the distribution is contained in the paragraphs that follow.

A TIME DISPLAYS

Jisual displays or clocks will be provided throughout the complex in accordance with the locations shown in Figures C-11 and C-12, and will be driven by the l-PFS pulse rate. Range time will be displayed in seconds up to 86,399 sec, and count, hold, and run time as well as time of day will be displayed in hours, minutes, and seconds up to 99 hours, 59 minutes and $59 \mathrm{sec}$. The count time clocks will also indicate negative (countdown) or positive (countup).

\section{B. ANALOG AND EVENTS RECORDING SYSTEM}

Five serial d-c shift codes will be directed to both systems for use with the strip chart, oscillograph, and event recorders. The pulse voltage level will be +3 volts and the load driving capability will be $40 \mathrm{ma}$. The analog magnetic tape recorder receives the modulated 1 kc carrier code. This code has a 3-to-l pulse-to-space amplitude ratio adjustable from 0 to 10 volts peak-topeak, and is capable of driving a $1000 \mathrm{ohm} \mathrm{load.}$ 
RN-S-0146

\begin{tabular}{|c|c|c|c|c|c|c|}
\hline $\begin{array}{l}\text { SUGGESTED } \\
\text { COOE } \\
\text { TYPE }\end{array}$ & $\begin{array}{l}\text { FRAME } \\
\text { LENGTH } \\
\text { (SECONDS) }\end{array}$ & $\begin{array}{l}\text { ELEMENT } \\
\text { PERIOD }\end{array}$ & $\begin{array}{l}\text { CODE } \\
\text { SCAN } \\
\text { RATE }\end{array}$ & $\begin{array}{l}\text { MOD. } \\
\text { FREQ }\end{array}$ & $\begin{array}{c}\text { "On } \\
\text { WIOTH }\end{array}$ & $\begin{array}{c}\text { "qn } \\
\text { wIOTH }\end{array}$ \\
\hline$A$ & 0.1 & 2 HS & $500 / \mathrm{SEC}$ & & $0.5 \mathrm{MS}$ & $1.5 \mathrm{MS}$ \\
\hline$B$ & 1.0 & $20 \mathrm{MS}$ & $50 / s E C$ & $1 \mathrm{kC}$ & $5 \mathrm{MS}$ & $15 \mathrm{MS}$ \\
\hline c & 10 & 200 MS & $5 /$ SEC & & $50 \mathrm{MS}$ & $150 \mathrm{MS}$ \\
\hline D & 100 & 2 SEC & $0.5 /$ SEC & & $500 \mathrm{MS}$ & $1.5 \mathrm{SEC}$ \\
\hline$E$ & 1000 & $20 \mathrm{SEC}$ & $.05 /$ SEC & & 5 SEC & $15 \mathrm{SEC}$ \\
\hline
\end{tabular}

Figure C-9

Serial Code Characteristics - Timing 
RN-S-0146

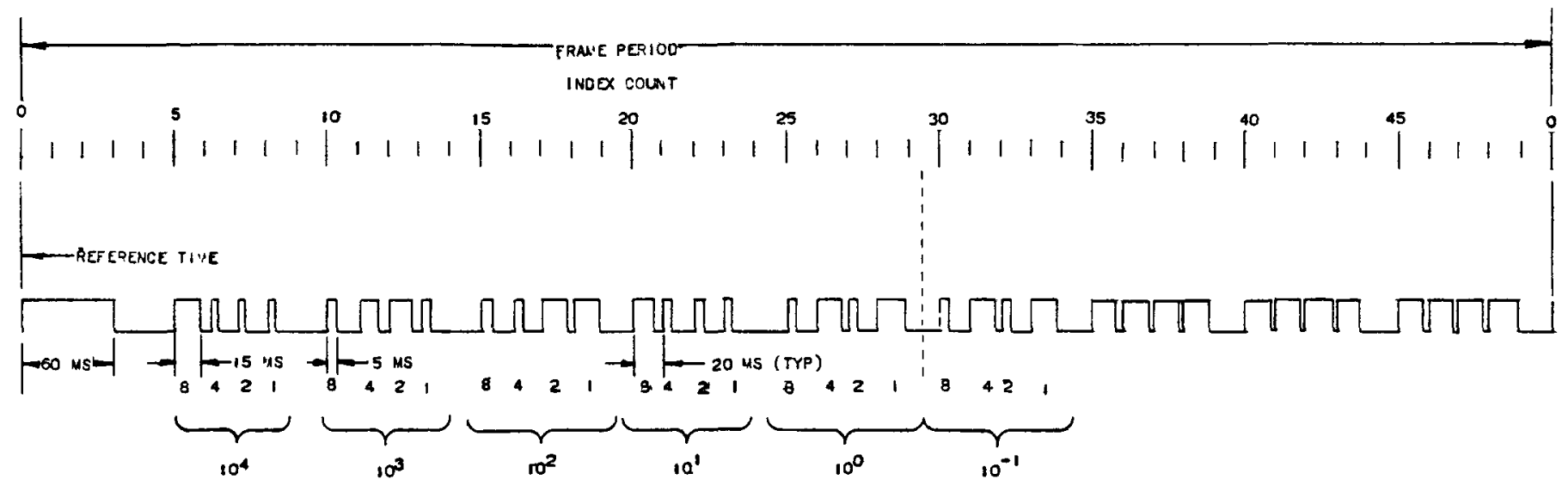

TIME REPRESENTED IS $86,385.5$ SECONDS, I SEGCNO COOE EXAMPLE SHOWN.

Figure $\mathrm{C}-10$

Typical Serial Time Code

$\mathrm{C}-34$ 


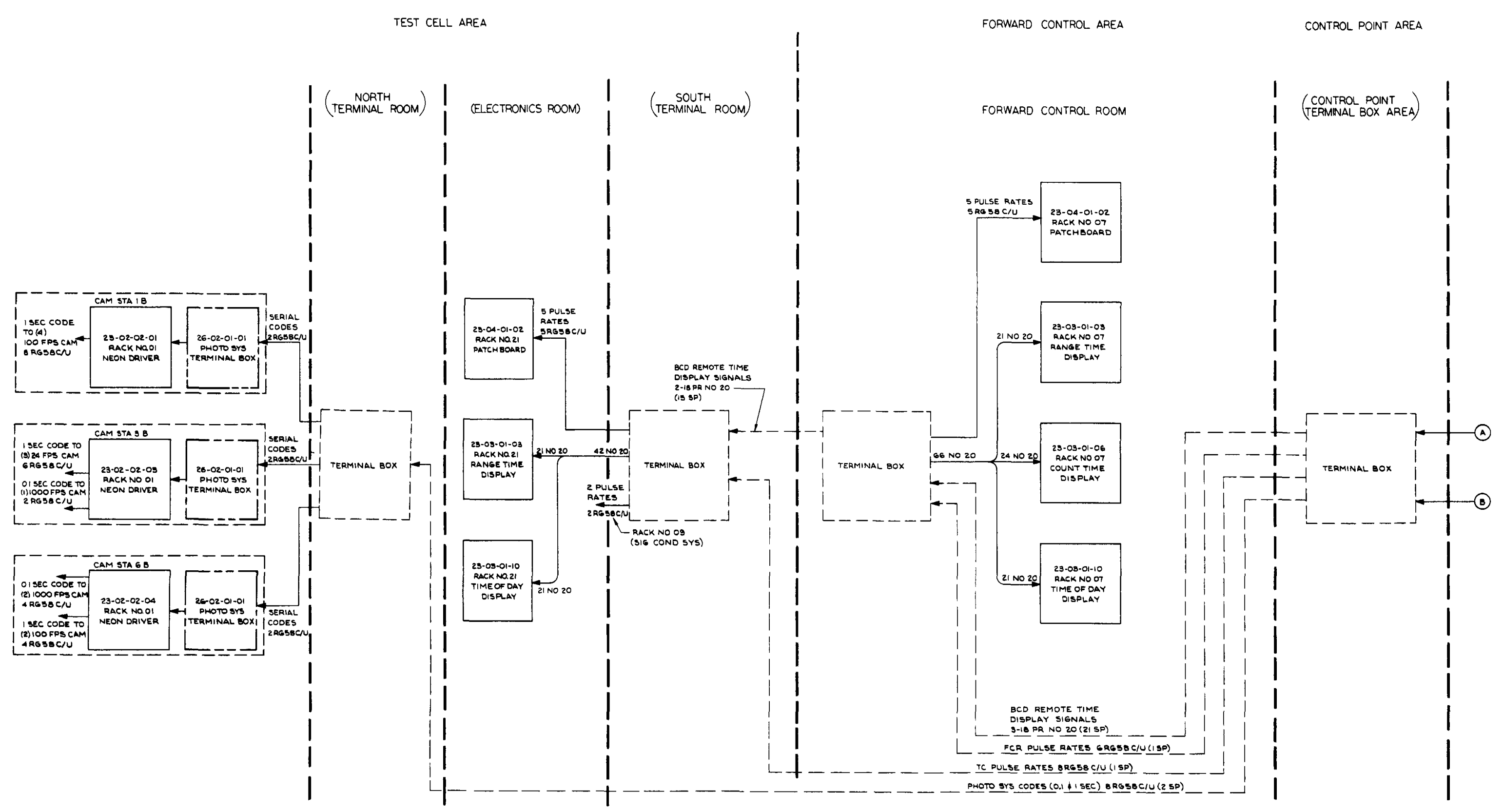

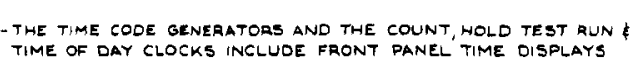

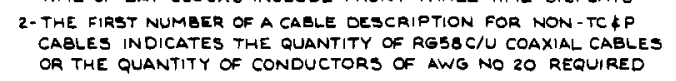

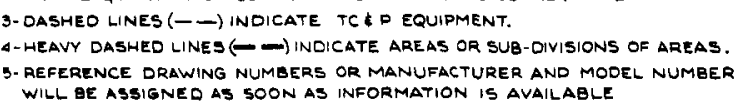

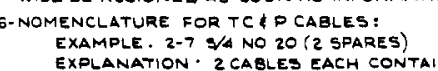

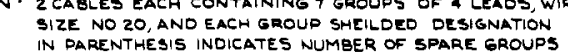

Figure $\mathrm{C}-11$

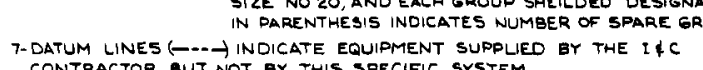




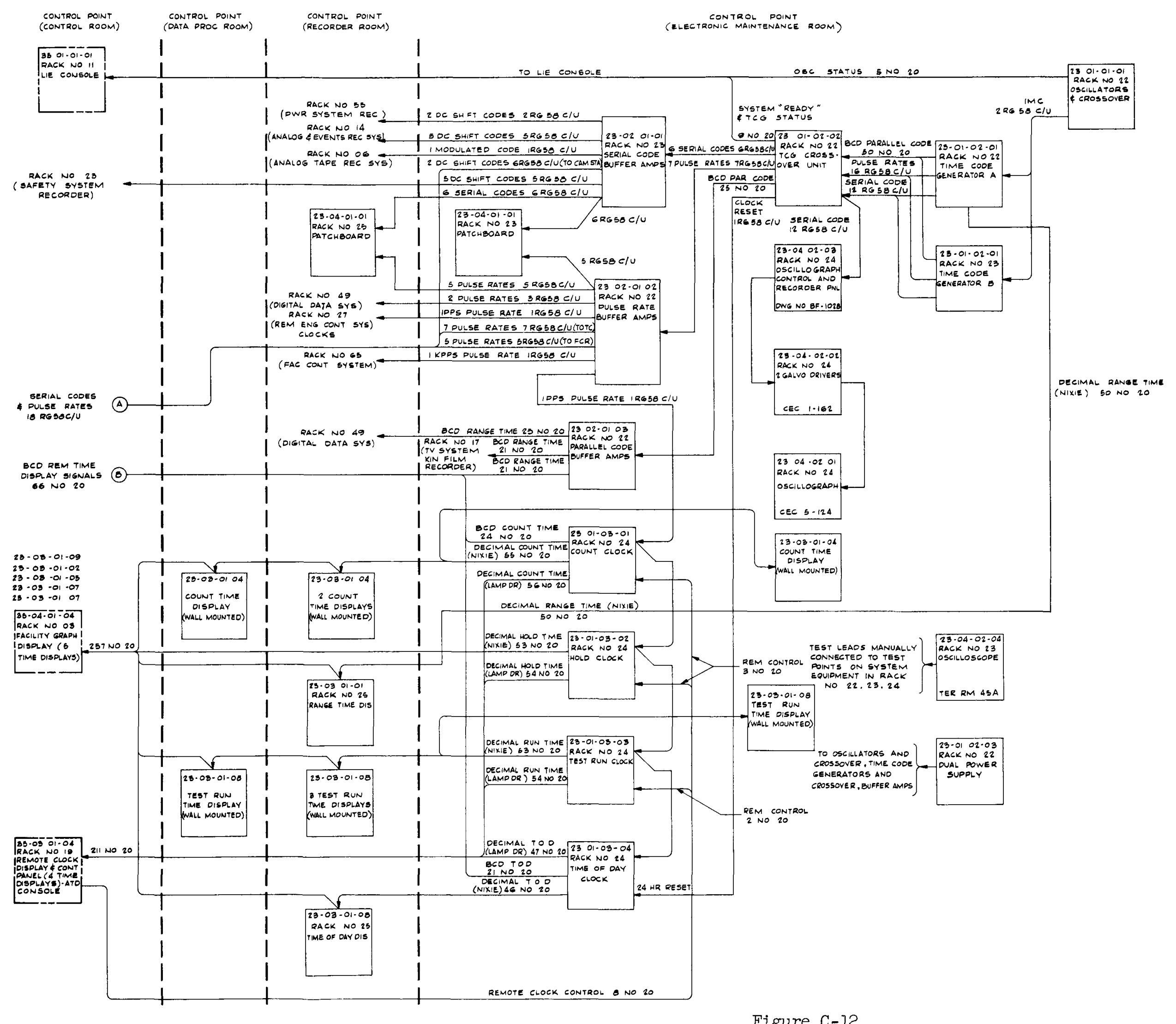

Figure $\mathrm{C}-12$

Second Timing System Block Diagram (From EG and G Dwg No. BF-1008 Sheet 2 of 2) 


\section{PATCHBOARDS}

Patchboards are provided at various locations to conveniently make available for special usage the serial codes and pulse rates. The five d-c shift codes and the five pulse rates $(1,10,1000,100,000$ and 1,000,000 PPS) are connected to the boards in the Recorder and Equipment Maintenance Room. Each output has the capability of driving a 1000-0hm load at an amplitude of -6 volts, and each has a zero baseline of \pm 0.4 volts. The modulated carrier code is also connected to these patchboards, and it has the capabilities described above. Two additional (total) patchboards are located in the Forward Control Room and Test Cell Building making available in those areas five of the pulse rates (same as above) for special applications.

\section{PHOTO SYSTEM}

The 0.1 sec and 1.0 sec $d-c$ shift serial codes will be directed to camera stations $1 B, 3 B$ and $6 B$. These codes will be amplified by neon driver amplifiers which will provide 150 volts at a load of 15 ma to each of the camera neon film marker lamps.

\section{E. DIGITAL DATA SYSTEM}

Range time is parallel 6-decade BCD format and pulse rates of 1,000,000 PPS will be furnished. The binary " 1 " level for the parallel code will be -6 volts \pm 0.6 volts, and "O" level will be zero \pm 0.4 volts. Pulse rate levels will be as described above.

\section{F。 T V SYSTEM}

Range time in 5-decade BCD parallel format will be furnished as 20 bits without complements representing time in straight seconds. The binary levels will be the same as those above.

\section{G. SIGNAL CONDITIONING SYSTEM}

Calibration of the speed and flow measuring channels is required. To accomplish this, two pulse rates (100 PPS and 10,000 PPS) will be directed to the Signal Conditioning System upon command. A frequency multiplier at that point will produce 200, 400 and 20,000 PPS for final use. 


$$
\mathrm{RN}-\mathrm{S}-\mathrm{O} 146
$$

H. REMOTE ENGINE CONTROL SYSTEM

Although this system is not within EG and $G$ 's contract, EG and $G$ will provide at the interface a 1 PPS pulse rate for driving three digital, elapsed-time clocks.

\section{FACILITY CONTROI SYSTEM}

In order to reduce the signal transmission lines from the duct and shield transducers to the graphic displays, the signals are multiplexed in the Test Cell Euilding and decommutated in the Control Point Building. The clock pulse used to drive the multiplexer logic is the 1000 PPS rate from the Timing System.

\section{J. SAFETY AND POWER SYSTEM}

Five d-c shift codes with the characteristics previously discussed will be directed to the Safety System for operation with the analog strip chart and multipoint recorders. Similarly, the Power System recorders will receive the 100 and 1000 sec serial codes.

Maximum reliability is achieved by providing a standby oscillator and a standby time code generator as noted in Figure C-12. The crossover unit will continuously monitor the oscillator output voltage level and a drop in voltage below a pre-set level will automatically cause a one-way crossover. Similarly, each of the five $a-c$ shift serial codes produced by the time code generator will be monitored, and a drop in voltage level will automatically transfer the standby TCG into the system. 
RN-S-0146

\section{APPENDIX D}

AN EXAMPLE OF ERROR PROPAGATION IN A HEAT-TRANSFER MODEL 


\section{APPENDIX D}

AN EXAMPLE OF ERROR PROPAGATION IN A HEAT-TRANSFER MODEI

\section{INTRODUCTION}

Consider again Equation D-1. The derivation of this equation was discussed in Section III,C,I.

$$
W c_{W} \frac{d T}{d t}=\dot{W} c_{p}\left(T_{i}-T_{0}\right)+Q_{F} Q_{m}
$$

where

$$
\begin{aligned}
& c_{p}=\text { specific heat of fluid, Btu/lb- }{ }^{\circ} \mathrm{R} \\
& c_{W}=\text { specific heat of wall, Btu/lb- }{ }^{\circ} \\
& Q_{F}=\text { spatial distribution factor } \\
& Q_{m}=\text { power level } \\
& T=\text { temperature, }{ }^{\circ} \\
& t=\text { time, sec } \\
& W=\text { mass of wall, } 1 b \\
& \dot{W}=\text { mass flow rate, } 1 b / \text { sec } \\
& \underline{\text { Subscripts }}
\end{aligned}
$$

$$
\begin{aligned}
& i \quad=\text { in (fluid entering) } \\
& 0=\text { out (fluid departing) } \\
& w=\text { wall }
\end{aligned}
$$

The derivative $\mathrm{dT}_{\mathrm{w}} / \mathrm{dt}$ in Equation $\mathrm{D}-1$ cannot be evaluated directly; however, a variety of numerical approximations are available. It suffices, for this section, to consider $\mathrm{dT}_{\mathrm{W}} / \mathrm{dt}$ as some function of the measurements, say $\mathrm{Z}(\mathrm{m})$, with expectation $E(Z)$ and variance $V(z)$. 
Under these conventions, Equation D-1 becomes

$$
W c_{W} Z(m)=\dot{w} c_{p}\left(T_{i}-T_{0}\right)+Q_{F} Q_{m}
$$

Solving for $\dot{w}$ in Equation D-2 yields

$$
\dot{w}=\frac{W c_{w} Z(m)}{c_{p}\left(T_{i}-T_{0}\right)}-\frac{Q_{T} Q_{m}}{c_{p}\left(T_{i}-T_{0}\right)}
$$

If a variable suffers a displacement, $\delta x$, then the accompanying change in $\dot{w}$ is given by $(\partial \dot{w} / \partial x)^{6} x$. The contribution to the approximate variance in $\dot{w}$ due to the variance of the single variable $x$ is given by

$$
\mathrm{V}(\dot{\mathrm{w}})_{\mathrm{x}}=\left(\frac{\partial \dot{\mathrm{w}}}{\partial \mathrm{x}}\right)^{2} \mathrm{~V}(\mathrm{x})
$$

Thus, the contribution to $V(\dot{w})$ due to $V(Z)$ is given by

$$
V(\dot{w})_{Z}=\left|\frac{\partial \dot{w}}{\partial Z}\right|^{2} V(Z)=\left|\frac{W c_{w}}{c_{p}\left(T_{i}-T_{0}\right)}\right|^{2} V(Z)
$$

For $\mathrm{T}_{i}$

$$
\mathrm{V}(\dot{\mathrm{w}})_{\mathrm{T}_{i}}=\left|\frac{\partial \dot{\mathrm{w}}}{\partial \mathrm{T}_{i}}\right|^{2} \mathrm{~V}\left(\mathrm{~T}_{i}\right)=\left|-\frac{\mathrm{Wc} \mathrm{W}(\mathrm{m})}{c_{\mathrm{p}}\left(\mathrm{T}_{i}-\mathrm{T}_{0}\right)^{2}}+\frac{\mathrm{Q}_{\mathrm{F}} \mathrm{Q}_{\mathrm{m}}}{\mathrm{c}_{\mathrm{p}}\left(\mathrm{T}_{i}-\mathrm{T}_{0}\right)^{2}}\right|^{2} \mathrm{~V}\left(\mathrm{~T}_{i}\right)
$$

and for $\mathrm{T}_{\mathrm{O}}$

$$
V(\dot{\mathrm{w}})_{\mathrm{T}_{0}}=\left|\frac{\partial \dot{\mathrm{w}}}{\partial \mathrm{T}_{0}}\right|^{2} \mathrm{~V}\left(\mathrm{~T}_{0}\right)=\left|\frac{\mathrm{Wc} \mathrm{w}(\mathrm{m})}{\mathrm{c}_{\mathrm{p}}\left(\mathrm{T}_{i}-\mathrm{T}_{0}\right)^{2}}-\frac{\mathrm{Q}_{\mathrm{F}} \mathrm{Q}_{\mathrm{m}}}{\mathrm{c}_{\mathrm{p}}\left(\mathrm{T}_{i}-\mathrm{T}_{0}\right)^{2}}\right|^{2} \mathrm{~V}\left(\mathrm{~T}_{0}\right)
$$

The contribution to $V(\dot{w})$ due to $V\left(Q_{m}\right)$ is given by

$$
V(\dot{w})_{Q_{m}}=\left|\frac{\partial \dot{w}}{\partial Q_{m}}\right| V\left(Q_{m}\right)=\left|\frac{Q_{F}}{c_{p}\left(T_{i}-T_{0}\right)}\right|^{2} V\left(Q_{m}\right)
$$


If $Q_{F}=0$, then the overall variance of $\dot{w}$ assumes the form

$$
\begin{aligned}
\mathrm{V}(\dot{\mathrm{w}})= & \mathrm{V}(\dot{\mathrm{w}})_{\mathrm{Z}}+\mathrm{V}(\dot{\mathrm{w}})_{\mathrm{T}_{i}}+\mathrm{V}(\dot{\mathrm{w}}) \mathrm{T}_{\mathrm{o}}=\left|\frac{\mathrm{Wc}}{\mathrm{c}_{\mathrm{p}}\left(\mathrm{T}_{i}-\mathrm{T}_{0}\right)}\right|^{2} \mathrm{~V}(\mathrm{z}) \\
& +\left|\frac{\mathrm{Wc} \mathrm{Z}(\mathrm{m})}{\mathrm{c}_{\mathrm{p}}\left(\mathrm{T}_{i}-\mathrm{T}_{0}\right)^{2}}\right|^{2}\left[\mathrm{~V}\left(\mathrm{~T}_{i}\right)+\mathrm{V}\left(\mathrm{T}_{0}\right)\right]
\end{aligned}
$$

If $Q_{F} \neq 0$, then the overall variance of $\dot{\mathrm{w}}$ is given by

$$
\begin{aligned}
V(\dot{w})= & V(\dot{w})_{Z}+V(\dot{w})_{T_{i}}+V(\dot{w})_{T_{0}}+V(\dot{w})_{Q_{m}}=\left|\frac{W c_{W}}{c_{p}\left(T_{i}-T_{0}\right)}\right|^{2} V(Z) \\
& +\left|\frac{W c z(m)}{c_{p}\left(T_{1}-T_{0}\right)^{2}}-\frac{Q_{F} Q_{m}}{c_{p}\left(T_{i}-T_{0}\right)^{2}}\right|^{2}\left[V\left(T_{1}\right)+V\left(T_{0}\right)\right]+\left|\frac{Q_{F}}{c_{p}\left(T_{1}-T_{0}\right)}\right|^{2} V\left(Q_{m}\right)
\end{aligned}
$$

When the fluid is two-phase, Equation $D-1$ is not valid and the fluid flow rates cannot be determined by temperature measurements alone.

The average heat-transfer coefficient, $h$, is defined by the equation

$$
h=\frac{Q / A}{T_{W}-T_{f}}=\frac{W c W(m)}{A\left(T_{f}-T_{W}\right)}-Q_{F} Q_{m} / A\left(T_{f}-T_{W}\right)
$$

where

$$
\begin{aligned}
A & =\text { area for heat transfer to fluid } \\
Q & =W c_{W} Z(m)-Q_{F} Q_{m}=\text { heat transferred } \\
T_{f} & =\frac{T_{0}+T_{i}}{2}=\text { average fluid temperature } \\
T_{W} & =\text { average wall temperature }
\end{aligned}
$$


Equation D-1I is a satisfactory definition of the heat-transfer coefficient if the average of the entering and exit temperature of the fluid is equal to the average temperature of the fluid, and if the average temperature of the solid material is the average temperature from which energy is transferred to the fluid. It should be noted that the use of the temperature difference, $T_{W}-T_{f}$, implies that this is the appropriate average temperature difference.

In view of Equation $D-2, Q$ may be defined as

$$
Q=\dot{w} c_{p}\left(T_{0}-T_{1}\right)
$$

so that, alternatively,

$$
h=\frac{\dot{w} c_{p}\left(T_{0}-T_{1}\right)}{A\left(T_{w}-T_{f}\right)}
$$

Consideration of the approximate varlance of $h$ as defined in Equation D-ll will be given first. The contribution to the approximate variance of $h$ due to the variance of $\mathrm{T}_{i}$ is given by

$$
V(h)_{T_{i}}=\left(\frac{\partial h}{\partial T_{i}}\right)^{2}\left[V\left(T_{i}\right)\right]=\left[-\frac{I}{2}\left(\frac{W c_{w} Z(m)}{A\left(T_{f}-T_{w}\right)^{2}}\right)+\frac{l}{2}\left(\frac{Q_{F} Q_{m}}{A\left(T_{f}-T_{w}\right)^{2}}\right)\right]^{2} V\left(T_{i}\right)
$$

If $Q_{F}=0$, then Equation $D-14$ reduces to

$$
V(h)_{T_{i}}=\frac{h_{1}^{2} V\left(T_{i}\right)}{4\left(T_{f}-T_{W}\right)^{2}}
$$

where $h_{1}$ is the quantity obtained from Equation D-ll by putting $Q_{F}=0$.

$$
\begin{aligned}
& \text { Similarly for } T_{0} \\
& V(h)_{T_{0}}=\left|\frac{\partial h}{\partial T_{0}}\right|^{2} V\left(T_{0}\right)=\left\lfloor-\frac{1}{2} \frac{W c_{W} Z(m)}{A\left(T_{f}-T_{W}\right)^{2}}+\frac{1}{2} \frac{Q_{F} Q_{m}}{A\left(T_{f}-T_{W}\right)^{2}}\right]^{-2} V\left(T_{0}\right)
\end{aligned}
$$

and if $Q_{F}=0$, then Equation $D-16$ reduces to

$$
V(h)_{T_{0}}=\frac{h_{l}^{2} V\left(T_{0}\right)}{4\left(T_{f}-T_{w}\right)^{2}}
$$


The contribution to $V(h)$ due to $V\left(T_{W}\right)$ is given by

y.

$$
V(h)_{T_{W}}=\left(\frac{\partial h}{\partial T_{W}}\right)^{2} V\left(T_{w}\right)=\left[\frac{W c_{w} Z(m)}{A\left(T_{f}-T_{W}\right)^{2}}-\frac{Q_{F} Q_{m}}{A\left(T_{f}-T_{W}\right)^{2}}\right]^{2} V\left(T_{W}\right)
$$

and if $Q_{F}=0$, then Equation $D-18$ reduces to

$$
V(h)_{T_{W}}=\frac{h_{1}^{2} V\left(T_{w}\right)}{\left(T_{f}-T_{W}\right)^{2}}
$$

For $Z(m)$

$$
V(h)_{Z}=\left(\frac{\partial h}{\partial Z}\right)^{2} V(z)=\left|\frac{W c}{A\left(T_{f}-T_{W}\right)}\right\rangle^{2} V(z)
$$

The contribution to $V(h)$ aue to $V\left(Q_{m}\right)$ is

$$
V(h)_{Q_{m}}=\left(\frac{\partial h}{\partial Q_{m}}{ }^{2} V\left(Q_{m}\right)=\left[-\frac{Q_{F}}{A\left(T_{f}^{-T_{W}}\right)}\right]^{2} V\left(Q_{m}\right)\right.
$$

IS

$10:$

$m_{n}$
If $A, W$, and $c_{W}$ are assumed free of measurement errors and $Q_{F} \neq 0$, then the overall variance of $h$ is given by

$$
\begin{aligned}
V(h)= & V(h)_{T_{i}}+V(h)_{T_{0}}+V(h)_{T_{W}}+V(h)_{Z}+V(h)_{Q_{m}} \\
= & \left.\frac{V\left(T_{i}\right)+V\left(T_{0}\right)}{4}+V\left(T_{W}\right)\right]\left[\frac{W c_{W} Z(m)-Q_{F} Q_{m}}{A\left(T_{f}-T_{W}\right)^{2}}\right]^{2} \\
& +V(z)\left[\frac{W c_{W}}{A\left(T_{f}-T_{W}\right)}\right]^{2} \\
& +V\left(Q_{m}\right) \frac{Q_{F}}{A\left(T_{f}-T_{W}\right)}
\end{aligned}
$$


and if $Q_{F}=0$, then Equation $D-22$ reduces to

$$
V(h)=\frac{V\left(T_{i}\right)+V\left(T_{0}\right)+4 V\left(T_{w}\right) h_{1}{ }^{2}}{4\left(T_{f}-T_{w}\right)^{2}}+\left[\frac{W c_{w}}{A\left(T_{f}-T_{w}\right)}\right]^{2} V(z)
$$

If $V\left(T_{i}\right)=V\left(T_{O}\right)$, then for $Q_{F} \neq 0$

$$
\mathrm{V}(\mathrm{h})_{\mathrm{T}_{i}}+\mathrm{V}(\mathrm{h})_{\mathrm{T}_{0}}=\frac{\mathrm{V}\left(\mathrm{T}_{\mathrm{O}}\right)}{2}\left[\frac{\mathrm{Wc} \mathrm{W}(\mathrm{m})-\mathrm{Q}_{\mathrm{F}} \mathrm{Q}_{\mathrm{m}}}{\mathrm{A}\left(\mathrm{T}_{\mathrm{f}}-\mathrm{T}_{\mathrm{W}}\right)^{2}}\right]^{2}
$$

and if $Q_{F}=0$

$$
V(h)_{T_{i}}+V(h)_{T_{0}} \quad=\frac{h_{1}^{2} V\left(T_{0}\right)}{2\left(T_{f}-T_{W}\right)^{2}}
$$

Thus, if $V\left(T_{i}\right)=V\left(T_{0}\right)$ and $Q_{F} \neq 0$, then Equation $D-22$ becomes

$$
\begin{aligned}
V(h)= & {\left[\frac{V\left(T_{o}\right)}{2}+V\left(T_{W}\right)\right]\left[\frac{W c_{W} Z(m)-Q_{F} Q_{m}}{A\left(T_{f}-T_{W}\right)^{2}}\right]^{2} } \\
& +V(z)\left[\frac{W c_{W}}{A\left(T_{f}-T_{W}\right)}\right]^{2}+V\left(Q_{m}\right)\left[\frac{Q_{F}}{A\left(T_{f}-T_{W}\right)}\right]^{2}
\end{aligned}
$$

and if $\mathrm{V}\left(\mathrm{T}_{1}\right)=\mathrm{V}\left(\mathrm{T}_{0}\right)$ with $Q_{\mathrm{F}}=0$, then Equation $\mathrm{D}-23$ becomes

$$
\mathrm{V}(\mathrm{h})=\frac{\left[\mathrm{V}\left(\mathrm{T}_{0}\right)+2 \mathrm{~V}\left(\mathrm{~T}_{\mathrm{w}}\right)\right] \mathrm{h}_{l}^{2}}{2\left(\mathrm{~T}_{\mathrm{f}}-\mathrm{T}_{\mathrm{w}}\right)^{2}}+\mathrm{V}(\mathrm{z})\left[\frac{\mathrm{Wc}}{\mathrm{A}\left(\mathrm{T}_{\mathrm{f}}-\mathrm{T}_{\mathrm{w}}\right)}\right]^{2}
$$

We now consider the approximate variance of $h$ when $Q$ is defined as

$$
Q=\dot{W} c_{p}\left(T_{0}-T_{i}\right)
$$

so that Equation D-13 is applicable. The contribution to the approximate variance of $h$ due to the variance to $T_{i}$ is given by 
$\mathrm{RN}-\mathrm{S}-0146$

$$
V(h)_{T_{i}}=\frac{\partial h}{\partial T_{i}} \quad V\left(T_{i}\right)=\left\{\frac{\dot{w} c_{p}}{A}\left[\frac{-\left(T_{w}-T_{f}\right)+\frac{1}{2}\left(T_{0}-T_{i}\right)}{\left(T_{w}-T_{f}\right)^{2}}\right]^{2} V\left(T_{i}\right)\right.
$$

Equation D-29 may be reduced to

$$
V(h)_{T_{i}}=V\left(T_{i}\right) h^{2} \quad \frac{-1}{\left(T_{0}-T_{i}\right)}+\frac{1}{2\left(T_{w}-T_{f}\right)} !^{\prime 2}
$$

In a similar fashion, for $\mathrm{T}_{\mathrm{O}}$

$$
V(h)_{T_{0}}=\left.i \frac{\partial h}{\partial T_{0}}\right|^{2} V\left(T_{0}\right)=\left\{\frac{\dot{w} c_{p}}{A} \frac{\left(T_{w}-T_{f}\right)+\frac{1}{2}\left(T_{0}-T_{i}\right)}{\left(T_{w}-T_{f}\right)^{2}}\right\}^{2} V\left(T_{0}\right)
$$

Equation D-3I may be reduced to

$$
V(h)_{T_{0}}=V\left(T_{0}\right) h^{2}\left[\frac{1}{\left(T_{0}-T_{i}\right)}+\frac{1}{2\left(T_{w}-T_{f}\right)}\right]^{2}
$$

The contribution to the approximate variance of' $h$ due to $\dot{w}$ is given by

$$
V(h)_{\dot{w}}=\left.\frac{\partial h}{\partial \dot{w}}\right|^{2} V(\dot{w})=i_{\frac{c_{p}\left(T_{0}-T_{i}\right)}{A\left(T_{W}-T_{f}\right)}}^{2} V(\dot{w})
$$

The variance $\mathrm{V}(\dot{\mathrm{w}})$ will be estimated by sample variance $\mathrm{S}(\dot{\mathrm{w}})$ so that Equation D-33 becomes

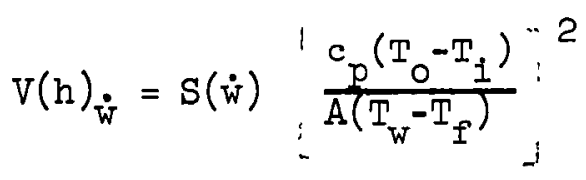

The contribution to the approximate variance of $h$ due to $T_{W}$ is given by

$$
V(h)_{T_{W}}=\left(\frac{\partial h}{\partial T_{W}}\right)^{2} V\left(T_{w}\right)=\left[\frac{\dot{w} c_{p}\left(T_{O}-T_{i}\right)}{A\left(T_{W}-T_{f}\right)^{2}}\right]^{2} V\left(T_{W}\right)
$$


Thus, assuming $c_{p}$ and $A$ are free of measurement errors, the overall variance of $h$, as defined by Equation D-13 is given by

$$
\begin{aligned}
V(h)= & V(h)_{T_{i}}+V(h)_{T_{0}}+V(h)_{\dot{w}}+V(h)_{T_{W}} \\
= & \left.V\left(T_{i}\right) h^{2}\left[\frac{-1}{\left(T_{0}-T_{i}\right)}+\frac{1}{2\left(T_{W}-T_{f}\right)}\right]^{2}+V\left(T_{0}\right) h^{2} \frac{1}{\left(T_{0}-T_{i}\right)}+\frac{1}{2\left(T_{W}-T_{f}\right)}\right]^{2} \\
& +S(\dot{w})\left[\frac{c_{p}\left(T_{0}-T_{f}\right)}{A\left(T_{W}-T_{f}\right)}\right]^{2}+\frac{V\left(T_{W}\right) h^{2}}{\left(T_{W}-T_{f}\right)^{2}}
\end{aligned}
$$

If $V\left(T_{i}\right)=V\left(T_{0}\right)$, then Equation $D-36$ may be written as

$$
V(h)=V\left(T_{0}\right) h^{2}\left[\frac{2}{\left(T_{0}-T_{i}\right)^{2}}+\frac{1}{2\left(T_{w}-T_{f}\right)^{2}}\right]+\frac{S(\dot{w}) h^{2}}{(\dot{w})^{2}}+\frac{V\left(T_{w}\right) h^{2}}{\left(T_{w}-T_{f}\right)^{2}}
$$

Since $V(h)$, as given by Equation $D-37$, is a function of the sample data it would be rather difficult to assess the relative merits of the two $V(h)$ a priori.

II. EFFECTS OF METHODS OF EVALUATING THE RATE OF TFMPFRATURE CHANGES

In the derivation of the variance for $\dot{w}$ and $h$ it was stated that the derivative $\mathrm{dT}_{\mathrm{w}} / \mathrm{dt}$ would be evaluated from sample data. Throughout the derivation this derivative was treated as some unspecified function of the measurements, $z(m)$, with expectation $E(Z)$ and variance $V(Z)$. This section will consider the practical evaluation of $\mathrm{dT}_{\mathrm{w}} / \mathrm{d} t$.

Given a discrete set of values, assumed to represent a continuous process, there are essentially two different methods avallable for approximating the slope of a line tangent to the sequence: (a) the two-point slope formula in which the slope of the line between two observations is determined; and ( $b$ ) the fitting of a polynomial to the observations by the method of least squares and evaluating the derivative of this polynomial at the time point of interest. 
A. THE TWO-POINT SLOPE METHOD

Consider the ratio, for $j-k$,

$$
\frac{T_{w j}-T_{w k}}{t_{j}-t_{k}}=\frac{\Delta T_{w}}{\Delta t}
$$

where

$$
\mathrm{T}_{\mathrm{wj}}=\mathrm{N}^{-1} \sum_{n=1}^{\mathrm{N}} \mathrm{T}_{\mathrm{wjn}}
$$

Let $\mathrm{N}$ be the number of measurements taken at wall temperature $T_{w}$ at times $j$ and $k$, and

$$
\mathrm{T}_{\mathrm{wk}}=\mathrm{N}^{-1} \sum_{\mathrm{n}=1}^{\mathrm{N}} \mathrm{T}_{\mathrm{wkn}}
$$

Equation D-38 gives the slope of the line determined by the points $\left(t_{j}, T_{w j}\right)$ and $\left(t_{k}, T_{w k}\right)$. Thus, at any point $t_{0}\left(\right.$ for $\left.t_{j}<t_{0}<t_{k}\right)$, Equation D-38 gives the rate of change along this line. Using this approximation to $\mathrm{dT}_{\mathrm{W}} / \mathrm{dt}$, Equation $\mathrm{D}-3$ becomes

$$
\dot{w}=\frac{W c_{W}}{c_{p}}\left[\frac{\left(T_{w j}-T_{W k}\right)}{\left(t_{j}-t_{k}\right)\left(T_{i}-T_{0}\right)}\right]-\frac{Q_{F} Q_{m}}{c_{p}\left(T_{i}-T_{0}\right)}
$$

The contribution to the approximate variance of $\dot{\mathrm{w}}$ due to $V\left(\mathrm{~T}_{\mathrm{wj}}\right)$ is given by

$$
V(\dot{w})_{T_{w j}}=\left(\frac{\partial \dot{w}}{\partial T_{w j}}\right)^{2} V\left(T_{w j}\right)=\left[\frac{W c_{W}}{c_{p}\left(t_{j}-t_{k}\right)\left(T_{i}-T_{0}\right)}\right]^{2} V\left(T_{w j}\right)
$$

and similarly for $\mathrm{T}_{\mathrm{wk}}$

$$
\mathrm{V}(\dot{\mathrm{w}})_{\mathrm{T}_{\mathrm{wk}}}={\frac{\partial \dot{\mathrm{w}}}{\partial \mathrm{T}_{\mathrm{wk}}}}^{2} \mathrm{~V}\left(\mathrm{~T}_{\mathrm{wk}}\right)=i_{-\mathrm{c}_{\mathrm{w}}\left(\mathrm{t}_{j}-\mathrm{t}_{\mathrm{k}}\right)\left(\mathrm{T}_{i}-\mathrm{T}_{0}\right)}^{2} \mathrm{~V}\left(\mathrm{~T}_{\mathrm{wk}}\right)
$$

Assuming that each of the $T_{w j n}$ are independently distributed with equal variances, 


$$
\mathrm{V}\left(\mathrm{T}_{\mathrm{wj}}\right)=\mathrm{N}^{-2} \sum_{\mathrm{n}=1}^{\mathbb{N}} \mathrm{V}\left(\mathrm{T}_{\mathrm{wjn}}\right)=\mathrm{N}^{-2}\left[\mathrm{NV}\left(\mathrm{T}_{\mathrm{wjn}}\right)\right]=\mathrm{N}^{-1}\left[\mathrm{~V}\left(\mathrm{~T}_{\mathrm{wjn}}\right)\right]
$$

and similarly for the $\mathrm{T}_{\mathrm{wkn}}$

$$
\mathrm{V}\left(\mathrm{T}_{\mathrm{wk}}\right)=\mathrm{N}^{-1} \mathrm{~V}\left(\mathrm{~T}_{\mathrm{wkn}}\right)
$$

Thus, using the two-point slope method, the overall variance of $\dot{w}$ is given by

$$
\begin{aligned}
& \mathrm{V}(\dot{\mathrm{w}})=\mathrm{V}(\dot{\mathrm{w}})_{\mathrm{T}_{\mathrm{wj}}}+\mathrm{V}(\dot{\mathrm{w}})_{\mathrm{T}_{\mathrm{wk}}}+\mathrm{V}(\dot{\mathrm{w}})_{\mathrm{T}_{\mathrm{o}}}+\mathrm{V}(\dot{\mathrm{w}})_{\mathrm{T}_{i}}+\mathrm{V}(\dot{\mathrm{w}})_{\mathrm{Q}_{\mathrm{m}}} \\
& \left.\left.V(\dot{w})=\frac{V\left(T_{w j n}\right)+V\left(T_{w k n}\right)}{\mathbb{N}}\right] \frac{W c_{w}}{\left[c_{p}\left(t_{j}-t_{k}\right)\left(T_{i}-T_{0}\right)\right.}\right]^{2} \\
& +\left[V\left(T_{0}\right)+V\left(T_{i}\right) \mid \frac{W_{w}\left(T_{w j}-T_{w k}\right)}{c_{p}\left(t_{j}-t_{k}\right)\left(T_{i}-T_{0}\right)^{2}}-\frac{Q_{F} Q_{m}}{c_{p}\left(T_{i}-T_{0}\right)^{2}}\right\rfloor^{2} \\
& +V\left(Q_{m}\right)\left[\frac{Q_{F}}{c_{p}\left(T_{i}-T_{o}\right)}\right]^{2}
\end{aligned}
$$

If $Q_{F}=0$,

$$
\begin{aligned}
& V(\dot{w})=\left.\frac{V\left(T_{w j n}\right)+V\left(T_{w k n}\right)}{N}: \frac{W c_{w}}{c_{p}\left(t_{j}-t_{k}\right)\left(T_{i}-T_{0}\right)}\right)^{2} \\
&+\mid V\left(T_{0}\right)+V\left(T_{i}\right) \\
& \frac{W c_{W}\left(T_{w j}-T_{w k}\right)}{c_{p}\left(t_{j}-t_{k}\right)\left(T_{i}-T_{0}\right)^{2}}
\end{aligned}
$$

Using the slope approximation, the average heat-transfer coefficient becomes

$$
h=\frac{W c_{W}\left(T_{w j}-T_{w k}\right)}{A\left(t_{j}-t_{k}\right)\left(T_{f}-T_{W O}\right)}-\frac{Q_{F} Q_{m}}{A\left(T_{f}-T_{W O}\right)}
$$

where $T_{\text {wo }}$ is defined for the time point to $\left(t_{j} t_{0} t_{k}\right)$ 
to be

$$
\mathrm{T}_{\text {wo }}=\mathrm{N}^{-1}\left\llcorner\mathrm{~T}_{\text {won }}\right.
$$

The variance of $\mathrm{T}_{\mathrm{wo}}$ is, then,

$$
\mathrm{V}\left(\mathrm{T}_{\mathrm{wO}}\right)=\mathrm{N}^{-1} \mathrm{~V}\left(\mathrm{~T}_{\mathrm{wOn}}\right)
$$

The contribution to the approximate variance of $h$ due to $V\left(T_{w O}\right)$ is given by

$$
\begin{aligned}
& \mathrm{V}(\mathrm{h})_{\mathrm{T}_{\text {wo }}}=\left(\left.\frac{\partial \mathrm{h}}{\partial \mathrm{T}_{\text {wO }}}\right|^{2} \mathrm{~V}\left(\mathrm{~T}_{\text {wo }}\right)\right. \\
& V(h)_{T_{\text {wo }}}=\frac{V\left(T_{w o n}\right)}{N}\left[\frac{W_{w}\left(T_{w j}-T_{w k}\right)}{A\left(t_{j}-t_{k}\right)\left(T_{f}-T_{w O}\right)^{2}}-\frac{Q_{F} Q_{m}}{A\left(T_{f}-T_{w o}\right)^{2}}\right]^{2}
\end{aligned}
$$

The variance contribution from $V\left(T_{w j}\right)$ is

$$
V(h)_{T_{w j}}=\left(\frac{\partial h}{\partial T_{w j}}\right)^{2} V\left(T_{w j}\right)=\frac{V\left(T_{w j n}\right)}{N}\left[\frac{W c_{w}}{A\left(t_{j}-t_{k}\right)\left(T_{f}-T_{w O}\right)}\right]^{2}
$$

and similarly for $\mathrm{T}_{\mathrm{wk}}$

$$
V(h)_{T_{w k}}=\left[\frac{\partial h}{\partial T_{w k}}{ }^{1} \quad V\left(T_{w k}\right)=\frac{V\left(T_{w k n}\right)}{N}\left[\frac{W c}{A\left(t_{j}-t_{k}\right)\left(T_{f}-T_{w O}\right)}\right]^{2}\right.
$$

Thus, using the slope approximation with

$$
\begin{aligned}
& \mathrm{V}(\mathrm{h})_{\mathrm{T}_{0}} \neq \mathrm{V}(\mathrm{h})_{\mathrm{T}_{i}} \text {, and } Q_{\mathrm{F}} \neq 0 \\
& V(h)=V(h)_{T_{0}}+V(h)_{T_{i}}+V(h)_{T_{w j}}+V(h)_{T_{w k}}+V(h)_{T_{w o}}+V(h)_{Q_{m}} \\
& V(h)=\left[\frac{V\left(T_{w o n}\right)}{N}+\frac{V\left(T_{0}\right)+V\left(T_{i}\right)}{4}\right]\left[\frac{W c_{w}\left(T_{w j}-T_{w k}\right)}{A\left(t_{j}-t_{k}\right)\left(T_{f}-T_{w o}\right)^{2}}-\frac{Q_{F} Q_{m}}{A\left(T_{f}-T_{w o}\right)^{2}}\right]^{2} \\
& +\left[\frac{V\left(T_{w j n}\right)+V\left(T_{w k n}\right)}{N}\right]\left[\frac{W c_{w}}{A\left(t_{j}-t_{k}\right)\left(T_{f}-T_{w O}\right)}\right]^{2}+V\left(Q_{m}\right)\left[\frac{Q_{F}}{A\left(T_{f}-T_{w O}\right)}\right]^{2}
\end{aligned}
$$


If $Q_{F}=0$, Equation $D-53$ becomes

$$
\begin{aligned}
V(h)= & \left.\frac{V(T)}{N}+\frac{V\left(T_{0}\right)+V\left(T_{i}\right)}{4} \mid \frac{W c_{w}\left(T_{w j}-T_{w k}\right)}{A\left(t_{j}-t_{k}\right)\left(T_{f}-T_{w O}\right)^{2}}\right]^{2} \\
& \left.\left.+\frac{V\left(T_{w j n}\right)+V\left(T_{w k n}\right)}{N}\right\rfloor \mid \frac{W c_{w}}{A\left(t_{j}-t_{k}\right)\left(T_{f}-T_{w O}\right)}\right]^{2}
\end{aligned}
$$

For $V(h)_{T_{0}}=V(h)_{T_{i}}$ and $Q_{F} \neq 0$,

$$
\begin{aligned}
& \left.V(h)=\left[\frac{V\left(T_{w O n}\right)}{N}+\frac{V\left(T_{o}\right)}{2}\right] \frac{W c_{w}\left(T_{w j}-T_{w k}\right)}{A\left(t_{j}-t_{k}\right)\left(T_{f}-T_{w O}\right)^{2}}-\frac{Q_{F} Q_{m}}{A\left(T_{f}-T_{w O}\right)^{2}}\right]^{2} \\
& \left.\left[+\frac{V\left(T_{w j n}\right)+V\left(T_{w k n}\right)}{N}\right] \frac{W c_{w}}{A\left(t_{j}-t_{k}\right)\left(T_{f}-T_{w O}\right)}\right]^{2}+V\left(Q_{m}\right)\left(\frac{Q_{F}}{A\left(T_{f}-T_{w O}\right)}\right)^{-2}
\end{aligned}
$$

If $V(h)_{T_{0}}=V(h)_{T_{i}}$, and $Q_{F}=0$,

$$
\begin{aligned}
V(h)= & \left.\frac{V\left(T_{w o n}\right)}{N}+\frac{V\left(T_{o}\right)}{2}: \frac{W c_{w}\left(T_{w j}-T_{w k}\right)}{A\left(t_{j}-t_{k}\right)\left(T_{w o}-T_{f}\right)^{2}}\right]^{2} \\
& +\frac{\left(v\left(T_{w j n}\right)+V\left(T_{w k n}\right)\right.}{N}\left[\frac{W c_{w}}{A\left(t_{j}-t_{k}\right)\left(T_{f}-T_{w 0}\right)}\right]^{2}
\end{aligned}
$$

\section{B. THE LEAST-SQUARES METHOD}

In the least-squares method, wall temperature measurements as observed by $\mathbb{N}$ thermocouples at various times $t_{j}(j=1, \ldots, J)$ will again be considered. Let $T_{w j n}$ denote the wall temperature as measured by thermocouple, $n$, at time $t_{j}$. The equations

$$
\mathrm{T}_{w j}=N^{-1} \sum_{n=1}^{N} T_{w j n}
$$


and

$$
\bar{t}=J^{-1} j_{j=1}^{J} t_{j}
$$

define $\mathrm{T}_{\mathrm{wj}}$ and $\overline{\mathrm{t}}$.

To obtain an estimate of $\mathrm{dT}_{\mathrm{W}} / \mathrm{dt}$, a second-degree polynomial

$$
\mathrm{T}_{\mathrm{w}}=\mathrm{a}+\mathrm{b}(\mathrm{t}-\overline{\mathrm{t}})+\mathrm{c}(\mathrm{t}-\overline{\mathrm{t}})^{2}
$$

will be fitted to $m$ successive values of the $T_{w j}$ by the method of least squares. The slope of the line tangent to $T_{W}$ at any point, $t_{0}$, interior to the span of m-fitted values is then given by

$$
\begin{aligned}
& \left.\frac{d T}{d t}\right|_{t=t_{0}}=b+2 c\left(t_{0}-\bar{t}\right) \text { for }\left(t_{1}-t_{0}-t_{m}\right) \\
& \text { The variance of } \frac{d T_{w}}{d t} \text {, is } \\
& V\left|\frac{d T_{W}}{d t}\right|=V(b)+4\left(t_{0}-\bar{t}\right)^{2} V(c)
\end{aligned}
$$

and, if Equation $D-6 I$ is evaluated at $t_{0}=\bar{t}$,

$$
v: \frac{d T}{d t}=V(b)
$$

It is well known that

$$
V(b)=\frac{\sigma_{e}^{2}}{c_{i i}}
$$

where $\sigma_{e}^{2}$ is the variance of the residuals between the data points and curve, and $c_{i i}$ is a diagonal element of the variance-covariance matrix. If orthogonal polynomials are used, the values of $c_{i i}$ may be obtained from The Biometrika Tables For Statisticians (see Reference 31 of the text). The following short table gives values of the $c_{i i}$ for selected values of $n$. 


\begin{tabular}{|c|c|c|c|}
\hline $\mathrm{n}$ & $c_{i i}$ & $\mathrm{n}$ & ${ }^{c}{ }_{i i}$ \\
\hline 11 & 110 & 31 & 2480 \\
\hline 13 & 182 & 41 & 5740 \\
\hline 23 & 1012 & 43 & 6622 \\
\hline 27 & 1638 & 51 & 11050 \\
\hline 29 & 2030 & & \\
\hline
\end{tabular}

Thus, at any point $t_{0}\left(t_{1}-t_{0}-t_{m}\right)$, Equation D-60 yields the rate of change along the curve $T_{W}$. Using the least-squares approximation, Equation $D-3$ becomes

$$
\dot{w}=\frac{W c_{W}\left[b+2 c\left(t_{0}-\bar{t}\right)\right.}{c_{p}\left(T_{i}-T_{0}\right)}-\frac{Q_{F} Q_{m}}{c_{p}\left(T_{i}-T_{0}\right)}
$$

given by

The contribution to the approximate variance of $\dot{w}$ due to $v\left(\frac{d T_{\mathrm{w}}}{d t}\right)$ is

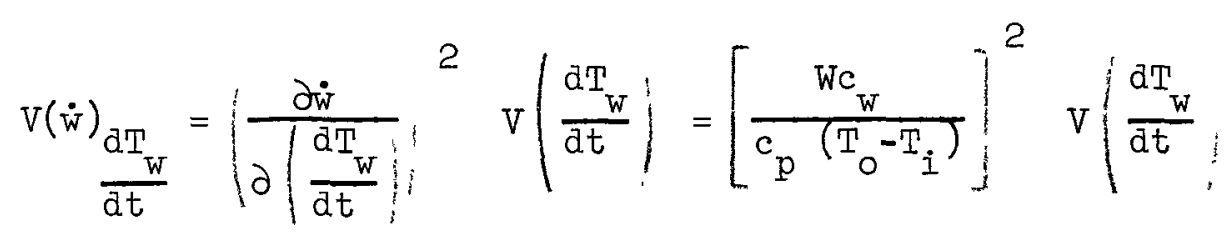

and at $t_{0}=\bar{t}$, Equation $D-65$ becomes

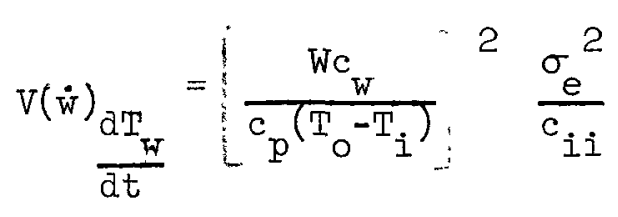

Thus, using the least-squares method, the overall variance of $\dot{w}$ is given by

$$
\begin{aligned}
& \mathrm{V}(\dot{\mathrm{w}})=\mathrm{V( \dot { \textrm {w } } ) _ { \frac { \mathrm { dT } } { \mathrm { w } } }}+\mathrm{V( \dot { \textrm {w } } ) _ { \mathrm { T } _ { \mathrm { O } } }}+\mathrm{V( \dot { \textrm {w } } ) _ { \mathrm { T } _ { i } }}+\mathrm{V( \dot { \textrm {w } } )} \mathrm{Q}_{\mathrm{m}}
\end{aligned}
$$

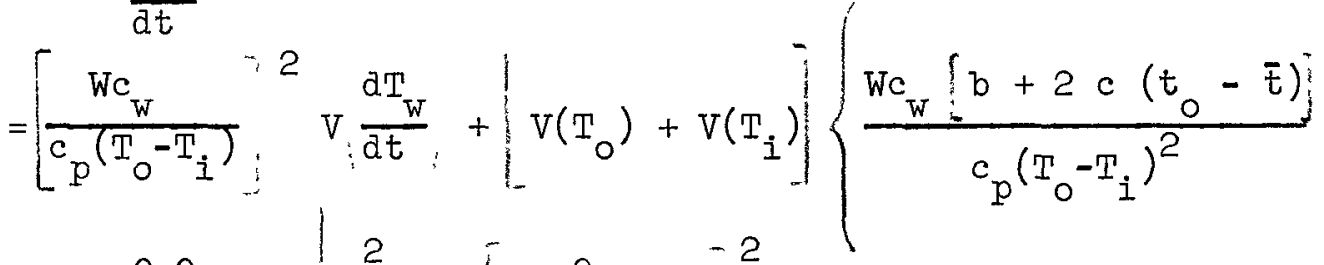

$$
\begin{aligned}
& \left.-\frac{Q_{F} Q_{m}}{c_{p}\left(T_{0}-T_{i}\right)^{2}}\right\}^{2}+\frac{Q_{F}}{c_{p}\left(T_{0}-T_{i}\right)} V^{-2}\left(Q_{m}\right)
\end{aligned}
$$


If $Q_{F}=0$ and $t_{0}=\bar{t}$, Equation $D-67$ reduces to

$$
V(\dot{w})=\left[\frac{W c_{w}}{c_{p}\left(T_{0}-T_{i}\right)}\right]^{2} \frac{\sigma_{e}^{2}}{c_{i i}}+\left[\frac{W c_{w} b}{c_{p}\left(T_{0}-T_{i}\right)^{2}}\right]^{2}\left[V\left(T_{0}\right)+V\left(T_{i}\right)\right]
$$

With use of the least-squares method, the average heat-transfer coefficient becomes

$$
h=\frac{W c_{W}\left[b+2 c\left(t_{o}-\bar{t}\right)\right]}{A\left(T_{f}-T_{W O}\right)}-\frac{Q_{F} Q_{m}}{A\left(T_{f}-T_{W O}\right)}
$$

where $T_{\text {wo }}$ is again obtained directly from data and is defined for the time point, $t_{0}$, to be

$$
\mathrm{T}_{\text {wO }}=\mathrm{N}^{-1} \sum^{-} \mathrm{T}_{\text {won }}
$$

As before, the variance of $\mathrm{T}_{\text {wo }}$ is given (see Equation D-49) by

$$
\mathrm{V}\left(\mathrm{T}_{\text {wo }}\right)=\mathrm{N}^{-1} \mathrm{~V}\left(\mathrm{~T}_{\text {won }}\right)
$$

The contribution to the approximate variance of $h$ due to $V\left(d T_{w} / d t\right)$ is given by

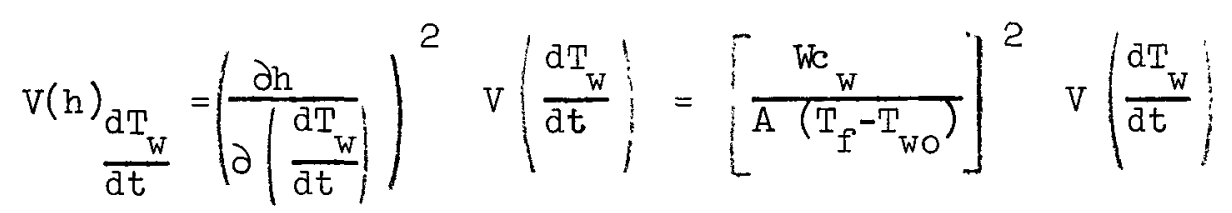

If $t_{0}=\bar{t}$, Equation $D-72$ reduces to

$$
V(h)_{\frac{d T_{w}}{d t}}=\left[\frac{W c_{w}}{A\left(T_{f}-T_{w O}\right)}\right]^{2} \frac{\sigma_{e}^{2}}{c_{i i}}
$$


The variance contribution from $\mathrm{V}\left(\mathrm{T}_{\text {wo }}\right)$ is

$$
V(h)_{T_{\text {WO }}}=V\left(T_{\text {wo }}\right)\left|\frac{\partial h}{\partial T_{\text {WO }}}\right|^{2}=\frac{V\left(T_{W O n}\right.}{N}\left\{\frac{W c_{W}\left[\mathrm{~b}+2 c\left(t_{O}-\bar{t}\right)\right]}{A\left(T_{f}-T_{W O}\right)^{2}}-\frac{Q_{F} Q_{m}}{A\left(T_{f}-T_{W O}\right)^{2}}\right\}^{2}
$$

Thus, using the polynomial approximation with $\mathrm{V}(\mathrm{h})_{\mathrm{T}_{\mathrm{O}}} \neq \mathrm{V}(\mathrm{h})_{\mathrm{T}_{\mathrm{i}}}$ and $\mathrm{Q}_{\mathrm{F}} \neq 0$,

$$
\begin{aligned}
& V(h)=V(h)_{\frac{d T}{d t}}+V(h)_{T_{W O}}+V(h)_{T_{0}}+V(h)_{T_{i}}+V(h)_{Q_{m}} \\
& \left.=\frac{\mathrm{Wc}}{\mathrm{A}\left(\mathrm{T}_{\mathrm{f}}-\mathrm{T}_{\mathrm{WO}}\right)^{2}}\right)^{2} \mathrm{~V}\left|\frac{\mathrm{dT}}{\mathrm{dt}}\right| \\
& \left.+\left[\frac{V\left(T_{\text {Won }}\right)}{N}+\frac{V\left(T_{0}\right)+V\left(T_{i}\right)}{4}\right] \frac{W c_{W}\left[b+2 c\left(t_{o}-\bar{t}\right)\right.}{A\left(T_{f}-T_{W O}\right)^{2}}-\frac{Q_{F} Q_{m}}{A\left(T_{f}-T_{W O}\right)^{2}}\right]^{2} \\
& +V\left(Q_{m}\right)\left[\frac{Q_{F}}{A\left(T_{f}-T_{W O}\right)}\right]^{2}
\end{aligned}
$$

If $V(h)_{T_{O}} \neq V(h)_{T_{i}}$ and $Q_{F}=0$, Equation $D-75$ becomes

$$
\begin{aligned}
& V(h)=\left[\frac{\mathrm{Wc}_{\mathrm{w}}}{\mathrm{A}\left(\mathrm{T}_{\mathrm{f}}-\mathrm{T}_{\mathrm{wO}}\right)}\right]^{2} \mathrm{~V}\left(\frac{\mathrm{dT}}{\mathrm{dt}}\right) \\
& +\frac{V\left(T_{\text {won }}\right)}{N}+\frac{V\left(T_{0}\right)+V\left(T_{i}\right) !}{4} \cdot \frac{W_{W}\left[b+2 c\left(t_{o}-\bar{t}\right)\right\}^{-2}}{A\left(T_{f}-T_{W O}\right)^{2}}
\end{aligned}
$$

If $V(h)_{T_{O}}=V(h)_{T_{i}}$ and $Q_{F} \neq 0$, Equation $D-75$ becomes 
$\mathrm{RN}-\mathrm{S}-0146$

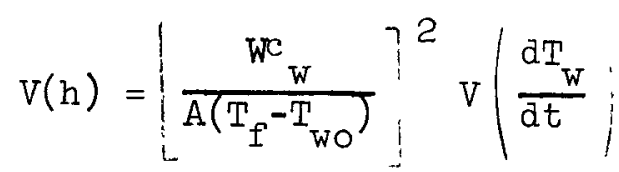

$$
\begin{aligned}
& \left.+\frac{i V\left(T_{w o n}\right)}{N}+\frac{V\left(T_{0}\right)}{2}\right]\left[\frac{w_{W}\left[b+2 c\left(t_{0}-\bar{t}\right)\right]}{A\left(T_{f}-T_{w O}\right)^{2}}-\frac{Q_{F} Q_{m}}{A\left(T_{f}-T_{w O}\right)^{2}}\right]^{2} \\
& +V\left(Q_{m}\right)\left[\frac{Q_{F}}{A\left(T_{f}-T_{W O}\right)}\right]^{2}
\end{aligned}
$$

If $V(h)_{T_{0}}=V(h)_{T_{i}}$ and $Q_{F}=0$, Equation $D-77$ reduces to

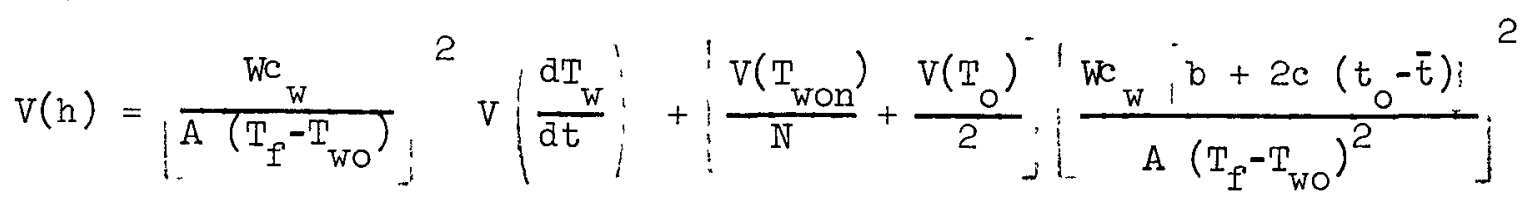

If $t_{0}=\bar{t}$, Equation $D-75$ becomes

$$
\begin{aligned}
V(h)= & \left.\frac{W c_{w}}{A\left(T_{f}-T_{w O}\right)} \frac{\sigma_{e}^{2}}{c_{i i}}+\frac{V\left(T_{w O n}\right)}{N}+\frac{V\left(T_{0}\right)+V\left(T_{i}\right)}{4}\right] \frac{W c_{w} b}{A\left(T_{f}-T_{w O}\right)^{2}} \\
& -\frac{Q_{F} Q_{m}}{A\left(T_{f}-T_{W O}\right)^{2}}: \\
& =2
\end{aligned}
$$

Similarly, if $t_{0}=\bar{t}$, Equation $D-76$ becomes

$$
V(h)=\frac{W c_{w}}{A\left(T_{f}-T_{w O}\right)} \frac{\sigma_{e}^{2}}{c_{i i}}+\frac{V\left(T_{w o n}\right)}{N}+\frac{V\left(T_{0}\right)+V\left(T_{i}\right)}{4} \frac{W c_{w} b}{A\left(T_{f}-T_{w o}\right)^{2}}
$$

and, if $t_{0}=\bar{t}$, Ecuation $\mathrm{D}-77$ becomes

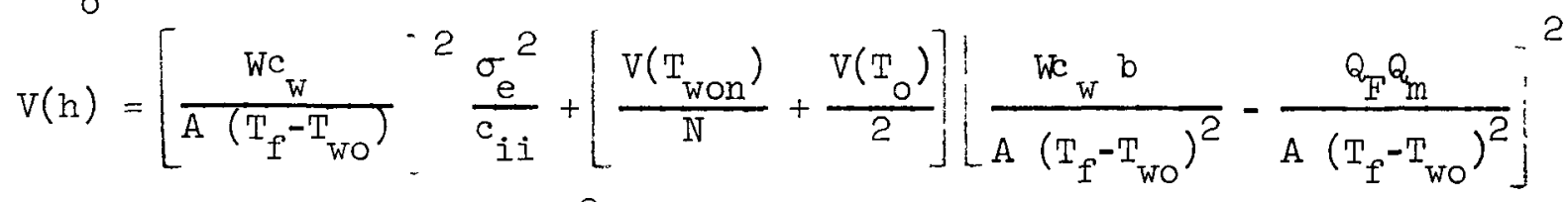

$$
\begin{aligned}
& +V\left(Q_{m}\right): \frac{Q_{F}}{A\left(T_{f}-T_{W O}\right)}
\end{aligned}
$$


and similarly Equation D-78 becomes

$$
V(h)=\left[\frac{W_{w}}{A\left(T_{f}-T_{w O}\right)} j^{2} \frac{\sigma_{e}^{2}}{c_{i i}}+\frac{V\left(T_{w o n}\right)}{N}+\frac{V\left(T_{o}\right)}{2}: \frac{W c_{w} b}{A\left(T_{f}-T_{w O}\right)^{2}}-2\right.
$$

which relates the variance of the temperature measurements with the variance of the calculated heat-transfer coefficient. 